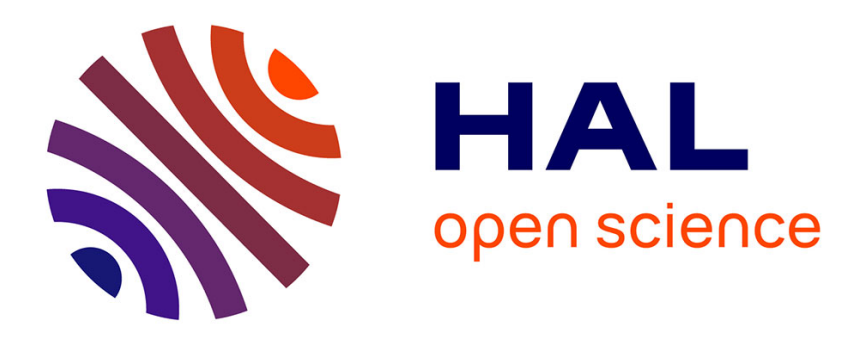

\title{
Study of the mechanical behavior of recycled fibers. Applications to papers and paperboards. Imtiaz Ali
}

\section{To cite this version:}

Imtiaz Ali. Study of the mechanical behavior of recycled fibers. Applications to papers and paperboards.. Other. Université de Grenoble, 2012. English. NNT : 2012GRENI075 . tel-00872112

\section{HAL Id: tel-00872112 \\ https://theses.hal.science/tel-00872112}

Submitted on 11 Oct 2013

HAL is a multi-disciplinary open access archive for the deposit and dissemination of scientific research documents, whether they are published or not. The documents may come from teaching and research institutions in France or abroad, or from public or private research centers.
L'archive ouverte pluridisciplinaire HAL, est destinée au dépôt et à la diffusion de documents scientifiques de niveau recherche, publiés ou non, émanant des établissements d'enseignement et de recherche français ou étrangers, des laboratoires publics ou privés. 


\section{UNIVERSITÉ DE GRENOBLE}

THÈSE

POUR OBTENIR LE GRADE DE

DOCTEUR DE L'UNIVERSITÉ DE GRENOBLE

Spécialité: Mécanique des fluides, Prodcédés, Energétique

Arrêté ministériel : 7 août 2006

Présentée PAR

IMTIAZ ALI

ThÈse dirigée Par Jean-Françis BLOCH et

CODIRIgÉE PAR RAPHAËL PASSAS

PRÉPARÉE AU SEIN DU

Laboratoire de LGP2/Grenoble INP-Pagora/CNRS

DANS L'École Doctorale:I-MEP ${ }^{2}$

\section{Contribution à l'étude du comportement mécanique des fibres recyclées. Applications aux support fibreux.}

ThÈSE SOUtenUe PUbLiQUeMENT Le 28 SEPTEMBRe 2012,

DEVANT LE JURY COMPOSÉ DE:

\section{Christian GEINDREAU}

PR, Université Joseph Fourier (Président)

Mme. Ana Paula COSTa

PR, Université de Beira Interior (Rapporteur)

M. WILliAM SAMPSON

PR, Université de Manchester (Rapporteur)

M. Jean-Françis BLOCH

MCF HDR, Grenoble-INP (Membre)

M. RAPHÄ̈L PASSAS

ING, AGEFPI (Membre) 
Dedicated to my teachers 


\section{Acknowledgements}

I would like to express my sincere gratitude towards Mr. Jean-Francis Bloch and Mr. Raphaël Passas. I feel myself lucky in having the opportunity to collaborate and get benefited from their knowledge. They have been very supportive throughout this work. They have given me the confidence and the ability to always move forward. Ensuring my professional development, they have always listened and given time to blossom my skills freely. I sincerely thank Mrs. Sabine Rolland du Roscoat for her help. Her unwavering commitment impresses me a lot. I would like to thank Mr. Pierre Dumont for his useful suggestions. For the tedious ESEM manipulations, I would like to acknowledge the help of Mrs. Bertine Khelifi. I would also like to thank Mr. Denis Curtil, Mr. Mohamed Aichi, Mr. Stéphane Dufreney, Mr. Marc Butel and Mrs. Cécile Bruzzese for their technical support. I want to thank the friendly support of Cyril, Satyajit, Robin, Eder, Preetha, Praveen, Kumar and Seema. Thanks to the service technique, service informatique, service bibliothèque, service scolarité and service comptabilité for making life easier.

And of course, a very special thank to my family for their support. An affectionate thought for Fatima and Hamza for being faithful despite the distance. Once again, I would like to thank Mr. Raphaël Passas for his pivotal role, endless support, knowledge, vision and to whom I owe more than any body else. 


\begin{abstract}
Incorporation of recycled fibres in high value paper products can reduce cost and environmental loads. Papermaking potential of cellulosic fibres decreases with recycling. The phenomenon of fibre hornification during pressing and drying is normally held responsible for the loss in strength. To study the impacts of recycling on pulp, fibre and paper properties some non conventional characterisation techniques like fibre saturation point, X-rays microtomography, environmental scanning electron microscopic observations, atomic force microscope (PeakForce QNM mode) and inverse size exclusion chromatography (ISEC) were used. In order to achieve good reproducibility of ISEC measurements, a semi-automatic column fabrication pilot system was built. The techniques were first validated on refining process before being applied to the recycling process. In this study, it was found that fibre hornification alone can not fully explain loss in strength during recycling. The loss in strength is much more complex and it is required to understand the morphological and ultra structural changes associated with recycling. Fibre width, cell wall thickness, curl, kink, irregularities decreased during recycling. Fibre became hard and brittle in dry state. Number of weak points in the fibre wall were increased initially and in the later recyclings. The increase in wet breaking length indicates increased surface friction and capillary forces with recycling. Decrease in bonded area during first recycle may be caused by the loss of fines and fibre flexibility whereas the increase afterwards may be linked to the lumen collapse. The strength of fibres did not decrease with recycling as shown by zero-span breaking lengths therefore the quality of bond may be deteriorated. It was thought that the partially delaminated P/S1 layers may be responsible for the loss of paper strength. It is suggested since the significant change is associated with the pressing and drying of never dried pulp therefore the drying process needs to be revisited. The delaminated layer should be restored so as to increase the recyclability of the recovered fibres for high value paper. Influence of recycled pulp blends on physical properties of paper was also studied. It was revealed that small quantity of recycled pulp can be used without significantly affecting the mechanical strength properties.
\end{abstract}

Keywords: recycling, refining, hornification, fibre morphology, shrinkage, Fibre Saturation Point, pore size distribution, weak points 


\section{Contents}

1 Introduction 1

2 Literature review 2

2.1 Forest outlook ....................... 2

2.2 Wood and fibres . . . . . . . . . . . . . . . 4

2.3 Pulp refining . . . . . . . . . . . . . . . 25

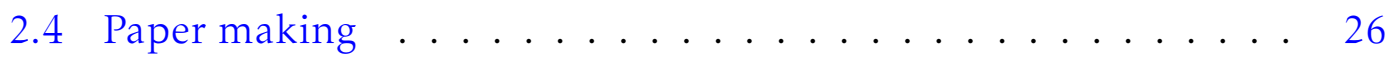

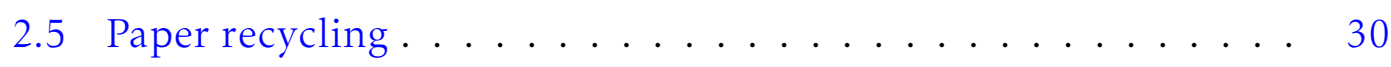

3 Experimental methods 34

3.1 Refined pulps and fibres ............... 35

3.2 Morphological properties of refined pulps and fibres . . . . . . 37

3.3 Dewatering of refined pulps and fibres . . . . . . . . . . . . 43

3.4 Water retention value of refined pulps and fibres . . . . . . . 49

3.5 Fibre saturation point of refined pulps and fibres . . . . . . . 51

3.6 Fibre wall pore size distribution of refined pulps and fibres . . . 56

3.7 Wet breaking length of refined pulps and fibres . . . . . . . . 59

3.8 Weak points in fibre wall of refined pulps and fibres . . . . . . . 61

3.9 Conclusions of pulp refining . . . . . . . . . . . 68

$\begin{array}{lll}4 & \text { Recycled pulps and fibres } & 70\end{array}$

4.1 Chemical properties of recycled pulps and fibres . . . . . . . 72

4.2 Morphological properties of recycled pulps and fibres . . . . . 73

4.3 Surface analysis . . . . . . . . . . . . . . . . 89

4.4 Dewatering of recycled pulps and fibres . . . . . . . . . . . 95

4.5 Specific water retention of recycled pulps and fibres . . . . . . 96

4.6 Hornification of recycled pulps and fibres . . . . . . . . . . 98 
4.7 Pore size distribution of recycled fibres . . . . . . . . . . . 100

4.8 Width shrinkability of recycled fibres . . . . . . . . . . . 102

4.9 Wet breaking length of recycled pulps and fibres . . . . . . . . 103

4.10 Weak points in fibre wall of recycled fibres . . . . . . . . . . . . . 104

4.11 Single fibre tensile properties . . . . . . . . . . . 108

5 Physical Properties 112

5.1 Properties of handsheets made from recycled pulps . . . . . . . . 113

5.2 Properties of handsheets made from recycled pulp blends . . . . 137

6 Conclusions 153

$\begin{array}{lll}7 & \text { Perspectives } & 156\end{array}$

$\begin{array}{lr}\text { A Filtration resistance } & 157\end{array}$

$\begin{array}{ll}\text { B Gel filtration chromatography } & 161\end{array}$

$\begin{array}{ll}\text { C Weak points in fibre wall } & 165\end{array}$

$\begin{array}{lr}\text { D Thermoporosimetry } & 167\end{array}$

$\begin{array}{ll}\text { E Résumé de la thèse en Français' } & 171\end{array}$

$\begin{array}{ll}\text { References } & 214\end{array}$

$\begin{array}{ll}\text { Index } & 238\end{array}$ 


\section{List of Figures}

2.1 Hierarchy of wood structure . . . . . . . . . . . . 5

2.2 Schematic cell wall layers model of a latewood tracheid . . . . 6

2.3 Schematic model of a cellulose microfibril . . . . . . . . . 8

2.4 Distribution of principal chemical constituents in softwood cell wall layers . . . . . . . . . . . . . . 9

2.5 Schematic depiction of fibre shapes during bonding $\ldots \ldots . .11$

2.6 Internal fibrillation of fibre wall as the swelling increases . . . 12

2.7 Dissolution of lignin out of the fibre wall . . . . . . . . . 13

2.8 Fibre length and cell wall thickness in pulpwood logs depending on the location in the stem . . . . . . . . . . . . . . . 14

2.9 Nomenclature of morphological properties . . . . . . . . . . 15

2.10 Principle of solute exclusion technique for the measurement of fibre saturation point . . . . . . . . . . . . . . 19

2.11 Slip plane and compression failure . . . . . . . . 22

2.12 Elastic modulus vs microfibril angle . . . . . . . . . . . . 24

2.13 Shapes of stress-strain curves due to fibre deformations . . . . . 24

2.14 Schematic representation of refining effects on fibre characteristics 25

2.15 Schematic diagram of a Fourdrinier paper machine . . . . . . 26

2.16 Effect of morphological properties on wet webs strength . . . . 27

2.17 Interactions responsible for specific bond strength . . . . . . 28

2.18 Schematic depiction of fibre collapse during drying . . . . . . 28

2.19 Fibre straightening during sheet drying . . . . . . . . . . . . 29

2.20 Structural changes involved in the Jentzen effect at fibre wall level 29

2.21 Evolution of worldwide production of wood pulps . . . . . . 30

2.22 Evolution of recycling rates of Europe and USA . . . . . . . . 32 
3.1 Preparation of refined pulps and fibres . . . . . . . . . 35

3.2 Working principle of PFI mill . . . . . . . . . . . . 36

3.3 Working principle of Valley beater . . . . . . . . . . 37

3.4 Working principle of morphological analyser . . . . . . . . . 38

3.5 Evolution of length-weighted fibre length during refining . . . . 39

3.6 Effect of refining on fibre length distributions . . . . . . . . . 39

3.7 Effect of refining on fibre width distribution . . . . . . . . . . . 40

3.8 Evolution of curl index during refining . . . . . . . . . . . . . . . . 40

3.9 Effect of refining on curl index distribution . . . . . . . . . . . 41

3.10 Evolution of kink number during refining . . . . . . . . . . 41

3.11 Evolution of broken content during refining . . . . . . . . . . . 42

3.12 Evolution of SR during refining . . . . . . . . . . . . . 44

3.13 Evolution of fibrillation index during refining . . . . . . . . . . 45

3.14 Evolution of broken and fines content of pulps during refining . 45

3.15 Schematic diagram of dynamic freeness tester . . . . . . . . . . 46

3.16 Filtration kinetics of refined pulps and fibres . . . . . . . . . . . . . . . . . . . 47

3.17 Filtration kinetics of refined pulps and fibres . . . . . . . . 47

3.18 Evolution of water retention value during refining . . . . . . 50

3.19 Making of the fibrous column for gel filtration chromatographic measurements .................... 52

3.20 Experimental set-up for chromatographic measurements . . . . . 53

3.21 Calibration curve of differential refractive index detector for glucose ............................ 54

3.22 Cumulative moving average fibre saturation point . . . . . . 55

3.23 Evolution of fibre saturation point of pulps during refining . . . 56

3.24 Cumulated apparent pore volume of refined fibres . . . . . . . 58

3.25 Effect of refining on apparent pore size distribution of fibres . . 59

3.26 Wet breaking length measurement device . . . . . . . . . . 60

3.27 Evolution of wet breaking length during refining . . . . . . . . 61

3.28 Measurement of cleavages per fibre during refining . . . . . . . . 62

3.29 Evolution of length-weighted length of cleaved refined fibres . . 62

3.30 Effect of $\mathrm{HCl}$ cleavage on fibre length distributions of unrefined and refined fibres . . . . . . . . . . . . . 63

3.31 Evolution of average width of cleaved refined fibres . . . . . . . . 64

3.32 Effect of $\mathrm{HCl}$ cleavage on width distribution of fibres . . . . . . . 64

3.33 Evolution of curl index of cleaved refined fibres . . . . . . . . 65

3.34 Evolution of fines content of cleaved refined fibres . . . . . . 66 
3.35 Evolution of cleavages per fibre during refining . . . . . . . . 66

3.36 Schematic diagram of probable fibre cell wall transformations during refining by $\mathrm{HCl}$ cleavage . . . . . . . . . . . . . . 68

4.1 Preparation of recycled paper, pulps and fibres . . . . . . . . 71

4.2 Evolution of length-weighted fibre length during recycling . . . 74

4.3 Effect of recycling on fibre length distribution . . . . . . . . . . 74

4.4 Evolution of fibre width during recycling . . . . . . . . . . . . . . . 75

4.5 Effect of recycling on width distributions . . . . . . . . . 76

4.6 Length-width distribution of never dried fibres and ten times recycled fibres . . . . . . . . . . . . . . 76

4.7 Evolution of fines content during recycling . . . . . . . . . . . 77

4.8 Evolution of curl index during recycling . . . . . . . . . . 78

4.9 Effect of recycling on curl index distributions . . . . . . . . . 78

4.10 Evolution of kink number during recycling . . . . . . . . . . 79

4.11 Evolution of thickenings and thinnings per fibre during recycling 79

4.12 Evolution of fibrillation index and fibril length during recycling $\quad 80$

4.13 Operating principle of SEM . . . . . . . . . . . . 81

4.14 SEM micrograph of never dried fibres and ten times recycled fibres 82

4.15 SEM micrograph of earlywood fibre perforations . . . . . . . . 82

4.16 Prepared sample holder for kink analysis . . . . . . . . . . . 83

4.17 ESEM images of once dried, twice and five times rewetted and dried kinked fibre at $5 \mathrm{RH} \ldots \ldots . \ldots . \ldots . . \ldots 84$

4.18 Schematic diagram of curl and kink reduction during recycling . 84

4.19 Working principle of cell wall thickness measurement device . . 85

4.20 Evolution of cell wall thickness and distribution of fibres during recycling ........................ 86

4.21 Prepared sample holder for cross section analysis . . . . . . . . 86

4.22 ESEM cross section images of once and twice air dried fibre . . . 87

4.23 Schematic diagram of fibre cross section and corresponding round attributes ........................ 88

4.24 Schematic diagram of fibre wall shrinkage during recycling . . . 89

4.25 SEM micrographs showing the surface characteristics of fibres during recycling . . . . . . . . . . . . . . . . . 990

4.26 Schematic diagram of probable transformations of fibre cell wall layers and surface during recycling . . . . . . . . . . . 91 
4.27 Schematic diagram of probable stitching and repairing of P/S1 layers by cross-linkers . . . . . . . . . . . . . . . 91

4.28 Schematic diagram of probable peeling off and fibrillation by refining .......................... 92

4.29 Working principle of AFM . . . . . . . . . . . . 93

4.30 Characteristic curve of PeakForce QNM tapping mode . . . . . . 93

4.31 Effect of recycling on fibre surface adhesion . . . . . . . . . . 94

4.32 Schematic diagram of AFM tip interactions with never dried and recycled fibre surface . . . . . . . . . . . . . . 95

4.33 Filtration kinetics of never dried pulp and fibres . . . . . . . 996

4.34 Evolution of specific water retention of pulps during recycling • 97

4.35 Evolution of specific water retention of fibres during recycling . 97

4.36 Evolution of fibre hornification based on water retention value during recycling . . . . . . . . . . . . . . . . . . 98

4.37 Evolution of fibre hornification based on fibre saturation point during recycling . . . . . . . . . . . . . . 100

4.38 Cumulated specific apparent pore volume of recycled fibres . . . 101

4.39 Effect of recycling on apparent pore size distribution of fibres . . 101

4.40 ESEM images of never dried fibre at $85 \mathrm{RH}$ and at $10 \mathrm{RH}$. . . . . 102

4.41 Width shrinkage of recycled fibres . . . . . . . . . . . 103

4.42 Wet breaking length of recycled fibres . . . . . . . . . . . . . 104

4.43 Effect of $\mathrm{HCl}$ cleavage on length distribution of never dried fibres 105

4.44 Evolution of average fibre width of cleaved recycled fibres . . . . 105

4.45 Effect of $\mathrm{HCl}$ cleavage on width distribution of never dried fibres 106

4.46 Cleavages per fibre of fibres during recycling . . . . . . . . 106

4.47 Schematic diagram of probable transformations of fibre cell wall during recycling by $\mathrm{HCl}$ cleavage . . . . . . . . . . . . . 107

4.48 Single fibre tensile test . . . . . . . . . . . . . . . 108

4.49 SEM micrograph of a fibre cross section . . . . . . . . . . . . 109

4.50 Stress-strain curve of air dried never dried and ten times recycled fibres . . . . . . . . . . . . . . . . . 109

4.51 Schematic diagram of probable reduction in MFA with recycling 111

5.1 Bulk of handsheets made from recycled pulps . . . . . . . . . . . 114

5.2 Prepared sample holder for X-rays microtomography . . . . . . . 116

5.3 Experimental set-up of ID-19 at ESRF . . . . . . . . . . . 116 
5.4 Microtomographs of handsheets produced from never dried and ten times recycled pulps . . . . . . . . . . . . . . . 117

5.5 Porosity profiles of handsheets made from recycled pulps . . . . 117

5.6 Measuring projected fibre intersection area from microtomographs 118

$5.7 z$ projection of $(7 \mu \mathrm{m})$ sub-stacked microtomographs . . . . . . . 119

5.8 Fibre cross section . . . . . . . . . . . . . . . 120

5.9 Slice of 10RF from re-sliced stack . . . . . . . . . . . . . 121

5.10 Zero-span breaking length of handsheets made from recycled pulps ........................... 123

5.11 Wet zero-span breaking length of handsheets made from recycled pulps . . . . . . . . . . . . . . . . 124

5.12 Variation of breaking lengths with span separation of paper produced from ten times recycled pulp . . . . . . . . . . . . . . . 124

5.13 Tensile index of handsheets made from recycled pulps . . . . . . 126

5.14 Elongation of handsheets made from recycled pulps . . . . . . . 127

5.15 Young's modulus of handsheets made from recycled pulps . . . . 128

5.16 Tensile energy absorption of handsheets made from recycled pulps 128

5.17 Fibre network activation of recycled pulps . . . . . . . . . . . . . 129

5.18 Tensile index as a function of activation coefficient . . . . . . . 130

5.19 Burst index of handsheets made from recycled pulps . . . . . . . 131

5.20 Tear index of handsheets made from recycled pulps . . . . . . . 132

5.21 Mechanical properties of handsheets made from recycled pulps . 134

5.22 Brightness of handsheets made from recycled pulps . . . . . . . 135

5.23 Opacity of handsheets made from recycled pulps . . . . . . . . 135

5.24 Scattering coefficient of handsheets made from recycled pulps . 136

5.25 Bulk of of handsheets made from recycled pulp blends . . . . . . 137

5.26 Zero-span breaking length of handsheets made from recycled pulp blends . . . . . . . . . . . . . . . . 138

5.27 Wet zero-span breaking length of handsheets made from recycled pulp blends . . . . . . . . . . . . . . . . 139

5.28 Tensile index of handsheets made from recycled pulp blends . . 139

5.29 Percentage change in tensile index of handsheets made from recycled pulp blends . . . . . . . . . . . . . . . . . 141

5.30 Elongation of handsheets made from recycled pulp blends . . . . 141

5.31 Percentage change in elongation of handsheets made from recycled pulp blends . . . . . . . . . . . . . . . . . 142

5.32 Young's modulus of handsheets made from recycled pulp blends 143 
5.33 Percentage change in young's modulus of handsheets made from recycled pulp blends . . . . . . . . . . . . . . . . . . . . . . 144

5.34 Tensile energy absorption of handsheets made from recycled pulp blends . . . . . . . . . . . . . . . . . . . . . . . . . 144

5.35 Percentage change in TEA of handsheets made from recycled pulp blends . . . . . . . . . . . . . . . . 145

5.36 Burst index of handsheets made from recycled pulp blends . . . 146

5.37 Percentage change in burst index of handsheets made from recycled pulp blends . . . . . . . . . . . . . . . . . . . . . 147

5.38 Tear index of handsheets made from recycled pulp blends . . . . 147

5.39 Percentage change in tear index of handsheets made from recycled pulp blends . . . . . . . . . . . . . . . . . . 148

5.40 Brightness of handsheets made from recycled pulp blends . . . . 149

5.41 Opacity of handsheets made from recycled pulp blends . . . . . 150

5.42 Scattering coefficient of handsheets made from recycled pulp blends . . . . . . . . . . . . . . . . . . 150

A.1 Schematic diagram of dewatering apparatus . . . . . . . . . . 157

A.2 Characteristic drainage curve . . . . . . . . . . . . 158

B.1 Column containing glass beads . . . . . . . . . . . . 161

B.2 Mass balance for FSP . . . . . . . . . . . . . . . . . 162

D.1 DSC curve of solvent exchanged ORF . . . . . . . . . . . 169 


\section{List of Tables}

2.1 Trends in extent of forest 1990-2010 . . . . . . . . . 3

2.2 Worldwide wood utilisation in 2010 . . . . . . . . . . 4

2.3 Average thickness of cell wall layers in spruce tracheids . . . . . 7

2.4 Average primary cell wall constituents in temperate softwoods and hardwoods ...................... 9

2.5 Production and trade of recovered pulp and paper in 2010 . . . 31

3.1 Morphological properties of Valley beater refined pulps and fibres 43

3.2 Evolution of specific filtration resistance during PFI refining . . 48

3.3 Evolution of specific filtration resistance during Valley beater refining . . . . . . . . . . . . . . . . . . 49

3.4 Calibration equations of probe molecules . . . . . . . . 54

3.5 Characteristics of probe molecules . . . . . . . . . . 57

4.1 Average DP values of cellulose obtained from molecular mass distribution ....................... . . 72

4.2 Properties of recycled pulps . . . . . . . . . . . 73

4.3 Evolution of kink angle during rewetting and drying cycles . . . 84

4.4 Evolution of fibre wall cross section area during rewetting and drying cycles . . . . . . . . . . . . . . . 87

4.5 Effect of rewetting and drying cycles on fibre wall dimensions . . 88

4.6 Effect of recycling on single fibre tensile properties . . . . . . . 110

5.1 Evolution of projected intersection area with recycling . . . . . . 119

5.2 Lumen collapse index as a function of recycling . . . . . . . . 121

5.3 Evolution of indices with recycle number . . . . . . . . . . 125

5.4 Mechanical properties of handsheets made from recycled pulps . 133 
5.5 Tensile index of handsheets made from recycled pulp blends . . 140

5.6 Elongation of handsheets made from recycled pulp blends . . . . 142

5.7 Young's modulus of handsheets made from recycled pulp blends 143

5.8 Tensile energy absorption of handsheets made from recycled pulp blends . . . . . . . . . . . . . . . . . . 145

5.9 Burst index of handsheets made from recycled pulp blends . . . 146

5.10 Tear index of handsheets made from recycled pulp blends . . . . 148

5.11 Cost analysis of recycled pulp blends . . . . . . . . . . . . . 151

5.12 Environmental impact estimates of recycled pulp blends in copy

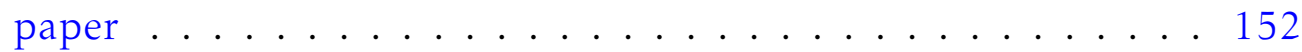

A.1 Specific filtration resistance of recycled pulps and fibres . . . . 160

B.1 Apparent pore volume of recycled pulps . . . . . . . . . . . . 164

B.2 Apparent pore volume of recycled fibres . . . . . . . . . . . . . 164 


\section{List of Notations}

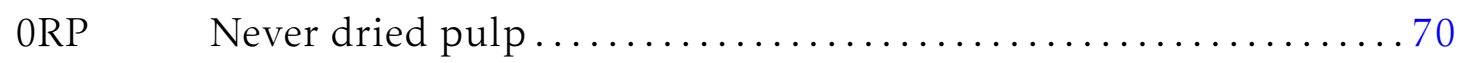

3D Three dimensional ................................ 115

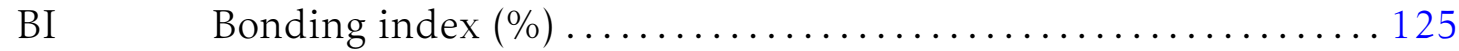

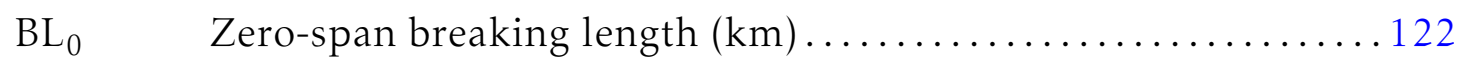

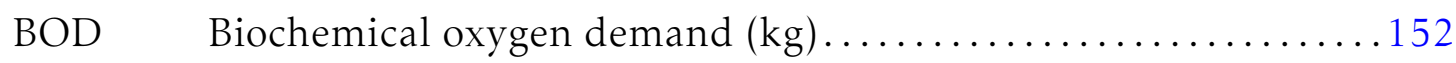

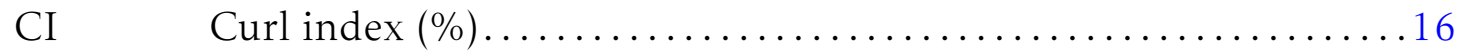

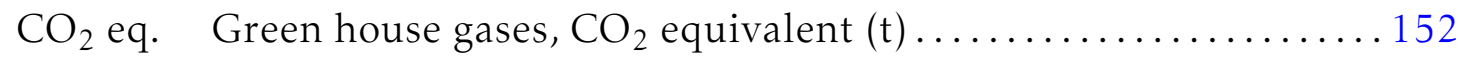

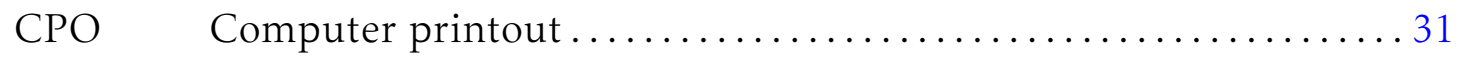

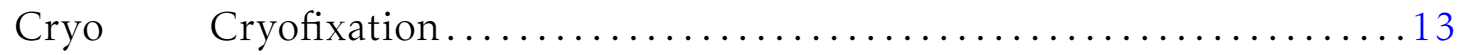

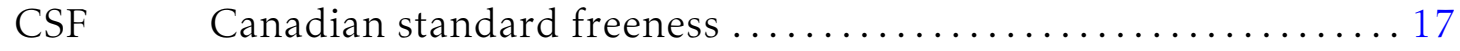

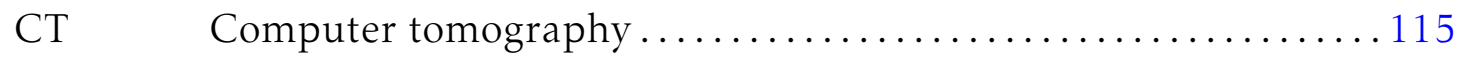

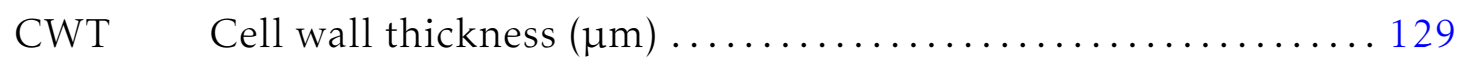

DFT Dynamic freeness tester ............................ 46

DSC Differential scanning calorimetry ...................... 167

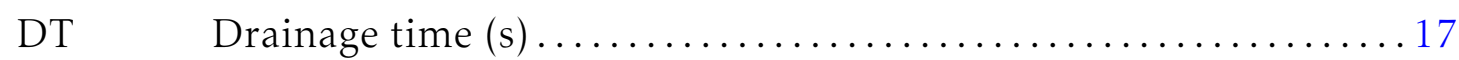

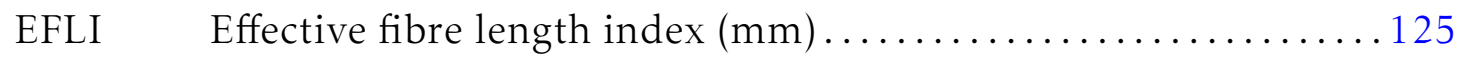

ESEM Environmental scanning electron microscope............... 81

ESRF European synchrotron radiation facility ................. 116

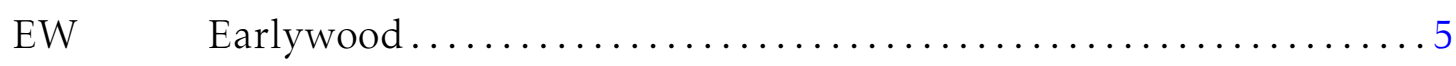

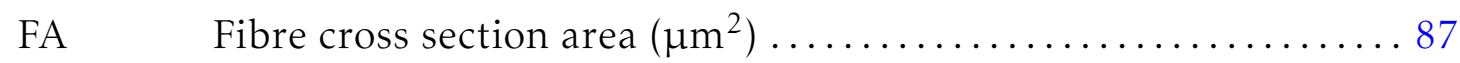

FAO Food and agriculture organization $\ldots \ldots \ldots \ldots \ldots \ldots \ldots \ldots \ldots \ldots$

FBW Freezing bound water .............................. 168

FE-SEM Field emission scanning electron microscopy $\ldots \ldots \ldots \ldots \ldots \ldots 13$

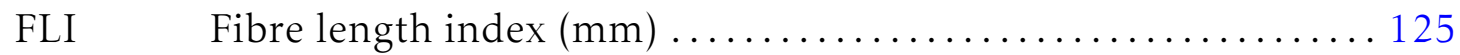

FP Fibre cross section perimeter $(\mu \mathrm{m}) \ldots \ldots \ldots \ldots \ldots \ldots \ldots \ldots \ldots$

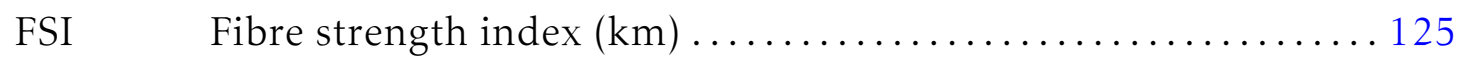


FSP Fibre saturation point $\left(\mathrm{mL} \cdot \mathrm{g}^{-1}\right) \ldots \ldots \ldots \ldots \ldots \ldots \ldots \ldots \ldots \ldots$

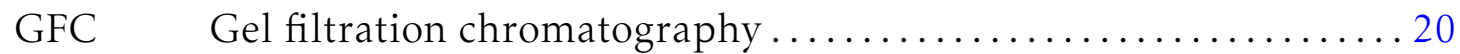

GPC Gel permeation chromatography ..................... 20

ISEC Inverse size exclusion chromatography $\ldots \ldots \ldots \ldots \ldots \ldots \ldots$

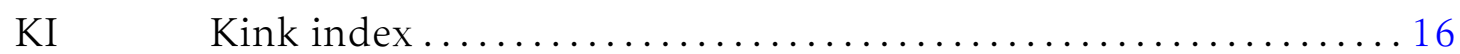

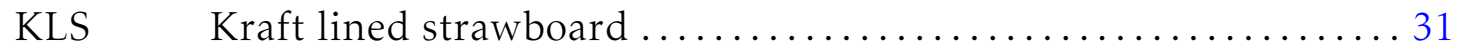

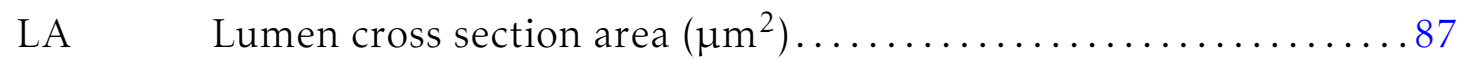

LP Lumen cross section perimeter $(\mu \mathrm{m}) \ldots \ldots \ldots \ldots \ldots \ldots \ldots$

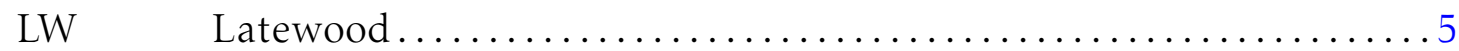

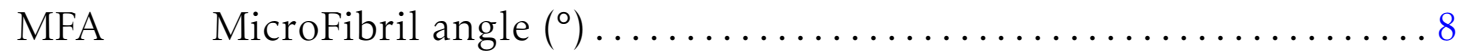

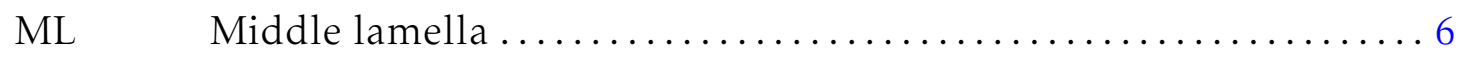

MOW $\quad$ Mixed office waste .................................... 31

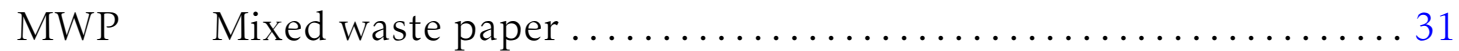

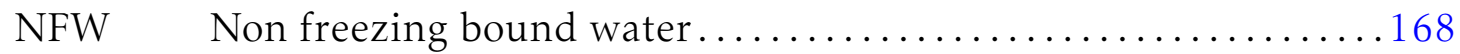

NMR Nuclear Magnetic Resonance ........................ 20

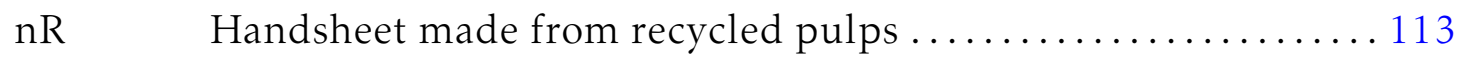

nRF Fibres obtained from the corresponding $n R P \ldots \ldots \ldots \ldots \ldots . \ldots 1$

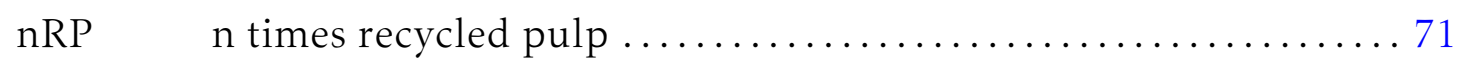

OCC Old corrugated containers .......................... 31

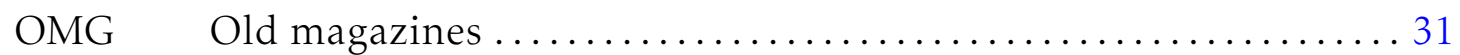

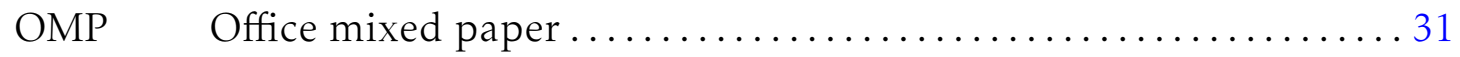

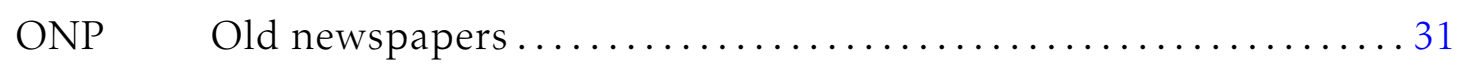

P100 Fraction of elements passed through 100 mesh screen .......... 36

RCF Relative centrifugal force, $105 \mathrm{~m} \cdot \mathrm{s}^{-2} \ldots \ldots \ldots \ldots \ldots \ldots \ldots \ldots$

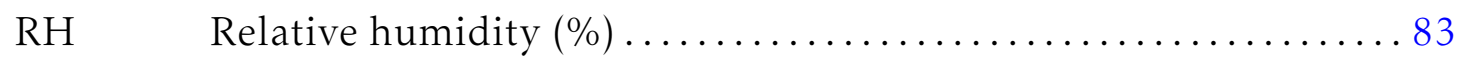

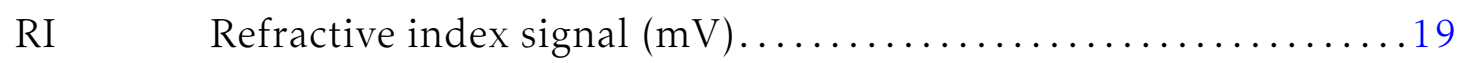

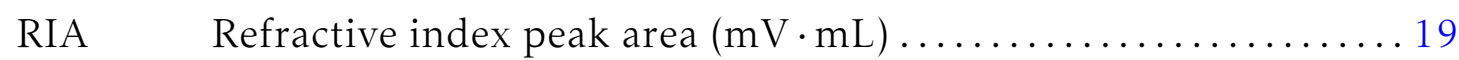

S1 Outer secondary cell wall layer $\ldots \ldots \ldots \ldots \ldots \ldots \ldots \ldots \ldots \ldots$

S2 Middle secondary cell wall layer .................... 6

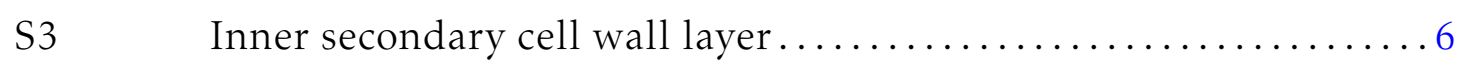

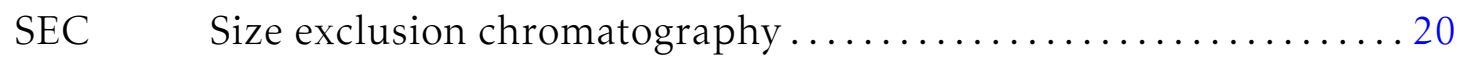

SEM Scanning electron microscope .......................... 115

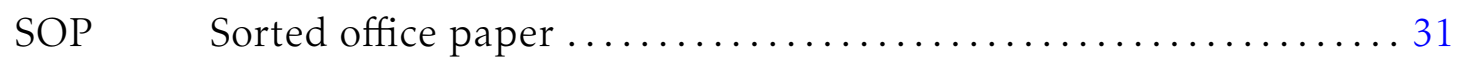

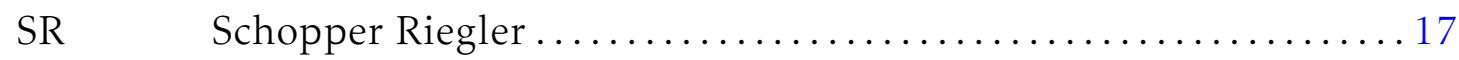

TEA Tensile energy absorption $\left(\mathrm{J} \cdot \mathrm{m}^{-2}\right) \ldots \ldots \ldots \ldots \ldots \ldots \ldots \ldots \ldots \ldots \ldots \ldots$

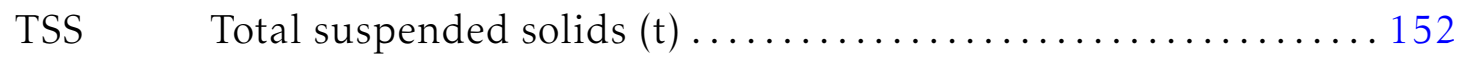

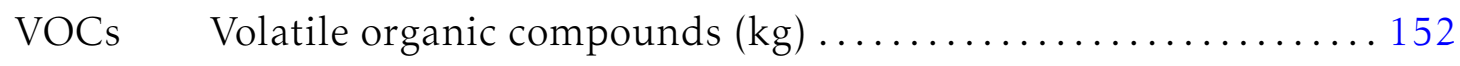


Voxel Volumetric pixels .............................. 116

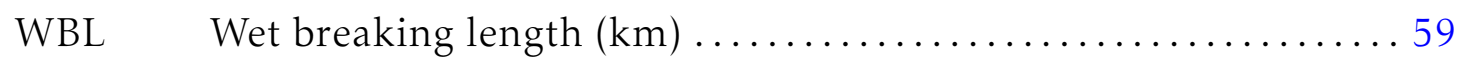

$\mathrm{WBL}_{0} \quad$ Wet zero-span breaking length $(\mathrm{km}) \ldots \ldots \ldots \ldots \ldots \ldots \ldots \ldots \ldots$

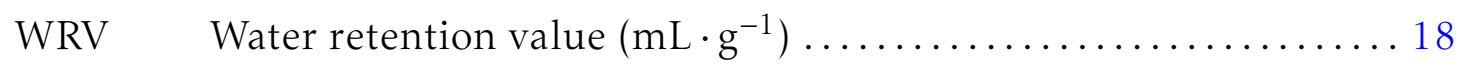

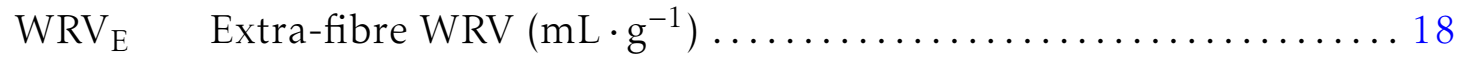

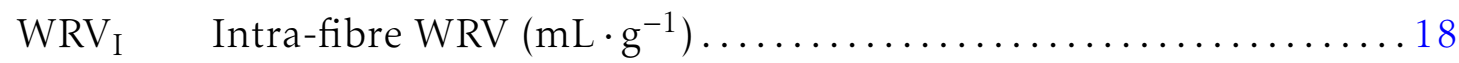

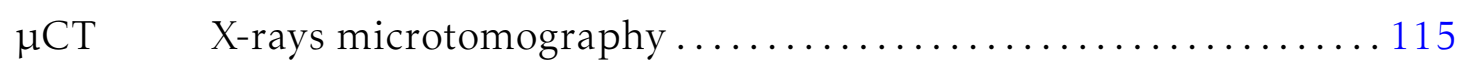




\section{List of Symbols}

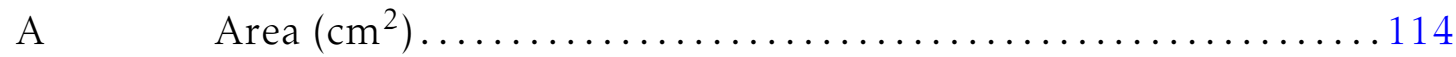

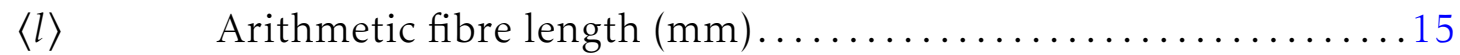

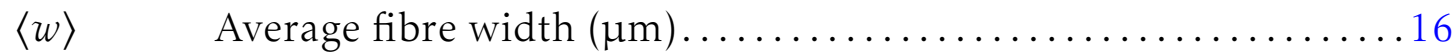

$k_{b} \quad$ Boltzmann's constant $\left(1.381 \times 10^{-23} \mathrm{~m}^{2} \cdot \mathrm{kg} \cdot \mathrm{s}^{-2} \cdot \mathrm{K}^{-1}\right) \ldots \ldots \ldots .20$

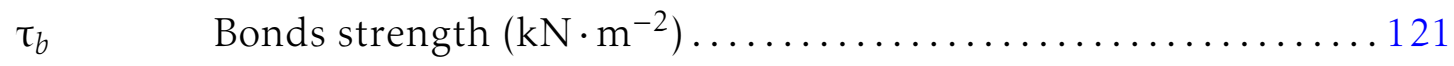

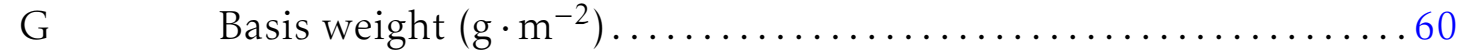

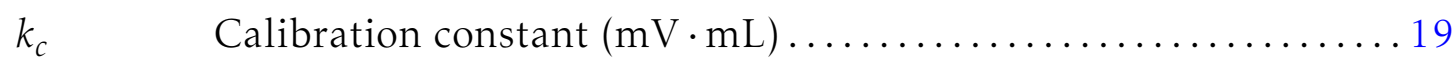

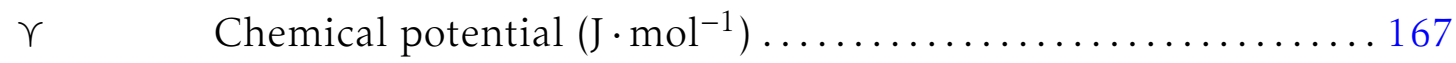

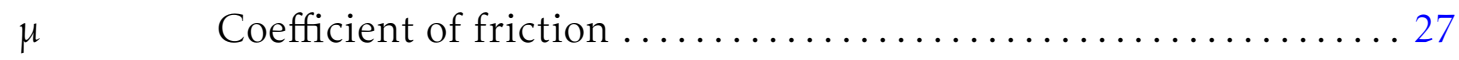

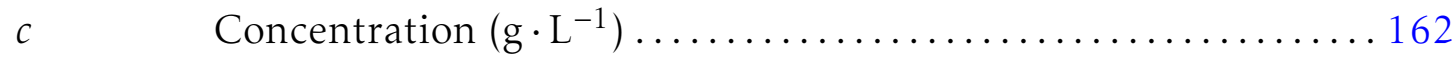

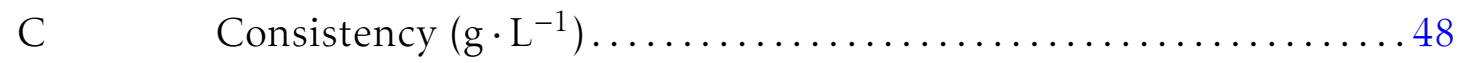

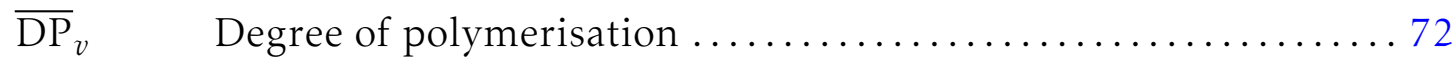

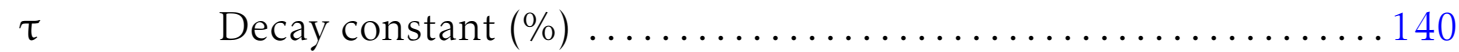

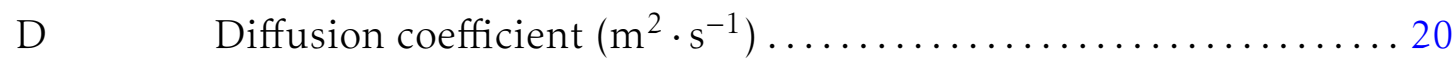

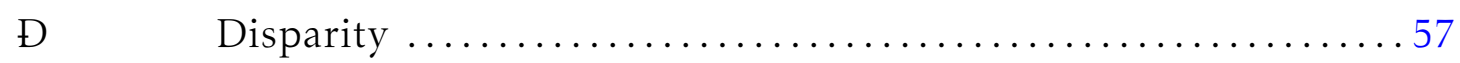

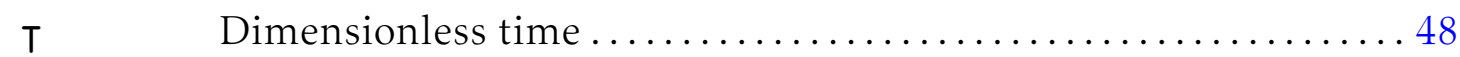

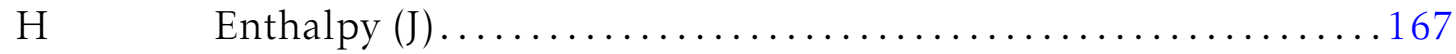

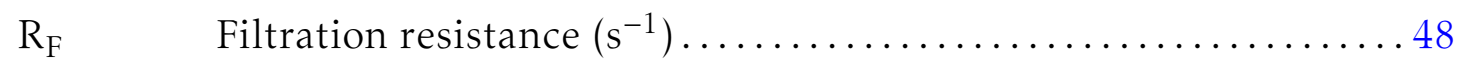

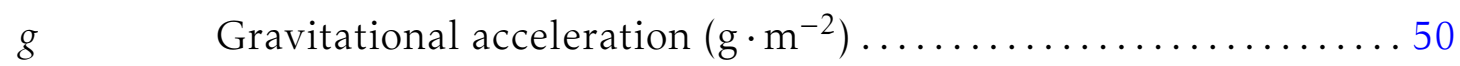

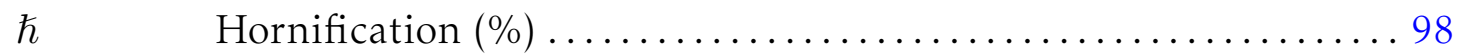

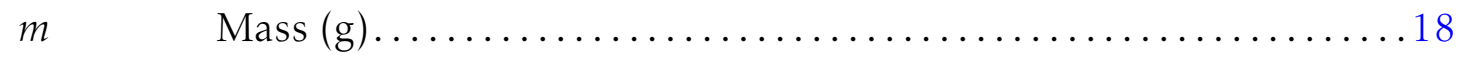

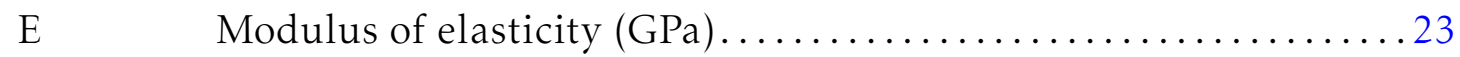

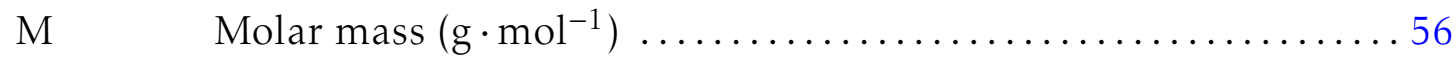

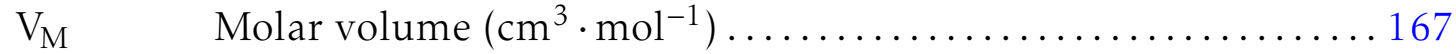

$n_{i} \quad$ Number of fibres in $\mathrm{i}^{\text {th }}$ class .......................... 15

$\mathrm{N}_{k} \quad$ Number of kinks................................. 16 


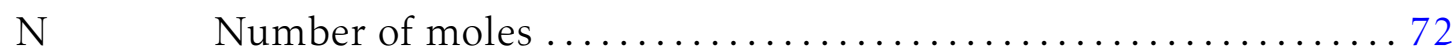

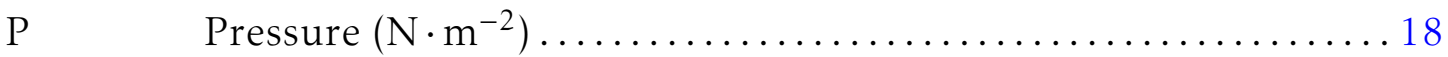

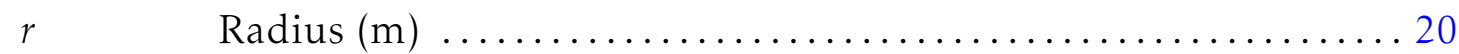

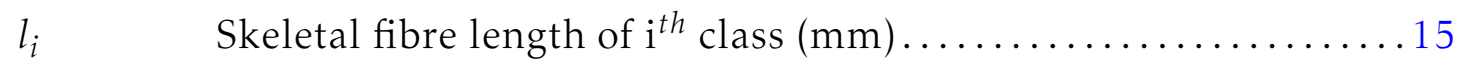

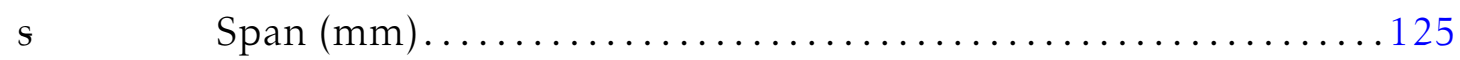

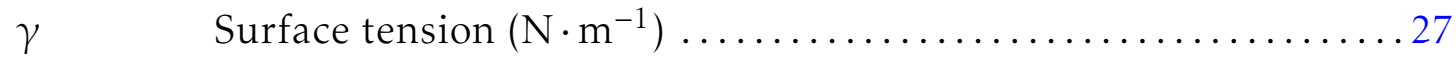

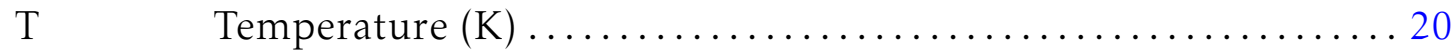

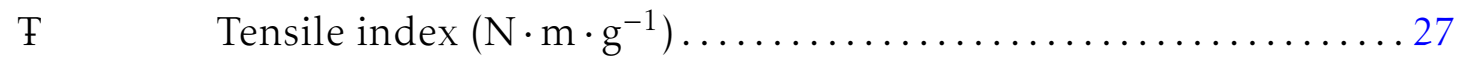

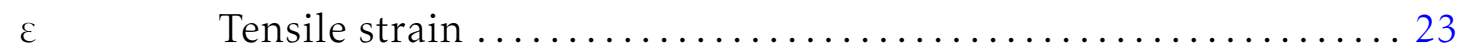

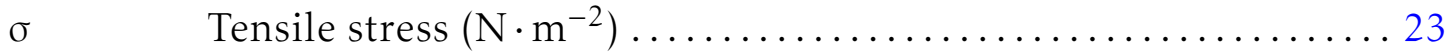

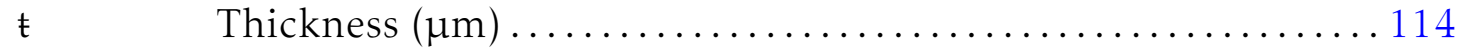

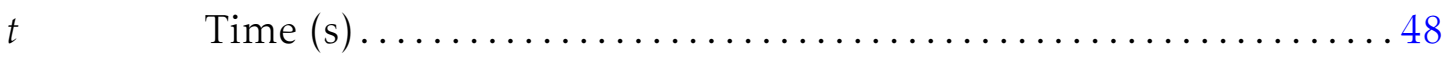

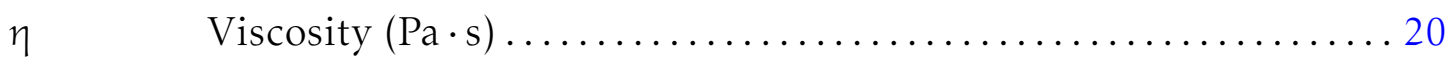

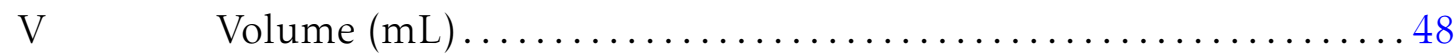

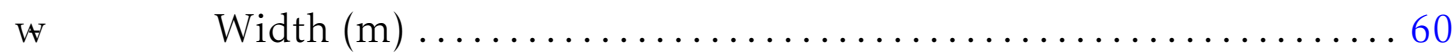

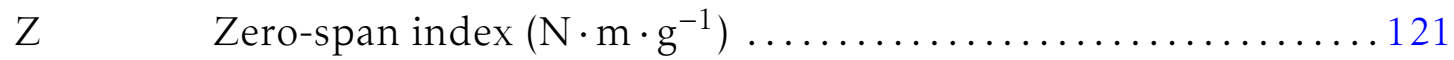




\section{canent 1}

\section{Introduction}

Recycled pulp is considered as an important secondary raw material for the production of paper and paper board. Replacement of virgin with recycled pulp is increasing day by day. The increased use of recycled pulp is partly caused by the increased price of the virgin pulp and partly by the enforcement of rigorous regulations from the environmental protection agencies in order to establish low-carbon and waste-free society.

During the past few decades, substantial amount of research in the field of paper recycling had been carried out. The focus of research was to improve its properties by mechanical and/or chemical treatments so that the end product should conform to the acceptable quality standards. Indeed, recycling tends to modify fibre morphology and affect physical properties of the fibres, pulp and paper. In order to introduce recovered fibres in high value paper it is required to better understand morphological changes and the phenomenon of fibre hornification associated with recycling.

The purpose of this study is to characterise, quantify and correlate the physical changes in fibres, pulps and paper due to recycling.

The process of refining was used to optimise the characterisation procedures for pulp and fibres. 
${ }_{\text {Chapter }} 2$

\section{Literature review}

\section{Contents}

2.1 Forest outlook ................. 2

2.2 Wood and fibres .................. 4

2.2.1 Anatomical structure . . . . . . . . . . . . . 4 4

2.2.2 Chemical constituents . . . . . . . . . . . 8

2.2.3 Morphological characteristics . . . . . . . . . . . . 14

2.2.4 Dewatering . . . . . . . . . . . . . 17

2.2.5 Water retention value . . . . . . . . . . 18

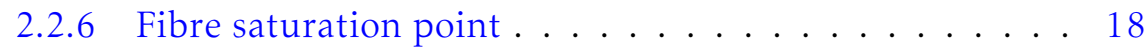

2.2.7 Fibre wall pore size distribution . . . . . . . . . 20

2.2.8 Weak-points in fibre wall . . . . . . . . . . . . . 21

2.2.9 Single fibre stress-strain properties . . . . . . . . 22

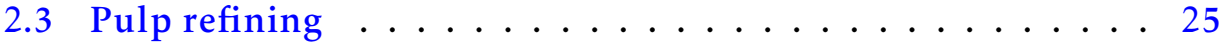

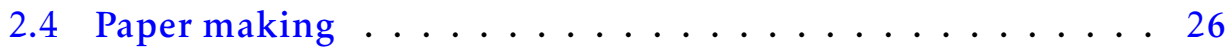

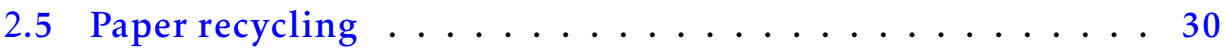

2.5.1 Recycling rate . . . . . . . . . . . . . 32

2.5.2 Effects of recycling on pulp, fibres and paper properties 33

\subsection{Forest outlook}

Forests cover $31 \%$ of the total land area of the world. Forests of Russian federation, Brazil, Canada, the United States of America and China alone accounts 
for more than half of the total world's forest area. Data from the Food and Agriculture Organization (FAO) [167] on forested areas and its annual change rate worldwide is given in Table 2.1.

Table 2.1: Trends in extent of forest 1990-2010 [167]

\begin{tabular}{l|ccc|cc|cc}
\multirow{2}{*}{ Region } & \multicolumn{3}{|c|}{ Forest area $(1000 \mathrm{ha})$} & \multicolumn{4}{c}{ Annual change rate } \\
\cline { 2 - 7 } & \multirow{3}{*}{2090} & 2000 & 2010 & \multicolumn{2}{c}{$1990-2000$} & \multicolumn{2}{c}{$2000-2010$} \\
\cline { 6 - 9 } & & & & 1000 ha & $\%$ & 1000 ha & $\%$ \\
\hline Africa & 749238 & 708564 & 674419 & -4067 & -0.56 & -3415 & -0.48 \\
Asia & 576110 & 570164 & 592512 & -595 & -0.10 & 2235 & 0.39 \\
Europe & 989471 & 998239 & 1005001 & 877 & 0.09 & 676 & 0.07 \\
America & 1654837 & 1609819 & 1569744 & -4502 & -0.27 & -4008 & -0.25 \\
Oceania & 198744 & 198381 & 191384 & -36 & -0.02 & -700 & -0.35 \\
\hline World & 4168399 & 4085168 & 4033060 & -8323 & -0.20 & -5211 & -0.13
\end{tabular}

Although, large scale tree plantation and natural expansion has significantly reduced the net loss of forests in the previous decade but still deforestation rate is alarming. Deforestation in Africa, Oceania and Latin America was the main cause for the net loss of forests in the previous decade. The reasons of deforestation are mainly social, political and economic. In Africa the main cause of deforestation is the dependence of its population on wood as a source of energy [24].

Production of wood and wood products starts with felling, skidding and onsite processing of round-wood logs. Round-wood is classified into industrial round-wood and fuel-wood.

Industrial round-wood includes timber logs, pulpwood and other industrial woods. Large-diameter, good-quality timber logs are used for the production of sawn-wood and veneer sheets. Lower-quality timber is generally used in the manufacture of pulp, particle board and fibreboard. Remaining industrial round-wood is used in the manufacture of poles, piling, posts or fencing. Fuel-wood is the wood of lower quality obtained from the trunks and branches of trees. It is used as fuel for cooking, heating and charcoal production. Secondary, lower-quality wood processing residues (chips and particles etc.) are 
either used as a fuel or for making pulp, particle board, or pellets, briquettes and other solid fuel.

These basic and primary wood products are further transformed into a range of products, many of which are consumer goods. Data obtained from the FAO [167] on the uses of wood for 2010 is given in Table 2.2.

Table 2.2: Worldwide wood utilisation in 2010 [167]

\begin{tabular}{l|cccccc}
\multirow{2}{*}{ Region } & \multicolumn{7}{|c}{ Production } \\
\cline { 2 - 7 } & Industrial & Wood & Sawnwood & $\begin{array}{c}\text { Wood-based } \\
\text { panels, } \mathrm{Mm}^{3}\end{array}$ & $\begin{array}{c}\text { Wood } \\
\text { pulp, Mt }\end{array}$ & $\begin{array}{c}\text { Paper and } \\
\text { paperboard, Mt }\end{array}$ \\
\hline Africa & 72.70 & 602.47 & 8.30 & 2.67 & 2.61 & 3.59 \\
America & 628.15 & 326.24 & 138.23 & 58.86 & 89.98 & 109.18 \\
Asia & 241.18 & 770.71 & 88.77 & 131.80 & 26.82 & 169.70 \\
Europe & 510.13 & 150.28 & 138.90 & 71.56 & 47.88 & 107.76 \\
Oceania & 50.48 & 10.71 & 8.55 & 3.68 & 2.84 & 4.12 \\
\hline World & 1502.65 & 1860.40 & 382.75 & 268.58 & 170.13 & 394.35
\end{tabular}

In 2010, significant quantity of wood was used as fuel in Asia and Africa. America, Asia and Europe were the major consumers of wood for the production of pulp and paper.

\subsection{Wood and fibres}

Wood is a heterogeneous, hygroscopic, cellular and anisotropic material present beneath the bark in the stems or branches of trees or shrub. It keeps the plants upright, helps in its growth, serves as storage tissue for reserve material, transport water and nutrients to the leaves. Earliest discovered plant containing wood dates back 400 million years [163]. Woods are commonly classified in the field of pulp and paper as either softwoods or hardwoods. Gymnosperms are the source of softwoods whereas hardwoods come from broad-leaved trees (angiosperms).

\subsubsection{Anatomical structure}

The hierarchy of wood structure is shown in Figure 2.1. 


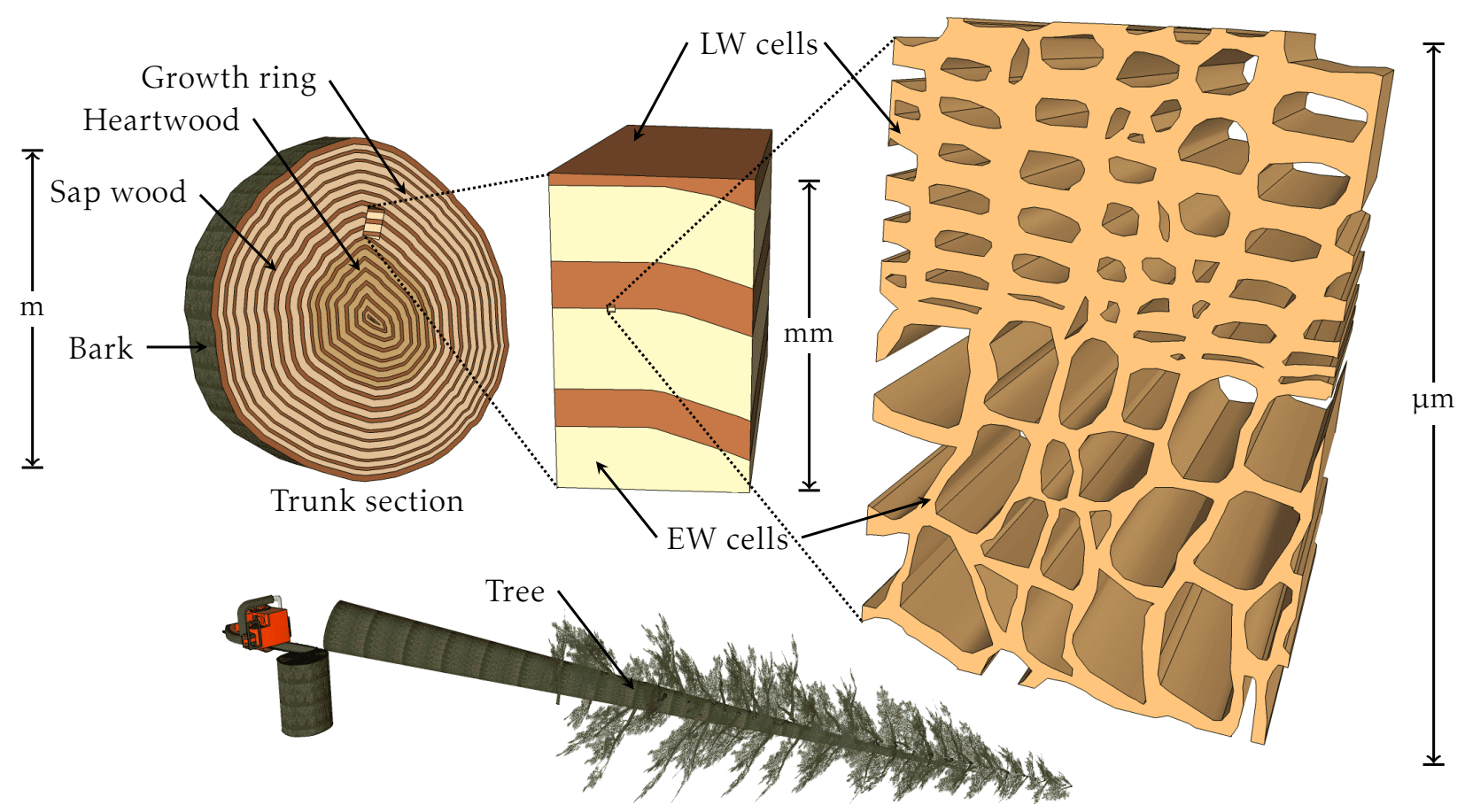

Figure 2.1: Hierarchy of wood structure

Wood grows in the form of concentric layers generally referred as growth rings or tree rings. The growth of wood is a periodic process which is restricted to the vegetation period. The parts of the world where the seasonal changes are annual and discrete, the rings are called the annual rings. An annual ring consists of a light coloured earlywood (EW) or springwood and a dark coloured latewood (LW) or summerwood ring. The annual early season growth accounts for 40 to $80 \%$ of a ring's growth in width [75]. In autumn, the growth rate normally slows down. In tropical and subtropical regions the growth increments in rings reflect alternating wet and dry seasons. Parts of the world where there are no distinct seasons, growth rings are either absent or unidentifiable.

Heartwood is the part of the wood that has become more resistant to decay due to a naturally occurring chemical transformation. The process of heartwood formation is spontaneous and is genetically programmed. Sapwood is the younger and outermost part of the wood.

Availability of water, temperature, sunlight conditions, soil conditions and the slope of the hill side affects the formation of EW and LW layers in the annual ring $[137,138,228]$. 


\section{Cell wall ultra-structure}

In softwoods, more than $90 \%$ of the total cell volume is occupied by tracheids, the rest being the parenchymas [112]. Tracheids are long, narrow and hollow softwood fibres. Figure 2.2 shows a schematic cell wall layer model of LW tracheid. The common layer holding adjacent cells together is called middle lamella (ML).

The hollow centre of the wood cell is called lumen. The cell wall is composed of layers, namely primary cell wall layer (P) and secondary cell wall layers (S). Secondary cell wall is subdivided into an outer secondary cell wall layer (S1), a relatively thick middle secondary cell wall layer (S2), and an inner secondary cell wall layer (S3).

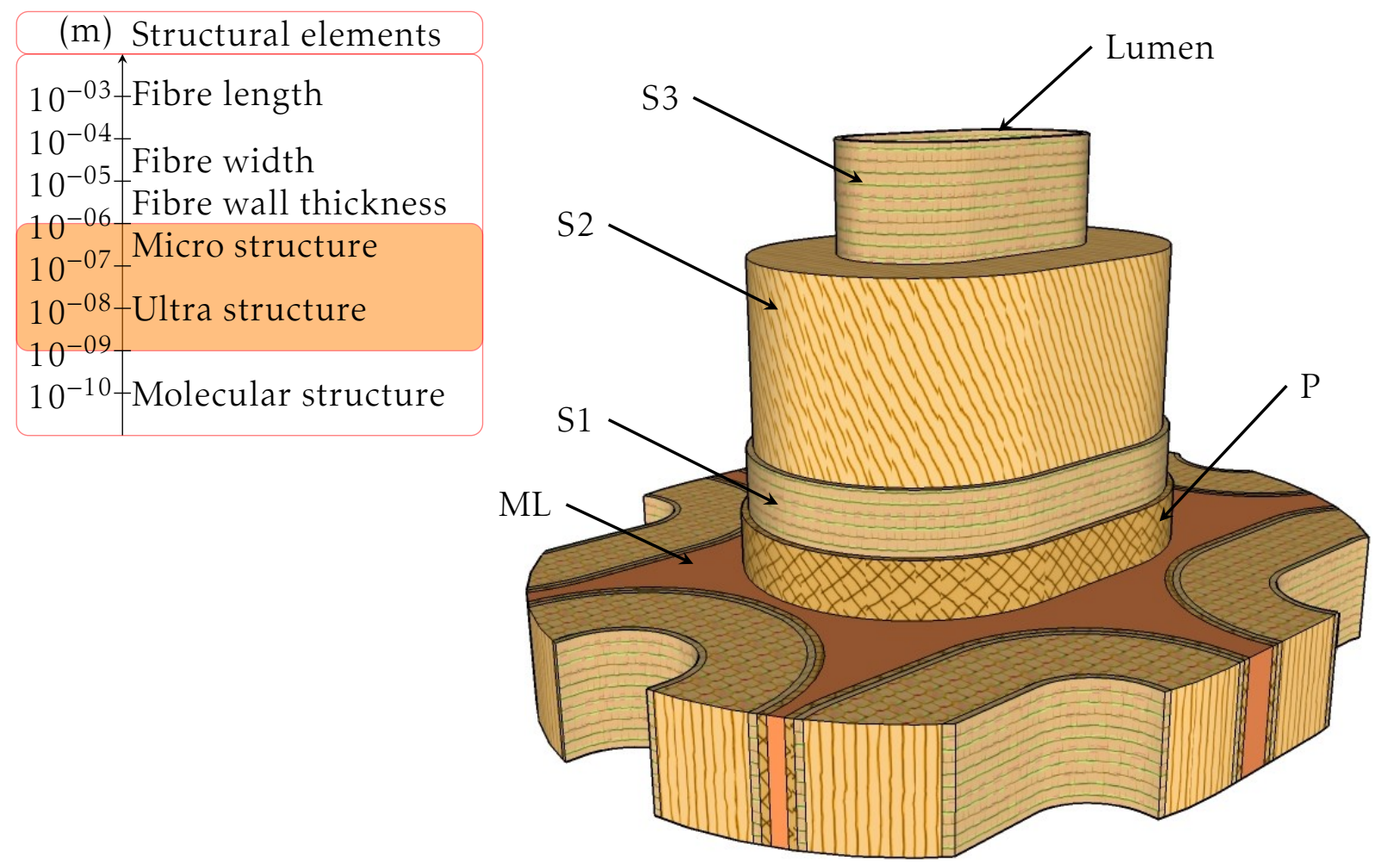

Figure 2.2: Schematic cell wall layers model of a latewood tracheid adapted from [159]

Secondary wall is composed of cellulose with small amounts of lignin and hemicellulose [104]. S1 and S3 layers are homogeneous and rigid [49]. Thickness of these layers varies between 0.1 to $0.3 \mu \mathrm{m}[46,86]$. In EW tracheids, the thickness of S2 layer is about $1 \mu \mathrm{m}$ and in LW tracheids it is around $5 \mu \mathrm{m}$ [86]. Cell wall of EW tracheids is thinner and lumen is wider [177] than those of LW 
tracheids. They are meant for sap conduction. LW trachieds are thick walled and provide mechanical strength to the structure [206, 189, 234, 219]. Average thickness of cell wall layers in spruce tracheids is presented in Table 2.3.

Table 2.3: Average thickness of cell wall layers in spruce tracheids [86]

\begin{tabular}{l|cc}
\multirow{2}{*}{ Layer } & \multicolumn{2}{|c}{ Average cell wall layer thickness, $\mu \mathrm{m}$} \\
\cline { 2 - 3 } & Earlywood & Latewood \\
\hline ML/P & 0.09 & 0.09 \\
S1 & 0.26 & 0.38 \\
S2 & 1.66 & 3.69 \\
S3 & 0.09 & 0.14 \\
\hline Total & 2.1 & 4.3
\end{tabular}

EW/LW ratio affects the pulp and paper properties [240, 169, 226]. For example, lower EW/LW ratio gives higher compressibility, stiffness, porosity, absorbency, tear strengths and lower tensile strengths [169].

Hardwoods contain substantial amounts of vessels and parenchyma along with fibres [207, 100]. Vessels are meant for sap conduction. Hardwood fibres are shorter and narrower than softwood fibres. They also have a smaller lumen which imparts mechanical strength.

In the cell wall, the intra and intermolecular hydrogen bonds aggregate cellulose molecules in the form of bundles, which are termed as microfibrils [90, $84,77,110]$. Figure 2.3 shows schematic model of cellulose microfibril containing accessible and inaccessible surfaces. Microfibrils have crystalline and para-crystalline (having short and medium range regularity in its lattice) regions. The former is located in the interior whereas the later is present on the surface of the microfibrils $[140,237]$. The cross section of a microfibril in wood is around $4 \times 4 \mathrm{~nm}^{2}[236]$. 


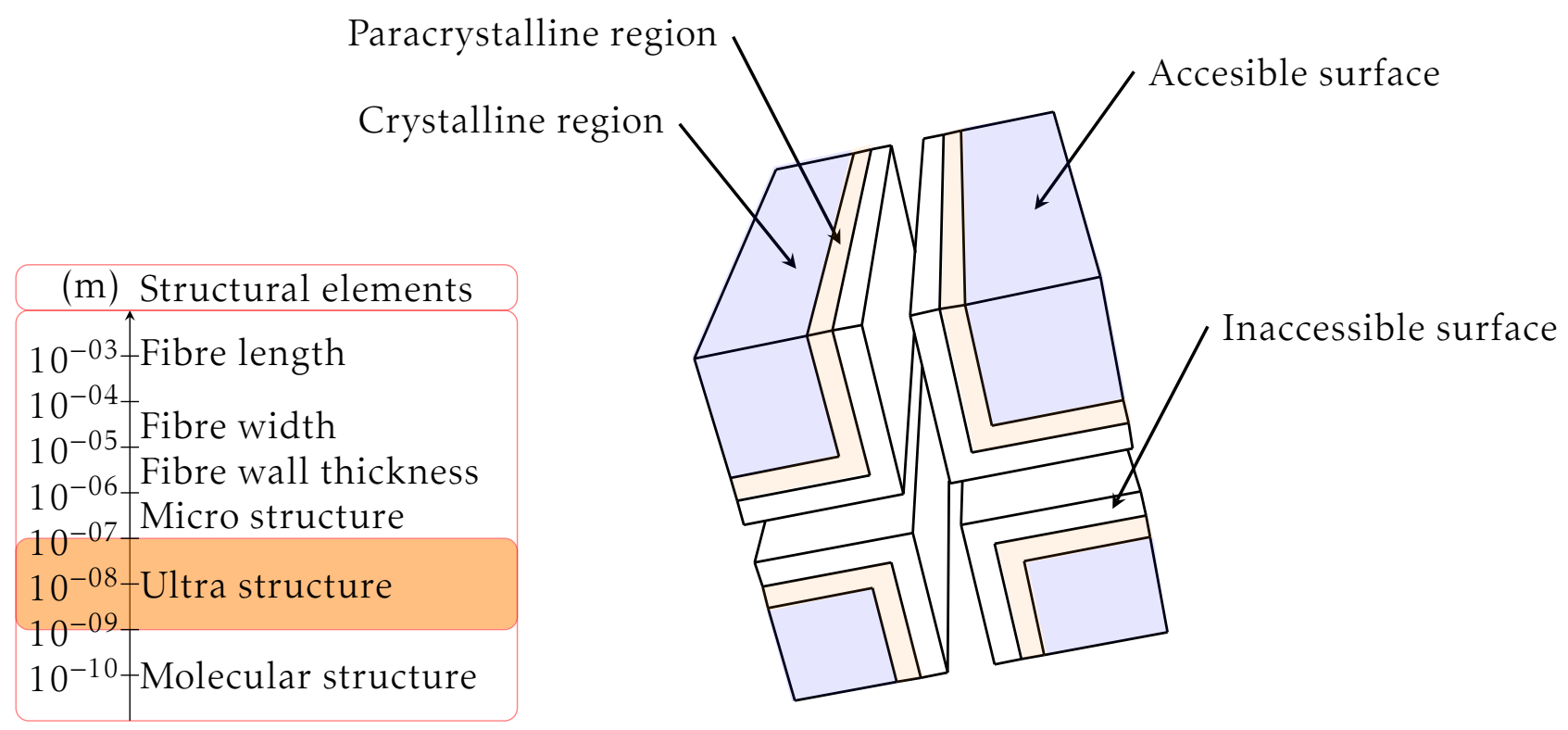

Figure 2.3: Schematic model of a cellulose microfibril adapted from [236]

The angle between the principal cell axis and the microfibrils is called the microfibrils angle (MFA). Since the S2 layer makes most of the secondary cell wall therefore MFA refers to the angle between the microfibrils of the S2 layer and the cell wall axis. The orientation of cellulose microfibrils is very important as it governs the physical properties of the wood cell [37]. For example, higher MFA in juvenile wood (a zone of wood extending outward from the pith produced in early growth period) gives low stiffness therefore higher proportion of juvenile wood renders it unsuitable for use as high-grade structural timber. The MFA in hardwoods is between 0 to $30^{\circ}$ while that of softwoods is higher [39]. The MFA in LW tracheids is slightly lower than that of EW tracheid $[156,191]$. The microfibrils of the S1 and S3 layers provide transverse stiffness [40] whereas the microfibrils of S2 layer provide stiffness and strength to the fibre in the longitudinal direction. The amount of lignin in EW is relatively higher than in LW $[240,87]$.

The microfibrils in the S2 layer are aggregated into a larger structural unit, termed as macrofibril. The cross section of the macrofibril is around $20 \times 20 \mathrm{~nm}^{2}$ [84, $77]$.

\subsubsection{Chemical constituents}

Cellulose, hemicellulose, lignin, extractives and inorganic minerals are the main constituents of wood. Average amounts of cell wall constituents in temperate 
woods are presented in Table 2.4 .

Table 2.4: Average primary cell wall constituents in temperate softwoods and hardwoods $[85,100]$

\begin{tabular}{l|cc}
\multirow{2}{*}{ Constituents } & \multicolumn{2}{|c}{ Proportion in dry matter, \% } \\
\cline { 2 - 3 } & Softwoods & Hardwoods \\
\hline Cellulose & $40-45$ & $40-45$ \\
Hemicellulose & $25-30$ & $25-35$ \\
Lignin & $25-35$ & $20-25$
\end{tabular}

Cellulose and hemicellulose falls in the category of carbohydrates (polysaccharides). Cellulose is a structural homopolysaccharide of $1,4-\beta$-glucosidic bonded D-glucose linear chains $[220,68]$. It is partially crystalline, strong, and resistant to hydrolysis. Hemicellulose is a matrix-polysaccharide. It is an amorphous, weak and is easily hydrolysed by dilute acids or bases or hemicellulases.

Lignin is a complex polymer containing phenylpropane units [26]. It imparts hydrophobic characteristics due to the presence of aromatic rings in its structure. It is an amorphous and is insoluble organic polymer which is very difficult to isolate in its natural state.

Figure 2.4 shows the typical composition of a conifer fibre across its cell wall.

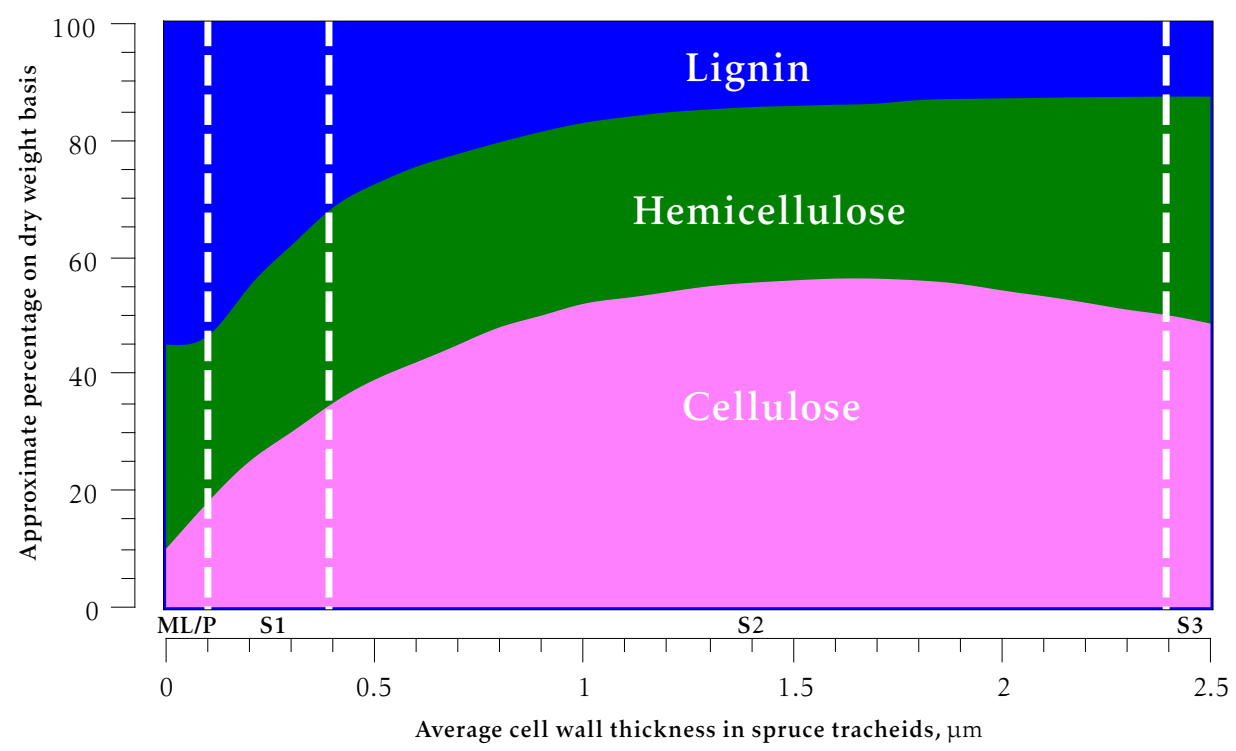

Figure 2.4: Distribution of principal chemical constituents in softwood cell wall layers adapted from [100, 177, 235, 193] 
Concentration of lignin is highest in the ML/P layer and it declines towards the lumen.

\section{Wood pulps}

Wood is the most important raw material for the production of pulp. Nonwood, annual plants are also used in parts of the world where wood is scarcely available. Debarking and chipping operations are carried out prior to the chemical digestion or pulping. Pulping is carried out in continuous or batch digester. Chemical digestion can be classified into alkaline, sulphite and other pulping processes. Kraft or sulphate pulping process has mostly replaced the older sodium hydroxide pulping process because of its higher selectivity towards the delignification. Sulphite processes can be further classified into acid bi-sulphite, bi-sulphite, neutral sulphite and alkaline sulphite processes. Mechanical properties of kraft pulps are superior to that of sulphite pulps. Other pulping processes include nitric acid, organic solvents and organic acids.

During chemical digestion of lignocelluloses (wood and other annual plants), lignin and the hemicelluloses are largely dissolved out of the fibre matrix. As a consequence, the cohesion of the structural elements is lost. Fibres are separated from each other and pores are created in the cell wall of the fibres. The fibrous material containing cellulose thus obtained is called pulp [179].

During this process, fibres become flexible, compressible and hence their bonding potential is increased (cf. Figure 2.5). 


\begin{tabular}{|c|l|}
\hline$(\mathrm{m})$ & \multicolumn{1}{|c|}{ Structural elements } \\
\hline $10^{-03}-$ & Fibre length \\
$10^{-04}$ & \\
$10^{-05}$ & Fibre width \\
$10^{-06}$ & Fibre wall thickness \\
$10^{-07}-$ & Micro structure \\
$10^{-08}-$ Ultra structure \\
$10^{-09}-$ \\
$10^{-10}-$ Molecular structure \\
\hline
\end{tabular}

Flexibility

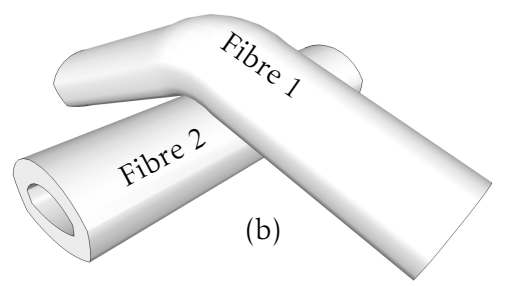

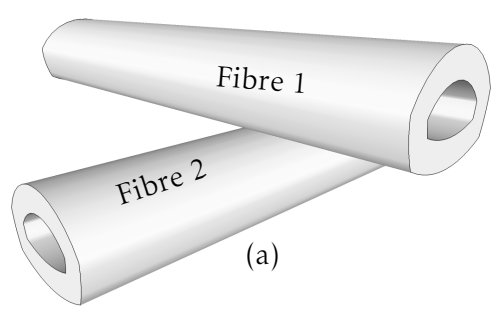

Conformability

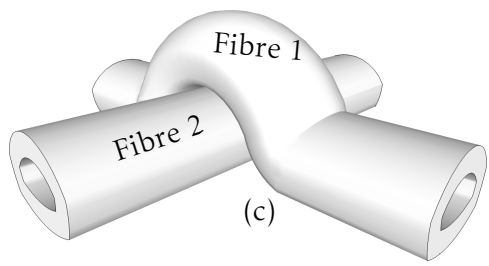

Collapsibility

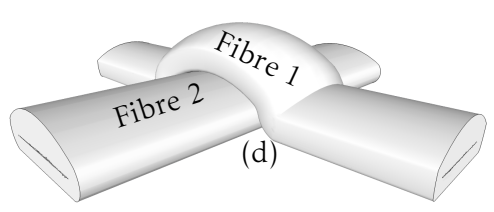

Figure 2.5: Schematic depiction of fibre shapes during bonding. In (a) both fibres are stiff, in (b) Fibre 1 is flexible, in (c) Fibre 1 is conformable whereas in (d) both fibres are collapsible

Collapsibility, conformability, flexibility [208], coarseness [200], shape [162] and fibrillation of the fibres influence the fibre networks. Conformability of fibres depends on chemical and morphological properties of the fibre wall. Dissolution of lignin and hemicelloses during cooking process make fibre wall structure porous. Average pore size increases with the decrease in yield of pulp. Stone and Scallan $[211,212]$ proposed a structural model of fibre wall where they defined macropores as inter-lamellar pores of size 25 to $300 \AA$ and micropores as intra-lamellar pores of size 5 to $25 \AA$ A. Later on, Scallan [197] presented a lamellar model of internal fibrillation with fibre wall swelling (cf. Figure 2.6). 


\begin{tabular}{|c|}
\hline$(\mathrm{m})$ Structural elements \\
$10^{-03}-$ Fibre length \\
$10^{-04}-$ Fibre width \\
$10^{-05}-$ Fibre wall thickness \\
$10^{-06}-$ Micro structure \\
$10^{-07}-$ Itra structure \\
$10^{-08}-$ Ultra \\
$10^{-09}$ \\
$10^{-10}-$ Molecular structure \\
\hline
\end{tabular}
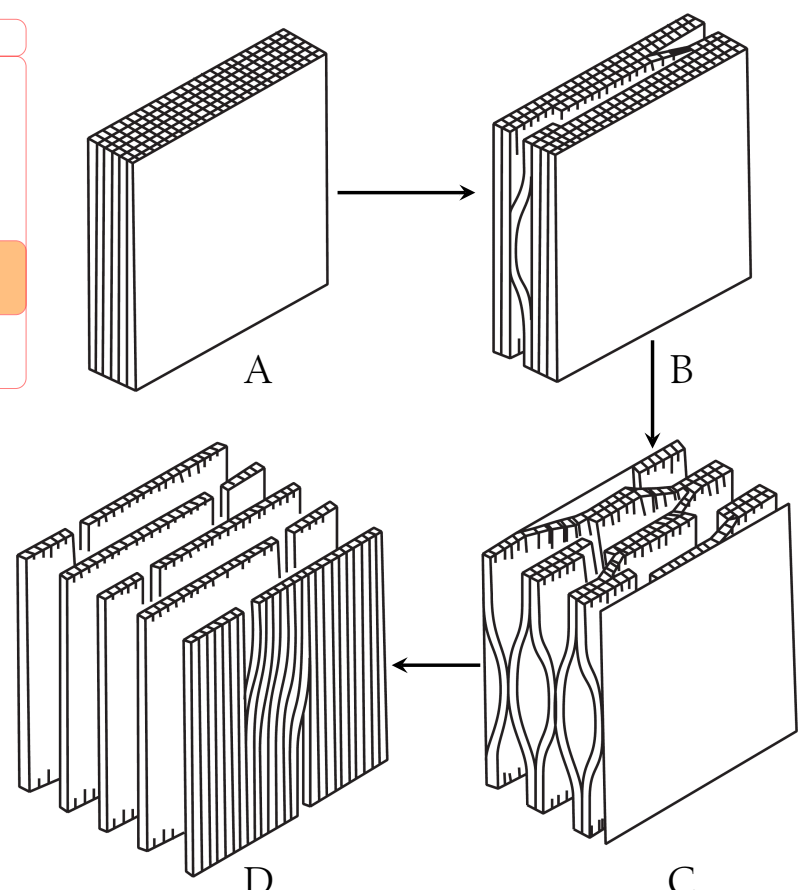

$\mathrm{C}$

Figure 2.6: Internal fibrillation of fibre wall as the swelling increases from A to $\mathrm{D}[197]$

Kerr and Goring [128] proposed a fibre wall model with the arrangement of chemical components. Delignification in the fibre wall takes place gradually, large pores are produced first which facilitates a rapid dissolution of lignin from the secondary layers [94].

Figure 2.7 is the speculative illustration of the dissolution of lignin from the fibre wall during delignification showing the creation of pores. 


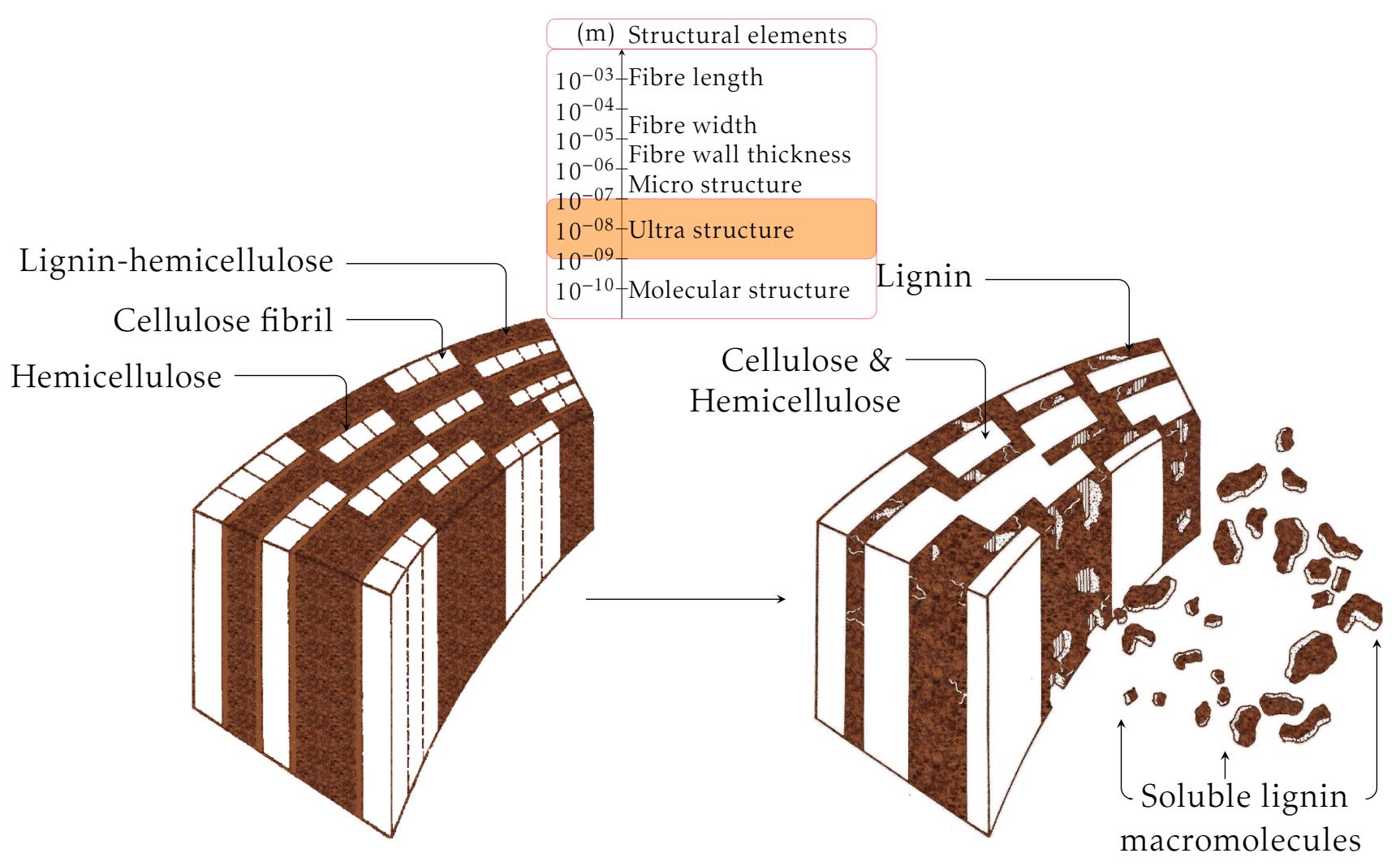

Figure 2.7: Dissolution of lignin out of the fibre wall adapted from [81]

Size distribution of the dissolved lignin $[82,239]$ may be related to the pore size distributions measured indirectly through solute exclusion [198] and inverse gel filtration chromatography [23, 42]. The pore size distribution affects fibre wall swelling in water [213], accessibility to macromolecules, the diffusion of chemicals across fibres, fibre shrinkage [232], water removal [123], colloidal interactions [188], etc.

Duchesne and Daniel [76] showed that fibre wall structure is much more open than previously described. Cryo-FE-SEM micrographs of never-dried chemical pulp revealed the inter-fibrils distance is around 10 to $50 \mathrm{~nm}$ whereas lateral dimension of fibril aggregates is around 10 to $20 \mathrm{~nm}$ [77]. Li et al. [142] used NMR relaxation measurement, Alince and van de Ven used polyeletrolyte adsorption method [28] and Maloney [150] used thermoporosmetric measurement to determine pore size and pore size distribution of pulp fibres.

Delignification during chemical digestion does not quantitatively reach to completion. A subsequent bleaching process is often required to remove residual lignin and the extractives. Partial or complete removal of hemicelluloses is also carried out if the pulp has to be used as a raw material for chemical products. 
Pulps used for this purpose are called dissolving pulps. Pulp properties depend on the raw material, the pulping process, the extent of delignification and the adopted bleaching sequences.

\subsubsection{Morphological characteristics}

Fibre morphology affects mechanical and optical properties of paper. Morphological properties of pulpwood fibres not only differ between the wood species but they also vary within the stem [147] (cf. Figure 2.8).

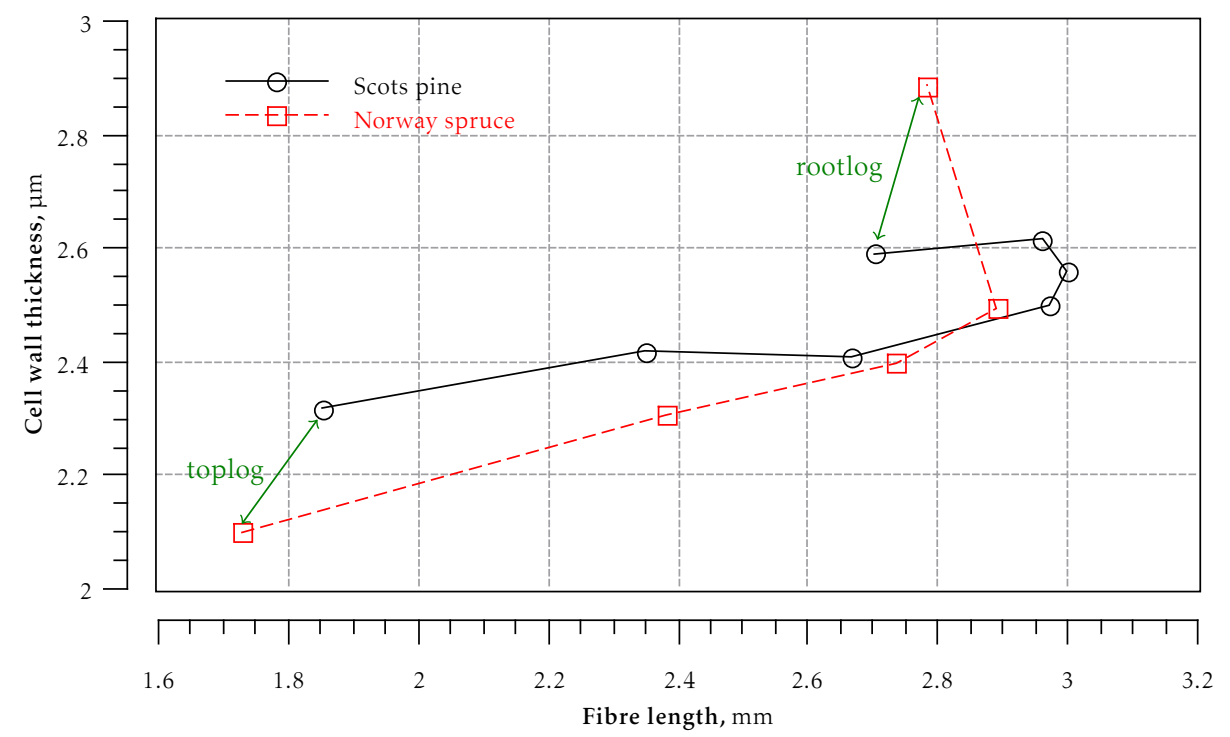

Figure 2.8: Fibre length and cell wall thickness in pulpwood logs depending on the location in the stem redrawn from [147]

Paper properties are correlated to fibre characteristics. For example, fibre length and cell wall thickness affect tensile and burst strengths whereas tearing strength is influenced by fibre wall cross section area [107]. There are also some apparent contradictions which result from the interplay of other factors. For example, cotton has longer fibres than softwood, but paper produced from it is relatively weaker. The reason to this anomaly is the deficiency of hemicelluloses. Similarly, higher lignin content reduces hydrogen bonding yet paper produced from unbleached softwood kraft is stronger than its bleached equivalent. Therefore, it can be said that properties of fibres and bonds are affected by the network structure they form during paper making process.

Morphological characteristics of fibre are presented in Figure 2.9. 


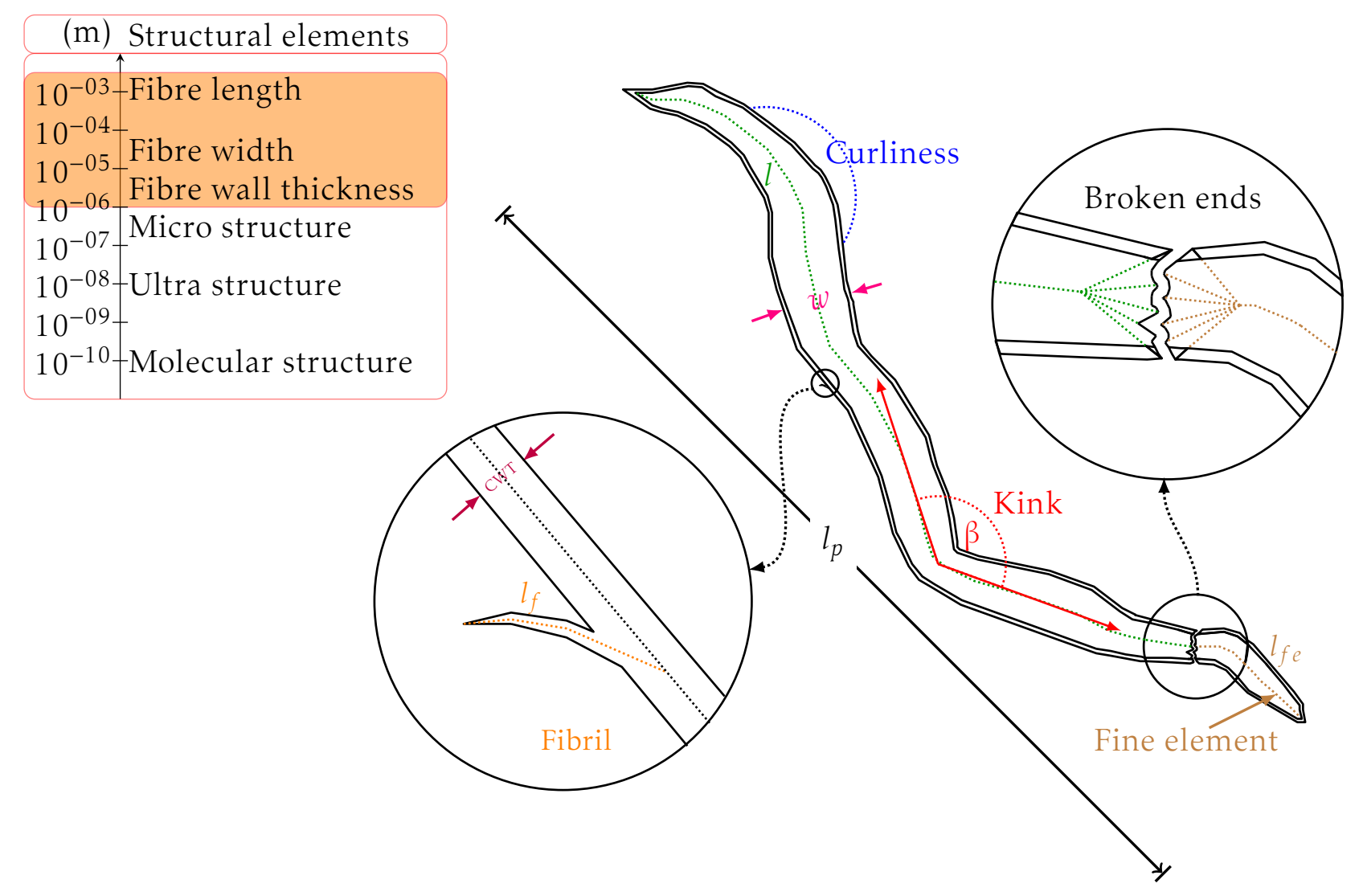

Figure 2.9: Nomenclature of morphological properties, where $l, l_{p}, l_{f e}, l_{f}, w$, $\beta$ and $c w t$ are the skeletal length, projected length, fine element length, fibril length, width, kink angle and cell wall thickness, respectively.

Arithmetic fibre length $\left\langle l_{n}\right\rangle$ can be represented as

$$
\left\langle l_{n}\right\rangle=\frac{\sum n_{i} \cdot l_{i}}{\sum n_{i}}
$$

where $l_{i}$ is the fibre length and $n_{i}$ is the number of fibres in $i^{\text {th }}$ class.

Mass-weighted average fibre length $\left\langle l_{m}\right\rangle$ can be described by the following expression

$$
\left\langle l_{m}\right\rangle=\frac{\sum m_{i} \cdot l_{i}}{\sum m_{i}}
$$

where $m_{i}$ is the mass of fibres in $\mathrm{i}^{\text {th }}$ class.

Coarseness $\langle\lambda\rangle$ of the fibres is described by the following expression

$$
\langle\lambda\rangle=\frac{\sum m_{i}}{\sum l_{i}}
$$


If coarseness of fibres is considered constant then mass-weighted average fibre length $\left\langle l_{m}\right\rangle$ coincides with length-weighted average fibre length $\left\langle l_{l}\right\rangle$ described as

$$
\left\langle l_{m}\right\rangle=\left\langle l_{l}\right\rangle=\frac{\sum n_{i} \cdot l_{i}^{2}}{\sum n_{i} \cdot l_{i}}
$$

If coarseness of fibres is proportional to fibre length then mass-weighted average fibre length becomes

$$
\left\langle l_{m}\right\rangle=\frac{\sum n_{i} \cdot l_{i}^{3}}{\sum n_{i} \cdot l_{i}^{2}}
$$

Average fibre width $\langle w\rangle$ is represented by the following relation

$$
\langle w\rangle=\frac{\sum n_{i} \cdot w_{i}}{\sum n_{i}}
$$

where $w_{i}$ is the fibre width of $\mathrm{i}^{\text {th }}$ class. Irregularities in fibre width can be described by thickenings (abrupt fibre width expansions) and thinnings (abrupt fibr width contractions).

Curl is the gradual and continuous curvature of a fibre [176]. It is usually expressed as curl index which can be represented by the following expression

$$
\mathrm{CI}(\%)=\left(1-\frac{l_{p}}{l}\right) \cdot 100
$$

Fibre kink is the abrupt change in fibre curvature. Kink angle is the obtuse angle between the two fibre segments. Kink index is usually defined by the Kibblewhite's equation [130]

$$
\mathrm{KI}=\frac{2 \mathrm{~N}_{k\left(21^{\circ}-45^{\circ}\right)}+3 \mathrm{~N}_{k\left(46^{\circ}-90^{\circ}\right)}+4 \mathrm{~N}_{k\left(91^{\circ}-180^{\circ}\right)}}{\sum l_{i}}
$$

where $\mathrm{N}_{k}$ is the number of kinks with specified kink angle range.

Number of kinks per fibre is described by kink number. It can be expressed by the following expression

$$
\text { Kink number }=\frac{\sum \mathrm{N}_{k}}{\sum n_{k}}
$$

where $n_{k}$ is the number of kinked fibres. 
Curl and kink arise from the various stresses to which fibres are exposed. They reduce the ability of fibres to carry and transmit loads however they increase deformations [105].

During mechanical treatments local damages in the fibre wall cause delamination of cell wall layers both internally (internal fibrillation) and externally (external fibrillation). The external fibrillation is determined by the fibrillation index, which can be described by the following expression

$$
\text { Fibrillation index }(\%)=\frac{\sum l_{f i}}{\sum l_{i}} \cdot 100
$$

Fines are defined here as the fragments shorter than $200 \mu \mathrm{m}$ and or slimmer than $5 \mu \mathrm{m}$. Fines content can be measured using following expression

$$
\text { Fine elements }(\%)=\frac{\sum l_{f e i}}{\sum l_{i}} \cdot 100
$$

Broken ends of fibre are the sawed-off fibre terminations [70] and are measured by Broken content which can be obtained from the following expression

$$
\text { Broken content }(\%)=\frac{\sum n_{b e}}{\frac{\sum n_{e}}{2}} \cdot 100
$$

where $n_{b e}$ and $n_{e}$ are the number of broken fibre ends and fibre ends, respectively. The denominator is equal to the number of fibres.

\subsubsection{Dewatering}

Water-fibre interactions are of great importance at all stages of paper making process (Formation, pressing and drying). Canadian standard freeness (CSF), is the ease with which water drains from pulp suspension. Schopper Riegler (SR), is the difficulty with which water drains from the pulp suspension. Drainage time (DT) is the time taken for the dewatering of pulp suspension in standard TAPPI hand-sheet former [20].

Drainage theory for the flow through the porous bed is based on the Darcy's equation [72].

$$
v=-\frac{\kappa}{\eta} \cdot \nabla \mathrm{P}
$$


where $v, \kappa, \eta$ and $\nabla \mathrm{P}$ are the Darcy's flux, permeability, dynamic viscosity and pressure gradient, respectively.

The Kozeny-Carman equation is a classical model for the flow through the fibrous bed [56].

$$
\kappa=\frac{\epsilon^{3}}{k \cdot S_{v}^{2}(1-\epsilon)^{2}}
$$

where $\epsilon, S_{v}$ and $k$ are the void volume, specific surface area and the Kozeny constant. The value of $k$ depends on the flow conditions and porous structure.

\subsubsection{Water retention value}

Water retention value (WRV) is defined as the ratio of the water retained by fibrous suspension after being centrifuged under standard conditions to its oven dry weight [121]. It describes the ease of water removal. It is a fast experimental method where the results depend upon the specific testing conditions. It may be split into the intra-fibre $\left(\mathrm{WRV}_{\mathrm{I}}\right)$ retained water and the extra-fibre $\left(W R V_{E}\right)$ bonded water. The intra-fibre $\left(W R V_{I}\right)$ retained water indicates fibre wall swelling while the extra-fibre $\left(W R V_{E}\right)$ water indicates fibrillation $[205,145,124]$.

The WRV can be expressed as

$$
\mathrm{WRV}=\left(\mathrm{WRV}_{\mathrm{E}}+\mathrm{WRV}_{\mathrm{I}}\right)=\frac{m_{1}-m_{2}}{m_{2}}
$$

where $m_{1}$ and $m_{2}$ are the mass of test specimen after centrifugation and its corresponding mass in grams after drying in an oven at $105^{\circ} \mathrm{C}$ for a constant weight, respectively. The mass of test specimen after centrifugation depends on relative centrifugal force (RCF), temperature and chemical conditions [168].

\subsubsection{Fibre saturation point}

Fibre saturation point (FSP) describes the swellability of the fibre wall. It was first defined by Tiemann [217] as the moisture content below which mechanical properties of wood increase. Later on, the definition of FSP was simplified as the moisture content at which cell wall is saturated with bound water whereas the cell wall cavities contains no free water. Babiak and Kúdela [35] described a brief review of the term and various indirect techniques used for measuring FSP.

Scallan and Stone [198] used solute exclusion to measure FSP. In this technique 
macromolecules (varying known sizes) are used to fill the pores of wet fibre wall. As the size of the macromolecule increases, the total pore volume accessible to them decreases. Thus the amount of inaccessible water increases and eventually reaches saturation. The saturation water content is called FSP. It is considered that probe molecules do not adsorb onto fibre surfaces. The principle of solute exclusion technique is shown in Figure 2.10.

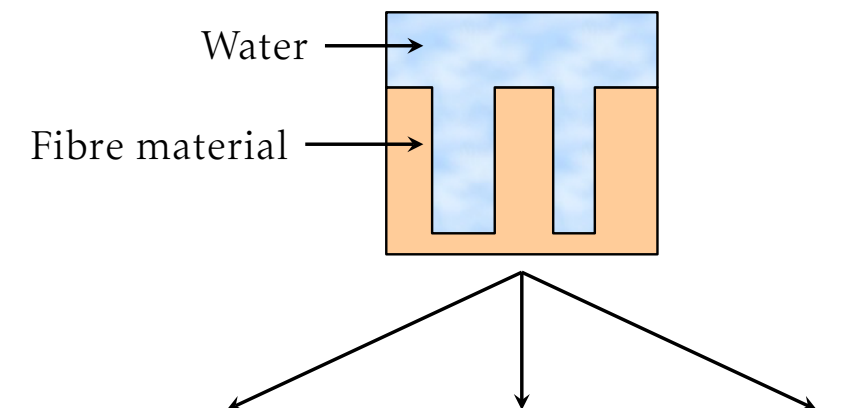

(m) Structural elements $10^{-03-F i b r e ~ l e n g t h ~}$ $10^{-04}$ Fibre width $10^{-05}$ Fibre wall thickness $10^{-06}$ Micro structure $10^{-08}$-Ultra structure $10^{-09}$

$10^{-10}-$ Molecular structure

Accessible water

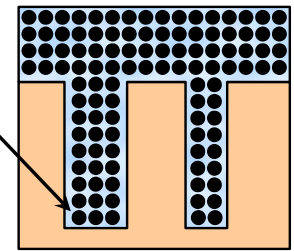

Case A

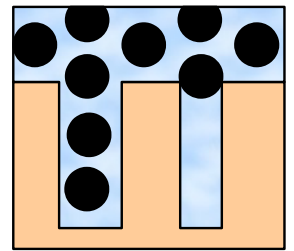

Case B

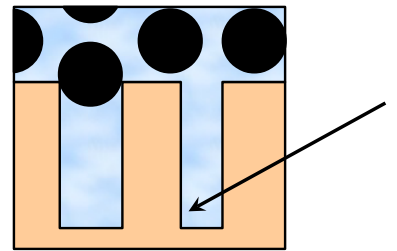

Case C

Figure 2.10: Principle of solute exclusion technique for the measurement of fibre saturation point [211]

In case $\mathrm{A}, \mathrm{B}$ and $\mathrm{C}$ the smaller sized macromolecule have total, the medium sized macromolecules have partial and larger sized macromolecules have no access to the pores of the stationary phase, respectively.

FSP is measured using following formula

$$
\mathrm{FSP}=\frac{m_{s}+m_{w}}{m}\left(1-\frac{m_{s}}{m_{s}+m_{w}} \times \frac{\mathrm{RIA}_{i}-k_{c}}{\mathrm{RIA}_{f}-k_{c}}\right)
$$

where $m_{s}, m_{w}, m, \mathrm{RIA}_{i}, \mathrm{RIA}_{f}$ and $k_{c}$ are the mass of probe solution, mass of water in pulp suspension, oven dry mass of fibres, refractive index peak area (RIA) of probe solution added, RI peak area of filtered probe solution and $k_{c}$ is the calibration constant of refractive index (RI) detector, respectively. Derivation of the expression (2.16) is described in Appendix B.

In the absence of any interaction with other macromolecules, the size of the 
macromolecule in solution is described by the Stokes-Einstein relation

$$
r_{h}=\frac{k_{b} \cdot \mathrm{T}}{6 \cdot \pi \cdot \eta \cdot \mathrm{D}}
$$

where $r_{h}, k_{b}, \mathrm{~T}, \eta$ and $\mathrm{D}$ are the hydrodynamic radius, Boltzmann's constant, temperature, solvent viscosity and diffusion coefficient, respectively. The hydrodynamic radius is an important dimension in case of flexible chains.

\subsubsection{Fibre wall pore size distribution}

Indirect methods for the determination of apparent pore volume and the pore size distribution of pulp fibres includes electron microscopy [98], mercury porosimetry, solute exclusion [214], gas adsorption [181], X-ray diffraction [141], Nuclear Magnetic Resonance (NMR) [142], Inverse Size Exclusion Chromatography (ISEC) [42] and thermoporosimetry [150].

\section{Size exclusion chromatography}

Liquid chromatography $[115,148]$ is a separation technique [218] which is based on the differential partitioning of analyte molecules between a stationary and a liquid mobile phase.

Size exclusion chromatography (SEC) is a chromatographic technique with which analyte molecules are separated on the basis of their molecular sizes [158]. Gel permeation chromatography (GPC) applies when the mobile phase contains an organic solvent. However, if the mobile phase contains water then the term gel filtration chromatography (GFC) is used. Gel filtration chromatography was first introduced by Porath and Flodin [180].

Size of the analyte molecules depends on the type of the solvent used. The selectivity of the system depends entirely on the accessibility of the pores of the stationary phase, if all interactions with the stationary phase are suppressed. The stationary phase contains tightly controlled pore sizes. Large molecules of the analyte cannot penetrate the pores and elute first while very small molecules have access to almost all intra particle volumes of the stationary phase and hence are eluted at the end.

\section{Inverse gel filtration chromatography}

Inverse gel filtration chromatography or inverse size exclusion chromatography (ISEC) [23] is a dynamic version of solute exclusion. It is a porosimetric 
chromatographic technique [93] where molecular probes of known sizes are eluted in a mobile phase through a column to determine pore dimensions of column packing. Laine [136] used this technique to determine FSP of bleached kraft fibres.

Fabrication of a homogeneous fibre bed column posed problems. Columns were either filled with ground and swollen fibres $[155,44,154]$ or with a pile of punched out discs $[224,50]$. In both techniques fibres were dried at some stage. Berthold and Salmén [42] devised an automated packing procedure with which packing of the column with never dried fibres was possible.

The limitation of ISEC is that the accessibility of the probe molecules is determined by the opening in the fibre wall [42]. Other factors influencing pore volumes are the shape of the pores, osmotic pressure and Donnan effect. Therefore it is unlikely that the pore size distribution obtained corresponds to the actual pore size distribution of the fibre wall.

\subsubsection{Weak-points in fibre wall}

In material science, a dislocation [229] is considered as an irregularity in crystal structure. Robinson [186] was first to observe the dislocated regions in longitudinally compressed wood samples under polarised light. These deformations are developed during the handling, processing and converting operations to which wood, pulp and paper is exposed. Fractures in EW fibres were found relatively higher than in LW fibres [129]. However dislocations appeared more pronounced in LW fibre due to their thicker fibre wall $[89,43]$. At the dislocated region, fibre cell wall is loosened up resulting in a change of MFA [101] and separation of microfibrils. Therefore, the accessibility of cell wall elements at the dislocations to the chemical hydrolysis by the acids, enzymes and other agents is increased $[96,31,32]$. Figure 2.11 illustrates fibre cell wall deformations. 


\begin{tabular}{|c|c|}
\hline$(\mathrm{m})$ & Structural elements \\
\hline $10^{-03}$ & Fibre length \\
\hline $\begin{array}{l}10^{-04} \\
10^{-05} \\
10^{-06} \\
10^{-07}\end{array}$ & $\begin{array}{l}\text { Fibre width } \\
\text { Fibre wall thickness } \\
\text { Micro structure }\end{array}$ \\
\hline $\begin{array}{l}10^{-08} \\
10^{-09} \\
10^{-10}\end{array}$ & $\begin{array}{l}\text {-Ultra structure } \\
\text {-Molecular structure }\end{array}$ \\
\hline
\end{tabular}

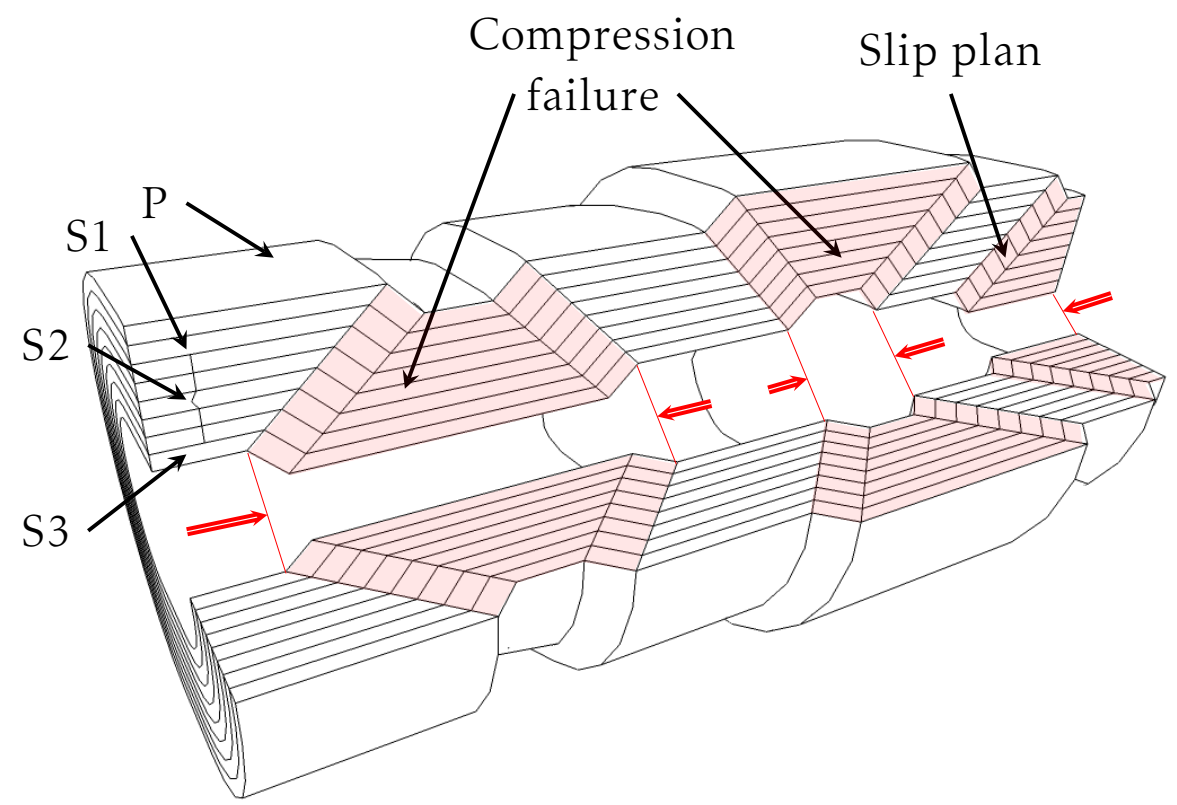

Figure 2.11: Slip plane and compression failure adapted from [227, 125]

Fibre wall deformations has been observed and characterised by polarised light [186, 227], stains [118], enzymes [31], swelling agents [209] and acid cleavage [29]. Fibre wall deformations with reference to wood fibres has been reviewed by Wilkins [238] whereas the review from Nyholm et al. [166] was focused on pulp fibres. The presence of large number of dislocations lowers the strength of fibres [174]. It has also been reported that dislocation increases with wet pressing and refining [27].

Ander and Daniel [29] quantified weak points in terms of cleavages per fibre using hydrochloric acid $(\mathrm{HCl})$. They described cleavages per fibre by the following expression [30]

$$
\text { Cleavages per fibre }=\frac{\left\langle l_{l}\right\rangle}{\left\langle l_{l c}\right\rangle}-1
$$

where $\left\langle l_{l}\right\rangle$ and $\left\langle l_{l c}\right\rangle$ are the length-weighted average fibre lengths of uncleaved and cleaved fibres, respectively.

\subsubsection{Single fibre stress-strain properties}

Measurement of single fibre property is often seen as a tedious task. Firstly, it is time consuming and secondly the variability of the test results obtained is quite large. To avoid any error in measurement and damage to the fibre, great 
attention is required while preparing and mounting the samples for testing. Fibre deforms, the internal structure rearranges and finally breaks under the action of the applied tensile stress. Hooke's law applies to the initial elastic region

$$
\sigma=\mathrm{E} \cdot \varepsilon
$$

where $\sigma, \varepsilon$ and $\mathrm{E}$ are tensile stress, tensile strain and the modulus of elasticity, respectively. E is the measure of the fibre's resistance towards the change in length under the application of tensile stress.

Tensile stress is defined by the following expression

$$
\sigma=\frac{\mathrm{F}}{\mathrm{A}}
$$

where $\mathrm{F}$ and $\mathrm{A}$ are the applied force and fibre cell wall cross section area (excluding lumen) under tensile stress.

Strain is the measure of deformation. Tensile strain can be represented by the following expression

$$
\varepsilon=\frac{\Delta l}{l}
$$

where $\Delta l$ and $l$ are the change in length and initial length of fibre under tensile stress, respectively.

Cintrón et al. [60] summarised various method used to determine the elastic modulus of native cellulose both experimentally and theoretically from molecular modelling. Experimentally determined values of the cellulose crystalline region vary from 120 to $143 \mathrm{GPa}$ in the longitudinal direction [192, 157, 165, 216].

Page et al. [175] have shown that the modulus of elasticity of the defect free fibres lowers with the increase of MFA (cf. Figure 2.12). Fibres with a higher modulus of elasticity show higher tensile strengths [172]. 


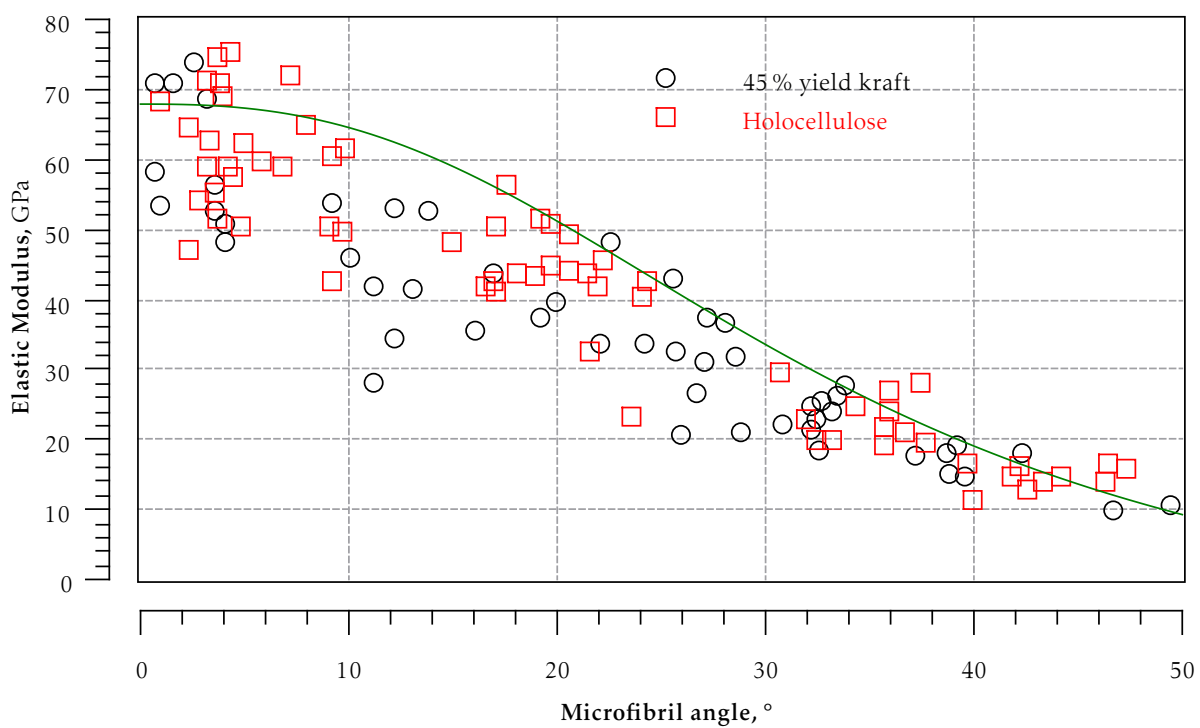

Figure 2.12: Elastic modulus vs microfibril angle redrawn from [175]

Due to higher percentage of S2 layer and lower MFA, the tensile strength and modulus of elasticity of LW fibres is generally higher than that of EW fibres [194]. Fibre defects like curl, kink, crimps and micro-compressions cause non linearity in the stress-strain curve (cf. Figure 2.13) [176].
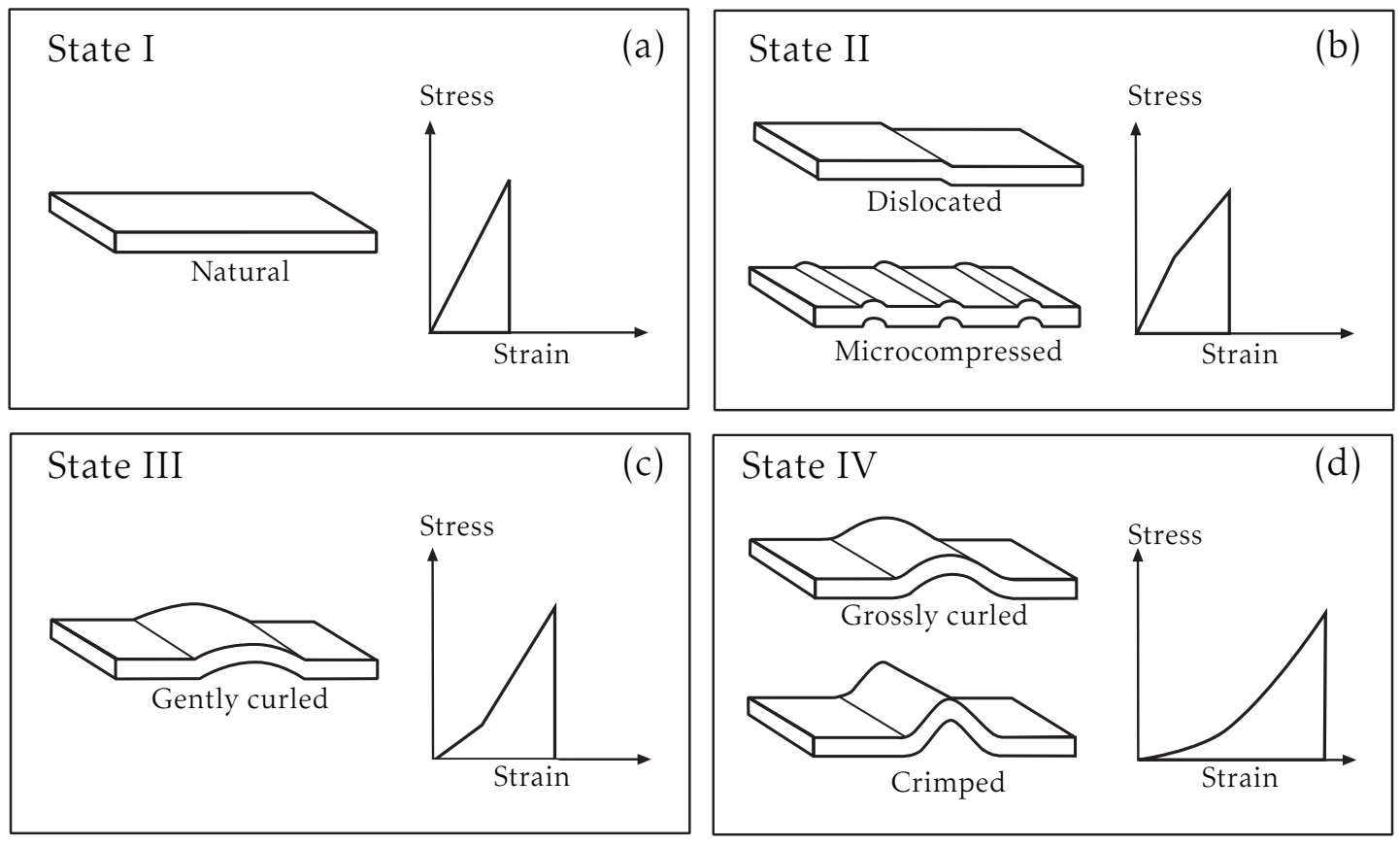

Figure 2.13: Shapes of stress-strain curves due to fibre deformations [176, 103]

Weak points in fibre wall like pores, dislocations and cracks lower modulus of elasticity. The increase in elastic modulus after drying and rewetting is due to 
the development of hydrogen bonding between the microfibrils [199].

\subsection{Pulp refining}

Pulp is refined to achieve properties suitable for paper making. In this process, pulp suspension is forced to pass through a narrow gap between two surfaces containing protruding bars and grooves. The gap clearance between the bars in low consistency ( 3 to $5 \%$ ) refining is around $100 \mu \mathrm{m}$ [146]. In a refiner, either one surface rotates or both of them rotate.

During refining, several mechanisms occur simultaneously [171] ; fibres undergo compression, bending, shear and tension. Resulting in internal fibrillation, external fibrillation [92], cutting, generation of secondary fines, fibre bruising, fibre curling or straightening and redistribution of hemicellulose in the fibre wall (cf. Figure 2.14) [126]. Internal fibrillation increases fibre flexibility and hence relative bonded area (RBA) is increased [146]. The effect of refining on wet fibre flexibility is however much smaller than that of pulping yield [102].

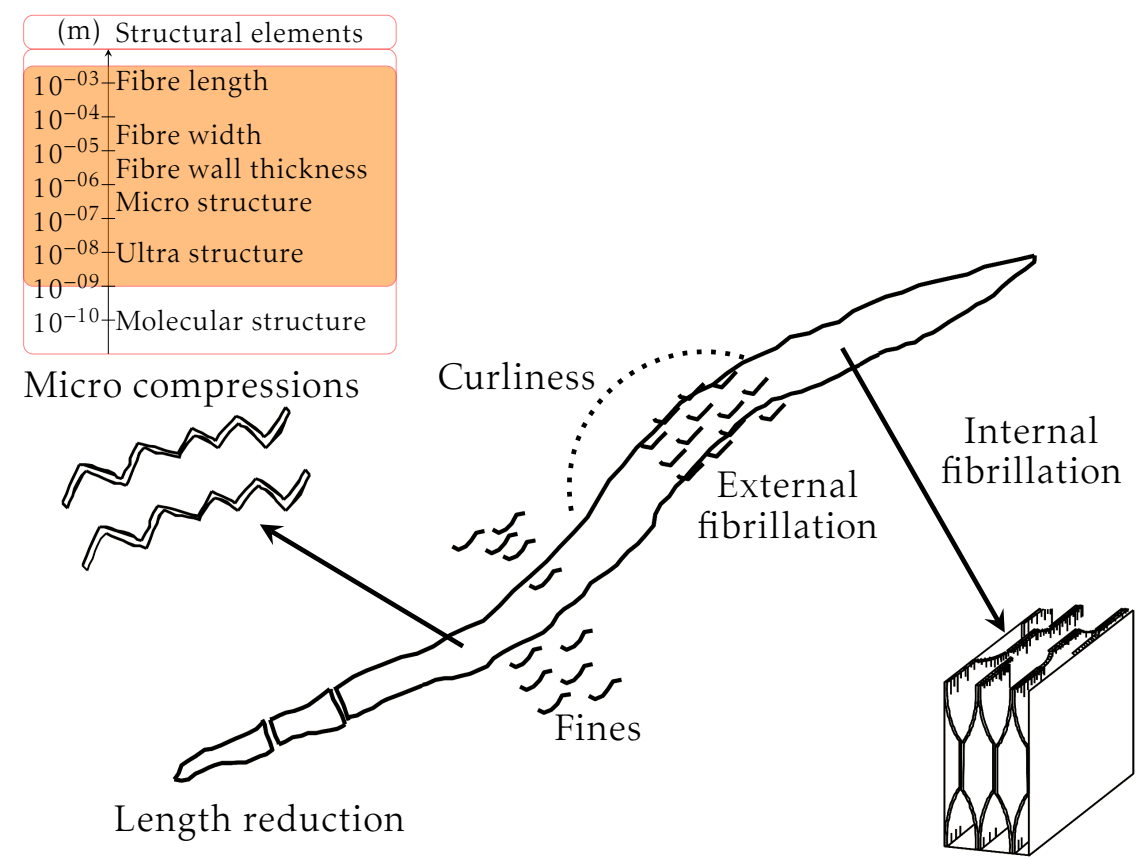

Figure 2.14: Schematic representation of refining effects on fibre characteristics $[126,132]$

The process of refining can be described by comminution theories as proposed by Rittinger's [184], Kick's [131] and Bond's [45]. 
Rittinger's law states that the energy consumed is proportional to the new surface created. Kick's theory relates it to the relative reduction in size whereas Bond's theory considers it proportional to the total length of new crack formed during size reduction.

Refining of fibres involves smaller particles therefore Rittinger's law [106] is often considered applicable when breaking happens due to the failure of bonds in the plastic region [57].

Mechanical properties and strength of chemical pulp fibres change inconsistently with refining. Harsh industrial refining induce fibre deformations whereas gentle laboratory refining may remove structural discontinuities [183].

\subsection{Paper making}

Figure 2.15 shows a typical paper machine where a dilute pulp suspension (99\% water content) is distributed from the headbox at an even flow rate across the width of a moving wire.

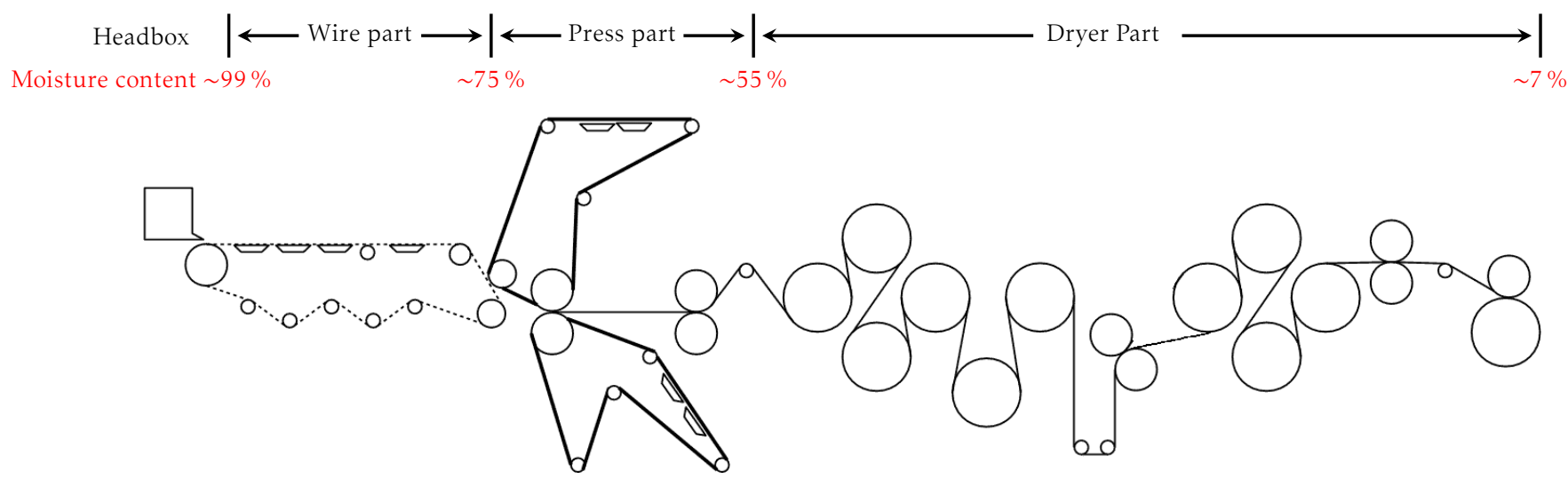

Figure 2.15: Schematic diagram of a Fourdrinier paper machine

Dewatering in the wire part takes place under the action of gravity, the blades, the pressure of forming rolls (twin wire former) and the vacuum. Retention of fines and flocculation affect dewatering. Densification of the fibrous mat intensifies in press section. The applied stress during pressing is balanced by the hydraulic pressure and the network stress. While hydraulic pressure is responsible for the flow of water, the network stress deforms the fibre network accordingly. Runnability of the paper machine is dependant on wet web strength. Web strength improves with the increase in the solid content. Figure 2.16 summarises the impact of morphological properties on wet web strength. 


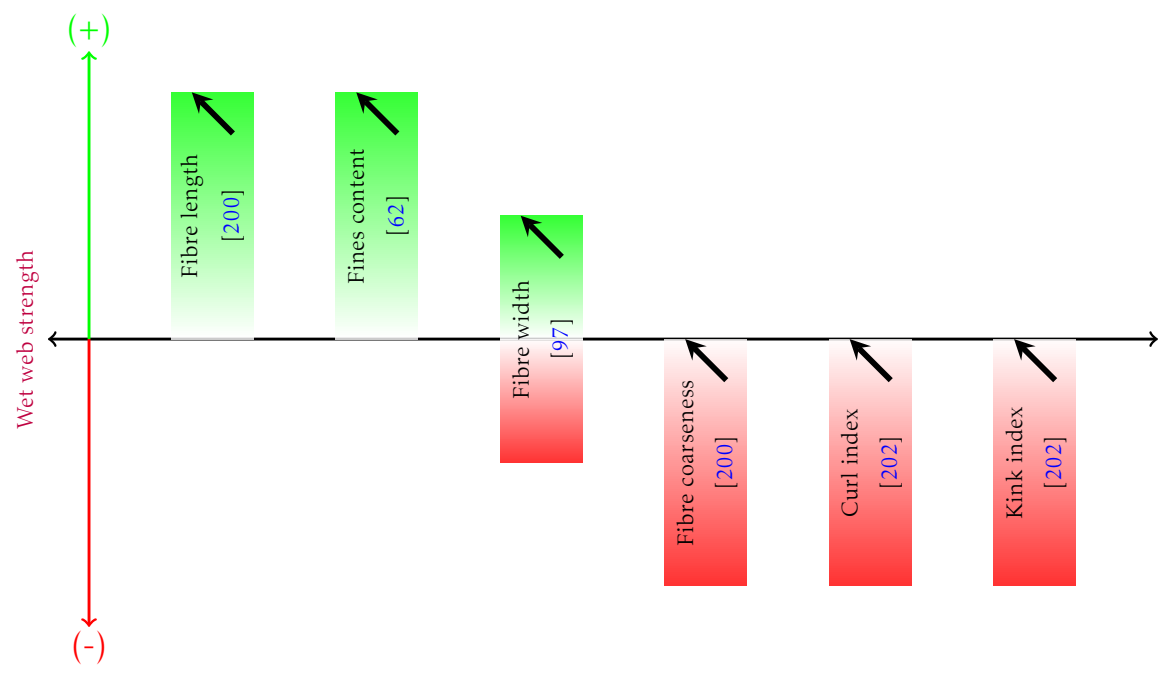

Figure 2.16: Effect of morphological properties on wet webs strength

Lyne and Gallay [149] showed that wet strength of a web (20 to $30 \%$ solids) is controlled by surface tension. Page [173] described wet web strength by following expression

$$
\mathrm{F}=\frac{\mu \cdot \gamma \cdot \mathrm{FP} \cdot l \cdot \mathrm{RBA}}{12 \mathrm{FA} \cdot \rho \cdot r}
$$

where $\mathrm{F}, \mu, \gamma, \mathrm{FP}, l, \mathrm{RBA}, \mathrm{FA}, \rho$ and $r$ are tensile index, coefficient of friction, surface tension, fibre cross section perimeter, fibre length, the proportion of fibre surface contained within a water meniscus of radius $r$, fibre cross section area, fibre density, radius of curvature of water meniscus, respectively.

It seems generally accepted that surface tension pulls fibre closer through Campbelleffect [52]. With the gradual removal of water the Campbell-effect transforms into intermolecular forces which enhances the web consolidation [221]. During drying, water is removed by evaporation and vapour transport. The mechanism of forming bonds between the two fibres is still a speculative issue. Figure 2.17 shows the probable mechanisms responsible for fibre-fibre bonds in the contact area. Mechanical interlocking arise from the irregularities of the two surfaces. Interdifusion is the situation when polymer molecules co-resides in two opposite bulk phases. Hydrogen bond, induced dipoles and ionic bonds are the intermolecular interactions. 


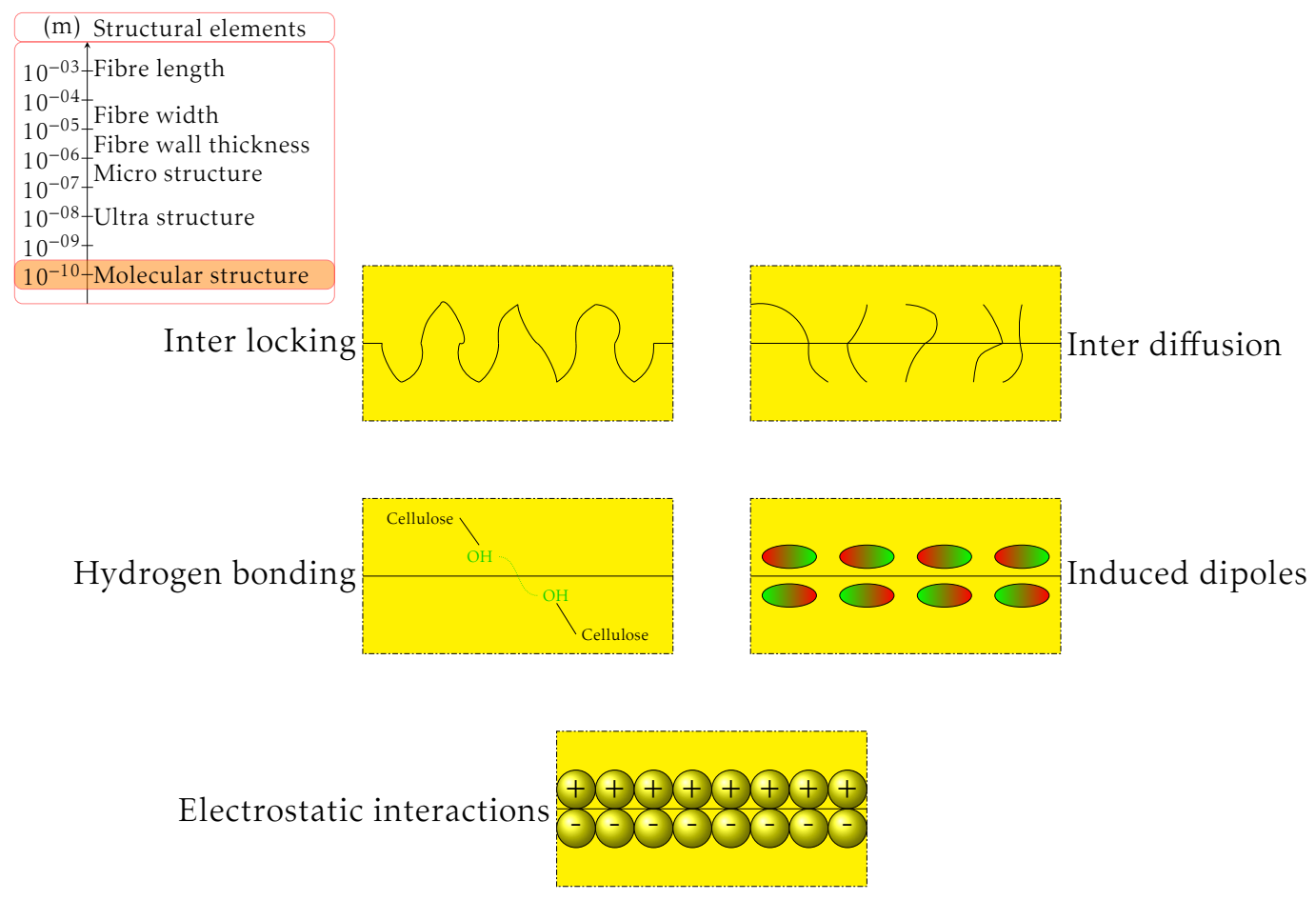

Figure 2.17: Interactions responsible for specific bond strength adapted from [144]

During drying, EW fibres tend to collapse whereas LW maintain their shape due to their thick wall (cf. Figure 2.18).

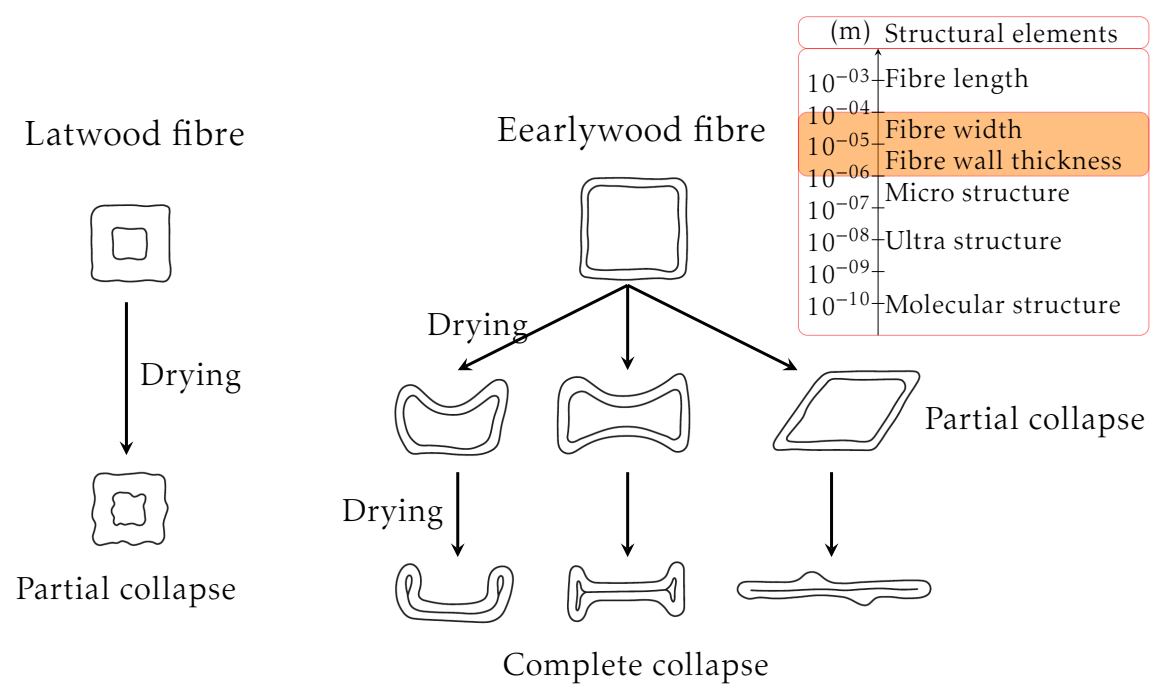

Figure 2.18: Schematic depiction of fibre collapse during drying [119]

Since the drying is restrained, the lateral shrinkage of fibres is transmitted to the axial shrinkage of the connected fibres in the bonded area. The slack fibre segments are straightened (cf. Figure 2.19). This fibre segment activation 
behaviour is called Giertz effect [91].

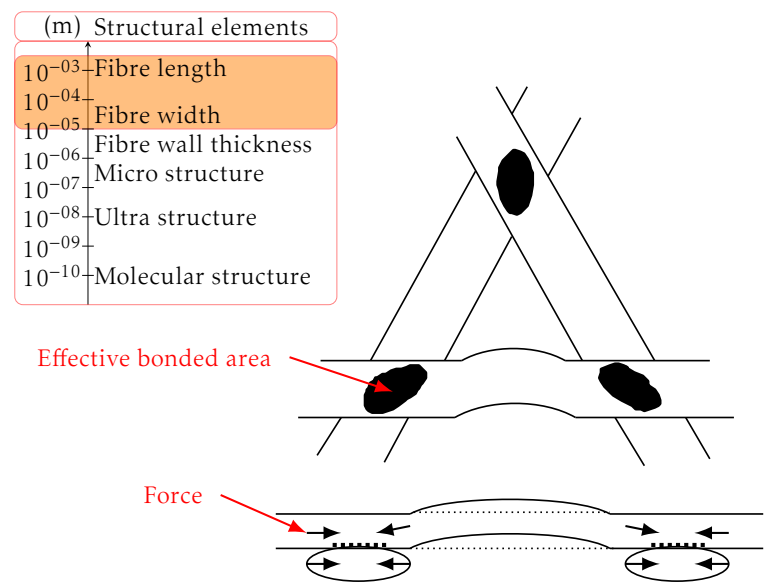

Figure 2.19: Fibre straightening during sheet drying [201]

Fibre straightens, the dislocations and other local fibre defects disappear and microfibril angle decreases during restrained drying. The internal rearrangement of the structure caused by the internal stresses during restrained drying (cf. Figure 2.20) is called Jentzen effect [122]. As a result, the tensile strength and the elastic modulus of paper is increased.

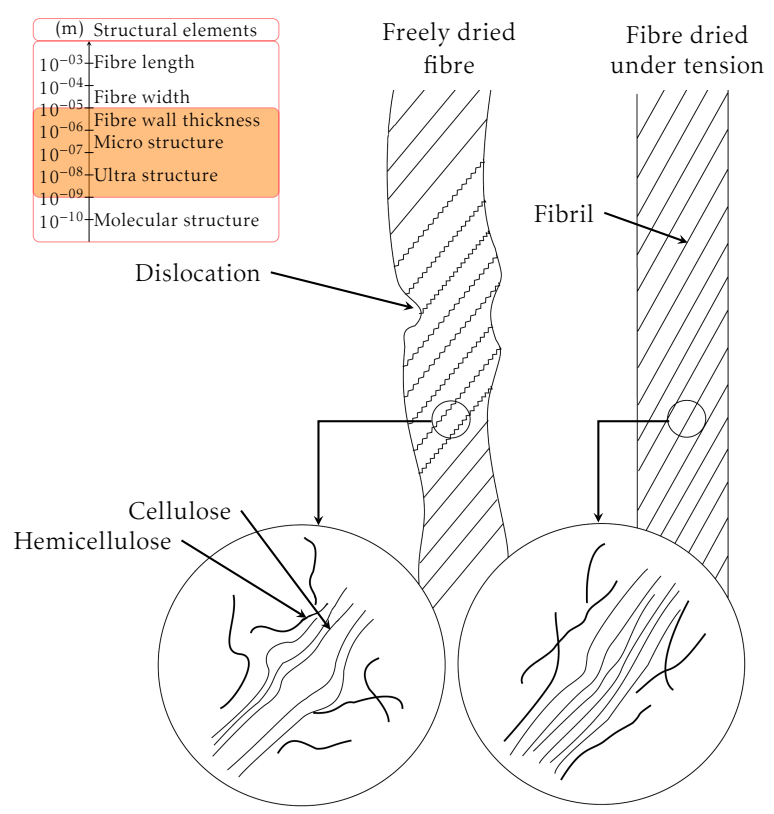

Figure 2.20: Structural changes involved in the Jentzen effect at fibre wall level [183]

The structural and molecular rearrangement of the fibre wall during drying is influenced by the amount of hemicellulose contained in the matrix. Pulkkinen 
and Alopaeus [182] have recently defined and correlated activation coefficient (see subsection 5.1.2) to the tensile properties of the paper produced from refined pulps.

\subsection{Paper recycling}

Figure 2.21 shows the comparison of the total wood pulp production with reference to the area of utilisation. It can be seen that currently only about $5 \%$ of the total wood pulp is used in the chemical industry. This ratio will increase in future with the development of biorefineries.

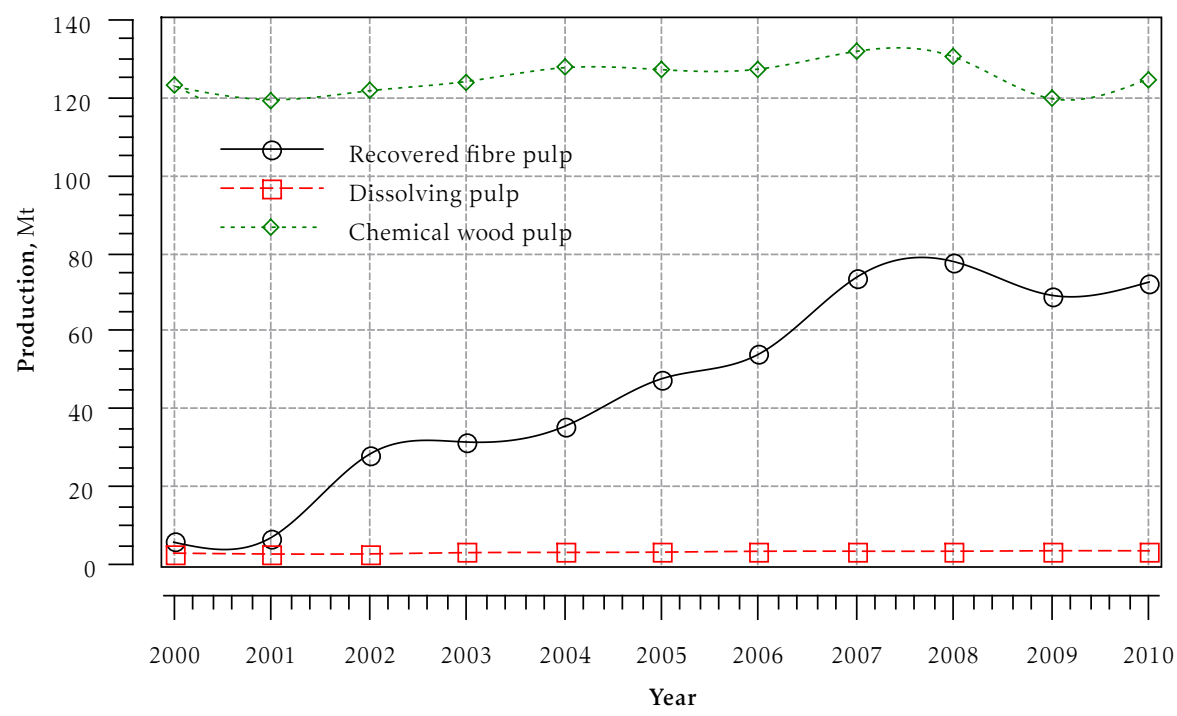

Figure 2.21: Evolution of worldwide production of wood pulps [80]

Paper recycling is the process of reusing recovered waste paper. One complete recycle includes reslushing/disintegration, deinking, washing, refining, pressing and drying operations. Besides refining, pressing and drying operations, all other operations have little or no significant effect on the morphology and/or physical characteristics of recovered fibres. Strength, swelling and optical properties, tend to deteriorate during recycling process. Modern deinking systems can efficiently remove contaminants, mechanical refining regenerates surface fibrils while modern paper making machines can enhance sheet strength and surface properties to some extent.

Although additives and modern processing technology compensate the deteriorated quality of recovered fibres, they contribute to higher product cost.

Waste paper can be classified on the basis of origin, quality and usage. Pre- 
consumer waste comes from paper converting plants whereas post-consumer waste is collected by municipal from supermarkets, department stores, other large shops, households and offices.

The four main categories of waste paper are

1. Pulp substitutes: converting and printing machines trimmings, shavings etc.

2. Deinking grades: old newspapers (ONP), old magazines (OMG), woodfree printed grades, computer printout (CPO), mixed office waste (MOW) or office mixed paper (OMP), sorted office paper (SOP) etc.

3. Brown kraft grades: corrugating plant waste, old corrugated containers (OCC), kraft lined strawboard (KLS), used kraft sacks, and converting waste etc.

4. Mixed waste paper (MWP): other than the previously described.

For the classification of waste paper, the standards followed in America and Europe are PS-2006 and BS EN 643:2002, respectively.

Almost all earth's resources are limited, efficient use along with recycling is required more than ever before. A lot of emphases is given on using recovered fibres as a replacement of virgin fibres due to economical and environmental reasons. Data from the FAO [167] on the production and trade of recovered pulp and paper for 2010 is given in Table 2.5.

Table 2.5: Production and trade of recovered pulp and paper in 2010 [167]

\begin{tabular}{l|ccc|ccc}
\multirow{2}{*}{2010} & \multicolumn{3}{|c|}{ Recovered fibre pulp, Mt } & \multicolumn{3}{c}{ Recovered paper, Mt } \\
\cline { 2 - 7 } & Production & Imports & Exports & Production & Imports & Exports \\
\hline Africa & - & - & 0.01 & 1.61 & 0.08 & 0.09 \\
America & 3.06 & 0.03 & 0.11 & 40.61 & 5.46 & 23.96 \\
Asia & 54.73 & 0.07 & 0.01 & 86.04 & 35.87 & 7.25 \\
Europe & 12.83 & 0.16 & 0.25 & 55.43 & 14.84 & 23.20 \\
Oceania & 1.99 & - & - & 1.99 & 0.01 & 1.59 \\
\hline World & 72.62 & 0.26 & 0.38 & 185.68 & 56.26 & 56.07
\end{tabular}

Asia was the main importer and consumer of recovered pulp and paper. 


\subsubsection{Recycling rate}

Recycling rate is an indicator used to express the recycling of recovered paper and paper-board. Recycling rate [63] is defined by the following expression

$$
\text { Recycling rate }=\frac{\text { mass of recovered paper used for recycling }}{\text { mass of paper consumed }}
$$

Recovery rate represents the apparent consumption of paper that is collected as waste paper.

$$
\text { Recovery rate }=\frac{\text { mass of waste paper recovered }}{\text { mass of paper consumed }}
$$

The term recycling rate in the European Declaration is comparable to the term recovery rate used in the U.S. and their evolutions are shown in Figure 2.22.

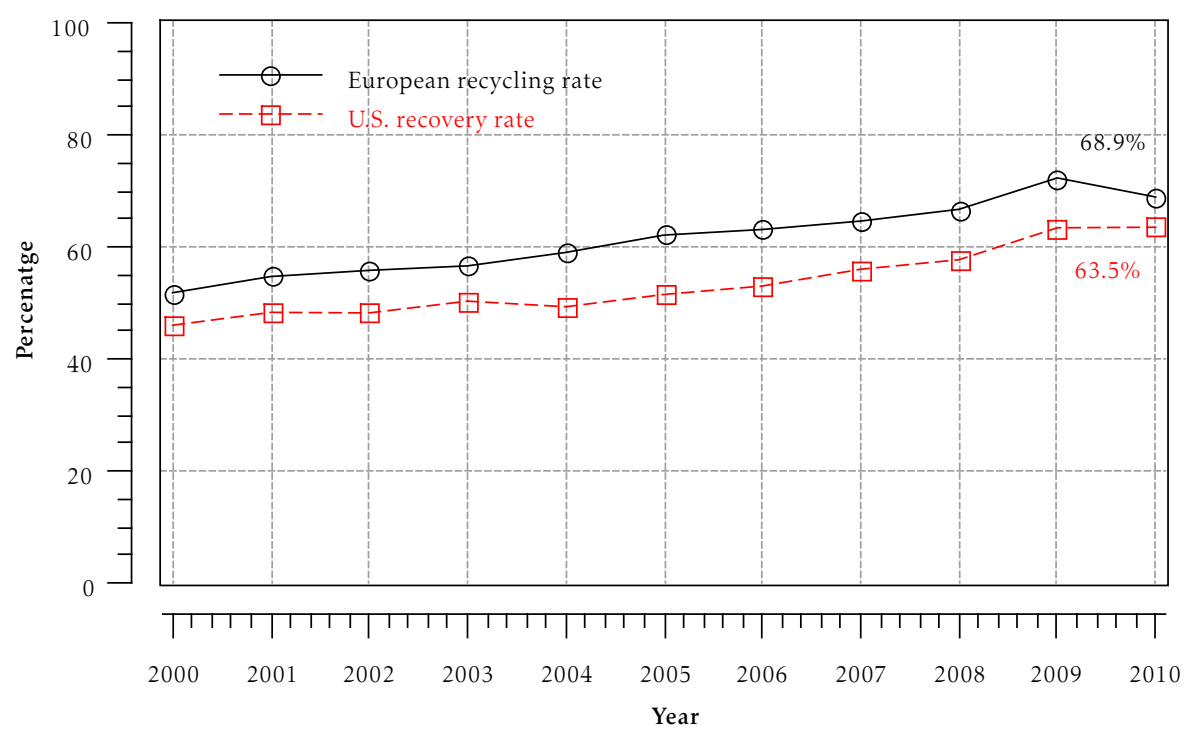

Figure 2.22: Evolution of recycling rates of Europe and USA [64],[65]

In 2006, European recycling rate was $63.4 \%$ and it reached its highest point of $72.2 \%$ in 2009. In 2010, it was slightly lowered to 68.9\% [64]. Similarly, recovery rate of U.S. consumed paper reached highest point ever, 63.5\% [65] in 2010.

The utilisation rate describes the amount of recycled fibres present in paper.

$$
\text { Utilisation rate }=\frac{\text { mass of recovered paper consumed }}{\text { mass of paper produced }}
$$

In 2010, the utilisation rate of recovered paper in Europe was 50.7\%. Only 
$11.4 \%$ of recovered paper was used in high grade papers [61].

\subsubsection{Effects of recycling on pulp, fibres and paper properties}

The irreversible loss of wet fibre flexibility and bonding ability of lignocellulosic materials caused by the removal of water during pressing and drying is generally attributed to fibre hornification [231]. The term hornification, expressed as the drop in WRV was first coined in 1944 by Jayme [117]. Once hornified, fibres can not regain their original swollen state even after rewetting [160]. The drop in WRV with multiple recycling relates to the fibre wall thickness. Its correlation with fibre width was however not established [38, 233]. Some studies show that irreversible fibre wall collapsing, pore closure [210, 211] and reduction in surface area [133] during drying are responsible for the drop in WRV. Other studies describe the aggregation of cellulose microfibrills $[120,111,195]$, formation of lactones $[190,36,74]$ and an increase in crystallinity [120] during pressing [151, 54] and drying [185].

Most published works seem to agree that the strength loss in low yield pulps during recycling is generally caused by fibre hornification. This effect was originally thought to be induced by the removal of lignin [199]. However, many researchers have found that the pentosans present in the pulp fibre wall play a crucial role in the recyclability $[53,34]$. Loss of strength in pulps with higher pentosans content during recycling is lower than the pulps with relatively lower amounts of pentosans $[53,58]$. Pressing and drying close most of the macropores whereas micropores of the cell wall remain unaffected [214]. However, it is also reported that hornification can partially collapse the micropores [150,153]. Similar effects have also been reported for non-wood fibres but at a reduced intensity because of the presence of higher pentosan content [59]. Recycling has been reported to decrease tensile and burst strengths whereas tear strength improves. Higher pentosan content not only hinders the decrease in tensile and burst strengths but it also prevents increase in tear strength [58]. Drop in tensile strength of hardwoods is relatively higher compared to softwoods. The irreversible reduction in pore volume and pores size are the responsible factors for the increase in dewatering, evaporation and vapour diffusion rates [25].

Howard and Bichard described in details the basic effects of recyclings on pulp properties [108]. A comprehensive review on the effect of paper making and recycling processes on the cellulosic fibres can be found in [109]. 


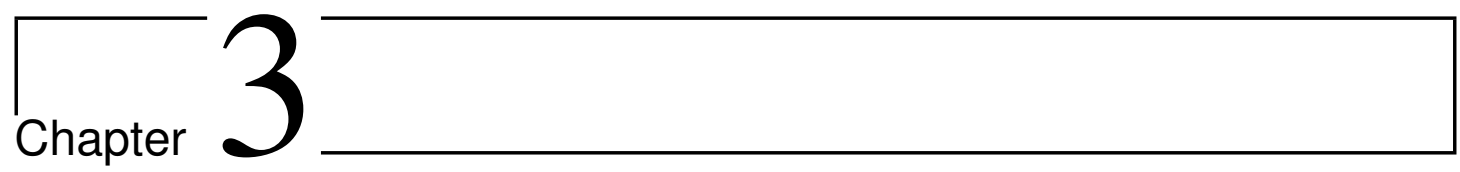

\section{Experimental methods}

\section{Contents}

3.1 Refined pulps and fibres . . . . . . . . . . . . 35

3.1.1 PFI mill refining of pulps . . . . . . . . 35

3.1.2 Valley beater refining of pulps . . . . . . . . 36

3.2 Morphological properties of refined pulps and fibres . . . . 37

3.3 Dewatering of refined pulps and fibres . . . . . . . . 43

3.3.1 Schopper Riegler of refined pulps and fibres . . . . . . . 43

3.3.2 Dewatering rate of refined pulps and fibres . . . . . . 46

3.3.3 Specific filtration resistance of refined pulps and fibres 48

3.4 Water retention value of refined pulps and fibres . . . . . . 49

3.5 Fibre saturation point of refined pulps and fibres . . . . . . 51

3.5.1 Preparation of mobile phase . . . . . . . . . . . 51

3.5.2 Fabrication of the column ........... 51

3.5.3 Calibration of differential refractive index detector . . . 53

3.5.4 Solute exclusion . . . . . . . . . . . . . . 55

3.5.5 Gel filtration chromatography . . . . . . . . . . . 55

3.6 Fibre wall pore size distribution of refined pulps and fibres . 56

3.7 Wet breaking length of refined pulps and fibres . . . . . . 59

3.8 Weak points in fibre wall of refined pulps and fibres . . . . 61

3.9 Conclusions of pulp refining $\ldots \ldots \ldots \ldots$

In order to characterise recycled pulp and fibres, the available methods and techniques were optimised for refined pulps and fibres. 


\subsection{Refined pulps and fibres}

Air dried sheets of pulp (SK100) produced from norway spruce and maritime pine were used as a starting material. Sheet borders were discarded and the left overs were hand-shredded into small pieces ( 2 to $4 \mathrm{~cm}$ ).

Block diagram of the preparation process is shown in Figure 3.1.

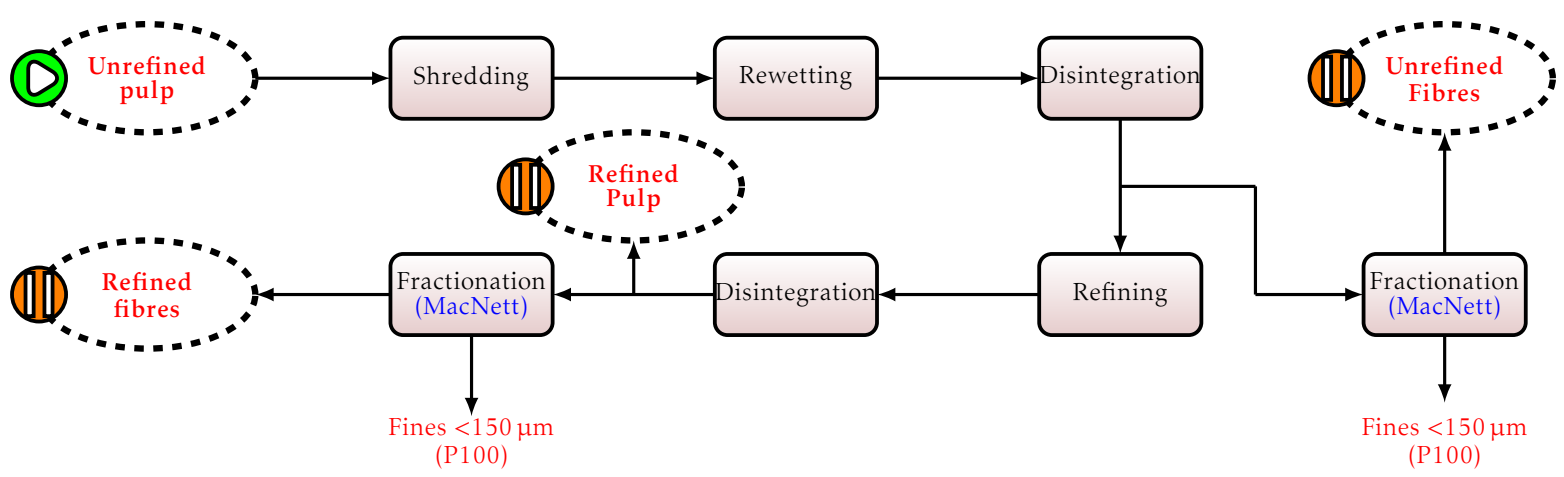

Figure 3.1: Preparation of refined pulps and fibres

Hereafter pulp will be used in place of whole pulp and fibres in place of the fibre fraction of the whole pulp obtained after fractionation.

\subsubsection{PFI mill refining of pulps}

PFI mill is a laboratory refiner. A compressive shear force between a bar roll and a smooth housing beat medium consistency pulp. The degree of refining is controlled by the number of revolutions and is generally reported as PFI revolutions. Refining action of PFI mill is different than that of conical and disc refiners. It was found that PFI mill applies more compressive and shear stress causing higher internal fibrillation and less fibre shortening than industrial refiners [127].

$32 \mathrm{~g}$ of air dried pulp excluding the edges was soaked overnight in two litres of normal tap water. Pre-soaked pulp was then disintegrated in Adamel Lhormargy disintegrator for 30000 revolutions [14]. Extra water was removed using $589^{1}$ Black ribbon S\&S filter paper placed on Büchner funnel fitted with a suction flask. Consistency of the pulp suspension was adjusted to $100 \mathrm{~g} \cdot \mathrm{L}^{-1}$ using normal tap water and mixed well. Refining of the pulp was carried out in Metrotec NPFI 01/04 PFI mill refiner [12]. Pulp was spread evenly along the inside wall of the refiner housing. The cover was placed and pressed firmly over the beater housing. Pulp was beaten at the required number of revolutions 
between the roll bars and the housing, both rotating in the same direction, but at different peripheral speeds under a standard load (cf. Figure 3.2). The beating process stops at the pre-set number of revolutions, automatically. Refined pulp samples were taken out from the beater housing carefully.

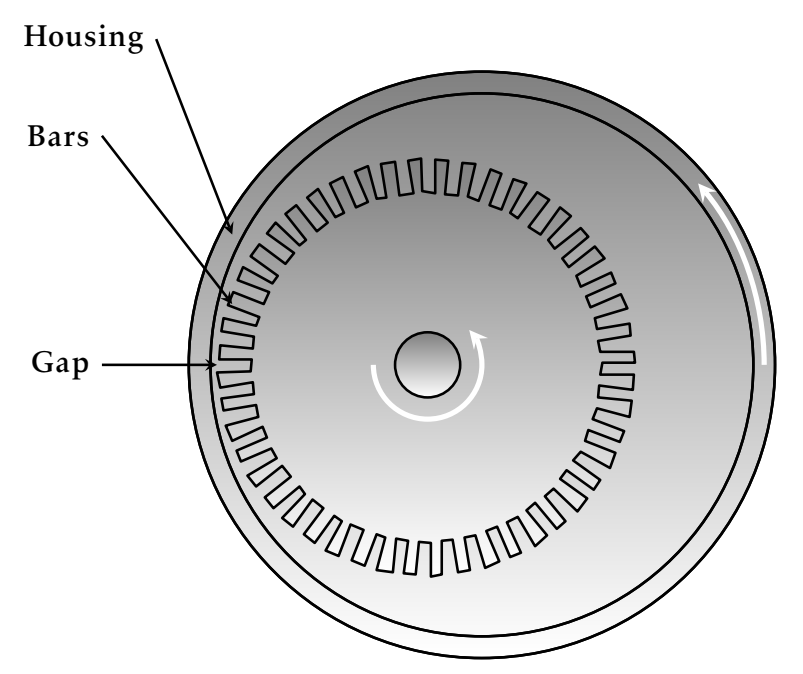

Figure 3.2: Working principle of PFI mill adapted from [114]

Consistency of the refined pulp was reduced to $15 \mathrm{~g} \cdot \mathrm{L}^{-1}$ and disintegration was carried out using Adamel Lhormargy disintegrator for 6000 revolutions. Fines were removed from refined pulp using Bauer Bros McNETT fibre classifier [17]. P100 represent fine elements (smaller than $150 \mu \mathrm{m}$ ) as a 100 mesh screen was used for their removal. Dewatering was carried out using $589^{1}$ Black ribbon S\&S filter paper placed on Büchner funnel fitted with a suction flask. Consistency was adjusted to $100 \mathrm{~g} \cdot \mathrm{L}^{-1}$ using normal tap water.

\subsubsection{Valley beater refining of pulps}

Besides PFI mill, Valley beater is another refiner which is used in the laboratories. Frequent calibration to maintain standardization adds to its disadvantage over PFI mill. Pulp at low consistency is circulated through beater roll where a bed plate connected to a lever arm applies load (cf. Figure 3.3). Fibres undergo refining while passing through the beater roll and bed plate. The degrees of refining is controlled by applied load and beating time. Refining action of Valley beater is also different than that of industrial refiners.

$436 \mathrm{~g}$ of air dried pulp excluding the edges was soaked overnight in $26 \mathrm{~L}$ of normal tap water. Pre-soaked pulp suspension was then disintegrated in "pulpeur inox ALLIBE" disintegrator for 5 min (6000 revolutions). Pulp was then slowly 
poured into the "VOITH Valley laboratory" beater [1]. A weight of $5.5 \mathrm{~kg}$ was positioned at the end of the bed-plate lever arm which corresponds to a load of $105 \mathrm{~N}$ upwards against the roll. $100 \mathrm{~cm}^{3}$ of sample were drawn at regular intervals to check the degree of refining. When the required degree of refining was attained the required amount of the pulp was withdrawn. Load on the arm was then reapplied and the same steps were repeated to obtain pulps of various refining degrees.

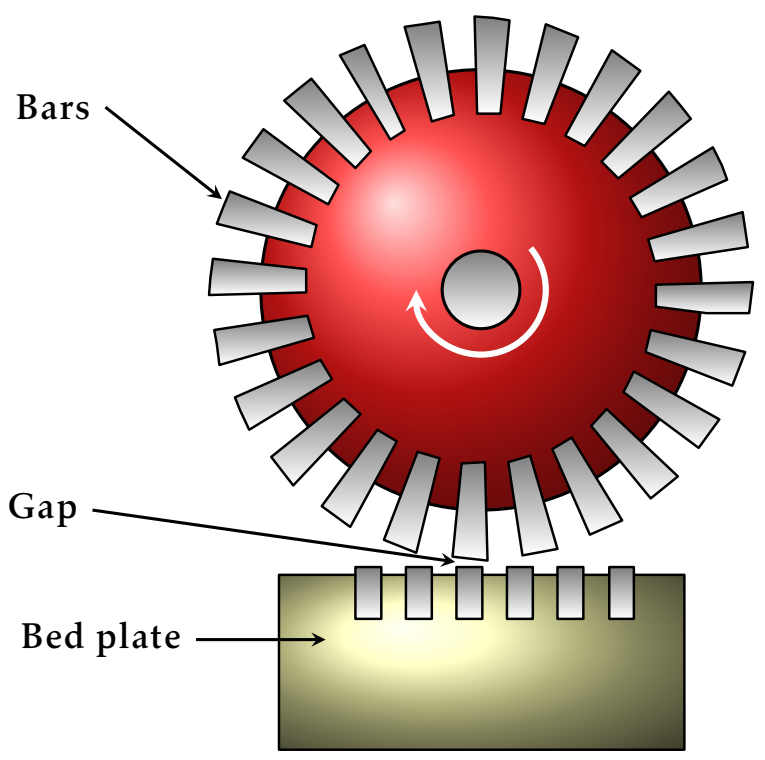

Figure 3.3: Working principle of Valley beater

At the end of the beating, rest of the pulp was taken out from the beater. Disintegration was carried out using "pulpeur inox ALLIBE" disintegrator for $5 \mathrm{~min}$ (6000 revolutions). Fines were removed from refined pulp using Bauer Bros McNETT fibre classifier. Dewatering was carried out using $589^{1}$ Black ribbon S\&S filter paper placed on Büchner funnel fitted with a suction flask. Consistency was adjusted to $100 \mathrm{~g} \cdot \mathrm{L}^{-1}$ using normal tap water.

\subsection{Morphological properties of refined pulps and fibres}

Morphological analysis of refined pulps and their corresponding fibres was carried out using MorFi LB01 [178]. The pulp suspension is recirculated and the flow go to the measurement cell where images are acquired. Specific image analysis is performed to determine the morphological parameters like length, 
width, curl etc. (cf. Figure 3.4). It is an apparatus to completely characterise fibres, shives and fine elements. As the measurement is carried out in water, all the morphological results are given for wet fibres.

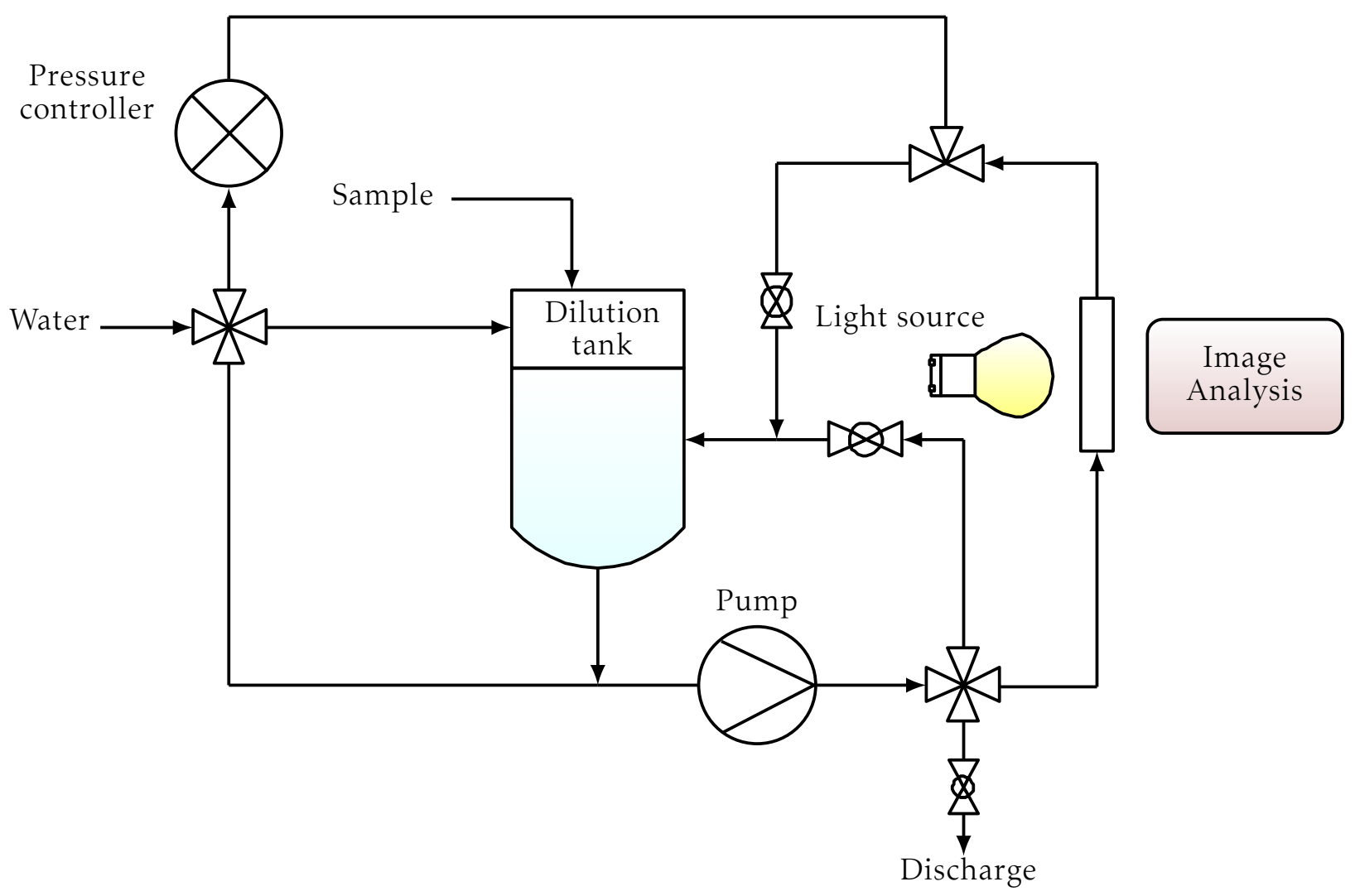

Figure 3.4: Working principle of morphological analyser (MorFi LB01)

Disintegrated pulp and fibres was diluted to form a suspension of $0.3 \mathrm{~g} \cdot \mathrm{L}^{-1}$. $1 \mathrm{~L}$ of the prepared suspension was used for one measurement. Three measurements were carried out for every sample. The value is represented as an average of three measurements. For each measurement more than 5000 fibres are analysed. Fibres are defined as objects 0.2 to $10 \mathrm{~mm}$ long and 5 to $75 \mu \mathrm{m}$ wide. Here it is important to note that morphological analysis of pulp takes into account the fibre portion of the whole pulp.

The effect of refining on length-weighted fibre length is shown in Figure 3.5. 


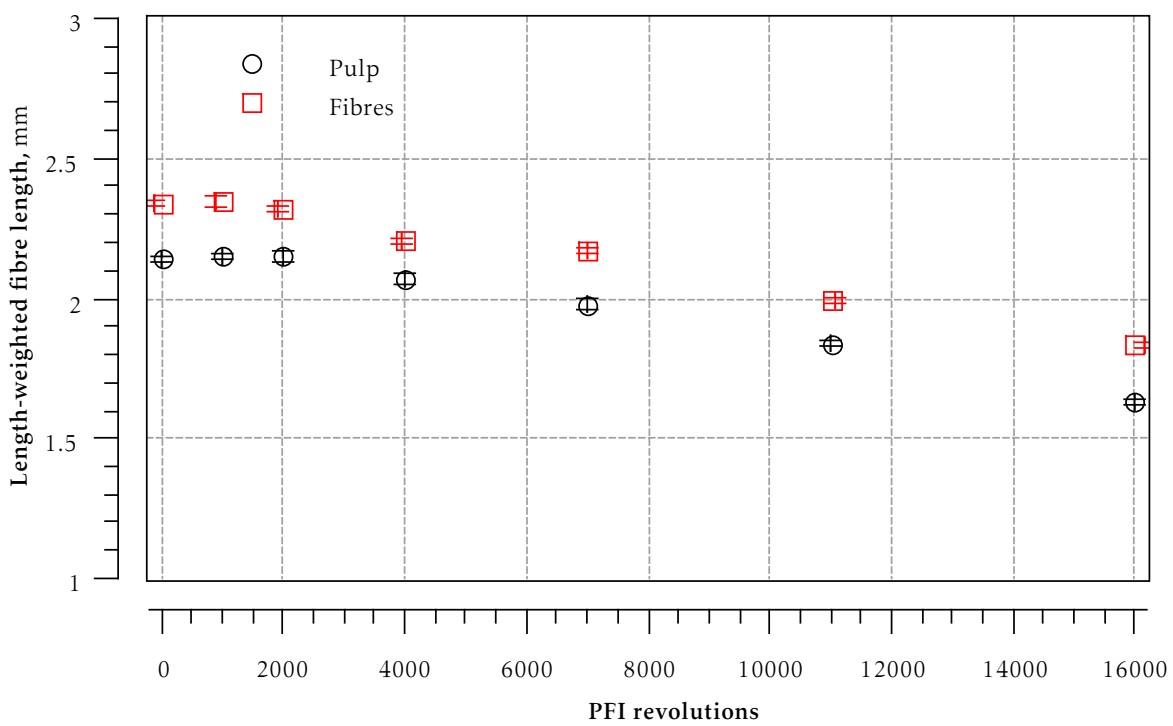

Figure 3.5: Evolution of length-weighted fibre length of pulps and corresponding fibres during refining

Length-weighted fibre length did not changed until 2000 PFI revolutions. Beyond that length-weighted fibre length decreased with refining. Therefore, it can be said that fibre shortening occurs only after 2000 PFI revolutions.

By interpreting the fibre length distributions (cf. Figure 3.6) it is clear that longer fibres (length $>1.7 \mathrm{~mm}$ ) underwent cleavage resulting in a frequency drift towards the shorter fibres $(0.2$ to $0.5 \mathrm{~mm})$. The relative frequency is calculated as the ratio of the number of fibres in a class of a given length to the total number of fibres. The difference between pulp and fibres is due to the loss of short fibres during fractionation.
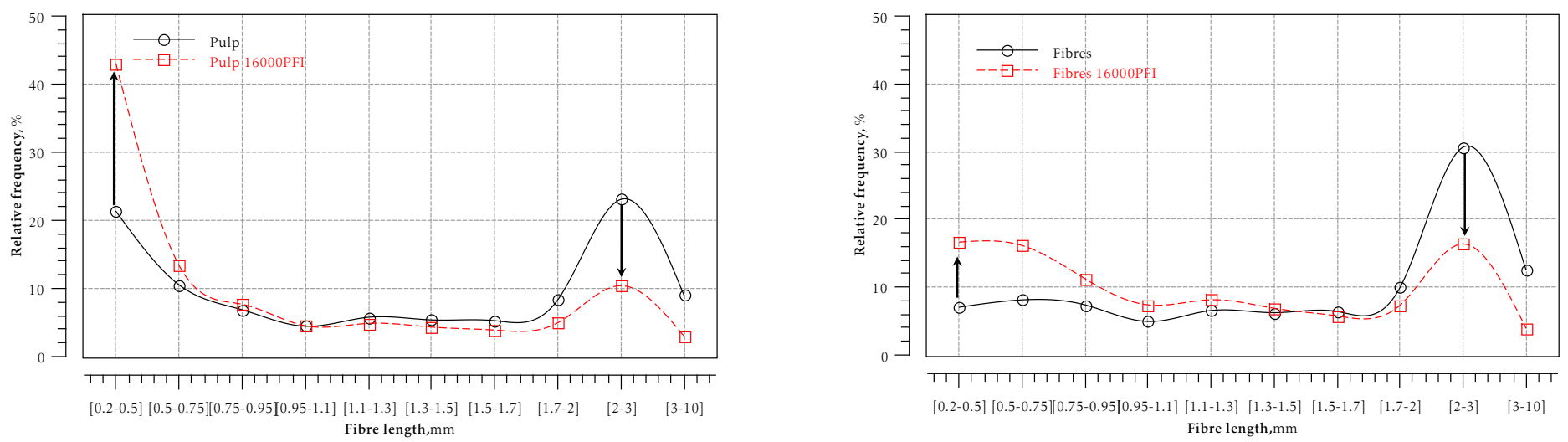

Figure 3.6: Effect of refining on fibre length distributions of pulps (left) and corresponding fibres (right) 
Average fibre width of pulps and fibres slightly increased with refining. The increase in average fibre width was $1 \mu \mathrm{m}$.

The effect of refining on fibre width distributions is shown in Figure 3.7.
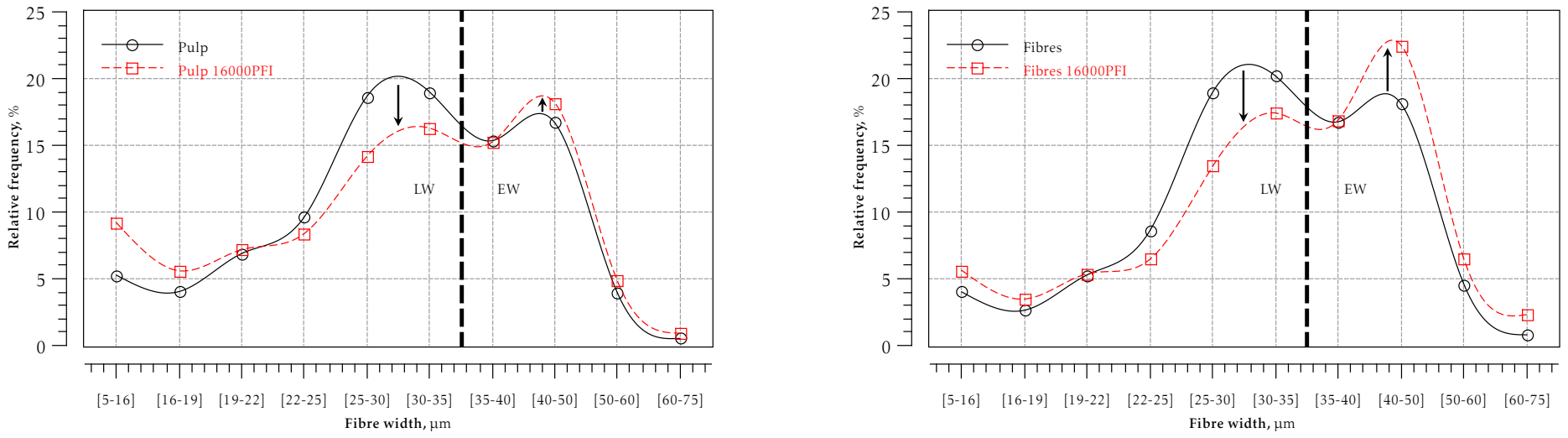

Figure 3.7: Effect of refining on fibre width distribution of pulps (left) and corresponding fibres (right)

Earlywood fibre were distinguished from latewood fibres using optical microscopy. Latewood fibres were mostly 22 to $35 \mu \mathrm{m}$ wide whereas the width of earlywood fibres ranged between 35 to $60 \mu \mathrm{m}$. Number of fibres slimmer than $35 \mu \mathrm{m}$ were decreased during refining. Therefore the increase in average fibre width during refining may be mainly caused by the width expansion of LW fibres.

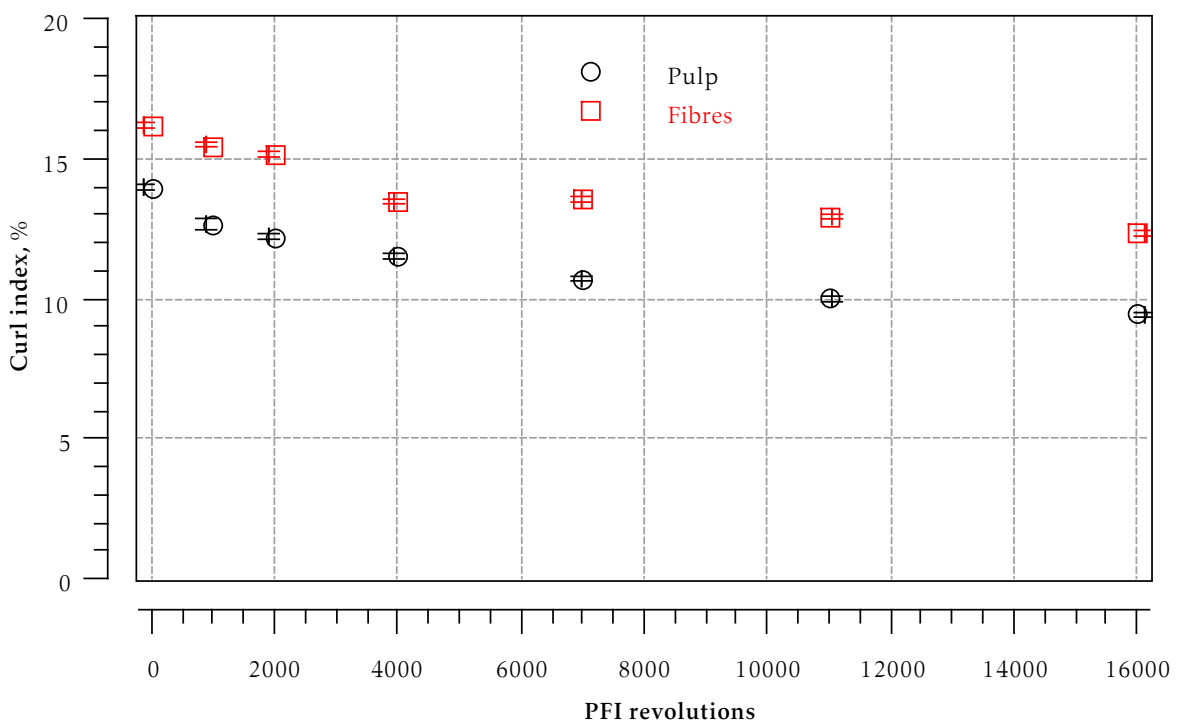

Figure 3.8: Evolution of curl index of pulps and fibres during refining

Curl index of pulps and fibres decreased with refining (cf. Figure 3.8). Curl in- 
dex of pulp is lower than corresponding fibres which is due to the loss of short fibres $(0.2$ to $0.5 \mathrm{~mm})$ during fractionation. Mohlin also reported fibre straightening during PFI refining [161].

A frequency drift can be seen from highly towards lowly curled fibres with refining (cf. Figure 3.9).
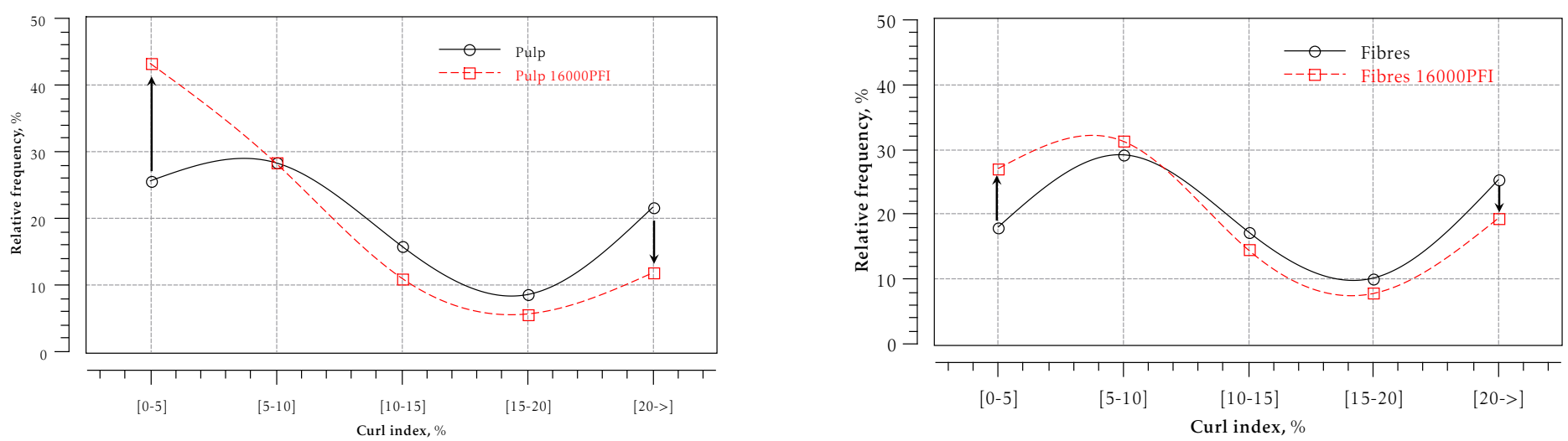

Figure 3.9: Effect of refining on curl index distribution of pulps (left) and fibres (right)

The evolution of kink number during refining is shown in Figure 3.10.

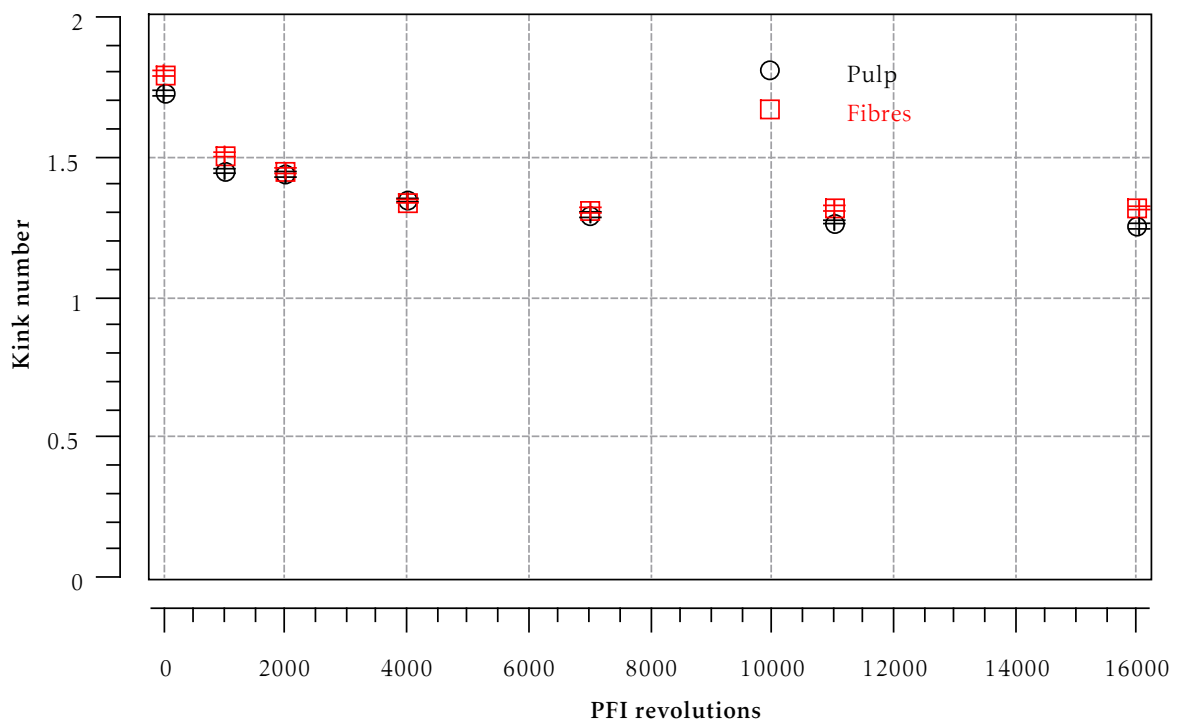

Figure 3.10: Evolution of kink number of pulps and corresponding fibres during refining

Kink number of pulps and fibres decreased with refining, essentially initially. The decrease in kink number from 1.8 to 1.4 accounts for $22 \%$ drop in initial 
value. It can also be noted here that the evolution of kink number of pulps and fibres with refining is identical. This implies that short and long fibres have more or less similar kink numbers.

The effect of refining on broken fibre content is shown in Figure 3.11.

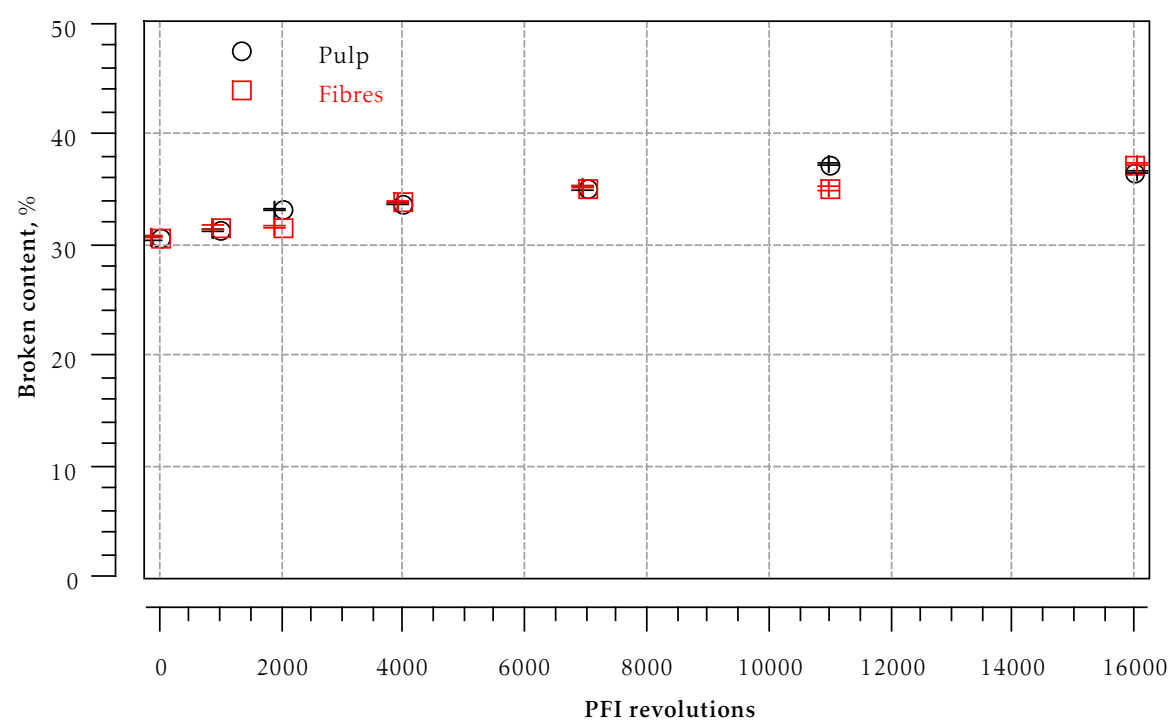

Figure 3.11: Evolution of broken content of pulps and corresponding fibres during refining

Broken content increased with refining due to the mechanical impacts. Same conclusion can be drawn for the evolution of broken content of pulps and fibres during refining as described for Figure 3.10.

Fibre width and broken ends increased while fibre length, kink and curl decreased during PFI refining. Morphological analysis of Valley beater refined pulp and corresponding fibres was performed to evaluate the associated changes in fibre morphology (cf. Table 3.3). 
Table 3.1: Morphological properties of Valley beater refined pulps and fibres, $\left\langle l_{l}\right\rangle,\langle w\rangle$, and CI are the length-weighted fibre length, average fibre width and curl index, respectively.

\begin{tabular}{c|cccc|cccc}
\multirow{2}{*}{$\begin{array}{c}\text { Valley beater } \\
\text { refining time, min }\end{array}$} & \multicolumn{5}{|c|}{ Pulp } & \multicolumn{4}{c}{ Fibres } \\
\cline { 2 - 9 } & $\left\langle l_{l}\right\rangle, \mathrm{mm}$ & $\langle w\rangle, \mu \mathrm{m}$ & $\mathrm{CI}, \%$ & Kink no. & $\left\langle l_{l}\right\rangle, \mathrm{mm}$ & $\langle w\rangle, \mu \mathrm{m}$ & $\mathrm{CI}, \%$ & Kink no. \\
\hline 0 & 2.21 & 27.8 & 13.07 & 1.62 & 2.34 & 31.3 & 15.62 & 1.70 \\
20 & 2.13 & 28.9 & 11.72 & 1.43 & 2.32 & 32.3 & 14.07 & 1.43 \\
30 & 2.04 & 29.3 & 11.39 & 1.40 & 2.23 & 33.4 & 13.77 & 1.45 \\
45 & 1.92 & 29.3 & 12.33 & 1.52 & 2.11 & 34.7 & 13.37 & 1.45 \\
80 & 1.70 & 29.4 & 11.27 & 1.43 & 1.83 & 34.5 & 12.44 & 1.38 \\
150 & 1.59 & 29.1 & 10.96 & 1.37 & 1.71 & 33.3 & 12.02 & 1.38
\end{tabular}

Fibre length shortened, width increased, curl and kink reduced during Valley beater refining.

\subsection{Dewatering of refined pulps and fibres}

SR [8] was measured using Schopper Riegler apparatus. $1 \mathrm{~L}$ of pulp suspension containing $2 \mathrm{~g}$ of oven dried pulp was drained through the fibre mat, formed on a wire screen, into a funnel and a side orifice (cf. Figure A.1 in Appendix A). The filtrate collected from the side orifice represents SR. The bone dry weight of the fibre mat was determined by drying the fibre mat in oven to constant weight at $105^{\circ} \mathrm{C}$. Corrected SR corresponding to $2 \mathrm{~g}$ was thus obtained from the weight correction curves.

\subsubsection{Schopper Riegler of refined pulps and fibres}

The evolution of SR during refining is shown in Figure 3.12. SR did not change until 2000 PFI revolutions. Beyond that, the difference in SR of pulps and fibres started to increase which can be attributed towards the increased surface area, fibrillation and fines content development (cf. Figure 3.13 and Figure 3.14). 

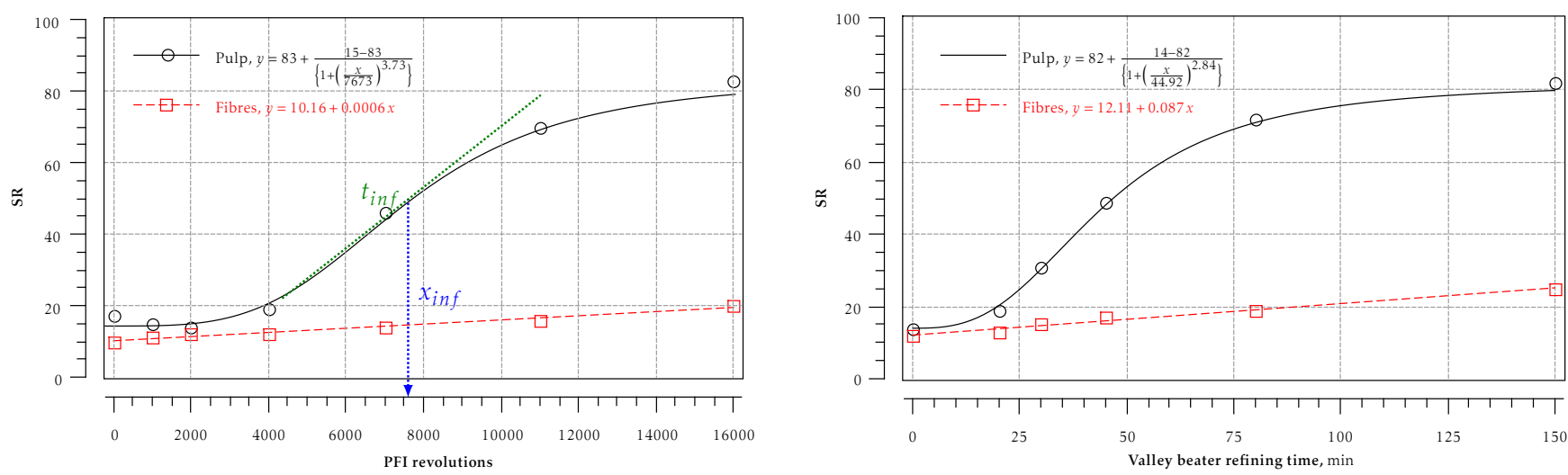

Figure 3.12: Evolution of SR of pulps and fibres during refining in PFI mill (left) and in Valley beater (right)

Evolution of pulp's SR during refining can be described by an S-shape logistic model.

Equation (3.1) is the general form of S-shape logistic model.

$$
y=y_{\max }+\frac{y_{\min }-y_{\max }}{\left\{1+\left(\frac{x}{x_{\text {inf }}}\right)^{t_{\text {inf }}}\right\}}
$$

where $y_{\text {max }}, y_{\text {min }}, x_{\text {inf }}$ and $t_{\text {inf }}$ are the maximum value of $y$, minimum value of $y$, inflection of $x$ in growth region and tangent at inflection, respectively. The higher value of tangent corresponds to the higher rate of growth. The value of tangent may be characteristic of fibre structure and the process conditions it has gone through.

In case of SR of pulps, the value of tangent corresponds to the rate of generation of fines during refining. The evolution of fibres' SR during refining seems to be linear $\left(R^{2}=0.98\right)$. The model equations can be used to estimate $S R$ of the pulp and fibres (SK100) refined to a given number of PFI revolutions and Valley beater refining time within the experimental conditions used here for refining. 

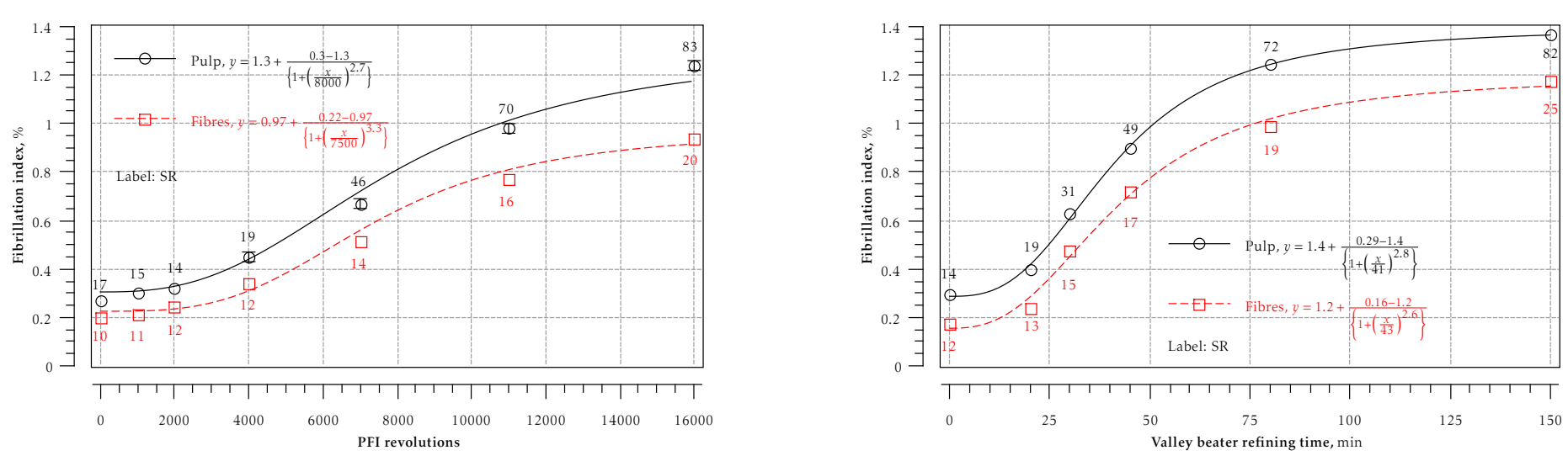

Figure 3.13: Evolution of fibrillation index of pulps and corresponding fibres during PFI (left) and Valley beater (right) refining

External fibrillation increased with refining (cf. Figure 3.13). The evolution of fibrillation index during refining can also be described by an S-shape logistic equation (3.1). The difference in fibrillation index of pulps and fibres increased with refining. The difference in pulp and fibres is the amount of short fibres. During refining short fibres were also fibrillated and during fraction they were lost as well. Therefore, the increase in the difference of fibrillation index between fibres and pulps during refining indicate the loss of fibrillated short fibres.

Broken and fines content increased during refining (cf. Figure 3.14).
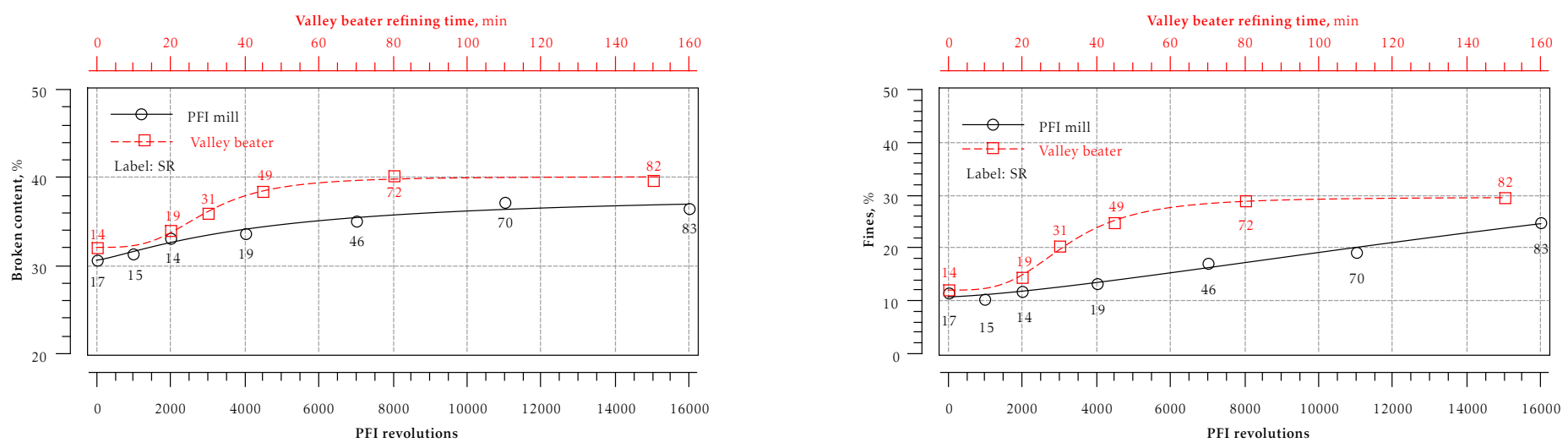

Figure 3.14: Evolution of broken (left) and fines (right) content of pulps during PFI and Valley beater refining 


\subsubsection{Dewatering rate of refined pulps and fibres}

Dewatering rate was measured using dynamic freeness tester (DFT 3000) [187, 188]. The operating principle of DFT 3000 is shown in Figure 3.15. Pulp suspension (20 to $50 \mathrm{~g} \cdot \mathrm{L}^{-1}$ ) is homogenised and transferred to the dilution chamber where the consistency is adjusted to $2 \mathrm{~g} \cdot \mathrm{L}^{-1}$, required for the drainage test. The diluted pulp is then transferred to the standard Tappi drainage tester. Integrated load sensors, present at the bottom of the volumetric cylinders, instantaneously record the mass of filtrate collected.

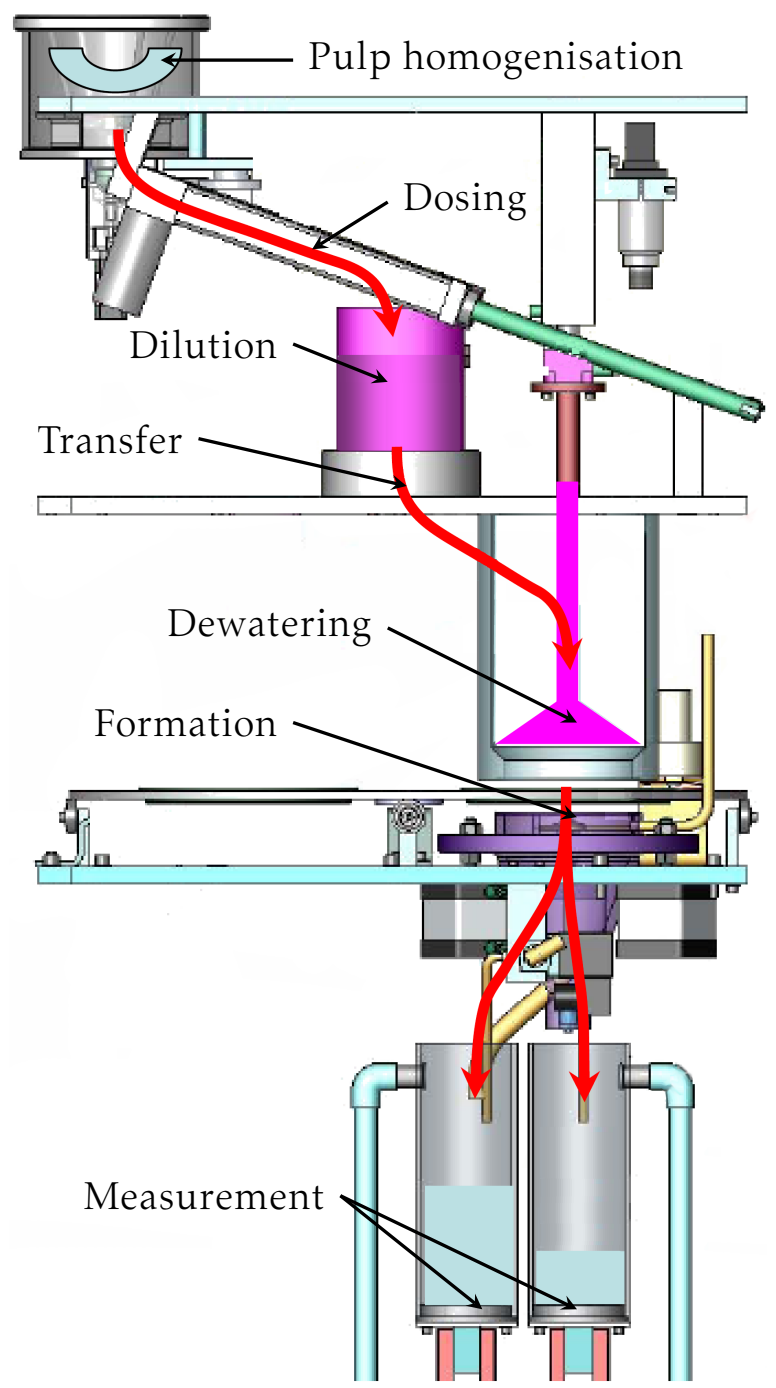

Figure 3.15: Schematic diagram of dynamic freeness tester (DFT 3000) [69]

Some example of dewatering curves with refining are shown in Figure 3.16. 

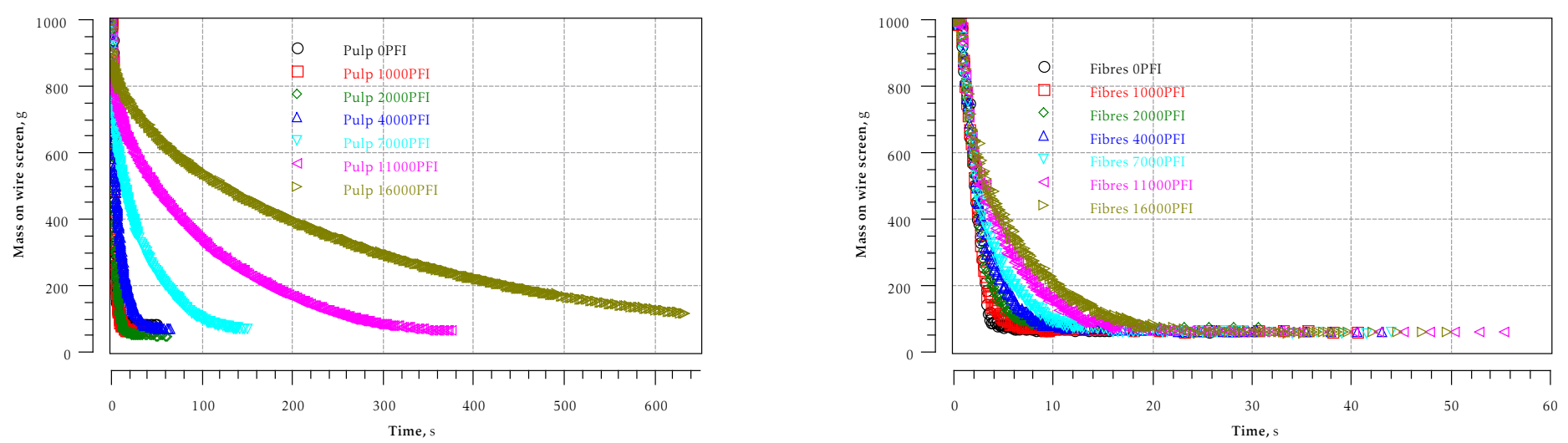

Figure 3.16: Filtration kinetics of refined pulps (left) and fibres (right)

Dewatering time of the pulps and fibres increased with refining. Significant increase in dewatering time of the pulp can be seen after 2000 PFI revolution which can be attributed to the increase in surface area.

Huge difference in the drainage rate of highly refined pulp and corresponding fibres can be seen in Figure 3.17 which is due to the presence of fines.

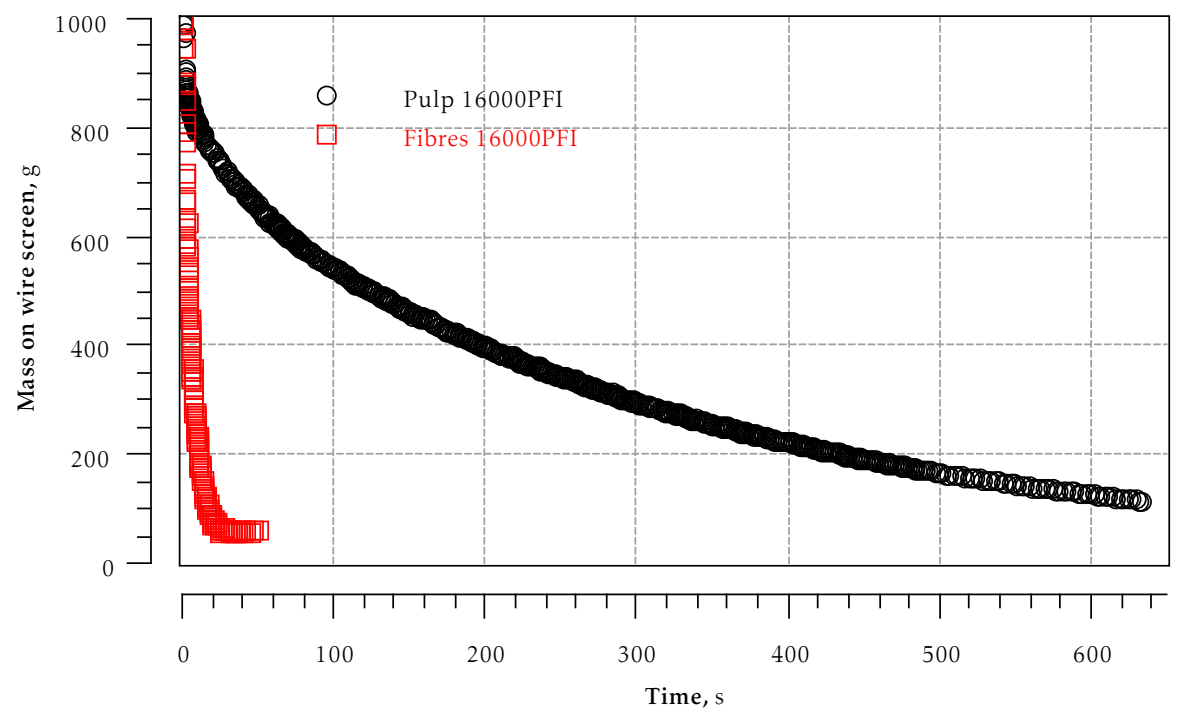

Figure 3.17: Filtration kinetics of refined pulp and corresponding fibres

Similar dewatering curves were obtained for the Valley beater refined pulps and fibres (SK100). 


\subsubsection{Specific filtration resistance of refined pulps and fibres}

Specific filtration resistance $\left(\mathrm{R}_{S \mathrm{~F}} \boldsymbol{T}_{\mathrm{T}}\right)$ was calculated from the following expression (see Appendix A for derivation)

$$
\mathrm{R}_{s \mathrm{~F}{ }_{\mathrm{T}}}=\frac{98 \cdot \mathrm{R}_{\mathrm{F}}}{\mathrm{C}_{0}\left\{\left(\mathrm{~V}_{0}-\mathrm{V}_{f}\right) \cdot e^{\left(\frac{-\mathrm{T} \cdot t_{f}}{\mathrm{R}_{\mathrm{F}}}\right)}+\mathrm{V}_{f}\right\}}
$$

where $\mathrm{R}_{\mathrm{F}}, \mathrm{C}_{0}, \mathrm{~V}_{0}$ and $\mathrm{V}_{f}$ are filtration resistance from characteristic drainage curve, consistency of the slurry at $t_{0}$, volume of the slurry at $t_{0}$ and volume of the slurry at $t_{f}$, respectively. $t_{f}$ is the moment in time when the discharge is zero. $\mathrm{T}$ corresponds to dimensionless time $\left(\mathrm{T}=\frac{t}{t_{f}}\right)$

Specific filtration resistances of PFI refined pulps and fibres are reported in Table 3.2.

Table 3.2: Evolution of specific filtration resistance of pulps and corresponding fibres during PFI refining

\begin{tabular}{c|ccccc|ccccc}
\multicolumn{7}{|c|}{ Pulp } & \multicolumn{5}{c}{ Fibres } \\
\hline 0 & 0 & 0.25 & 0.5 & 0.75 & 1 & 0 & 0.25 & 0.5 & 0.75 & 1 \\
\hline 1000 & 1.0 & 14.2 & 14.5 & 14.5 & 14.5 & 0.5 & 9.6 & 9.7 & 9.7 & 9.7 \\
2000 & 1.2 & 19.8 & 20.1 & 20.1 & 20.1 & 0.7 & 13.2 & 13.2 & 13.2 & 13.2 \\
4000 & 1.8 & 30.7 & 31.3 & 31.3 & 31.3 & 1.0 & 19.0 & 19.8 & 19.9 & 19.9 \\
7000 & 1.1 & 33.7 & 35.4 & 35.4 & 35.4 & 1.2 & 22.6 & 23.2 & 23.2 & 23.2 \\
11000 & 19.2 & 117.5 & 150.5 & 152.3 & 152.4 & 1.6 & 25.3 & 26.1 & 26.1 & 26.1 \\
16000 & 185.6 & 299.4 & 670.2 & 923.6 & 1008.0 & 2.2 & 30.3 & 31.5 & 31.5 & 31.5 \\
& 181.0 & 351.3 & 612.5 & 924.0 & 1200.6 & 3.2 & 40.2 & 43.1 & 43.1 & 43.1
\end{tabular}

Specific filtration resistance of PFI refined pulp and fibres increased with refining. Increase in filtration resistance with refining (16000PFI) due to fibrillation alone was $33 \mathrm{~s}^{-1}$ whereas due to fibrillation and fines was $1186 \mathrm{~s}^{-1}$. Therefore the contribution of fines compared to fibrillation towards the slowness in dewatering was enormously higher. The increase in filtration resistance was calculated from the difference of filtration resistance of pulp or fibres refined at $16000 \mathrm{PFI}$ revolution to the filtration resistance of unrefined pulp or fibres at $t_{f}$. 
Similarly, specific filtration resistances of Valley beater refined pulps and fibres were calculated (cf. Table 3.3). Specific filtration resistance of Valley beater refined pulp and fibres increased with refining. Increase in filtration resistance with refining (150 min) due to fibrillation alone was $49 \mathrm{~s}^{-1}$ whereas from fibrillation and fines was $494 \mathrm{~s}^{-1}$.

Table 3.3: Evolution of specific filtration resistance of Valley beater refined pulps and fibres

\begin{tabular}{|c|c|c|c|c|c|c|c|c|c|c|}
\hline \multirow{2}{*}{$\underbrace{\mathrm{R}_{s \mathrm{~F}, \mathrm{~T}} \times 10^{5}, \mathrm{~s}^{-1}}_{\min }$} & \multicolumn{5}{|c|}{ Pulp } & \multicolumn{5}{|c|}{ Fibres } \\
\hline & 0 & 0.25 & 0.5 & 0.75 & 1 & 0 & 0.25 & 0.5 & 0.75 & 1 \\
\hline 0 & 1.4 & 16.0 & 17.1 & 17.2 & 17.2 & 0.9 & 11.8 & 12.9 & 12.9 & 12.9 \\
\hline 20 & 2.3 & 19.2 & 22.3 & 22.4 & 22.4 & 1.0 & 15.2 & 17.2 & 17.2 & 17.2 \\
\hline 30 & 8.4 & 27.9 & 55.3 & 70.0 & 74.2 & 1.2 & 19.2 & 20.4 & 20.4 & 20.4 \\
\hline 45 & 19.5 & 48.6 & 94.5 & 136.6 & 159.8 & 1.5 & 21.6 & 21.9 & 21.9 & 21.9 \\
\hline 80 & 53.1 & 108.7 & 201.5 & 322.9 & 439.4 & 2.2 & 31.6 & 39.0 & 39.1 & 39.1 \\
\hline 150 & 81.7 & 157.0 & 269.7 & 399.5 & 510.9 & 3.1 & 49.1 & 61.3 & 61.6 & 61.6 \\
\hline
\end{tabular}

Similar conclusions can be drawn for Valley beater refined pulps and fibres as that of PFI refined pulps and fibres.

Although broken and fines content (cf. Figure 3.14) of Valley beater refined pulp was relatively higher than PFI mill refined pulp at the same SR, the specific filtration resistance of PFI refined pulp was comparatively higher (cf. Table 3.2 and Table 3.3). This might have been caused by the increased internal and external fibrillation. Thereby making fibres refined in PFI mill more flexible, conformable and collapsible. The filtration mat thus produced was compact and highly resistant.

\subsection{Water retention value of refined pulps and fi- bres}

Refined pulps and their corresponding fibres were checked for their ability to retain water. Test specimens for WRV were prepared on Tappi handsheet former [204] containing 150 mesh wire screen. A rubber frame was placed over 
the screen containing three open compartments of equal area $\left(\sim 33 \mathrm{~cm}^{2}\right.$ each). Pulp slurry containing $1.2 \mathrm{~g}$ oven dried pulp was poured in the cylinder of the handsheet former, previously half filled with water. Three vertical movements with a standard agitator were carried out. After agitation, the pulp suspension was allowed to drain. Each of the test specimen from the compartment of the rubber frame was rolled and placed into the centrifugation tube containing three small cylindrical holes with retaining sieves at the other end. Centrifugation was carried out in Sigma 3-16K centrifuge for 15 minute at $3000 \times g$ (relative centrifugal force). Dry mass of every test specimen was around $0.2 \mathrm{~g}$. After centrifugation, pulp samples were immediately weighed on a precision balance to the nearest $0.1 \mathrm{mg}$. The centrifuged and weighed test specimens were then dried in oven at $105^{\circ} \mathrm{C}$ to a constant weight. WRV was calculated using equation (2.15).

The evolution of WRV with refining is presented in Figure 3.18. WRV of pulp was slightly higher than that of fibres at the same levels of refining which was due to the presence of fines. Since fibre fraction does not contain fines therefore the increase in WRV after 7000 PFI revolutions may be attributed to the capillary effect of fines.

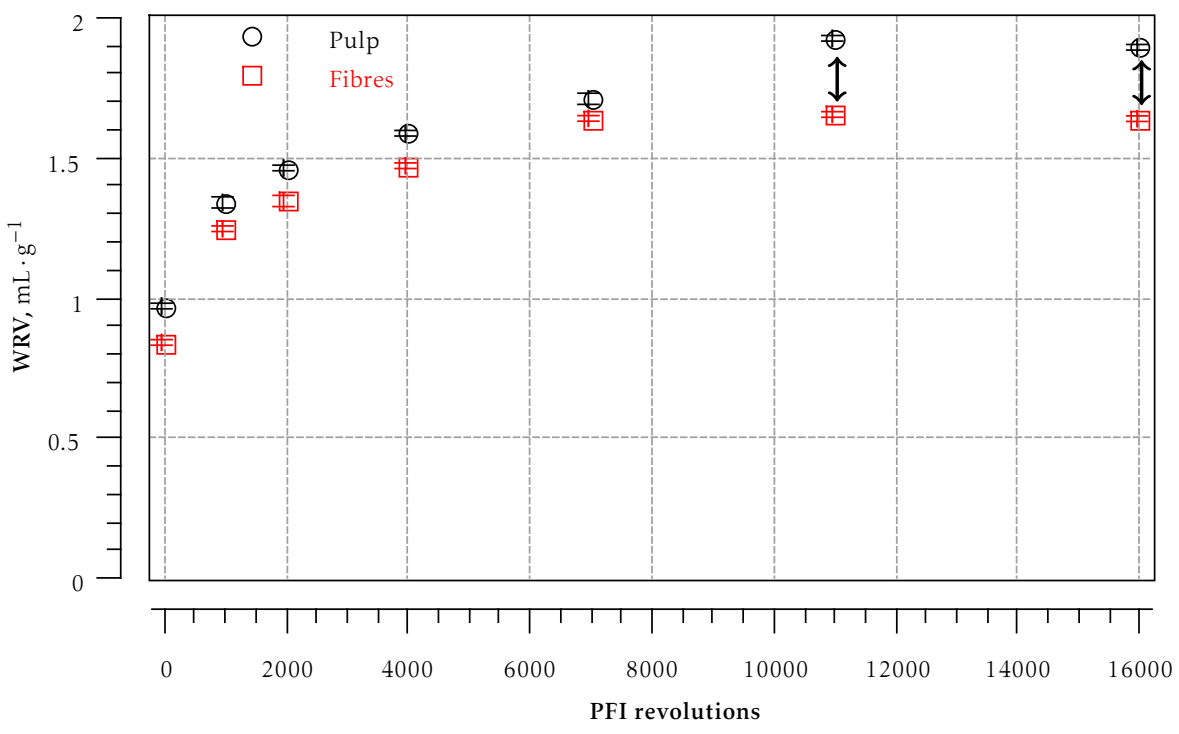

Figure 3.18: Evolution of water retention value during refining 


\subsection{Fibre saturation point of refined pulps and fi- bres}

\subsubsection{Preparation of mobile phase}

Deionized water was ultra purified using Milli-Q system of EMD Millipore Ltd. Ultra-purified-type-1 [2] water was then filtered through $0.45 \mu \mathrm{m}$ milipore filter paper using all glass filter holder. Filtered-ultra-purified-type-1 water was used as mobile phase. $0.5 \mathrm{~g} \cdot \mathrm{L}^{-1}$ of sodium azide (Product code 438456 SigmaAldrich) was added to inhibit bacterial growth.

Degassing of the mobile phase was of utmost importance for smooth operation. It was achieved by sparging $\mathrm{N}_{2}(\mathrm{~g})$ through the mobile phase for $5 \mathrm{~min}$. $\mathrm{He}(\mathrm{g})$ at low flow rate was used for in-situ sparging.

\subsubsection{Fabrication of the column}

Preparation of uniformly dense, stable, reproducible and efficient column is of utmost importance. Inter and intra fibre porosities are present in the bed of the fibrous column.

Inter fibre porosity is the fraction of the overall geometrical volume of the column available to mobile phase. Intra fibre porosity includes both the open and closed pores present in the structure of the fibres. During the fabrication of column it is required to minimise inter fibre porosity.

Packing of the column was carried out in accordance to the procedure described by Berthold and Salmén [42]. Figure 3.19 shows steps involved in the fabrication of the column containing fibrous bed. A disintegrated fibrous suspension containing $5 \mathrm{~g}$ oven dried mass was mixed with $2 \mathrm{~L}$ aqueous $\mathrm{NaCl}$ solution $\left(2 \mathrm{~mol} \cdot \mathrm{L}^{-1}\right)$. Addition of salt stabilises the swelling behaviour over a wide range of $\mathrm{pH}$ [95]. After $15 \mathrm{~min}$, fibrous suspension was drained on a fine mesh screen and washed with deionised water. The fibrous suspension was then transferred to $15 \mathrm{~L}$ reservoir. Again deionised water was used for dilution. Agitation at a very slow speed was started and a flow rate was adjusted to $120 \mathrm{~g} \cdot \mathrm{L}^{-1}$ with the help of rotameter and vacuum controller. Made sure that the flow rate during fabrication remain constant. The column was provided with a $20 \mu \mathrm{m}$ porosity polyethylene bed support. $12 \mathrm{~L}$ of fibrous suspension was filtered, the column was removed and the bed was pressed at $15.7 \mathrm{kPa}$ for 15 min to complete the process of column fabrication. 


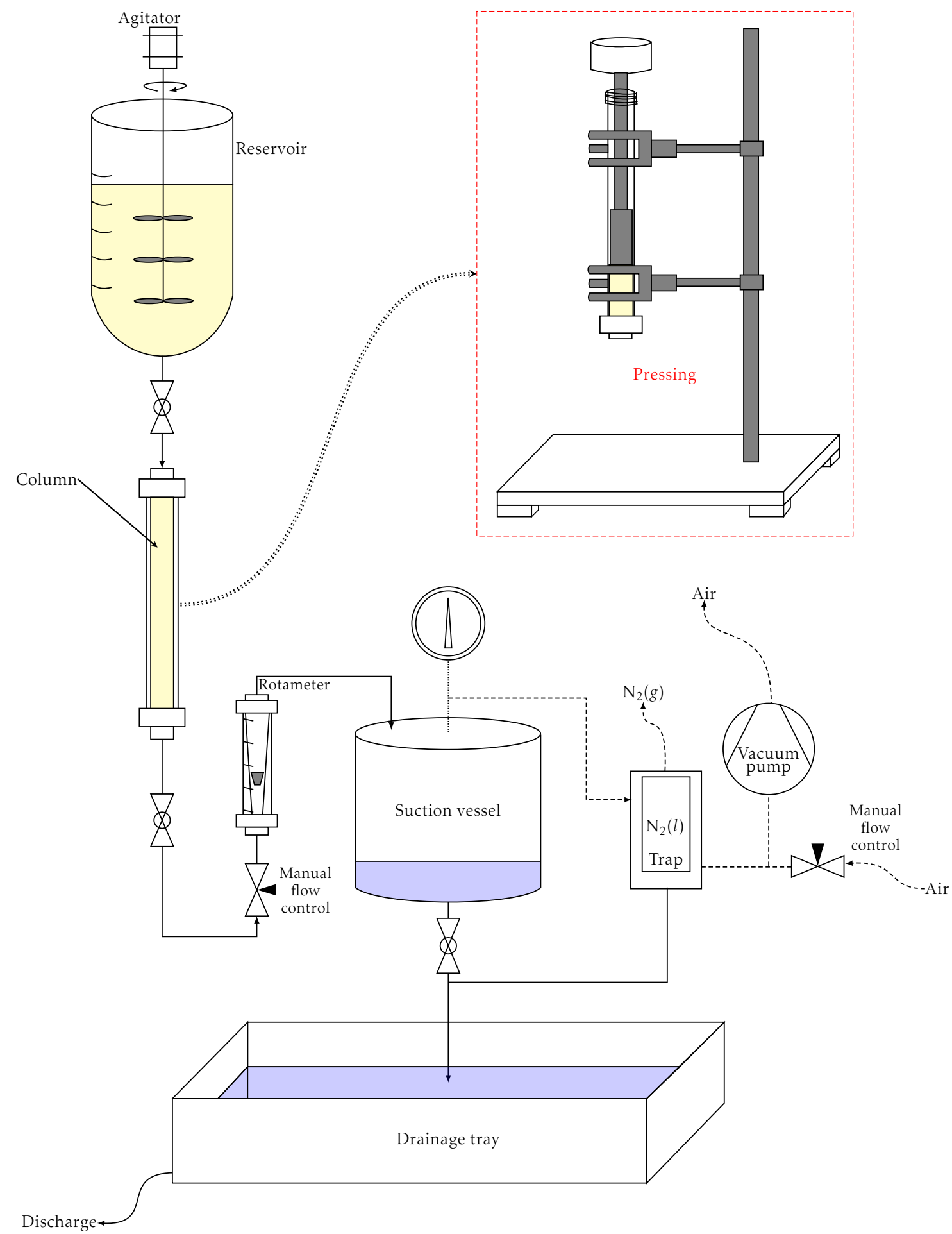

Figure 3.19: Making of the fibrous column for gel filtration chromatographic measurements

Characteristics of the columns used are described in Appendix B.

A representative chromatographic system containing a mobile phase reservoir, 
a high/medium pressure pump, an injection device, a fibrous column, a detector, a waste collector and a data recording system is shown in Figure 3.20.

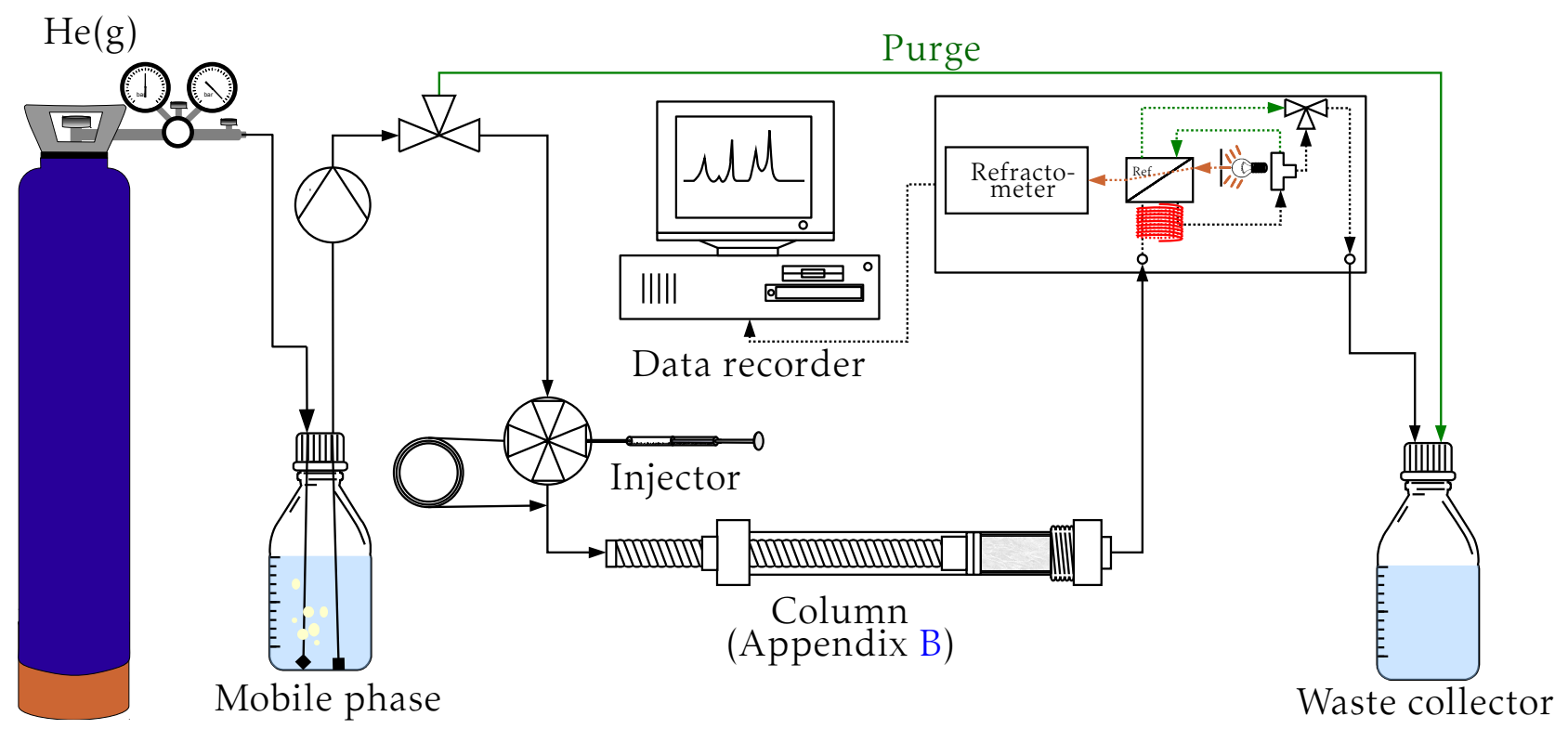

Figure 3.20: Experimental set-up for chromatographic measurements

\subsubsection{Calibration of differential refractive index detector}

Differential refractive index detector (RI detector) is commonly used in exclusion chromatography. Measurement is based on light beam deflection through a split optical cell, one side of the cell contains static reference of the mobile phase while the other side of it contains flowing analyte in the mobile phase. Control of temperature is very important as refractive index strongly depends on temperature. 3580 Viscotek from Malvern Instruments Ltd was used as differential RI detector. The detector is equipped with OmniSEC software for instrumental control, data acquisition and analysis.

For the calibration curves, measurements were made without column [143], with column containing glass beads and with column containing fibrous bed. Without column, the response of the detector was non linear above $10 \mathrm{~g} \cdot \mathrm{L}^{-1}$ which might have been caused by the plug flow, upper limit of the detector and/or the discrepancy in the measurement of the area under refractive index peak (RIA). Column filled with glass beads was used to obtain dispersed flow. Due to difficulty in removing trapped air inside glass beads, a fibrous bed column of smaller diameter and longer bed height was preferred for gel filtration 
chromatographic (GFC) measurements.

The flow rate of the mobile phase was $1.5 \mathrm{~mL} \cdot \mathrm{min}^{-1}$ and the injection volume of the probe solution was $100 \mu \mathrm{L}$. Calibration curves of RI detector for glucose with and without fibrous column is shown in Figure 3.21.

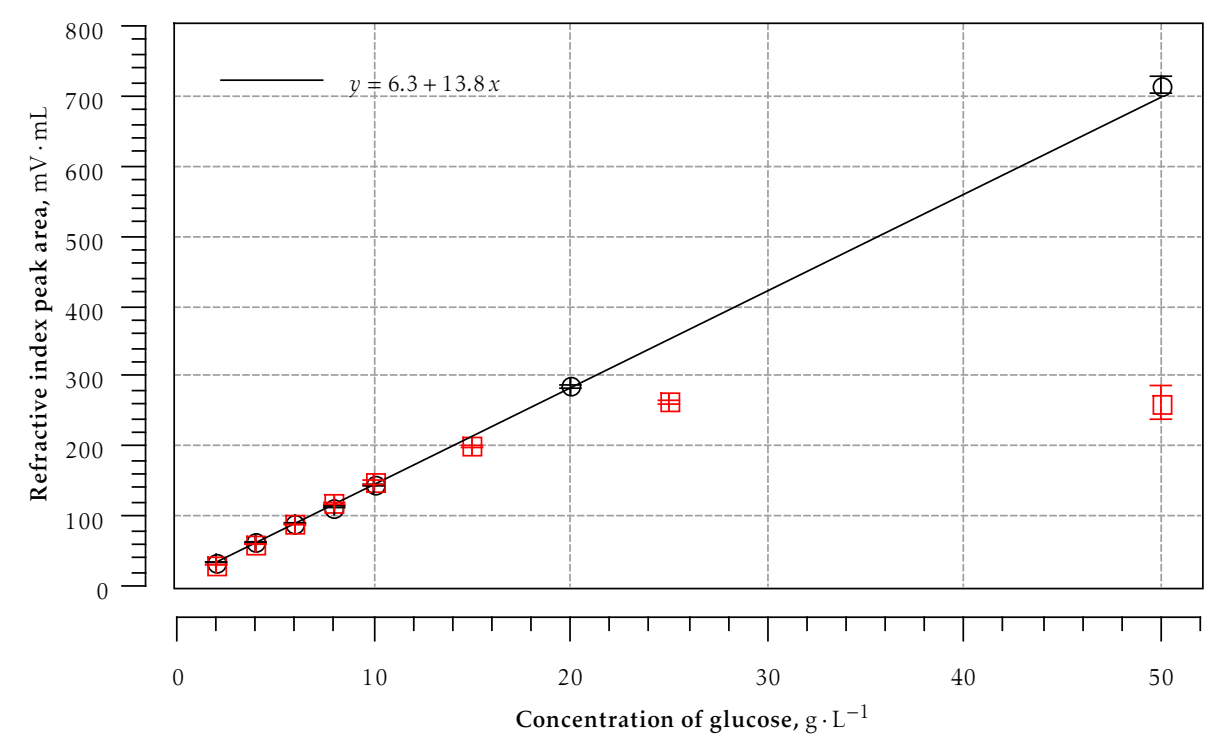

Figure 3.21: Calibration curve of differential refractive index detector for glucose, (O) corresponds to the GFC measurements using fibrous bed column whereas $(\square)$ corresponds to GFC measurements without column

Similar calibration curves were obtained for other probe solutions as well. It was found that RIA value was linearly related to the concentration of probes solutions (with column and within the range of concentration) (cf. Table 3.4).

Table 3.4: Calibration equations of probe molecules

\begin{tabular}{l|c|cc}
\multirow{2}{*}{ Probe molecules } & Weight average & \multicolumn{2}{|c}{$y=k_{c}+a x$} \\
\cline { 3 - 4 } & molar mass, Da & $k_{c}$ & $a$ \\
\hline Glucose & 180 & 6.3 & 13.8 \\
PSS-ddxt1.3k & 1320 & 1.0 & 13.2 \\
PSS-ddxt3m & 3500000 & -0.9 & 13.9
\end{tabular}

where $x, y, a, k_{c}$ are the concentration of probe solution $\left(g \cdot \mathrm{L}^{-1}\right)$, RIA value $(\mathrm{mV} \cdot \mathrm{mL})$, slope and calibration constant, respectively. 


\subsubsection{Solute exclusion}

Aqueous solution of dextran 2000 (product code 95771, Sigma Aldrich) at a concentration of $7 \mathrm{~g} \cdot \mathrm{L}^{-1}$ was used for FSP measurements.

Pulp suspension containing $1.5 \mathrm{~g}$ oven dried pulp was poured in a $60 \mathrm{~mL}$ preweighed PET bottle. $40 \mathrm{~mL}$ of $7 \mathrm{~g} \cdot \mathrm{L}^{-1}$ aqueous dextran solution was added to it. The bottle was tightly capped and shaken vigorously for one minute. The bottle was turned over from time to time, every day for three consecutive days. The suspension was filtered using $0.45 \mu \mathrm{m}$ membrane filter and the concentration was checked using GFC.

\subsubsection{Gel filtration chromatography}

FSP was calculated from equation (2.16). In order to see mean value stabilisation, a cumulative moving average curve of FSP was plotted against the number of measurements (cf. Figure 3.22).

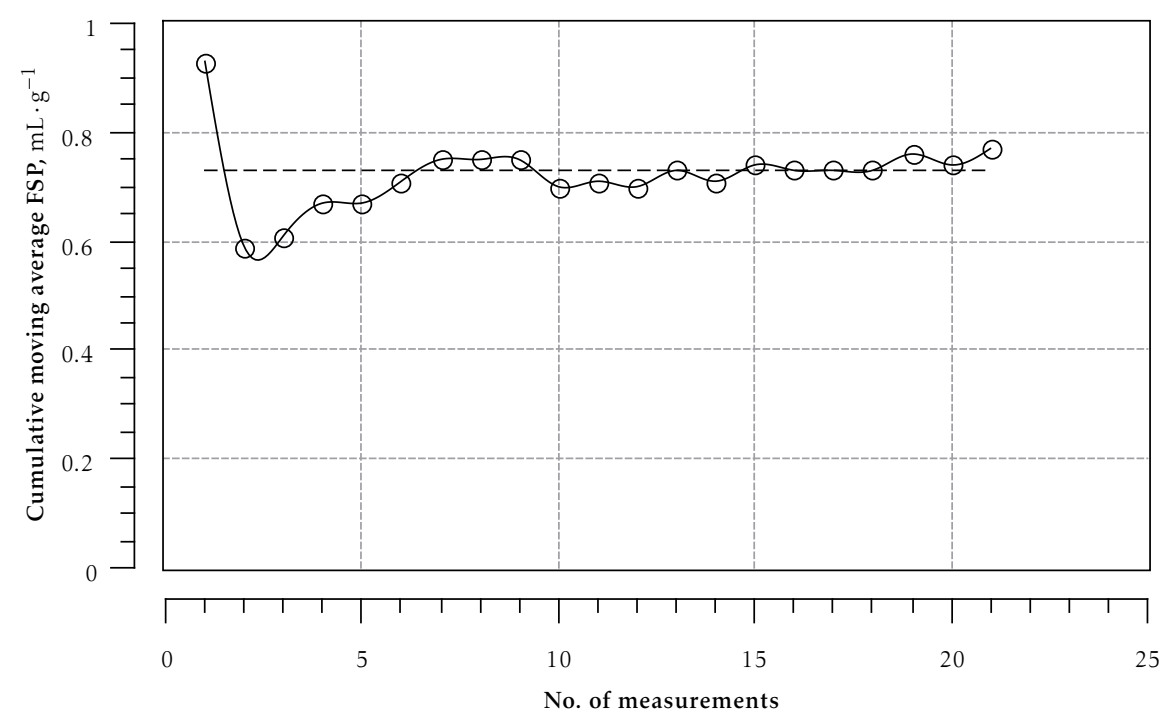

Figure 3.22: Cumulative moving average fibre saturation point

It was found that at least ten GFC measurements were required to obtain a stable FSP value (variation $<9 \%$ ). Therefore for every sample, ten GFC measurements were carried out.

The effect of refining on FSP of pulp is presented in Figure 3.23. FSP did not change until 2000 PFI revolutions. Beyond 2000 PFI revolutions FSP of pulp increased with refining. The increase in FSP with refining might have been 
caused by the delamination (internal fibrillation) of the fibre wall [171]. Therefore it is believed that internal fibrillation started around 2000 PFI revolutions and continuously increased with refining.

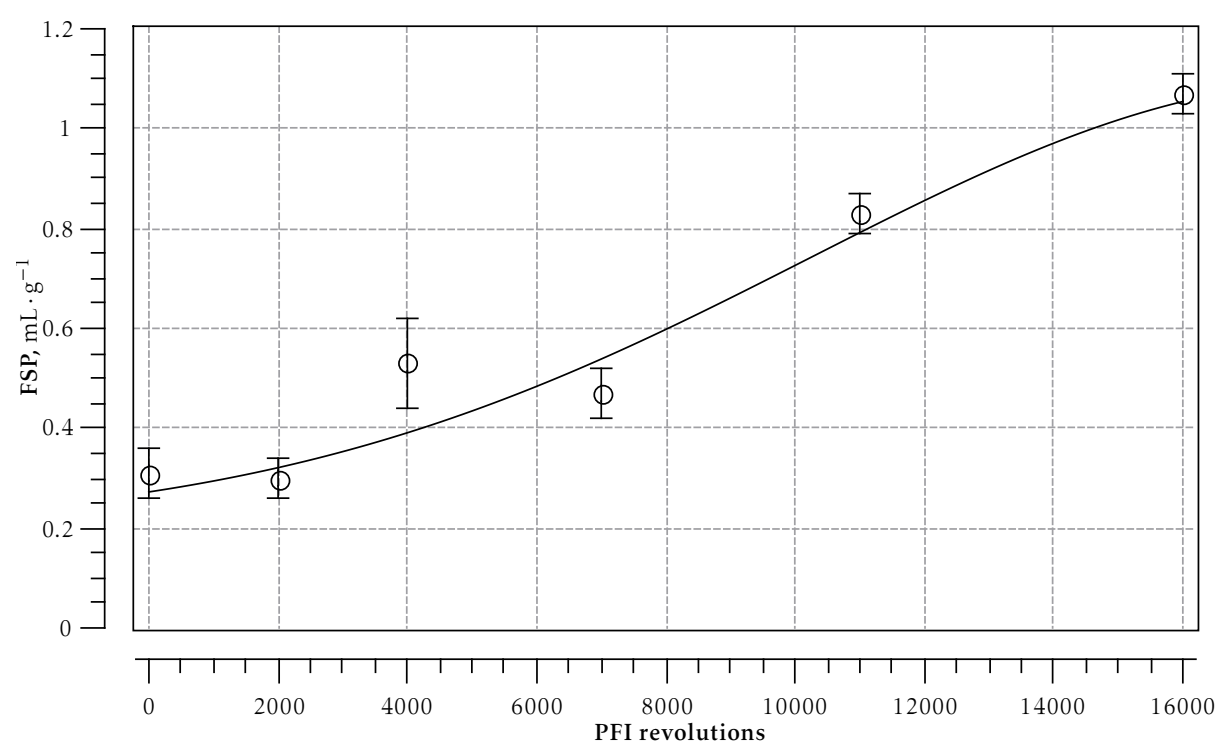

Figure 3.23: Evolution of fibre saturation point of pulps during refining

\subsection{Fibre wall pore size distribution of refined pulps and fibres}

DIN certified standard dextrans were obtained from PSS (Polymer Standard service $\mathrm{GmbH})$, Mainz Germany.

Viscosity radius $r_{\eta}(\AA)$ of dextran [99] in aqueous solution was calculated from molar mass of dextran at the peak maximum $\mathrm{M}_{p}(\mathrm{Da})$ provided by the dextran supplier using following relation

$$
r_{\eta}=0.271 \mathrm{M}_{p}^{0.498}
$$

Table 3.5 represents characteristics of probe molecules used for ISEC measurement. 
Table 3.5: Characteristics of probe molecules

\begin{tabular}{l|ccccc} 
Probe molecules & $\mathrm{M}_{w}, \mathrm{Da}$ & $\mathrm{M}_{n}, \mathrm{Da}$ & $\mathrm{M}_{p}, \mathrm{Da}$ & $\mathrm{D}=\frac{\mathrm{M}_{w}}{\mathrm{M}_{n}}$ & $r_{\eta}, \AA$ \\
\hline Glucose & 180 & - & - & - & 3.9 \\
PSS-ddxt1.3k & 1320 & 1080 & 1080 & 1.23 & 8.8 \\
PSS-ddxt12k & 11600 & 8100 & 9900 & 1.43 & 26.5 \\
PSS-ddxt80k & 80900 & 55500 & 66700 & 1.46 & 68.5 \\
PSS-ddxt150k & 148000 & 100000 & 124000 & 1.47 & 93.2
\end{tabular}

where $\mathrm{M}_{w}, \mathrm{M}_{n}$ and $Đ$ are weight average molar mass, number average molar mass and disparity, respectively. $Đ$ indicates the narrowness of molar mass distribution.

Total volume of the bed can be represented by the following expression

$$
\mathrm{V}_{\text {total }}=\mathrm{V}_{\text {inter }}+\mathrm{V}_{\text {intra }}+\mathrm{V}_{\text {fibres }}
$$

where $\mathrm{V}_{\text {inter }}, \mathrm{V}_{\text {intra }}$ and $\mathrm{V}_{\text {fibres }}$ are the inter-fibre, intra-fibre and fibre volumes, respectively. $\left(\mathrm{V}_{\text {inter }}+\mathrm{V}_{\text {intra }}\right)$ is the total accessible volume to the eluent. It is assumed that the inter- and intra-fibre volumes are totally accessed by glucose probe molecules. Whereas, dextran probe molecules can access to interfibre volume and depending on their molecular size to the intra-fibre volumes. Therefore, the specific accessible pore volume $(\mathrm{V})$ to dextran probe molecules can be represented by the following expression

$$
\mathrm{V}=\frac{\left(\mathrm{V}_{g}-\mathrm{V}_{d}\right)}{m}
$$

where $V_{g}, V_{d}$ and $m$ are the accessible volume to glucose probe molecules, accessible volume to dextran probe molecules and the oven dried mass of fibrous bed, respectively. If we consider that the volume is given by the multiplication of flow rate and time then equation (3.5) can be rewritten as

$$
\mathrm{V}=\frac{\left(t_{g}-t_{d}\right)}{m} \cdot v
$$

where $t_{g}, t_{d}, v$ and $m$ are the elution time of glucose solution, elution time of dextran solution, the flow rate of eluent, respectively. $t_{g}$ and $t_{d}$ are determined 
experimentally from the chromatograms, $v$ is fixed during the experiment and $m$ is obtained by drying the fibrous bed in oven to constant weight at $105^{\circ} \mathrm{C}$ once the experiment is finished.

A probe concentration of $10 \mathrm{~g} \cdot \mathrm{L}^{-1}$ was chosen for chromatographic porosimetry. Cumulated apparent pore volume of refined fibres is shown in Figure 3.24.

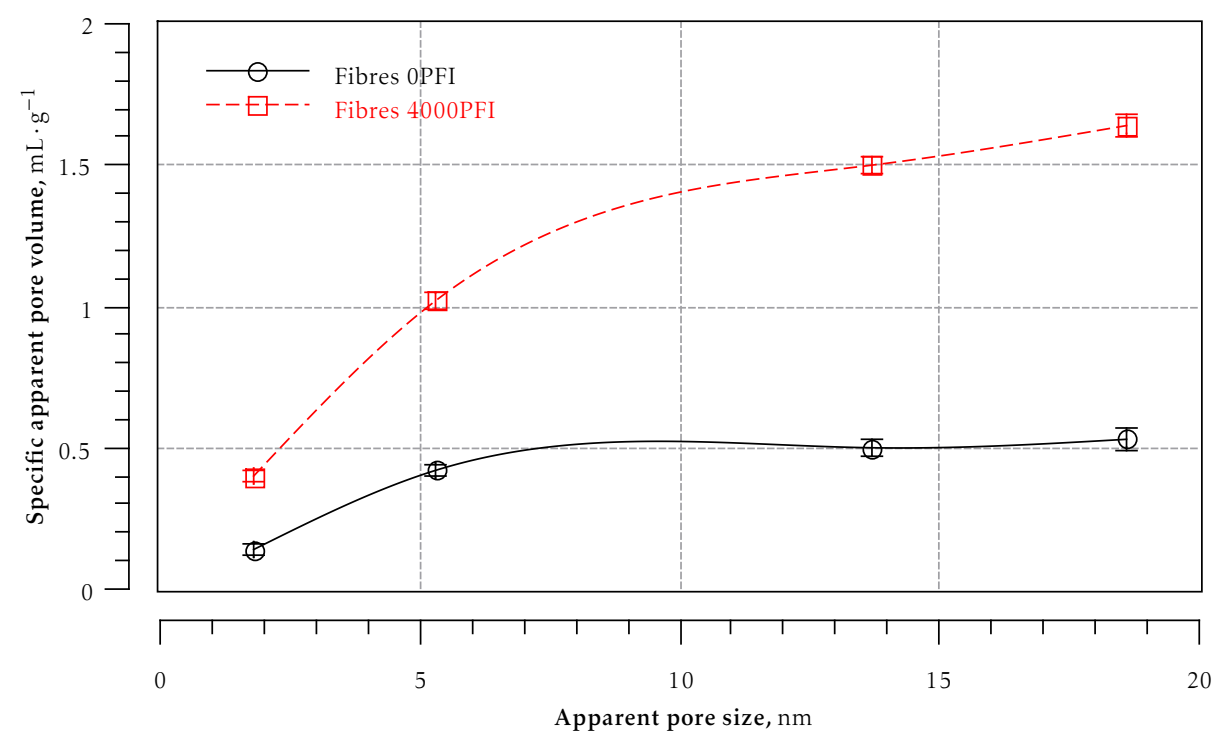

Figure 3.24: Cumulated apparent pore volume of refined fibres

Apparent pore volume of fibres increased with refining. The results obtained in this study follow similar evolution with refining as has been reported earlier by Stone et al. [213]. Increase in pore volume with refining reported previously [213] was 1.2 to 1.4 times than that of unrefined fibres whereas in our study it is 2.5 to 3 times than that of unrefined fibres.

The evolution of apparent pore size distribution during refining is presented in Figure 3.25. Average pore size increased from $6.4 \mathrm{~nm}$ to $8.1 \mathrm{~nm}$ with refining. Increase in pore size was due to the relative increase in specific apparent volume of 5.3 to $18.6 \mathrm{~nm}$ size pores. 


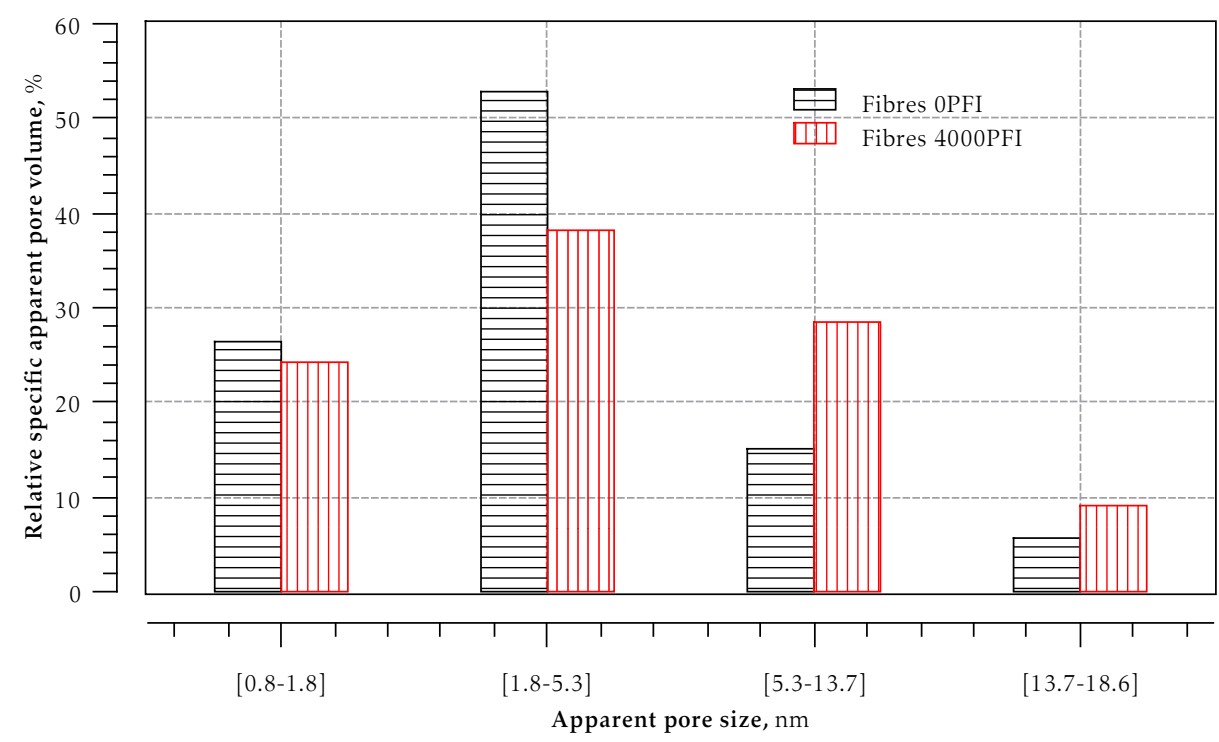

Figure 3.25: Effect of refining on apparent pore size distribution of fibres

\subsection{Wet breaking length of refined pulps and fibres}

The instrument used for measuring wet breaking length (WBL) of wet fibre strip is shown in Figure 3.26. The platform of WBL instrument was levelled with the help of level tool and levelling screws. The dead weight was adjusted to balance the weight of empty bucket. Volumetric cylinder was filled with water. Wet fibrous test specimen strips were formed on Karl Frank Rapid-Köthen handsheet former using a frame of five compartments of $9.5 \times 1.5 \mathrm{~cm}^{2}$ each. To avoid any possible structural changes caused by fibre hornification, the wet web was used without any pressing contrary to the standard fabrication of handsheets. The wet web test specimens produced from refined pulps and fibres were instantly placed between the jaws of the instrument. Initial span of the jaws was $0.5 \mathrm{~cm}$ with a clamping pressure of $94.6 \mathrm{kN} \cdot \mathrm{m}^{-2}$. Jaws retaining bolt was lifted and flow was started by opening the valve provided at the bottom of the volumetric cylinder. The quantity of water accumulated in the bucket increased so the load on the strip was increased. The point was reached when the strip was unable to withstand any further added load and ruptures. At that point, flow was stopped and drop in water level was noted. 


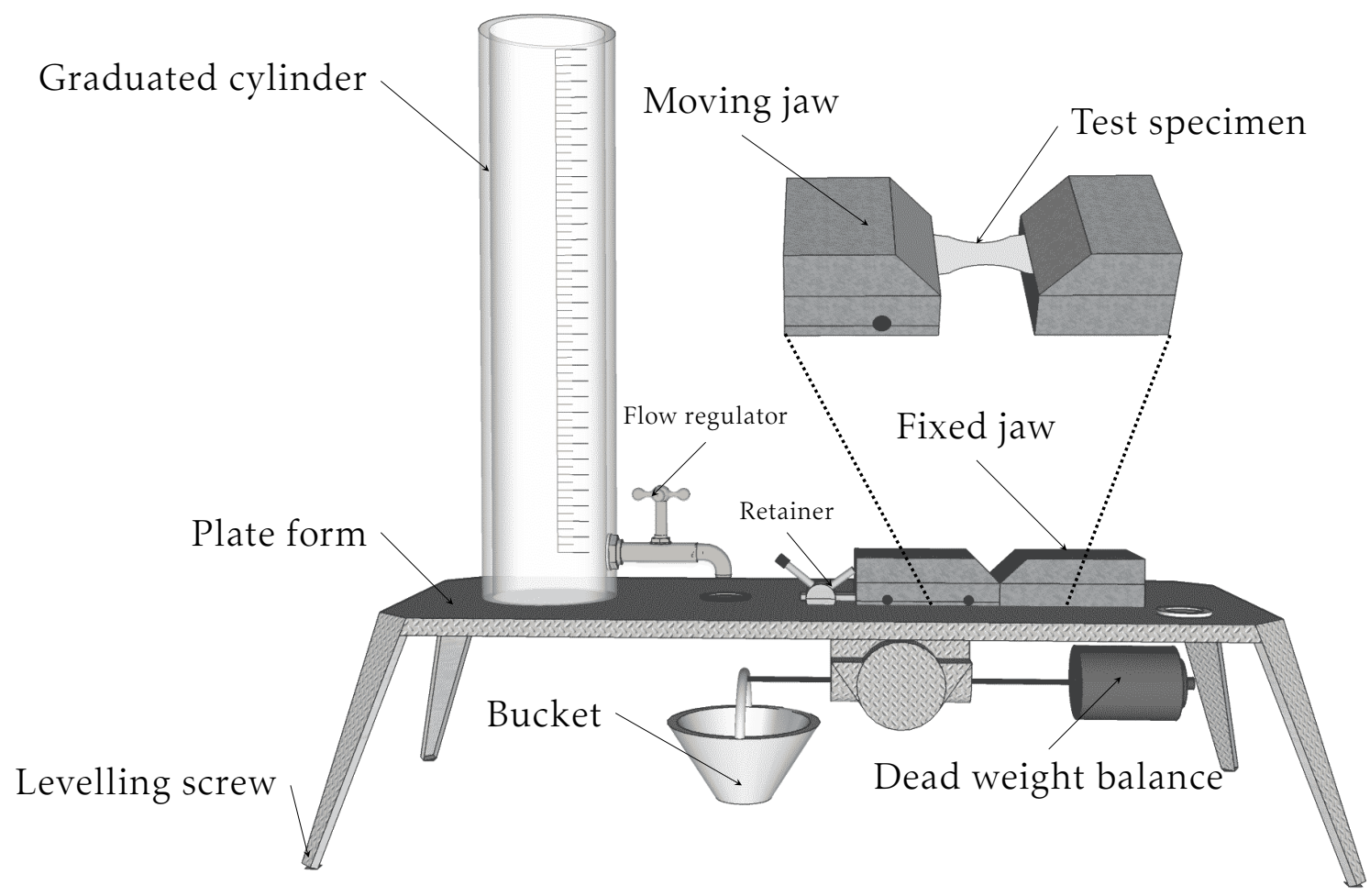

Figure 3.26: Wet breaking length measurement device

WBL was calculated from the following expression

$$
\mathrm{WBL}=\frac{m_{w}}{W \cdot G}
$$

where $m_{w}, w$ and $G$ are the mass of water, width and basis weight of the test strip, respectively.

Effect of refining on WBL of pulps and fibres is shown in Figure 3.27. Moisture content of the test specimens was 75 to $80 \%$. Mean values are based on ten measurements. 


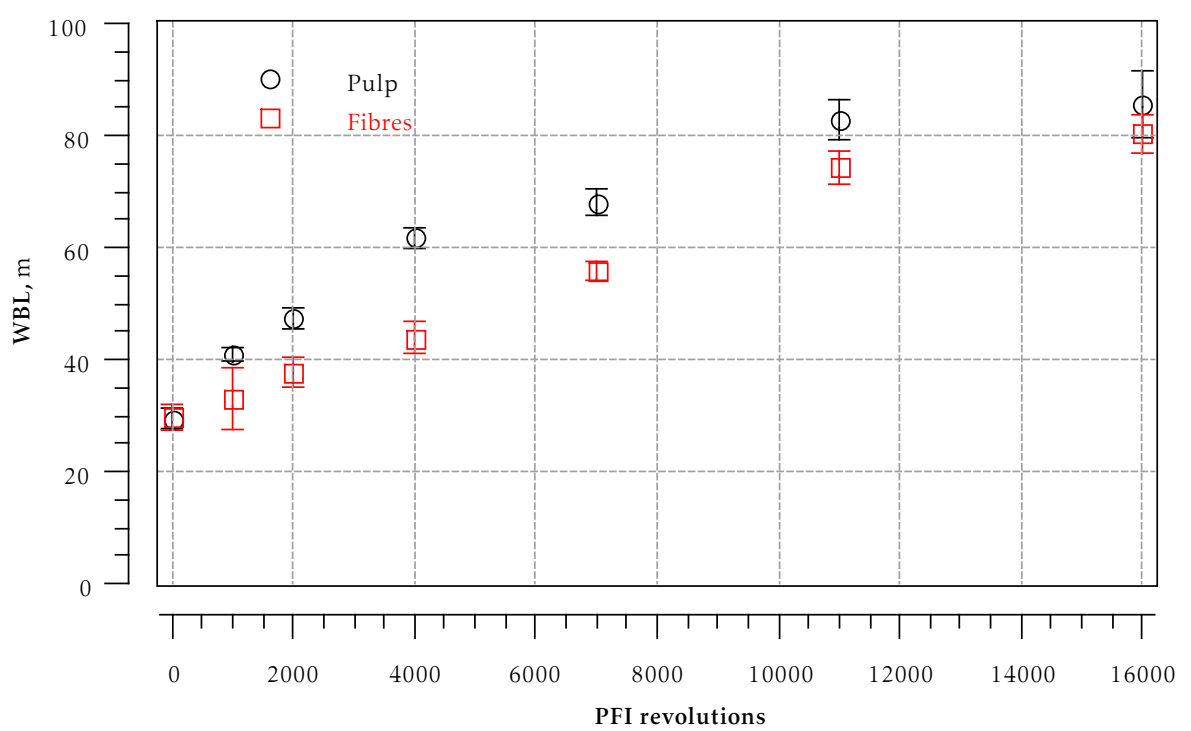

Figure 3.27: Evolution of wet breaking length of pulps and corresponding fibres during refining

WBL of pulp and fibres increased with refining. Refined pulps have higher WBL compared to fibres at the same level of refining. Internal fibrillation increased flexibility of the fibres. Fibrils and fines present in the inter-fibre spaces of network increased friction and surface tension forces (cf. equation 2.22). Little difference in wet strengths of fibres and pulps at the initial and final stage of refining can be explained on the basis of fines content. Low fine content initially offered lesser friction and surface tension. Drop in wet strength with higher fines content indicate a state of saturation where fines content reach a critical value (critical fines content). The results are in accordance to the previous study of Corson [62]. Since fines carry huge specific surface therefore they also have a lot of "surface water" which lubricate fines-fines contact and therefore wet strength is decreased.

\subsection{Weak points in fibre wall of refined pulps and fibres}

Strength of the fibres is affected by the presence of dislocated regions (weak points) in the fibre wall. Quantification of the weak points in the fibre wall is not simple. Ander and Daniel [29] used cleavages per fibre as a relative measure for their quantification using $\mathrm{HCl}$. 


\section{Cleavages per fibre of refined pulps and fibres}

Process flow diagram of fibre cleavage measurement during refining is shown in Figure 3.28. Refined air dried pulp was shredded, disintegrated, refined and fractionated in accordance to the procedure described in subsection 3.1.1. Steps carried out for $\mathrm{HCl}$ treatment are described in Appendix C. After washing, the cleaved fibres were characterised for the morphological changes.

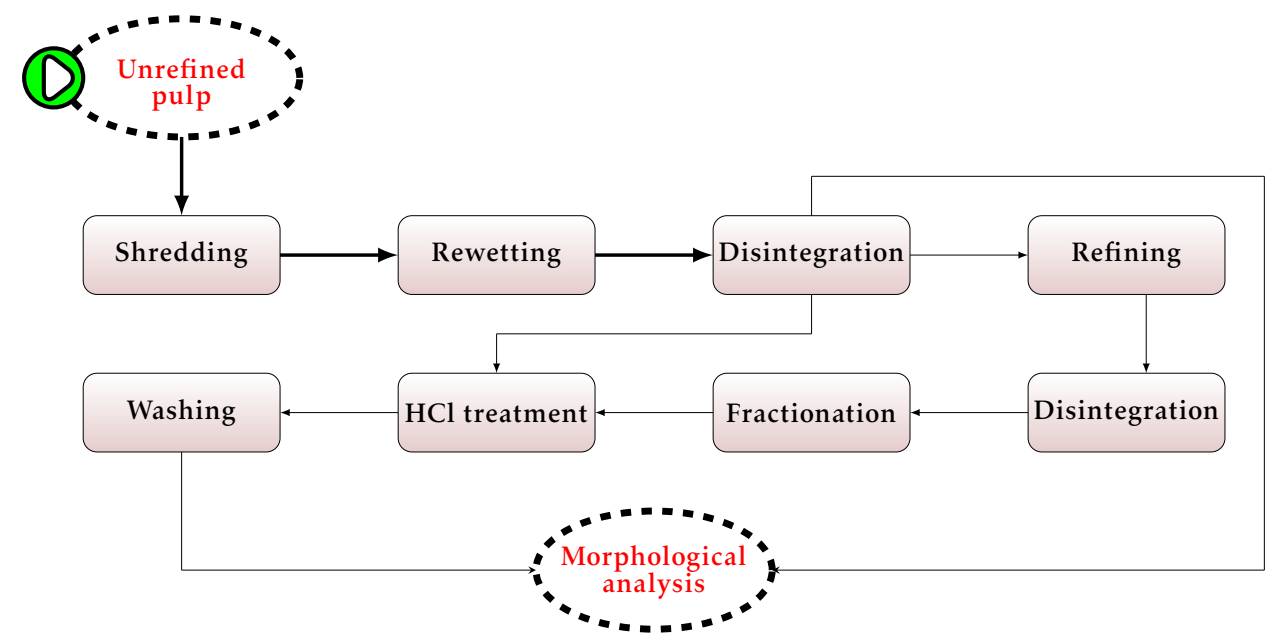

Figure 3.28: Measurement of cleavages per fibre during refining

Length-weighted length of uncleaved and cleaved fibres with $1 \mathrm{M} \mathrm{HCl}(\mathrm{pH}=0)$ decreased with refining. With $2 \mathrm{M} \mathrm{HCl}(\mathrm{pH}=-0.3)$, length-weighted fibre length did not change until 11000 PFI revolutions, beyond that mean length-weighted fibre length increased slightly (cf. Figure 3.29).

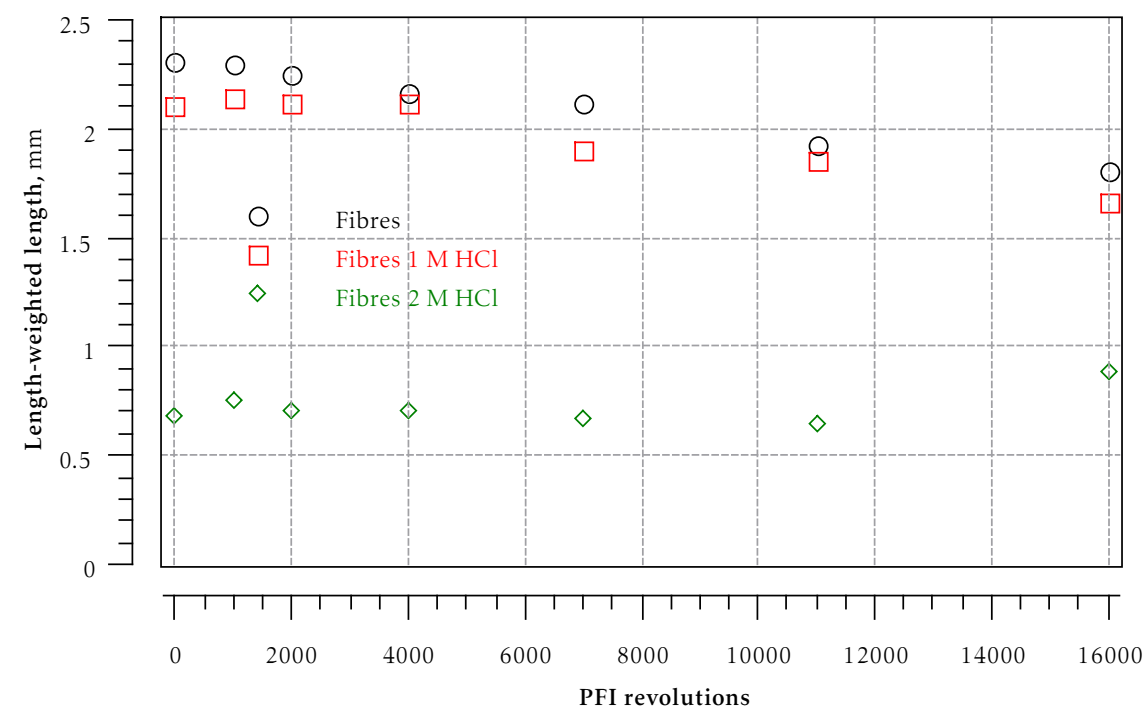

Figure 3.29: Evolution of length-weighted length of cleaved refined fibres 
Fibre length distributions of cleaved unrefined and refined fibres are shown in Figure 3.30. Refining increased short fibre content $(0.2$ to $0.5 \mathrm{~mm})$ due to the mechanical impacts. Mild treatment of unrefined and refined fibres with $1 \mathrm{M} \mathrm{HCl}$ did not alter length distributions. However, number of long fibres significantly decreased when treated with $2 \mathrm{M} \mathrm{HCl}$ resulting in a relative increase of short fibres in length distributions. The increase in short fibre content is relatively higher in unrefined fibres than in refined fibres after treatment with $2 \mathrm{M} \mathrm{HCl}$.
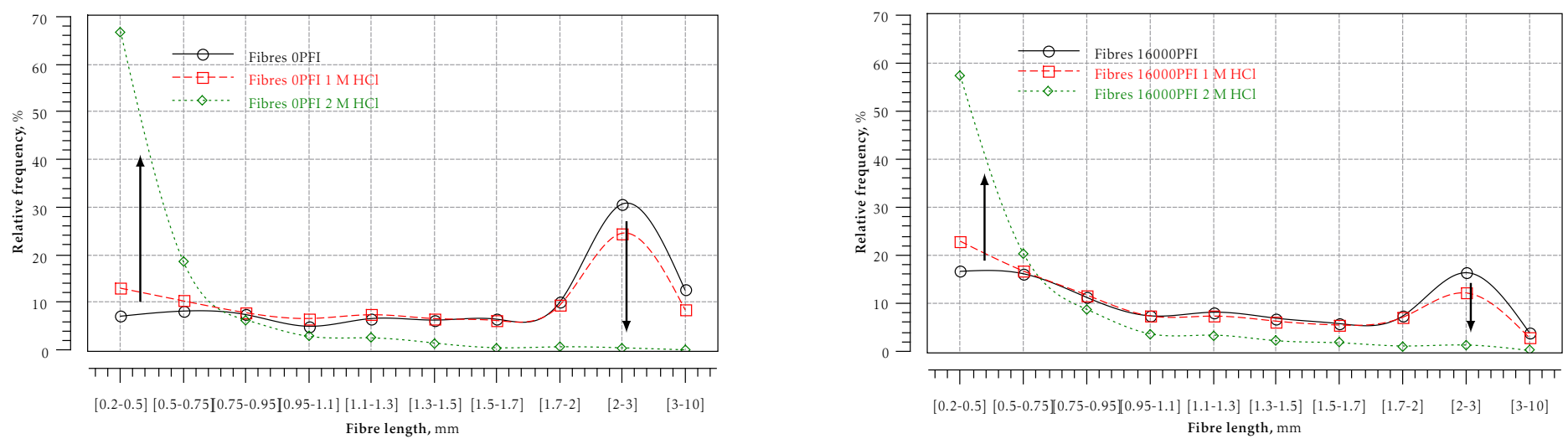

Figure 3.30: Effect of $\mathrm{HCl}$ cleavage on fibre length distributions of unrefined (left) and refined (right) fibres

Fibre width decreased only when treated with $2 \mathrm{M} \mathrm{HCl}$. It can be said that $2 \mathrm{M} \mathrm{HCl}$ attacked outer layers (P/S1 and S2) of fibre wall (cf. Figure 3.31). 


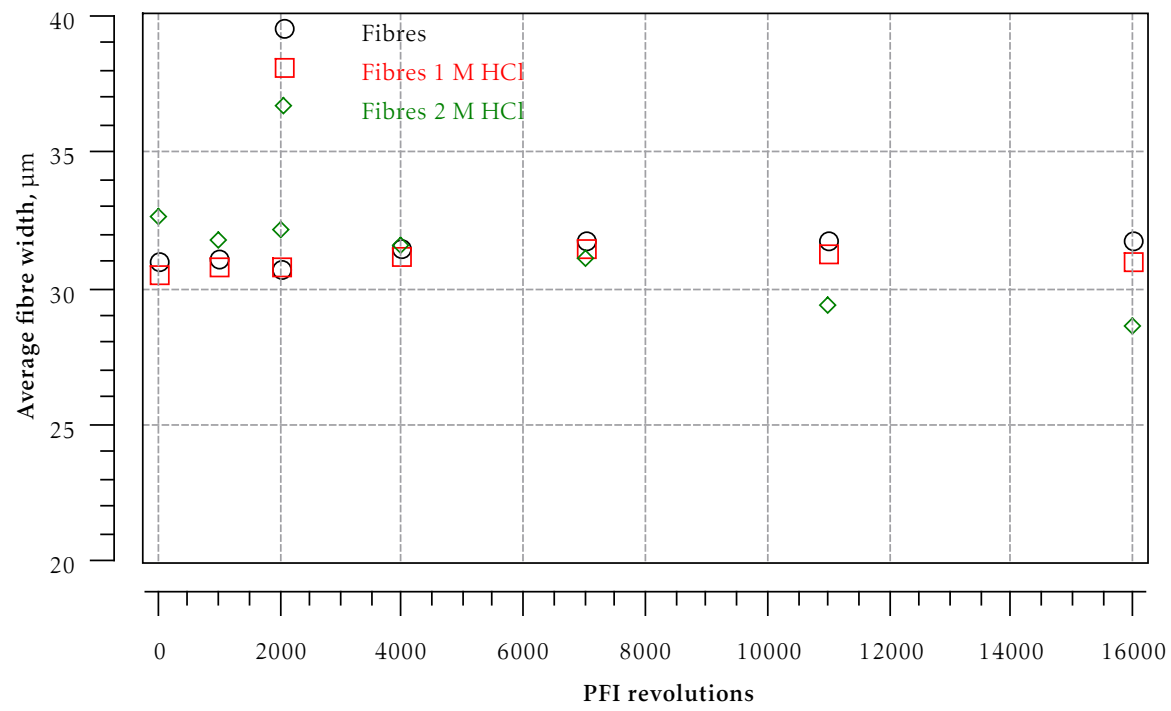

Figure 3.31: Evolution of average width of cleaved refined fibres

Fibre width distributions of cleaved fibres are shown in Figure 3.32. Number of fibres slimmer than $35 \mu \mathrm{m}$ were decreased during $\mathrm{HCl}$ treatment resulting in relative increase of fibres wider than $35 \mu \mathrm{m}$ in the distribution. It can be said that expansion of unrefined fibres when treated with $2 \mathrm{M} \mathrm{HCl}$ was caused by LW fibres. However, in case of refined fibres the relative increase in frequency of slimmer fibres ( 5 to $16 \mu \mathrm{m}$ ) was more pronounced when treated with $2 \mathrm{M} \mathrm{HCl}$ than that of unrefined fibres.
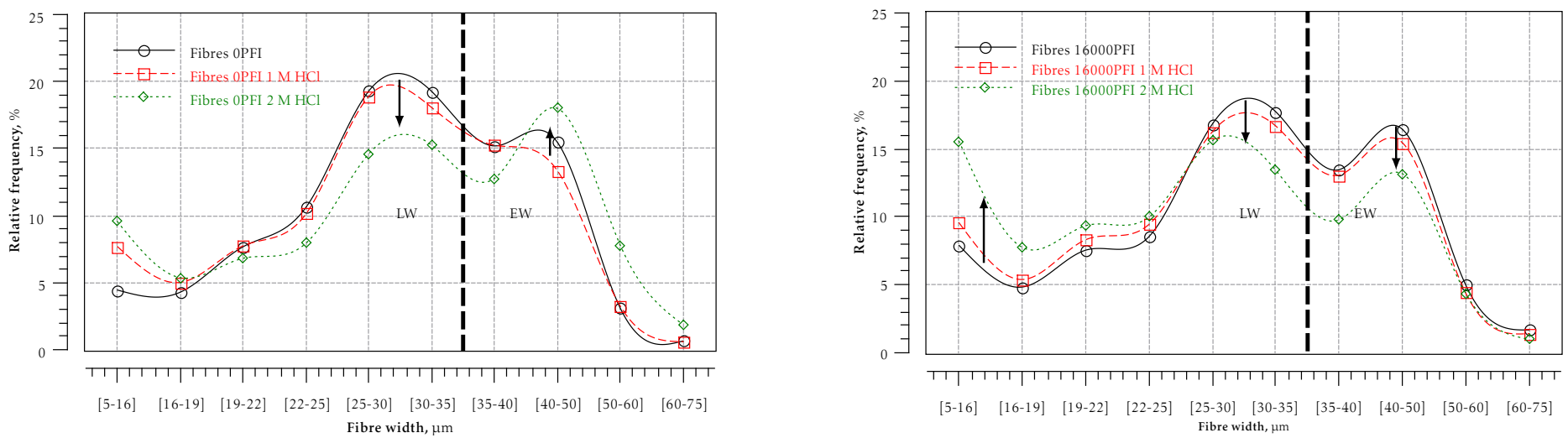

Figure 3.32: Effect of $\mathrm{HCl}$ cleavage on width distribution of unrefined (left) and refined (right) fibres

Average fibre curl index of cleaved refined fibres is shown in Figure 3.33. 


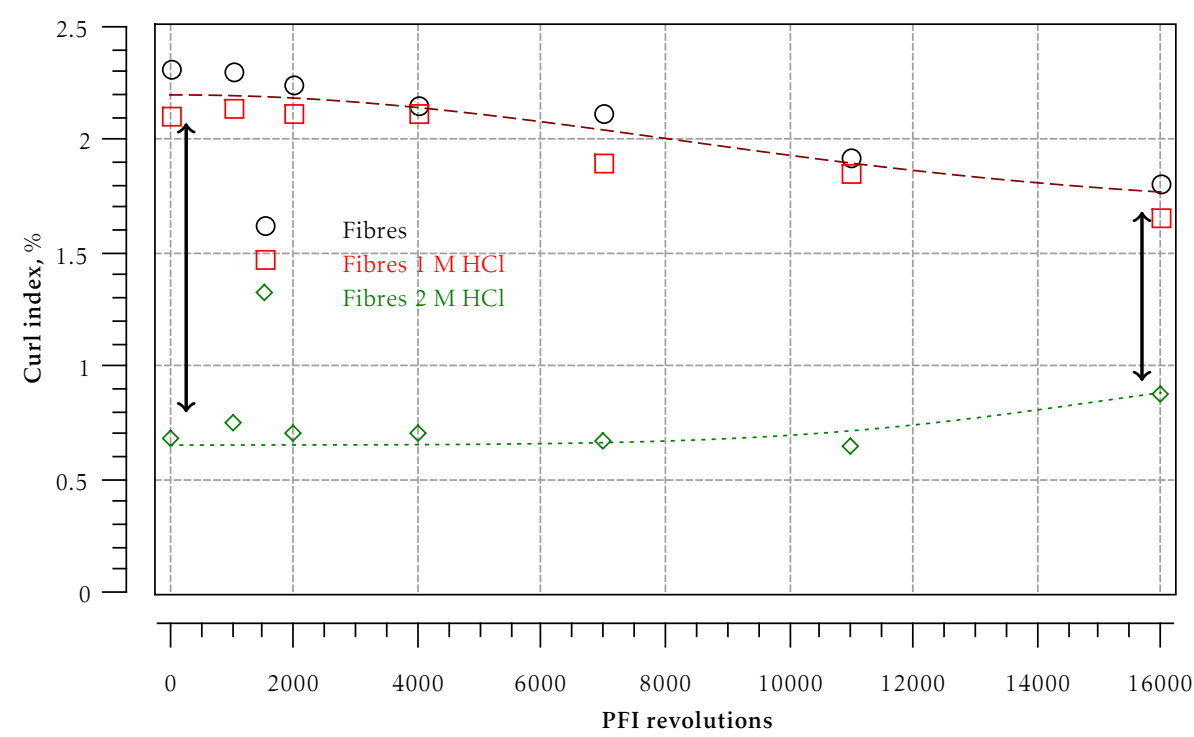

Figure 3.33: Evolution of curl index of cleaved refined fibres

Curl index of untreated and treated fibres with $1 \mathrm{M} \mathrm{HCl}$ decreased during refining. The decrease in curl index during refining was due to the increase of fibre flexibility and shortening. Strong cleavage with $2 \mathrm{M} \mathrm{HCl}$ resulted in the drop of curl index as expected. Curl index of fibres treated with $2 \mathrm{M} \mathrm{HCl}$ during refining increased only slightly until 11000 PFI revolutions, however after 11000 PFI revolutions curl index increased significantly.

When treated with $2 \mathrm{M} \mathrm{HCl}$, fines content increased with refining until 11000 PFI revolutions. Decrease in fines content after 11000 PFI revolutions (cf. Figure 3.34) indicates drop in cleavages. 


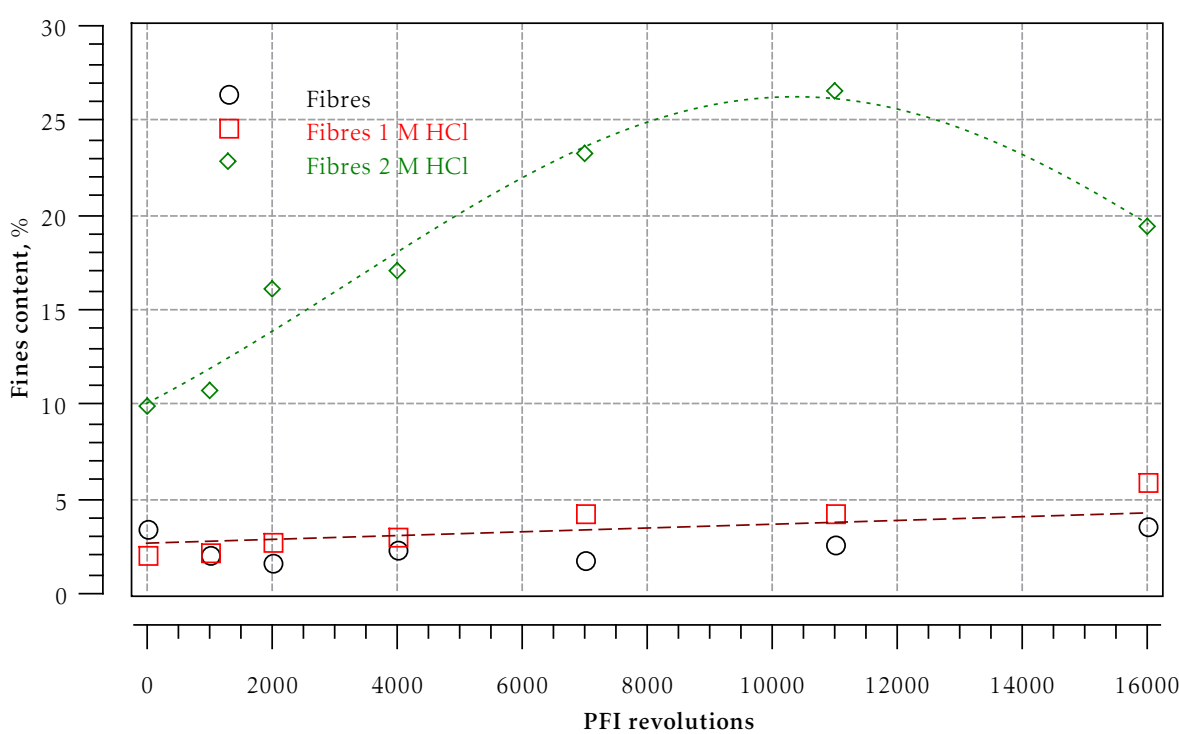

Figure 3.34: Evolution of fines content of cleaved refined fibres

Cleavages per fibre of refined fibres is shown in Figure 3.35 for two $\mathrm{HCl}$ concentrations. Dislocations present in the fibre wall serve as weak points for acid attack. Fibre wall cleavage takes place at these weak fibre wall areas. Fibres were cleaved when treated with $2 \mathrm{M} \mathrm{HCl}$. Cleavages per fibre slightly decreased until 11000 PFI revolutions however, beyond 11000 PFI revolutions cleavages per fibre decreased strongly.

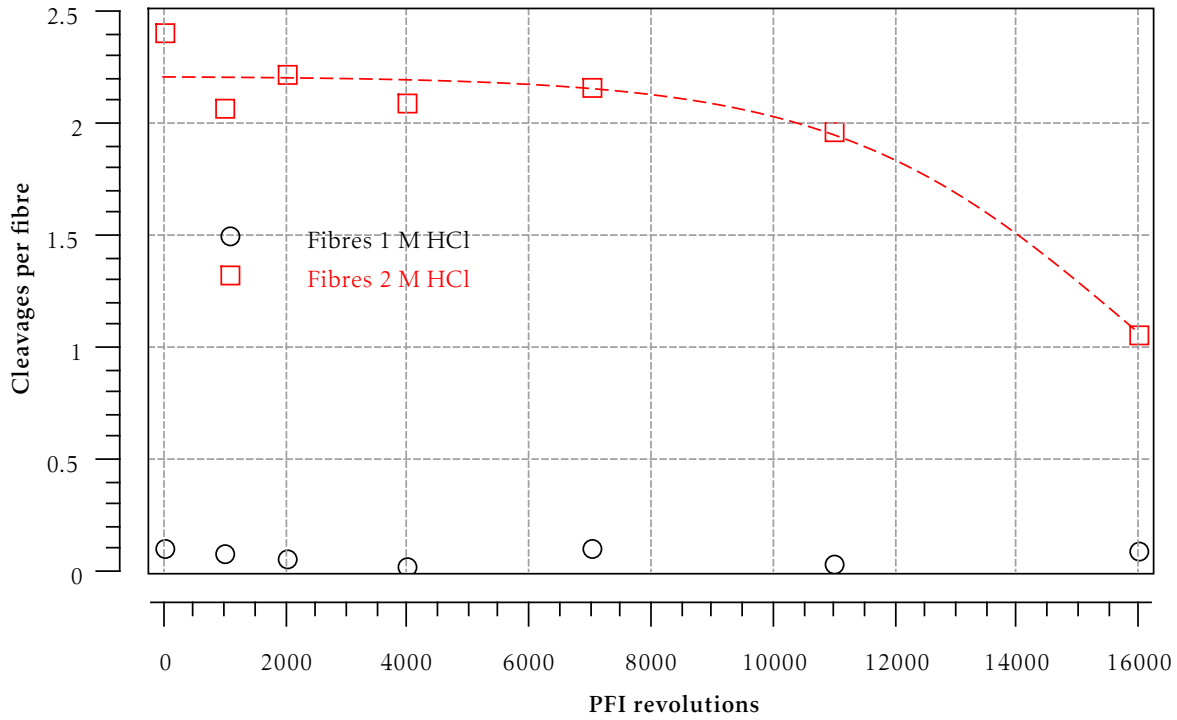

Figure 3.35: Evolution of cleavages per fibre during refining

During refining, fibres were broken at already present weak points resulting in the production of fines and fibre shortening. Outer cell wall layers were 
loosened and ruptured by refining, the accessibility to inner layers hence increased. The results indicate that the number of dislocations per unit fibre length decreased with refining however the decrease in width indicates the attack on outer S2 layers. Inner S2 and S3 layers kept fibre intact during acid attack. The results are in contradiction to the previously reported [27] increase in dislocations with refining and wet pressing.

Probable transformations in cell wall layers (radial section) during refining due to cleavage are summarised in Figure 3.36. Cell wall is cleaved (b) by $\mathrm{HCl}$ at dislocation (a). Refining removes dislocation (c) to certain extent. $\mathrm{HCl}$ attacks and removes outer layers of refined fibre wall (d). Cooked and delignified fibres are more flexible and less brittle in the absence of lignin. Laboratory refining removes dislocations in fibre wall to some extent. 


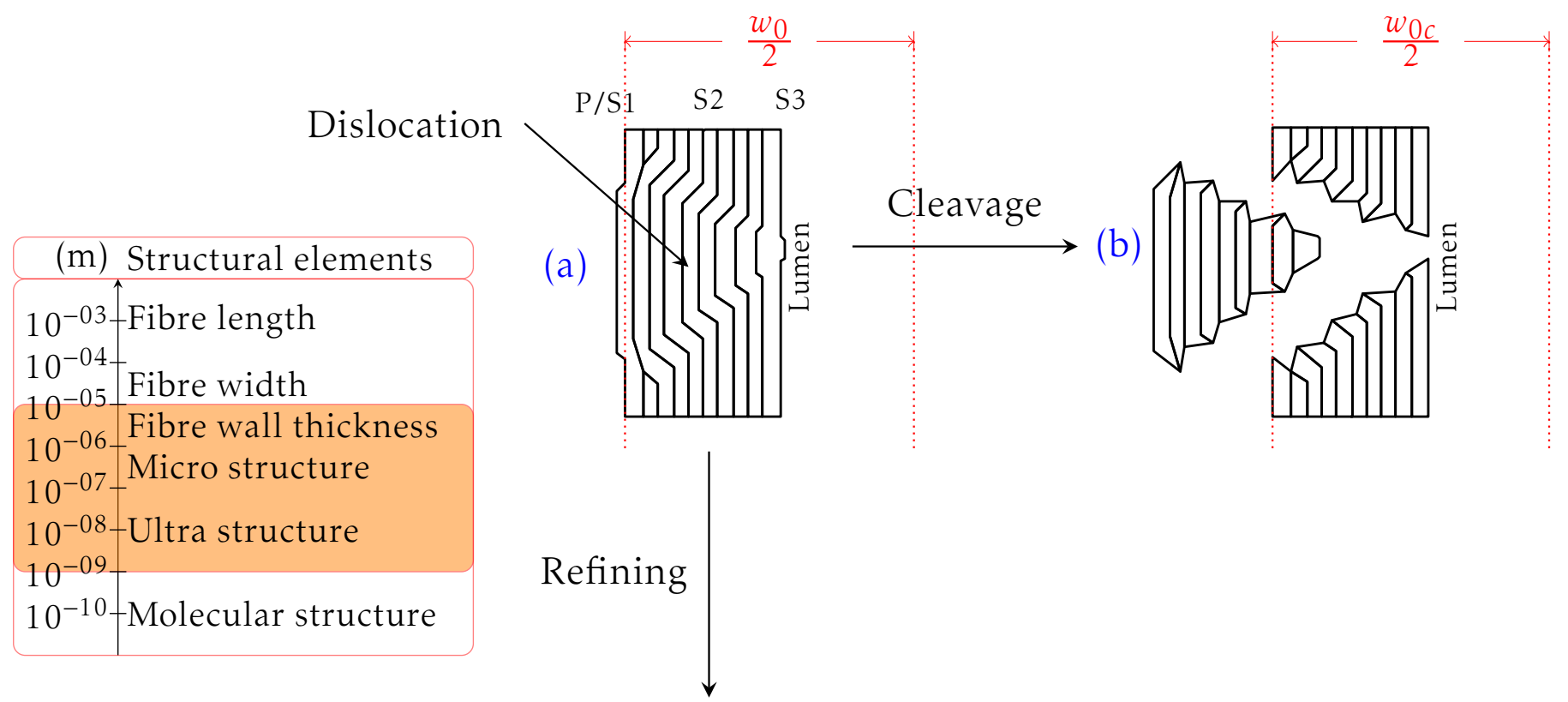

External fibrillation

Internal fibrillation

(delamination)
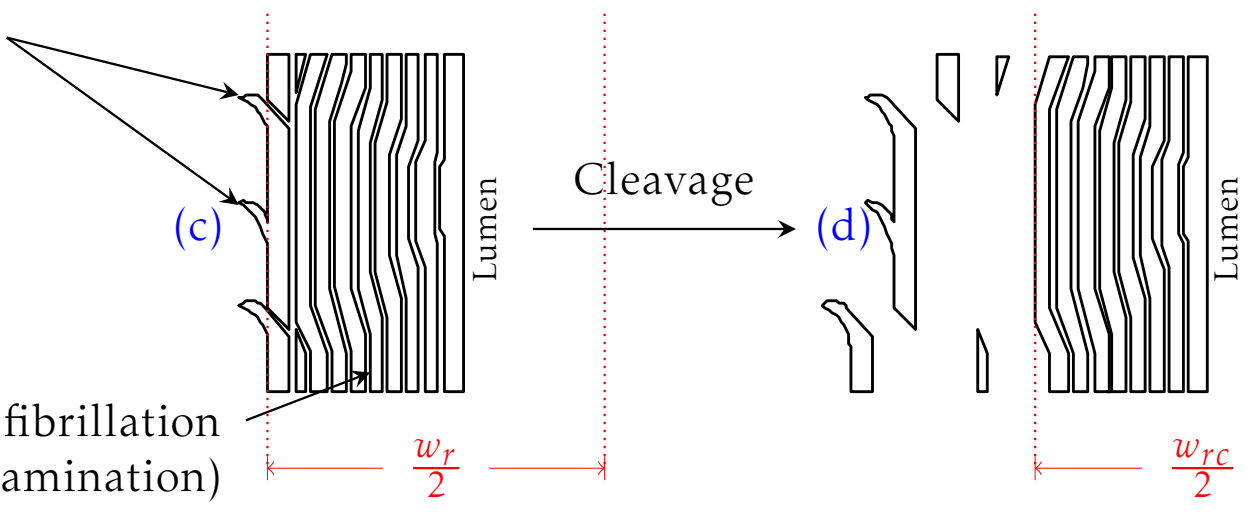

Figure 3.36: Schematic diagram of probable fibre cell wall transformations during refining by $\mathrm{HCl}$ cleavage. $w_{0}, w_{r}, w_{0 c}$ and $w_{r c}$ are fibre widths of unrefined, refined, unrefined cleaved and refined cleaved fibres.

\subsection{Conclusions of pulp refining}

Refining study was used to validate the protocols for morphological analysis especially cleavages per fibre, fibre saturation point and inverse size exclusion chromatography. In order to have good reproducibility of inverse size exclusion chromatographic measurements, a semi automatic column fabrication device was developed. Results obtained during refining are in accordance with the literature.

Fibre lengths decreased with refining. Latewood fibres underwent width expansion causing increase in average fibre width. External fibrillation and broken ends were increased whereas curl, kink and kinked fibres were decreased. 
Pulps and fibres refined in PFI mill were morphologically different than those refined in Valley beater.

SR and drainage time increased with refining. Pulps and fibres refined with PFI mill formed compact and highly resistant filtration mat.

Water retention valaue of pulps and fibres increased with refining. Fibre saturation point was also increased by refining. Fibre wall porosity and average pore size (ISEC) increased with refining.

Wet breaking length increased with refining. Below critical fines content, fines increase wet strength. Weak points per fibre decreased with refining. 
$\Gamma_{\text {Chapter }}$

\section{Recycled pulps and fibres}

\section{Contents}

4.1 Chemical properties of recycled pulps and fibres . . . . . 72

4.2 Morphological properties of recycled pulps and fibres . . . 73

4.3 Surface analysis . . . . . . . . . . . . . . 89

4.4 Dewatering of recycled pulps and fibres $\ldots \ldots \ldots$

4.5 Specific water retention of recycled pulps and fibres . . . 9 96

4.6 Hornification of recycled pulps and fibres . . . . . . . 98

4.7 Pore size distribution of recycled fibres . . . . . . . . 100

4.8 Width shrinkability of recycled fibres . . . . . . . . 102

4.9 Wet breaking length of recycled pulps and fibres . . . . 103

4.10 Weak points in fibre wall of recycled fibres . . . . . . . . 104

4.11 Single fibre tensile properties $\ldots \ldots \ldots \ldots$. . . . . 108

Never dried bleached mixed softwood kraft pulp (0RP), a mixture of norway spruce, douglas fir, scots pine, maritime and pine pulps was obtained from Tembec.

Main unit operations involved in mild laboratory recycling are shown in Figure 4.1. Process of recycling was repeated 10 times to obtain repeatedly recycled pulps, fibres and papers. 


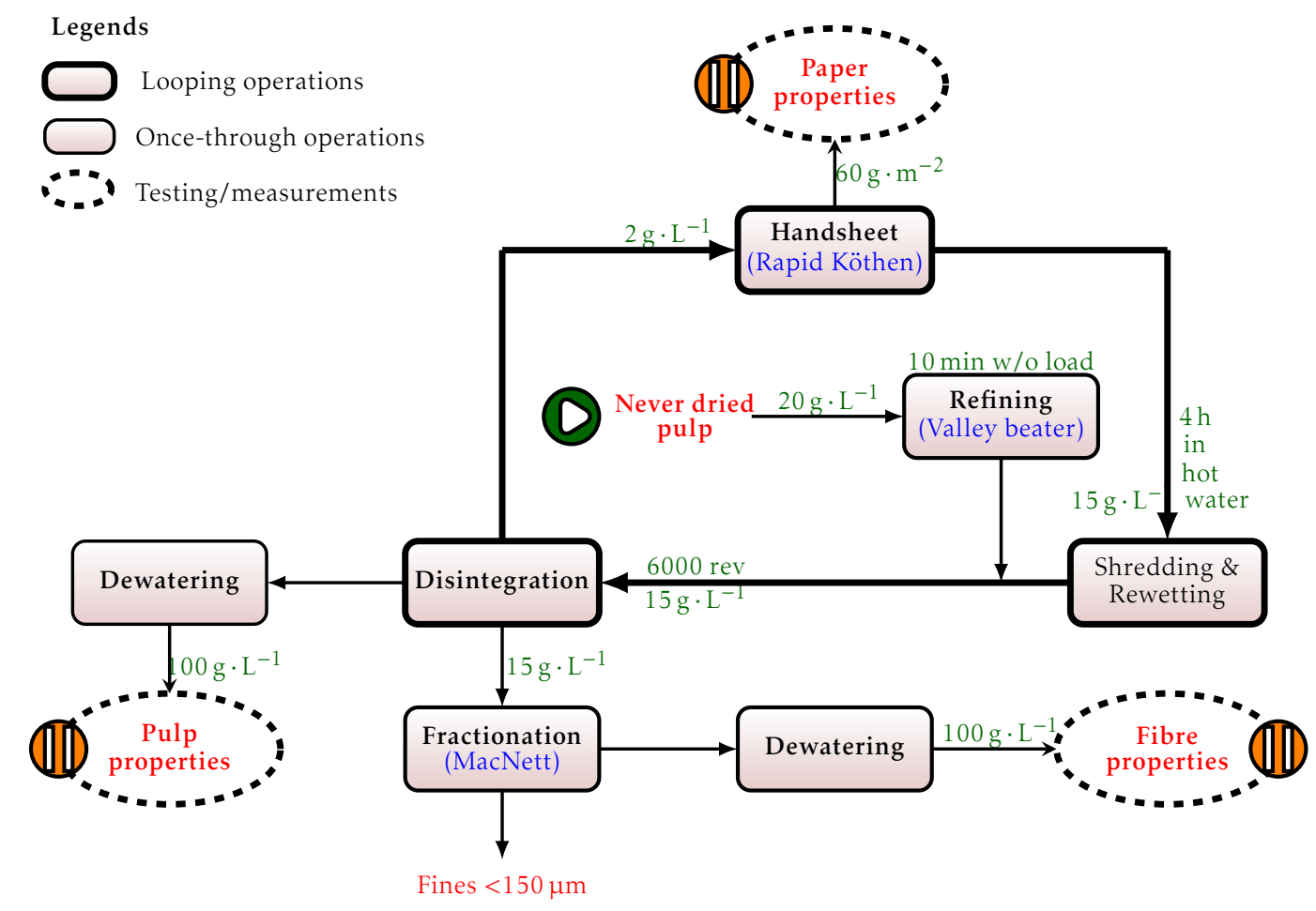

Figure 4.1: Preparation of recycled paper, pulps and fibres

Consistency of never dried pulp (0RP) was set to $20 \mathrm{~g} \cdot \mathrm{L}^{-1}$. Pulp was homogenised using "VOITH valley laboratory" beater [1] for $10 \mathrm{~min}$ without applying any load. Later on consistency of pulp was reduced to $15 \mathrm{~g} \cdot \mathrm{L}^{-1}$ and disintegration [14] was carried out in "pulpeur inox ALLIBE" disintegrator for $5 \mathrm{~min}$. Consistency of the pulp suspension was then reduced to $2 \mathrm{~g} \cdot \mathrm{L}^{-1}$ for handsheet formation. "Karl Frank Rapid- Köthen" [15] an automatic sheet machine was used to form isotropic circular handsheets of $2 \mathrm{~g}\left(60 \mathrm{~g} \cdot \mathrm{m}^{-2}\right.$ oven-dry). Wet handsheet was couched from the wire screen with the help of blotters, pressed and dried in dryer with almost complete prevention of shrinkage. The temperature in the dryer was $80^{\circ} \mathrm{C}$. This temperature is lower than the industrial ones reaching roughly $120^{\circ} \mathrm{C}$. This might modify the hornification behaviour.

Handsheets were shredded and immersed in hot water for $4 \mathrm{~h}$. Consistency was maintained at $15 \mathrm{~g} \cdot \mathrm{L}^{-1}$. Soaked pulp suspension was then disintegrated in "pulpeur inox ALLIBE" disintegrator for 5 min (6000 revolutions). P100 was removed using "Bauer-McNett classifier". Looping operations were followed to obtain recovered pulps (nRPs) and fibres (nRFs). nRP is $n$ times recycled pulp and $\mathrm{nRF}$ is the corresponding fibre fraction obtained after fractionation. 


\subsection{Chemical properties of recycled pulps and fi- bres}

The number of repeating units in a polymeric chain is termed as degree of polymerisation (DP). The DP of cellulose depends on the type of wood specie and the process conditions (cf. Table 4.1).

Table 4.1: Average DP values of cellulose obtained from molecular mass distribution [241]

\begin{tabular}{l|c} 
Cellulose & Degree of polymerisation \\
\hline Wood & $6000-10000$ \\
Pulp & $500-2000$ \\
Sulphate pulp & $950-1300$ \\
Chemical pulp bleached & 700
\end{tabular}

Average DP of cellulose based on viscosity $\left(\overline{\mathrm{DP}}_{v}\right)$ can be described by the following expression

$$
\overline{\mathrm{DP}}_{v}=\frac{\mathrm{M}_{v}}{\mathrm{M}_{g}}=\frac{\frac{\sum \mathrm{N}_{i} \cdot \eta_{i}}{\sum \mathrm{N}_{i}}}{\mathrm{M}_{g}}
$$

where $\mathrm{M}_{v}, \mathrm{M}_{g}, \mathrm{~N}_{i}$ and $\eta_{i}$ are the viscosity-average molecular weight, molecular weight of anhydrous glucose, number of moles and viscosity of $i^{\text {th }}$ fraction, respectively.

$\overline{\mathrm{DP}}_{v}$ of cellulose can be calculated using Mark-Houwink-Sakurada (MHS) equation described by Sihtola et al. [203].

$$
\overline{\mathrm{DP}}_{v}^{0.905}=0.75 \eta_{s}
$$

where $\eta_{s}$ is the SCAN viscosity $\left(\mathrm{mL} \cdot \mathrm{g}^{-1}\right)[7]$.

$$
\eta_{s}=954 \cdot \log (\eta)-325
$$

Viscosity of cellulose dissolved in cupri-ethylenediamine (CED) solution ( $\eta$, $\mathrm{mPa} \cdot \mathrm{s})$ was measured using Schott's Cannon-Fenske reverse flow viscometer (Type 51113 of capillary number 150).

Pentosans [11] were hydrolysed and quantified colorimetrically.

Residual lignin content of wood pulp is represented by Kappa number. Kappa 
number of the pulps was measured using standardised analytical method [13].

Properties of recycled pulps are presented in Table 4.2.

Table 4.2: Properties of recycled pulps

\begin{tabular}{c|ccc}
$\begin{array}{c}\text { Recycle } \\
\text { number }\end{array}$ & $\overline{\mathrm{DP}}_{v}$ & $\begin{array}{c}\text { Pentosans } \\
\%\end{array}$ & $\begin{array}{c}\text { Kappa } \\
\text { number }\end{array}$ \\
\hline 0 & 910 & 3.5 & 0.9 \\
1 & 891 & 3.9 & 0.9 \\
10 & 891 & 3.6 & 0.8
\end{tabular}

$\overline{\mathrm{DP}}_{v}$, pentosan content and Kappa number did not change during the coarse of recycling. Bouchard and Douek [47] reported depolymerisation of cellulose during recycling. However, they arrived at a conclusion that there was no direct relation between the change in chemical properties to the change in physical properties (strength properties) of fibres during recycling.

\subsection{Morphological properties of recycled pulps and fibres}

As mentioned in section 3.2, the morphological analysis was performed in order to asses the effect of recycling on fibre length, width, curl, kink and discontinuities etc.

Figure 4.2 shows the effect of recycling on length-weighted fibre length of pulps and corresponding fibres. 


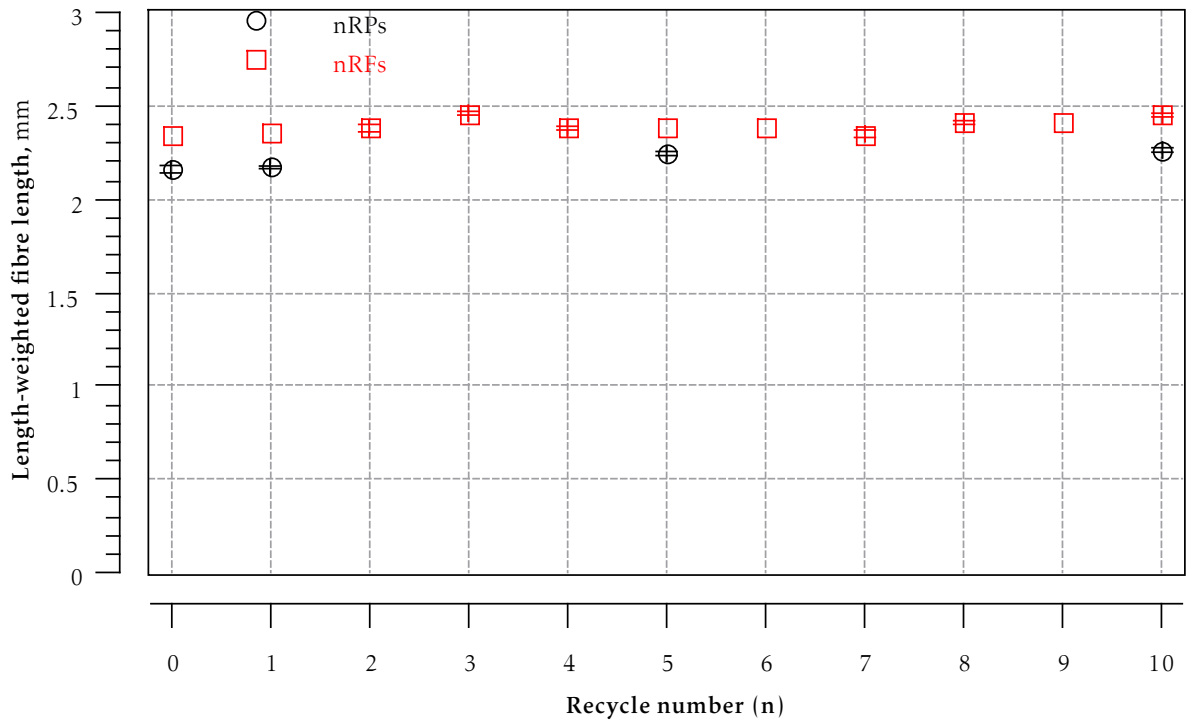

Figure 4.2: Evolution of length-weighted fibre length of pulps and corresponding fibres during recycling

Arithmetic and length-weighted fibre length of pulps were slightly increased during repeated cycles of recycling.

The effect of recycling on length distribution of pulps and corresponding fibres is presented in Figure 4.3. Relative frequency of shorter fibres $(0.2$ to $0.5 \mathrm{~mm})$ in pulps decreased during recycling. However, length distribution of the fibres remained unaffected by recycling (cf. Figure 4.3).
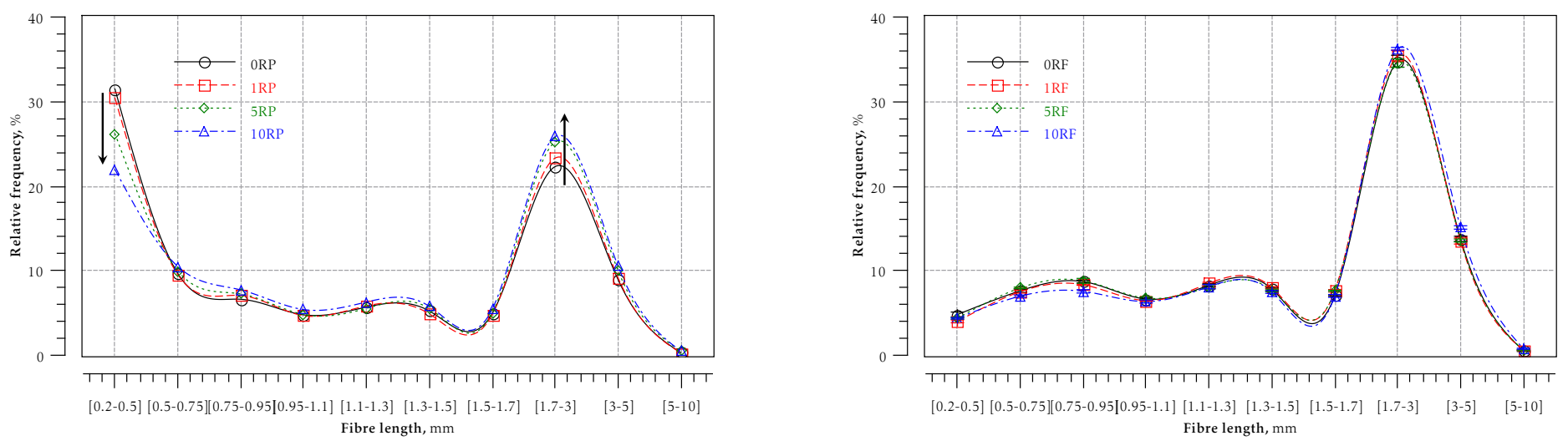

Figure 4.3: Effect of recycling on fibre length distribution of pulps (left) and corresponding fibres (right)

Therefore it can be said that the difference in lengths of pulps and fibres in Figure 4.2 is due to the loss of short fibres $(0.2$ to $0.5 \mathrm{~mm})$ during fractionation. 
The effect of recycling on fibre width of pulps and the corresponding fibres is shown in Figure 4.4 .

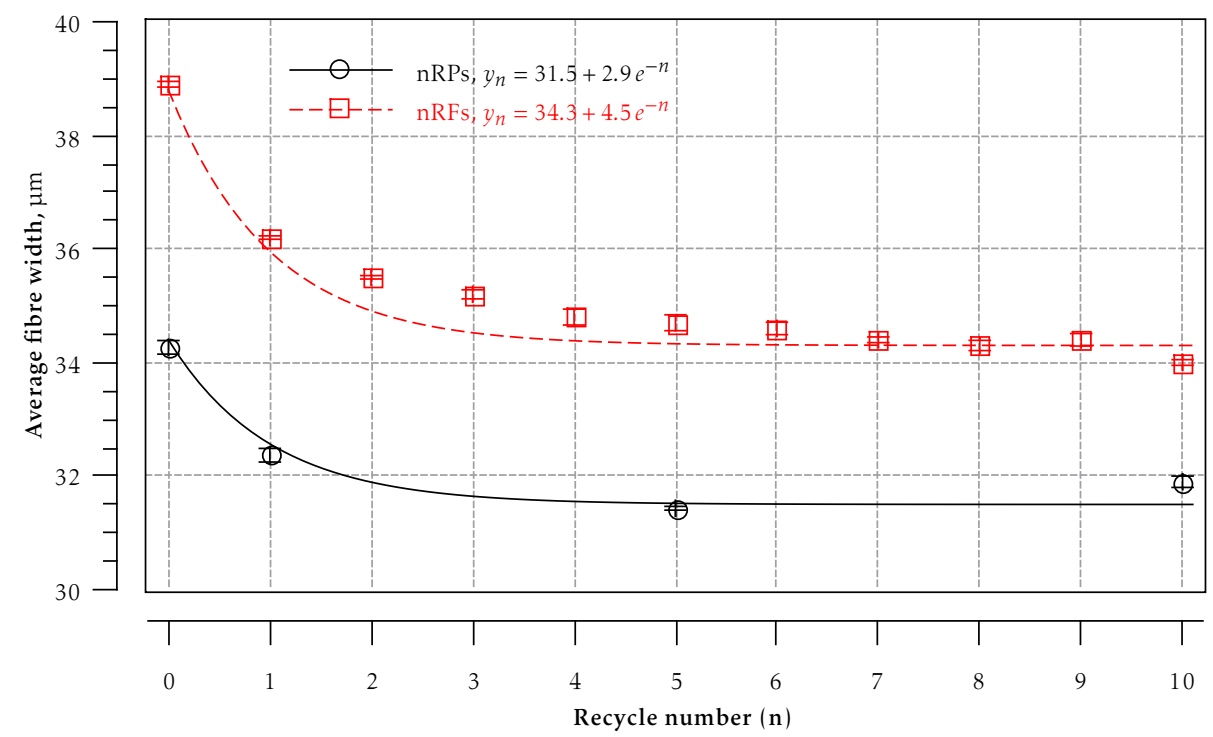

Figure 4.4: Evolution of fibre width of pulps and corresponding fibres during recycling

Contribution of short fibres (difference in average width of fibres and pulp) towards width shrinkage decreased with recycling.

Fibre width shrinkage can be described by the following expression

$$
\text { Width shrinkage }=\frac{\left\langle w_{n}\right\rangle-\left\langle w_{0}\right\rangle}{\left\langle w_{0}\right\rangle} \cdot 100
$$

where $\left\langle w_{n}\right\rangle$ is the average width of $n$ times recycled pulps or fibres. The value of $n$ (recycle number) varies between 0 to $10 .\left\langle w_{0}\right\rangle$ is the average width of never dried pulps or fibres. 6 to $8 \%$ fibre width shrinkage took place during first cycle of recycling which represents 60 to $65 \%$ of total shrinkage. After that average width decreased only slightly. The evolution of average widths during recycling can be described by a first order exponential decay.

$$
\left\langle w_{n}\right\rangle=\left\langle w_{10}\right\rangle+\left\{\left\langle w_{0}\right\rangle-\left\langle w_{10}\right\rangle\right\} e^{-n}
$$

where $\left\langle w_{10}\right\rangle$ is the average width of ten times recycled pulp or fibres.

The effect of recycling on fibre width distributions of pulps and corresponding fibres is shown in Figure 4.5. 


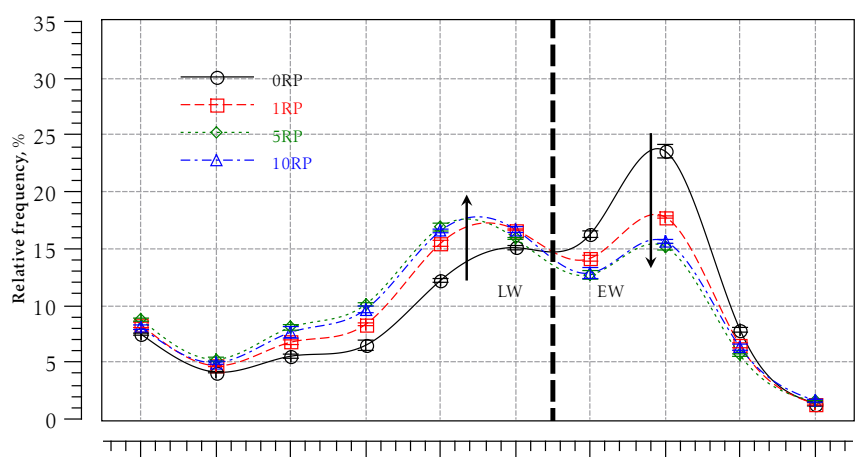

$\left[\begin{array}{llllllllll}{[5-16]} & {[16-19]} & {[19-22]} & {[22-25]} & {[25-30]} & {[30-35]} & {[35-40]} & {[40-50]} & {[50-60]} & {[60-75]}\end{array}\right.$

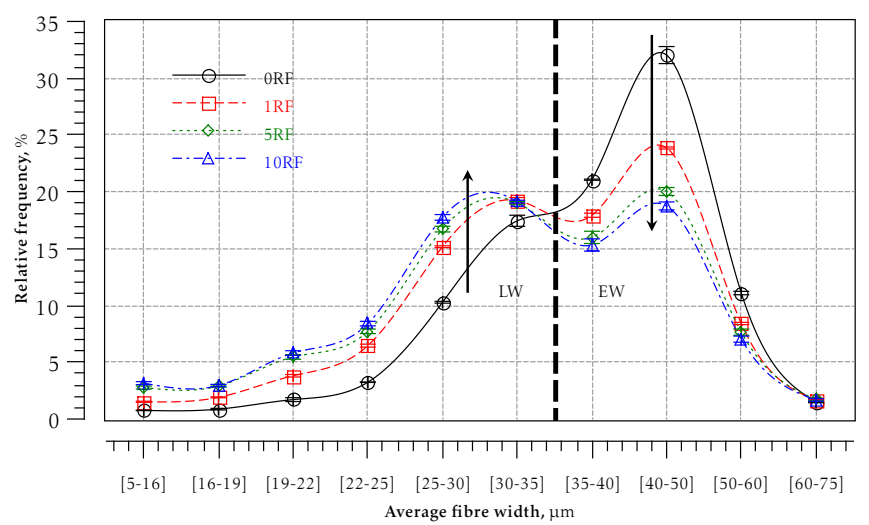

Average fibre width, $\mu \mathrm{m}$

Figure 4.5: Effect of recycling on width distributions of pulps (left) and corresponding fibres (right)

A frequency drift from thicker to thinner fibres can be seen. Number of fibres thinner than $35 \mu \mathrm{m}$ were increased while fibres wider than $35 \mu \mathrm{m}$ decreased during recycling. It can be said that EW fibres underwent strong width shrinkage. The effect of recycling on width distribution of both pulps and fibres is different than the one due to refining (cf. Figure 3.7). Furthermore, the nature of the different fibres (mixture of softwoods) should have been analysed to validate the assumption of the distinction between earlywood and latewood fibres.

To check if EW and LW fibres have same length distributions, length-width distributions of ORF and 10RF were plotted. By comparing both graphs, it can be said that length distribution of EW and LW fibres are similar (cf. Figure 4.6).
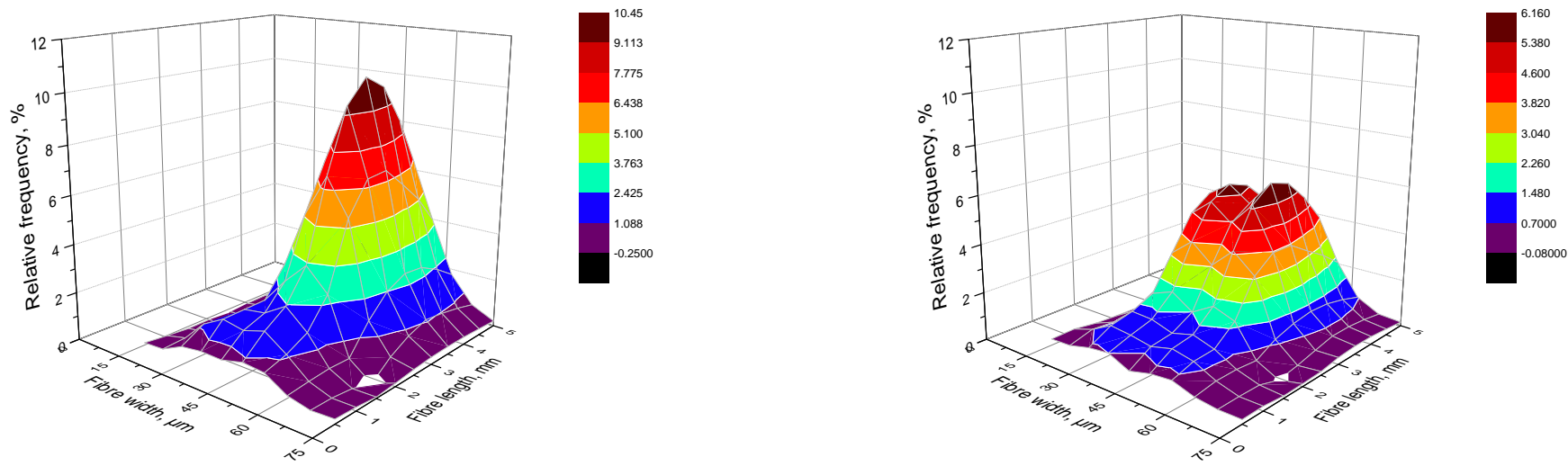

Figure 4.6: Length-width distribution of never dried fibres (left) and ten times recycled fibres (right) 
The effect of recycling on the amount of fines of pulps and corresponding fibres is presented in Figure 4.7.

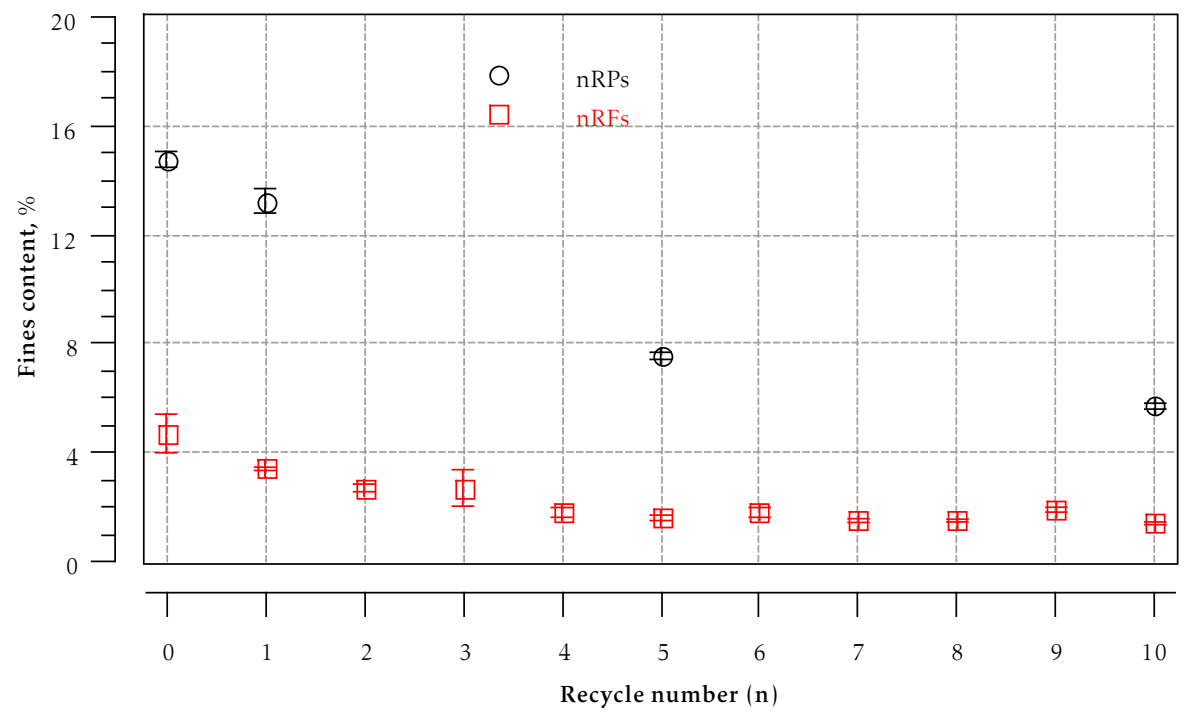

Figure 4.7: Evolution of fines content of pulps and corresponding fibres during recycling

Significant decrease in fines until fifth cycle of recycling is followed by minor variations. The decrease in fines content can be attributed to their drainage during sheet forming in the hand sheet former. Distribution of fines in fibres remained unaffected as expected. Similarly broken content also reduced with recycling. The chosen protocol leads to a decrease of fines content, contrary to the industrial treatments leading to an increase of fines: many mechanical actions modify the pulp during the different unit operations such as trituration.

Curl index decreased during recycling as it induced straightening in fibres (cf. Figure 4.8). The difference in the curl of fibres and pulps is normal as length is inversely related to the curl. Since average fibre length remained unchanged therefore curl is linked to the fibre flexibility. It can be said that the straightening might have been resulted from the hornification (loss of swelling) and/or stiffening of fibres . 


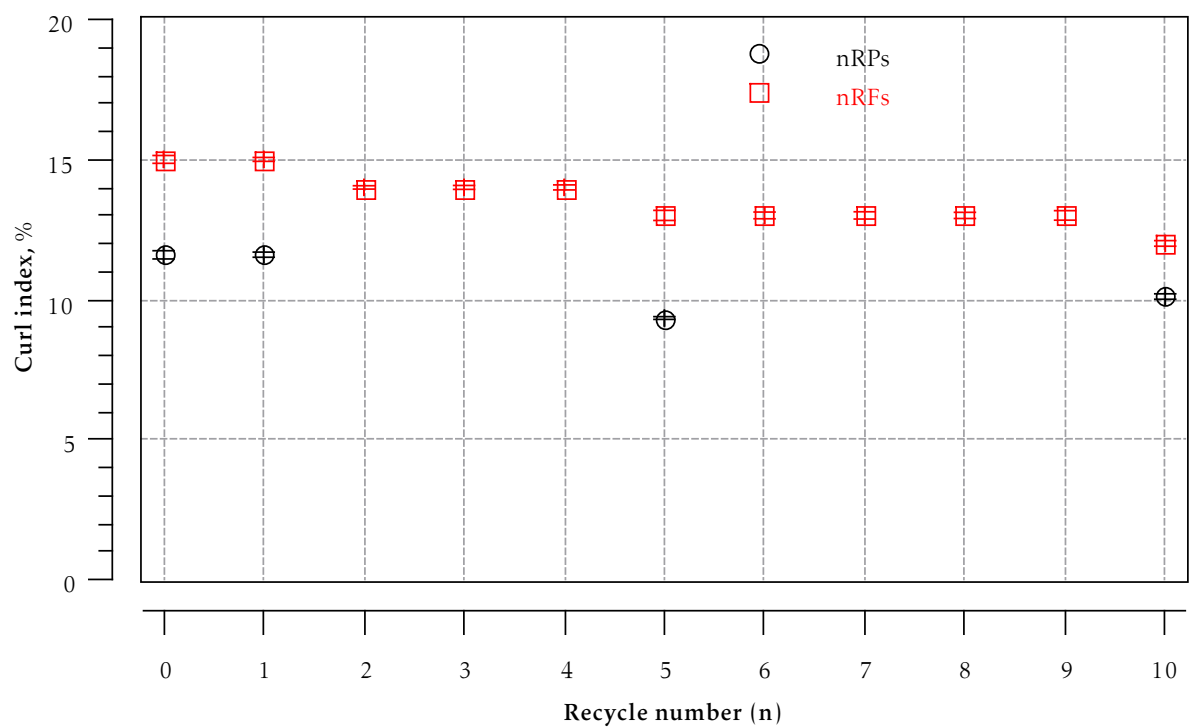

Figure 4.8: Evolution of curl index of pulps and corresponding fibres during recycling

A uniform frequency drift from highly towards lowly curled fibres during repeated cycles of recycling can be seen in the Figure 4.9. Pulp containing fines and short fibres have higher proportion of lowly curled fraction.
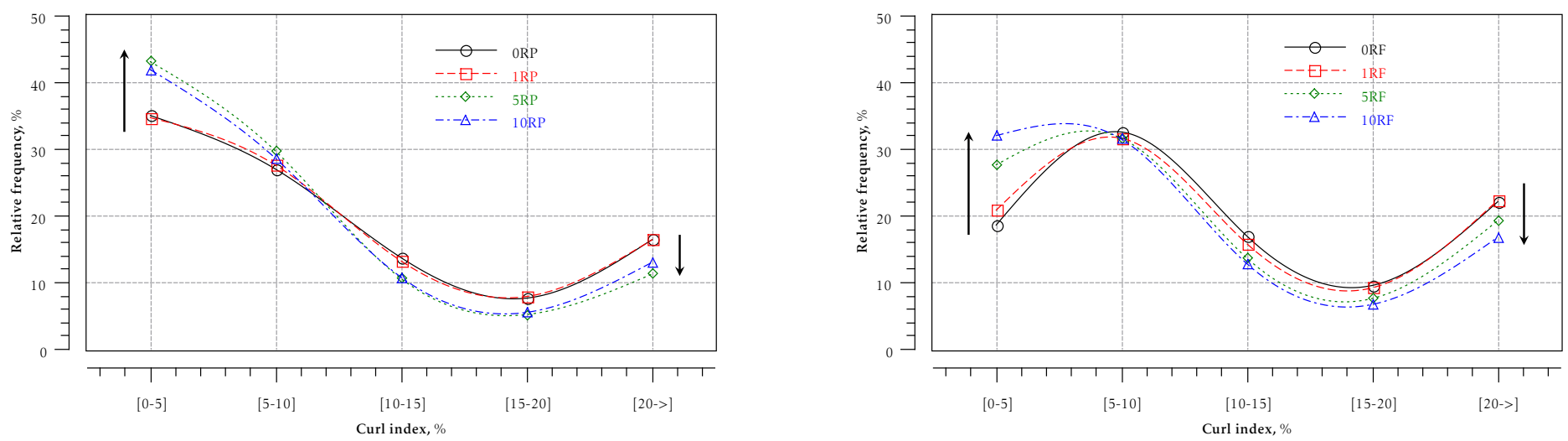

Figure 4.9: Effect of recycling on curl index distributions of pulps (left) and corresponding fibres (right)

Kink number decreased during repeated cycles of recycling (cf. Figure 4.10). First three recycles brought about $17.6 \%$ loss in kink number. Kinked fibres decreased whereas kink angle increased slightly with recycling. 


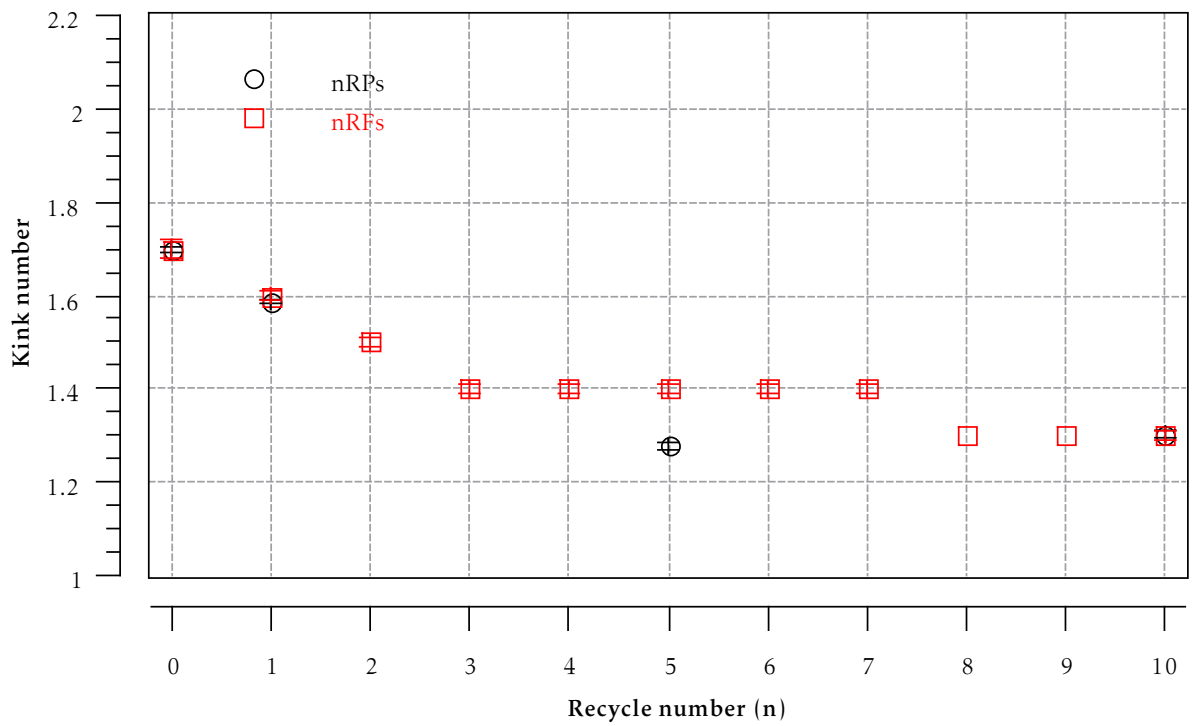

Figure 4.10: Evolution of kink number of pulps and corresponding fibres during recycling

Thickenings and thinnings per fibre and fraction of fibres containing irregularities decreased with repeated recycling (cf. Figure 4.11). The drop in irregularities during initial recyclings was significantly higher.
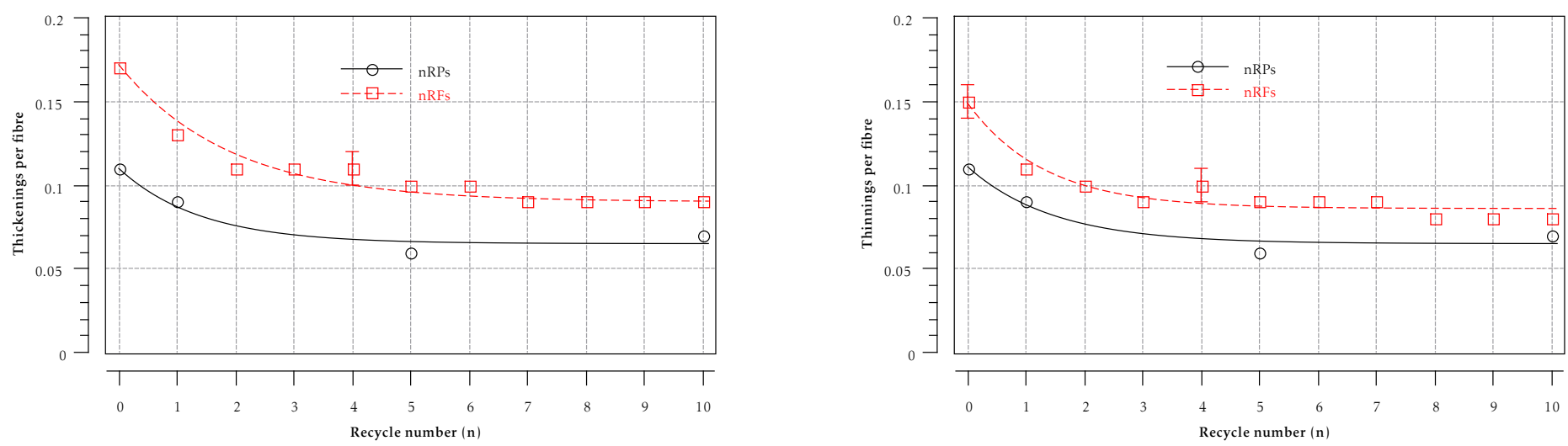

Figure 4.11: Evolution of thickenings (left) and thinnings (right) per fibre of pulps and corresponding fibres during recycling

The difference in pulps and fibres is the short fibre content. The decrease in the difference of fibre and pulps implies that the irregularities in short fibres also decrease with recycling.

Fibrillation index decreases whereas fibril length stays more of less the same during recycling (cf. Figure 4.12). 

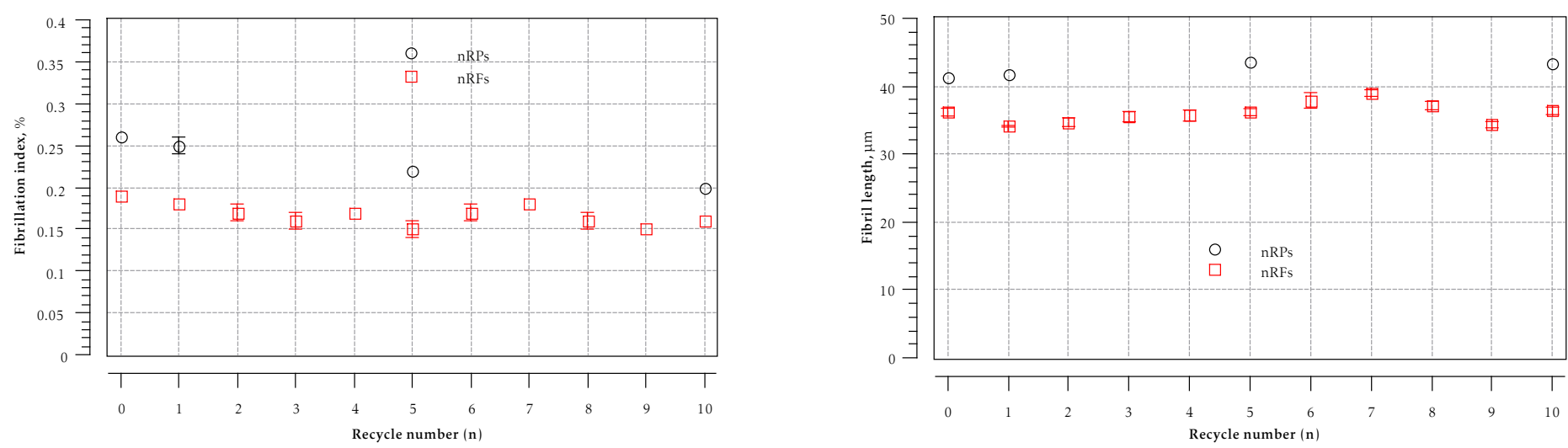

Figure 4.12: Evolution of fibrillation index (left) and fibril length (right) of pulps and corresponding fibres during recycling

\section{Study of morphological changes by Environmental scanning elec- tron microscopic observations}

Collision of electrons with a bulk material produce secondary electrons, scattered electrons, Auger electrons and electromagnetic radiations. SEM is based on the principles of electrons-matter interactions where electrons are bombarded on a sample under investigation. The scanning electron micrographs are either produced by detecting backscattered or secondary electrons. The contrast depends on the orientation of the surface of the sample therefore the surface points facing towards the detector appear brighter in the micrographs.

The operating principle of SEM is shown in Figure 4.13. Electron beam produced by the filament is focused onto the sample with the help of condenser and the objective lenses. Final beam diameter is controlled by the apertures. 


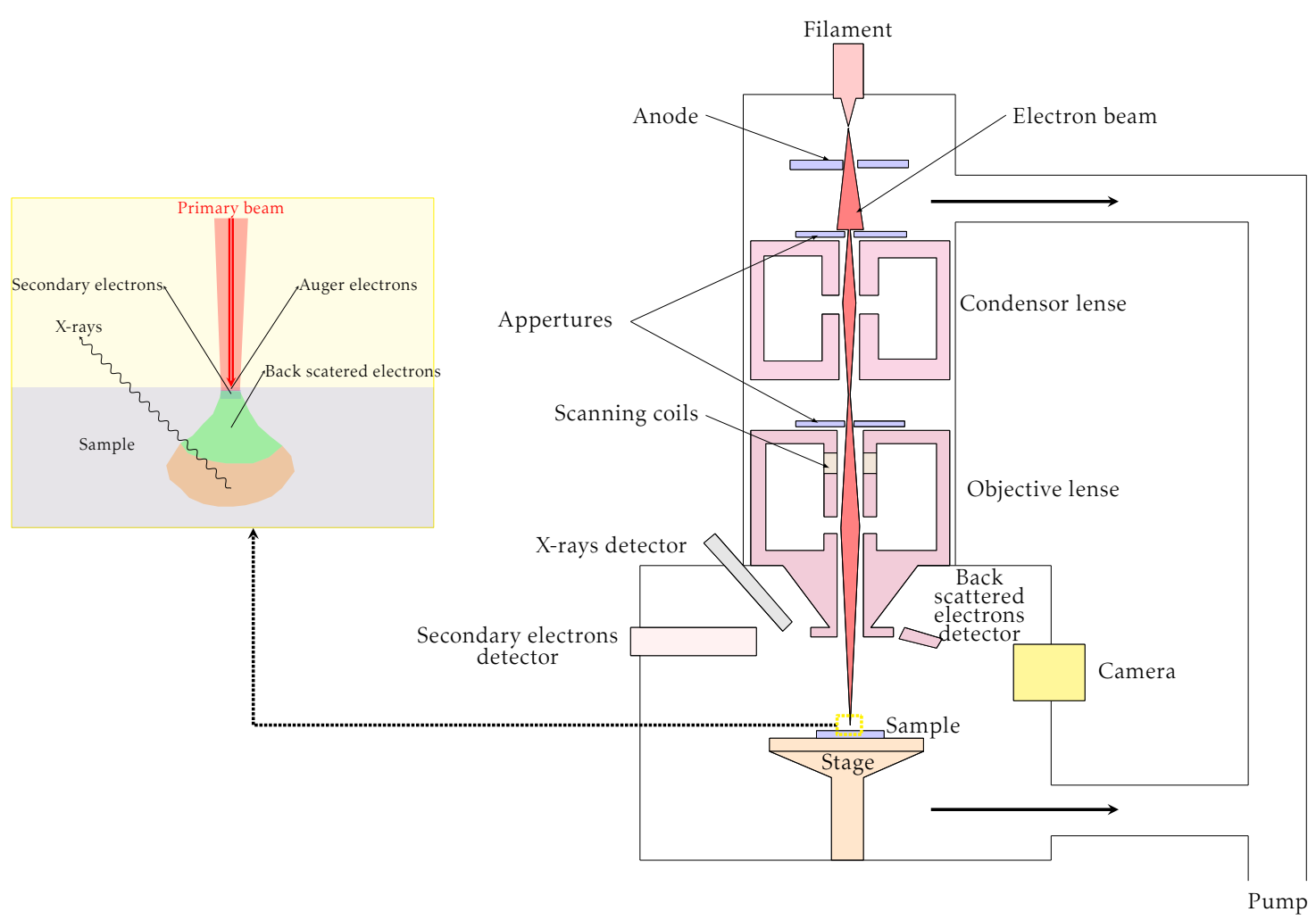

Figure 4.13: Operating principle of SEM

SEM operates in ultra-high vacuum. It has been proved an effective tool for surface analysis of wood fibres [73]. In environmental mode, samples can be analysed in wet state and at higher pressure. The gaseous secondary electron detector (GSED) along with differential pumping systems allow the transfer of the electron beam from the high vacuum in the column area to the high pressure in gaseous environment of specimen chamber. The column is separated from specimen chamber by pressure-limiting apertures (PLA). Depending on the pressure in the chamber, the nature of the gaseous environment and the applied voltage the electron beam looses electron because of their scattering by gas molecules in specimen chamber [71].

Environmental Scanning Electron Microscope (ESEM QUANTA 200) was used for topological analysis. Figure 4.14 shows the SEM micrographs of never dried fibres and ten times recycled fibres at a magnification of $100 \times$. 

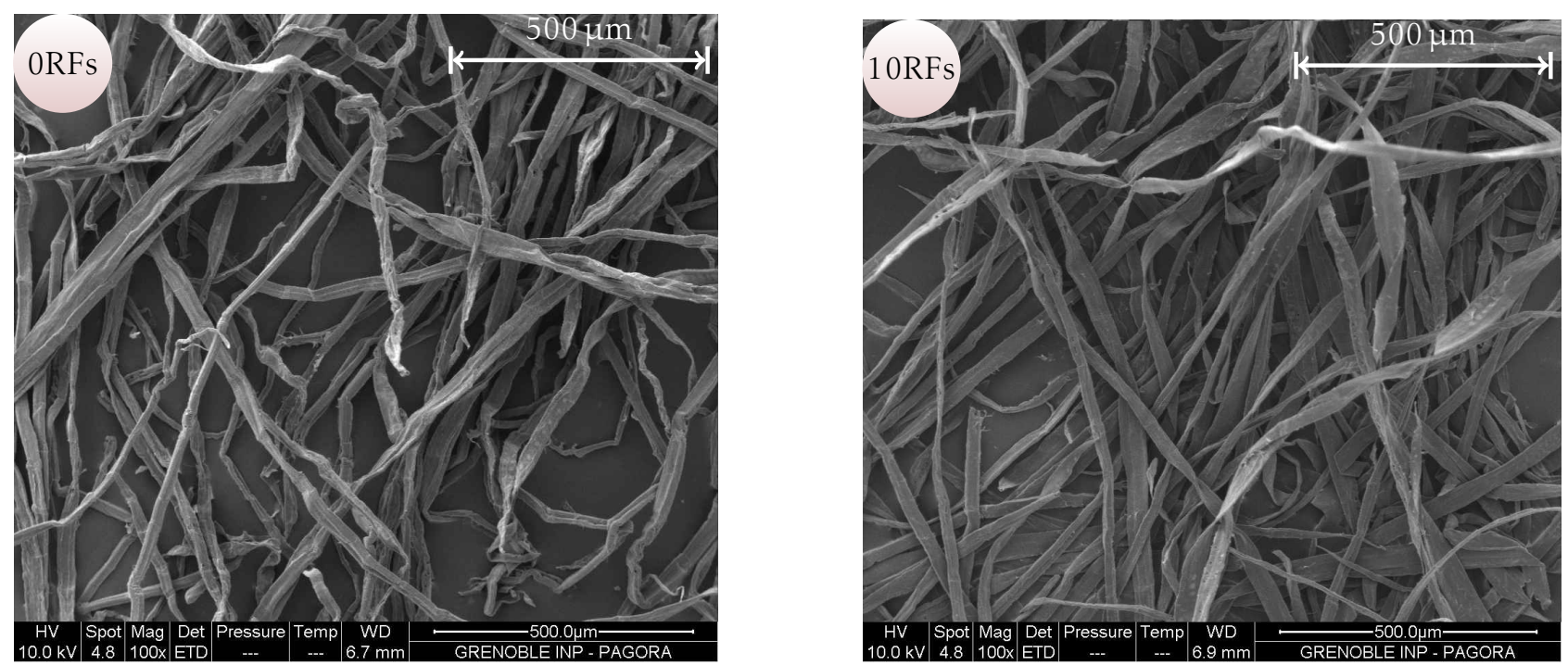

Figure 4.14: SEM micrograph of never dried fibres (left) and ten times recycled fibres (right)

It is clear from the SEM micrographs (cf. Figure 4.14) that the recycled fibres became flat and the irregularities seems to disappear.

Broken ends in EW fibres appear to be mostly from the ruptured perforations (cf. Figure 4.15).
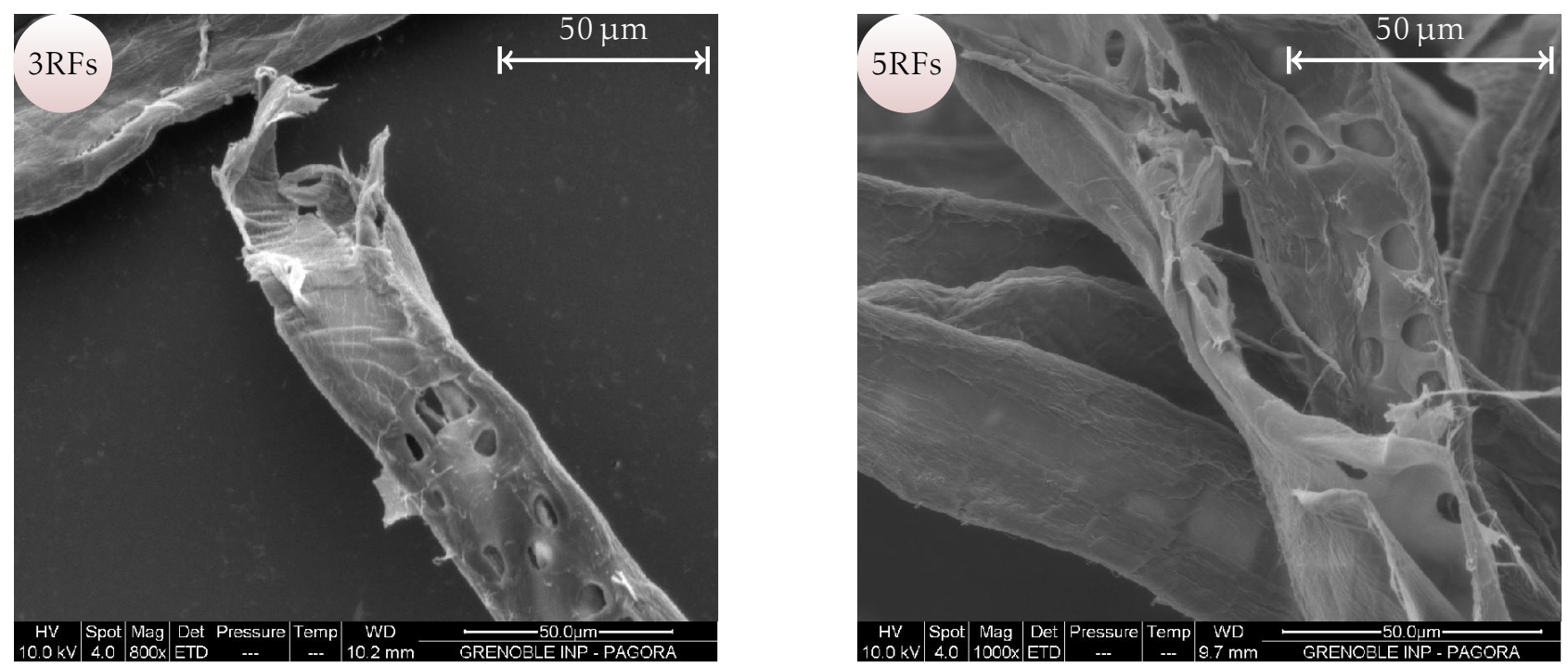

Figure 4.15: SEM micrograph of earlywood fibre perforations

For the evolution of kink angle during rewetting and drying cycles, air dried ORF were placed on sample holder as shown in Figure 4.16. The movement of 
fibre in $\mathrm{z}$-direction was restricted by placing a pin $1 \mathrm{~mm}$ away from the conductive double adhesive carbon taped end of the fibre. Wider pin was placed on the borders of the sample holder so that fibres can move freely without touching the conductive adhesive carbon tape.

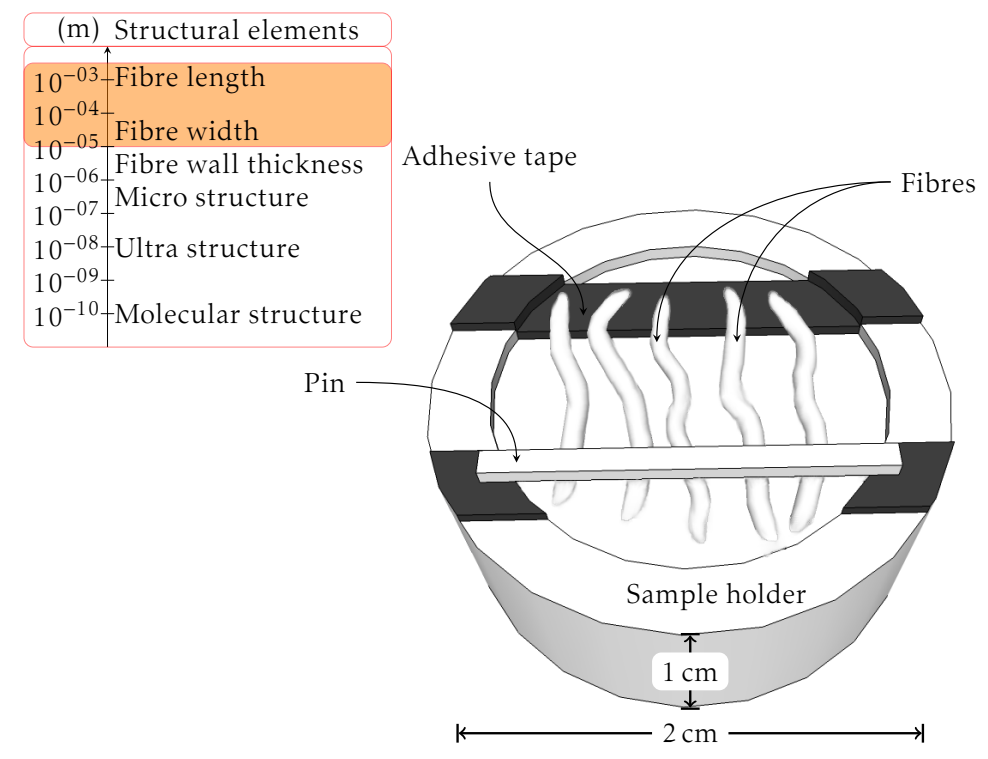

Figure 4.16: Prepared sample holder for kink analysis

Images were taken at $5 \%$ Relative Humidity $(\mathrm{RH})$. To achieve dry condition (5 RH) within the chamber, the temperature was set at $18^{\circ} \mathrm{C}$ and the pressure at $0.107 \mathrm{kPa}$. After $5 \mathrm{~min}$ the pressure in the chamber was increased to $1.027 \mathrm{kPa}$ to obtain $50 \mathrm{RH}$. Fibres were kept in this condition for another $5 \mathrm{~min}$ before wetting the sample at $100 \mathrm{RH}$.

Wet fibres were rested for $15 \mathrm{~min}$ and the same route of conditions was adopted in reverse order to dry the fibres. Fibres were dried for $15 \mathrm{~min}$ to attain equilibrium and micrographs at a magnification of $600 \times$ were registered.

Five cycles of rewetting and drying were carried out in-situ in ESEM.

Figure 4.17 shows the ESEM micrographs of in-situ rewetting and drying of once air dried kinked fibre. The evolution of kink angle with repeated rewetting and drying cycles was determined from the micrographs and the results are presented in Table 4.3. 

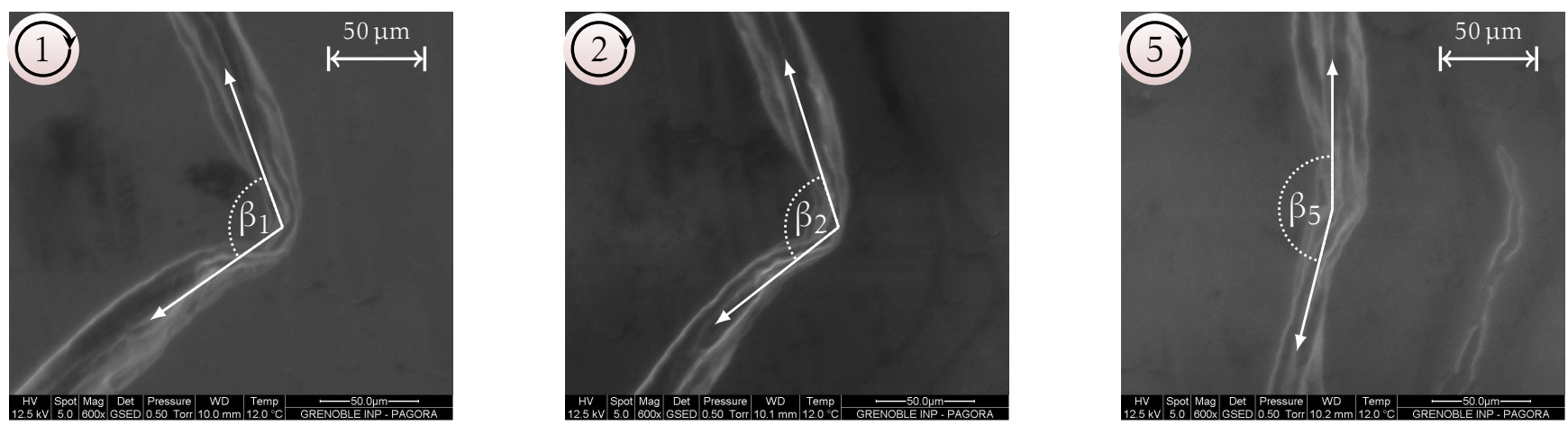

Figure 4.17: ESEM images of once dried kinked fibre at $5 \mathrm{RH}$, first cycle (left), second cycle (middle) and fifth cycle (right)

Table 4.3: Evolution of kink angle during rewetting and drying cycles

\begin{tabular}{l|ccccc} 
No. of cycles & 1 & 2 & 3 & 4 & 5 \\
\hline Kink angle, $\beta^{\circ}$ & 106 & 114 & 146 & 159 & 157
\end{tabular}

It was verified that kink angle increased with repeated rewetting and drying cycles as expected from the morphological analysis. Figure 4.18 shows the probable schematic diagram of fibre straightening.

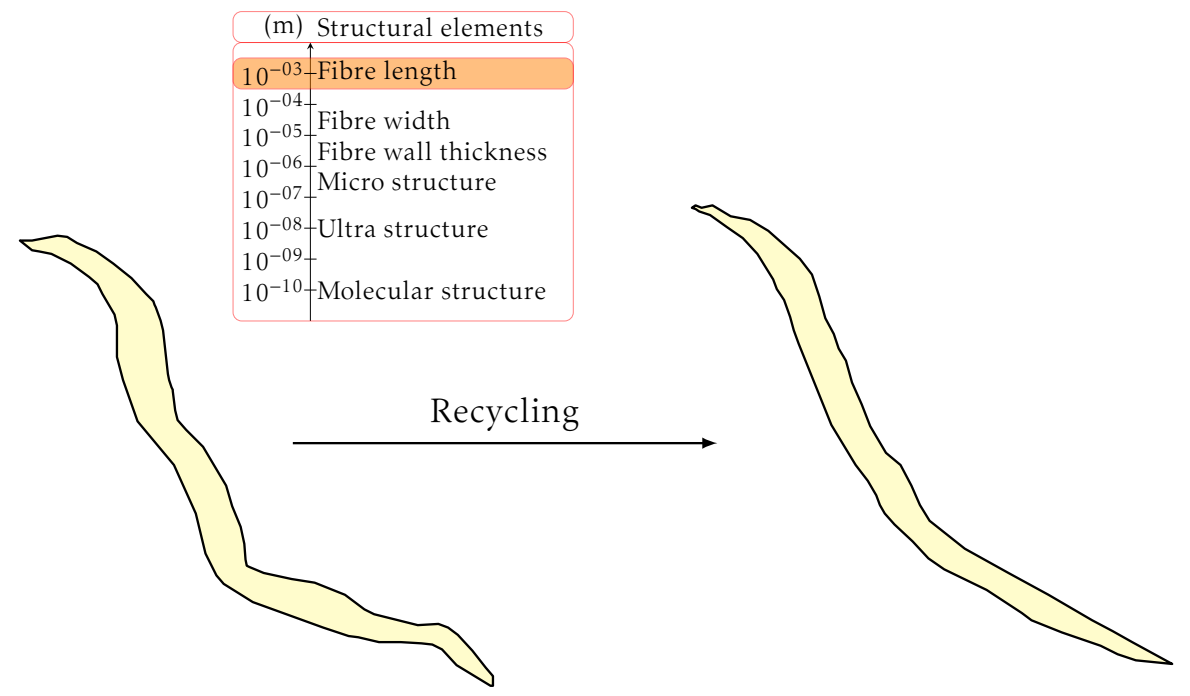

Figure 4.18: Schematic diagram of curl and kink reduction during recycling 


\section{Cell wall thickness of recycled fibres}

Cell wall thickness was measured using MorFi wall thickness device (see Figure 4.19). The working principle is based on optical microscopy in transmitted light with compensated lens. It is equipped with a camera containing high resolution microscopic lens $(0.7 \mu \mathrm{m})$.

Pulp suspension at a very low consistency $\left(1 \mathrm{mg} \cdot \mathrm{L}^{-1}\right)$ is pumped from the beaker to the measurement cell where fibres are imaged and their cell wall thickness is recorded.

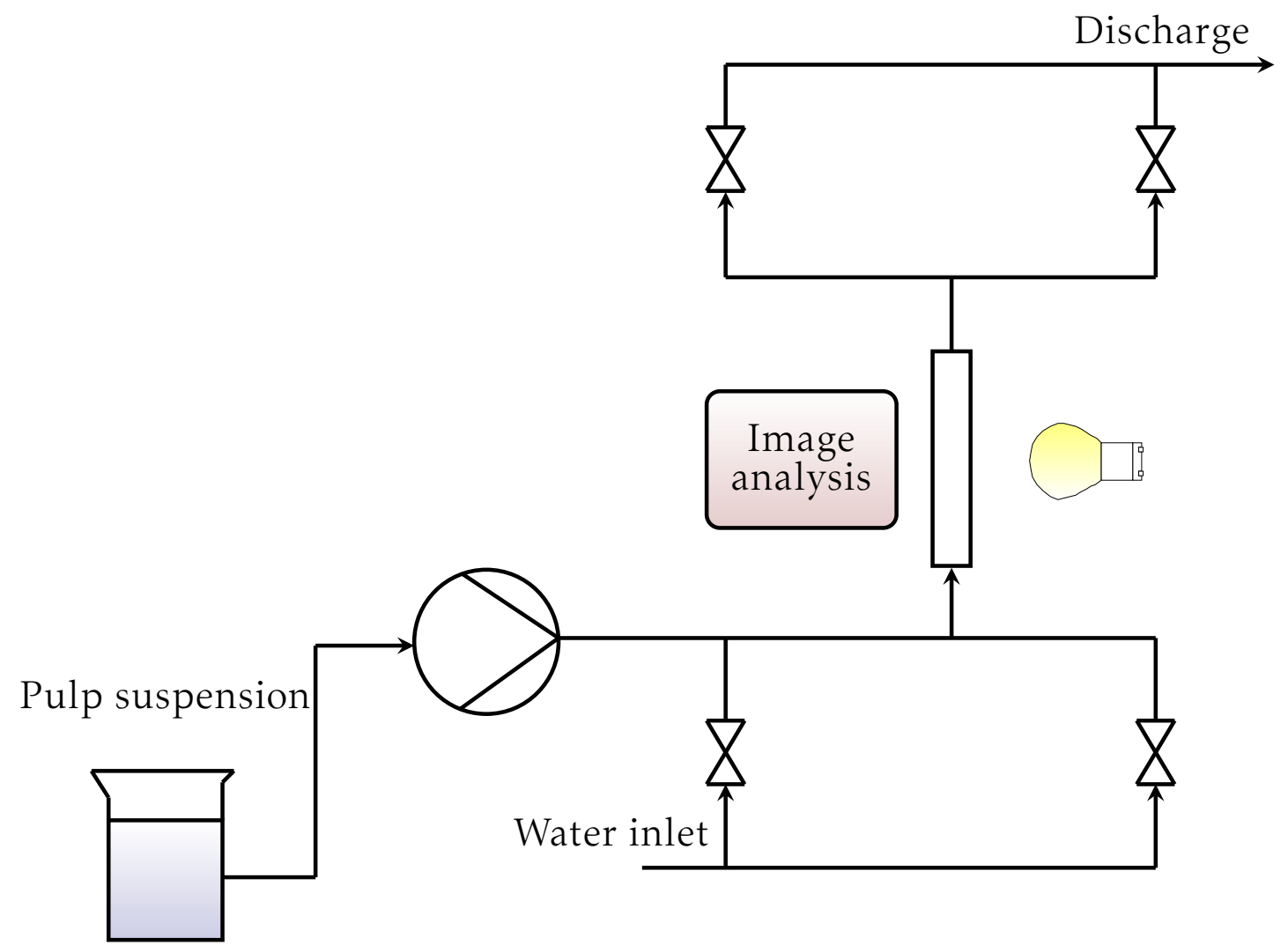

Figure 4.19: Working principle of cell wall thickness measurement device (MorFi WT)

100 frames per sample were analysed. At an average, 200000 to 300000 thickness measurements per sample were recorded. Every sample was analysed thrice, only those two cell wall thickness values were taken into account where the difference was less than $0.1 \mu \mathrm{m}$.

The effect of recycling on the fibre cell wall thickness is shown in Figure 4.20. 

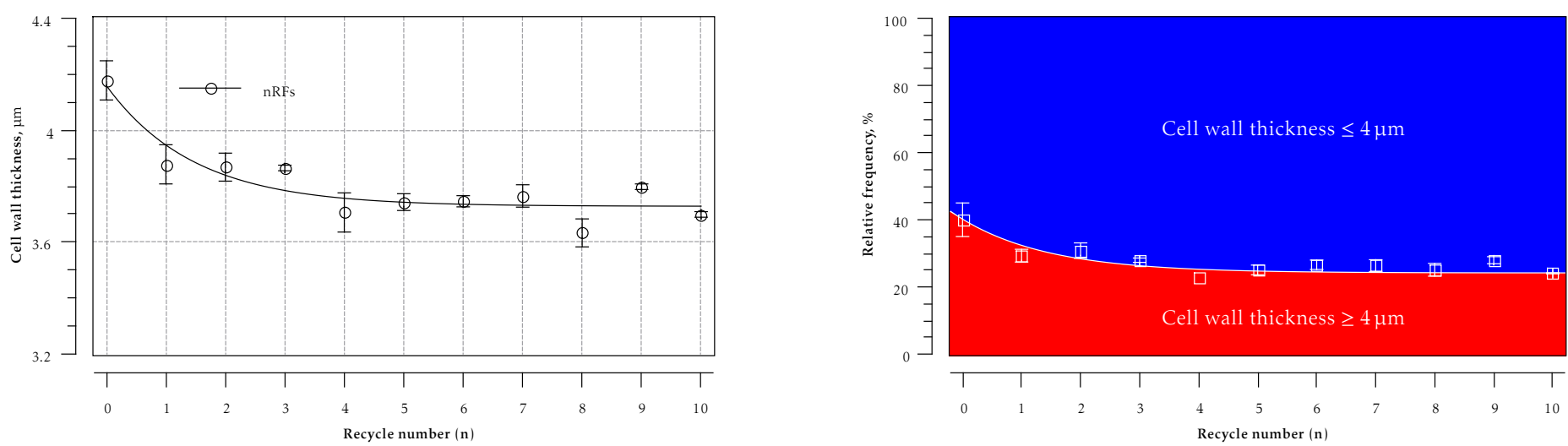

Figure 4.20: Evolution of cell wall thickness of fibres (left) and distribution (right) during recycling

It is evident from Figure 4.20 that fibre wall shrinked with repeated recycling. Drop in cell wall thickness during first recycle was $0.3 \mu \mathrm{m}$ which accounts for $60 \%$ of total shrinkage. From the distribution, it can be seen that the relative frequency of thick cell wall fibres decreased with recycling.

The evolution of fibre wall cross section during rewetting and drying was also carried out in ESEM chamber. For this purpose air dried fibres were cut with razor blade. Fibres were placed vertically on the sample holder as shown in Figure 4.21.

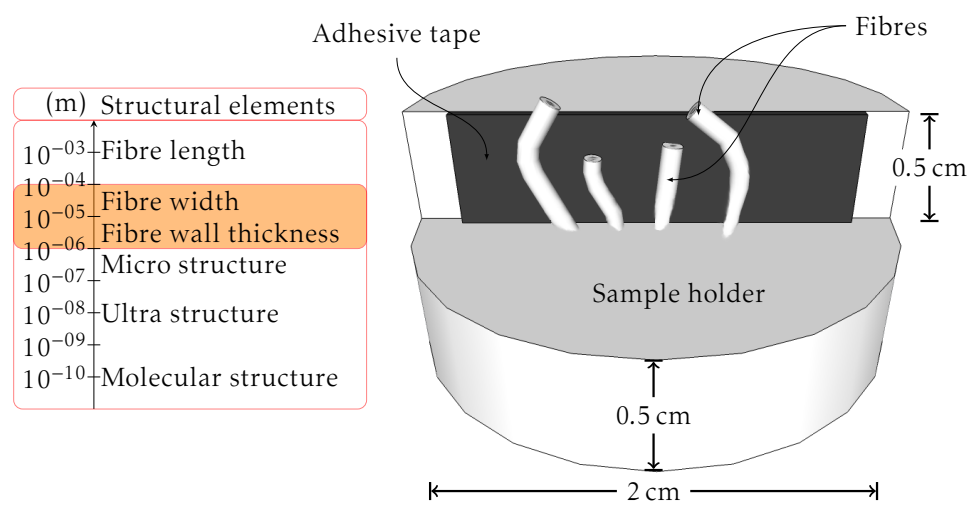

Figure 4.21: Prepared sample holder for cross section analysis

Rewetting and drying of the fibres was carried out according to the previously described procedure in ESEM chamber. Micrographs were registered at a magnification of $1200 \times$ (cf. Figure 4.22). 

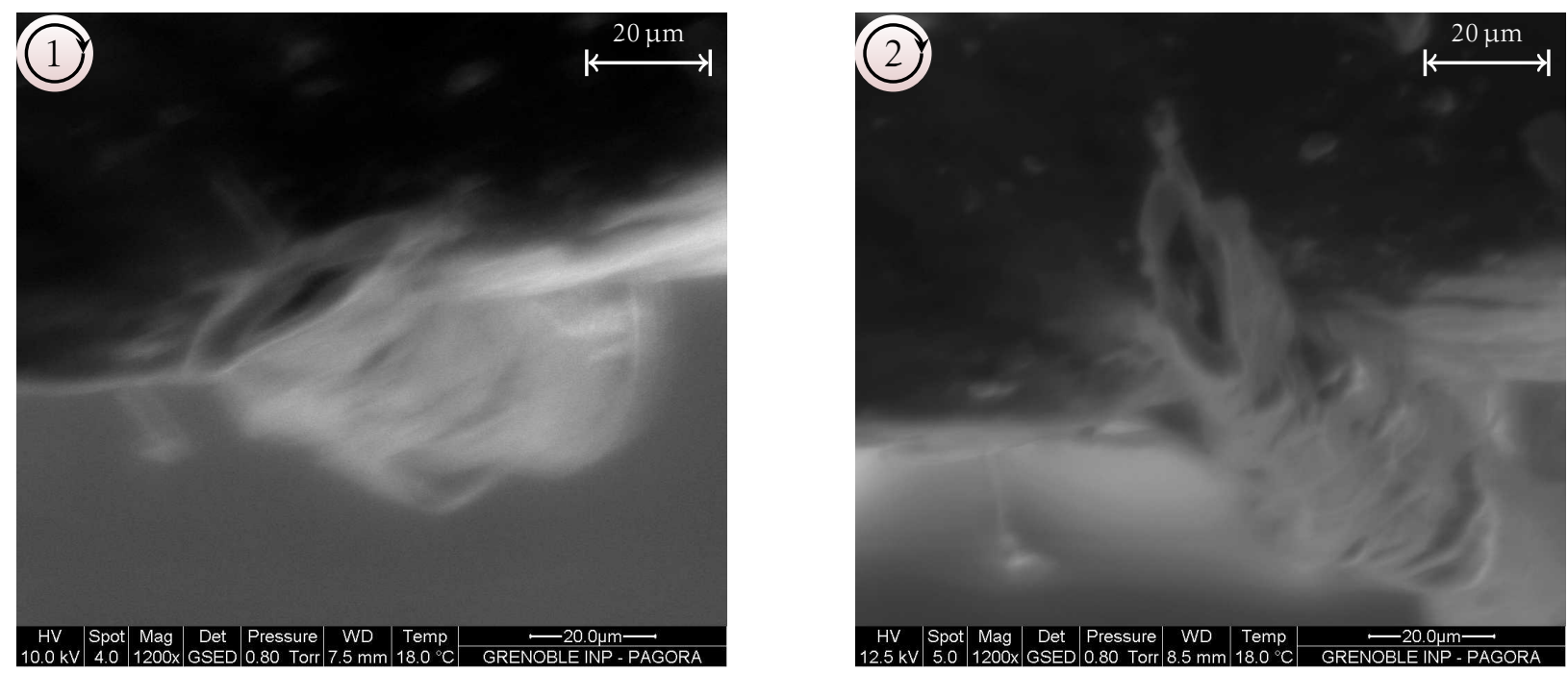

Figure 4.22: ESEM cross section images of once (left) and twice air dried (right) fibre

Based on Figure 4.22, it is possible to determine fibre wall cross section area $(\triangle \mathrm{A})$ according to equation (4.6)

$$
\Delta \mathrm{A}=\mathrm{FA}-\mathrm{LA}
$$

where FA and LA are the fibre and lumen cross section areas, respectively.

The quantitative image analysis was performed with ImageJ[22]. The evolution of fibre wall cross section area of never dried fibres due to rewetting and drying cycles is presented in Table 4.4. Mean values are based on the measurement of twelve fibres.

Table 4.4: Evolution of fibre wall cross section area during rewetting and drying cycles

\begin{tabular}{l|ccccc} 
Number of cycles & 1 & 2 & 3 & 4 & 5 \\
\hline Fibre wall cross section area, $\mu \mathrm{m}^{2}$ & $263 \pm 28$ & $189 \pm 28$ & $189 \pm 25$ & $191 \pm 26$ & $190 \pm 31$
\end{tabular}

Lumen of the fibres were collapsed during cutting (sample preparation), that was the reason why investigation of the evolution of lumen cross section perimeter (LP) with rewetting and drying cycles was not possible. However, It was verified that cell wall cross section area (without lumen) decreased with repeated rewetting and drying cycles. The drop in fibre wall cross section area after first cycle was significant and irreversible. 
Figure 4.23 is a schematic representation of the fibre cross section.

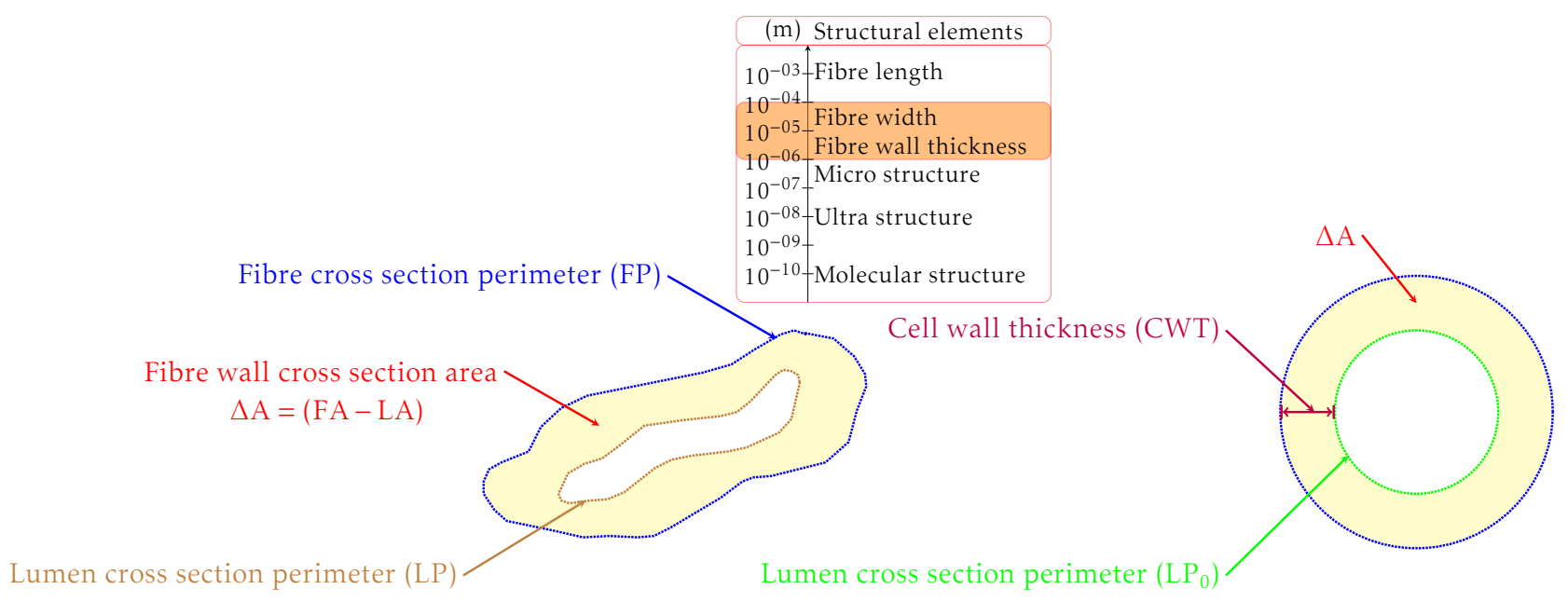

Figure 4.23: Schematic diagram of fibre cross section (left) and corresponding round attributes (right)

If we assume that the uncollapsed shapes of the fibre and lumen is round then lumen cross section perimeter $\left(\mathrm{LP}_{0}\right)$ can be calculated from the following expression

$$
\mathrm{LP}_{0}=\sqrt{\mathrm{FP}^{2}-4 \pi \Delta \mathrm{A}}
$$

where FP is fibre cross section perimeter.

Cell wall thickness (CWT) can be calculated from the following expression

$$
\mathrm{CWT}=\frac{\mathrm{FP}-\mathrm{LP}_{0}}{2 \pi}=\frac{\mathrm{FP}-\sqrt{\mathrm{FP}^{2}-4 \pi \Delta \mathrm{A}}}{2 \pi}
$$

The quantitative image analysis of the fibre with open lumen (Figure 4.22) is presented in Table 4.5.

Table 4.5: Effect of rewetting and drying cycles on fibre wall dimensions

\begin{tabular}{l|cc} 
Number of rewetting and drying cycles & 1 & 2 \\
\hline Fibre wall cross section area $(\Delta \mathrm{A}), \mu \mathrm{m}^{2}$ & 439 & 345 \\
Fibre cross section perimeter $(\mathrm{FP}), \mu \mathrm{m}$ & 107 & 92 \\
Cell wall thickness (CWT), $\mu \mathrm{m}$ & 10 & 7 \\
Lumen cross section perimeter $(\mathrm{LP}), \mu \mathrm{m}$ & 43 & 48
\end{tabular}

Fibre cross section perimeter (FP) decreased whereas lumen cross section perime- 
ter (LP) increased by repeated rewetting and drying cycles. It is clear from the qualitative and quantitative analysis that fibre wall shrinked with repeated rewetting and drying cycles. In other words we can say that the swelling ability of fibres wall is decreased. Figure 4.24 is a schematic representation of fibre wall shrinkage during recycling.
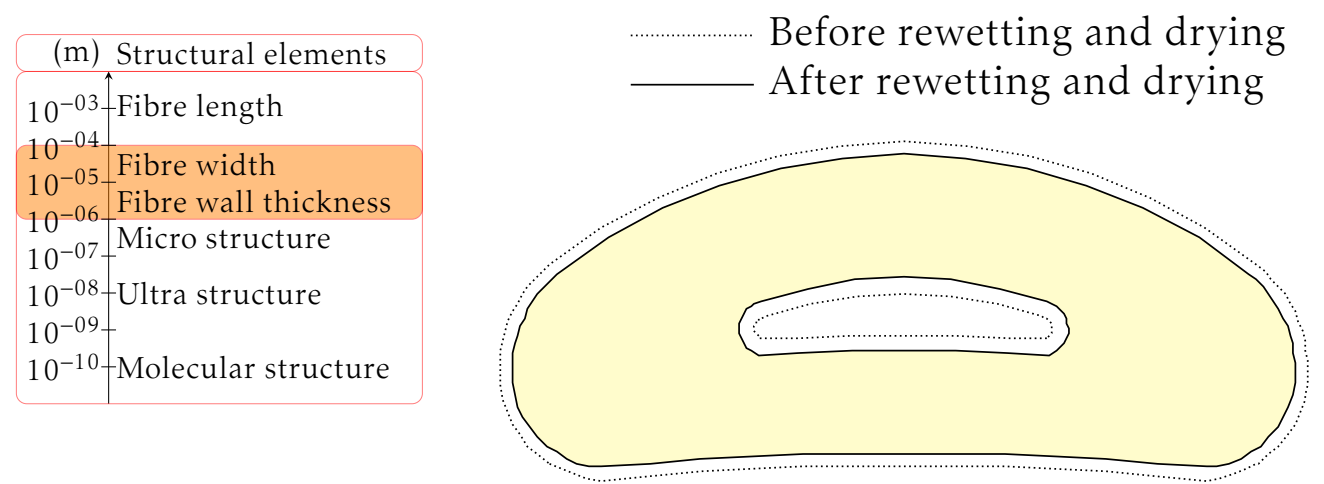

Figure 4.24: Schematic diagram of fibre wall shrinkage during recycling

\subsection{Surface analysis}

The surface of the fibres in the first few recycles was compacted see Figure 4.14. Picking of the outer layers started after fourth recycle. Delayering of the outer layers is visible in the later recyclings. Which means that the specific bond strength between fibres surpassed cohesive forces of the outer layers (between P/S1 and S2). There are two possibilities either specific bond strength [215] has been increased or fibre wall layer cohesion has been decreased by repeated recycling.

The second case gets its logic from microfibril angle (MFA). Since MFA of P/S1 layer is different than S2 layer therefore the shrinkage of the layers is also different. During repeated recyclings the layers are separated and end up in picking of P/S1 layer. 

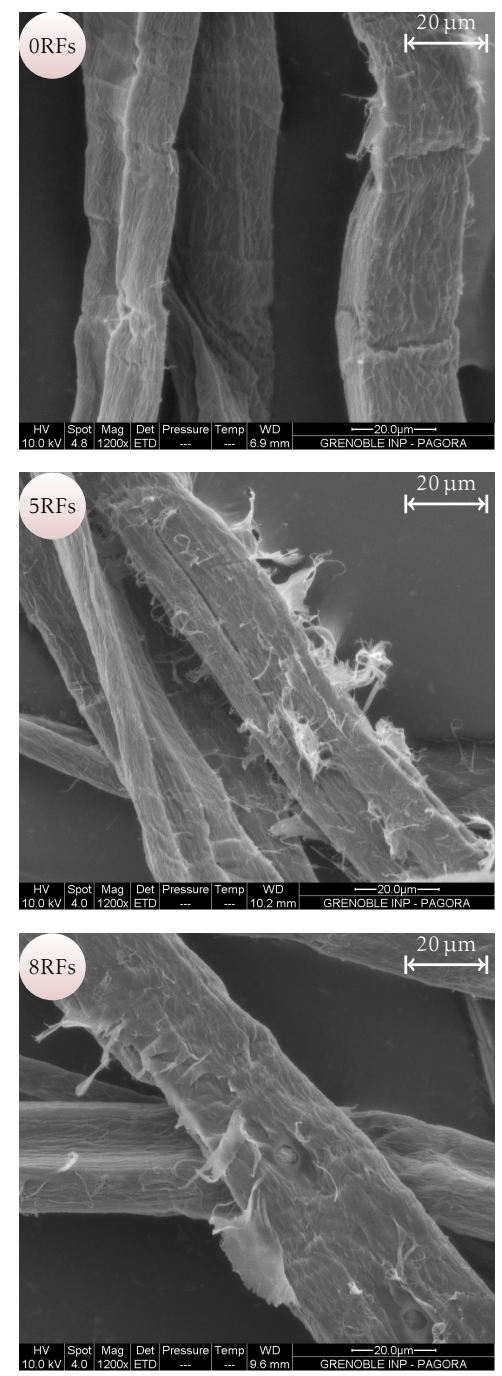
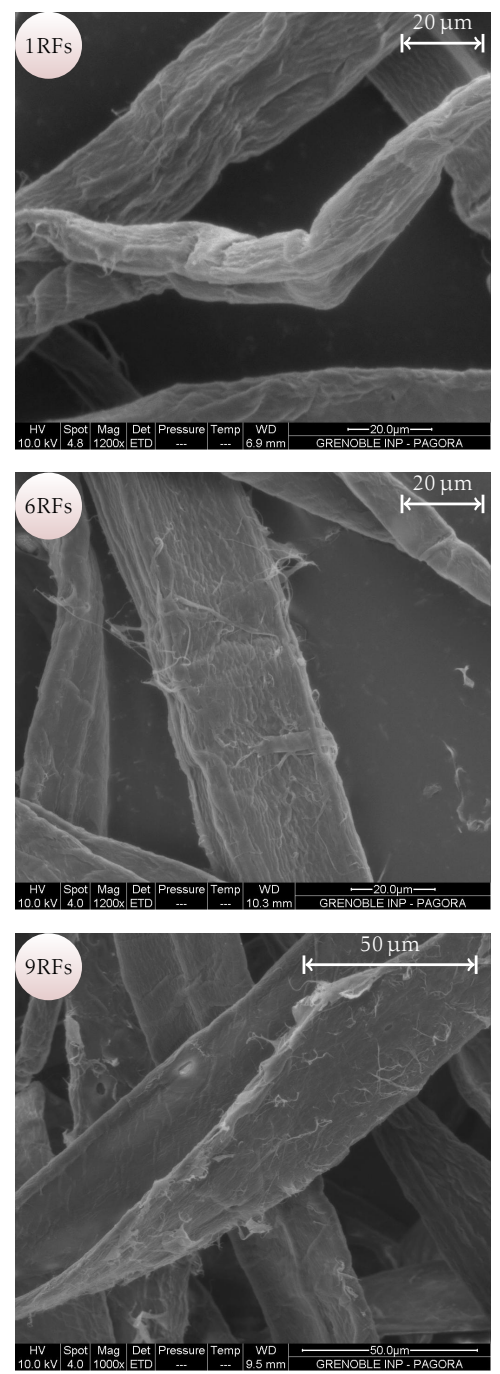
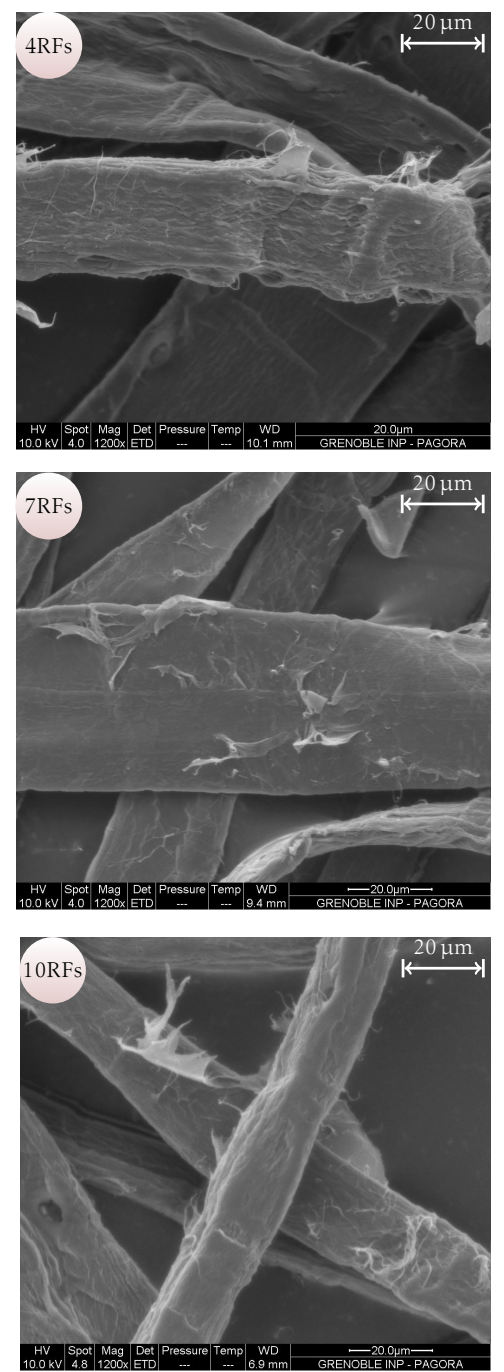

Figure 4.25: SEM micrographs showing the surface characteristics of fibres during recycling

Probable transformations in cell wall layers (radial section) and on fibre surface due to recycling are shown in Figure 4.26. The process may be divided into two stages. In stage I, S2 layer pulls P/S1 layer towards the lumen. Fibre wall shrinks and surface becomes smooth. In stage II, bond strength ruptures P/S1 layer however compaction of S2 and S3 layers continues. 


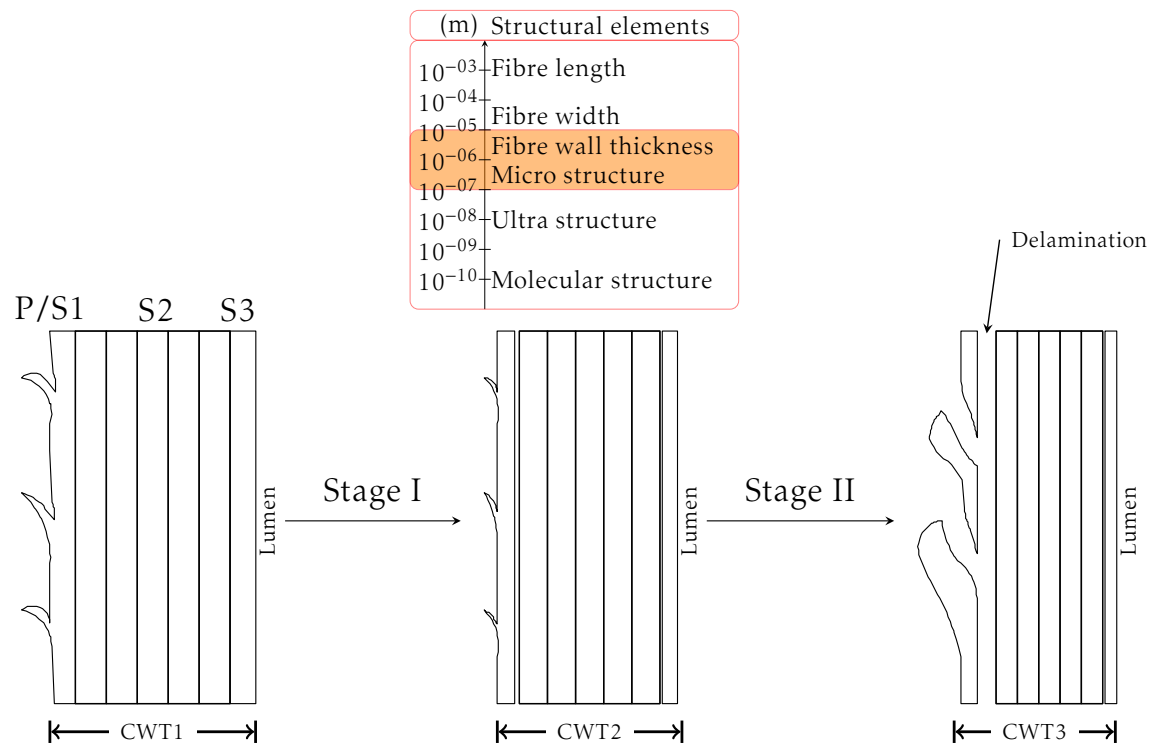

Figure 4.26: Schematic diagram of probable transformations of fibre cell wall layers and surface during recycling

Here it can also be explained how the cross linking (strength resins) agents increase the strength properties. Probable mechanism of P/S1 stitching through the addition of cross-linkers is shown in Figure 4.27. Cross-linkers enter through the ruptured openings of P/S1 layers. Cross links S2 and P/S1 layers as well as "repair" the ruptured openings.

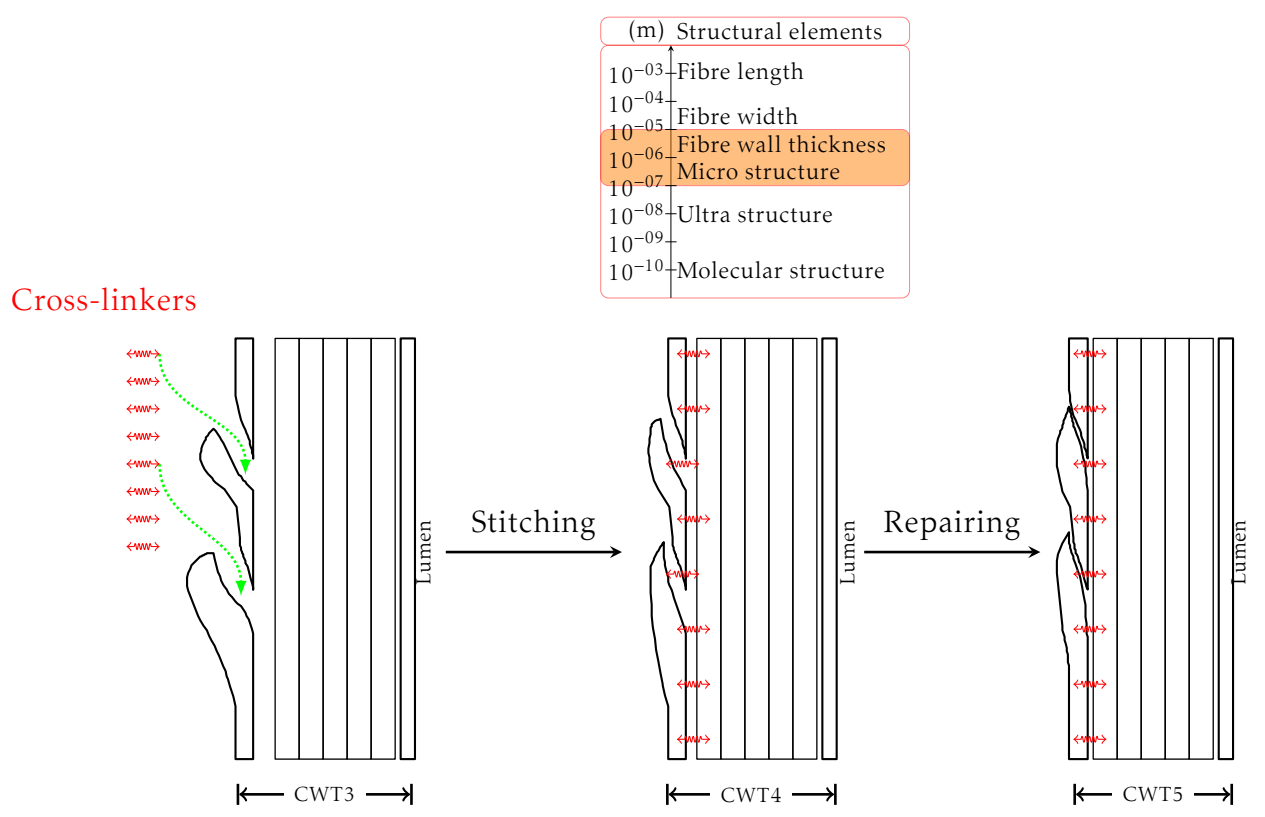

Figure 4.27: Schematic diagram of probable stitching and repairing of P/S1 layers by cross-linkers

Improvement in the strength properties of recycled fibres by refining can be 
explained as peeling off the weaker outer layer.

Probable mechanism of P/S1 peeling and fibrillation of recycled fibre cell wall layers (radial section) by refining is shown in Figure 4.28.

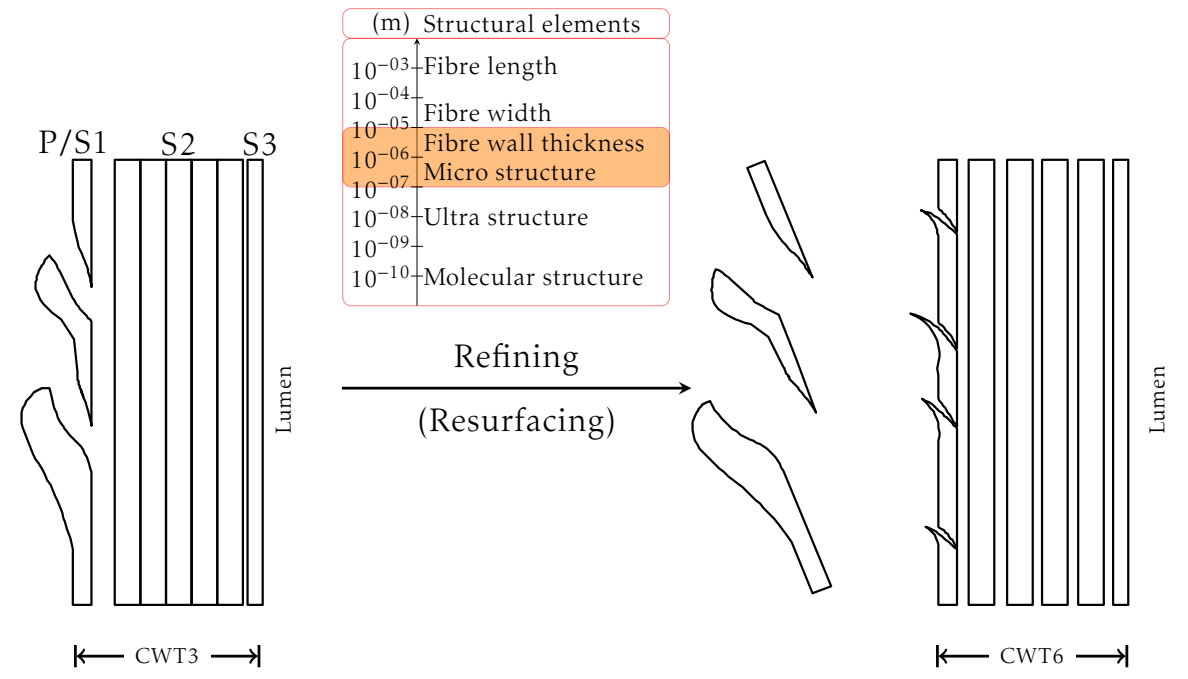

Figure 4.28: Schematic diagram of probable peeling off and fibrillation by refining

Refining may remove weaker ruptured and detached P/S1 layers. Furthermore it generates fibrils from S2 layer along with internal fibrillation.

Fibre surface was modified during recycling. In order to analyse fibre surface modifications, atomic force microscope (Dimension Icon Atomic Force Microscope System with ScanAsyst) was used.

\section{Atomic force microscopy (AFM)}

In atomic force microscopy, cantilever is oscillated by piezo-crystal at its resonance frequency. The surface of the sample is scanned with a sharp tip attached to the loose end of the cantilever. The reduction in the amplitude of the cantilever due to the interactions of tip to the surface are recorded by reflecting laser beam from cantilever surface onto a photo-detector (cf. Figure 4.29). 


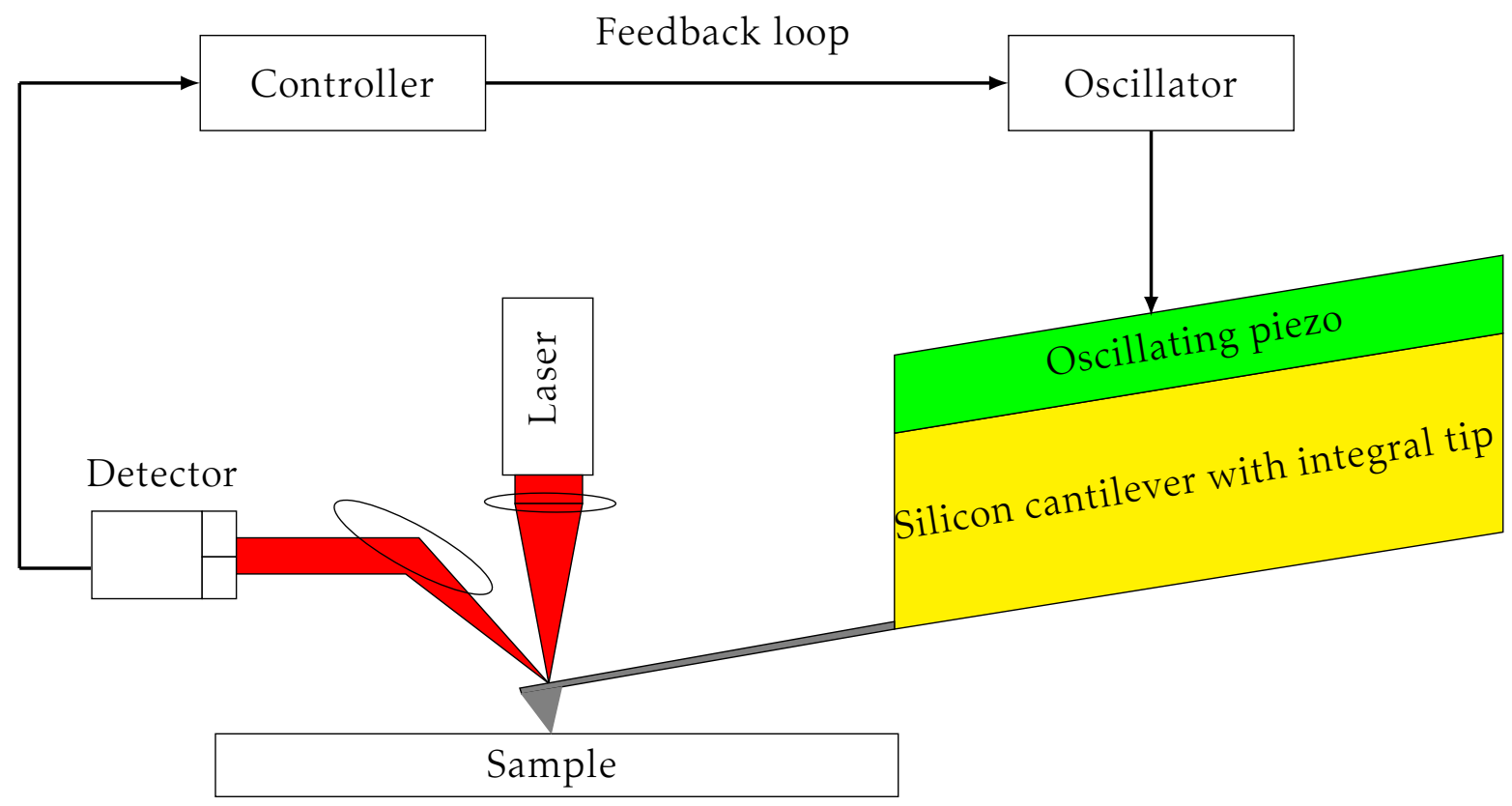

Figure 4.29: Working principle of AFM adapted from [135]

AFM operates in contact and dynamic mode. The dynamic mode is subdivided into tapping and non-contact modes.

The PeakForce QNM tapping mode allows measurement of modulus and adhesion of the material. Continuous series of force-distance curves are obtained as the cantilever is oscillated well below resonance. The deformation is determined from the cantilever deflection and the z-piezo displacement. For reliable quantitative characterisation a good calibration is required.

Characteristic curves of PeakForce QNM tapping mode are shown in Figure 4.30.
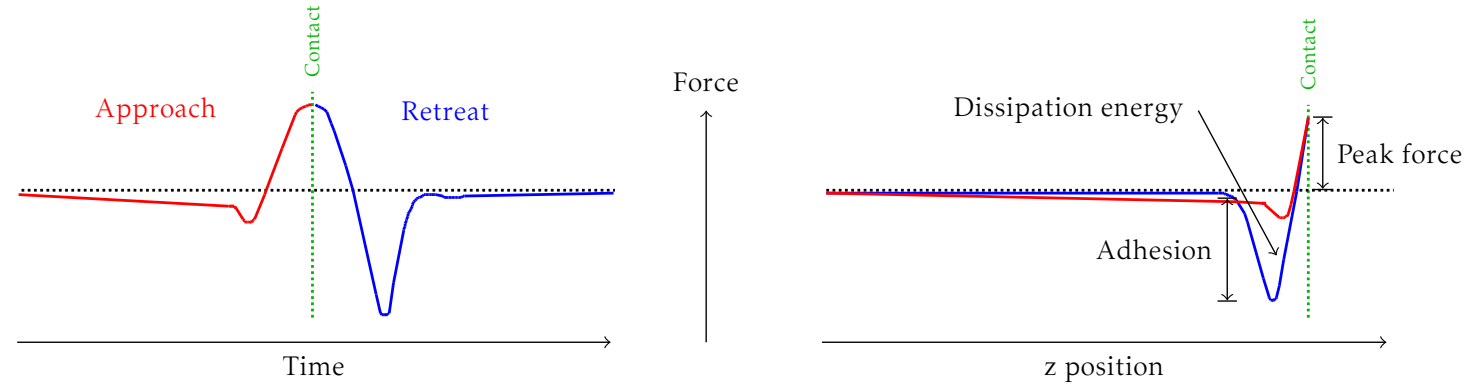

Figure 4.30: Characteristic curve of PeakForce QNM tapping mode

Hard rotated tip (Veeco TAP525A) suitable for materials having modulus 1 to 
20 GPa was used. Since calibration was not performed therefore the comparison of surface adhesion is presented in Figure 4.31 in terms of signal strengths.

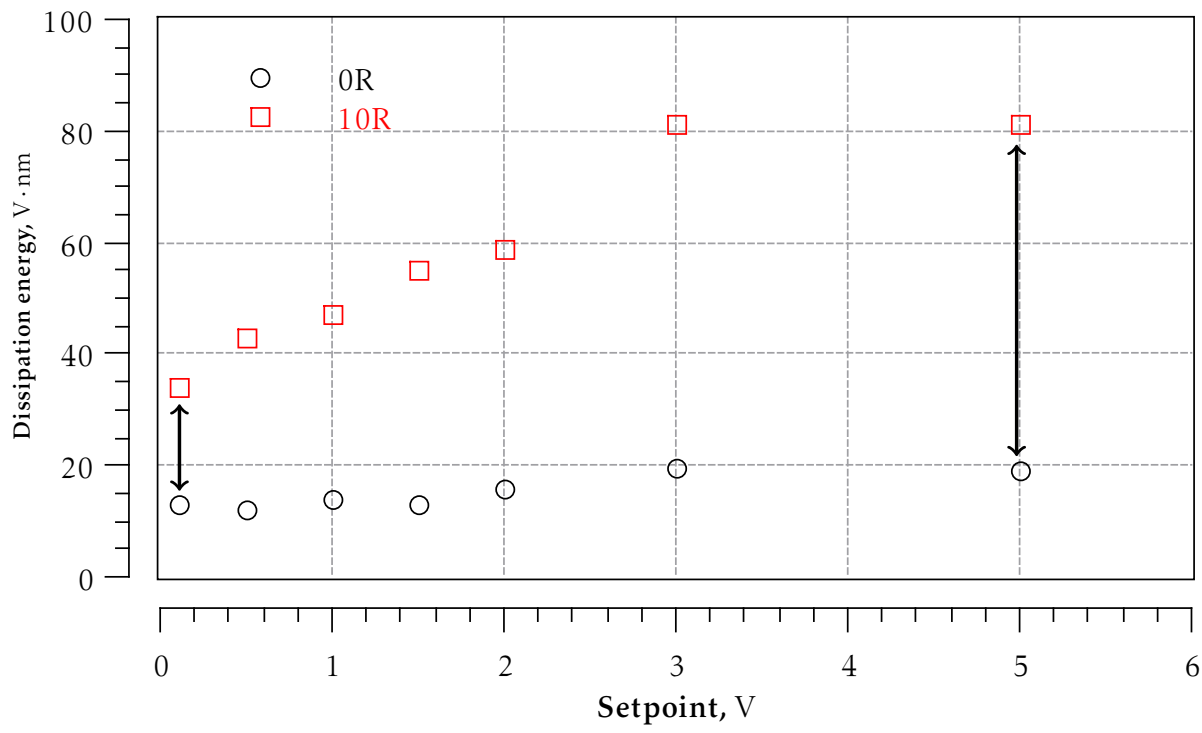

Figure 4.31: Effect of recycling on fibre surface adhesion

Set point (tapping force) describes the oscillation amplitude of the cantilever. The difference in dissipation energy of never dried and ten times recycled fibre indicates change in surface characteristics. Brancato regarded this behaviour a consequence of surface smoothness with recycling i.e. increase tip-fibre contact sites [48].

Dissipation energy of recycled fibres increased with the increase of set point whereas in case of never dried fibres there was no difference. The surface adhesion is may be due to the detached surface layers of recycled fibres.

Figure 4.32 is a speculative diagram of AFM tip interactions with the surface of never dried and recycled fibres. In case of never dried fibre; AFM tip approaches, makes contact and retreats without experiencing any adhesive forces. Whereas in case of recycled fibres the tip pushes P/S1 layer towards the lumen and while retreat the P/S1 layer is adhered. 


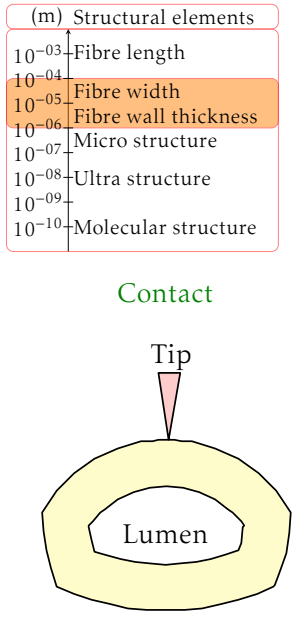

Never dried fibre

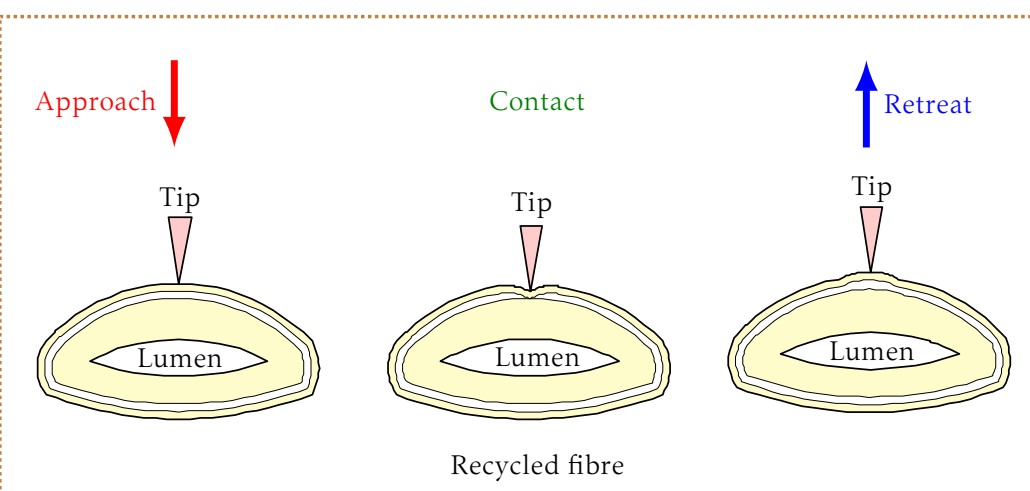

Figure 4.32: Schematic diagram of AFM tip interactions with never dried and recycled fibre surface

\subsection{Dewatering of recycled pulps and fibres}

SR values during recycling range between 12 to 14 . As we used unbeaten pulp containing limited amount of fines, therefore SR of pulps and fibres did not change with recycling. Dewatering rates of fibres and pulps during the coarse of recycling remained unchanged. In other words recycling does not affect water permeability through the fibre mat.

However, if we compare the drainage profile of never dried pulp and the corresponding fibres (cf. Figure 4.33) we can see some difference. 


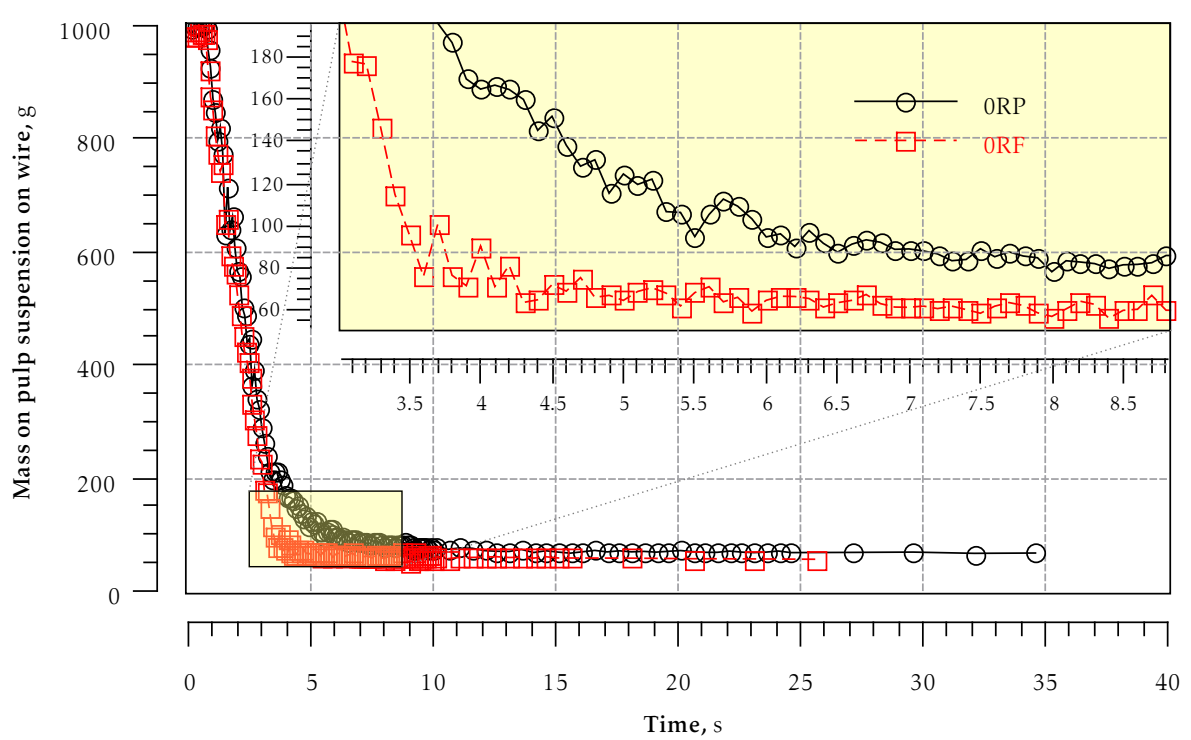

Figure 4.33: Filtration kinetics of never dried pulp and fibres

The differential zone is zoomed in Figure 4.33. Never dried pulp drains a little slower than corresponding fibres. This can be attributed to the fines and short fibres present in pulp. Specific filtration resistance of pulps and corresponding fibres did not change. The results are tabulated in Appendix A.

\subsection{Specific water retention of recycled pulps and fibres}

The evolution of specific water retained by pulps with recycle number is shown in Figure 4.34. Mean values are based on three WRV and ten FSP measurements. 


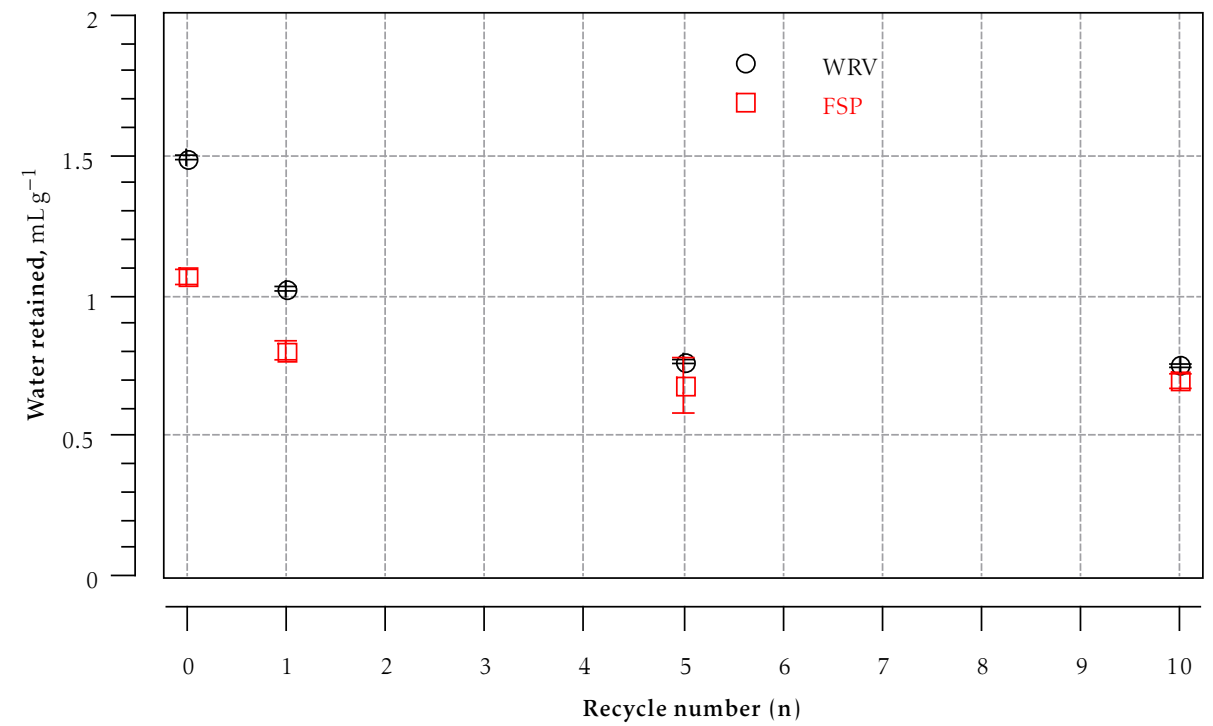

Figure 4.34: Evolution of specific water retention of pulps during recycling

WRV and FSP of pulps decreased with recycle number. The difference (WRVFSP) represents the amount of extra pore water. The amount of (WRV-FSP) water contained in pulps decreased with recycle number from 0.43 to $0.06 \mathrm{~mL} \cdot \mathrm{g}^{-1}$ (cf. Figure 4.34).

Dealing with the fibres, the specific water retention is presented in Figure 4.35.

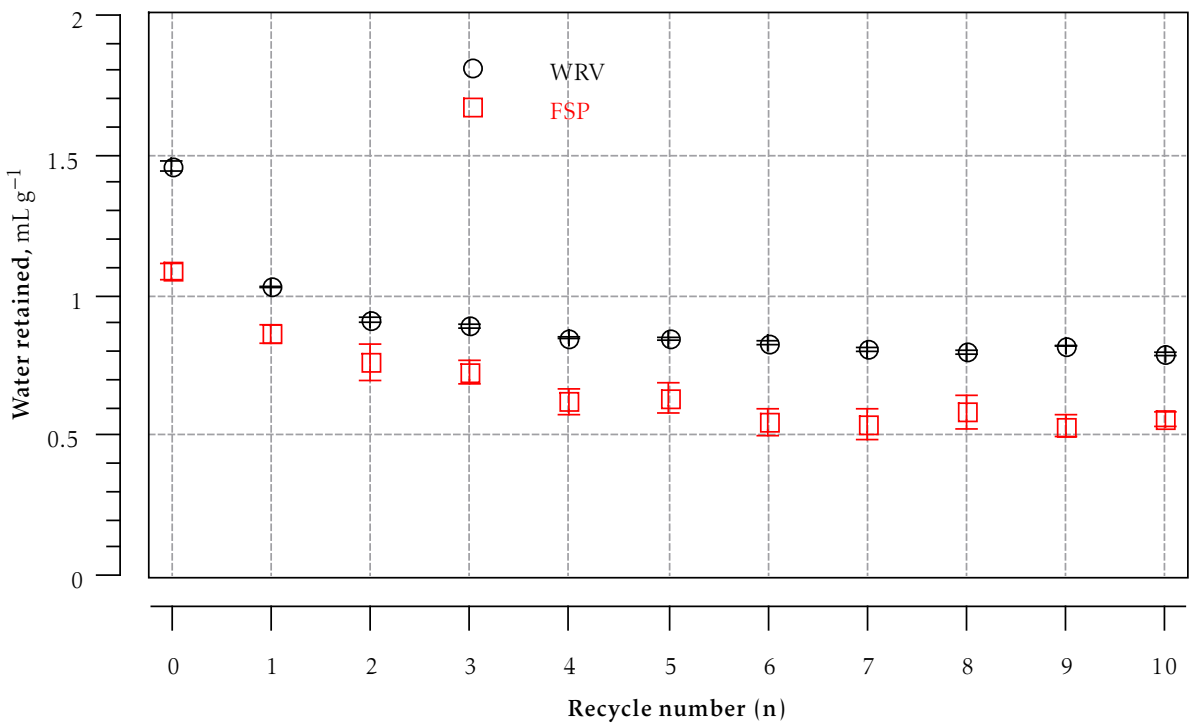

Figure 4.35: Evolution of specific water retention of fibres during recycling

WRV and FSP of fibres also decreased with recycle number. The decrease in WRV and FSP was significant during first few recycles. The amount of (WRV- 
FSP) water contained in fibres decreased until third recycle (cf. Figure 4.35). Both WRV and FSP values are constant after the fourth cycle.

Water carrying capacity of pulps and fibres includes extra and intra fibre waters. The reduction in intra fibre water may be caused by the pore closure and lumen collapse during recycling. In other words it can be said that fibre cell wall densified during recycling.

\subsection{Hornification of recycled pulps and fibres}

Fibre hornification $(\hbar)$ based on WRV can be represented by the following expression $[230,139]$

$$
\text { Fibre hornification }(\%)=\hbar_{\mathrm{WRV}}=\frac{\mathrm{WRV}_{0 \mathrm{RF}}-\mathrm{WRV}_{\mathrm{nRF}}}{\mathrm{WRV}_{0 \mathrm{RF}}} \cdot 100
$$

where $W R V_{0 R F}$ and $W R V_{n R F}$ are the water retention values of never-dried and $n$ times recycled fibres, respectively. Assuming the $\mathrm{WRV}_{0 \mathrm{RF}}$ corresponds to unhornified fibres whereas a WRV of zero describes complete hornification.

Hornification of pulps and fibres with recycle number is presented in Figure 4.36 .

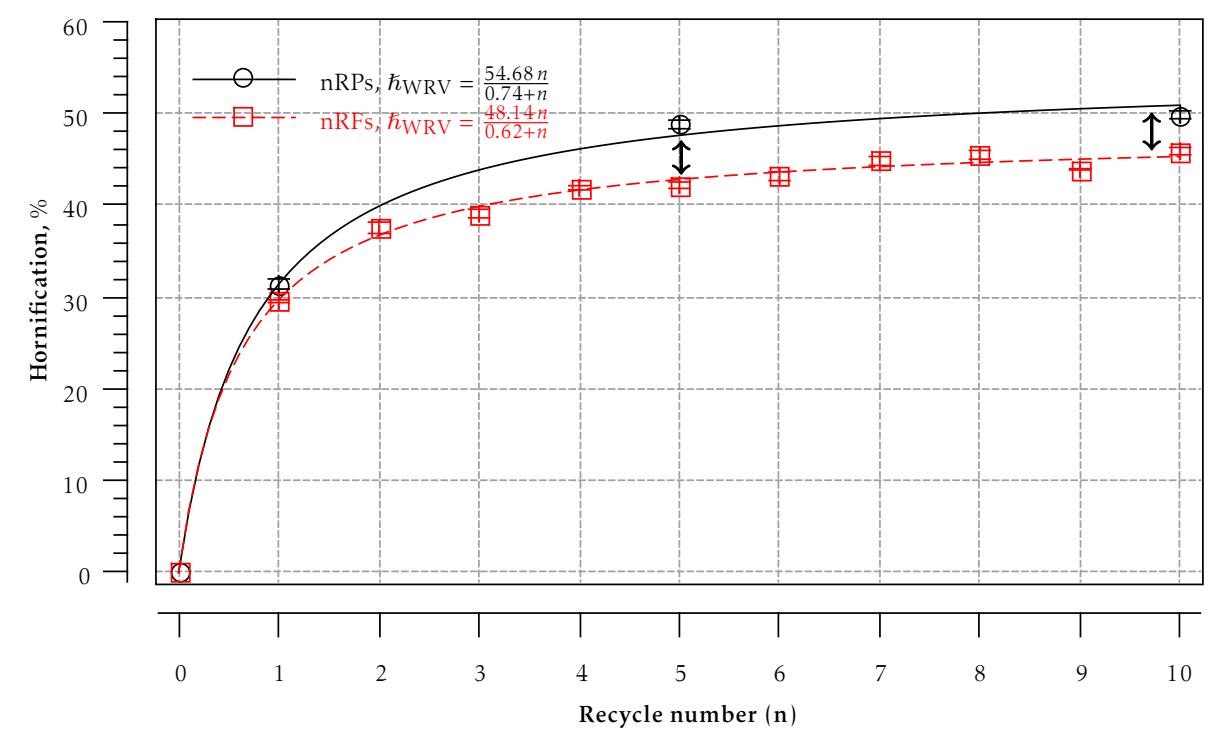

Figure 4.36: Evolution of fibre hornification based on water retention value of pulps and fibres during recycling

The evolution of the hornification may be characterised by the derivative of the previous curve (cf. Figure 4.36), that is to say the quantity: (WRV $(\mathrm{n}-1) \mathrm{RF}-$ 
$\mathrm{WRV}_{\mathrm{nRF}}$ ) [this evolution may be introduced in the final manuscript if possible]. Fibre hornification $(\hbar)$ followed a Michaelis-Menten kinetics (cf. equation (4.10)).

$$
\hbar=\frac{\hbar_{\text {max }} \cdot n}{n_{\hbar_{0.5 \max }}+n}
$$

where $\hbar_{\max }, n$ and $n_{\hbar_{0.5 \max }}$ are the maximum fibre hornification value, recycle number and recycle number corresponding to half of the maximum fibre hornification, respectively. $n_{\hbar_{0.5 \text { max }}}$ may be characteristic of a pulp, recycling process and measurement method. The higher the value of $n_{\hbar_{0.5 \max }}$, the slower is the rate of fibre hornification. The model equations can be used to estimate fibre hornification based on WRV of pulp and fibres during recycling within the experimental conditions used here. At the end of third recycle, fibre hornification reached and ceased at $42 \% .67 \%$ of the total loss in hornification was contributed by pressing and drying of never dried pulp. Fibre hornification of pulps is higher than corresponding fibres.

The hornification measured here by the WRV method charaterises both internal and external modifications of the fibres. In order to study the evolution of the internal structure, another experimental technique is used, namely the fibre saturation point. This leads to a complementary definition of the hornification based on the FSP measurements. Fibre hornification based on FSP $\left(\hbar_{\mathrm{FSP}}\right)$ can be represented by the following expression

$$
\text { Fibre hornification }(\%)=\hbar_{\mathrm{FSP}}=\frac{\mathrm{FSP}_{0 \mathrm{RF}}-\mathrm{FSP}_{\mathrm{nRF}}}{\mathrm{FSP}_{0 \mathrm{RF}}} \cdot 100
$$

where $\mathrm{FSP}_{\mathrm{ORF}}$ and $\mathrm{FSP}_{\mathrm{nRF}}$ are the fibre saturation points of never-dried and $\mathrm{n}$ times recycled fibres. Assuming the $\mathrm{FSP}_{0 \mathrm{RF}}$ corresponds to un-hornified fibres whereas a FSP of zero describes complete hornification.

Figure 4.37 shows the evolution of fibre hornification based on FSP of the pulps and the corresponding fibres. 


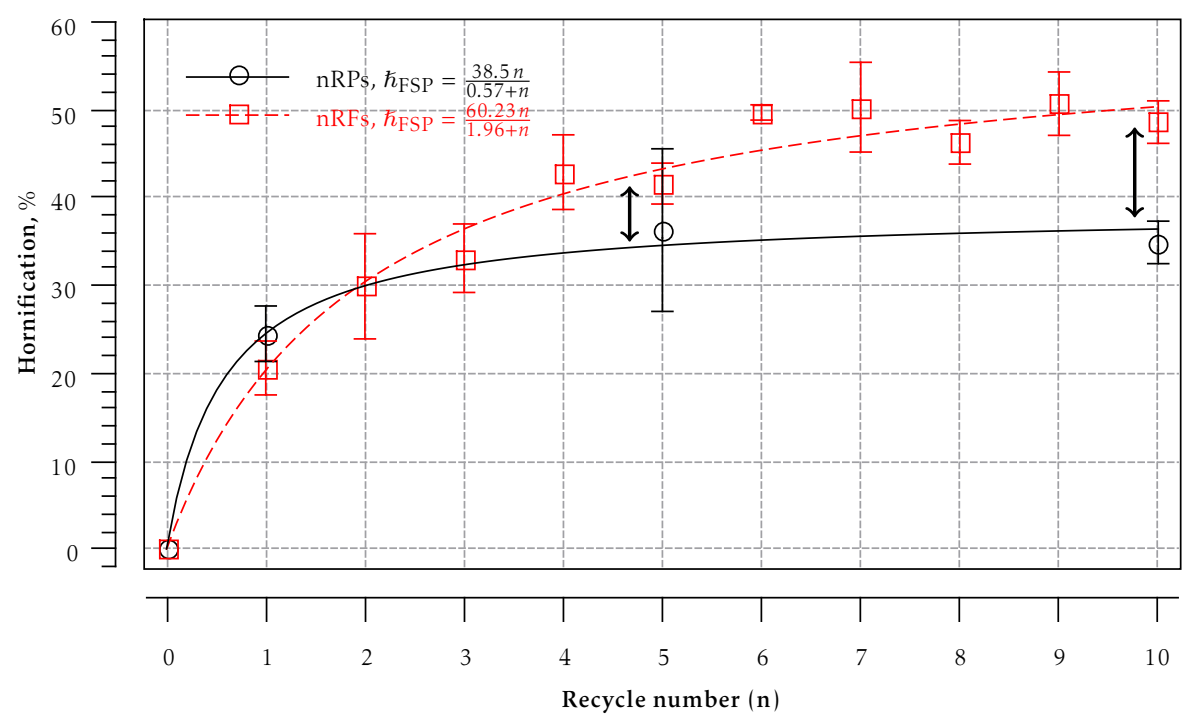

Figure 4.37: Evolution of fibre hornification based on fibre saturation point of pulps and fibres during recycling

The trend of hornification based on FSP with recycle number is similar to the one based on WRV. However, pulp containing fines and short fibres show lesser drop in hornification compared to the one based on WRV. The model equations can be used to estimate fibre hornification based on FSP of pulp and fibres during recycling within the experimental conditions used here.

\subsection{Pore size distribution of recycled fibres}

Figure 4.38 shows cumulative apparent pore volume of recycled fibres. Significant pore closure took place in the first recycle. From second recycle onwards cumulated apparent pore volume was slightly affected. 


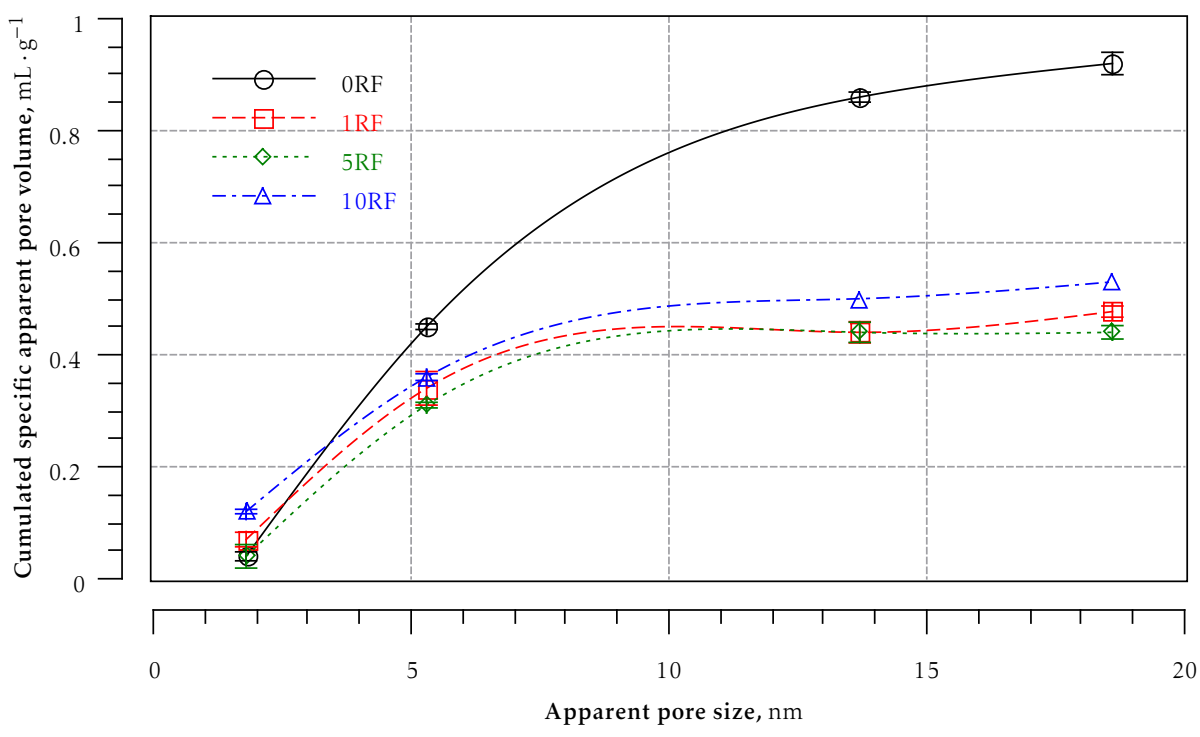

Figure 4.38: Cumulated specific apparent pore volume of recycled fibres

First recycle contributes to pore closure predominantly in pores of size 5.3 to $13.7 \mathrm{~nm}$ (cf. Figure 4.39). Although, cumulative pore volume of once and five times recycled fibres was the same, relative pore volumes of 0.8 to $1.8 \mathrm{~nm}$ and 13.7 to $18.6 \mathrm{~nm}$ pore sizes were slightly decreased whereas relative pore volume of 1.8 to $13.7 \mathrm{~nm}$ pore size was slightly increased. The relative increase in pore volume of ten times recycled fibres was due to pore openings of 0.8 to $1.8 \mathrm{~nm}$ pores sizes.

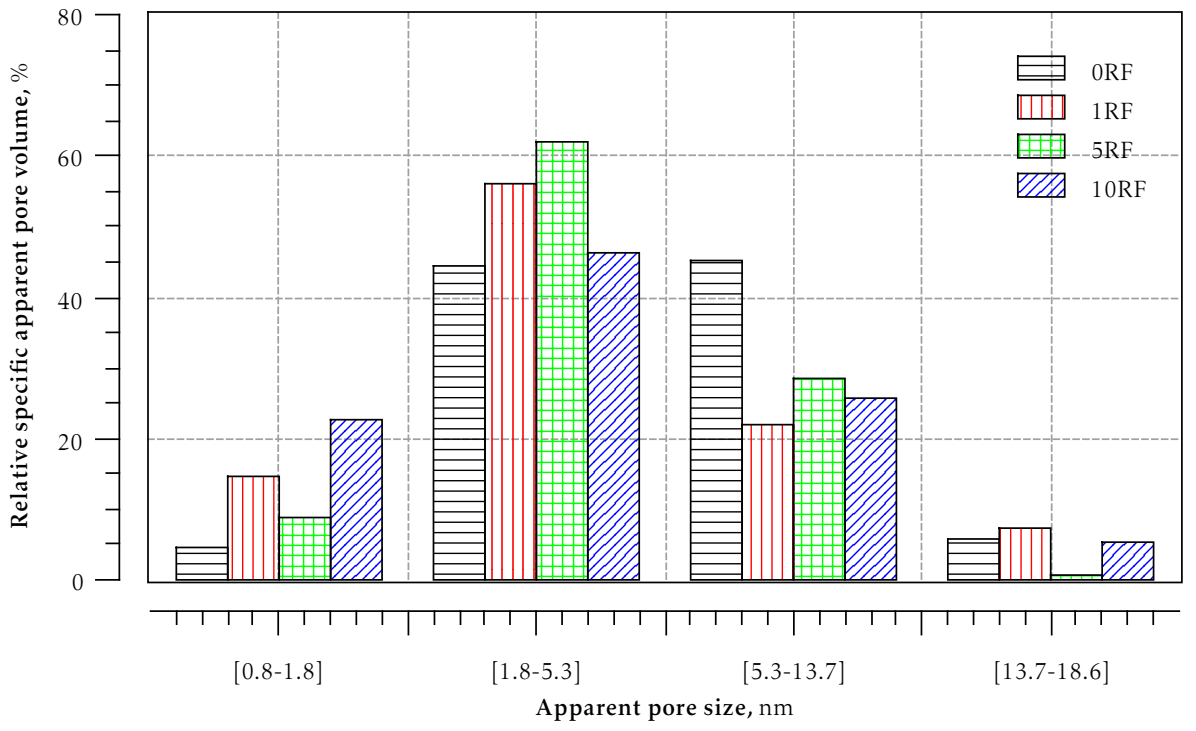

Figure 4.39: Effect of recycling on apparent pore size distribution of fibres

Specific apparent pore volume results are tabulated in Appendix B. 


\subsection{Width shrinkability of recycled fibres}

Fibre width shrinkage while drying is an indirect mean of quantifying fibre swellability. Ten fibres were imaged using ESEM to observe fibre width shrinkability while in-situ drying. Wet swollen conditions of $85 \mathrm{RH}$ correspond to a temperature of $5^{\circ} \mathrm{C}$ and a pressure of $0.733 \mathrm{kPa}$ whereas dry conditions of $10 \mathrm{RH}$ correspond to a temperature of $24^{\circ} \mathrm{C}$ and a pressure of $0.267 \mathrm{kPa}$. Fibres were kept for $30 \mathrm{~min}$ to attain equilibrium before taking micrographs at a magnification of $2400 \times$.

The effect of drying on fibre width of never dried fibres is shown in Figure 4.40. The quantitative image analysis was performed with ImageJ[22]. Fibre width decreased while drying.
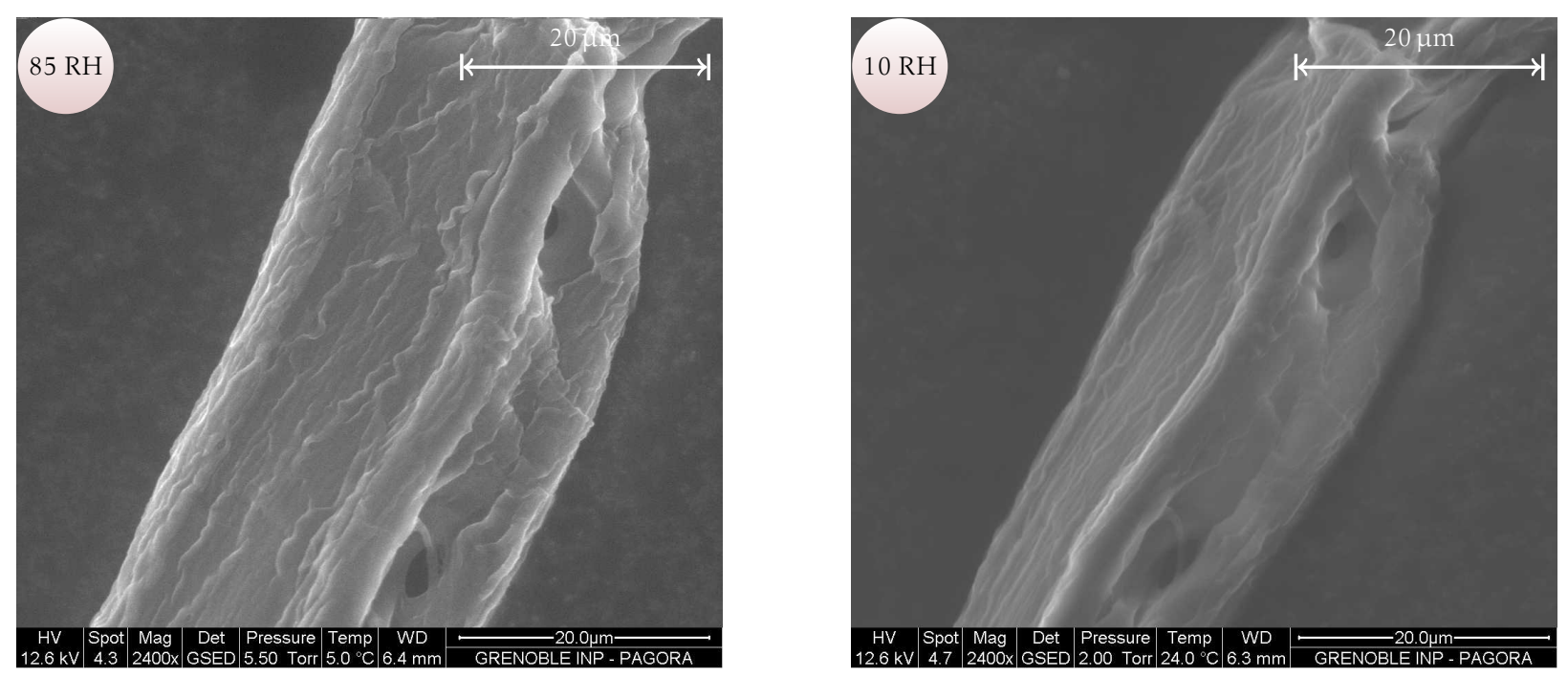

Figure 4.40: ESEM images of never dried fibre at $85 \mathrm{RH}$ (left) and at $10 \mathrm{RH}$ (right)

The evolution of fibre width shrinkability with recycling is shown as box-andwhisker plot in Figure 4.41. 


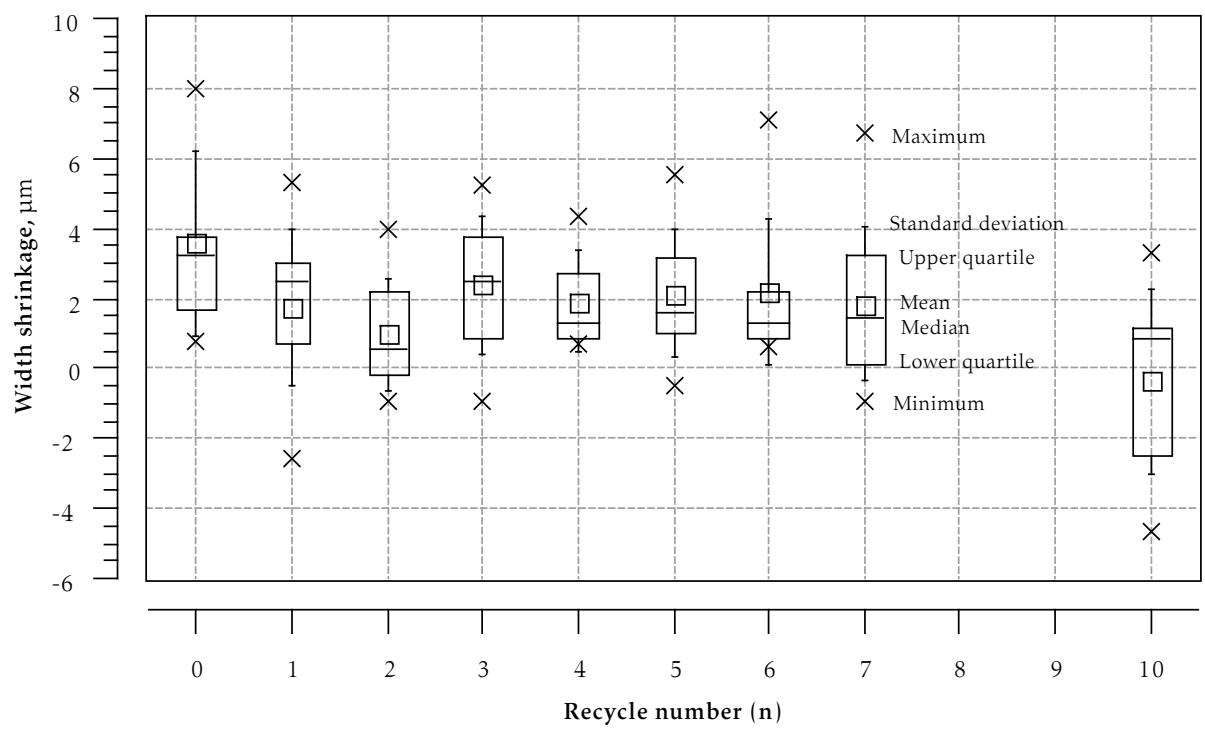

Figure 4.41: Width shrinkage of recycled fibres

No quantitative conclusion can be drawn on the evolution of fibre width shrinkability with recycling due to a high standard deviation, which can be attributed to the variety of fibres present in the pulp mix and above all to the twist of fibres during in-situ drying (cf. Figure 4.41). Twisting of some fibres resulted in negative shrinkability value (swelling) which can only be addressed if the measurements are carried out in 3D.

\subsection{Wet breaking length of recycled pulps and fi- bres}

The effect of recycling on wet breaking length of recycled fibres is presented in Figure 4.42. Moisture content of the test specimens was 75 to $80 \%$. Mean values are based on five measurements. 


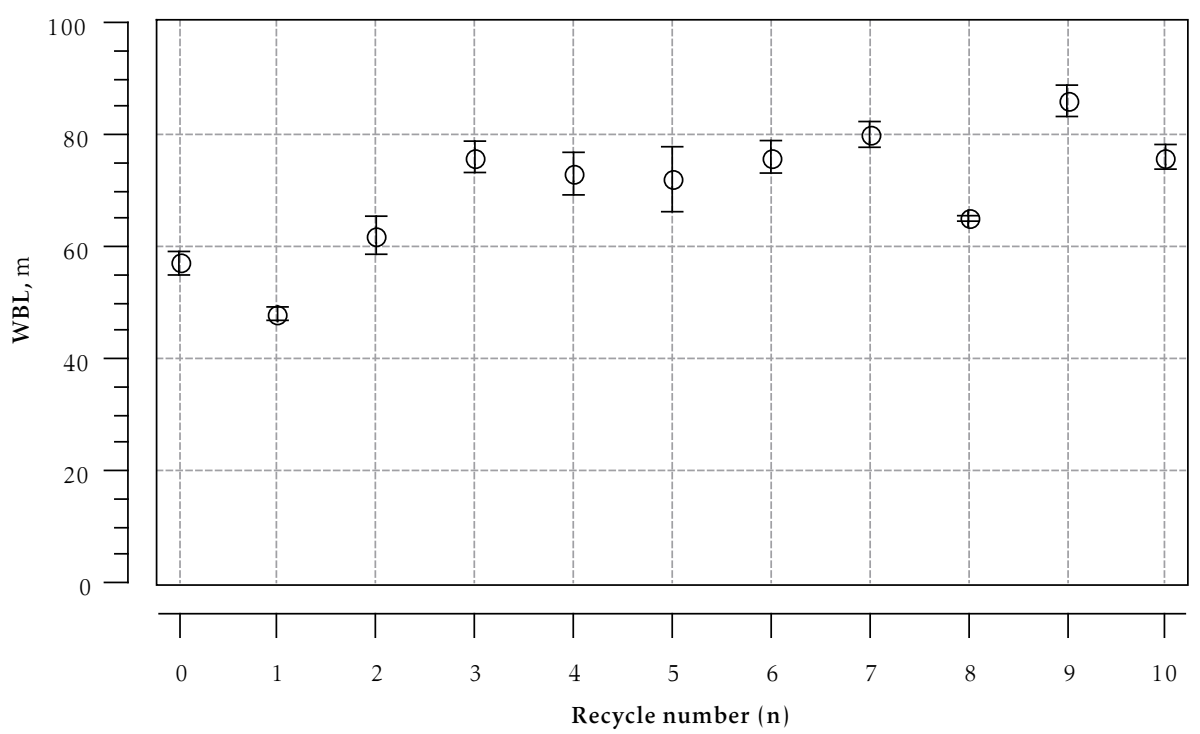

Figure 4.42: Wet breaking length of recycled fibres

WBL slightly decreased in first recycle, increased until third recycle and remained constant till seventh recycle. Thereafter, WBL slightly decreased followed by a slight increase in the next recycles. Overall we can say that WBL slightly increased with recycling. Despite the straightening, flattening and the drop in fibre flexibility with recycling, the increase in WBL may be caused by the ruptured outer layers (see section 4.3) offering an increased friction.

\subsection{Weak points in fibre wall of recycled fibres}

Weak points in fibre wall during recycling were quantified as cleavages per fibre. The procedure of $\mathrm{HCl}$ treatment is described in Appendix C.

Effect of recycling on fibre length distribution of cleaved never dried fibres is presented in Figure 4.43. Longer fibre fraction reduced as a result of the $\mathrm{HCl}$ treatment. Weak points present in the fibre wall came under acid attack causing cleavages. 


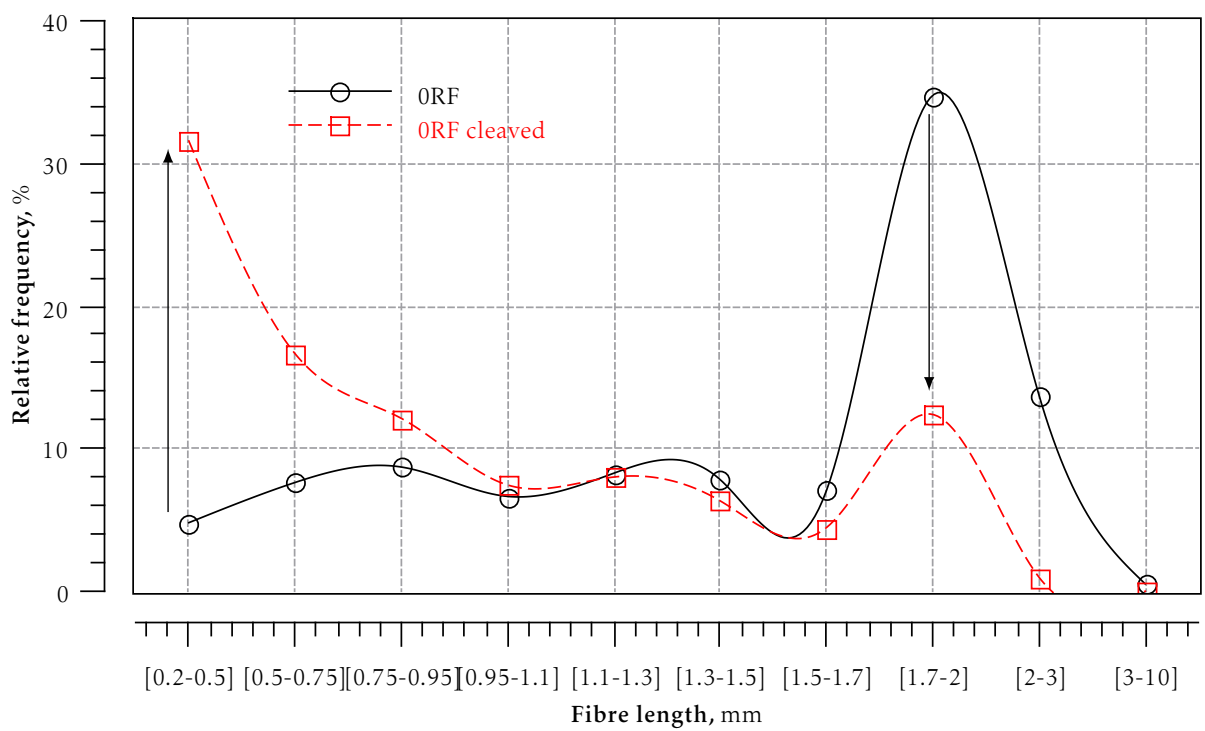

Figure 4.43: Effect of $\mathrm{HCl}$ cleavage on length distribution of never dried fibres

$\mathrm{HCl}$ attacked outer fibre wall layers (P/S1) (cf. Figure 4.44). Fibre width decreased until ninth recycle when treated with $\mathrm{HCl}$.

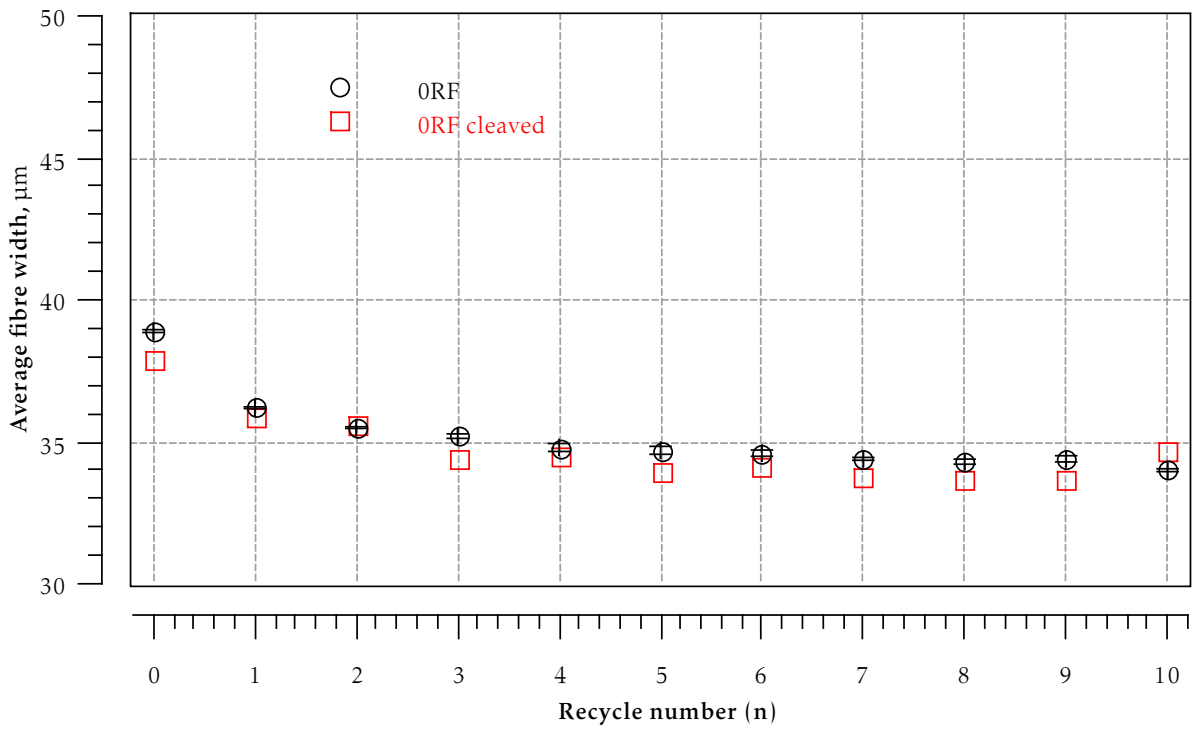

Figure 4.44: Evolution of average fibre width of cleaved recycled fibres

Effect of recycling on fibre width distribution of cleaved never dried fibres is shown in Figure 4.45. 


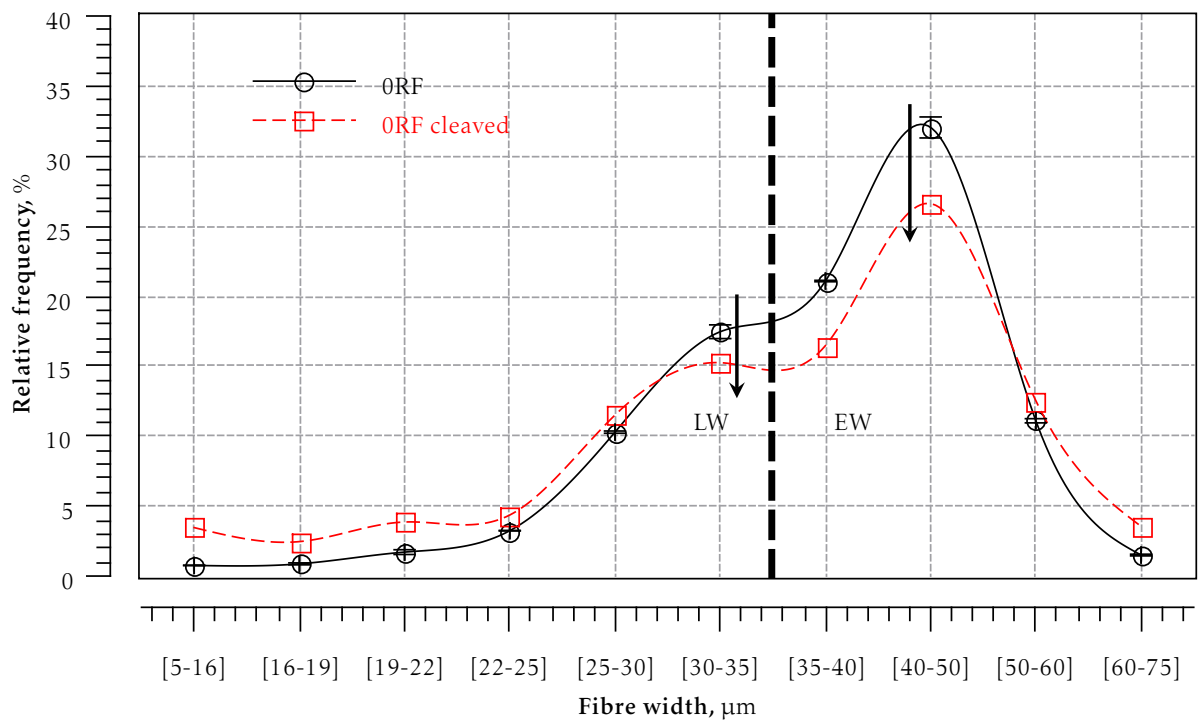

Figure 4.45: Effect of $\mathrm{HCl}$ cleavage on width distribution of never dried fibres

Weaker and ruptured outer layers of EW and LW fibres were detached by the acid attack (cf. Figure 4.45).

Effect of recycling on cleavages per fibre is shown in Figure 4.46.

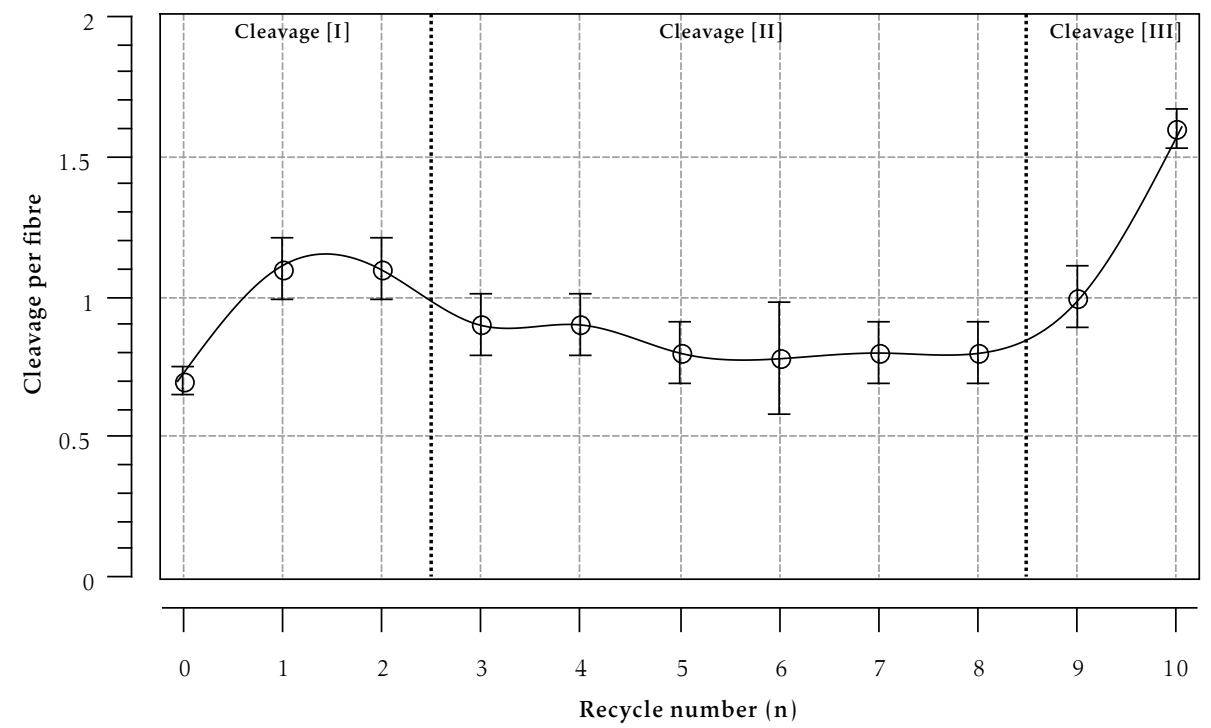

Figure 4.46: Cleavages per fibre of fibres during recycling

Cleavage [I] indicates growing region where fibre length is shortened along with the delayering of outer (P/S1) layer. Cleavage [II] is constant region where $\mathrm{S} 2$ layer is compacted while (P/S1) layers are detached further. $\mathrm{HCl}$ remove outer (P/S1) layers and cleaves fibre wall. In cleavage [III], the outer layer is al- 
ready detached with repeated recycling and fibre cleavage becomes much more easier (cf. Figure 4.46).

Probable transformations in cell wall layers (radial section) during recycling due to cleavage are summarised in Figure 4.47.

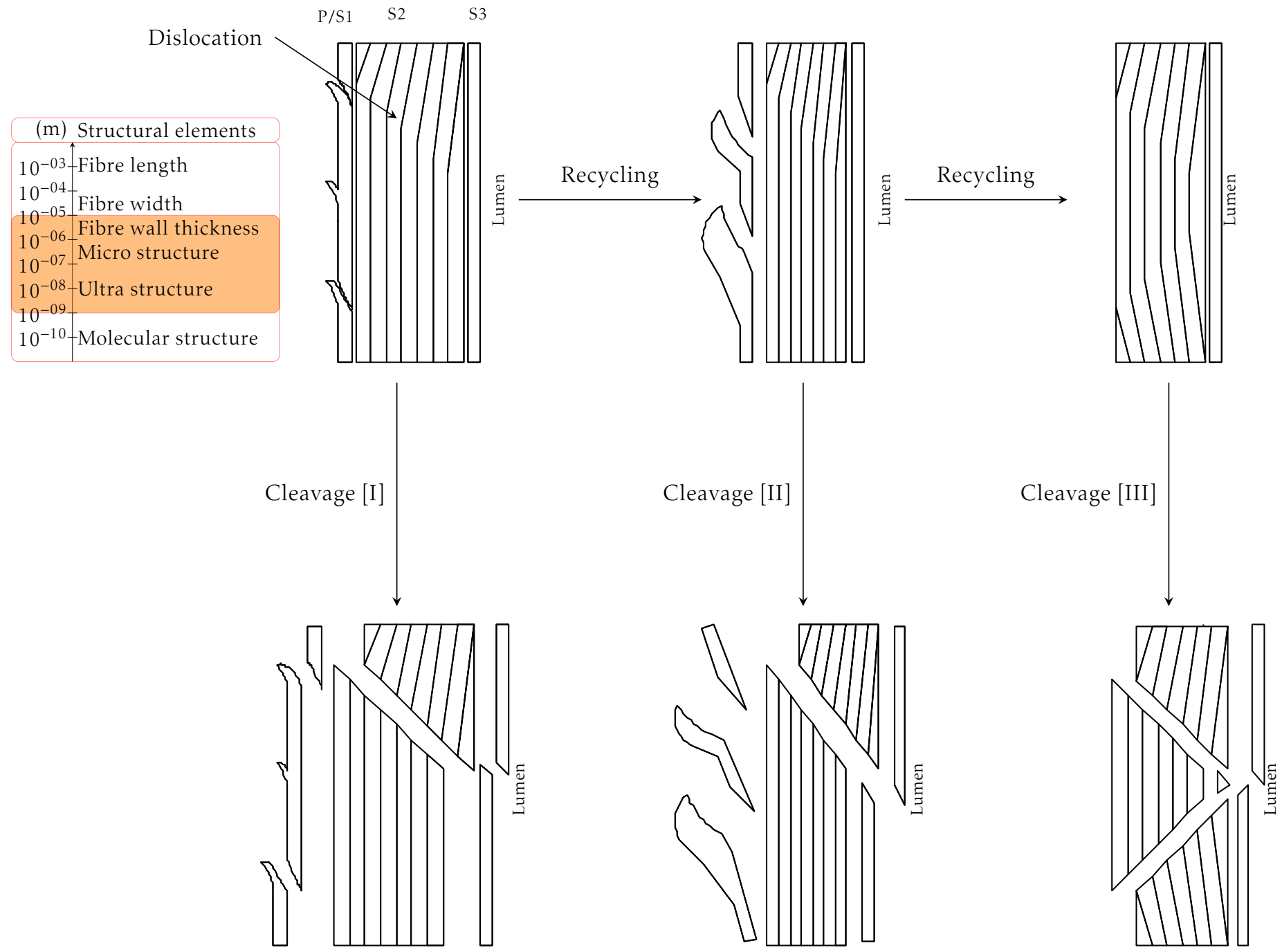

Figure 4.47: Schematic diagram of probable transformations of fibre cell wall during recycling by $\mathrm{HCl}$ cleavage

Recycling increased weak points in fibre wall. The outer (P/S1) layers play an important role, once the outer layers are detached the inner layers containing dislocations are exposed to acid. 


\subsection{Single fibre tensile properties}

Single fibre tensile properties were investigated on TA Instruments' Dynamic Mechanical Analyser (DMA RSA3) at ambient temperature. It measures the sample's response to the applied stress.

Fibres were carefully extracted from air dried pulp with the help of fine tweezers. The extremities of the fibre were glued with the help of SADER epoxy composite on a support. The setting and curing times were $2 \mathrm{~h}$ and $15 \mathrm{~h}$, respectively. Every step in the procedure was carried out under stereo-microscope. After curing the sample was mounted carefully between the jaws of the DMA.

Figure 4.48 shows the prepared sample for single fibre tensile testing.
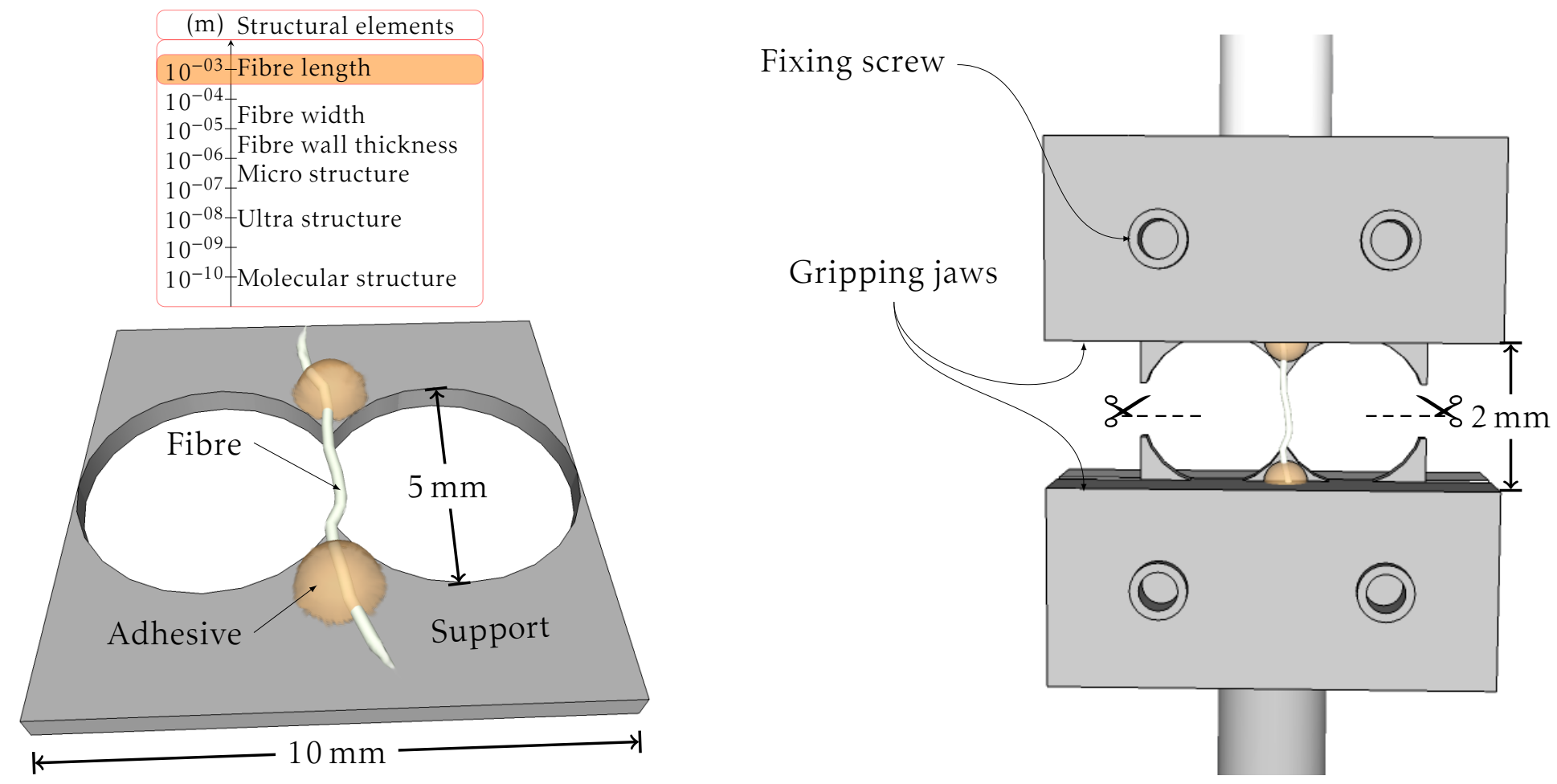

Figure 4.48: Single fibre tensile test

The initial span and the constant strain rate were $2 \mathrm{~mm}$ and $0.006 \mathrm{~mm} \cdot \mathrm{min}^{-1}$, respectively. Fibre was stretched under the application of tensile stress. The applied stress approached the ultimate strength of the fibre. Until a moment in time was reached when the fibre was unable to withstand any additional load, resulting in tensile failure. The broken fibre was preserved instantly and the cross section was analysed later by cutting it with a razor blade near the glued end. It was assumed that the fibre wall cross section was constant across the 
length of fibre under tensile stress. SEM micrograph of fibre wall cross section at $2400 \times$ is shown in Figure 4.49.

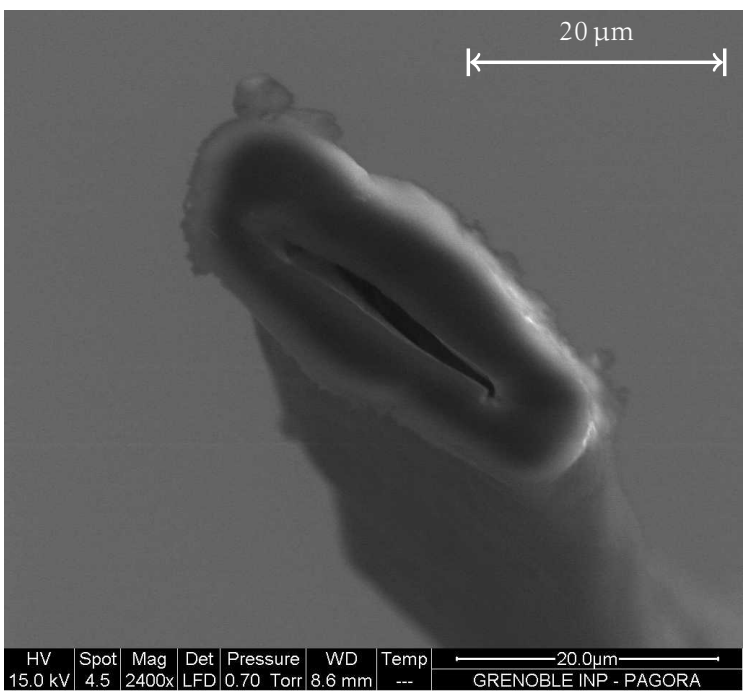

Figure 4.49: SEM micrograph of a fibre cross section

Fibre wall cross sectional area without lumen (equation (4.6)) was calculated from the micrographs using ImageJ [22].

The stress-strain curves of air dried never dried and ten times recycled fibres are presented in Figure 4.50.
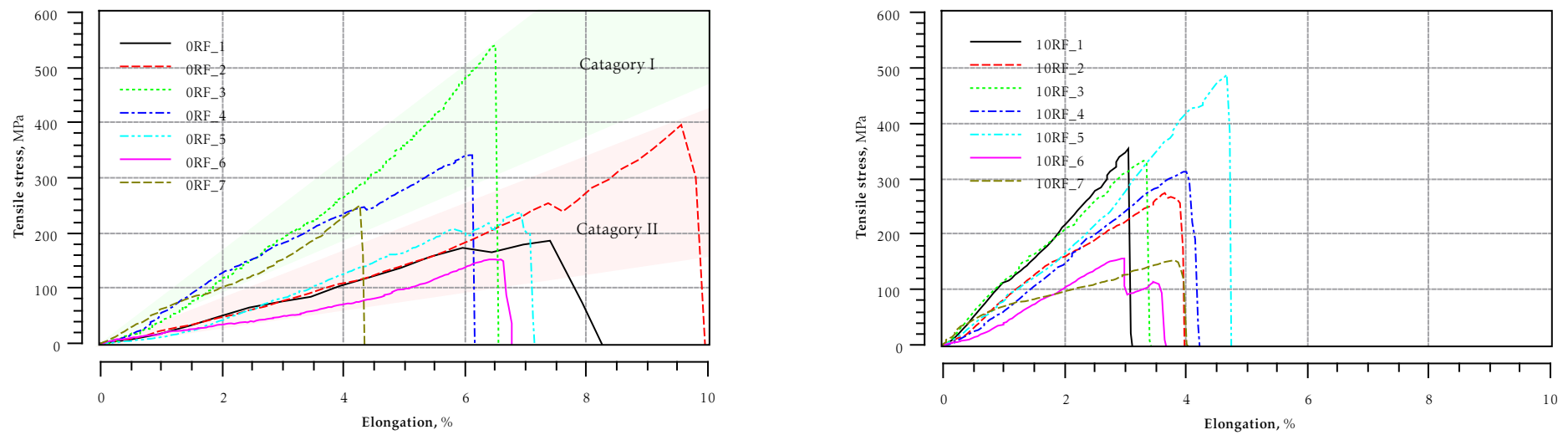

Figure 4.50: Stress-strain curve of air dried never dried fibres (left) and ten times recycled fibres (right)

Stress-strain curves of never dried fibres may be divided in two categories whereas stress-strain curves of ten times recycled fibres crowded together (cf. Figure 4.50). Modulus of elasticity was determined from the slope of stress-strain curve in 
the initial elastic region. The non-linearity in the stress-strain curves arose from the dislocations present in the fibre wall. Tensile energy absorbed represents area under stress-strain curve.

Table 4.6 summarises the effect of recycling on single fibre tensile properties.

Table 4.6: Effect of recycling on single fibre tensile properties

\begin{tabular}{l|cc} 
& 0RF & 10RF \\
\hline Elongation, \% & $6.8 \pm 0.8$ & $4.1 \pm 0.6$ \\
Stress at break, GPa & $0.32 \pm 0.06$ & $0.27 \pm 0.04$ \\
Elastic modulus, GPa & $5.27 \pm 1.56$ & $8.54 \pm 1.31$ \\
Tensile energy absorbed, $\mathrm{J} \cdot \mathrm{m}^{-2}$ & $2.09 \times 10^{-11} \pm 3.34 \times 10^{-12}$ & $1.19 \times 10^{-11} \pm 1.74 \times 10^{-12}$
\end{tabular}

Increase in modulus was $62 \%$ whereas elongation, stress at break and tensile energy absorbed were dropped by $40 \%, 16 \%$ and $43 \%$, respectively. The increase in modulus may be resulted from the densification of fibre wall (decrease in fibre wall cross section area), the straightness of fibre itself, alignment of microfibrils or reduction in MFA and/or aggregation of microfibrils (increased hydrogen bonding) [199]. The decrease in elongation, stress at break and area under stress-strain curve may be due to the weak points present in the fibre wall. It can be said that fibre became hard and brittle with recycling. The results are in accordance to the earlier findings where increase in elastic modulus and decrease in tensile strain during restrained drying were reported $[122,78]$. If we use the work of Page [172] to estimate the change in MFA then we arrive on a decrease in MFA by $4.5^{\circ}$. A schematic description of the change in MFA during recyclings is shown in Figure 4.51. 


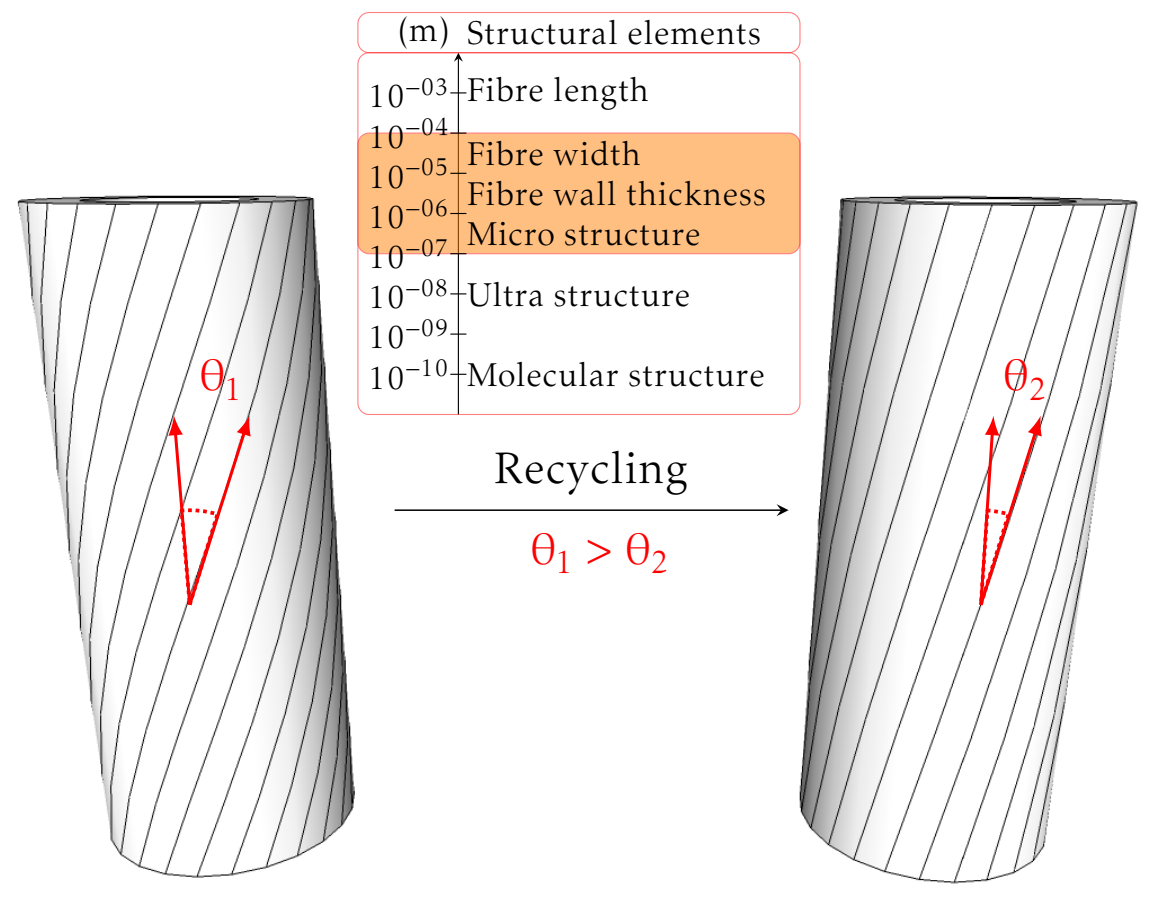

Figure 4.51: Schematic diagram of probable reduction in MFA with recycling 


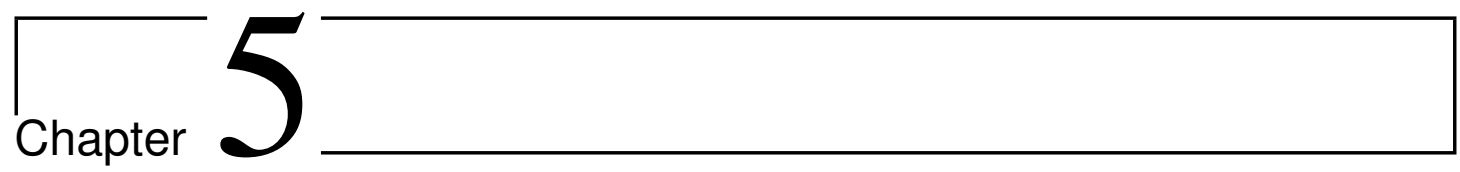

\section{Physical Properties}

\section{Contents}

5.1 Properties of handsheets made from recycled pulps . . . 113

5.1.1 Structural properties of handsheets made from recycled pulps . . . . . . . . . . . . . 113

5.1.2 Mechanical properties of handsheets made from recycled pulps . . . . . . . . . . . . . . 121

5.1.3 Optical properties of handsheets made from recycled

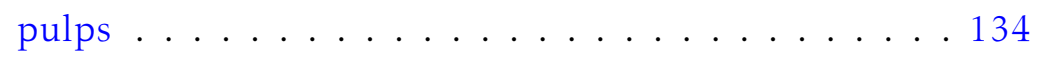

5.2 Properties of handsheets made from recycled pulp blends . . 137

5.2.1 Structural properties of handsheets made from recycled pulp blends . . . . . . . . . . . . . . 137

5.2.2 Mechanical properties of handsheets made from recycled pulp blends . . . . . . . . . . . . . . 138

5.2.3 Optical properties of handsheets made from recycled pulp blends ... . . . . . . . . . . . 149

5.2.4 Cost analysis of recycled pulp blends . . . . . . . . . 151

The term paper is strictly restricted to a sheet material that is made mainly from fibres of plant origin. Paper properties are related to the characteristics of raw materials and process parameters. Raw materials inherit individual characteristics while process parameters ameliorate and/or deteriorate their effects. Pulp fibres form the supporting structure of paper and board. 


\section{Handsheet formation}

In conventional handsheet making machine (sheet former), a low consistency wet disintegrated pulp [14] is used to form an isotropic sheet which is then couched from the wire screen with the help of blotters, wet pressed and then dried in contact with drying plate in conditioned air.

In another method, sheets are dried within the blotters. In this case, the sheet shrinks in accordance to the blotters but the anisotropy of the blotters may also influence the shrinkage of the sheet. In both lab drying techniques, the shrinkage differs from the conditions of paper machine.

In contrast to the conventional handsheet former, Rapid-Köthen [15] sheet former is also widely used in the laboratories. It is integrated with quick vacuum drying system. The pulp suspension is added to the forming cylinder. The sheet forming column comprises of a sheet supported sieve suction chamber, an evenly distributed water injection system and an external compressed air supply system for mixing and agitation. In the drying section the wet sheet is subjected to the pressure and dried with almost complete prevention of shrinkage.

Handsheet forming was carried out in automatic mode where the sequential fixed sheet forming and drying intervals were followed. Drying temperature was set at $80^{\circ} \mathrm{C}$. Handsheets (nRs) were conditioned at $23 \pm 1{ }^{\circ} \mathrm{C}$ and $50 \pm 2 \% \mathrm{RH}$ for a period of at least $24 \mathrm{~h}$ before testing [3].

\subsection{Properties of handsheets made from recycled pulps}

\subsubsection{Structural properties of handsheets made from recycled pulps}

\section{Bulk of handsheets made from recycled pulps}

Bulk is obtained from two basic properties of paper i.e. basis weight and caliper. Basis weight or grammage is the mass per unit area of the test specimen [6]. It also includes the amount of water present under existing environmental conditions. The mass of the test specimen was measured using Mettler H35AR, a precision balance with an accuracy of $0.1 \mathrm{mg}$. Grammage, $\mathrm{G}\left(\mathrm{g} \cdot \mathrm{m}^{-2}\right)$ was calculated from equation (5.1)

$$
\mathrm{G}=\frac{m}{\mathrm{~A}} \cdot 10000
$$


where $m(\mathrm{~g})$ and $\mathrm{A}\left(\mathrm{cm}^{2}\right)$ are the mass and surface area of the handhseet, respectively. Paper calliper or thickness is the perpendicular distance between the two opposite surfaces of a sheet of paper. Calliper gauge contains two parallel platens of determined surfaces, a constant pressure is applied at constant prescribed rate, the reading is taken after $2 \mathrm{~s}$ once the contact is made [16]. Calliper was measured on digital micrometer (Adamel Lhomargy MI20 N).

Bulk $\left(\mathrm{cm}^{3} \cdot \mathrm{g}^{-1}\right)$ is the inverse of density.

$$
\text { Bulk }=\frac{\hbar}{G}
$$

where $\ddagger(\mu \mathrm{m})$ is the thickness of the handsheet.

Density of pure cellulose is $1500 \mathrm{~kg} \cdot \mathrm{m}^{-3}$ whereas the density of paper varies between 300 to $1000 \mathrm{~kg} \cdot \mathrm{m}^{-3}$ [83].

The evolution of bulk during recycling is presented in Figure 5.1. The data comprised of a set of six thickness and one grammage measurements per handsheet for three handsheets.

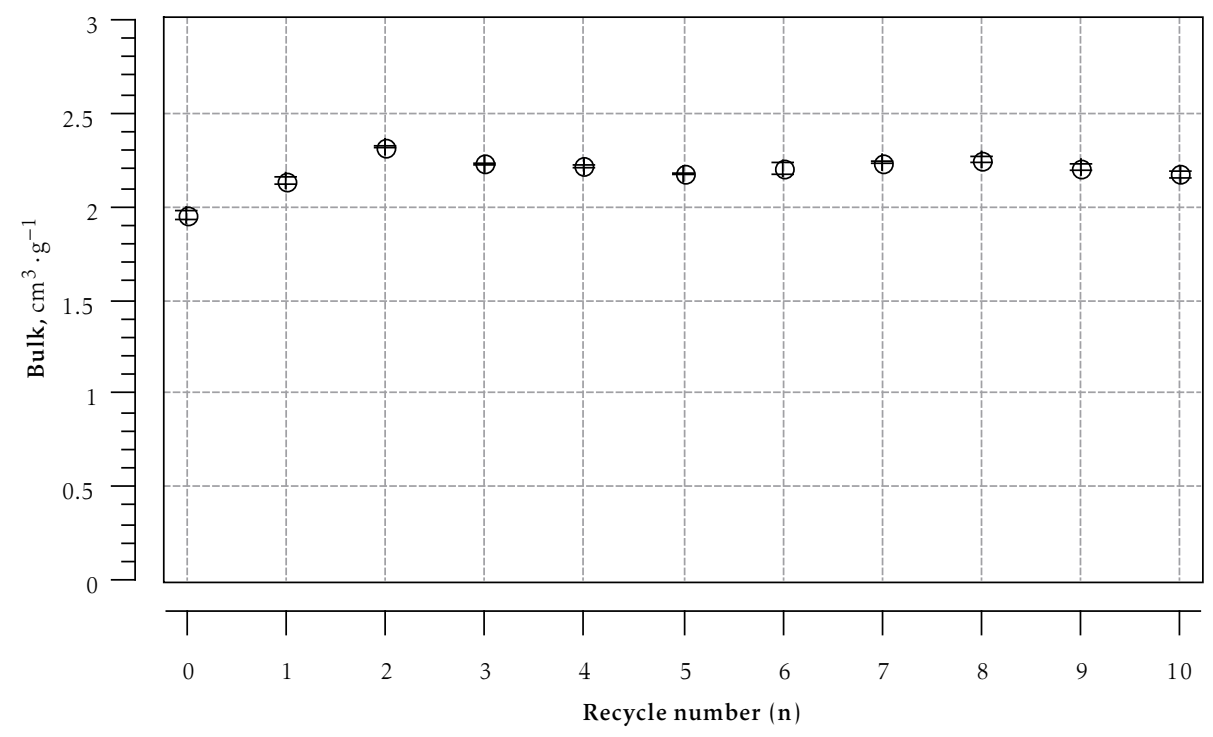

Figure 5.1: Bulk of handsheets made from recycled pulps

Bulk of the handsheets increased until second cycle of recycling. After that there was not any significant variation. The increase in bulk during first few recycles may be attributed to the loss of fines while handsheet formation and loss of fibre flexibility. Fines were lost during handsheet making, never dried pulp was rich in fines whereas ten times recycled pulp contained minimum amount of fines. The decrease in fines content was uniform during recycling 
whereas the loss of fibre flexibility was significant during first few recycles. Therefore, the contribution of fibre flexibility towards the increase in bulk may be more due to loss of fibre flexibility than fines.

\section{D fibre structure of handsheets made from recycled pulps}

Three dimensional (3D) structure carries a lot of useful information. 3D paper structure has previously been simulated as a function of raw material properties to predict end product properties [164]. Actual 3D pore structure of paper is far more complex. Therefore a simplified model is not sufficient to fully describe its microstructure. With the advent of technology, new experimental techniques have been developed to investigate paper in a better way.

Tomography refers to the 3D imaging of an object. 3D imaging of sample larger than few centimetres of length scale is called computer tomography (CT). Microtomography is the notation used for the $3 \mathrm{D}$ image acquisition of a sample having dimensions below centimetre range. X-ray imaging in absorption contrast mode is used in for example medical practices to differentiate bones and tissues.

Compared to scanning electron microscope (SEM), X-rays microtomography $(\mu \mathrm{CT})$ does not require a contrast material or an epoxy binder for analysis [196]. Cutting of the sample in SEM may damage fibres and resin may alter its 3D structure. $\mu \mathrm{CT}$ is a non destructive technique $[196,33]$. In $\mu \mathrm{CT}$ X-rays are projected onto the sample. Depending on the density and composition, proportion of X-rays are absorbed whereas the rest transmit through the sample. The sample holder rotates and a set of X-ray projections called sinogram columns are obtained. The sinograms are filtered and back projected to get a $2 \mathrm{D}$ reconstructed image called slice. A volume is obtained from a set of image acquisition projections.

Paper samples were first mounted on a capillary with help of wax. The capillary was then fixed on the sample holder (cf. Figure 5.2). The prepared sample holder was placed inside measurement chamber so that the sample adjust its humidity and temperature levels accordingly. 


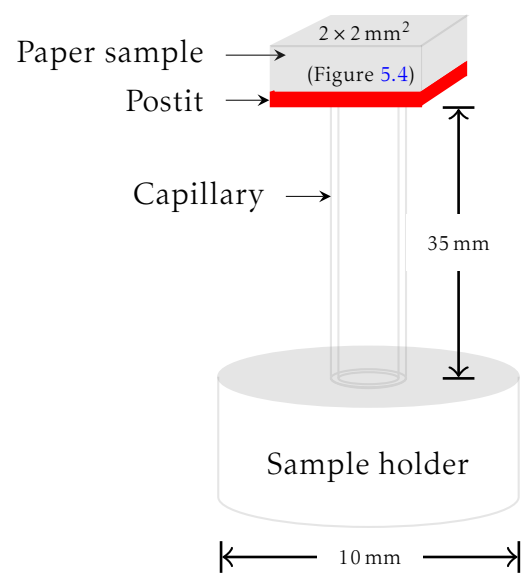

Figure 5.2: Prepared sample holder for X-rays microtomography

Figure 5.3 shows the experimental set-up at ID-19 of European synchrotron radiation facility (ESRF). X-rays from synchrotron are projected onto the paper sample. A volume of image stack is obtained from image acquisition of 1500 projections.

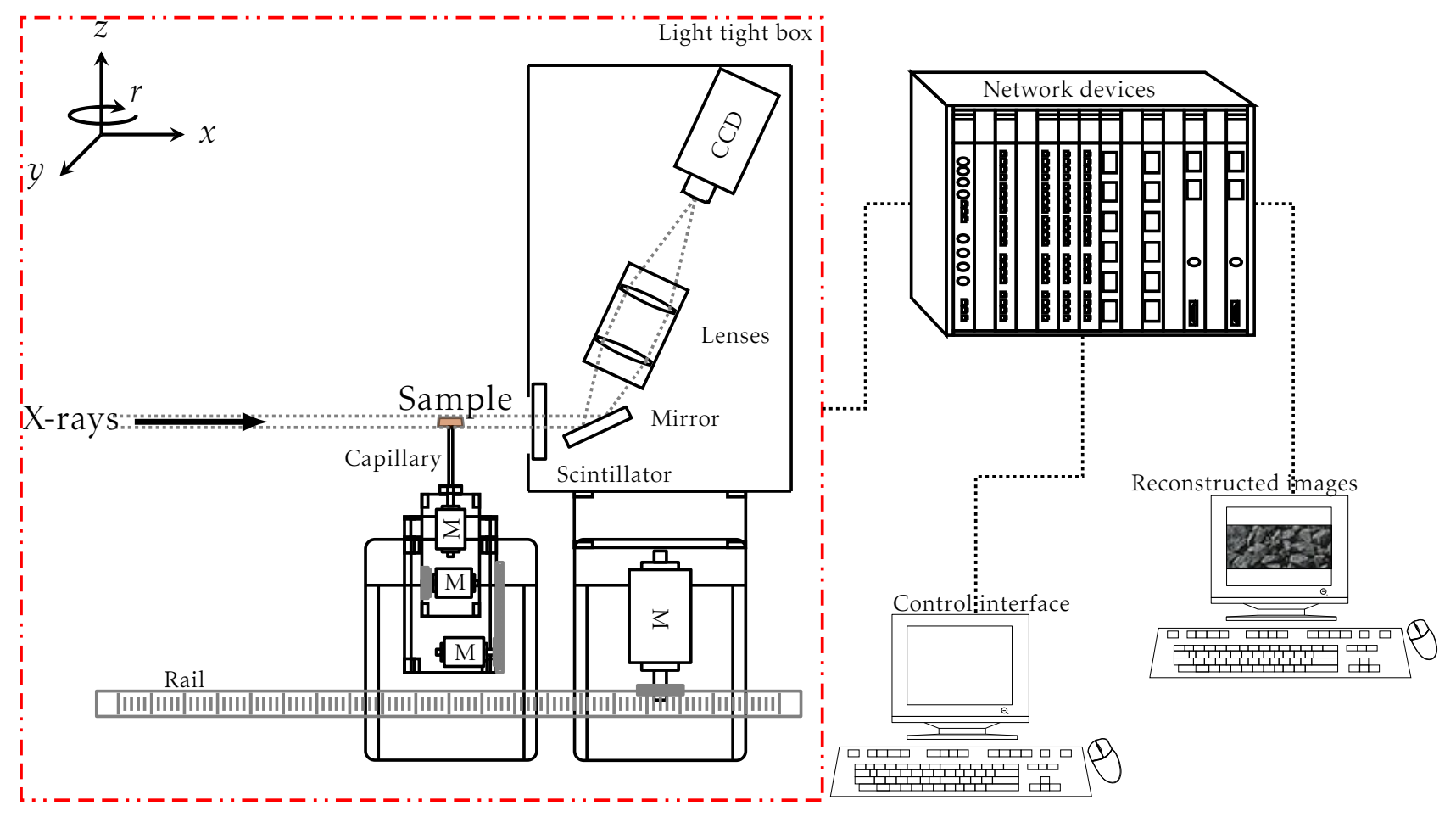

Figure 5.3: Experimental set-up of ID-19 at ESRF

Figure 5.4 shows the effect of recycling on 3D structure of paper. The voxel (volumetric pixels) size used to obtain X-rays micrographs was $0.7 \mu \mathrm{m}$ which 
allowed to acquire images of $2048 \times 2048 \times \notin$ voxels.
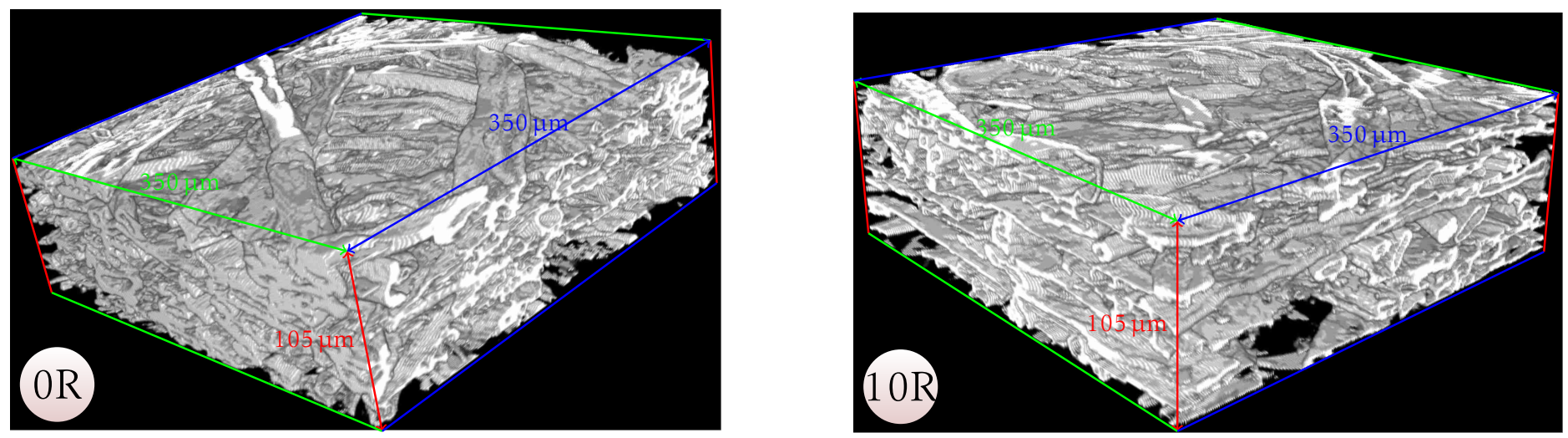

Figure 5.4: Microtomographs of handsheets produced from never dried (left) and ten times recycled (right) pulps

Based on the image stacks, it is possible to obtain porosity profiles by thresholding as shown in Figure 5.5. Also fibre intersection area, collapse index and aspect ratios can also be analysed from these stacks.

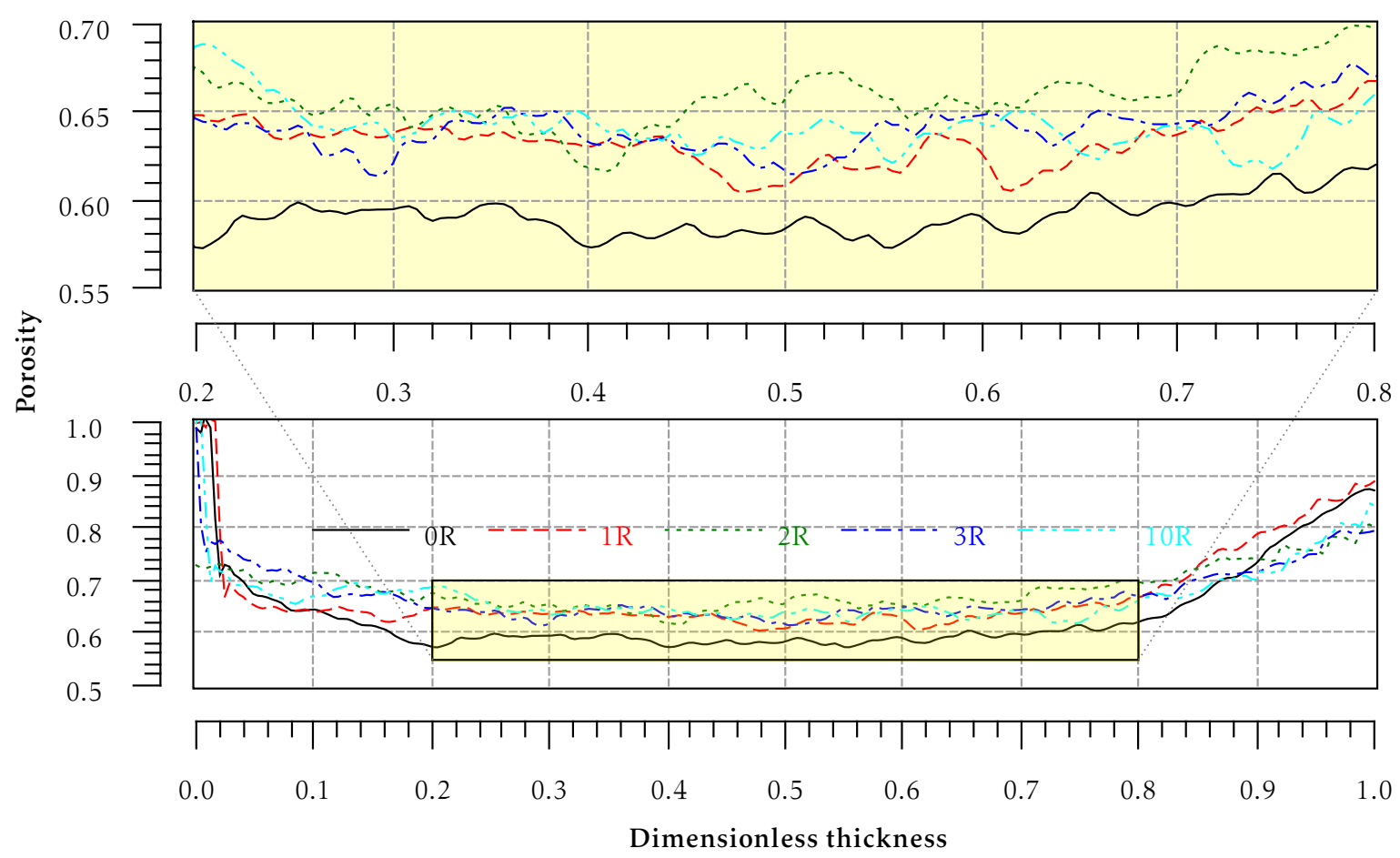

Figure 5.5: Porosity profiles of handsheets made from recycled pulps

To have a better understanding of the structural variations, porosity is plotted against dimensionless thickness $\left(\mathfrak{t}_{i} / \mathfrak{t}\right)$. $\mathfrak{t}_{i}$ is the coordinate position in the thickness direction. Porosity increases towards the middle. The top and bottom 
sides of the sheets are normally compacted. The increase in porosity of top and bottom sides resulted from the tilt of the test sample during image acquisition with respect to $z$-axis. However, if we ignore the extremities of thickness, a significant decrease in porosity can be seen in the first recycle. It might have been resulted from the loss of fines during handsheet making and fibre hornification. Later recycles slightly affected the 3D structure as indicated by the porosity profiles. The average porosity values corresponds to the evolution of bulk during recycling.

\section{Projected intersection area of recycled pulp fibres}

The adopted approach for calculating intersection area (area of fibre crossings) from microtomographs is presented in Figure 5.6. The stack of image slices was resliced and sub-stacked. The sub-stacks were then merged to get corresponding z projection images.

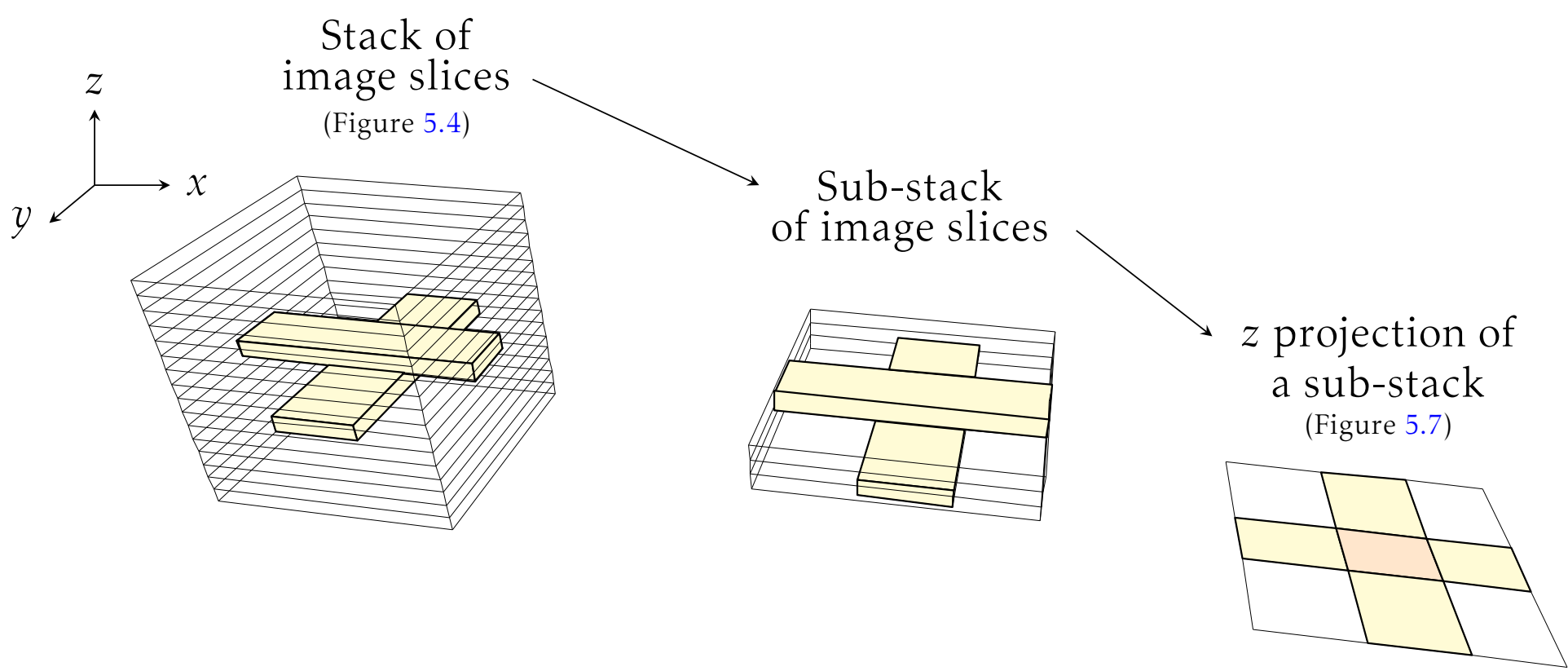

Figure 5.6: Measuring projected fibre intersection area from microtomographs

Fibre intersection area from $z$ projection of sub-stacked microtomographs is shown in Figure 5.7. 


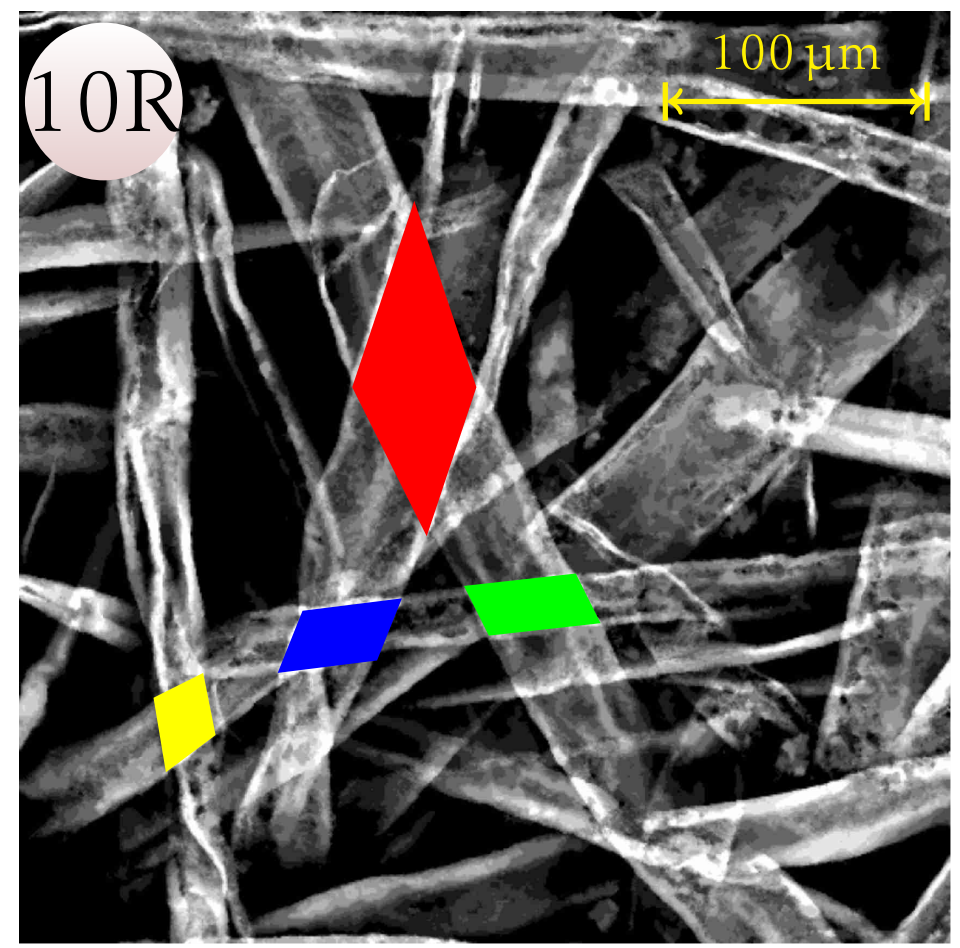

Figure 5.7: $z$ projection of $(7 \mu \mathrm{m})$ sub-stacked microtomographs

Evolution of projected intersection area of a volume of $350 \times 350 \times 105 \mu \mathrm{m}^{3}$ is described in Table 5.1.

Table 5.1: Evolution of projected intersection area with recycling

\begin{tabular}{c|cc} 
& $\begin{array}{c}\text { Number of } \\
\text { bonds analysed }\end{array}$ & $\begin{array}{c}\text { Projected bonded } \\
\text { area, } \mu \mathrm{m}^{2}\end{array}$ \\
\hline 0R & 244 & 272536 \\
1R & 188 & 243783 \\
2R & 218 & 268066 \\
3R & 232 & 271767 \\
10R & 261 & 343186
\end{tabular}

With the exception of first recycle, the fibre intersection area increased with recycling. The decrease in first recycle may be linked to the drop in fibre flexibility and loss of fines during handsheet making. 


\section{Lumen collapse of recycled pulp fibres}

Figure 5.8 is a representation of the fibre cross section.

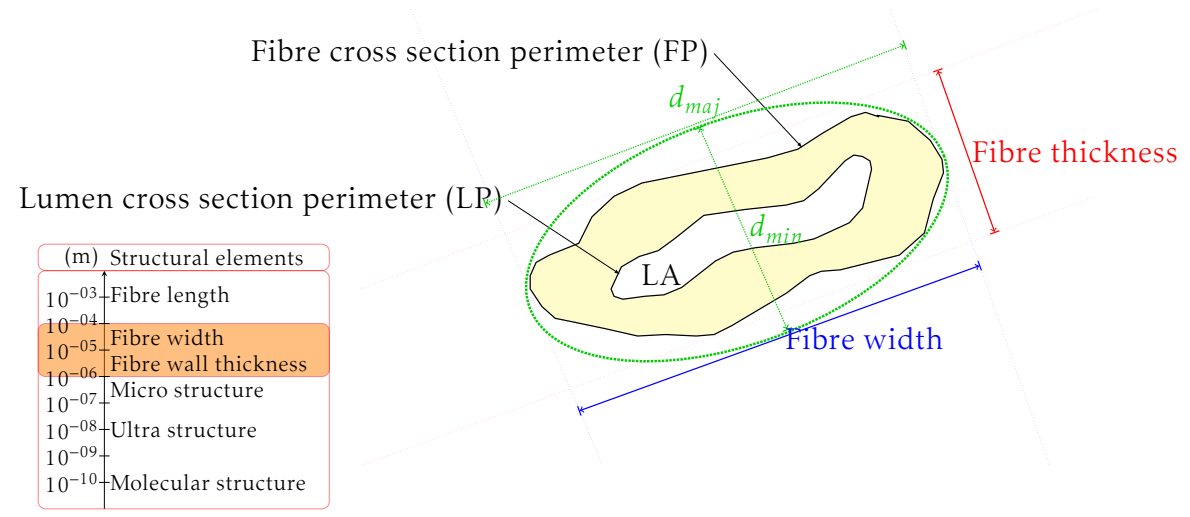

Figure 5.8: Fibre cross section, where LA is the lumen cross section area, $d_{\text {maj }}$ and $d_{\min }$ are the major and minor axis dimensions of best fit ellipse

Fibre lumen collapse can be described by collapse index [116].

$$
\text { Collapse index }=1-\frac{\mathrm{LA}}{\mathrm{LA}_{0}}
$$

where $\mathrm{LA}_{0}$ is the uncollapsed (intact) lumen cross section area having the same lumen perimeter (LP). Uncollapsed lumen is considered round. Therefore, the collapse index of unity represents fully collapsed lumen.

Shape of the fibre and lumen can be described by aspect ratio

$$
\text { Aspect ratio }=\frac{d_{m a j}}{d_{m i n}}
$$

Fibre cross sections were analysed from the re-sliced stack (cf. Figure 5.9). Only those fibre and lumen cross sections were taken into account which had axis perpendicular to the slice. Slice range of the fibre segments was noted where there was no vertical and horizontal movement of the fibre. 


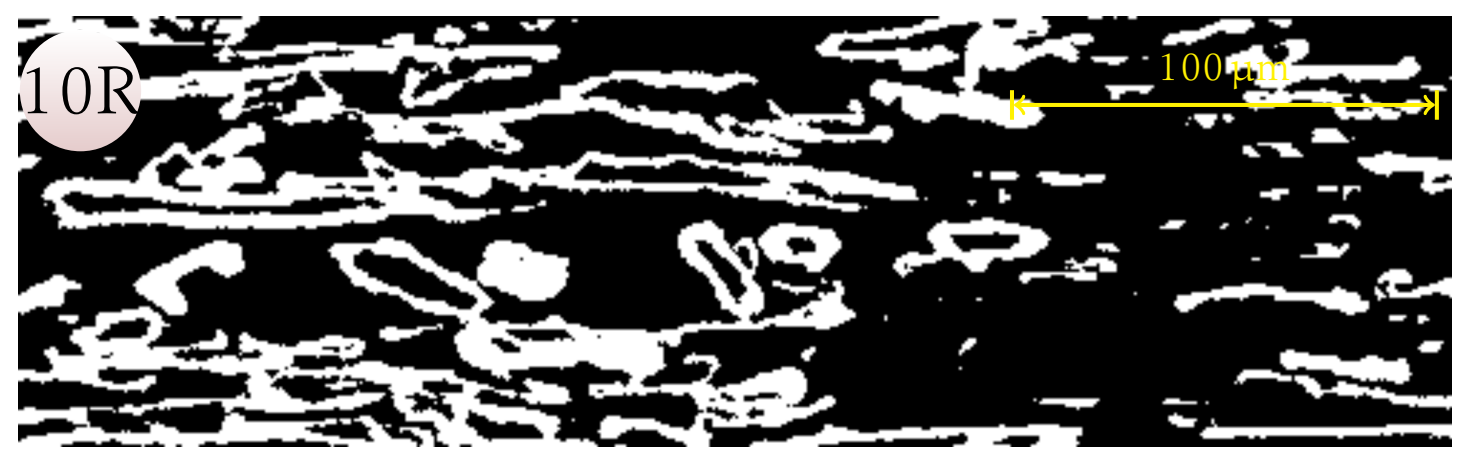

Figure 5.9: Slice of 10RF from re-sliced stack

Lumen collapse index is presented in Table 5.2.

Table 5.2: Lumen collapse index as a function of recycling

\begin{tabular}{c|cccc} 
& $\begin{array}{c}\text { Number of } \\
\text { measurements }\end{array}$ & $\begin{array}{c}\text { Collapse } \\
\text { index }\end{array}$ & $\begin{array}{c}\text { Aspect ratio } \\
\text { of fibres }\end{array}$ & $\begin{array}{c}\text { Aspect ratio } \\
\text { of lumen }\end{array}$ \\
\hline 0R & 110 & 0.47 & 2.46 & 3.89 \\
$10 \mathrm{R}$ & 132 & 0.55 & 2.14 & 4.07
\end{tabular}

Fibre lumen collapsed during recycling as expected. Aspect ratios indicate fibre and lumen width flattening.

\subsubsection{Mechanical properties of handsheets made from recy- cled pulps}

Strength properties of paper depends on fibre strength, bond strength and bonded area. Many researches tried to correlate tensile failure to fibre and bonding properties.

According to Page theory [170]

$$
\frac{1}{\mathrm{~F}}=\frac{9}{8 \mathrm{Z}}+\frac{12 \mathrm{FA} \cdot \rho}{\tau_{b} \cdot \mathrm{FP} \cdot l \cdot \mathrm{RBA}}
$$

where $\mathrm{F}, \mathrm{Z}, \mathrm{FA}, \rho, \tau_{b}, \mathrm{FP}, l$ and RBA are the tensile index, zero-span tensile index, fibre cross section area, fibre density, bond strength per unit area, fibre cross section perimeter, fibre length and relative bond area, respectively. The first term on right hand side of the equation (5.5) accounts for the fibre strength whereas the second term accounts for the contribution of bond strength and 
RBA. The theory however, does not include fibre shape, fibre length distribution, fibre flexibility, conformabililty, anisotropy in formation and the shape of the load-elongation curve (rate of loading).

Besides Page model, shear-lag model [55] is also used to predict and explain tensile failure mechanism. In shear-lag model it is assumed that the fibres carry axial load whereas the fibre-fibre interface transmits shear between the fibres. Based on fibre strength and specific bond strengths, critical length $\left(l_{c}\right)$ is defined.

$$
l_{c}=\frac{\sigma_{f} \cdot d_{f}}{2 \tau_{b}}
$$

where $\sigma_{f}, d_{f}$ and $\tau_{b}$ are the axial stress on fibre, fibre diameter and shear strength of fibre-fibre interface. If critical length is greater than fibre length then the failure is caused by pulling out of fibres otherwise the failure occurs due to fibre breakage.

\section{Zero-span breaking lengths of handsheets made from recycled pulps}

In this test, load is applied across a plane through the thickness of the test specimen [51] held between jaws with a span close to zero. During straining, some fibres reach their ultimate strengths before the others. The test specimen breaks when the applied stress reaches the ultimate stress. The maximum stress reached is called the zero-span tensile strength. Zero-span tensile strength is often used as a relative measure of fibre strength. Theoretically, at true zerospan fibre bonds become insignificant i.e. the forces between the grips of the jaws are transmitted to the fibres. The strength of the network and the strength of the fibres can thus be differentiated. Van der Akker et al. [222] were the first to correlate zero-span breaking strength of the paper with the strength of individual fibres and the number of fibres carrying the load considering all of them aligned in the direction of applied stress. Kinks and curl in fibre affects zero-span tensile strength. Mild refining removes fibre defects and therefore zero-span tensile strength may increase [160].

Zero-span strengths of the test specimens were measured on Pulmac TS-100. Zero-span breaking length $\left(\mathrm{BL}_{0}\right)$ can be represented by equation (5.7)

$$
\mathrm{BL}_{0}=\frac{\mathrm{F}_{b}}{\mathrm{w} \cdot \mathrm{G}}
$$


where $F_{b}, w$ and $G$ are the force at break $\left(\mathrm{kg}_{\mathrm{f}}\right)$, width of the test strip under the grip of the jaws $(\mathrm{m})$ and grammage $\left(\mathrm{g} \cdot \mathrm{m}^{-2}\right)$, respectively. The width of the test strip under the grip of the jaws was $15 \mathrm{~mm}$.

Figure 5.10 shows the effect of recycling on the zero-span breaking length. Mean values are based on twelve measurement.

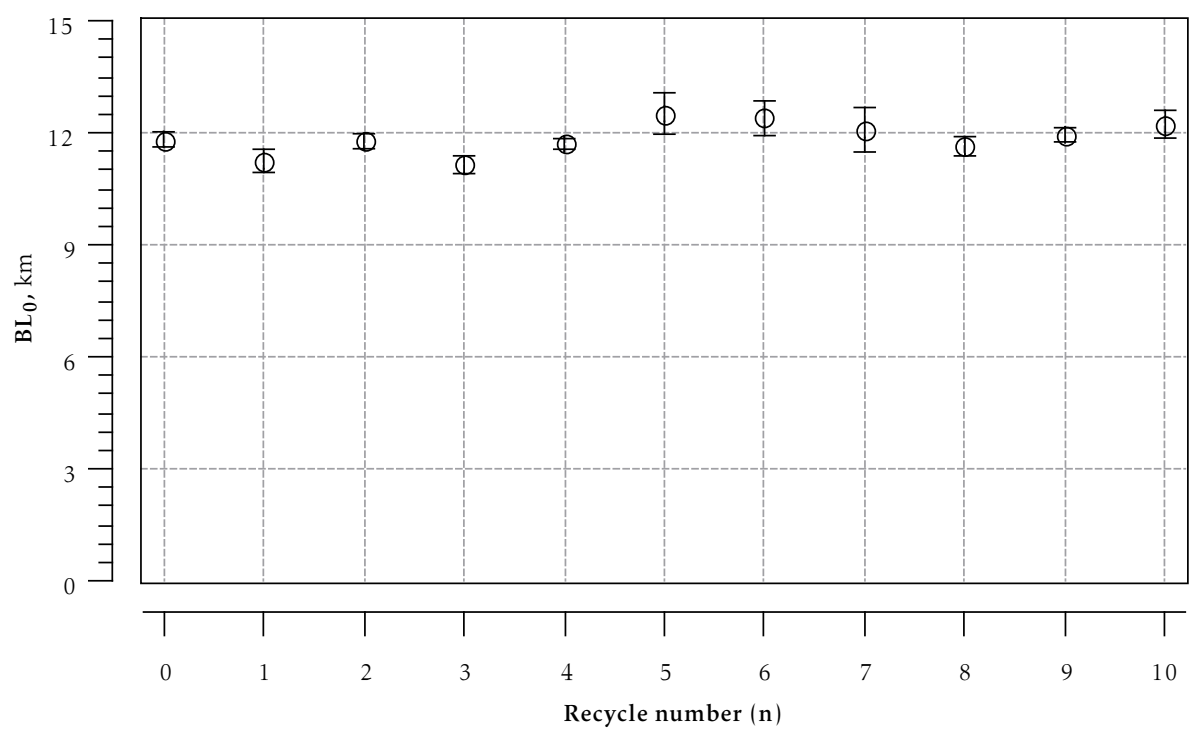

Figure 5.10: Zero-span breaking length of handsheets made from recycled pulps

Zero-span breaking length did not change with recycling. It means that during recycling the strength of fibres and bonds in dry condition remained constant during recycling. In order to eliminate the bonding effect, Cowan [66] proposed rewetting test specimen before zero-span breaking strength measurement.

Test strips of $25 \mathrm{~mm}$ wide and $10 \mathrm{~cm}$ long were soaked in deionised water for $10 \mathrm{~s}$. Wet strips were placed in between blotters for $5 \mathrm{~s}$ to remove excess water. Wet zero-span strengths of the test specimens were carried out on Pulmac TS100 .

Figure 5.11 shows the effect of recycling on the Wet zero-span breaking length $\left(\mathrm{WBL}_{0}\right)$. Mean values are based on twelve measurements. 


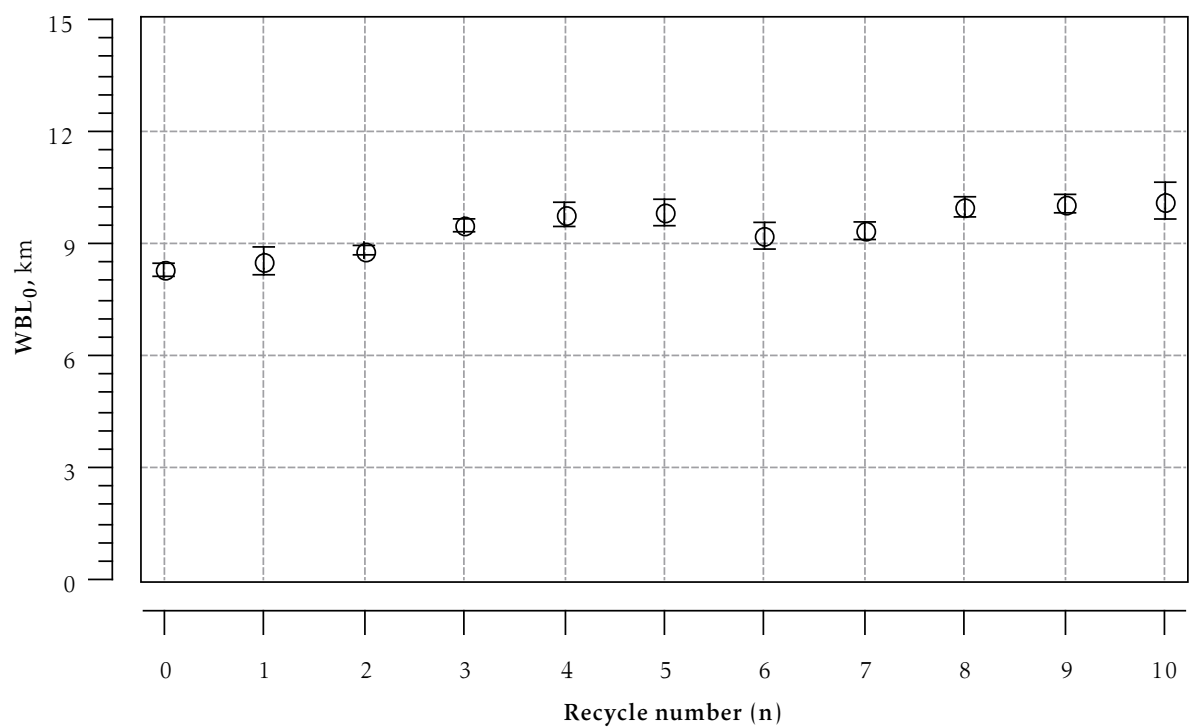

Figure 5.11: Wet zero-span breaking length of handsheets made from recycled pulps

Wet zero-span breaking length increased slightly with recycling. It might be due to the elimination of weak points in the cell wall structure. As a relative measure of fibre strength it hints towards the increase in fibre strength with recycling. Furthermore, Cowan and Cowdrey [67] defined strength components (FSI, FLI and BI ) using dry and wet zero/short-span tensile curves.

An example of dry and wet zero/short-span tensile curve is shown in Figure 5.12 .

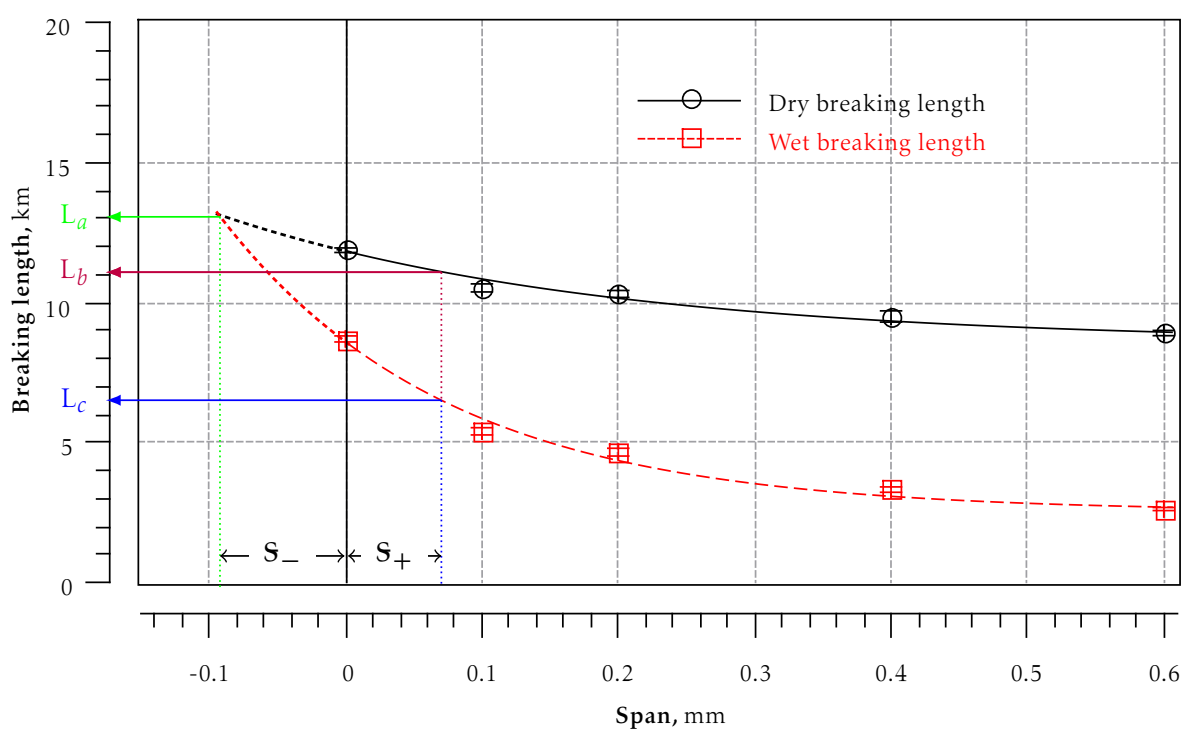

Figure 5.12: Variation of breaking lengths with span separation of paper produced from ten times recycled pulp 
where,

$\mathrm{L}_{a}$ is the intercept of wet and dry breaking length curves at true zero-span. The strength value corresponding to true zero-span is called fibre strength index (FSI, km).

$\mathrm{L}_{c}=\frac{\mathrm{L}_{a}}{2}$

$\mathrm{s}_{+}$is the span separation of $\mathrm{L}_{c}$.

$\mathrm{L}_{b}$ is the dry breaking length corresponding to $\mathrm{s}_{+}$.

$\mathrm{s}_{-}$is the span separation of $\mathrm{L}_{a}$.

Fibre length index (FLI) describes the fraction of fibres carrying load at wet short-span to the fraction of fibres carrying the load at wet zero-span.

$$
\operatorname{FLI}(\mathrm{mm})=\mathrm{s}_{-}+\mathrm{s}_{+}
$$

Effective fibre length index (EFLI) describes the fraction of fibres carrying load at wet short-span of $0.4 \mathrm{~mm}\left(\mathrm{WBL}_{0.4}\right)$ to the fraction of fibres carrying the load at wet zero-span $\left(\mathrm{WBL}_{0}\right)$ [9].

$$
\operatorname{EFLI}(\mathrm{mm})=\frac{\mathrm{WBL}_{0.4}}{\mathrm{WBL}_{0}}
$$

Bonding index $(\mathrm{BI})$ describe the ratio of dry short-span to wet short-span at $\mathrm{s}_{+}$ span separation.

$$
\mathrm{BI}(\%)=\left(\frac{\mathrm{L}_{b}}{\mathrm{~L}_{c}}-1\right) \cdot 100
$$

Results of strength component calculations during recycling are presented in Table 5.3.

Table 5.3: Evolution of indices with recycle number

\begin{tabular}{c|cccccccc} 
Recycle & FSI & $\mathrm{L}_{c}$ & $\mathrm{~L}_{b}$ & $\mathrm{~s}_{-}$ & $\mathrm{s}_{+}$ & $\mathrm{FLI}$ & EFLI & BI \\
number & $\mathrm{km}$ & $\mathrm{km}$ & $\mathrm{km}$ & $\mathrm{mm}$ & $\mathrm{mm}$ & $\mathrm{mm}$ & $\mathrm{mm}$ & $\%$ \\
\hline 0 & 13.16 & 6.58 & 11.14 & 0.09 & 0.07 & 0.16 & 0.38 & 69.32 \\
2 & 13.58 & 6.79 & 11.49 & 0.11 & 0.14 & 0.26 & 0.43 & 69.23 \\
5 & 13.81 & 6.90 & 10.97 & 0.06 & 0.14 & 0.20 & 0.45 & 58.92 \\
10 & 14.09 & 7.04 & 11.29 & 0.07 & 0.10 & 0.17 & 0.48 & 60.30
\end{tabular}

FSI and EFLI increased while BI decreased with recycling. Increase in FSI has 
already been indicated by the increase in wet breaking lengths. The decrease in BI may be caused by the decrease in WRV i.e. drop in fibre flexibility with recycling.

\section{Tensile index of handsheets made from recycled pulps}

Tensile properties of the test specimens were measured using L\&W tensile tester. A strip of the test specimen, clamped between the jaws is subjected to a specified rate of strain on the extensometer. The force per unit width required to break the test specimen is called tensile strength whereas the tensile index represents the tensile strength per unit basis weight of the test specimen.

The width of the strip was $15 \mathrm{~mm}$ and the strain rate was normalised at $10 \mathrm{~mm} \cdot \mathrm{min}^{-1}$ [5]. The effect of recycling on the tensile index is shown in Figure 5.13.

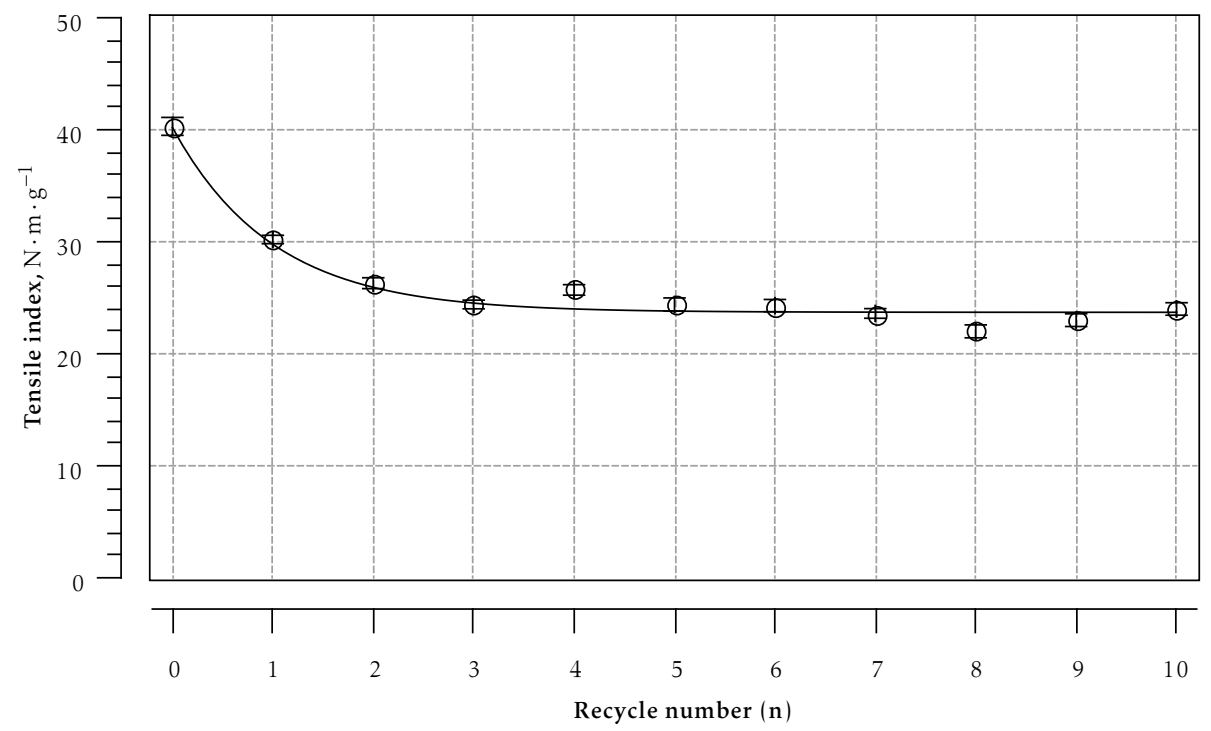

Figure 5.13: Tensile index of handsheets made from recycled pulps

Tensile index of the test specimens decayed exponentially with recycling. Since strength of fibre did not decreased as has been indicated by wet zero-span breaking lengths and dry/wet zero/short-span curves therefore the loss in strength originates from the drop of bond strength. The loss in tensile strength was higher in first few recycles which may be caused by the loss of fines and fibre flexibility. Therefore the number and the quality of the bonds reduced initially. Increase in bonded area and collapse index with recycling (see subsection 5.1.1) do not recover the loss of strength, which means the quality of bonds deteriorated. 


\section{Elongation at break of handsheets made from recycled pulps}

Elongation or stretch at rupture is the maximum amount of deformation which paper undergoes under tensile stress. It is reported as the percentage change in length at the time of rupture.

The effect of recycling on the elongation is shown in Figure 5.14.

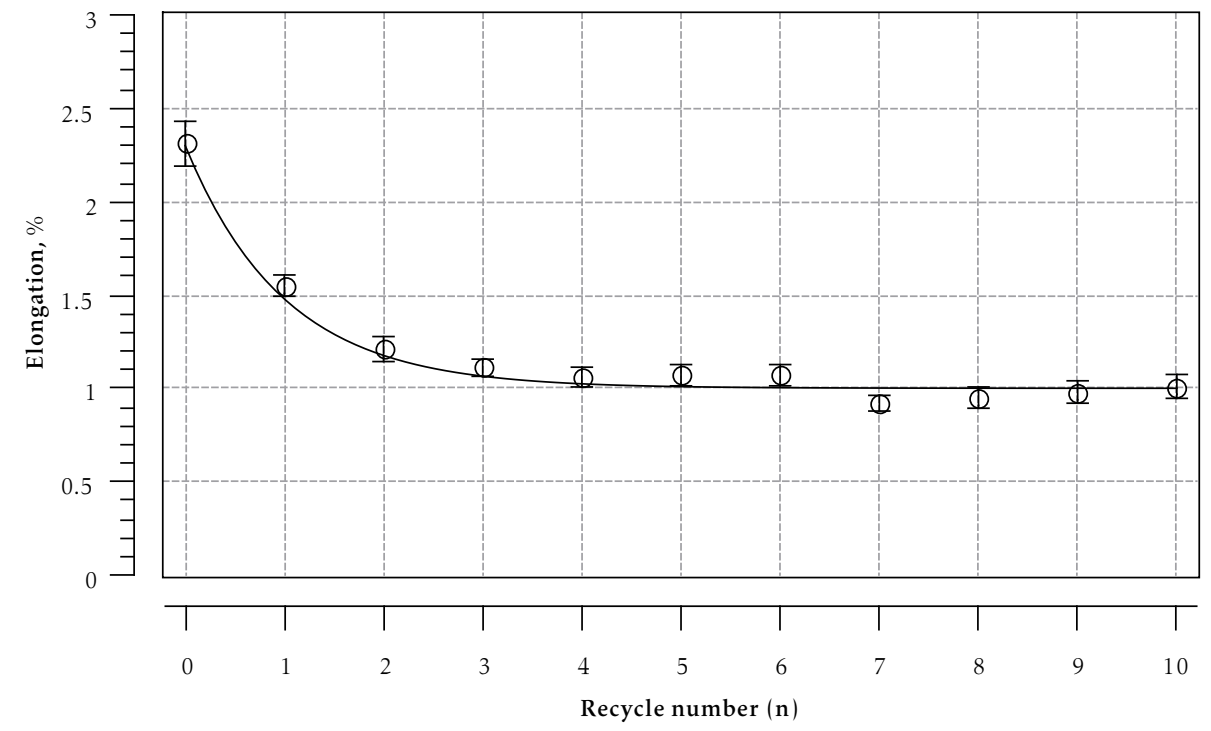

Figure 5.14: Elongation of handsheets made from recycled pulps

Elongation of the test specimens decayed exponentially with recycling. The decrease was higher in first few recycles which can be attributed to the loss of fibre flexibility and network activation. The reduction in elongation indicates the fragility of the network. The stress is transferred to the weak points of the network sooner.

\section{Modulus of elasticity of handsheets made from recycled pulps}

The slope of stress-strain curve within elastic region is called the modulus of elasticity or Young's modulus.

Figure 5.15 shows the effect of recycling on Young's modulus. 


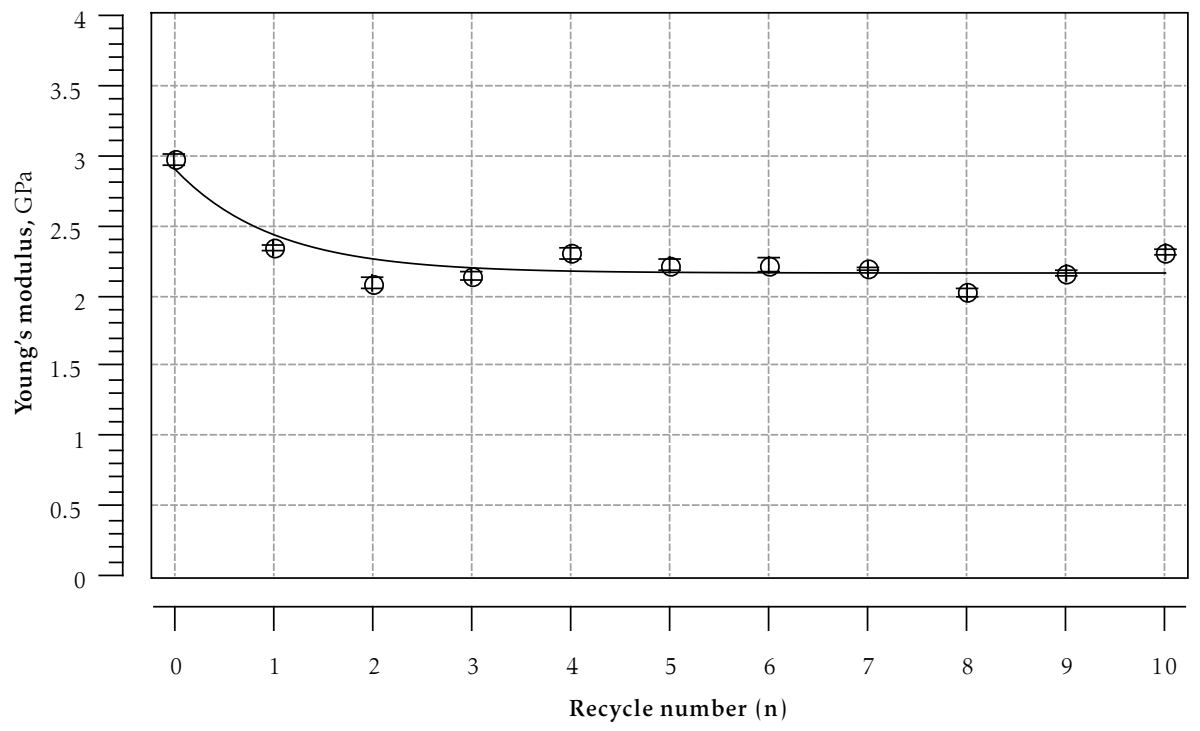

Figure 5.15: Young's modulus of handsheets made from recycled pulps

Young's modulus decayed exponentially with recycling. The decrease in modulus was higher in first few recycles.

\section{Tensile energy absorption of handsheets made from recycled pulps}

Area under the load elongation curve is called Tensile Energy Absorbed (TEA).

Figure 5.16 shows the effect of recycling on TEA.

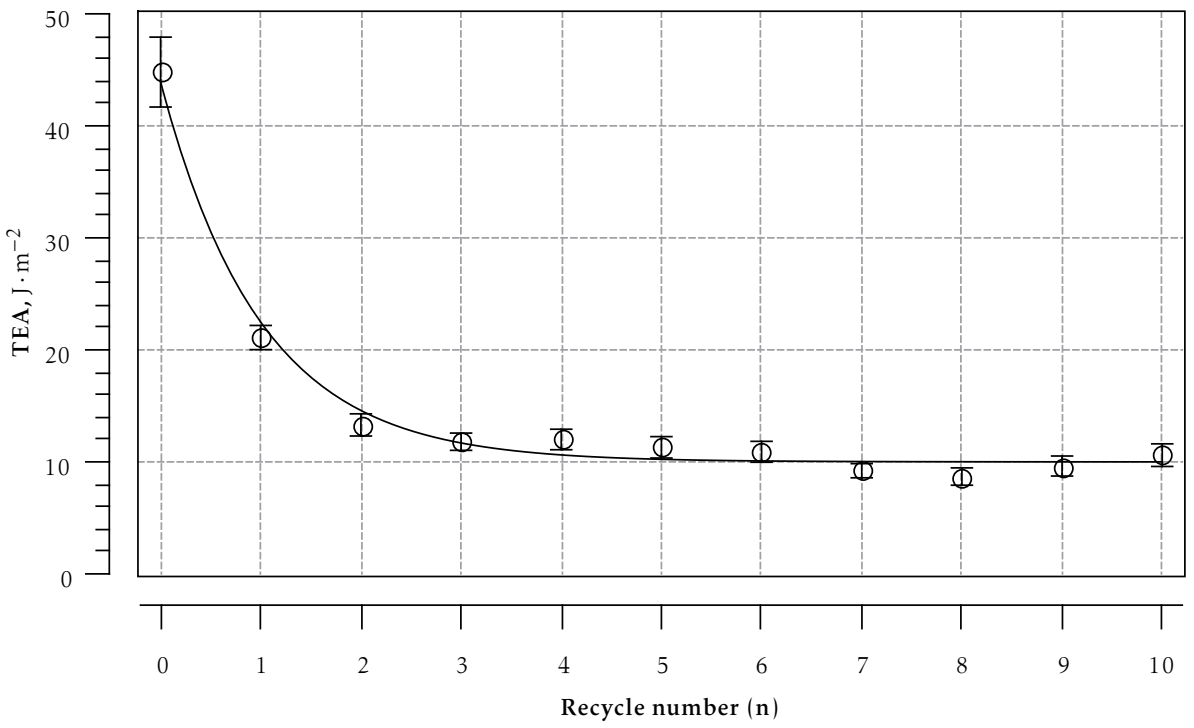

Figure 5.16: Tensile energy absorption of handsheets made from recycled pulps

TEA decayed exponentially with recycling. The capacity of fibre network to 
deform (cf. Figure 5.14) and to resist (cf. Figure 5.15) the applied stress deteriorated with recycling. The decrease in TEA was higher in first few recycles, after third recycle the variations in TEA became insignificant (cf. Figure 5.16).

\section{Fibre network activation while restrained drying}

Activation coefficient $\left\langle\mathrm{A}_{3}\right\rangle$ while restrained drying has previously been used to correlate technical properties of paper produced from pulp refined at different levels [182]. It describes the lack of fibre network activation and can be defined empirically by the following expression

$$
\left\langle\mathrm{As}_{s}\right\rangle=\left(\frac{\langle\mathrm{CWT}\rangle \cdot\langle\mathrm{CI}\rangle}{\left\langle l_{l}\right\rangle}\right)^{\left(\frac{1}{\langle\mathrm{WRV}\rangle}\right)}
$$

where $\langle\mathrm{CWT}\rangle,\langle\mathrm{CI}\rangle,\left\langle l_{l}\right\rangle$ and $\langle\mathrm{WRV}\rangle$ are the average cell wall thickness $(\mu \mathrm{m})$, average curl index $(\%)$, the length-weighted length $(\mathrm{mm})$ and the average water retention value $\left(\mathrm{mL} \cdot \mathrm{g}^{-1}\right)$, respectively.

Effect of recycling on network activation is represented in Figure 5.17.

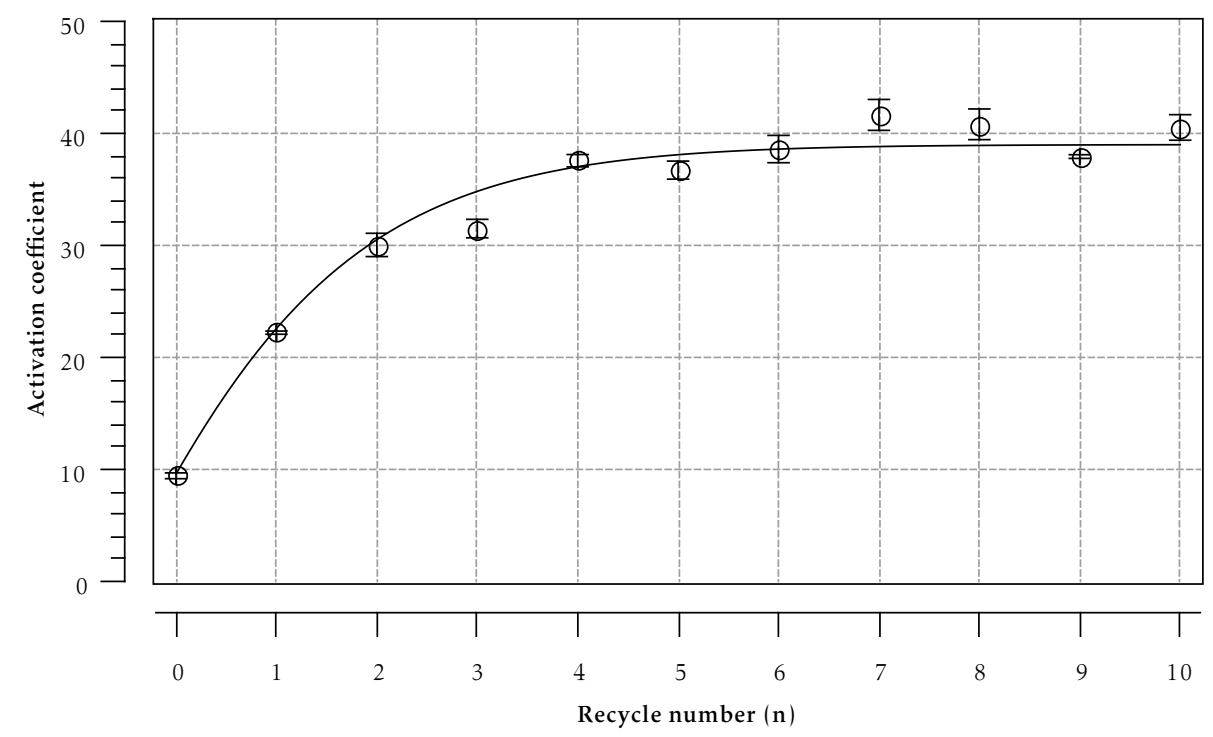

Figure 5.17: Fibre network activation of recycled pulps

Activation coefficient increased with recycling. The increase in activation coefficient during first few recycles was significant, reaching the asymptotic value of 40 after fourth recycle (cf. Figure 5.17). Since fibre length did not change with recycling, therefore from the definition of fibre network activation coeffi- 
cent the decrease in fibre network activation potential is caused by the decrease in curl index and water retention value.

A negative correlation between tensile index and activation coefficient exists (cf. Figure 5.18). Fibres became flat, straight and hornified during recycling. Their activation potential decreased with recycling. Fibre network activation model can be used to differentiate recycled pulps during first few recycles. Perhaps the asymptotic network activation value of 40 indicates the maximum loss of fibre network activation for the conditions of recyclings used for this study.

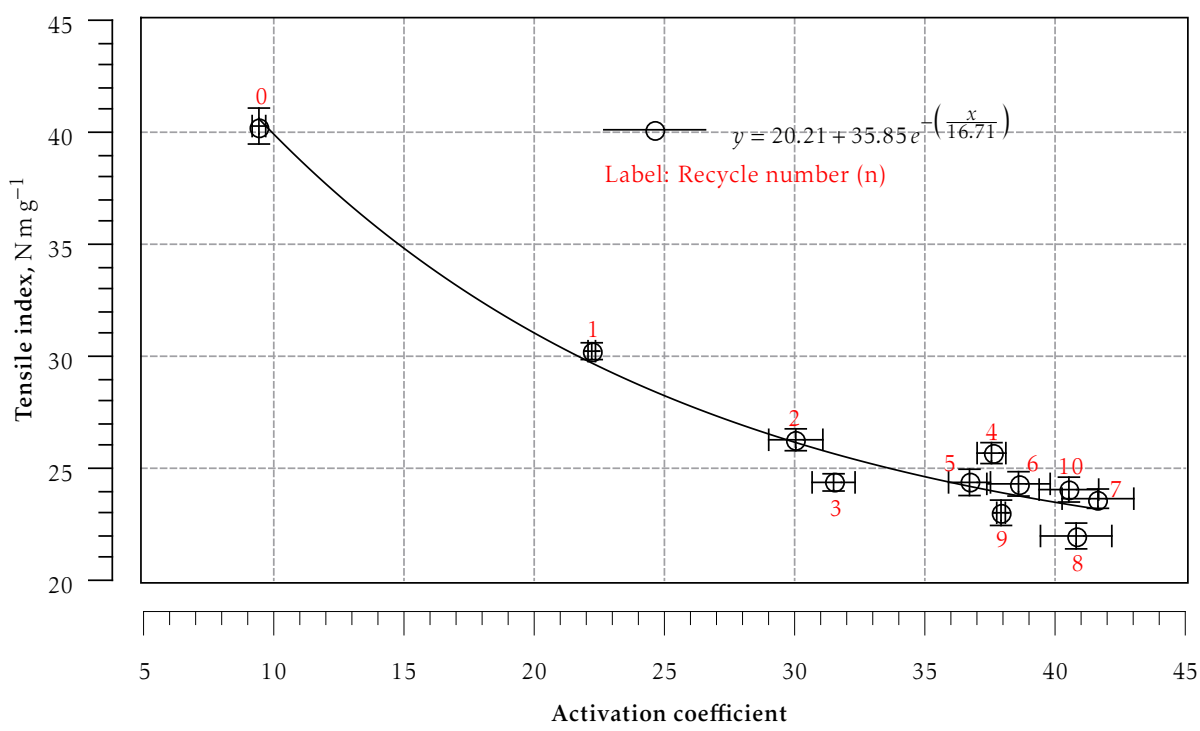

Figure 5.18: Tensile index as a function of activation coefficient

\section{Burst index of handsheets made from recycled pulps}

Burst strength is the pressure required to rupture the free area of the test specimen. The pressure is applied through a circular diaphragm of $7.298 \mathrm{~cm}^{2}$ area made of rubber. Burst strength of the test specimens was measured on MTS Admal Lhormargy EC.05 [10].Burst index is the ratio of burst strength to unit basis weight.

Burst index decayed exponentially with recycling (cf. Figure 5.19). As burst index depends on breaking length and elongation, the significant decrease in burst index initially is linked to the significant drop in breaking length and elongation during first recycle. 


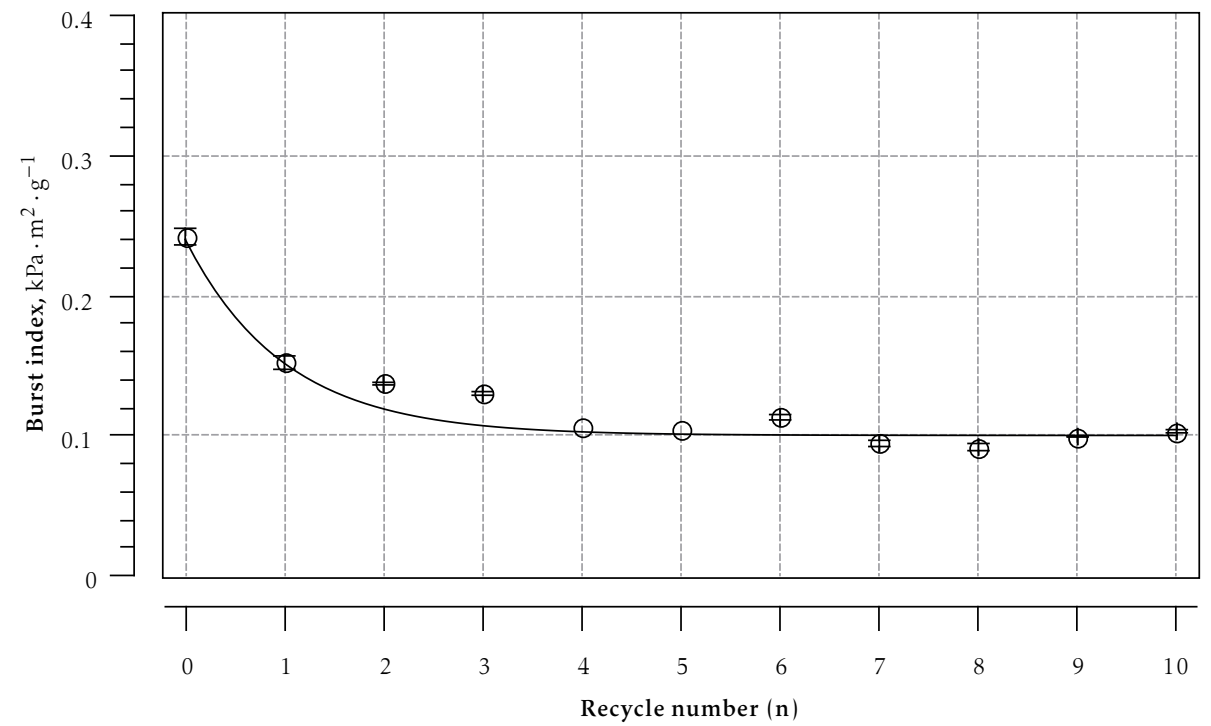

Figure 5.19: Burst index of handsheets made from recycled pulps

\section{Tear index of handsheets made from recycled pulps}

Tear strength is the amount of energy required to propagate an initial fracture to a specified distance. Tear index represents the tear strength per unit basis weight of the test specimen. It includes both, the amount of energy required to rupture the fibres along the tear line and also the amount of energy required to pull the fibres crossing the tear line. The tearing strength of a paper depends on fibre length, fibre strength, fibre stiffness, number of bonds, quality of bonds and the orientation of fibres.

Tear strength of the test specimens was measured on Adamel Lhormargy ED20. The test was carried out on a test piece of $65 \times 50 \mathrm{~mm}^{2}$ dimension [4].

Tear index decayed exponential during recycling (cf. Figure 5.20). Tear index is related to the density, the fibre length and the fibre strength. Fibre length and strength did not decrease with recycling therefore the observed significant drop in tearing strength in first recycle may be linked to the decrease in sheet density. This tendency is inversely related to that of refining in the initial zone where tear energy increase with the increase in sheet density [223]. 


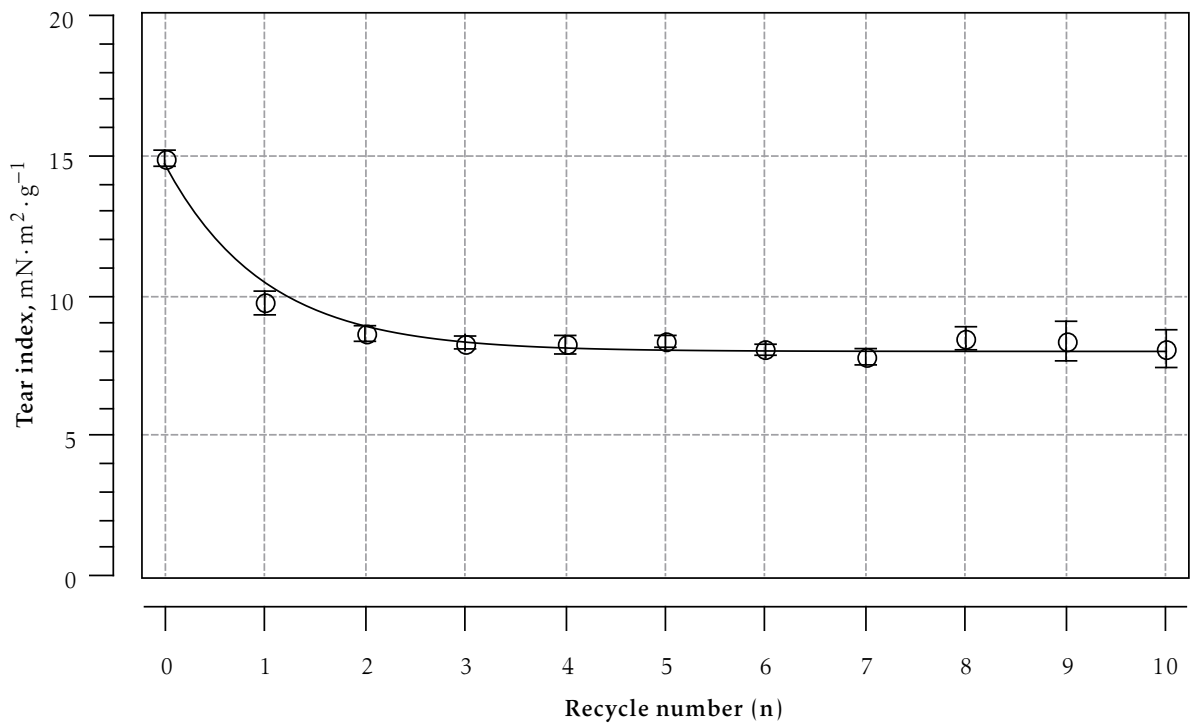

Figure 5.20: Tear index of handsheets made from recycled pulps

\section{Comparison of mechanical properties}

Mechanical properties except zero-span breaking lengths decayed exponentially with recycling. A first order exponential decay model was used to describe the evolution of mechanical properties with recycling. The value of decay constant was unity (cf. equation (5.12)).

$$
y_{n}=b+a \cdot e^{(-n)}
$$

where $y_{n}, b$ and $a$ are the strength property of $n$ times recycled pulp, y-offset value and amplitude, respectively.

Effect of recycling on mechanical properties is summarised in Table 5.4. 
Table 5.4: Mechanical properties of handsheets made from recycled pulps

\begin{tabular}{l|cc|c|cc|cc} 
& \multirow{2}{*}{$a$} & $b$ & $\mathrm{R}^{2}$ & \multicolumn{2}{|c|}{ Calculated } & \multicolumn{2}{c}{ Experimental } \\
\cline { 5 - 8 } Mechanical property & $a$ & $y_{0}$ & $y_{10}$ & $y_{0}$ & $y_{10}$ \\
\hline Tensile index, $\mathrm{N} \cdot \mathrm{m} \cdot \mathrm{g}^{-1}$ & 16.5 & 23.7 & 0.972 & 40.2 & 23.7 & 40.3 & 24.0 \\
Elongation, \% & 1.30 & 1.00 & 0.983 & 2.30 & 1.00 & 2.31 & 1.01 \\
Young's modulus, GPa & 0.75 & 2.16 & 0.825 & 2.91 & 2.16 & 2.97 & 2.31 \\
TEA, J $\cdot \mathrm{m}^{-2}$ & 33.9 & 10.0 & 0.990 & 43.9 & 10.0 & 44.8 & 10.6 \\
Burst index, $\mathrm{kPa} \cdot \mathrm{m}^{2} \cdot \mathrm{g}^{-1}$ & 0.14 & 0.10 & 0.940 & 0.20 & 0.10 & 0.20 & 0.10 \\
Tear index, $\mathrm{mN} \cdot \mathrm{m}^{2} \cdot \mathrm{g}^{-1}$ & 6.70 & 8.00 & 0.971 & 14.7 & 8.00 & 14.9 & 8.10
\end{tabular}

where $y_{0}, y_{10}$ and $\mathrm{R}^{2}$ are the strength of paper produced from never recycled pulp, strength of paper produced from ten times recycled pulp and the coefficient of determination, respectively. The value of determination coefficient of the measured strength properties is fairly high which indicates the goodness of the data fit.

The evolution of mechanical properties is of particular interest for paper makers. The percentage change in mechanical properties calculated according to equation (5.13) is shown in Figure 5.21.

$$
\text { Percentage change }=\frac{y_{n}-y_{0}}{y_{0}} \cdot 100
$$

The effect of recycling on the percentage change in mechanical properties is shown in Figure 


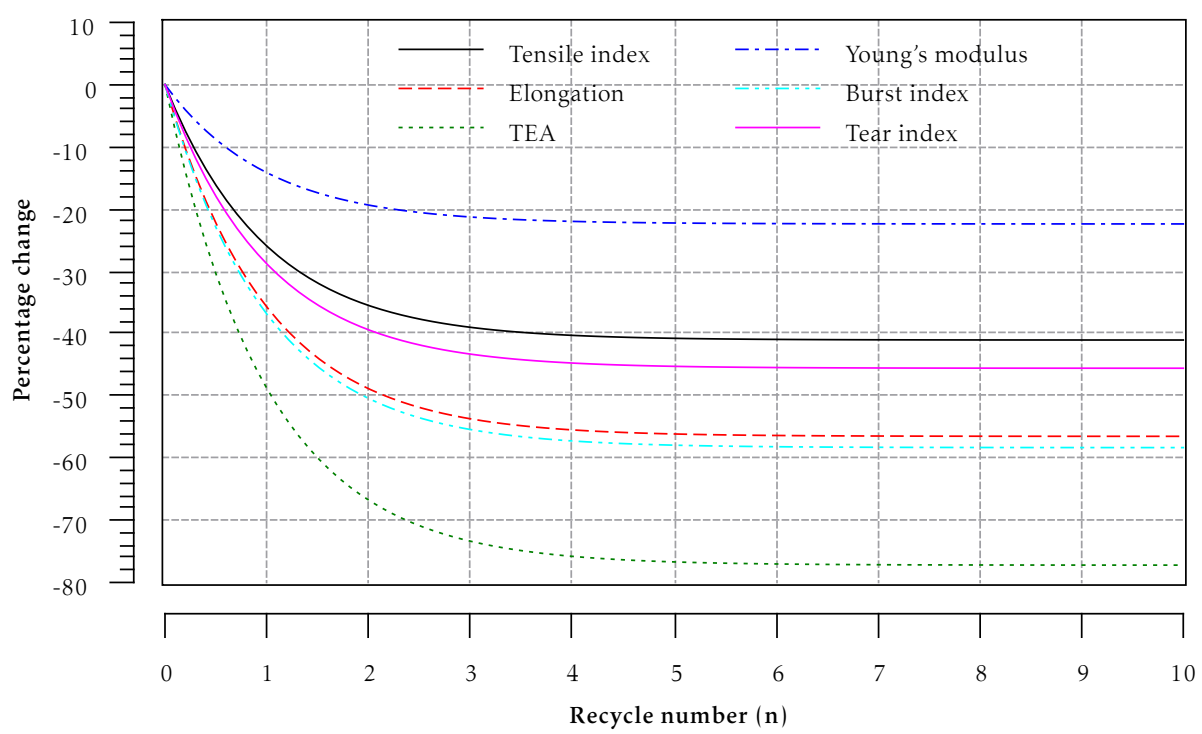

Figure 5.21: Mechanical properties of handsheets made from recycled pulps

After ten recycles, percentage change in tensile index, elongation, Young's modulus, TEA, burst index and tear index were $40 \%, 56 \%, 22 \%, 76 \%, 50 \%$ and $46 \%$, respectively.

\subsubsection{Optical properties of handsheets made from recycled pulps}

Optical properties like brightness and opacity were measured on spectrophotometer Colour Touch DATA COLOR from Technidyne Corporation [18].

\section{Brightness of handsheets made from recycled pulps}

ISO brightness is the intrinsic diffuse reflectance of blue light ( $457 \mathrm{~nm})$. The measurement is normally carried out on an opaque test specimen (several layers of folded sheets) [19].

Brightness slightly increased initially and then decreased with recycling (cf. Figure 5.22). The initial increase in light reflectance may be attributed to the loss of fines changing the network structure of paper. 


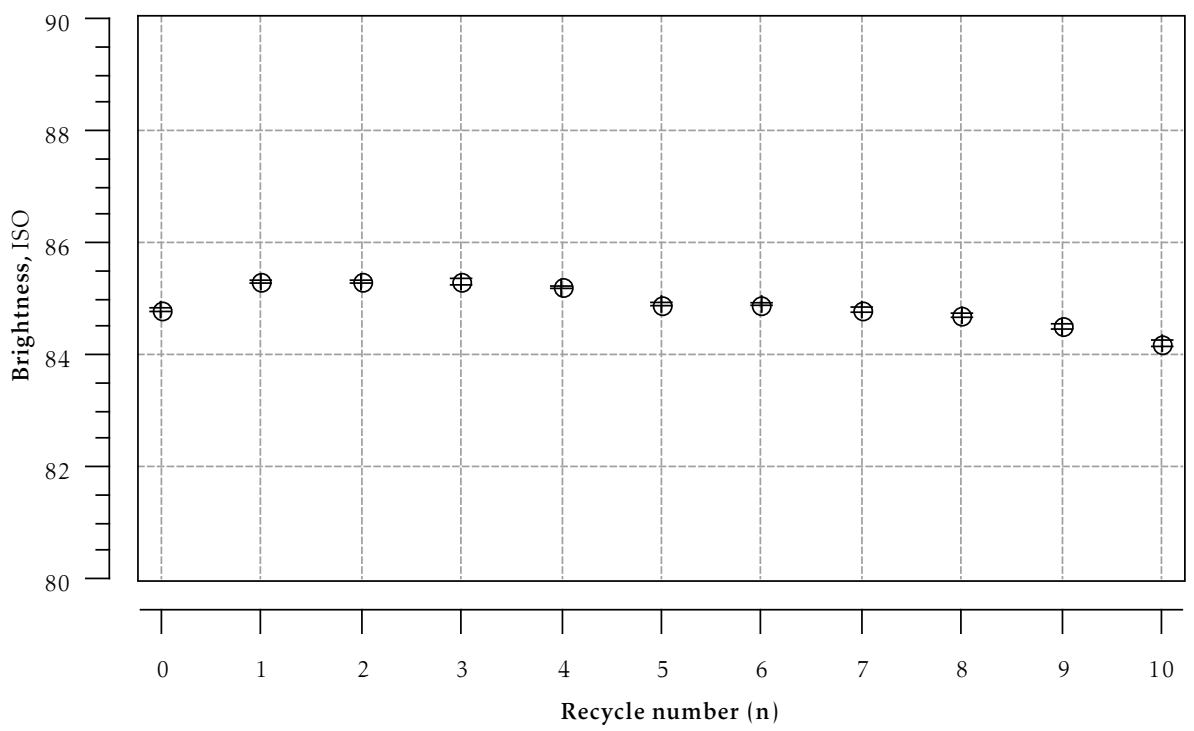

Figure 5.22: Brightness of handsheets made from recycled pulps

\section{Opacity of handsheets made from recycled pulps}

Opacity is the ratio of diffused reflectance of single sheet backed by a black body to the diffused reflectance of thick stack of the same sheets.

Opacity increased in first recycle and then remained constant during further recycling (cf. Figure 5.23). Removal of fines modified paper structure by increasing the reflectance therefore opacity increased initially.

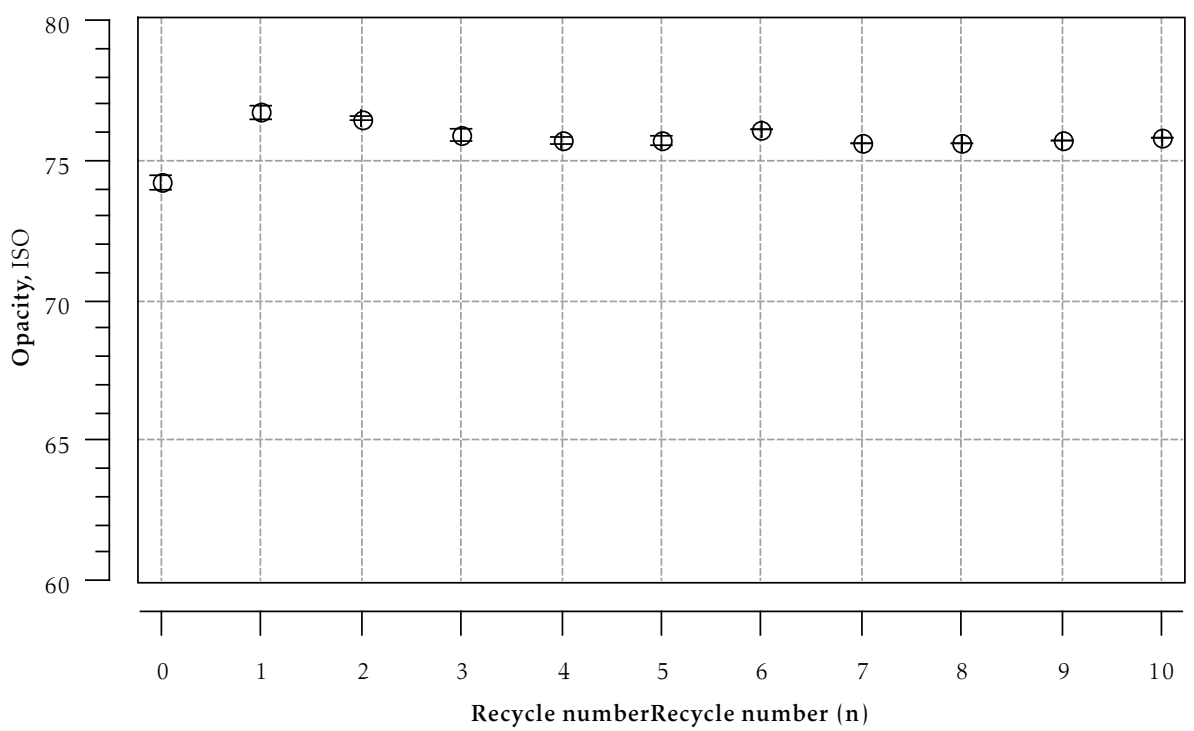

Figure 5.23: Opacity of handsheets made from recycled pulps 


\section{Scattering coefficient of handsheets made from recycled pulps}

Figure 5.24 displays the effect of recycling on the scattering coefficient.

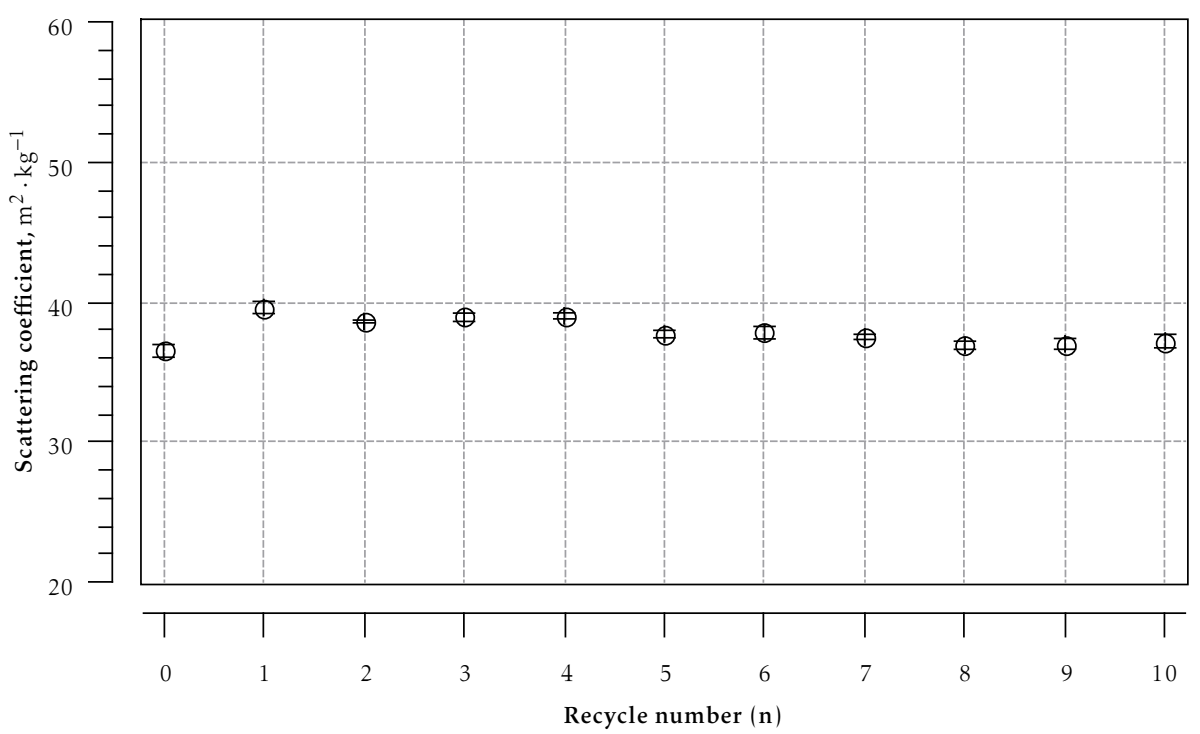

Figure 5.24: Scattering coefficient of handsheets made from recycled pulps

Scattering coefficient slightly increased after first recycle and then decreased with recycling. The initial increase in brightness and opacity might have been resulted from the aggregation of cellulose microfibrils. 


\subsection{Properties of handsheets made from recycled pulp blends}

Recycled pulp is rarely incorporated in high grade papers and its use is one way of cost reduction. Repeatedly recycled pulps were blended with never dried pulp to see the evolution of physical properties. Handsheets were made using $25 \%, 50 \%$ and $75 \%$ recycled pulps. Handsheets made from recycled pulp blends were conditioned at $23 \pm 1{ }^{\circ} \mathrm{C}$ and $50 \pm 2 \% \mathrm{RH}$ for a period of at least $24 \mathrm{~h}$ before testing [3].

\subsubsection{Structural properties of handsheets made from recycled pulp blends}

Bulk of handsheets made from recycled pulp blends

The effect of quantity and the number of recycled pulp used on bulk is presented in Figure 5.25.
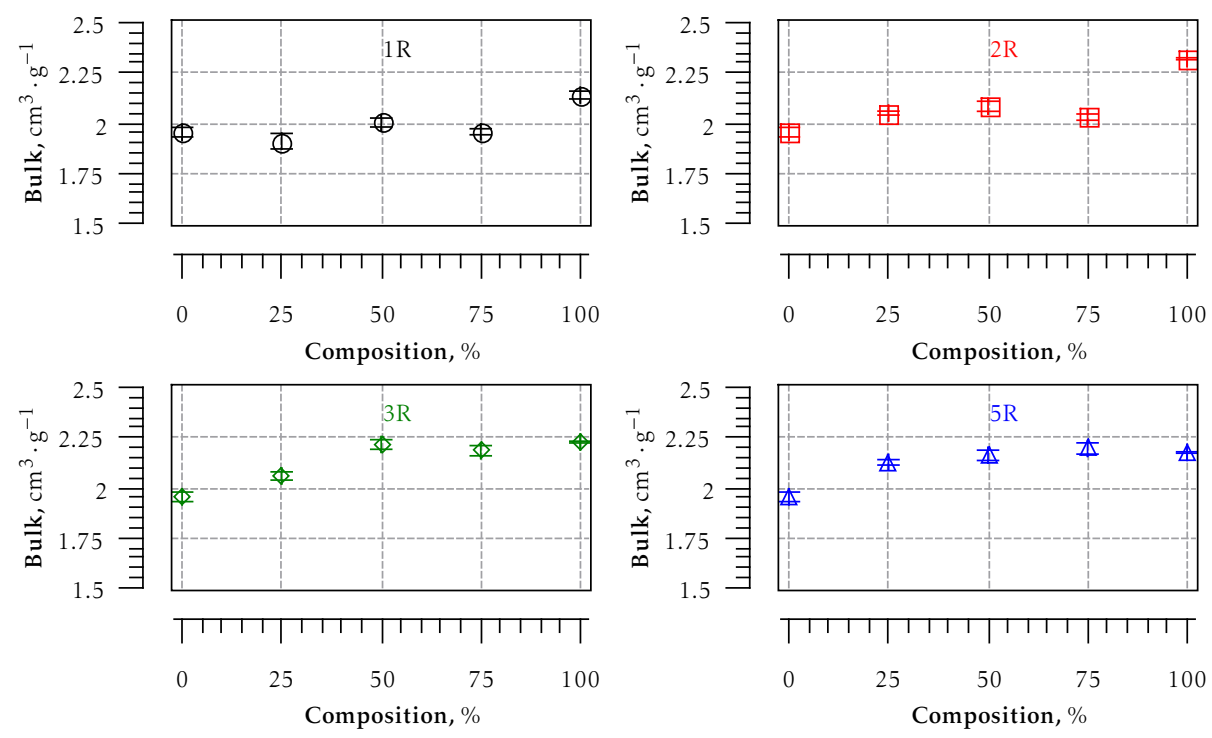

Figure 5.25: Bulk of of handsheets made from recycled pulp blends

Bulk of handsheets increased slightly with the increase of the quantity of recycled pulp used. 


\subsubsection{Mechanical properties of handsheets made from recy- cled pulp blends}

Zero-span breaking lengths of handsheets made from recycled pulp blends

The effect of quantity and the number of recycled pulp used on zero-span breaking length is presented in Figure 5.26.
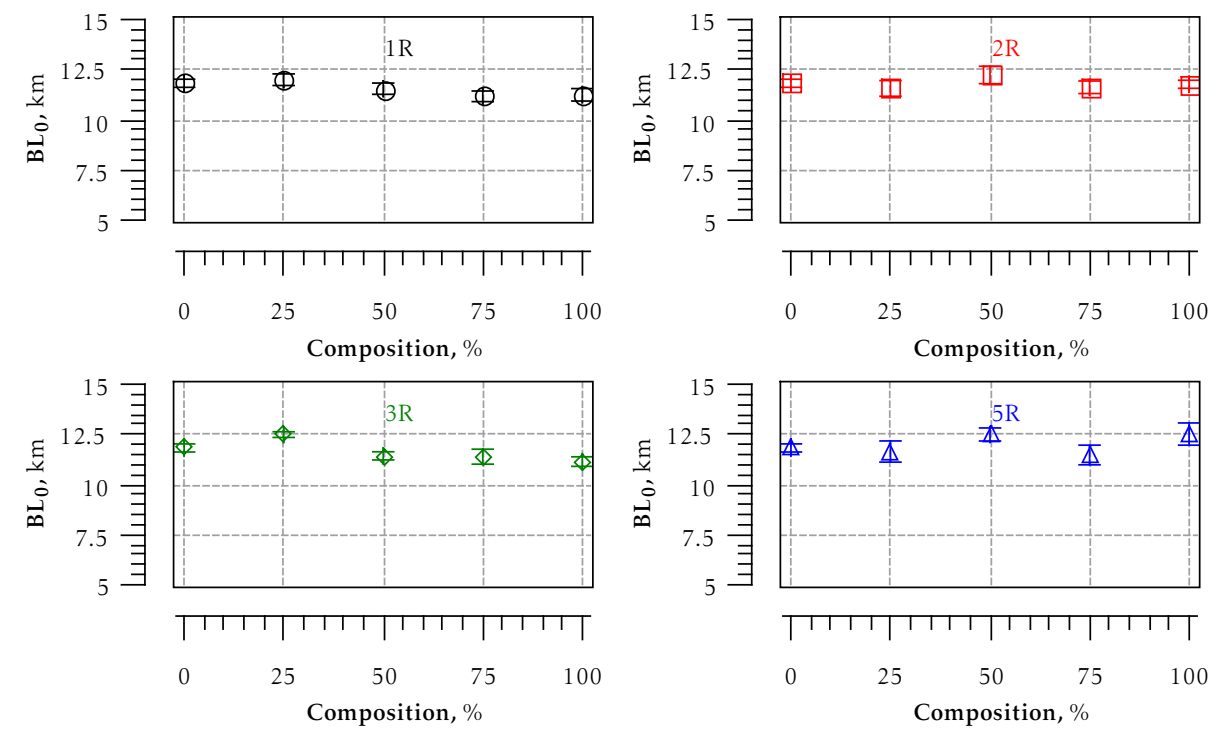

Figure 5.26: Zero-span breaking length of handsheets made from recycled pulp blends

Zero-span breaking length did not change with the amount of recycled pulp used. In order to eliminate the associated effect of bonding in dry zero-span breaking length measurement, the sheets were rewetted [66] according to the procedure described in subsection 5.1.2.

The effect of quantity and the number of recycled pulp used on wet zero-span breaking length is presented in Figure 5.27. 

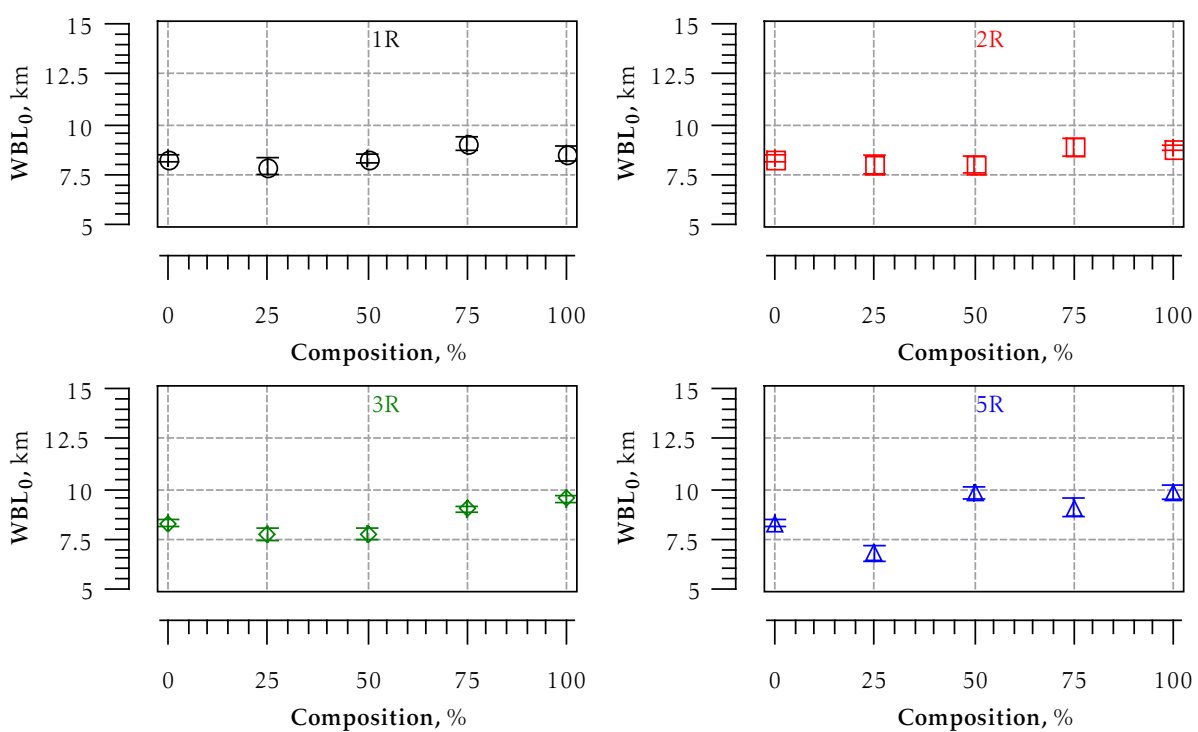

Figure 5.27: Wet zero-span breaking length of handsheets made from recycled pulp blends

Wet zero-span breaking length increased slightly when recycled pulp was used in excess of $50 \%$. Wet zero-span breaking length of recycled fibres was comparatively higher than virgin fibres (see subsection 5.1.2).

\section{Tensile index of handsheets made from recycled pulp blends}

The effect of quantity and the number of recycled pulp used on tensile index is presented in Figure 5.28.
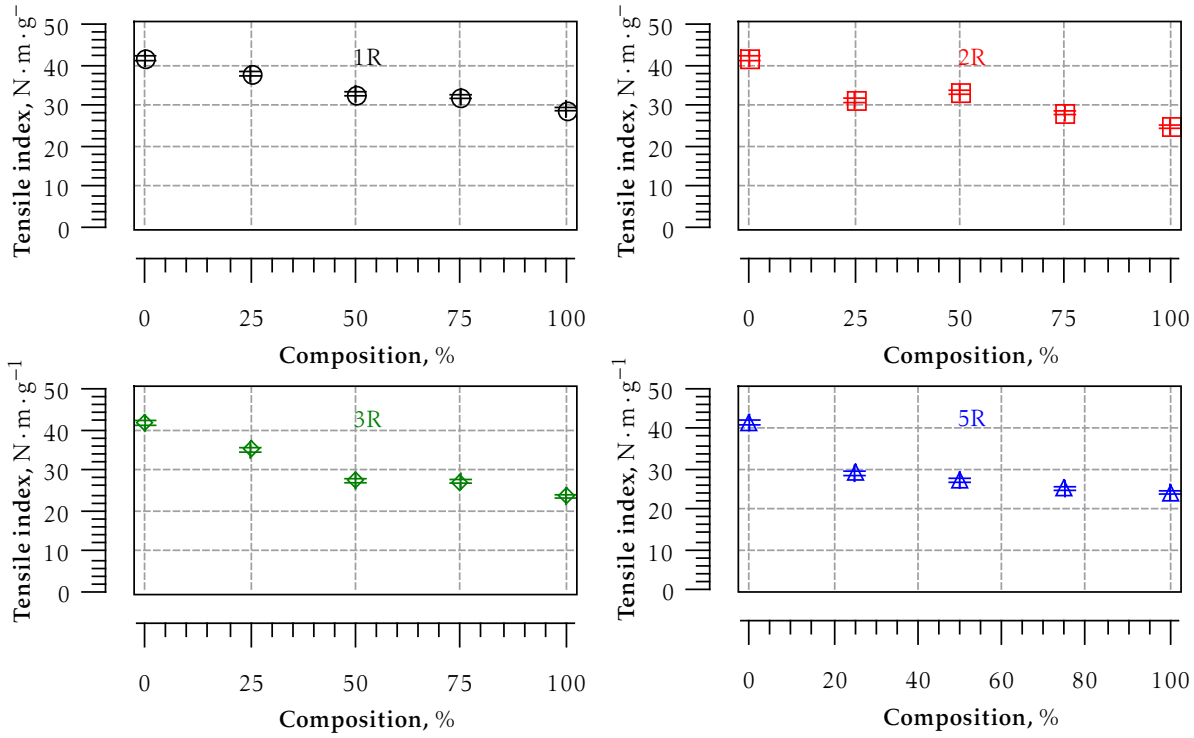

Figure 5.28: Tensile index of handsheets made from recycled pulp blends 
Evolution of tensile index with the quantity of recycled pulp used can be described by a first order exponential decay model.

General form of the first order exponential decay equation is

$$
y_{n}(x)=b+a \cdot e^{-\left(\frac{x}{\tau}\right)}
$$

where $y_{n}(x), b, a, \tau$ are the strength property of $x(\%)$ of $n$ times recycled pulp, $\mathrm{y}$-offset value, amplitude and decay constant, respectively.

The coefficients of equation (5.14) are summarised in Table 5.5.

Table 5.5: Tensile index of handsheets made from recycled pulp blends

\begin{tabular}{c|ccccc} 
Tensile index, & \multicolumn{5}{|c}{$y_{n}(x)=b+a \cdot e^{-\left(\frac{x}{\tau}\right)}$} \\
\cline { 2 - 6 } $\mathrm{N} \cdot \mathrm{m} \cdot \mathrm{g}^{-1}$ & $y_{1}(x)$ & $y_{2}(x)$ & $y_{3}(x)$ & $y_{4}(x)$ & $y_{5}(x)$ \\
\hline$b$ & 29.1 & 24.8 & 23.5 & 24.0 & 24.2 \\
$a$ & 12.5 & 16.9 & 18.1 & 17.6 & 17.4 \\
$\tau$ & 47 & 44 & 41 & 27 & 23 \\
\hline $\mathrm{R}^{2}$ & 0.96 & 0.87 & 0.97 & 0.98 & 0.99
\end{tabular}

where $y_{n}(x)$, and $\mathrm{R}^{2}$ are the tensile index of $x(\%)$ of $n$ times recycled pulp and coefficient of determination, respectively.

Decay constant decreased with the increase of recycle number, indicating that the use of higher recycle number pulp greatly reduces the tensile index which may be linked to the reduced bonding ability of repeatedly recycled pulps. The value of amplitude represents the difference between the tensile strengths of never dried and $n$ times recycled pulps. The increase in the amplitude indicate an increase in the magnitude of the strength loss with recycle number. The percentage change in tensile index calculated from equation (5.13) is shown in Figure 5.29. 


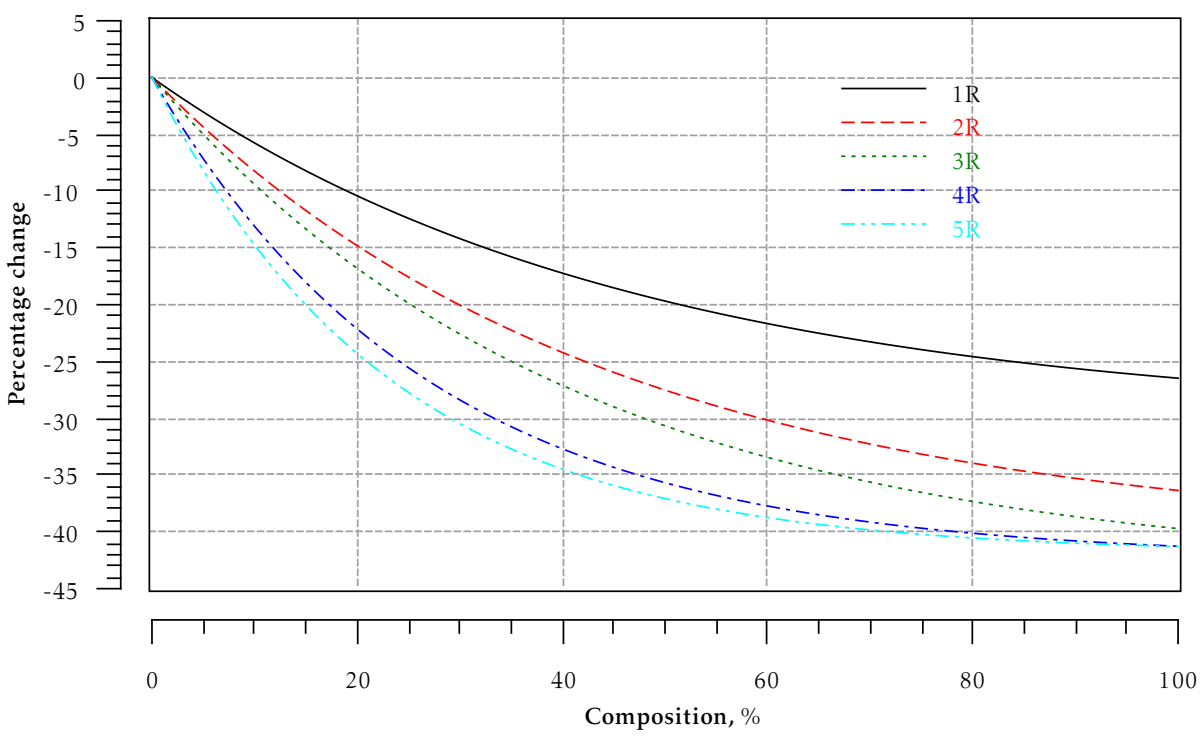

Figure 5.29: Percentage change in tensile index of handsheets made from recycled pulp blends

The loss of tensile strength is more if higher quantity of pulp with higher recycle number is blended.

\section{Elongation at break of handsheets made from recycled pulp blends}

The evolution of elongation followed first order exponential decay model and the coefficients of equation (5.14) are summarised in Table 5.6..
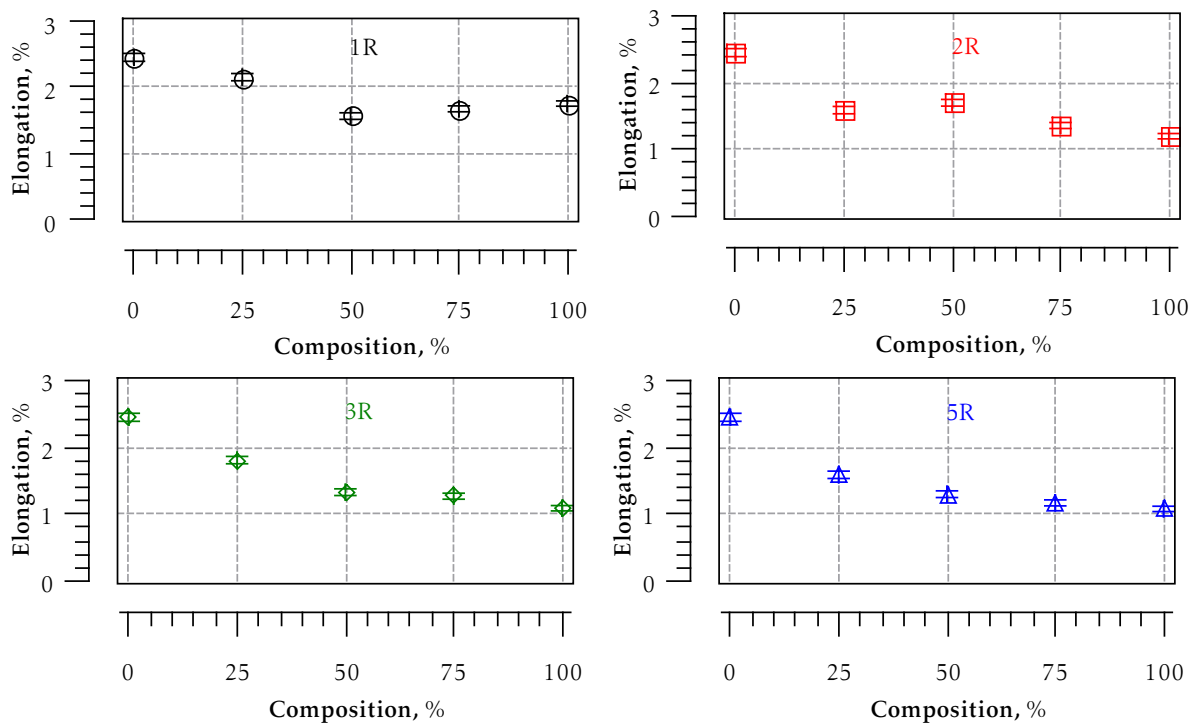

Figure 5.30: Elongation of handsheets made from recycled pulp blends 
Table 5.6: Elongation of handsheets made from recycled pulp blends

\begin{tabular}{c|ccccc} 
Elongation, & \multicolumn{5}{|c}{$y_{n}(x)=b+a \cdot e^{-\left(\frac{x}{\tau}\right)}$} \\
\cline { 2 - 6 }$\%$ & $y_{1}(x)$ & $y_{2}(x)$ & $y_{3}(x)$ & $y_{4}(x)$ & $y_{5}(x)$ \\
\hline$b$ & 1.8 & 1.2 & 1.1 & 1.0 & 1.1 \\
$a$ & 0.7 & 1.3 & 1.4 & 1.5 & 1.4 \\
$\tau$ & 25 & 34 & 34 & 35 & 26 \\
\hline $\mathrm{R}^{2}$ & 0.82 & 0.90 & 0.99 & 0.99 & 0.99
\end{tabular}

The magnitude of drop of elongation increase with recycling. Decay constant was lower when once and five times recycled pulps were used. The percentage change in elongation calculated from equation (5.13) is presented in Figure 5.31 .

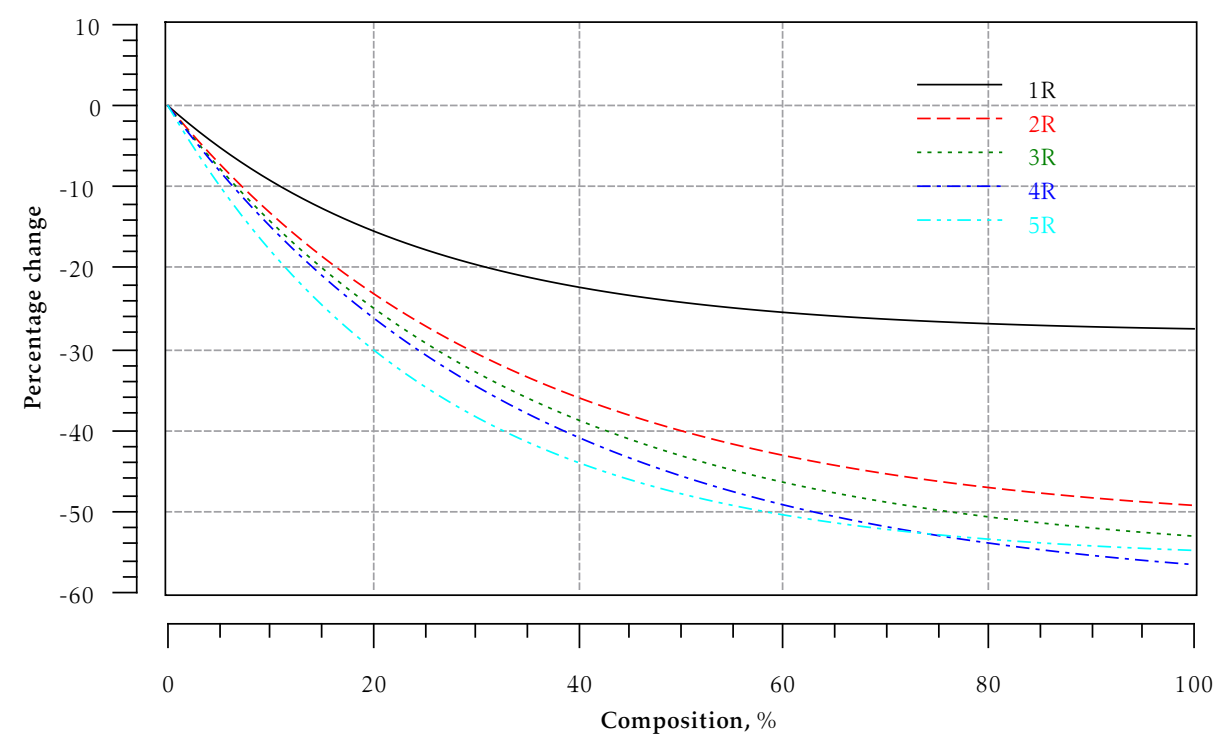

Figure 5.31: Percentage change in elongation of handsheets made from recycled pulp blends

The drop in elongation is more if higher quantity of pulp with higher recycle number is blended.

\section{Young's modulus of handsheets made from recycled pulp blends}

Evolution of Young's modulus with the quantity of recycled pulp followed first order exponential decay model. The coefficients of equation (5.14) are summarized in Table 5.7. 

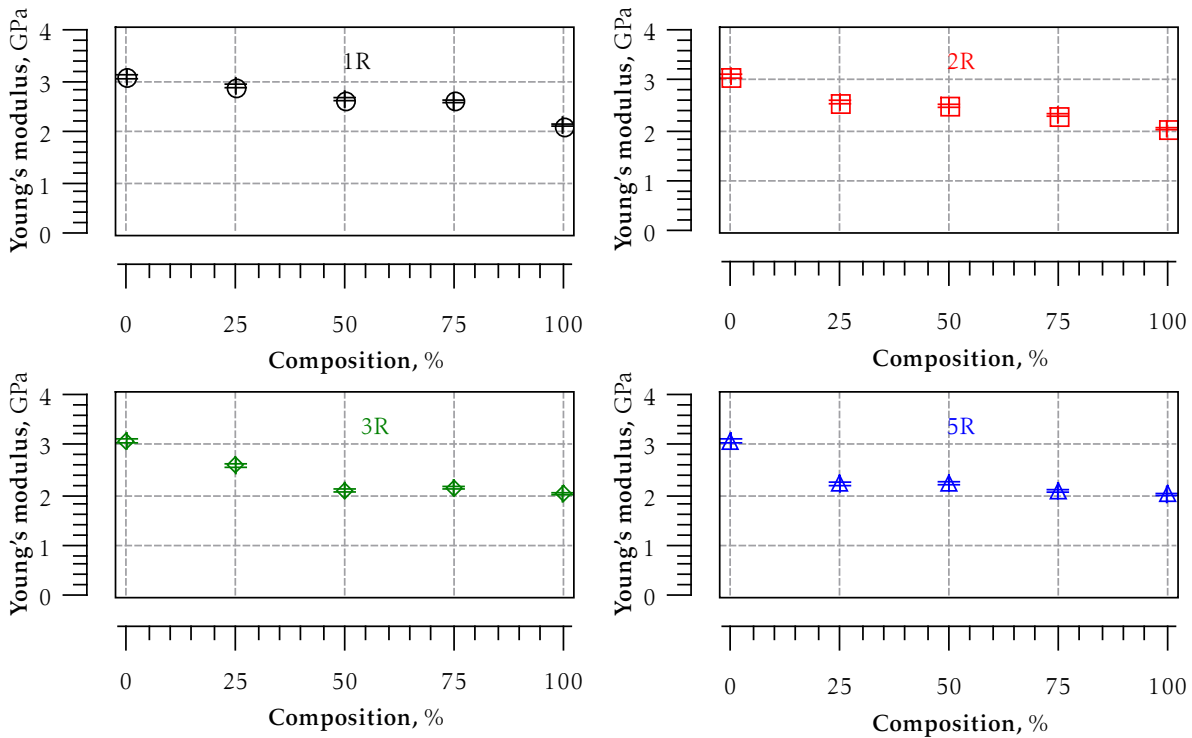

Figure 5.32: Young's modulus of handsheets made from recycled pulp blends

Table 5.7: Young's modulus of handsheets made from recycled pulp blends

\begin{tabular}{c|ccccc} 
Young's modulus, & \multicolumn{5}{|c}{$y_{n}(x)=b+a \cdot e^{-\left(\frac{x}{\tau}\right)}$} \\
\cline { 2 - 6 } GPa & $y_{1}(x)$ & $y_{2}(x)$ & $y_{3}(x)$ & $y_{4}(x)$ & $y_{5}(x)$ \\
\hline$b$ & 2.1 & 2.0 & 2.0 & 2.1 & 2.0 \\
$a$ & 1.0 & 1.0 & 1.1 & 1.0 & 1.1 \\
$\tau$ & 61 & 45 & 29 & 18 & 17 \\
\hline $\mathrm{R}^{2}$ & 0.84 & 0.94 & 0.96 & 0.97 & 0.96
\end{tabular}

Decay constant decreased with the increase of the recycle number, indicating that the use of higher recycle number pulp greatly reduces the Young's modulus. The percentage change in young's modulus calculated from equation (5.13) is presented in Figure 5.33 . 


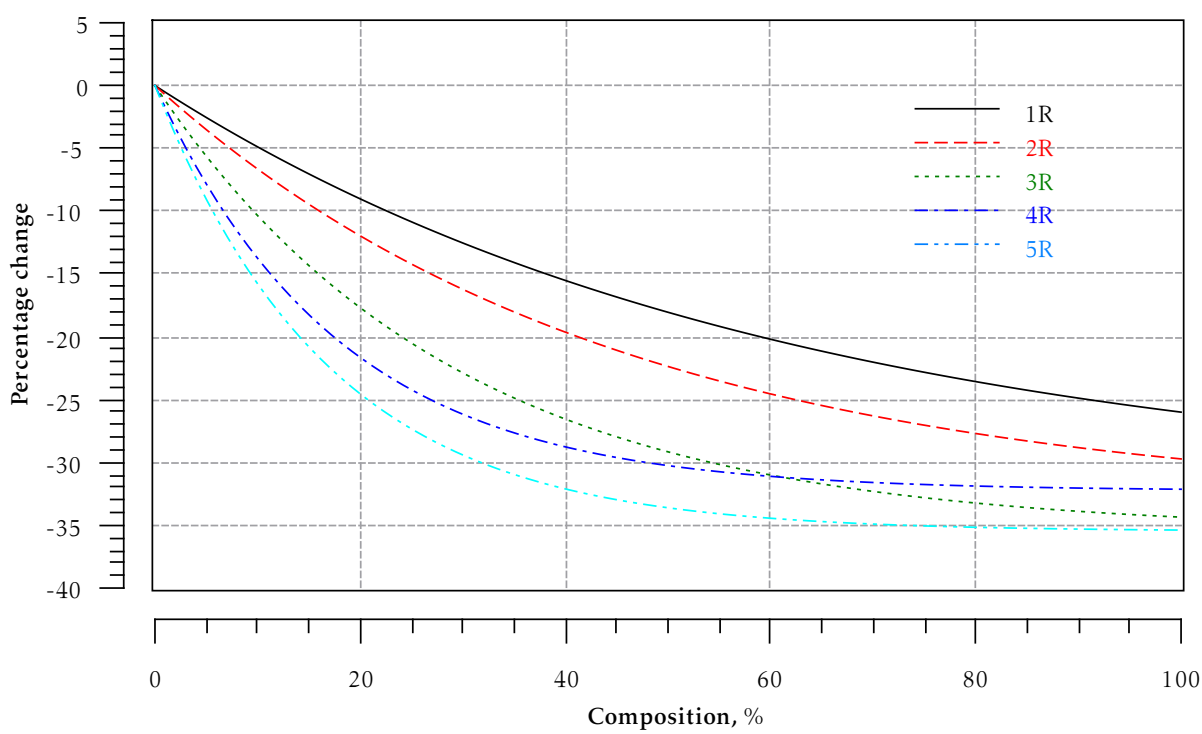

Figure 5.33: Percentage change in young's modulus of handsheets made from recycled pulp blends

The drop in modulus is more pronounced if higher quantity of pulp with higher recycle number is blended.

\section{Tensile energy absorption of handsheets made from recycled pulp blends}

Evolution of TEA with the quantity of recycled pulp followed first order exponential decay model. The coefficients of equation (5.14) are summarized in Table 5.8.
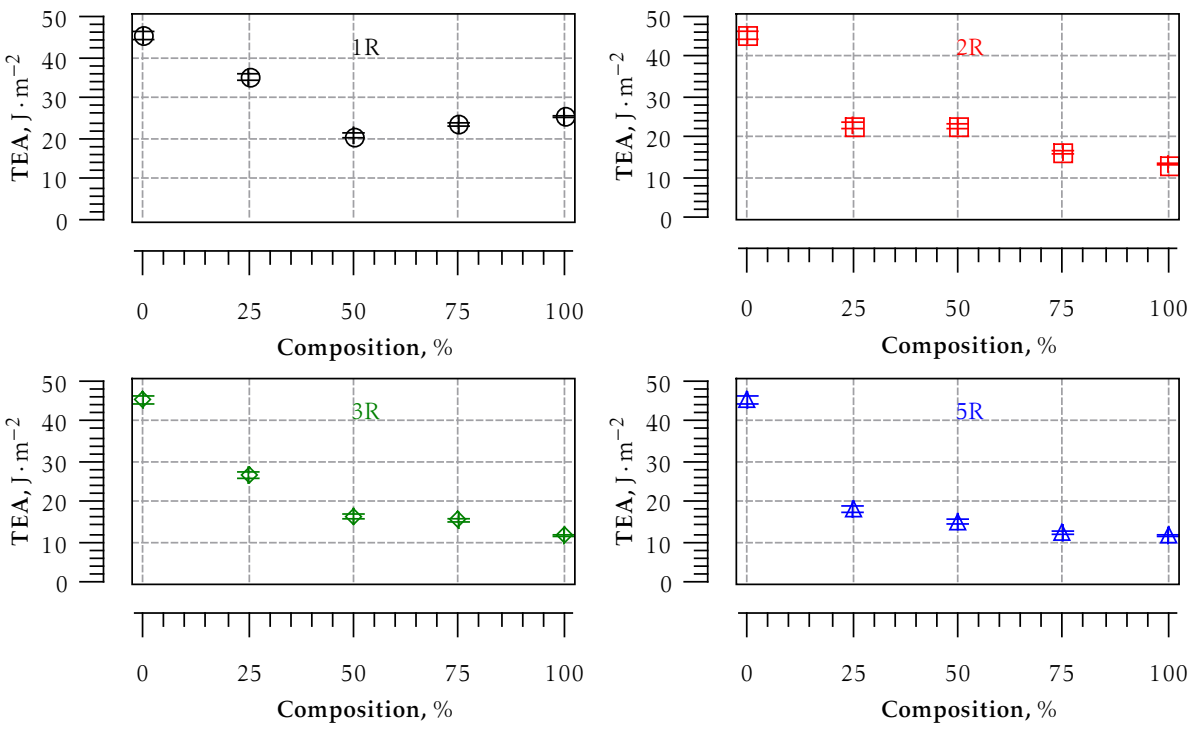

Figure 5.34: Tensile energy absorption of handsheets made from recycled pulp blends 
Table 5.8: Tensile energy absorption of handsheets made from recycled pulp blends

\begin{tabular}{c|ccccc}
\multirow{2}{*}{ TEA, } & \multicolumn{5}{|c}{$y_{n}(x)=b+a \cdot e^{-\left(\frac{x}{\tau}\right)}$} \\
\cline { 2 - 6 } $\mathrm{J} \cdot \mathrm{m}^{-2}$ & $y_{1}(x)$ & $y_{2}(x)$ & $y_{3}(x)$ & $y_{4}(x)$ & $y_{5}(x)$ \\
\hline$b$ & 22.3 & 13.2 & 11.5 & 10.2 & 11.5 \\
$a$ & 23.7 & 32.1 & 33.8 & 34.1 & 33.8 \\
$\tau$ & 27 & 28 & 29 & 24 & 21 \\
\hline $\mathrm{R}^{2}$ & 0.89 & 0.95 & 0.99 & 0.99 & 0.99
\end{tabular}

Decay constant of four and five times recycled pulps was lower. The percentage change in TEA calculated from equation (5.13) is presented in Figure 5.35.

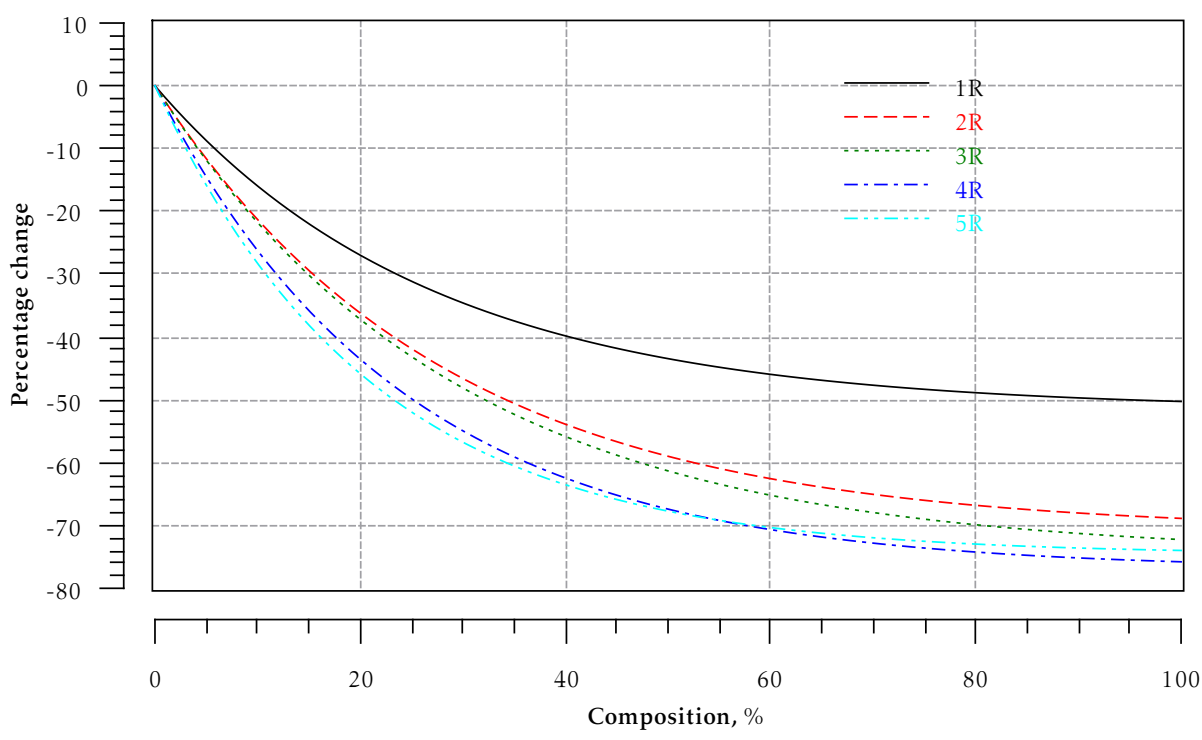

Figure 5.35: Percentage change in TEA of handsheets made from recycled pulp blends

The drop in TEA is more pronounced if pulp with higher recycle number is blended in excess.

\section{Burst index of handsheets made from recycled pulp blends}

The effect of quantity and the number of recycled pulp used on burst index is presented in Figure 5.36. 

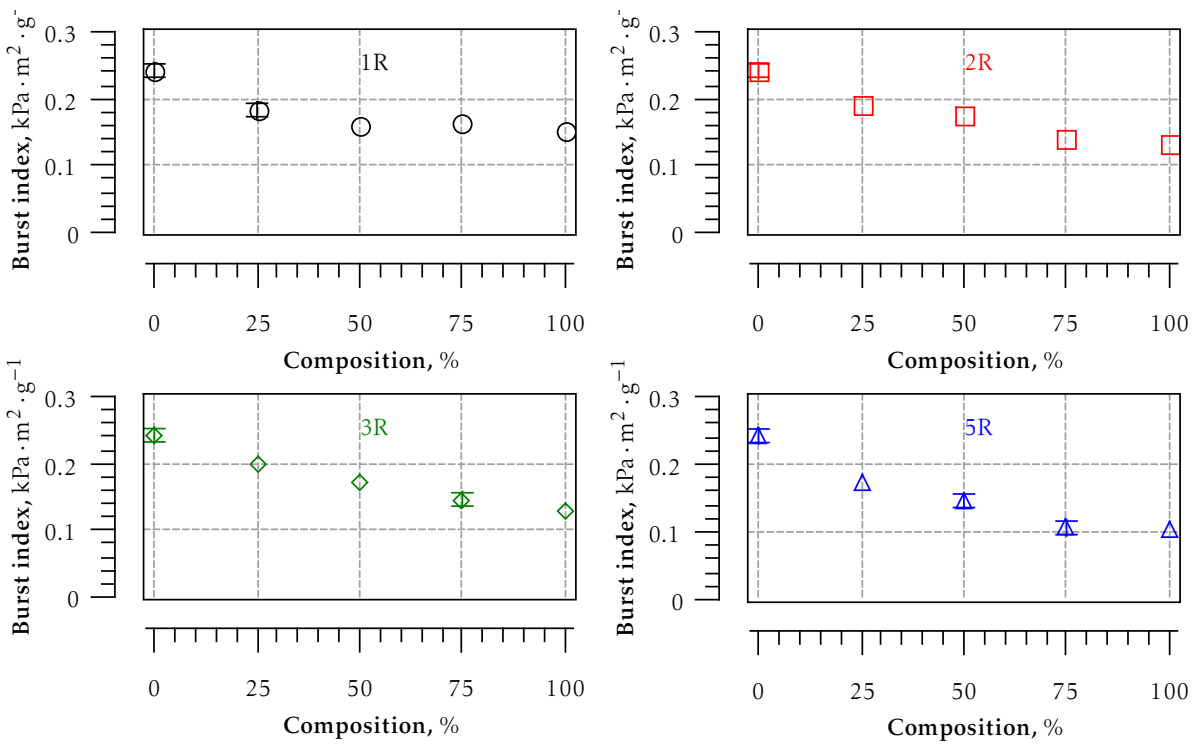

Figure 5.36: Burst index of handsheets made from recycled pulp blends

Evolution of burst index with the quantity of recycled pulp used followed first order exponential decay model. The coefficients of equation (5.14) are summarised in Table 5.9.

Table 5.9: Burst index of handsheets made from recycled pulp blends

\begin{tabular}{c|ccccc}
\multirow{2}{*}{$\begin{array}{c}\text { Burst index, } \\
\mathrm{kPa} \cdot \mathrm{m}^{2} \cdot \mathrm{g}^{-1}\end{array}$} & \multicolumn{5}{|c}{$y_{n}(x)=b+a \cdot e^{-\left(\frac{x}{\tau}\right)}$} \\
\cline { 2 - 6 } & $y_{1}(x)$ & $y_{2}(x)$ & $y_{3}(x)$ & $y_{4}(x)$ & $y_{5}(x)$ \\
\hline$b$ & 0.15 & 0.13 & 0.13 & 0.11 & 0.10 \\
$a$ & 0.09 & 0.11 & 0.11 & 0.14 & 0.14 \\
$\tau$ & 26 & 40 & 42 & 24 & 33 \\
\hline $\mathrm{R}^{2}$ & 0.98 & 0.97 & 0.999 & 0.99 & 0.97
\end{tabular}

Decay constant of once and four time recycled pulps was relatively lower. The percentage change in burst index calculated from equation (5.13) is presented in Figure 5.37. 


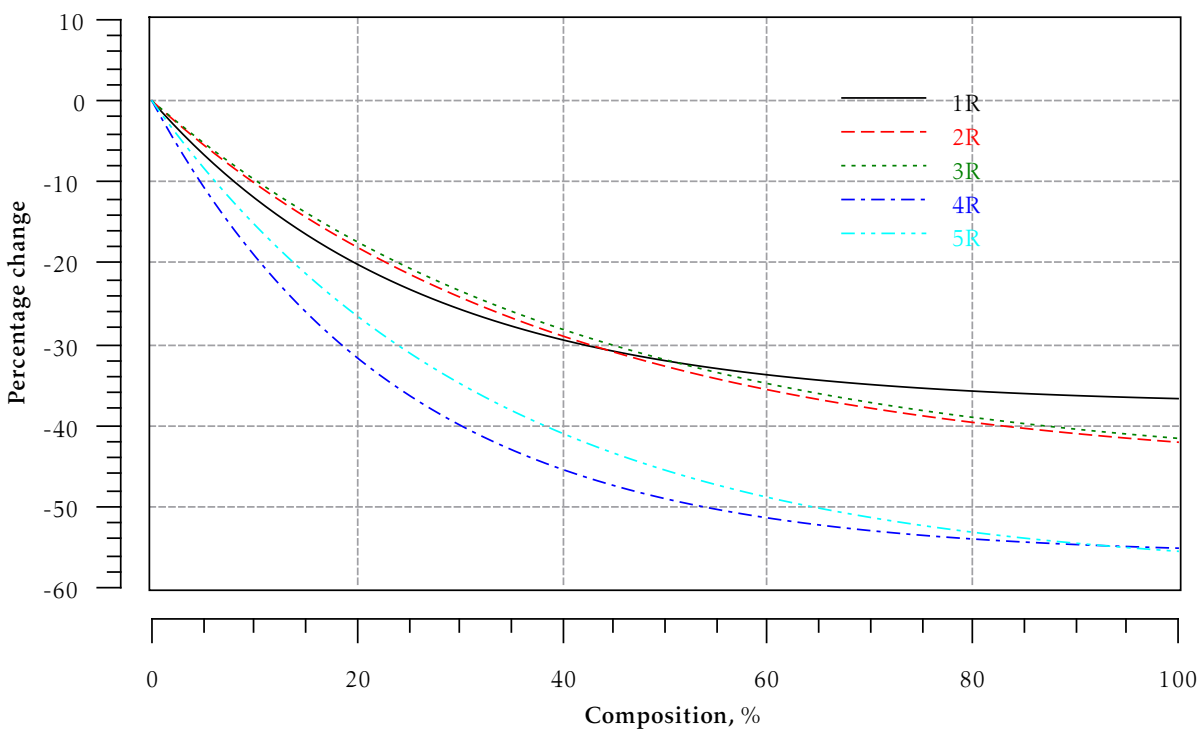

Figure 5.37: Percentage change in burst index of handsheets made from recycled pulp blends

The drop in burst index is more pronounced if four and five times recycled pulps are blended in excess.

\section{Tear index of handsheets made from recycled pulp blends}

The effect of quantity and the number of recycled pulp used on tear index is presented in Figure 5.38.
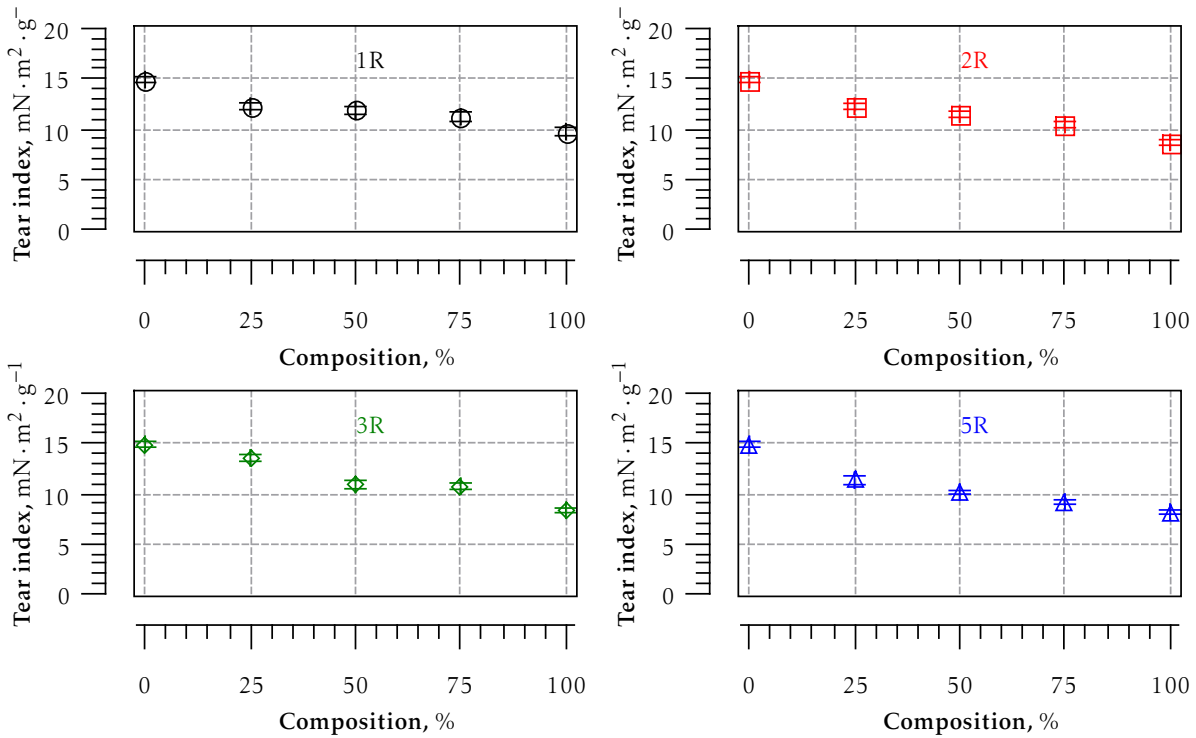

Figure 5.38: Tear index of handsheets made from recycled pulp blends

Evolution of tear index with the quantity of recycled pulp used can be de- 
scribed by first order exponential decay model. The coefficients of equation (5.14) are summarized in Table 5.10.

Table 5.10: Tear index of handsheets made from recycled pulp blends

\begin{tabular}{c|ccccc}
\multirow{2}{*}{$\begin{array}{c}\text { Tear index, } \\
\mathrm{mN} \cdot \mathrm{m}^{2} \cdot \mathrm{g}^{-1}\end{array}$} & \multicolumn{5}{|c}{$y_{n}(x)=b+a \cdot e^{-\left(\frac{x}{\tau}\right)}$} \\
\cline { 2 - 6 } & $y_{1}(x)$ & $y_{2}(x)$ & $y_{3}(x)$ & $y_{4}(x)$ & $y_{5}(x)$ \\
\hline$b$ & 9.7 & 8.6 & 8.3 & 8.3 & 8.2 \\
$a$ & 5.2 & 6.3 & 6.6 & 6.6 & 6.8 \\
$\tau$ & 44 & 47 & 55 & 39 & 35 \\
\hline $\mathrm{R}^{2}$ & 0.94 & 0.94 & 0.91 & 0.98 & 0.99
\end{tabular}

Decay constant of four and five times recycled pulps was lower. The percentage change in tear index calculated from equation (5.13) is presented in Figure 5.39 .

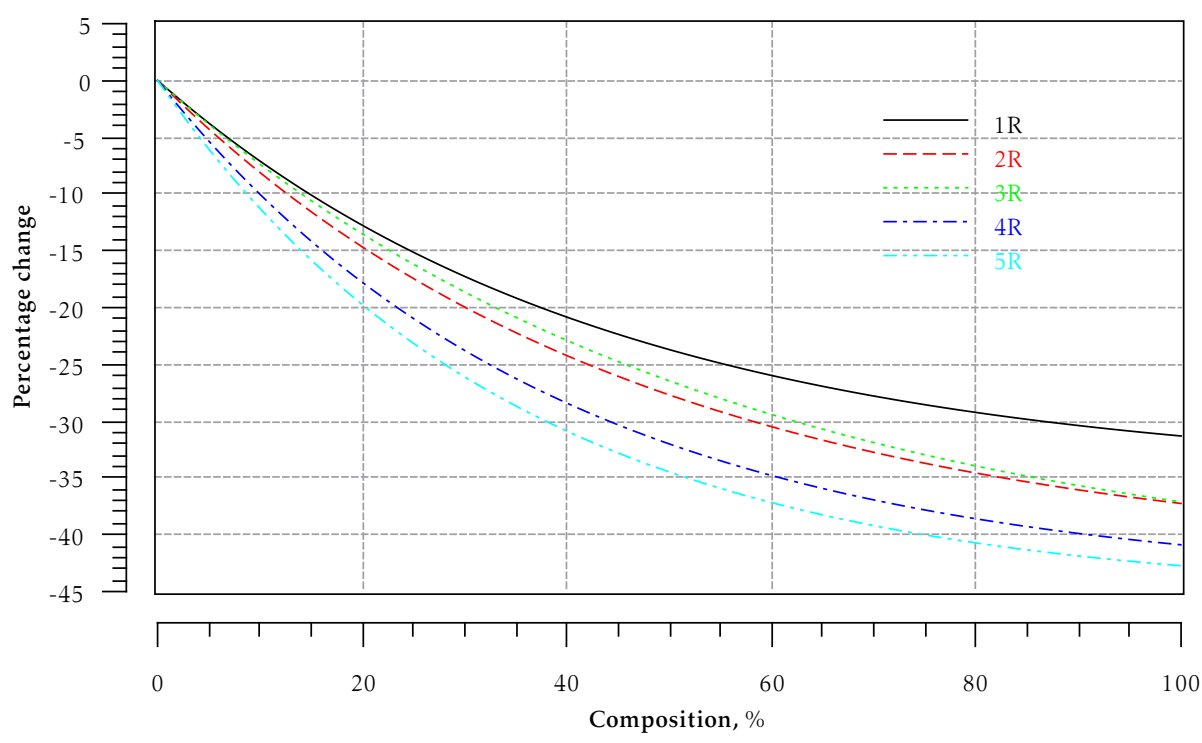

Figure 5.39: Percentage change in tear index of handsheets made from recycled pulp blends

The drop in tear index is more pronounced if pulp with higher recycle number is blended in excess. 


\subsubsection{Optical properties of handsheets made from recycled pulp blends}

Brightness of handsheets made from recycled pulp blends

The effect of quantity and the number of recycled pulp used on brightness is presented in Figure 5.40.
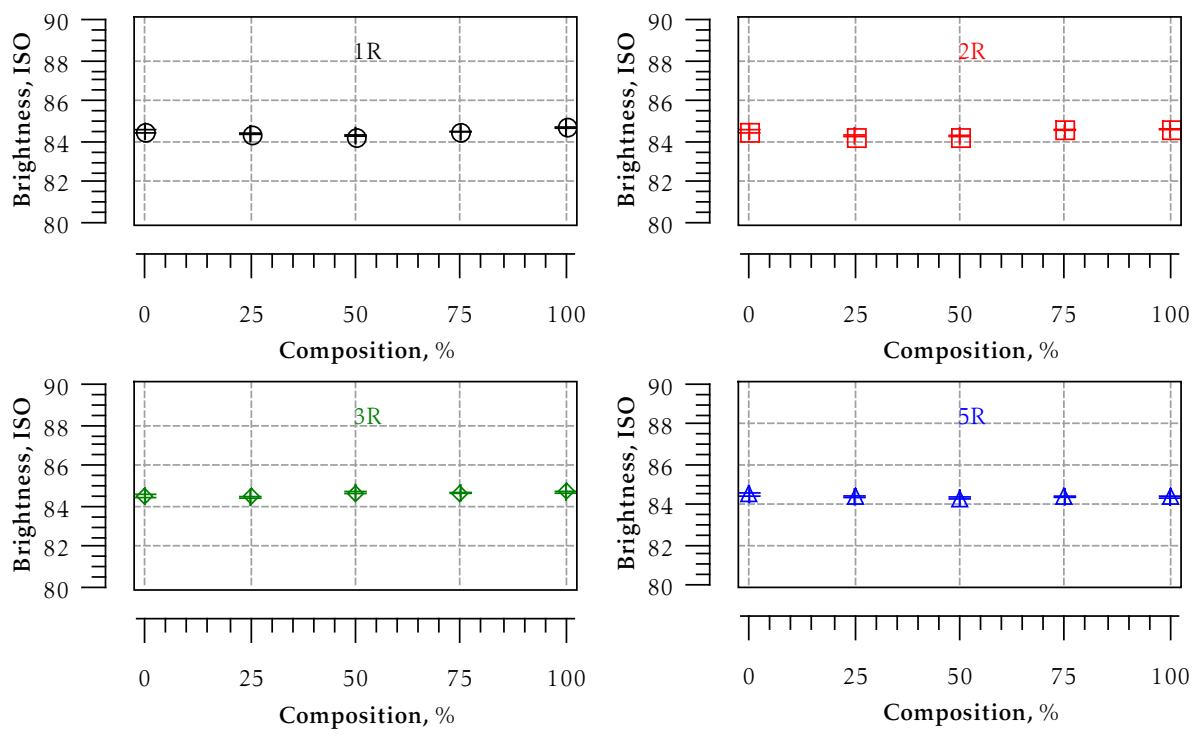

Figure 5.40: Brightness of handsheets made from recycled pulp blends

Brightness did not change with the quantity and the number of recycle pulp used.

\section{Opacity of handsheets made from recycled pulp blends}

The effect of recycled pulp quantity and number on opacity is presented in Figure 5.41. 

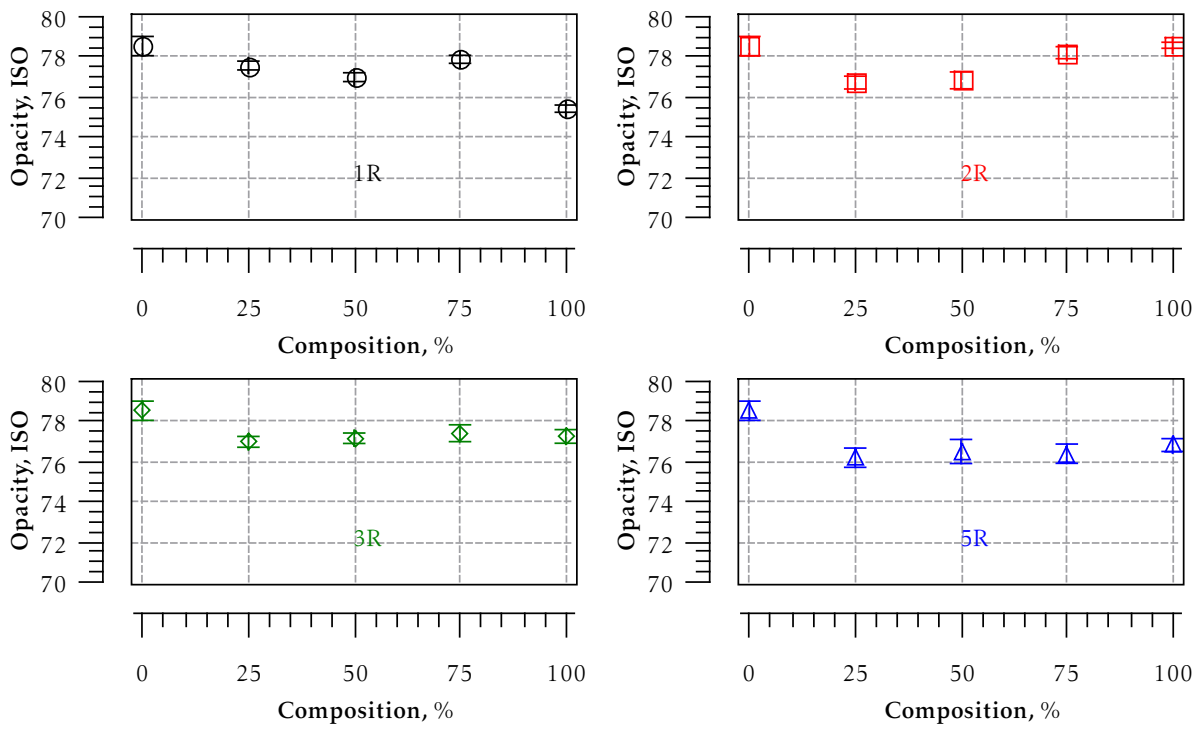

Figure 5.41: Opacity of handsheets made from recycled pulp blends

Opacity varied slightly with the quantity and the number of recycle pulp used.

\section{Scattering coefficient of handsheets made from recycled pulp blends}

The effect of quantity and the number of recycled pulp used on scattering coefficient is presented in Figure 5.42.
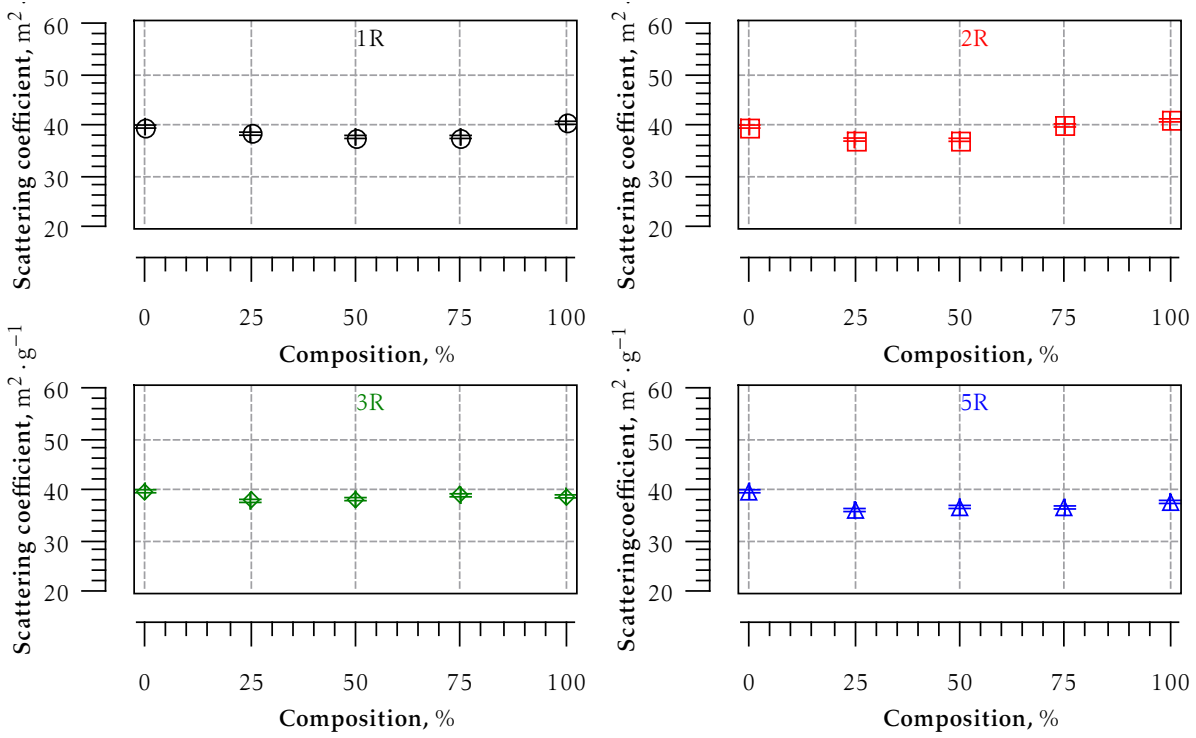

Figure 5.42: Scattering coefficient of handsheets made from recycled pulp blends

Scattering coefficient did not change with the quantity and the number of recycle pulp used. 
With the exception of zero-span breaking lengths, the incorporation of repeatedly recycled pulp blends deteriorated mechanical properties.

\subsubsection{Cost analysis of recycled pulp blends}

In Europe, out of 29.9 Mt chemical wood pulp, 23.3 Mt was bleached in 2010 [80]. On an average softwood accounts for $71.6 \%$ of total wood pulp consumption [61]. Which means 16.68 Mt of bleached softwood was used in Europe in 2010. High value paper grades rarely incorporate recovered fibres. Prices of softwood pulp and old newspapers and magazine paper are $€ 633.52 / \mathrm{t}$ and $€ 143.57 / \mathrm{t}$ respectively [88]. If we suppose that $12 \mathrm{Mt} \cdot \mathrm{a}^{-1}$ of softwood pulp is used in high value paper alone. The cost analysis of recycled pulp blends on the basis of blending ratios is described in Table 5.11.

Table 5.11: Cost analysis of recycled pulp blends

\begin{tabular}{l|ccccc} 
Recycle pulp & $0 \%$ & $25 \%$ & $50 \%$ & $75 \%$ & $100 \%$ \\
\hline$€ / \mathrm{t}$ & 633.52 & 511.03 & 388.55 & 266.06 & 143.57 \\
Savings, $€ / \mathrm{t}$ & 0 & 122.49 & 244.98 & 367.46 & 489.95 \\
\hline Savings, G€/a & 0 & 1.47 & 2.94 & 4.41 & 5.88
\end{tabular}

The use of recycled pulp fibres in high value paper can bring reasonable savings (cf. Table 5.11).

Table 5.12 summarises the environmental impacts estimated for one tonne of copy paper containing $84 \%$ fibre content [79]. 
Table 5.12: Environmental impact estimates of recycled pulp blends in copy paper [79]

\begin{tabular}{l|ccccc} 
Composition, \% & 0 & 25 & 50 & 75 & 100 \\
\hline Wood, $\mathrm{t}$ & 4 & 3 & 2 & 1 & 0 \\
Net energy, GJ & 34 & 32 & 28 & 25 & 23 \\
CO 2 equivalent, $\mathrm{t}$ & 3 & 2 & 2 & 2 & 2 \\
NOx, kg & 5 & 4 & 4 & 4 & 4 \\
Particulates, kg & 3 & 2 & 2 & 2 & 1 \\
HAPs, kg & 1 & 1 & 1 & 0.5 & 0.5 \\
VOCs, kg & 1 & 1 & 1 & 1 & 0.5 \\
Wastewater, kL & 84 & 73 & 62 & 51 & 39 \\
BOD, kg & 5 & 4 & 4 & 3 & 3 \\
TSS, $\mathrm{t}$ & 0.01 & 0.01 & 0.01 & 0.01 & 0.005
\end{tabular}

Keeping in view of the possible compromise on the final quality parameters, a blending ratio can be selected. It is clear from the cost and environmental impacts that the incorporation of recovered fibres in high value paper not only reduces cost but also reduces the environmental loads. 


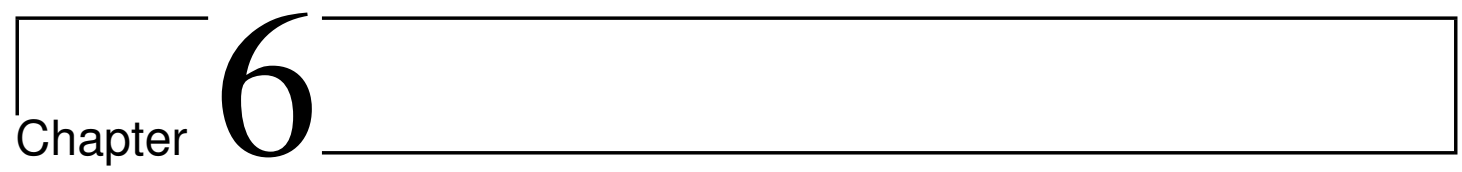

\section{Conclusions}

The maximum potential of fibres for paper making is still under investigation. A lot of time and resources are being spent to increase the recovery and utilisation rates of recycled fibres. With effective public awareness campaigns, adoption of commingled system and the improvement in production technology; the sorting, collection, recyclability and utilisation of recycled fibres has been increased over past few decades. However, the utilisation rate of recycled fibres in graphic papers is still negligible. The limitation of the use of recycled fibres in high value paper grades can only be addressed after knowing the physical changes in fibre, pulp and paper associated with multiple recyclings.

In this study, never dried pulp was recycled ten times. The characterisation of pulps and fibres was realised during recyclings. It was found that measured chemical properties of pulps did not change. However, the process of recycling did modified fibre morphology as shown by morphological analysis. The drop in fibre width was predominately caused by earlywood fibre width shrinkage. Curl and kink in fibres were also reduced thereby reducing the relative proportion of curled and kinked fibres in corresponding distributions. Fibres became flat as irregularities in fibre decreased. Fibre wall was densified and the contribution of latewood fibres towards fibre wall shrinkage was relatively higher. In situ rewetting and drying of fibres in enviromental scanning electron microscope verified the results obtained from morphological analysis.

Surface analysis of fibres revealed delayering of P/S1 layer during recycling. Surface adhesion of fibres increased with recycling as indicated by the increase in dissipation energy measured through atomic force microscopy. Increase in wet breaking length also indicated an increase in surface friction and capillary forces with recycling. 
To investigate the effect of recycling on fibre strength, single fibre tensile tests were realised. It was found that fibres became hard and brittle. Weak points in fibre wall increased initially and at the later recycles.

Fibre hornification was quantified using two experimental techniques (water retention value and fibre saturation point). Fibre hornification followed Michaelis Menten kinetics during recycling. Fines have to be excluded for the measurement of fibre hornification based on water retention value. To investigate pore size distribution, a semi automatic column fabrication system was developed. It was found that the specific apparent pore volume decrease whereas pores open and close during recyclings. Ultra structural changes were found significant during first recycle.

Dewatering and specific filtration resistance of pulp and fibres did not change during the coarse of recycling.

Physical properties of papers produced from never dried and recycled pulps were measured. X-rays microtomographical images indicated alteration in fibre network. Projected bonded (fibre intersection) area and collapse index changed with recycling.

With the exception of zero-span breaking lengths all other measured mechanical properties decayed exponential with repeated recycling. The increase in wet zero-span breaking length indicates fibre strength improvement with recycling. Strength loss in first recycle was due to the decrease in projected bonded area and flexibility. Despite the increase of projected bonded area, loss of strength was irrecoverable in later recycles. From the surface analysis of recycled fibres, it can be assumed that the tensile failure may be due to the failure of delaminated outer fibre cell wall layers. Therefore it can be said that the morphological and structural changes in pulp, fibres and network during recycling plays an important role in strength development.

Physical properties of papers produced from recycled pulp blends were carried out. Blending recycled with virgin pulp altered end product characteristics. Measured mechanical properties (with the exception of zero-span breaking lengths) decayed exponentially with the amount of recycled pulp used. The loss of strength was relatively higher when recycled pulps of higher recycled number were used. Optical properties remained unchanged. Bearing in mind of the compromise on final product qualities, incorporation of recovered fibres in high value paper can reduce cost and the environmental loads.

Evolution of physical changes was described by empirical models with coefficients corresponding to this raw material and to the experimental conditions 
used in this study. 


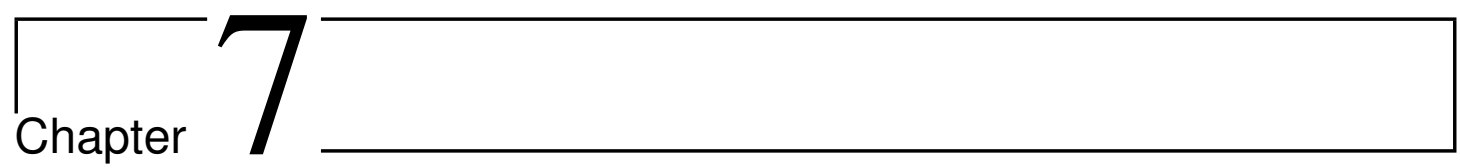

\section{Perspectives}

The behaviour of earlywood and latewood fibres towards recycling is dissimilar therefore it is required to distinguish their contribution to the change in physical properties. Although measuring single fibre properties is tedious, more fibres should be tested and the evolution of single fibre tensile properties and micro fibril angle with recycling needs to be established. Fibre surface changed with recycling as has been indicated by scanning electron microscopic and atomic force microscopic observations. However, the quantification of the evolution of surface adhesion and hardness with recycling needs to be established using atomic force microscope. X-rays microtomographs need further exploitation since the evolution of structural changes as a function of earlywood and latewood fibres is not addressed.

We have seen here that the contribution of first drying towards the structural changes is enormous. Therefore, the process of drying in non-integrated mills needs to be revisited. It is well established that fibre hornification of pulps containing higher amounts of hemicelluloses is comparatively lower hence their recyclability is better. With the advent of biorefining particularly the extraction of hemicelluloses from pulps will reduce the recyclability.

We studied the influence of the cycle of pressing and drying. The influence of beating on fibre hornification should be analysed in a futur work. 


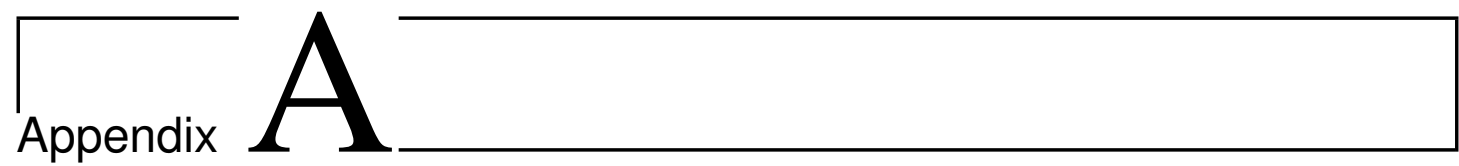

\section{Filtration resistance}

Schematic diagram of drainage tester is shown in Figure A.1.

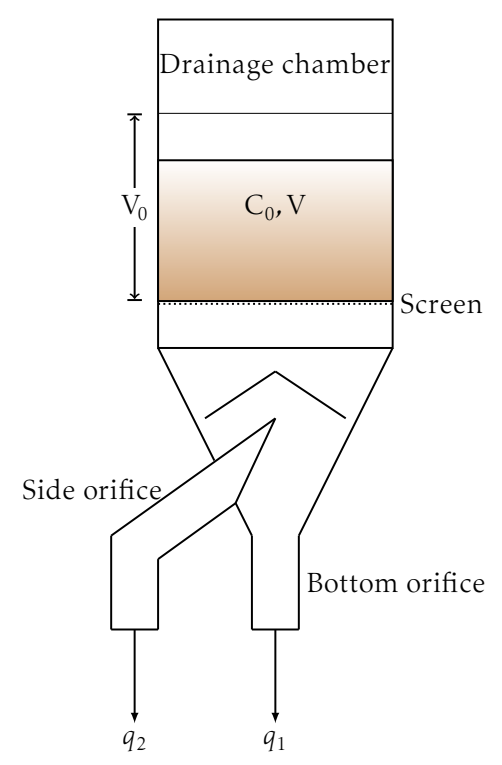

Figure A.1: Schematic diagram of dewatering apparatus

where $\mathrm{C}_{0}$ and $\mathrm{V}_{0}$ are the consistency and the volume of slurry on wire screen at $t_{0}$.

Total volumetric flow rate at any time can be represented as

$$
\begin{gathered}
q=q_{1}+q_{2} \\
q=\frac{d\left(\mathrm{~V}_{0}-\mathrm{V}\right)}{d t}=-\frac{\mathrm{V}}{\mathrm{R}_{\mathrm{F}}}
\end{gathered}
$$

integrating to get

$$
\mathrm{V}=\left(\mathrm{V}_{0}-\mathrm{V}_{f}\right) \cdot e^{\left(\frac{-t}{\mathrm{R}_{\mathrm{F}}}\right)}+\mathrm{V}_{f}
$$


where $\mathrm{R}_{\mathrm{F}}$ and $\mathrm{V}_{f}$ are the filtration resistances and volume of the slurry on wire screen at $t_{f}$.

Figure A.2 shows characteristic drainage curve of refined pulp

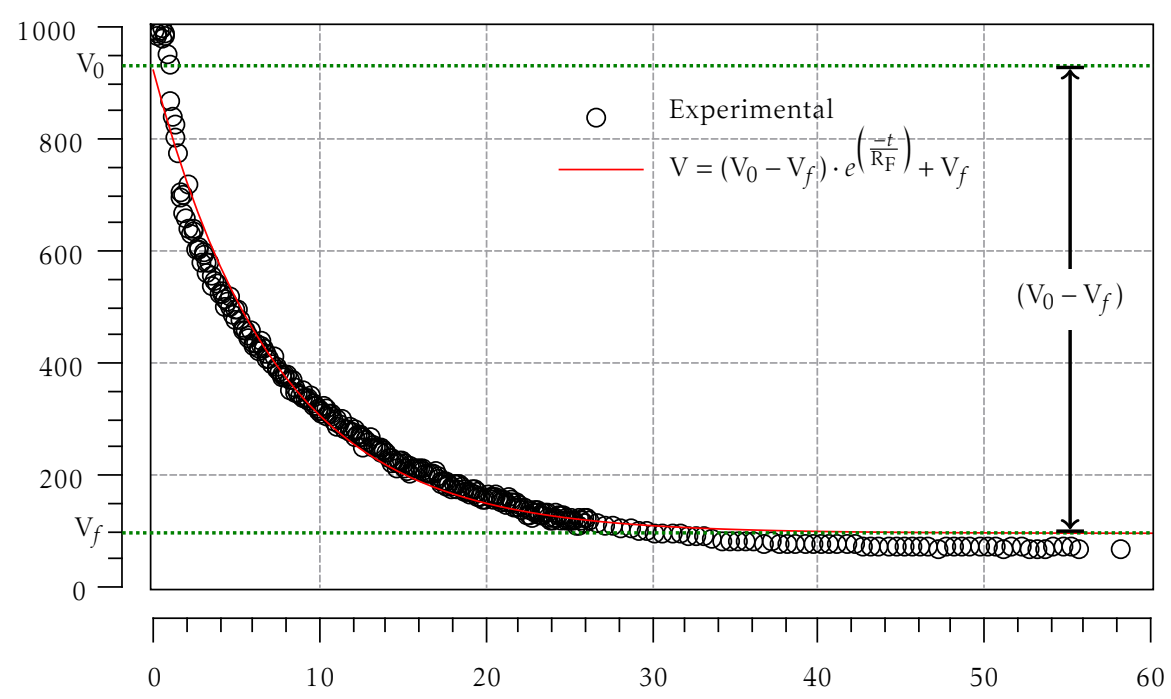

Figure A.2: Characteristic drainage curve

From Darcy law [72]

$$
\frac{d \mathrm{~V}}{d t}=-\frac{\mathrm{V}}{\mathrm{R}_{\mathrm{F}}}=-\frac{\mathrm{A} \cdot \Delta \mathrm{P}}{\mathrm{R}_{s \mathrm{~F}} \cdot \mathrm{W}}
$$

$\mathrm{R}_{s \mathrm{~F}}$ is the specific filtration resistance of the mat formed on the screen.

The basis weight of the mat can be described as

$$
\mathrm{W}=\frac{\mathrm{C}_{0}\left(\mathrm{~V}_{0}-\mathrm{V}\right)}{\mathrm{A}}
$$

From equation (A.4) and (A.5)

$$
\frac{\mathrm{V}}{\mathrm{R}_{\mathrm{F}}}=\frac{\mathrm{A}^{2} \cdot \Delta \mathrm{P}}{\mathrm{R}_{s \mathrm{~F}} \cdot \mathrm{C}_{0}\left(\mathrm{~V}_{0}-\mathrm{V}\right)}
$$

Rearranging

$$
\mathrm{R}_{s \mathrm{~F}}=\frac{\mathrm{A}^{2} \cdot \Delta \mathrm{P} \cdot \mathrm{R}_{\mathrm{F}}}{\mathrm{C}_{0} \cdot \mathrm{V}\left(\mathrm{V}_{0}-\mathrm{V}\right)}
$$

Hydraulic pressure can be described as

$$
\mathrm{A} \cdot \Delta \mathrm{P}=\rho \cdot g\left(\mathrm{~V}_{0}-\mathrm{V}\right)
$$


From equation (A.7) and (A.8)

$$
\mathrm{R}_{s \mathrm{~F}}=\frac{\mathrm{A} \cdot \rho \cdot g \cdot \mathrm{R}_{\mathrm{F}}}{\mathrm{C}_{0} \cdot \mathrm{V}}
$$

From equation (A.3) and (A.9)

$$
\mathrm{R}_{s \mathrm{~F}}=\frac{\mathrm{A} \cdot \rho \cdot g \cdot \mathrm{R}_{\mathrm{F}}}{\mathrm{C}_{0}\left\{\left(\mathrm{~V}_{0}-\mathrm{V}_{f}\right) \cdot e^{\left(\frac{-t}{\mathrm{R}_{\mathrm{F}}}\right)}+\mathrm{V}_{f}\right\}}
$$

Diameter of the screen $(d)=0.1125 \mathrm{~m}$

Area of the screen $(A)=\frac{\pi(0.1125)^{2}}{4}=0.01 \mathrm{~m}^{2}$

Density of the slurry $(\rho)=1000 \mathrm{~kg} \cdot \mathrm{m}^{-3}$

Gravitational constant $(g)=9.8 \mathrm{~m} \cdot \mathrm{s}^{-2}$

Putting the data in equation (A.10)

$$
\mathrm{R}_{s \mathrm{~F}}=\frac{98 \mathrm{R}_{\mathrm{F}}}{\mathrm{C}_{0}\left\{\left(\mathrm{~V}_{0}-\mathrm{V}_{f}\right) \cdot e^{\left(\frac{-t}{\mathrm{R}_{\mathrm{F}}}\right)}+\mathrm{V}_{f}\right\}}
$$

Dimensionless time $(\mathrm{T})=\frac{t}{t_{f}}$

Specific filtration resistance at $\mathrm{T}$ can be represented by the following expression

$$
\mathrm{R}_{s \mathrm{~F}, \mathrm{~T}}=\frac{98 \mathrm{R}_{\mathrm{F}}}{\mathrm{C}_{0}\left\{\left(\mathrm{~V}_{0}-\mathrm{V}_{f}\right) \cdot e^{\left(\frac{-\mathrm{T} \cdot t_{f}}{\mathrm{R}_{\mathrm{F}}}\right)}+\mathrm{V}_{f}\right\}}
$$

\section{Specific filtration resistance of recycled pulps and fi- bres}

Specific filtration resistances of $\mathrm{nRPs}$ and corresponding $\mathrm{nRF}$ as a function of recycle number $(\mathrm{n})$ have been reported in Table A.1. 
Table A.1: Specific filtration resistance of recycled pulps and fibres

\begin{tabular}{|c|c|c|c|c|c|c|c|c|c|c|}
\hline $\mathrm{R}_{s \mathrm{~F}, \mathrm{~T}} \times 10^{5}, \mathrm{~s}^{-1}$ & \multicolumn{5}{|c|}{ Fibres } & \multicolumn{5}{|c|}{ Pulps } \\
\hline$n$ & 0 & 0.25 & 0.5 & 0.75 & 1 & 0 & 0.25 & 0.5 & 0.75 & 1 \\
\hline 0 & 0.5 & 9.2 & 10.1 & 10.1 & 10.1 & 0.7 & 11.7 & 13.1 & 13.1 & 13.1 \\
\hline 1 & 0.4 & 15.4 & 16.5 & 16.5 & 16.5 & 0.4 & 8.8 & 9.7 & 9.7 & 9.7 \\
\hline 2 & 0.4 & 9.8 & 10.0 & 10.0 & 10.0 & - & - & - & - & - \\
\hline 3 & 0.5 & 22.9 & 23.0 & 23.0 & 23.0 & - & - & - & - & - \\
\hline 4 & 0.4 & 14.5 & 17.0 & 17.0 & 17.0 & - & - & - & - & - \\
\hline 5 & 0.5 & 13.8 & 14.0 & 14.0 & 14.0 & 0.4 & 10.2 & 10.5 & 10.5 & 10.5 \\
\hline 6 & 0.5 & 10.2 & 11.7 & 11.7 & 11.7 & - & - & - & - & - \\
\hline 7 & 0.4 & 12.5 & 14.2 & 14.2 & 14.2 & - & - & - & - & - \\
\hline 8 & 0.6 & 11.5 & 14.4 & 14.5 & 14.5 & - & - & - & - & - \\
\hline 9 & 0.5 & 15.4 & 16.2 & 16.2 & 16.2 & - & - & - & - & - \\
\hline 10 & 0.6 & 19.0 & 19.8 & 19.8 & 19.8 & 0.3 & 9.6 & 10.1 & 10.1 & 10.1 \\
\hline
\end{tabular}

Specific filtration resistance of nRPs and corresponding nRFs did not change. 
Appendix

\section{Gel filtration chromatography}

\section{Glass beads bed column}

Packing material $=$ spherical glass beads

Packing height $=1.63 \mathrm{~cm}$

Internal diameter of the column $=2.5 \mathrm{~cm}$

Diameter of single bead $=0.6 \mathrm{~cm}$

Number of beads $=49$

Porosity $=0.379$

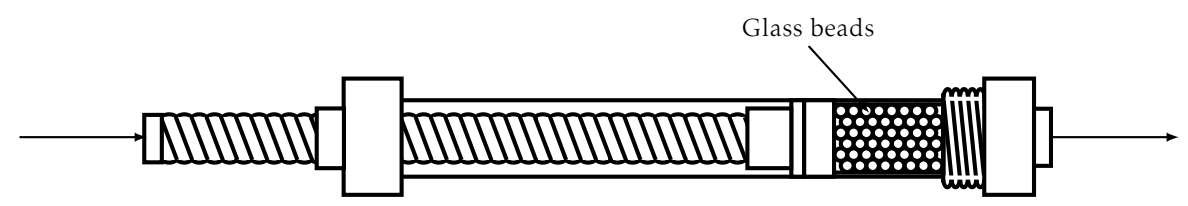

Figure B.1: Column containing glass beads

\section{Fibrous bed column}

Packing material $=$ bleached softwood kraft pulp fibres

Packing height $=4 \mathrm{~cm}$

Internal diameter of the column $=1 \mathrm{~cm}$

Mass of the oven dried fibres $=1 \mathrm{~g}$

Same column fabrication process was adopted. 


\section{Mass balance for FSP}

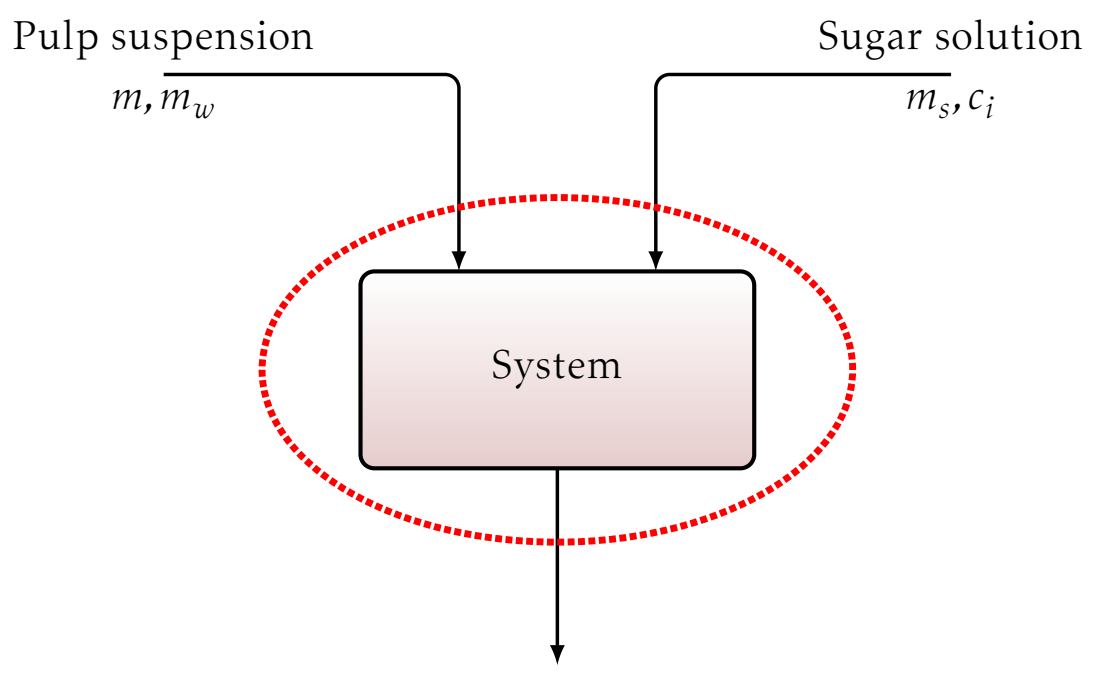

Filtered sugar solution

$$
m_{s f}, c_{f}
$$

Figure B.2: Mass balance for FSP

Mass of oven dried pulp $(\mathrm{g})=m$

Mass of water in pulp suspension $(\mathrm{g})=m_{w}$

Mass of sugar solution $(\mathrm{g})=m_{\mathrm{s}}$

Concentration of sugar solution prepared $\left(\mathrm{g} \cdot \mathrm{L}^{-1}\right)=c_{i}$

Mass of filtered sugar solution $(\mathrm{g})=m_{s f}$

Concentration of filtered sugar solution prepared $\left(\mathrm{g} \cdot \mathrm{L}^{-1}\right)=c_{f}$

Applying total mass balance around the system

$$
\text { In }- \text { out }=\text { Accumulation }
$$

$\mathrm{FSP}=$ specific inaccessible water $=$ specific accumulated water

Mass balance of water around system

$$
\text { mass of water in }- \text { mass of water out }=\text { mass of accumulated water }
$$

Mass of water into the system

$$
\begin{aligned}
\text { water in }= & \text { water in the pulp suspension }+ \text { water in solution } \\
& \text { mass of sugar in solution }(g)=\frac{m_{s} \cdot c_{i}}{1000}
\end{aligned}
$$




$$
\text { mass of water in } \operatorname{solution}(g)=m_{s}-\frac{m_{s} \cdot c_{i}}{1000}
$$

Putting (B.6) in (B.4)

$$
\text { mass of water in }=m_{w}+\left(m_{s}-\frac{m_{s} \cdot c_{i}}{1000}\right)
$$

If there is no accumulation of sugar in the system

$$
\begin{gathered}
\text { mass of sugar in filtered solution }(g)=\frac{m_{s} \cdot c_{i}}{1000} \\
\text { concentration of filtered solution }\left(\frac{g}{l}\right)=c_{f}=\frac{m_{s} \cdot c_{i}}{m_{s f}+\left(\frac{m_{s} \cdot c_{i}}{1000}\right)}
\end{gathered}
$$

Mass of water out of the system

$$
\text { mass of water in filtered solution }(g)=m_{s f}=\frac{m_{s} \cdot c_{i}}{c_{f}}-\frac{m_{s} \cdot c_{i}}{1000}
$$

Putting (B.7) and (B.10) in (B.3)

$$
\begin{gathered}
\text { mass of accumulated water }=m_{w}+m_{s}-\left(\frac{m_{s} \cdot c_{i}}{1000}\right)-\left(\frac{m_{s} \cdot c_{i}}{c_{f}}\right)+\left(\frac{m_{s} \cdot c_{i}}{1000}\right) \\
\text { mass of accumulated water }=m_{w}+m_{s}-m_{s} \cdot \frac{c_{i}}{c_{f}}
\end{gathered}
$$

From (B.2)

$$
\mathrm{FSP}=\frac{m_{w}+m_{s}}{m}-\frac{m_{s}}{m} \cdot \frac{c_{i}}{c_{f}}
$$

or

$$
\mathrm{FSP}=\frac{m_{s}+m_{w}}{m}\left(1-\frac{m_{s}}{m_{s}+m_{w}} \cdot \frac{c_{i}}{c_{f}}\right)
$$

Calibration of the differential refractometer gives

$$
\mathrm{RIA}=\text { slope } \cdot c+k_{c}
$$

where $c$ and $k_{c}$ are the concentration and constant of calibration, respectively. Finally, we obtain the following expression

$$
\mathrm{FSP}=\frac{m_{s}+m_{w}}{m}\left(1 \frac{m_{s}}{m_{s}+m_{w}} \cdot \frac{\mathrm{RIA}_{i}-k_{c}}{\mathrm{RIA}_{f}-k_{c}}\right)
$$




\section{Pore size distribution of recycled pulps and fibres}

Evolution of apparent pore volume of various pore sizes of pulps with recycling is presented in Table B.1.

Table B.1: Apparent pore volume of recycled pulps

\begin{tabular}{c|ccc|c} 
Recycle & \multicolumn{3}{|c|}{ Apparent pore volume, $\mathrm{mL} \cdot \mathrm{g}^{-1}$} & Average pore \\
\cline { 3 - 3 } number & 0.8 to $1.8 \mathrm{~nm}$ & 1.8 to $13.7 \mathrm{~nm}$ & 13.7 to $18.6 \mathrm{~nm}$ & size, $\mathrm{nm}$ \\
\hline 0 & $0.00 \pm 0.00$ & $0.56 \pm 0.04$ & $0.21 \pm 0.01$ & 12.9 \\
1 & $0.11 \pm 0.00$ & $0.54 \pm 0.01$ & $0.01 \pm 0.00$ & 8.7 \\
5 & $0.19 \pm 0.04$ & $0.27 \pm 0.04$ & $0.00 \pm 0.00$ & 7.0 \\
10 & $0.31 \pm 0.03$ & $0.36 \pm 0.03$ & $0.00 \pm 0.00$ & 4.6
\end{tabular}

Evolution of apparent pore volume of various pore sizes with recycling is presented in Table B.2

Table B.2: Apparent pore volume of recycled fibres

\begin{tabular}{c|ccc|c} 
Recycle & \multicolumn{3}{|c|}{ Apparent pore volume, $\mathrm{mL} \cdot \mathrm{g}^{-1}$} & $\begin{array}{c}\text { Average pore } \\
\text { number }\end{array}$ \\
\cline { 3 - 4 } & 0.8 to $1.8 \mathrm{~nm}$ & 1.8 to $13.7 \mathrm{~nm}$ & 13.7 to $18.6 \mathrm{~nm}$ & size, $\mathrm{nm}$ \\
\hline 0 & $0.04 \pm 0.01$ & $0.820 \pm 0.01$ & $0.05 \pm 0.03$ & 9.7 \\
1 & $0.07 \pm 0.02$ & $0.37 \pm 0.05$ & $0.03 \pm 0.02$ & 8.4 \\
2 & $0.23 \pm 0.01$ & $0.33 \pm 0.03$ & $0.03 \pm 0.02$ & 5.5 \\
3 & $0.15 \pm 0.03$ & $0.28 \pm 0.02$ & $0.06 \pm 0.01$ & 7.7 \\
4 & $0.09 \pm 0.05$ & $0.29 \pm 0.03$ & $0.00 \pm 0.00$ & 6.2 \\
5 & $0.04 \pm 0.04$ & $0.40 \pm 0.04$ & $0.00 \pm 0.00$ & 7.5 \\
6 & $0.25 \pm 0.02$ & $0.38 \pm 0.03$ & $0.02 \pm 0.01$ & 5.8 \\
7 & $0.07 \pm 0.02$ & $0.34 \pm 0.01$ & $0.03 \pm 0.01$ & 7.9 \\
8 & $0.16 \pm 0.01$ & $0.49 \pm 0.04$ & $0.00 \pm 0.00$ & 7.0 \\
9 & $0.15 \pm 0.01$ & $0.40 \pm 0.01$ & $0.06 \pm 0.01$ & 8.2 \\
10 & $0.12 \pm 0.01$ & $0.38 \pm 0.01$ & $0.03 \pm 0.00$ & 7.4
\end{tabular}




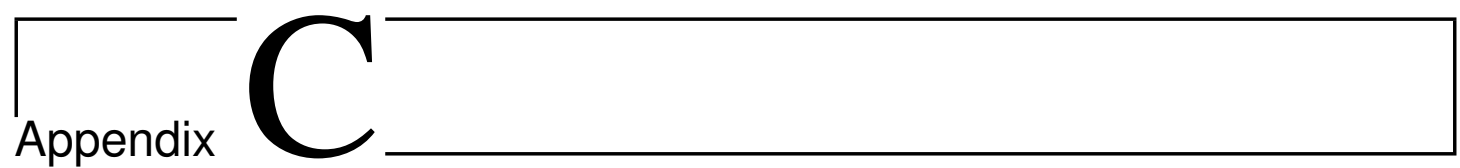

\section{Weak points in fibre wall}

\section{Preparation of $\mathrm{HCl}$ solutions}

$\mathrm{HCl}$ solution was prepared by diluting concentrated $\mathrm{HCl}$ solution (CAS Number: 7647-01-0). Density of the $36.5 \%$ concentrated $\mathrm{HCl}$ solution was $1.189 \mathrm{~g} \cdot \mathrm{mL}^{-1}$. So the concentration of the mother solution was $12.2 \mathrm{M}$.

To make $1 \mathrm{~L}$ of $1 \mathrm{M} \mathrm{HCl}$ solution, $82 \mathrm{~mL}$ of $12.2 \mathrm{M} \mathrm{HCl}$ solution was added slowly to $750 \mathrm{~mL}$ of deionised water taken in a volumetric flask of $1 \mathrm{~L}$. Deionised water was added to complete the volume to $1 \mathrm{~L}$. Similarly to make $1 \mathrm{~L}$ of $2 \mathrm{M} \mathrm{HCl}$ solution, $164 \mathrm{~mL}$ of $12.2 \mathrm{M} \mathrm{HCl}$ was added to $836 \mathrm{~mL}$ of deionised water.

\section{Preparation of phosphate buffer solution $(\mathrm{pH} 7)$}

$31.2 \mathrm{~g}$ of $\mathrm{NaH}_{2} \mathrm{PO}_{4} \cdot 2 \mathrm{H}_{2} \mathrm{O}$ (CAS Number: $13472-35-0$ ) was added to a $1 \mathrm{~L}$ measuring flask. Deionised water was added to make a volume upto $1 \mathrm{~L}$. In another $1 \mathrm{~L}$ measuring flask $53.61 \mathrm{~g}$ of $\mathrm{Na}_{2} \mathrm{HPO}_{4} \cdot 7 \mathrm{H}_{2} \mathrm{O}$ (CAS Number: 7782-85-6) was added. The volume was made up to the mark by adding deionised water. The solutions were stirred on IKAMAG ${ }^{\circledR}$ Reo magnetic stirring machine.

To make $1 \mathrm{~L}$ of $0.2 \mathrm{M}$ buffer solution, $625 \mathrm{~mL}$ of first solution was mixed with $375 \mathrm{~mL}$ of the second solution. 


\section{$\mathrm{HCl}$ treatment}

$3 \mathrm{~g}$ of $100 \mathrm{~g} \cdot \mathrm{L}^{-1}$ pulp and pulp fibres were taken in $100 \mathrm{ml}$ glass bottle. $17 \mathrm{~mL}$ of water and $40 \mathrm{~mL}$ of the $\mathrm{HCl}$ solution was added to the bottle. It was then tightly capped and wrapped in a polythene bag. It was then sealed in another stronger polythene bag and placed in preheated heating water bath.

Temperature of the bath was maintained at $80^{\circ} \mathrm{C}$ during the whole manipulation. After $4 \mathrm{~h}$, the bottle was withdrawn and uncapped under fume hood. Magnetic stirrer was added to the bottle containing $\mathrm{HCl}$ treated fibrous suspension. IKAMAG ${ }^{\circledR}$ Reo magnetic stirring machine was used. Stirring at $150 \mathrm{rpm}$ was carried out for $30 \mathrm{~min}$.

After removing magnetic stirrer, the fibrous suspension was filtered on Whatman GF/C glass microfibre filter paper. Filtered fibrous suspension was washed with $0.2 \mathrm{M}$ phosphate buffer solution. Finally the fibrous suspension was washed with deionised water. 


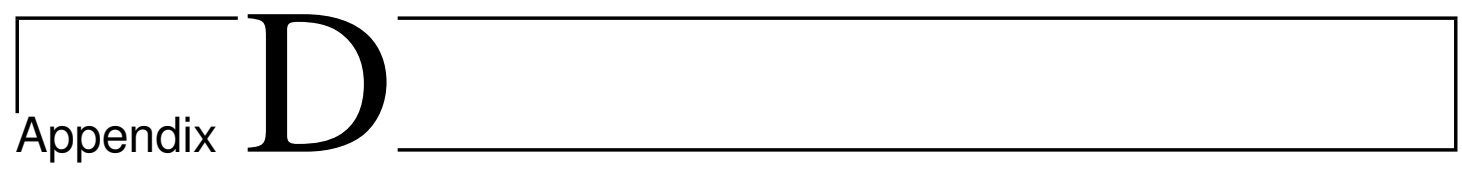

\section{Thermoporosimetry}

Aqueous fibrous suspension contains free [41] and bound water. Free water also referred as bulk water carries ordinary thermodynamic properties. It is found in inter-fibre (between the fibres) or intra-fibre (inside the lumen and in the fibre wall macropores).

Bound water can be freezing bound water (in micropores) or non freezing bound water (chemically attached to hydroxylic and carboxylic acid groups). The freezing bound water is water present in micropores.

Different waters can be differentiated using differential scanning calorimetry (DSC). Pore diameter can be calculated using Gibbs-Thomsan equation assuming cylindrical shape of the pores.

$$
d=\frac{4 \cdot \mathrm{V}_{\mathrm{M}} \cdot \mathrm{T}_{0} \cdot \Upsilon_{1 \mathrm{~s}}}{\mathrm{H}_{m} \cdot \Delta \mathrm{T}}=\frac{k}{\Delta \mathrm{T}}
$$

where $V_{M}$ is the molar volume, $T_{0}$ is the melting point at standard temperature and pressure, $\Delta \mathrm{T}$ is melting temperature depression, $\mathrm{H}_{m}$ is the latent heat of melting, $\curlyvee_{l s}$ is the surface energy at the liquid-solid interface.

$$
d_{n}(n m)=\frac{43}{\Delta \mathrm{T}_{n}}
$$

where $\Delta \mathrm{T}_{n}$ is melting point depression of $\mathrm{n}^{\text {th }}$ step. For cyclohexane $k=117 \mathrm{~nm} \cdot{ }^{\circ} \mathrm{C}$ in working form of Gibbs-Thomsan equation.

Thermoposrosimetry is based on the principles of DSC. This technique has been used for measuring pore size distribution of silica gel [113]. In order to avoid the thermal lag, isothermal step melting procedure was adopted [153] 
for measuring PSD.

Choosing the first step where there is no melting, the supplied heat is used to increases the temperature alone.

$$
\mathrm{Q}_{0}=m \cdot c_{p} \cdot \Delta \mathrm{T}_{0}
$$

where $\mathrm{m}, c_{p}$ and $\Delta \mathrm{T}_{0}$ are the mass, heat capacity and temperature change of sample.

The heat required to melt and increase temperature of the next step can be represented by the following expression

$$
\mathrm{Q}_{1}=m \cdot c_{p} \cdot \Delta \mathrm{T}_{1}+m_{1} \cdot \mathrm{H}_{m}
$$

where $\Delta \mathrm{T}_{1}=\mathrm{T}_{1}-\mathrm{T}_{0}$ and $m_{1}$ is the molten mass.

Rearranging to get

$$
m_{1}=\frac{1}{\mathrm{H}_{m}}\left\{\mathrm{Q}_{1}-\mathrm{Q}_{0} \cdot \frac{\Delta \mathrm{T}_{1}}{\Delta \mathrm{T}_{0}}\right\}
$$

The heat required to melt and increase temperature of the next step can be represented by the following expression

$$
\mathrm{Q}_{2}=m \cdot c_{p} \cdot \Delta \mathrm{T}_{0}+m_{2} \cdot \mathrm{H}_{m}
$$

where $\Delta \mathrm{T}_{2}=\mathrm{T}_{2}-\mathrm{T}_{1}$ and $m_{2}$ is the molten mass.

$$
m_{2}=\frac{1}{\mathrm{H}_{m}}\left\{\mathrm{Q}_{2}-\mathrm{Q}_{0} \cdot \frac{\Delta \mathrm{T}_{2}}{\Delta \mathrm{T}_{0}}\right\}
$$

Similarly the specific pore volume can be calculated by using the following expression

$$
\mathrm{V}_{n}=\frac{m_{n}}{m_{f}}=\frac{1}{\mathrm{H}_{m} \cdot m_{f}}\left(\mathrm{Q}_{n}-\mathrm{Q}_{0} \frac{\Delta \mathrm{T}_{n}}{\Delta \mathrm{T}_{0}}\right)
$$

where $\mathrm{Q}_{n}, \Delta \mathrm{T}_{n}$ and $m_{f}$ are the heat, melting point depression of $\mathrm{n}^{\text {th }}$ step and the dry mass of pulp fibres, respectively.

Maloney [153] applied it to pulp fibres for measuring the amount of freezing bound water (FBW) and non freezing bound water (NFW) present in the micropores of the fiber wall. 
Drawbacks in using thermoporosimetry for pore analysis of aqueous pulp suspension includes distortion of cell wall by ice crystal growth, depression of freezing point of water due to osmotic pressure and inadequate range to cover macropores [152]. Smaller liquid droplets having higher surface to volume ratio exhibit higher effective vapour pressure. This effect can cause strong depression of the freezing point of liquids which are dispersed in fine porous materials.

The issues related to the freezing of water were addressed by replacing water with acetone and then acetone with cyclohexane [225]. FSP was described as

$$
\begin{gathered}
\mathrm{FSP}=\mathrm{NFB}_{\text {chex }}+\mathrm{FB}_{\text {chex }}+\mathrm{B}_{\text {chex }} \\
\mathrm{TB}_{\text {chex }}=\mathrm{NFB}_{\text {chex }}+\mathrm{FB}_{\text {chex }}
\end{gathered}
$$

Where $\mathrm{NFB}_{\text {chex }}, \mathrm{FB}_{\text {chex }}, \mathrm{B}_{\text {chex }}$ and $\mathrm{TB}_{\text {chex }}$ are the non-freezing cyclohexan, freezebound cyclohexan, bulk cyclohexane and total-bound cyclohexan, respectively.

\section{Remarks}

Differential scanning calorimetry measurements were carried out on DSC Q100 equipped with $\mathrm{N}_{2}(1)$ cooling system (TA Instruments, New Castle). 6 to $10 \mathrm{mg}$ of solvent exchanged fibrous suspension was sealed hermetically in an aluminium pan. Chex:Fibres ratio was kept around 80:20. Sealed pans were heated from -30 to $7^{\circ} \mathrm{C}$ using isothermal step melting procedure.
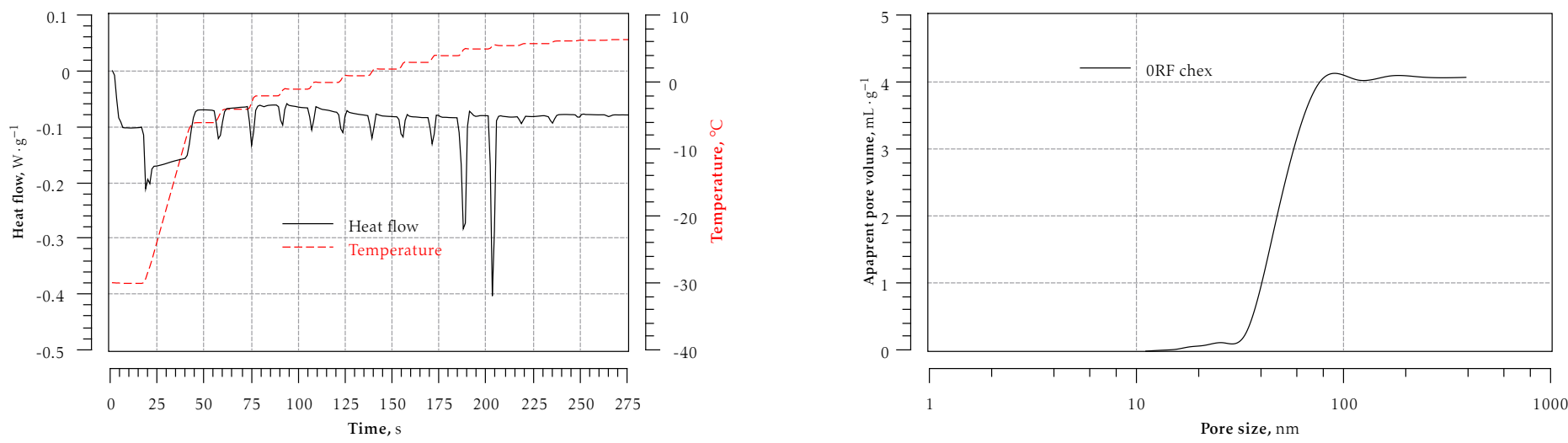

Figure D.1: DSC curve of solvent exchanged ORF

Melting occurred mostly between $5^{\circ} \mathrm{C}$ and $5.5^{\circ} \mathrm{C}$ (as shown by two peaks). 
Firstly it was thought that perhaps the final two peaks were the peaks of bulk melting, therefore it was required to recalibrate the system. After recalibration similar peaks were obtained at the same temperature. It was therefore thought that perhaps melting is taking place between $5{ }^{\circ} \mathrm{C}$ and $5.5^{\circ} \mathrm{C}$ so more steps were required in the described temperature range. The noise was subtracted from the resulting pore volume as Maloney suggested. Even with that the amount of pore volume obtained was quite high. The only thing required as suggested by Maloney is to calibrate DSC using cyclohexane to obtain a melting temperature with an accuracy of $\pm 0.02^{\circ} \mathrm{C}$. 


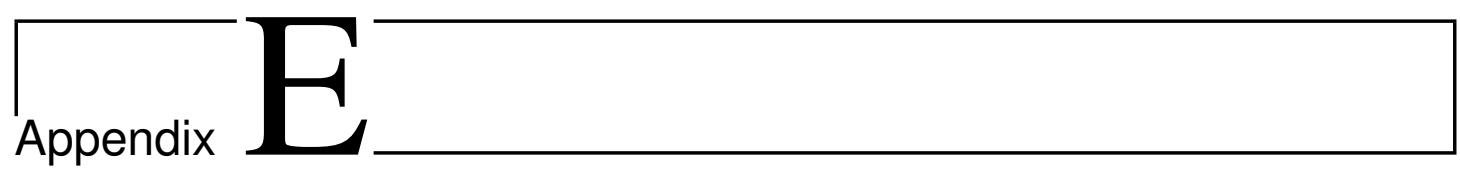

\section{Résumé de la thèse en Français'}

\section{Déforestation}

Les forêts comme toutes les ressources naturelles sont limitées. La déforestation est le terme utilisé pour décrire la diminution de l'espace forestier. Le taux de déforestation de 1990 à 2000 était de 8,23 Mhectare par an, puis de 5,21 Mhectare par an de 2000 à 2010 (voir la figure E.1) [80]. La déforestation est la plus importante aux Etats-Unis et en Afrique. La raison principale de la déforestation en Afrique est la dépendance de la population pour le bois comme source d'énergie [24]. La situation a été améliorée lors de la dernière décade en raison de plantation d'arbres à grandes échelles. La notion de la durabilité des forêts est acceptée partout mais la disparition de 5,21 Mh / an de terres forestières est alarmante et nécessite davantage d'efforts en vue du développement durable.
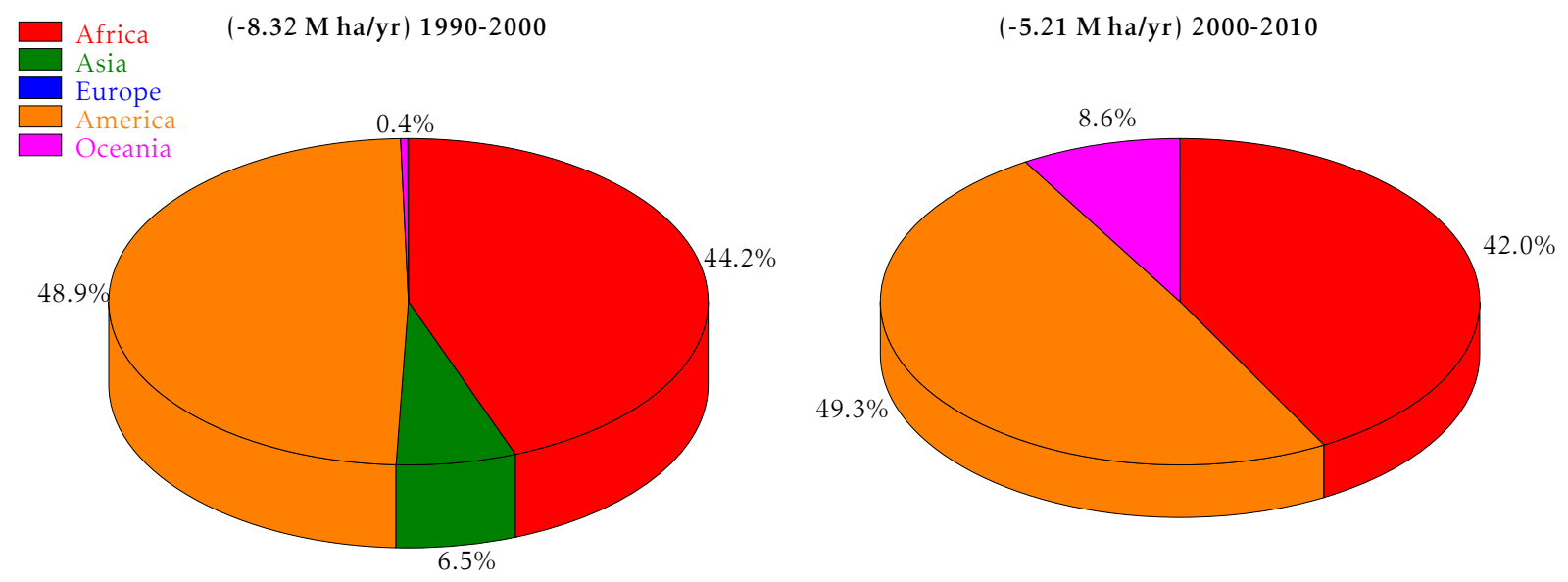

Figure E.1: Annual deforestation rate 


\section{Pâtes de bois}

Le bois est la principale matière première cellulosique utilisée pour la production de pâtes et papiers. La production annuelle de pâte chimique varie entre 120 à 130 Mt / an (cf. figure E.2) [80]. L'utilisation de la pâte recyclée a été considérablement augmentée en raison de la réglementation des agences de protection de l'environnement en vue d'établir des sociétés à faibles empreintes carbone. La production de pâte à papier recyclée a augmenté jusqu'en 2010 pour se stabiliser à environ $73 \mathrm{Mt} /$ an. La stabilisation de la production de pâte recyclée est liée à la limitation de son utilisation en raison de la détérioration de la résistance et des propriétés optiques des papiers recyclés.

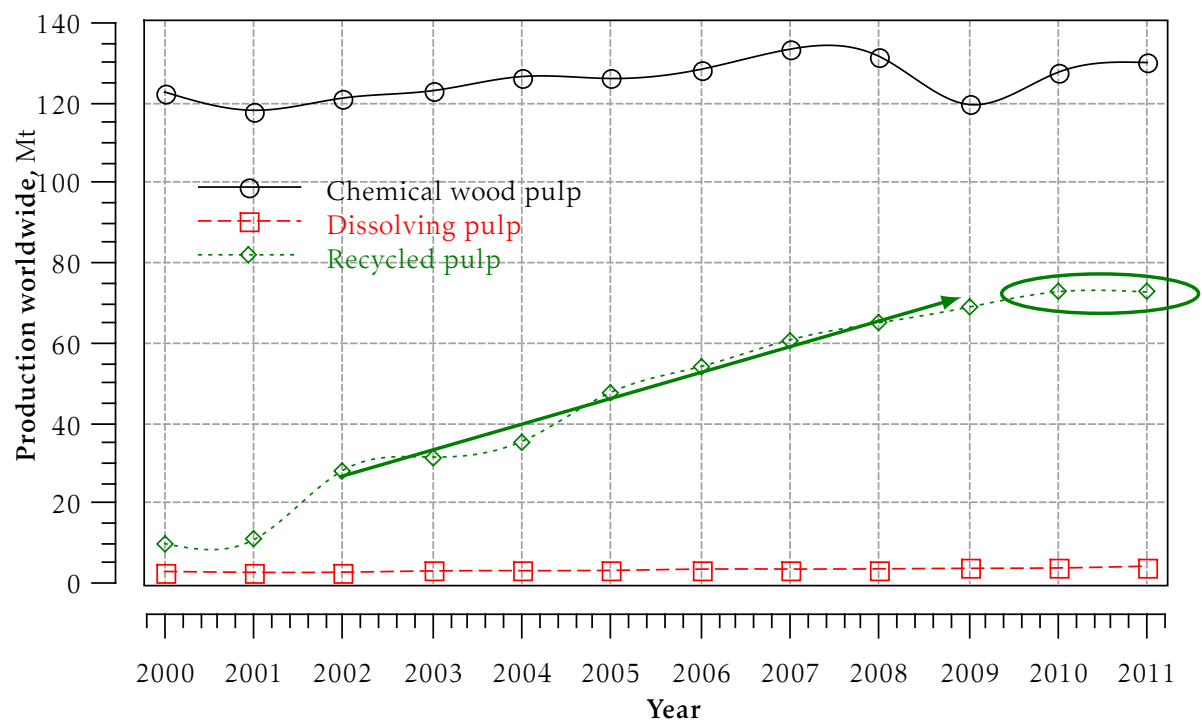

Figure E.2: Production of wood pulps

\section{Taux de recyclage}

Le recyclage est généralement décrit en termes de taux de recyclage. Il est défini comme étant le rapport entre la masse des papiers de récupération pour le recyclage et la consommation de papier.

$$
\text { Recycling rate }=\frac{\text { mass of recovered paper used for recycling }}{\text { mass of paper consumed }}
$$

Le taux de récupération est le terme utilisé aux Etats-Unis qui est équivalent au taux de recyclage utilisé en Europe. Le taux de recyclage pour l'Europe était de 70.4\% et le taux de recouvrement des Etats-Unis était de 66.8\% en 2011 [21]. Le recyclage du papier en Europe et aux États-Unis a augmenté au cours de la 
décennie précédente. A partir de 2009, le taux de recyclage de l'Europe a été stabilisé à 70\% environ comme indiqué dans la figure E.3.

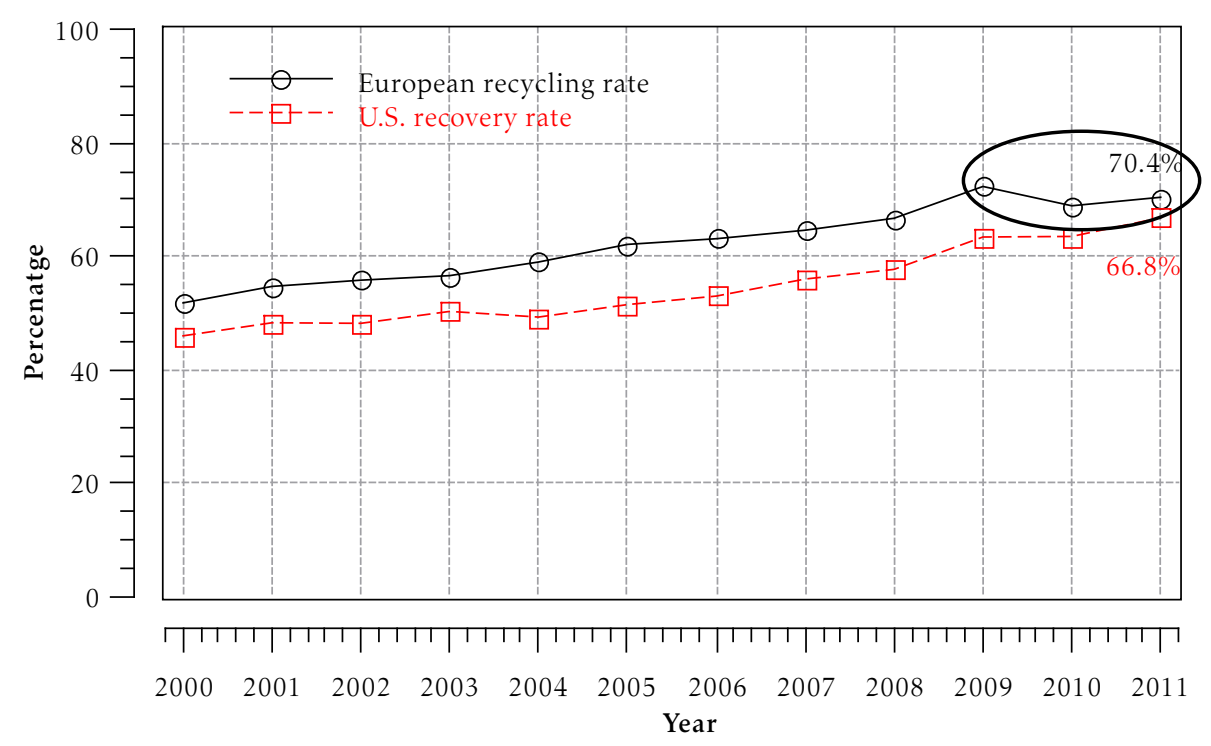

Figure E.3: Recycling rate of Europe and USA

L'autre terme utilisé pour décrire l'utilisation de la pâte recyclée est le taux d'utilisation. Il peut être défini par l'expression suivante

$$
\text { Utilisation rate }=\frac{\text { mass of recovered paper consumed }}{\text { mass of paper produced }}
$$

Le taux d'utilisation en Europe était de 50.1\% en 2011 [21]. Le taux d'utilisation de la pâte recyclée dans des papiers à haute valeur ajoutée est très faible. Les papiers à usage graphique hors papier journal atteint $31.6 \%$ du volume du marché des papiers et cartons en Europe. Le taux d'utilisation de la pâte recyclée pour les papiers graphiques à l'exclusion du papier journal a été de 10.5\% en 2011 [21]. L'utilisation de pâte recyclée dans du papier de haute qualité peut engendrer des gains financiers et, par conséquent augmenter le taux d'utilisation. Si nous prenons $€$ 633,52/t et $€$ 143,57/t [88] comme les prix de la pâte de résineux et de vieux journaux et du papier magazine respectivement, les économies réalisées en termes d'euros par tonne peuvent être évaluées comme suit 
Table E.1: Cost analysis of recycled pulp blends

\begin{tabular}{l|cccc} 
Recycled pulp & $25 \%$ & $50 \%$ & $75 \%$ & $100 \%$ \\
\hline Savings, €/t & 71.0 & 193.5 & 316.0 & 438.5
\end{tabular}

D'autres bénéfices à considérer consistent en la réduction de la demande de pâte de bois, du besoin de produits chimiques, des effluents, une diminution en besoins énergétiques et en eau.

Le bois est utilisé comme matière première pour l'industrie des pâtes et papiers. Il se développe sous forme de couches concentriques, appelées cernes annuels. La croissance dépend essentiellement des variations saisonnières. La croissance en début de saison constitue un anneau de bois de printemps par opposition au bois d'automne (cf. figure E.4).

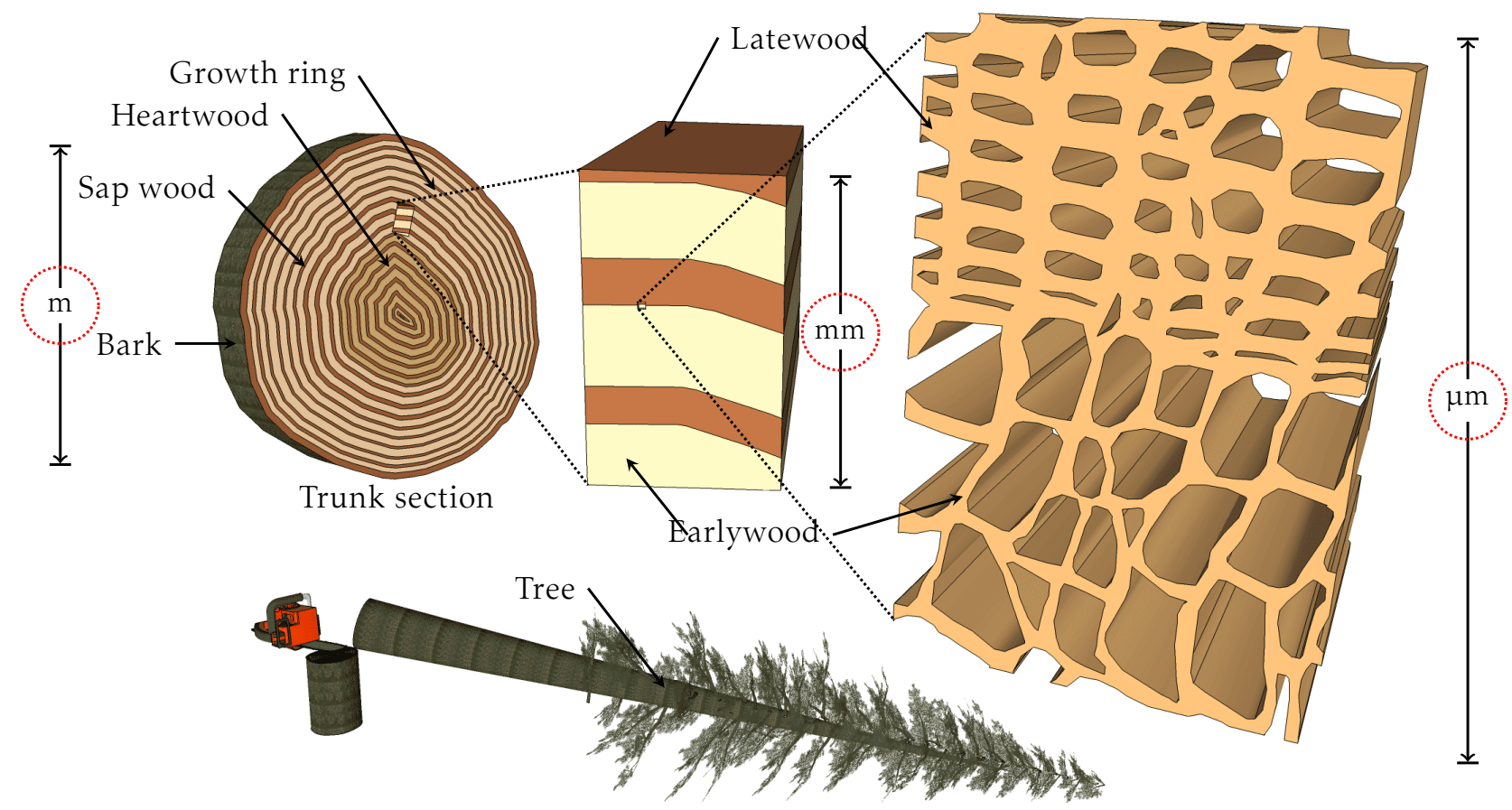

Figure E.4: Hierarchy of wood structure

Dans le bois, les fibres sont liées entre elles par la lamelle mitoyenne composée majoritairement de lignine (ML). La fibre de bois peut être divisée en une couche primaire extérieure $(\mathrm{P})$ et dans une couche secondaire $(\mathrm{S})$. La partie creuse à l'intérieur est appelée lumen. Les couches secondaires peuvent être subdivisées en couches S1, S2 et S3 (cf. figureE.5) [159]. 


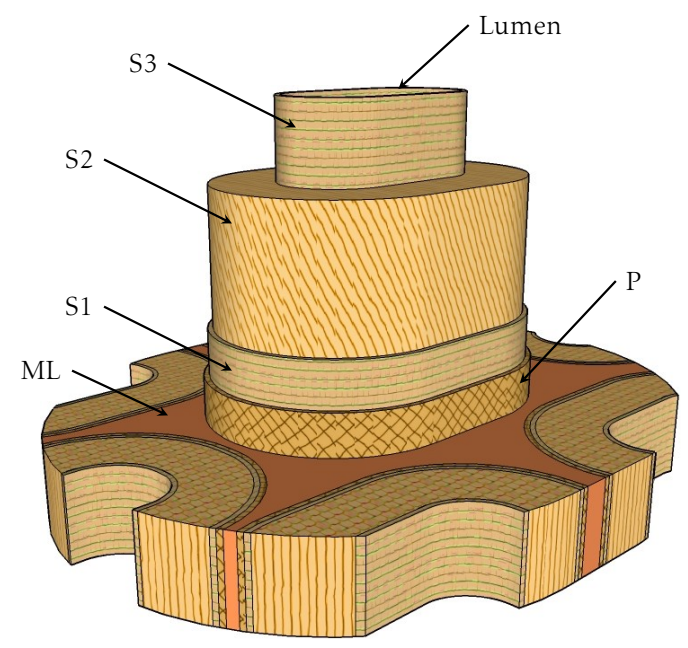

Figure E.5: Schematic cell wall layers model of a latewood tracheid adapted from [159]

L'épaisseur de la couche S2 correspond à 80\% de l'épaisseur totale de la paroi cellulaire (cf. figure E.6). Par conséquent, tout changement de structure et de composition de cette couche S2 modifie de façon significative les caractéristiques mécaniques de la fibre. Les distinctions entre couches sont liées à l'orientation des microfibrilles. Celles-ci sont constituées de chaines de cellulose agrégées [90, 84, 77, 110]. L'orientation des microfibrilles de cellulose est très importante car elle régit les propriétés physiques des cellules du bois [37].

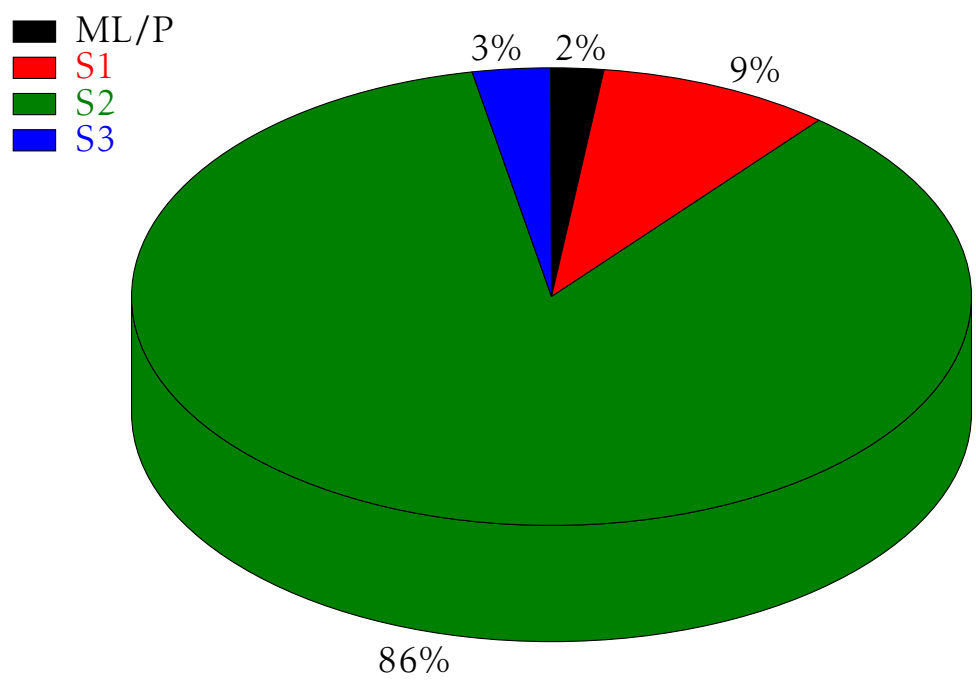

Figure E.6: Schematic cell wall layers model of a latewood tracheid adapted from [159]

Pendant la cuisson, la lignine est éliminée de la lamelle mitoyenne ML ainsi les fibres se séparent les unes des autres. La délignification de la paroi des fibres 
a lieu par étapes. La méthode Kerr décrit la localisation des constituants chimiques et leur dissolution de la paroi de la fibre [81]. La lignine est dissoute dans la paroi de la fibre en même temps que les hémicelluloses. La paroi des fibres devient poreuse et les fibrilles sont agrégées pour former des macrofibrilles (cf. figure E.7).

Lignin-hemicellulose
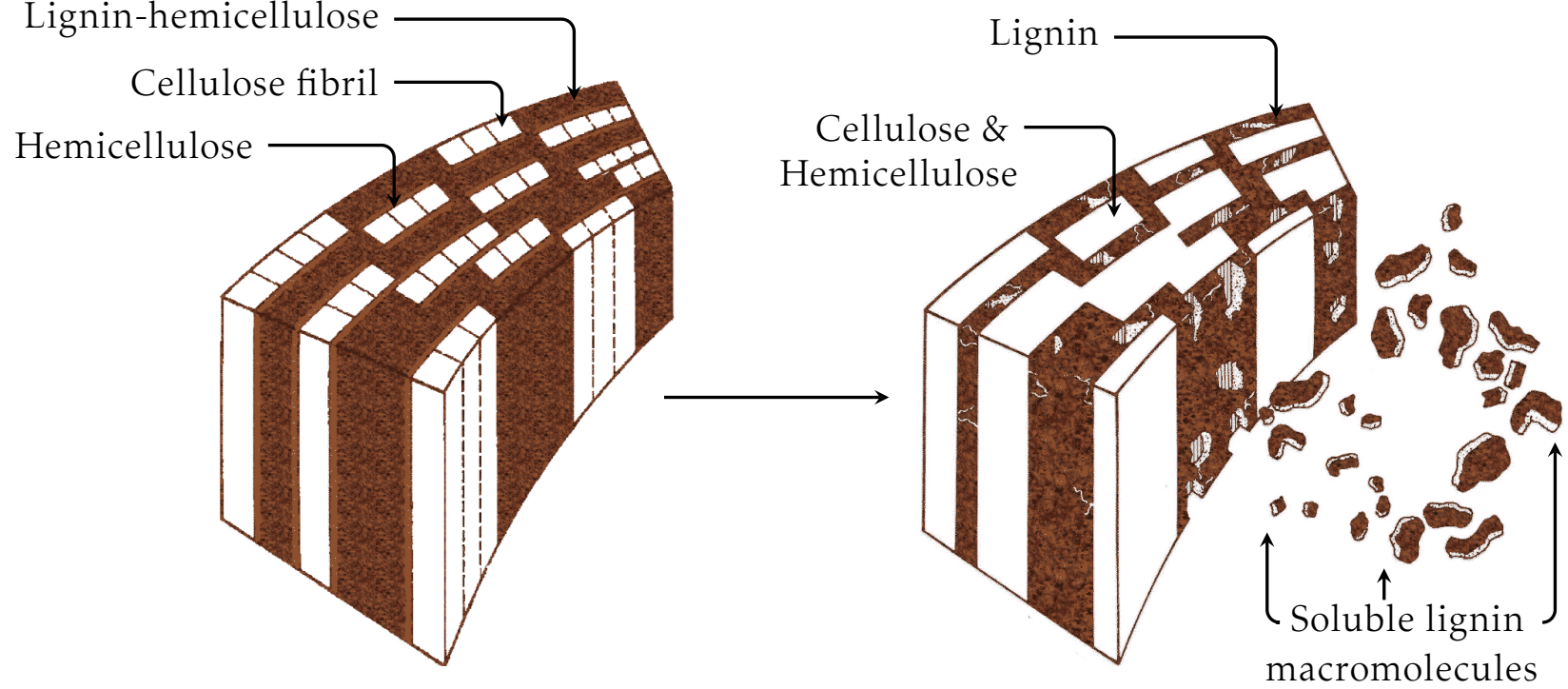

Figure E.7: Dissolution of lignin out of the fibre wall adapted from [81]

Les fibres deviennent flexibles, conformables et peuvent se collapser. Le potentiel de liaison des fibres est en conséquence amélioré (cf. figure E.8).

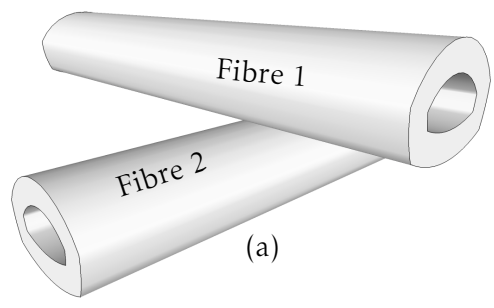

Flexibility

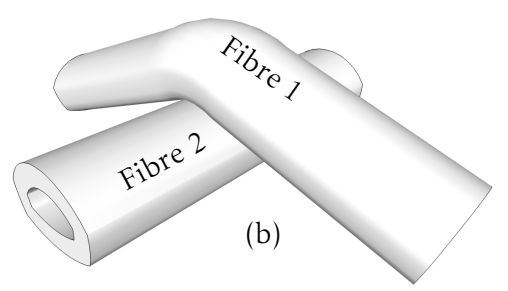

Conformability

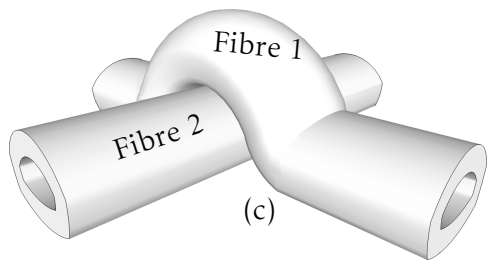

Collapsibility

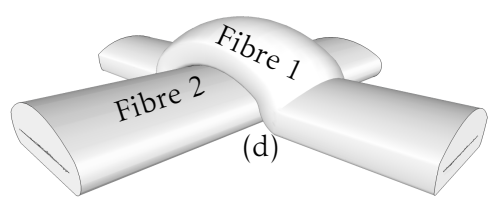

Figure E.8: Schematic depiction of fibre shapes during bonding. In (a) both fibres are stiff, in (b) Fibre 1 is flexible, in (c) Fibre 1 is conformable whereas in (d) both fibres are collapsible 
Les fibres subissent de nombreuses contraintes durant leur croissance dans le bois et lors de leur mise en pâte. En conséquence, les couches dans les parois des fibres peuvent présenter des dislocations. Elles causent des microcompressions et des ruptures. En raison de ces dislocations localisées, les parois des fibres se déforment et il apparaît une courbure globale, des coudes et des points faibles. La capacité de résistance au chargement mécanique des fibres est donc fortement diminuée. La courbe contrainte-déformation d'une fibre sans défaut est élastique, alors que celle d'une fibre déformée est non linéaire et inélastique (cf. figure E.9).

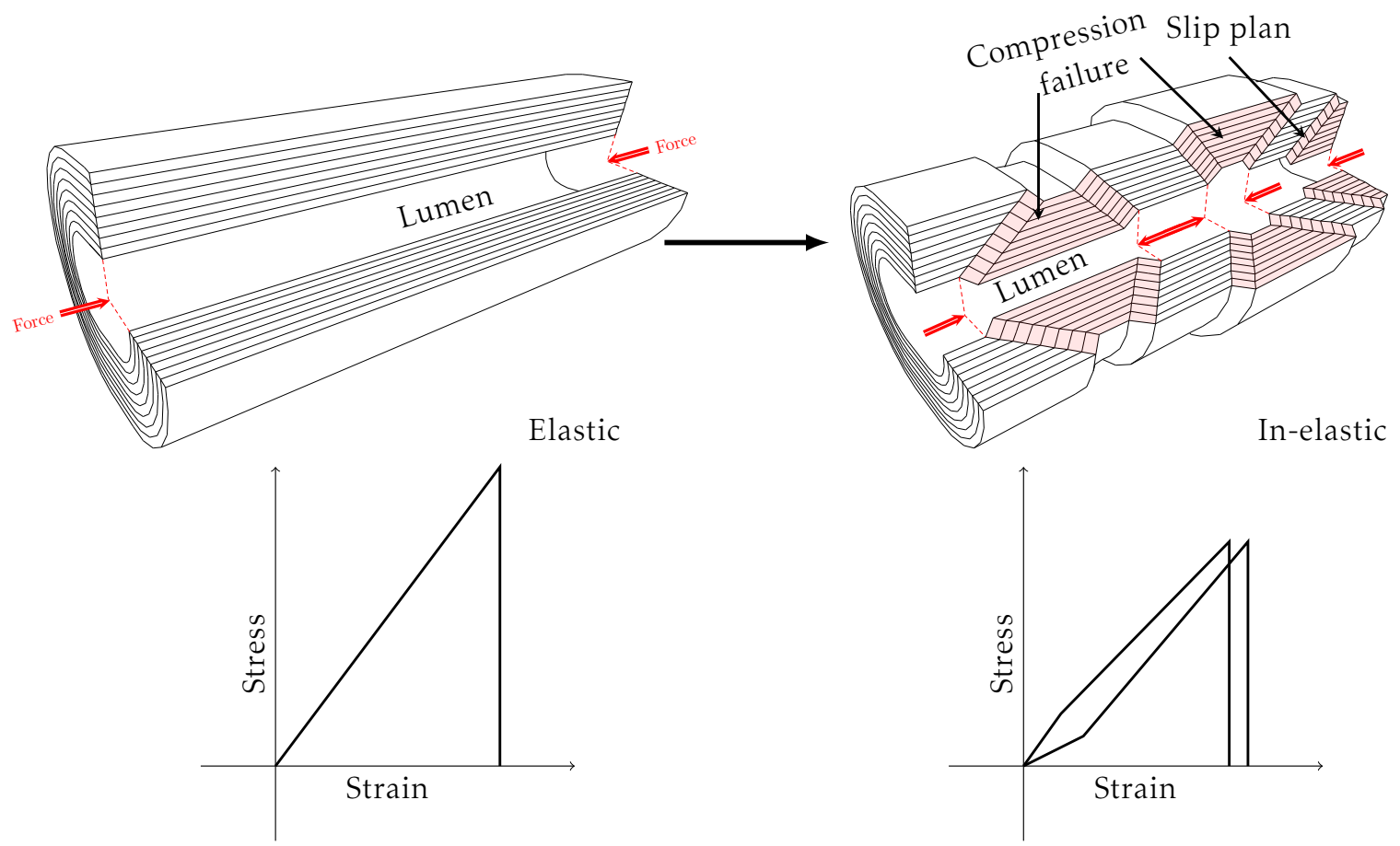

Figure E.9: Slip plane and compression failure adapted from [227, 125]

La souplesse des fibres humides est généralement liée au gonflement de la fibre. Ce dernier est défini en termes de capacité des fibres pour 'retenir' l'eau. Pour des fibres humides, l'eau est présente dans le lumen, à l'intérieur des pores et liée à la surface (cf. figure E.10). 


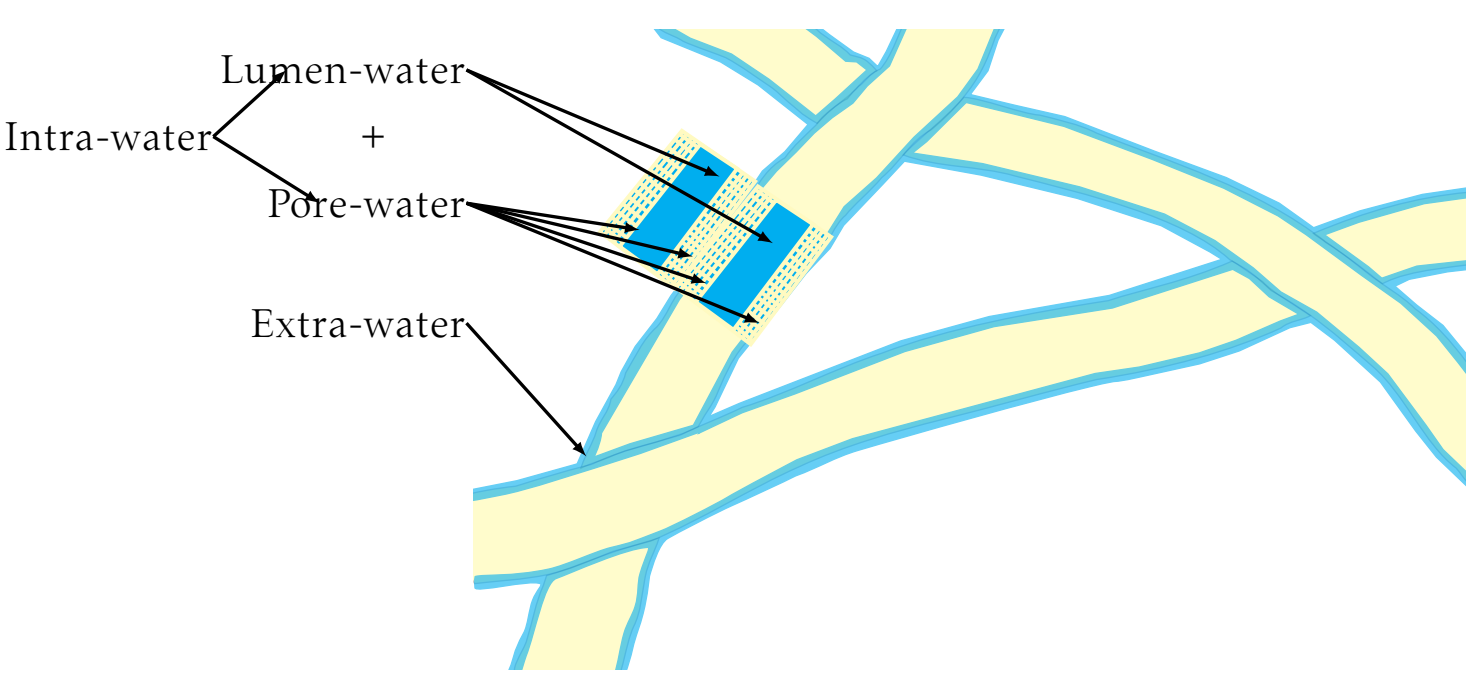

Figure E.10: Wet swollen fibres

La valeur de rétention d'eau (WRV) donne la quantité d'eau retenue par la suspension de pâte après centrifugation tandis que le point de saturation des fibres (FSP) mesure l'eau interstitielle seule. Le FSP est mesuré en utilisant une technique d'exclusion de soluté. Le principe de mesure du FSP est illustré à la figure E.11.
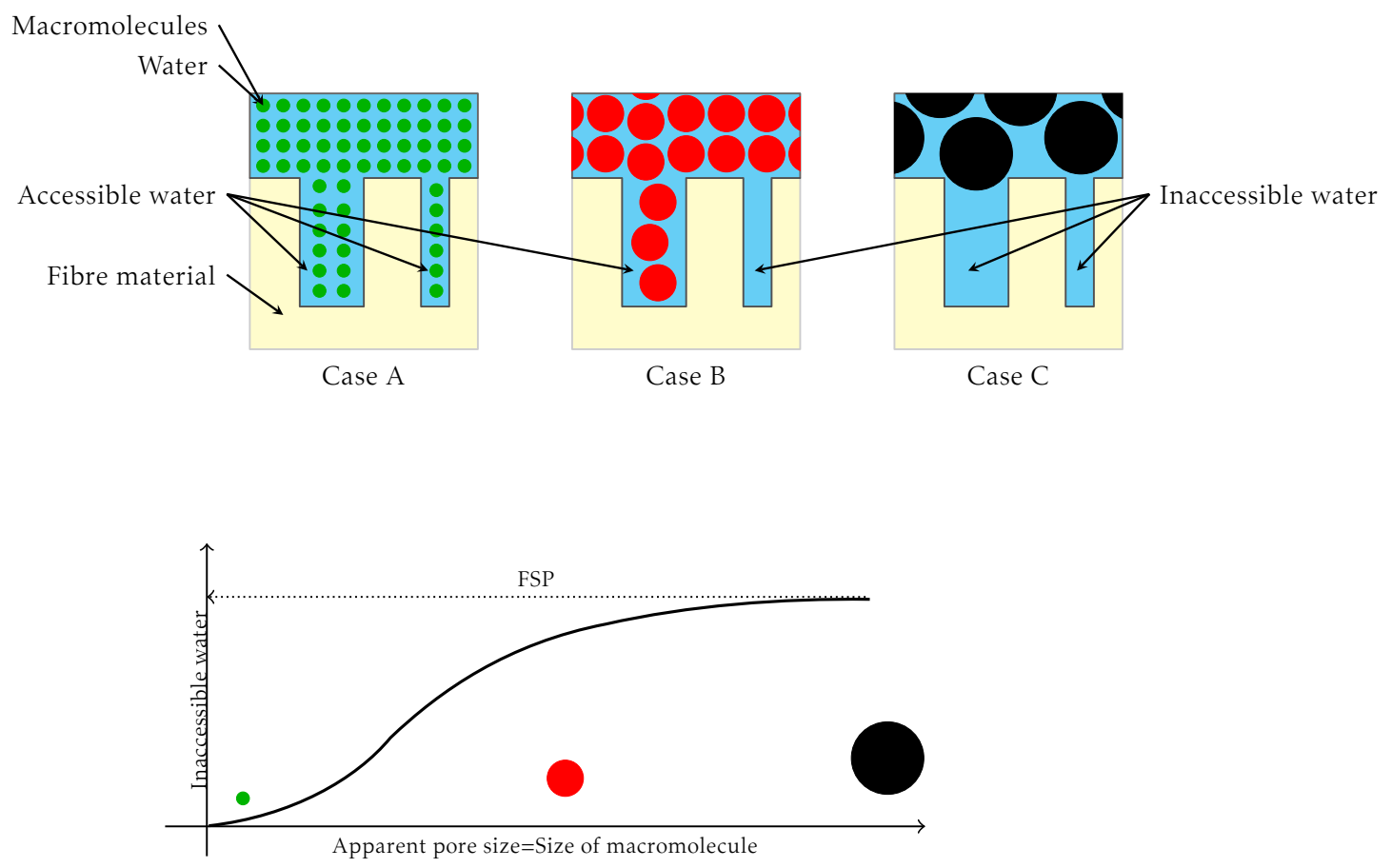

Figure E.11: Principle of solute exclusion technique for the measurement of fibre saturation point adapted from [211]

Des molécules de soluté, n’interagissant pas, et de dimensions connues, sont 
utilisées pour remplir les pores de la paroi cellulaire de la fibre. À partir de la différence des concentrations de la solution de soluté, le volume de l'eau interstitielle inaccessible est calculé. Le volume des pores inaccessibles augmente avec une augmentation de la taille des molécules de soluté. Le point de saturation correspondant au volume poreux total est appelé point de saturation des fibres (FSP).

\section{Etude des fibres et des pâtes}

Le racornissement de la fibre est le terme utilisé pour décrire précisément cette tendance. Par définition, il correspond à la diminution de la valeur de rétention de l'eau au cours de recyclages répétés. Le phénomène de racornissement de la fibre est normalement tenu pour responsable de la perte de résistance de la fibre et/ou des liaisons au cours de recyclages.

En situation réelle, le produit final contient des fibres vierges et des fibres recyclées, obtenues à partir d'un nombre variable de recyclage (cf. figure E.12). L'incorporation de fibres recyclées est principalement contrôlée par les caractéristiques requises du produit final. La résistance mécanique détériorée et les propriétés optiques constituent les verrous quant à son utilisation dans du papier de grande valeur ajoutée.

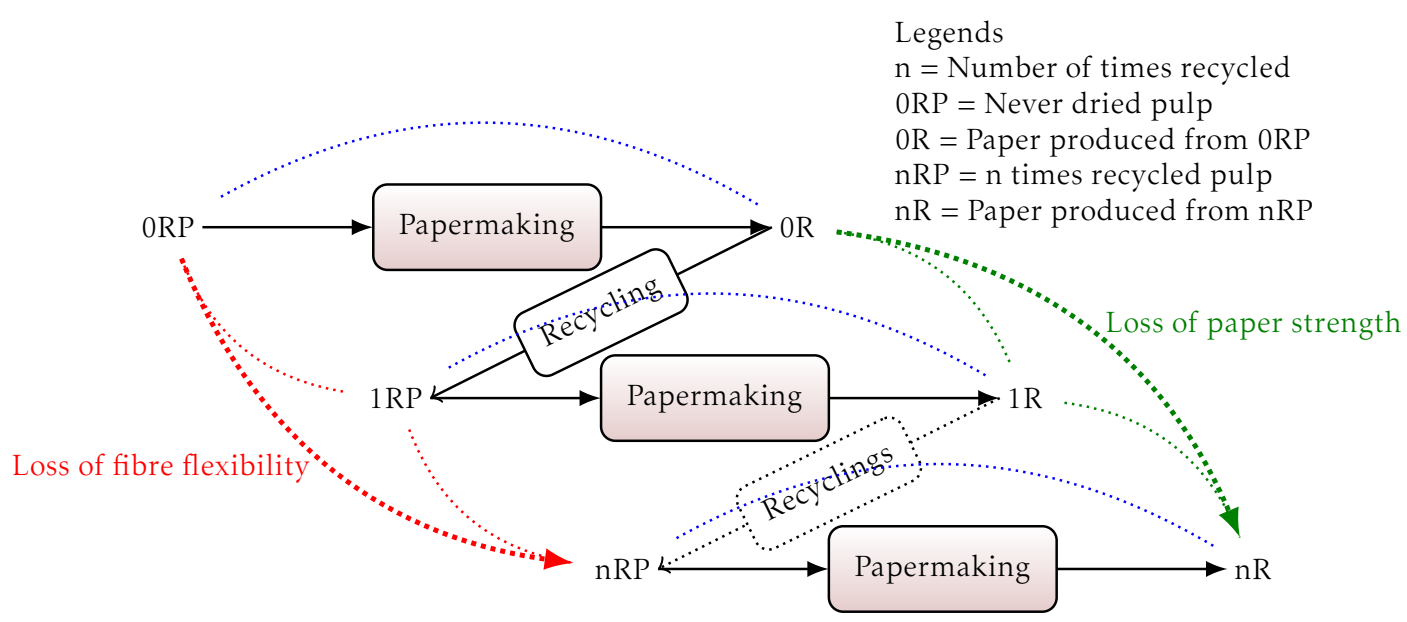

Figure E.12: Cycles of recycling

La question centrale de cette étude était : "Peut-on utiliser de la pâte recyclée dans le papier de grande valeur et quels sont les limitations en termes de qualité?". La démarche adoptée a consisté à caractériser les modifications de la structure des fibres, des pâtes et des papiers en fonction des recyclages. 
Pour cette étude, une pâte industrielle, jamais séchée, constituée d'un mélange de fibres de résineux, a été homogénéisé dans un raffineur Valley, puis désintégrée. Pour obtenir des fractions de fibres, les fines (les éléments de moins de 150 microns) ont été retirées de la pâte à l'aide d'un fractionnement sur un appareil Macnett. Les formettes ont été réalisées à l'aide d'un appareil Rapid Köthen à partir de la pâte désintégrée pour obtenir un papier. Les feuilles d'essai ont été déchiquetées et remouillées pendant 4h dans l'eau chaude. Le processus de désintégration, le fractionnement, la prise de feuille d'essai, le broyage et le remouillage ont été réalisés afin d'obtenir des pâtes, des fibres et des papiers recyclés jusqu'à 10 fois (cf. figure E.13).

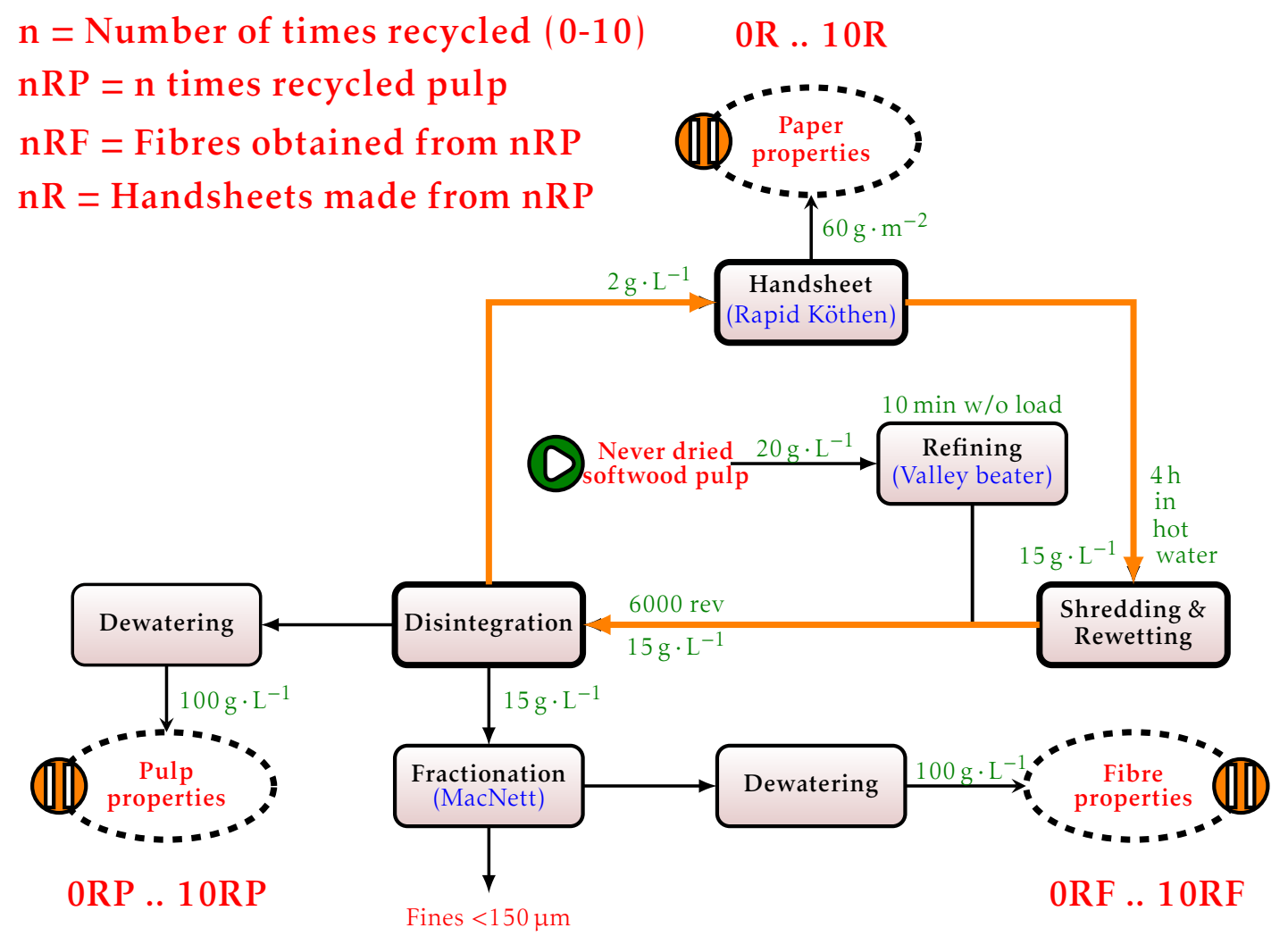

Figure E.13: Block diagram of the preparation process of recycled pulps, fibres and papers

Les analyses chimiques, principalement le degré de polymérisation de la cellulose $\left(\overline{\mathrm{DP}}_{v}\right)$, la teneur en pentosanes et la teneur en lignine résiduelle, ont été effectuées. Il a été constaté que le processus de recyclage ne modifie pas la quantité de constituants chimiques mentionnés ci-dessus (cf. tableau E.2). 
Table E.2: Chemical properties of pulps

\begin{tabular}{c|ccc}
$\begin{array}{c}\text { Recycle } \\
\text { number }\end{array}$ & $\overline{\mathrm{DP}}_{v}$ & $\begin{array}{c}\text { Pentosans } \\
\text { (SCAN-CM 15:88) }\end{array}$ & $\begin{array}{c}\text { Kappa number } \\
\text { (T 223 cm-10) }\end{array}$ \\
\hline 0 & 910 & 3.5 & 0.9 \\
1 & 891 & 3.9 & 0.9 \\
10 & 891 & 3.6 & 0.8
\end{tabular}

\section{Morphologie}

L'analyse morphologique a été effectuée à l'aide de l'appareil MorFi LB01. Un échantillon de pâte en suspension contenant $0,3 \mathrm{~g}$ de masse sèche, est introduit dans la cuve de dilution puis il est automatiquement dilué et homogénéisé. Elle passe ensuite à travers la cellule de mesure, où les images sont acquises. L'analyse d'image est effectuée pour calculer les propriétés morphologiques.

La nomenclature concernant la morphologie des fibres est indiquée dans la figure E.14.

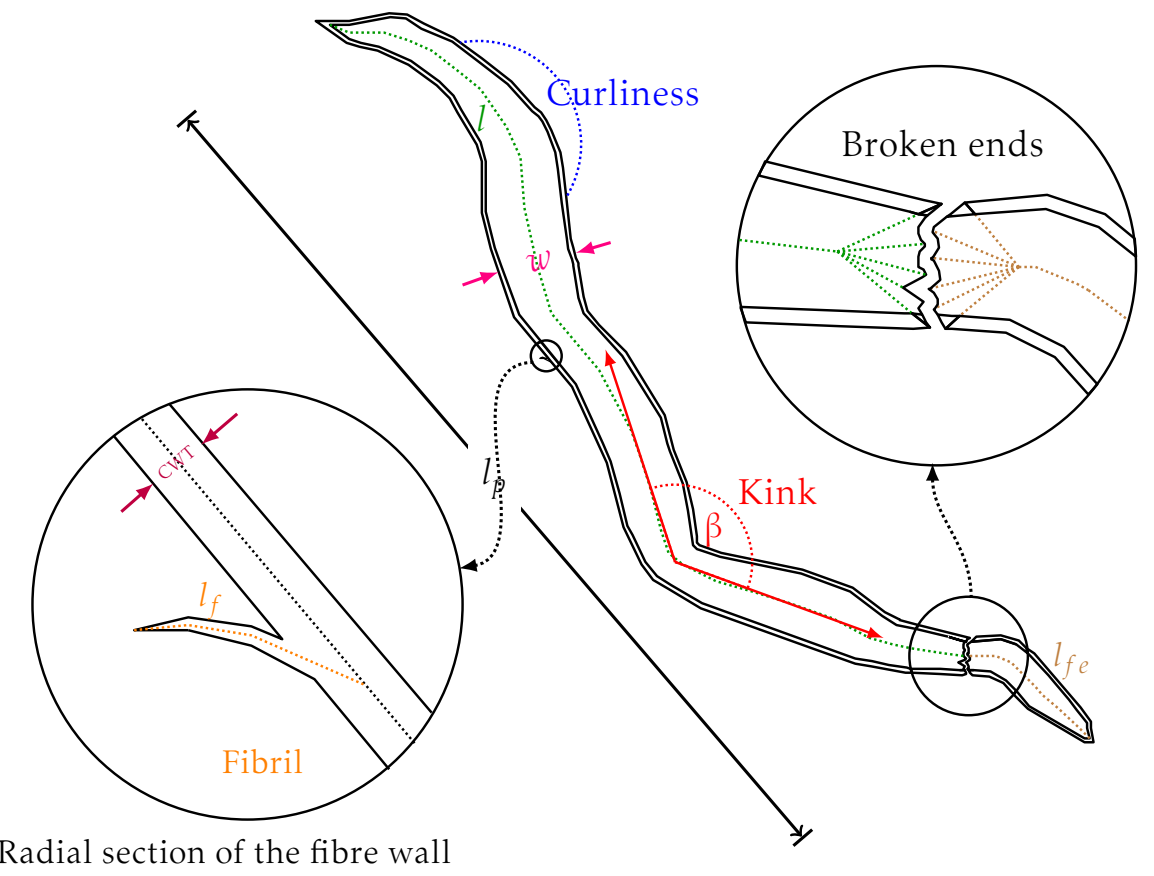

Figure E.14: Nomenclature of morphological properties, where $l, l_{p}, l_{f e}, l_{f}, w$, $\beta$ and $c w t$ are the skeletal length, projected length, fine element length, fibril length, width, kink angle and cell wall thickness, respectively.

La longueur moyenne des fibres et les distributions des longueurs n'ont pas 
évolué au cours des recyclages. La largeur des fibres a toutefois diminué comme le montre la figure E.15. Le retrait des fibres lié au premier recyclage représente 60 à $65 \%$ du retrait total. La différence entre la largeur des fibres seules et des fibres contenues dans la pâte, est due à la perte de fibres courtes durant le fractionnement.

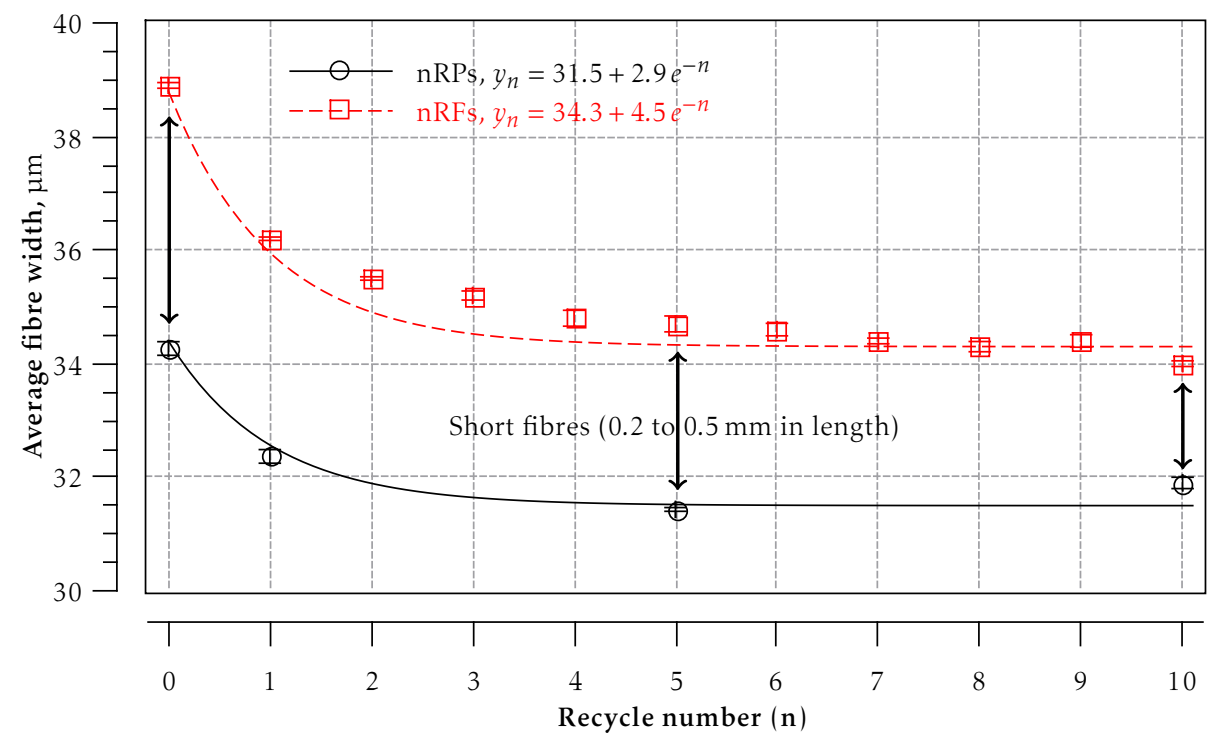

Figure E.15: Evolution of fibre width of pulps and corresponding fibres during recycling

Une observation approfondie des résultats a révélé que la fraction des fibres ayant une largeur de fibre supérieure à $35 \mu \mathrm{m}$, est réduite avec les recyclages (cf. figure E.16).

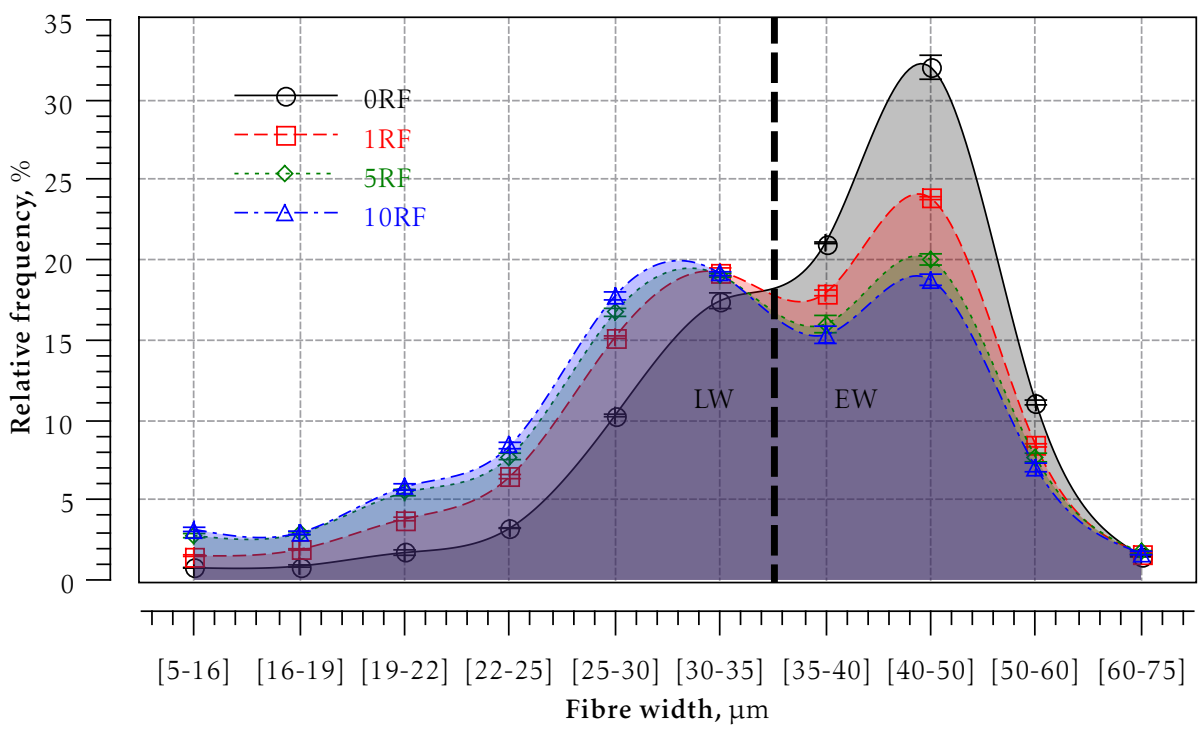

Figure E.16: Effect of recycling on width distributions of fibres 
A partir des distributions de longueur-largeur de 0RF et 10RF, il est montré que les longueurs des fibres d'été et d'automne, sont similaires (cf. figure E.17).
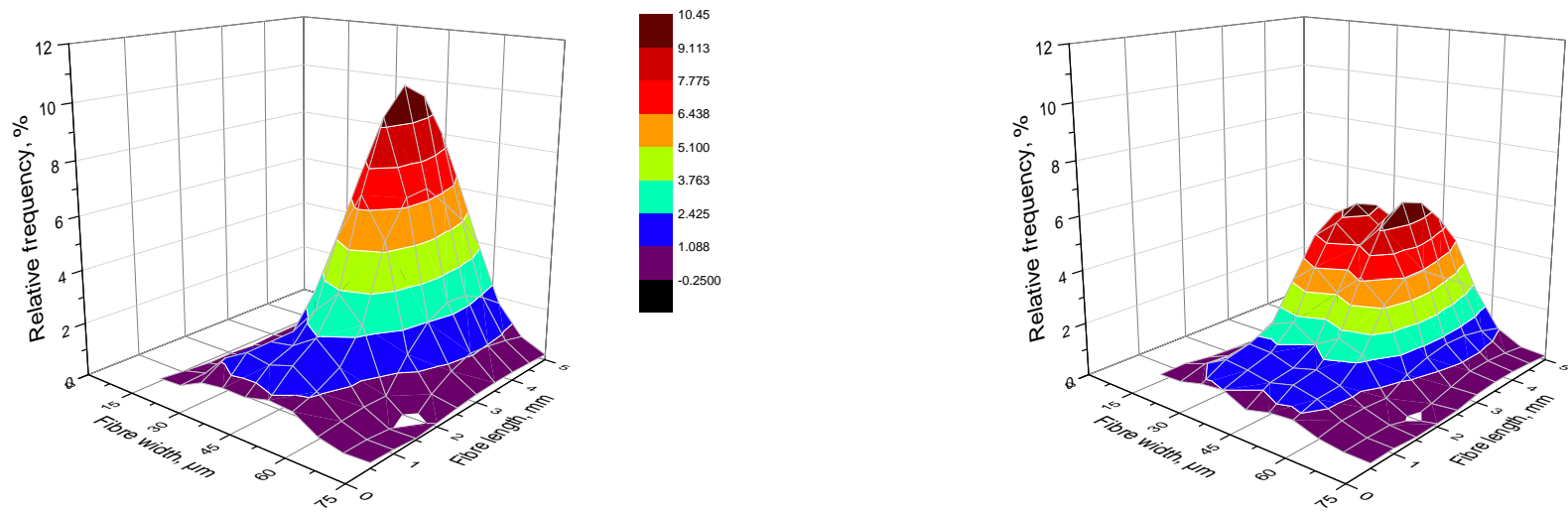

Figure E.17: Length-width distribution of never dried fibres (left) and ten times recycled fibres (right)

La caractérisation de l'épaisseur de la paroi de la fibre a été réalisée en utilisant le dispositif MORFI CWT. Une diminution de l'épaisseur de la paroi cellulaire avec le recyclage a été constatée. La réduction d'épaisseur de la paroi cellulaire était importante lors du premier recyclage. Environ 60\% de retrait de la paroi cellulaire a eu lieu lors du premier recyclage (cf. figure E.18).

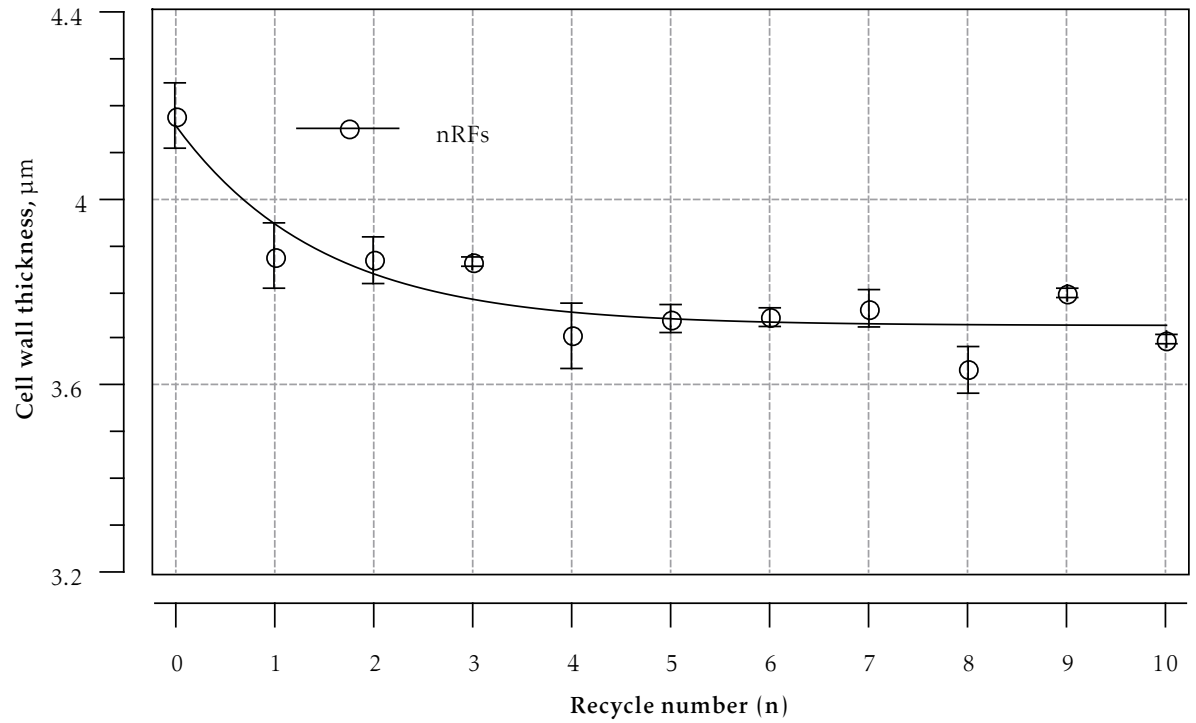

Figure E.18: Evolution of cell wall thickness of fibres during recycling

La proportion de fibres ayant une épaisseur de paroi cellulaire supérieure à 
$5 \mu \mathrm{m}$ a diminué dans la distribution (cf. figure E.19). Par conséquent, on peut dire que les parois des fibres LW se contractent avec le recyclage.

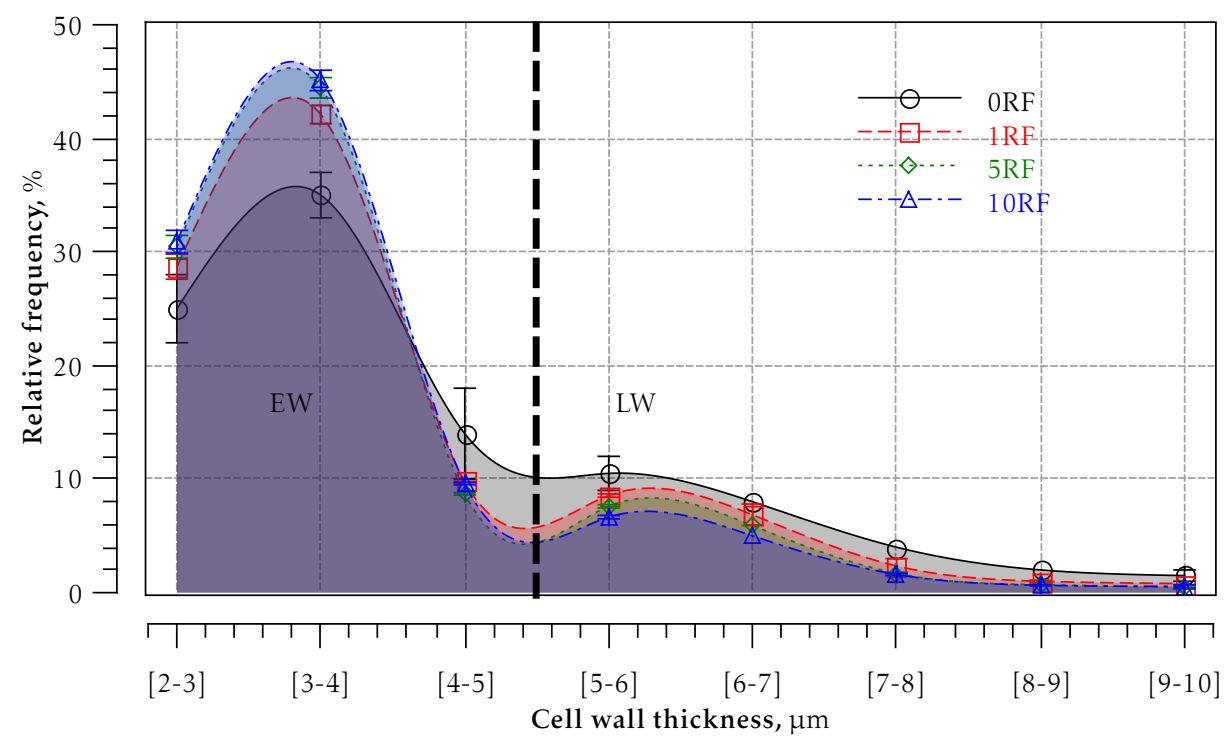

Figure E.19: Effect of recycling on cell wall thickness distribution of fibres

Pour valider la densification de fibres avec le recyclage, l'ESEM a été utilisée. Les fibres séchées une fois ont d'abord été coupées par une lame de rasoir, puis montées à la verticale sur le porte-échantillon, comme indiqué à la Figure E.20.

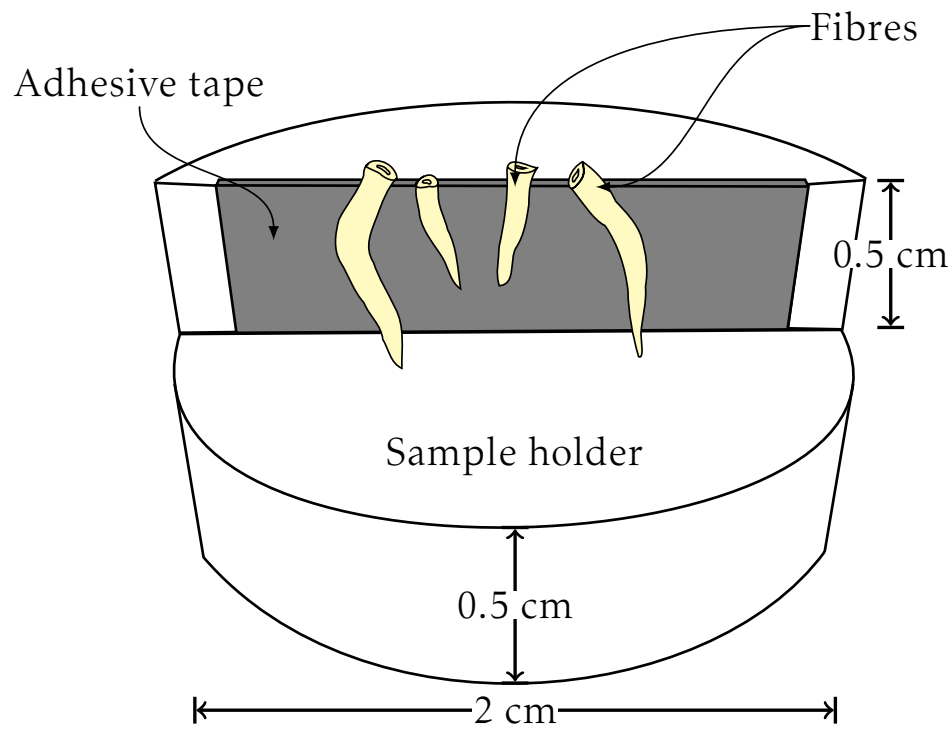

Figure E.20: Prepared sample holder for cross section analysis

Une étude cinétique de la stabilisation des dimensions de la section des fibres lors du séchage a été réalisée afin de pouvoir imager les fibres dans un état sta- 
tionnaire. En accord avec la figure E.21, un délai de 15 minutes a été considéré entre le début du séchage et la prise de vue.

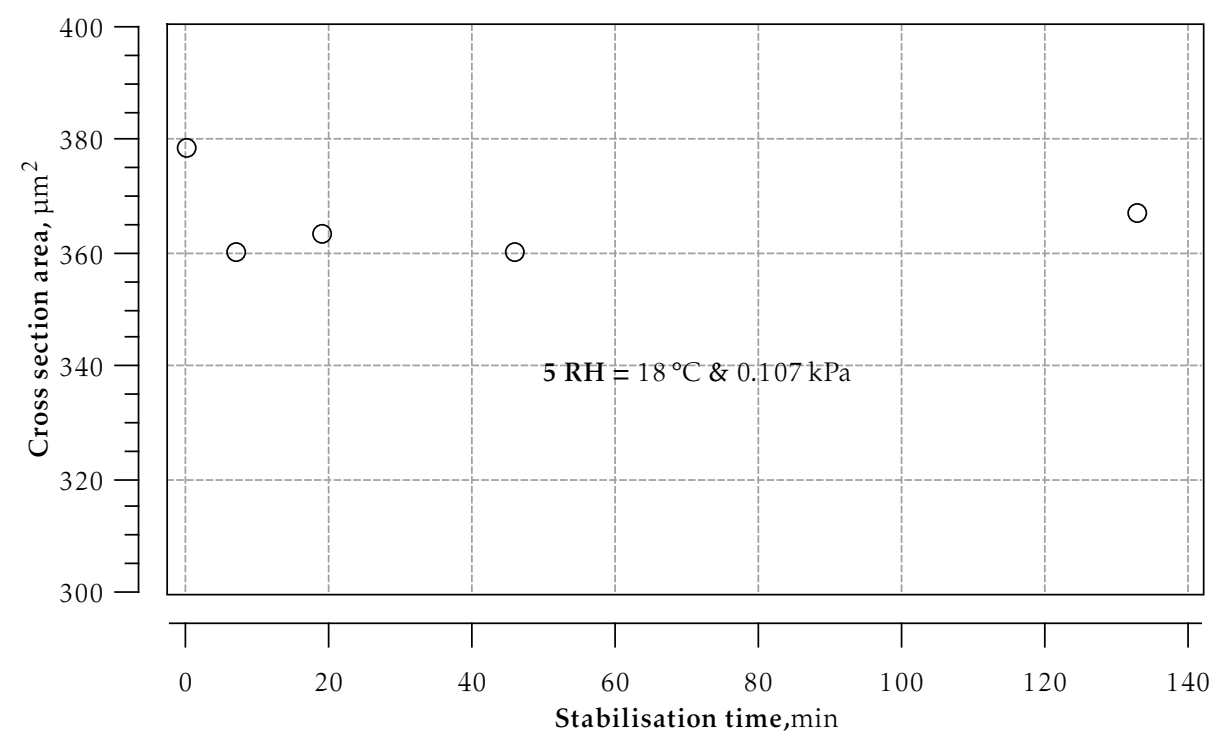

Figure E.21: Evolution of fibre cross section area with time of drying

Les conditions de température et de pression dans la chambre de l'ESEM ont été ajustées afin d'humidifier complètement les fibres pendant le remouillage et de les sécher complètement lors des cycles de séchage (cf. figure E.22).

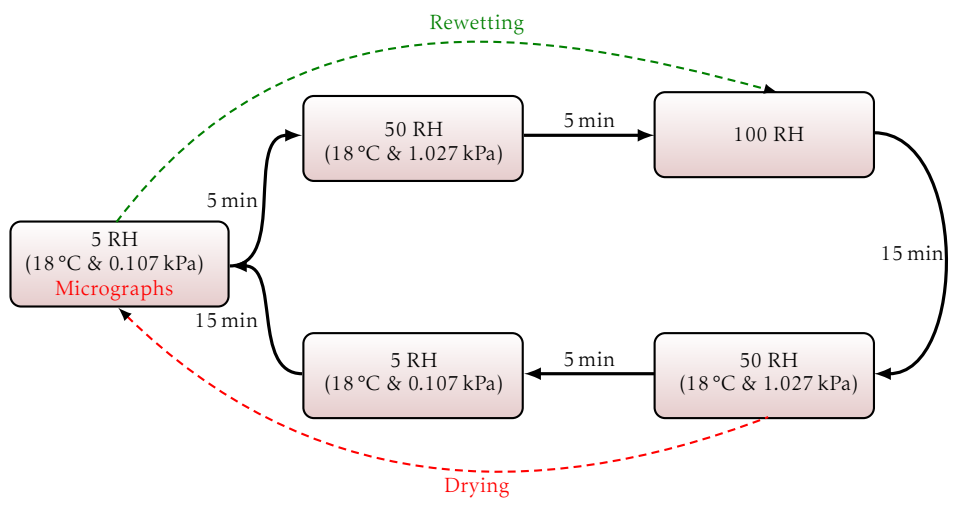

Figure E.22: Evolution of fibre cross section area with time of drying

Les micrographies des fibres à l'état sec ont révélées des résultats similaires à ceux obtenus lors des analyses morphologiques. Les parois des fibres rétrécissaient au fur et à mesure des cycles de remouillage et de séchage (cf. figure E.23). 

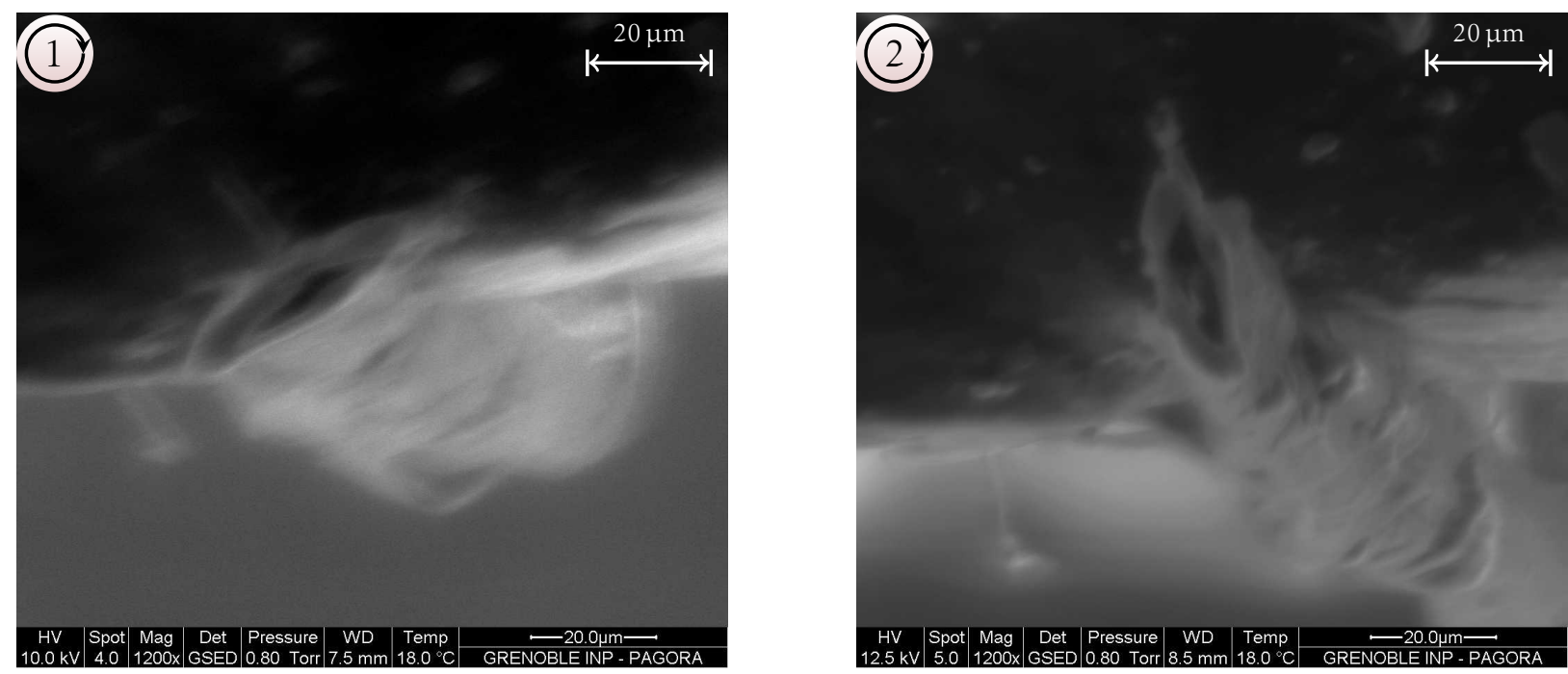

Figure E.23: ESEM cross section images of once (left) and twice air dried (right) fibre

Des paramètres de forme de sections de fibre ont été définis afin de quantifier l'évolution de la paroi lors du recyclage (cf. figure E.24).
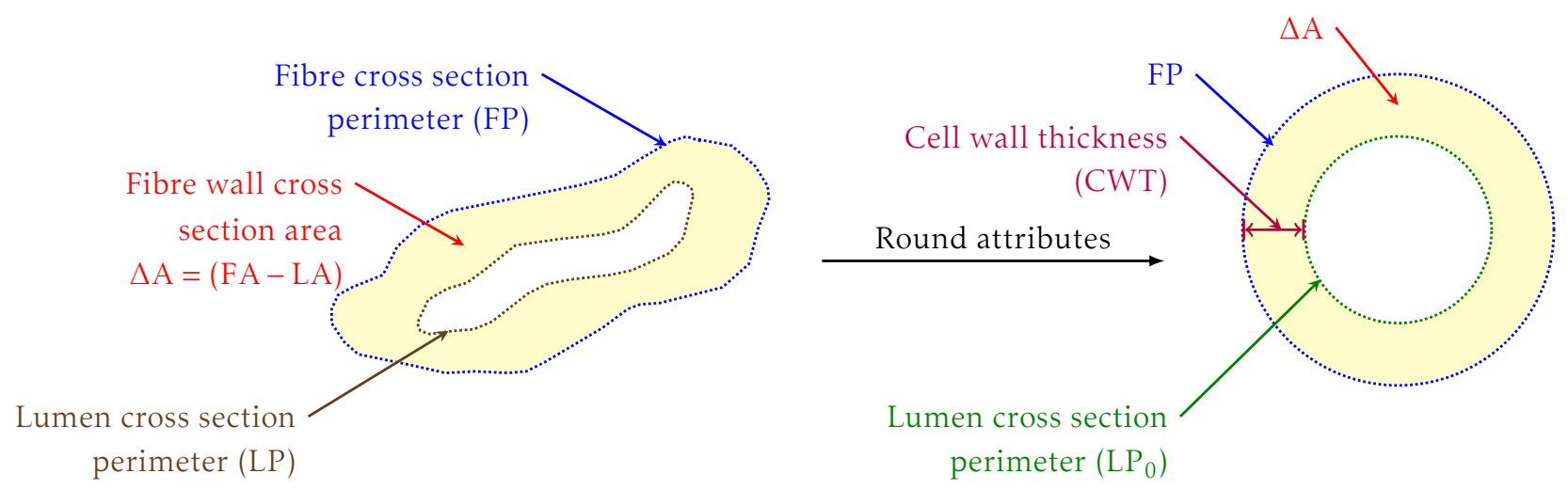

Figure E.24: Round cell wall cross section attributes

Le périmètre de la section des fibres non closes $\left(\mathrm{LP}_{0}\right)$ peut être défini en considérant une forme cylindrique de la fibre et du lumen.

$$
\mathrm{LP}_{0}=\sqrt{\mathrm{FP}^{2}-4 \pi \Delta \mathrm{A}}
$$

L'épaisseur de la paroi (CWT) peut être décrite par l'expression

$$
\mathrm{CWT}=\frac{\mathrm{FP}-\mathrm{LP}_{0}}{2 \pi}=\frac{\mathrm{FP}-\sqrt{\mathrm{FP}^{2}-4 \pi \Delta \mathrm{A}}}{2 \pi}
$$

Les résultats de l'analyse d'image réalisée à l'aide du logiciel ImageJ sont décrits 
dans le tableau E.3. L'aire de la section transversale de la fibre, le périmètre de la section transversale de la fibre et le CWT a diminué avec la réhumidification et le séchage alors que le périmètre de la section transversale du lumen augmente avec le remouillage et le séchage.

Table E.3: Effect of rewetting and drying cycles on fibre wall dimensions

\begin{tabular}{l|cc} 
Number of cycles & 1 & 2 \\
\hline Fibre wall cross section area $(\Delta \mathrm{A}), \mu \mathrm{m}^{2}$ & 439 & 345 \\
Fibre cross section perimeter $(\mathrm{FP}), \mu \mathrm{m}$ & 107 & 92 \\
Cell wall thickness (CWT), $\mu \mathrm{m}$ & 10 & 7 \\
Lumen cross section perimeter $(\mathrm{LP}), \mu \mathrm{m}$ & 43 & 48
\end{tabular}

Le périmètre de la section de la fibre diminue alors que le périmètre du lumen augmente durant le remouillage et le séchage. La figure E.25 est une représentation schématique des changements prenant place dans la section droite des fibres.

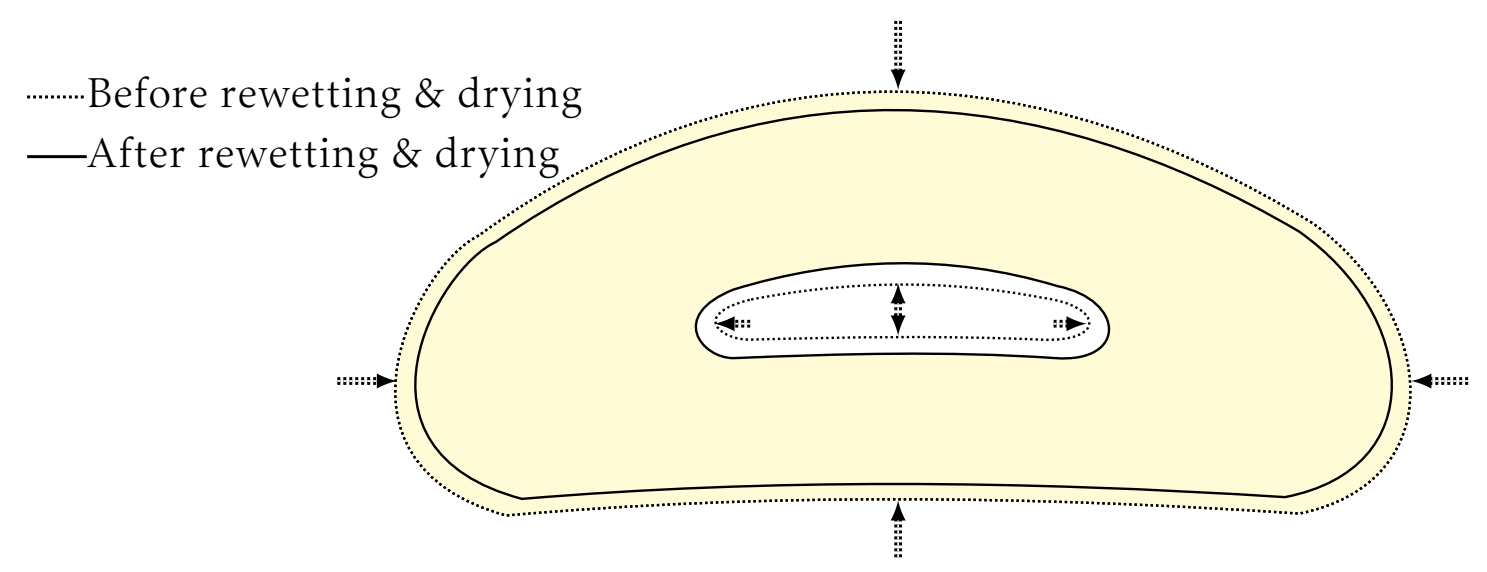

Figure E.25: Schematic diagram of fibre wall shrinkage during recycling

La forme globale d'une fibre est normalement décrite en termes de courbure et de coudes. La courbure est un changement graduel de l'orientation de l'axe central de la fibre alors qu'un coude correspond à un changement abrupt. L'indice de courbure peut être représenté par l'expression suivante

$$
\text { Curl index }(\%)=\left(1-\frac{l_{p}}{l}\right) \cdot 100
$$


L'indice de courbure diminue avec les recyclages (cf. figure E.26). La différence de l'indice de courbure des fibres et des pâtes est due à la courbure toujours plus faible des fibres.

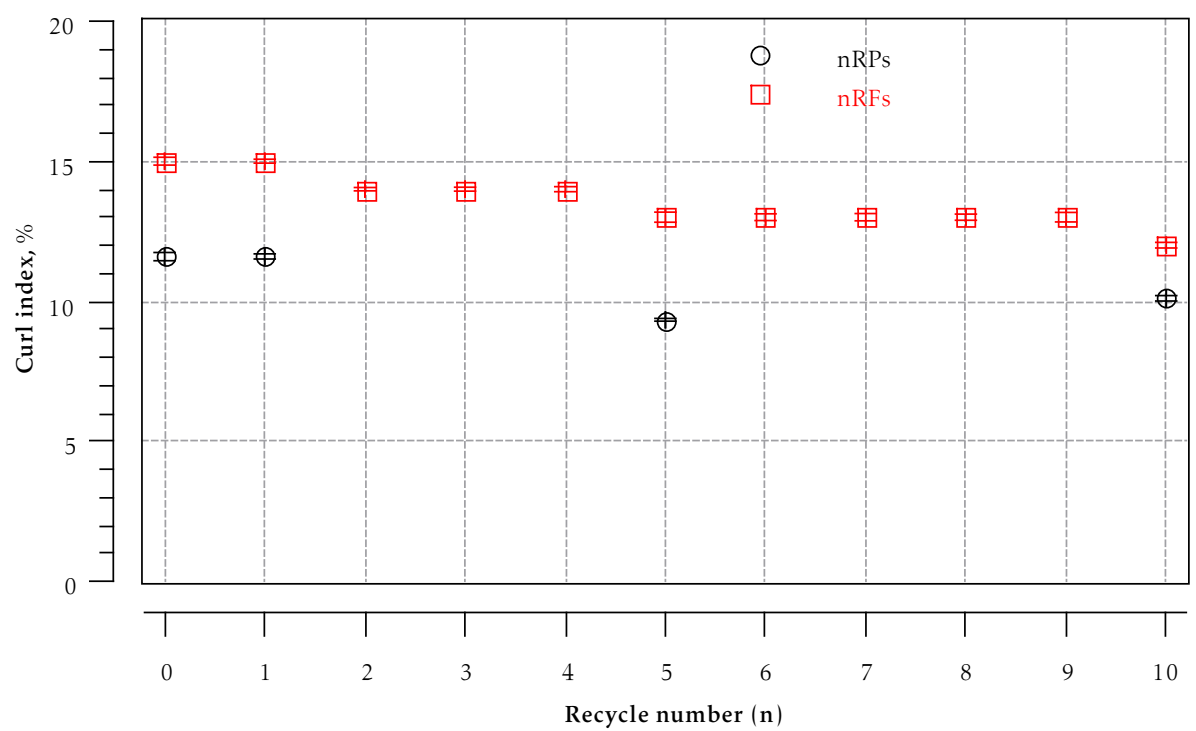

Figure E.26: Evolution of curl index of pulps and corresponding fibres during recycling

La distribution de l'indice de courbure est représentée dans la figure E.27. Les fibres fortement courbées ont été transformées en fibres moins courbées.

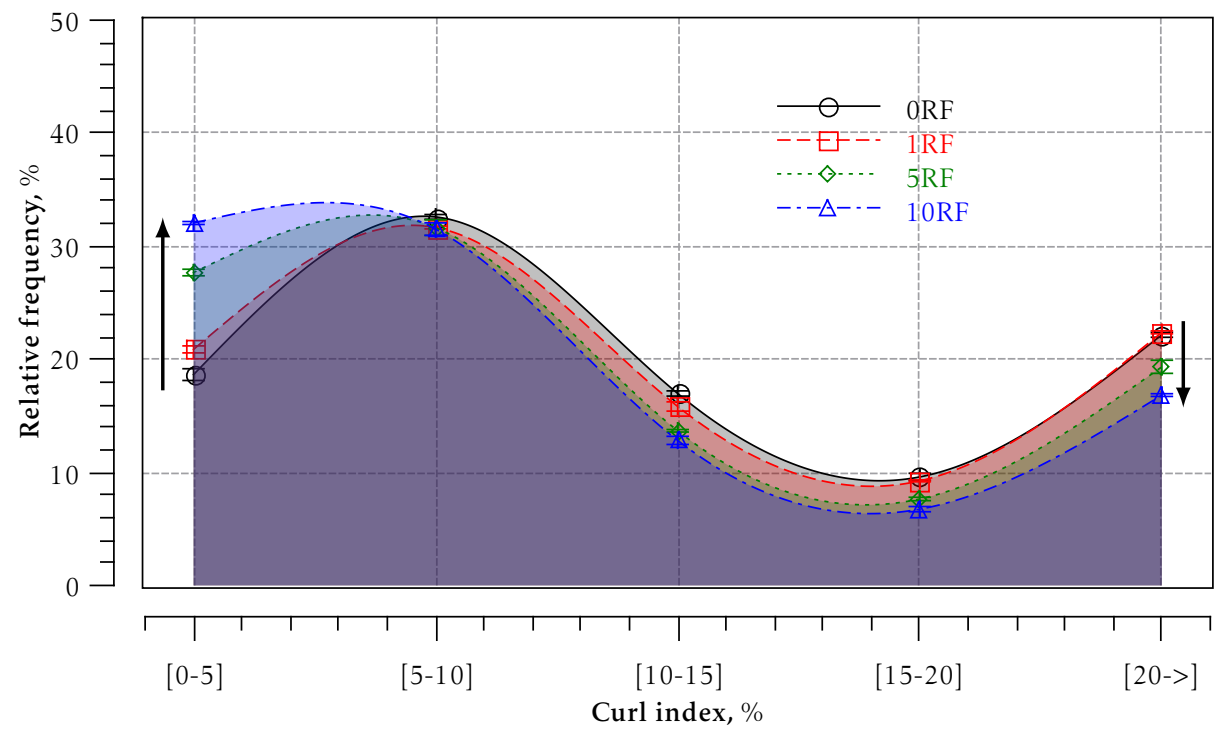

Figure E.27: Effect of recycling on curl index distributions fibres

Afin de vérifier le changement de forme, l'ESEM a été utilisée dans les mêmes conditions que précédemment. Les fibres sont placées horizontalement. Une 
extrémité de la fibre est fixée à l'aide d'un adhésif tandis que l'autre est libre de tout mouvement. Une barre a été placée à $1 \mathrm{~cm}$ de l'extrémité libre de façon à empêcher tout mouvement vertical hors du plan imagé (cf. figure E.28)

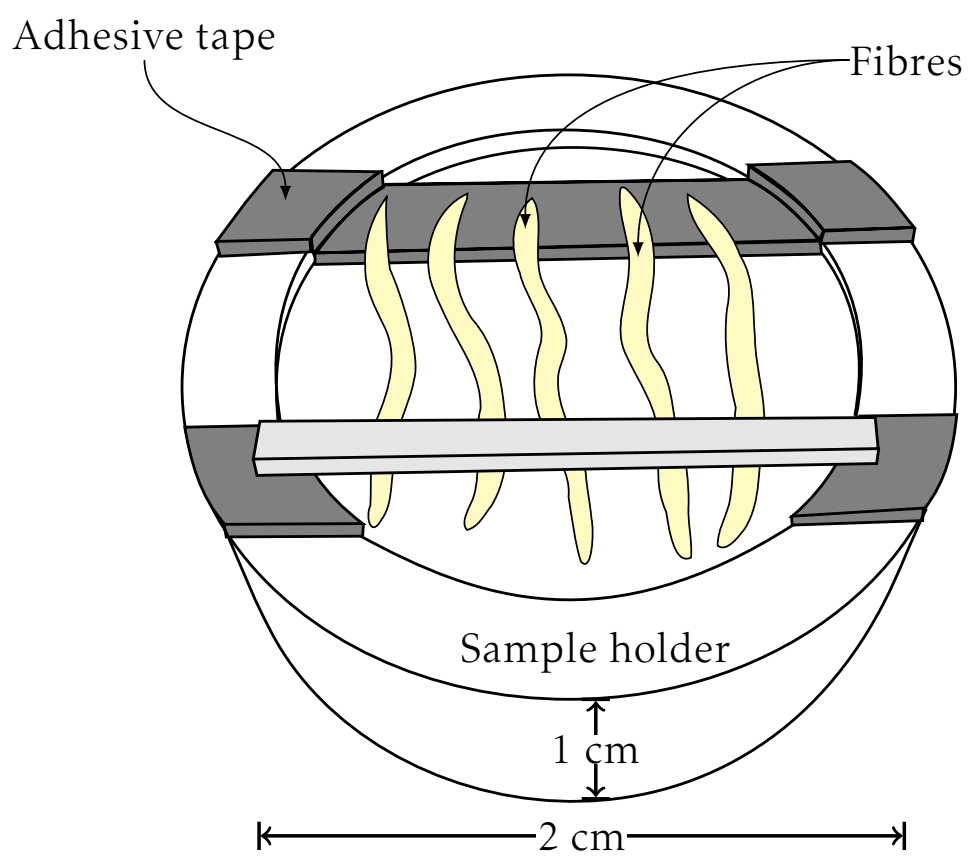

Figure E.28: Prepared sample holder for fibre shape

La figure E.29 présente les vues obtenues pour 1, 2 et 5 cycles. Les résultats issus de l'analyse des images sont regroupés dans le tableau E.4.
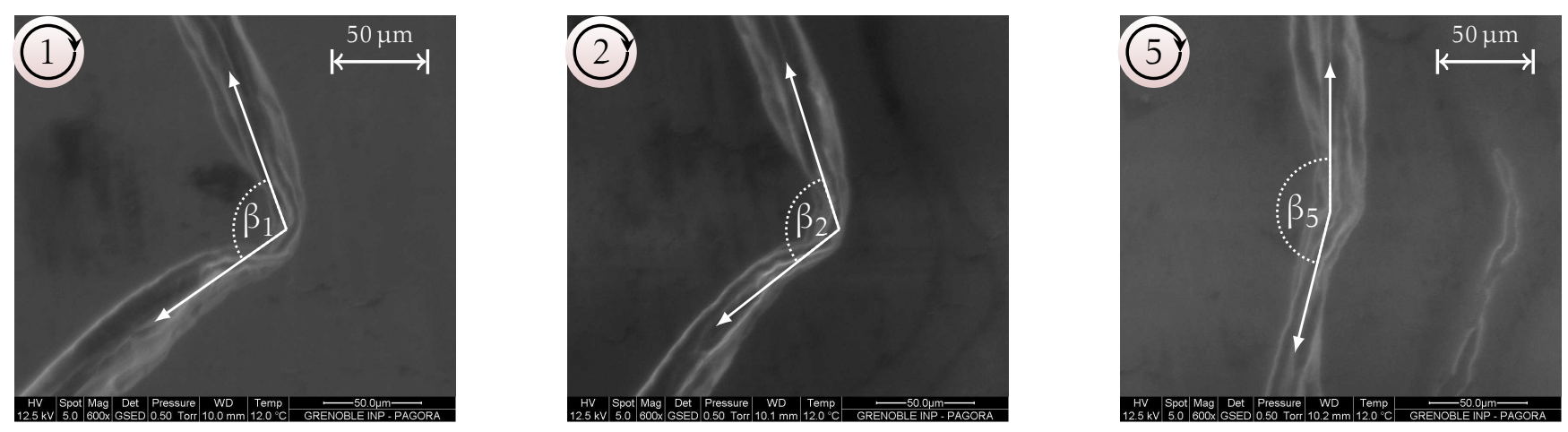

Figure E.29: ESEM micrographs of once dried kinked fibre at $5 \mathrm{RH}, 1$ st (left), 2nd (middle) and 5th cycle (right) 
Table E.4: Evolution of kink angle during rewetting and drying cycles

\begin{tabular}{l|ccccc} 
No. of cycles & 1 & 2 & 3 & 4 & 5 \\
\hline Kink angle, $\beta^{\circ}$ & 106 & 114 & 146 & 159 & 157
\end{tabular}

Lorsque les fibres sont soumises à des cycles successifs d'hydratation et de séchage, l'angle moyen des coudes augmente. Les différents effets des changements probables des fibres lors du recyclage sont schématisés sur la figure E.30.

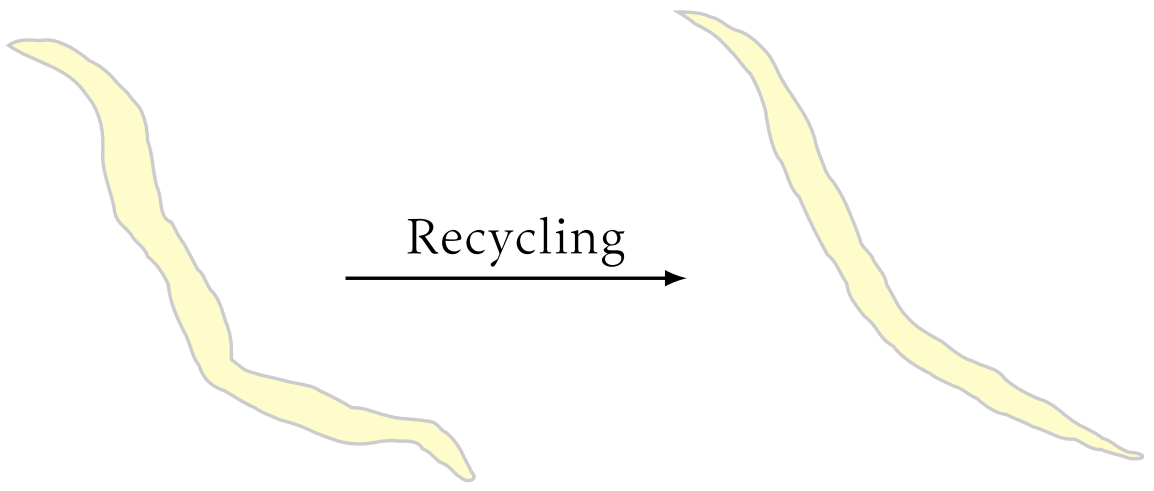

Figure E.30: Schematic diagram of curl and kink reduction during recycling

\section{Gonflement des fibres}

Le calcul de l'hornification peut se faire en utilisant les mesures de rétention d'eau (WRV) comme indiqué dans l'équation (E.6).

$$
\text { Fibre hornification }(\%)=\hbar_{\mathrm{WRV}}=\frac{\mathrm{WRV}_{0 \mathrm{RF}}-\mathrm{WRV}_{\mathrm{nRF}}}{\mathrm{WRV}_{0 \mathrm{RF}}} \cdot 100
$$

Où $W R V_{0 R F}$ et $W R V_{n R F}$ sont les valeurs de rétention d'eau respectivement pour les fibres jamais séchées et pour celles qui ont été recyclées $n$ fois.

Lors du recyclage, l'hornification des fibres évolue surtout lors du premier cycle (cf. figure E.31). La présence de morceaux de fibre et des fines dans la pâte augmente encore cet effet. Les fines générées lors du recyclage sont différentes de celles produites dans l'opération de raffinage avec respectivement plus de particules lamellaires (Flakes) et plus de fibrilles. Ceci se traduit par une surface spécifique des fines plus faible dans le cas du recyclage. 


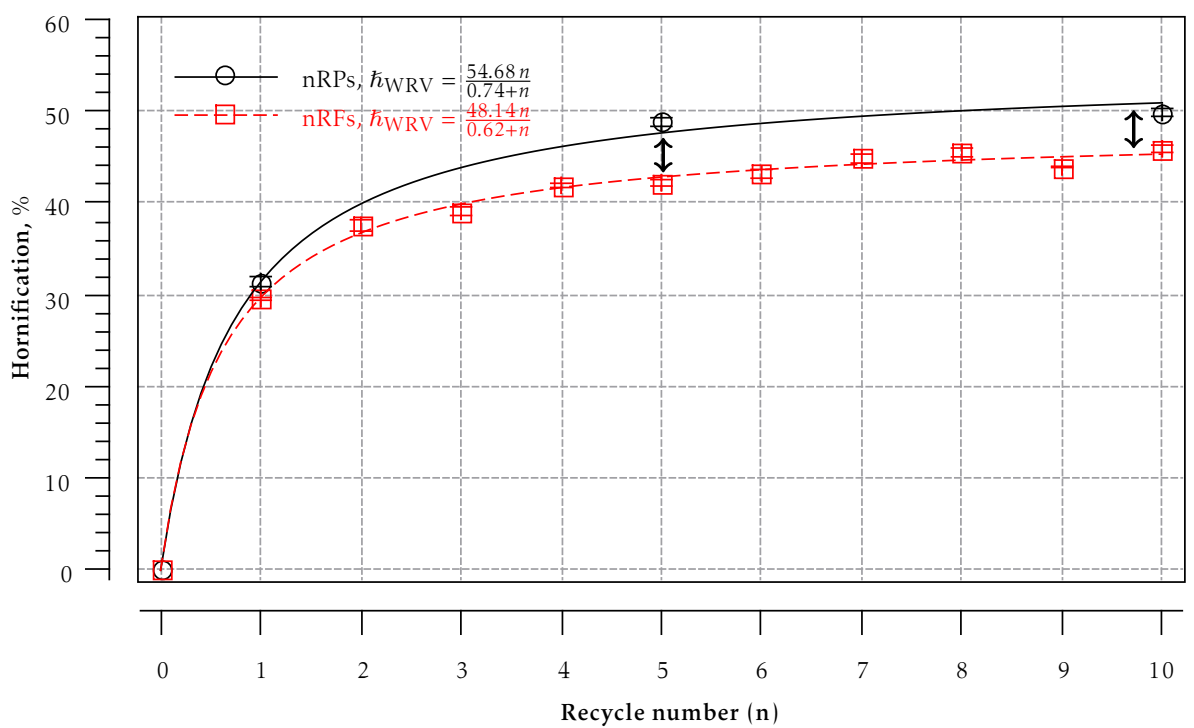

Figure E.31: Evolution of fibre hornification based on water retention value of pulps and fibres during recycling

L'hornification, calculée à partir du WRV, est due au collapse du lumen, à la fermeture des pores et à la réduction de la quantité d'eau liée à la surface. Cependant, pour prendre en compte seulement l'évolution liée à la fermeture des pores, il est nécessaire de calculer l'hornification en se basant sur le point de saturation des fibres (cf. equation (E.7)).

$$
\text { Fibre hornification }(\%)=\hbar_{\mathrm{FSP}}=\frac{\mathrm{FSP}_{0 \mathrm{RF}}-\mathrm{FSP}}{\mathrm{nRF}} \cdot 100
$$

avec $\mathrm{FSP}_{0 \mathrm{RF}}$ et $\mathrm{FSP}_{\mathrm{nRF}}$ les valeurs des points de saturation des fibres jamais séchées et recyclées $n$ fois respectivement.

L'hornification des pâtes est plus faibles que celles des fibres ce qui indique que la fermeture des pores des flakes et des fibres courtes est relativement plus faible que celle des fibres (cf. figure E.32). 


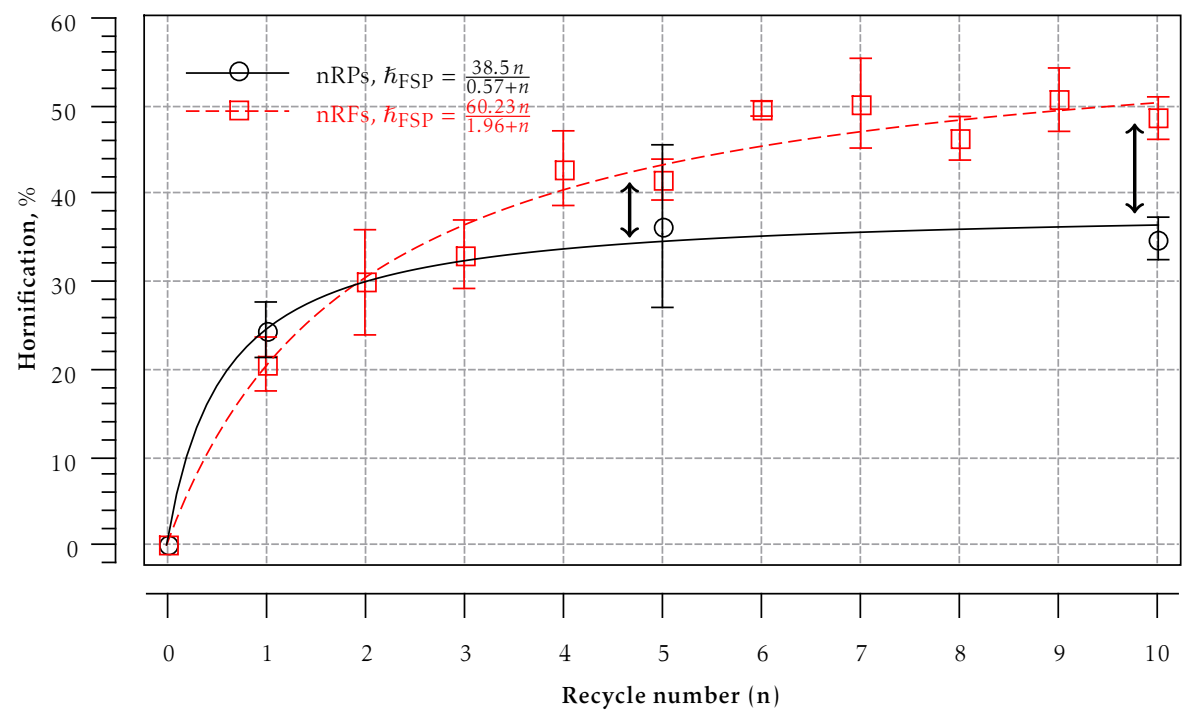

Figure E.32: Evolution of fibre hornification based on fibre saturation point of pulps and fibres during recycling

Une autre méthode indirecte pour déterminer le gonflement des fibres est la mesure de leur retrait par observation en microscopie électronique environnemental à balayage. Les conditions de réalisation des observations sont précisées sur la figure E.33 et des exemples d'image sont visibles sur la figure E.34.

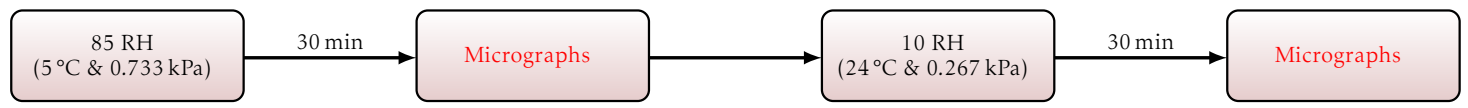

Figure E.33: Block diagram of the conditions used for shrinkability
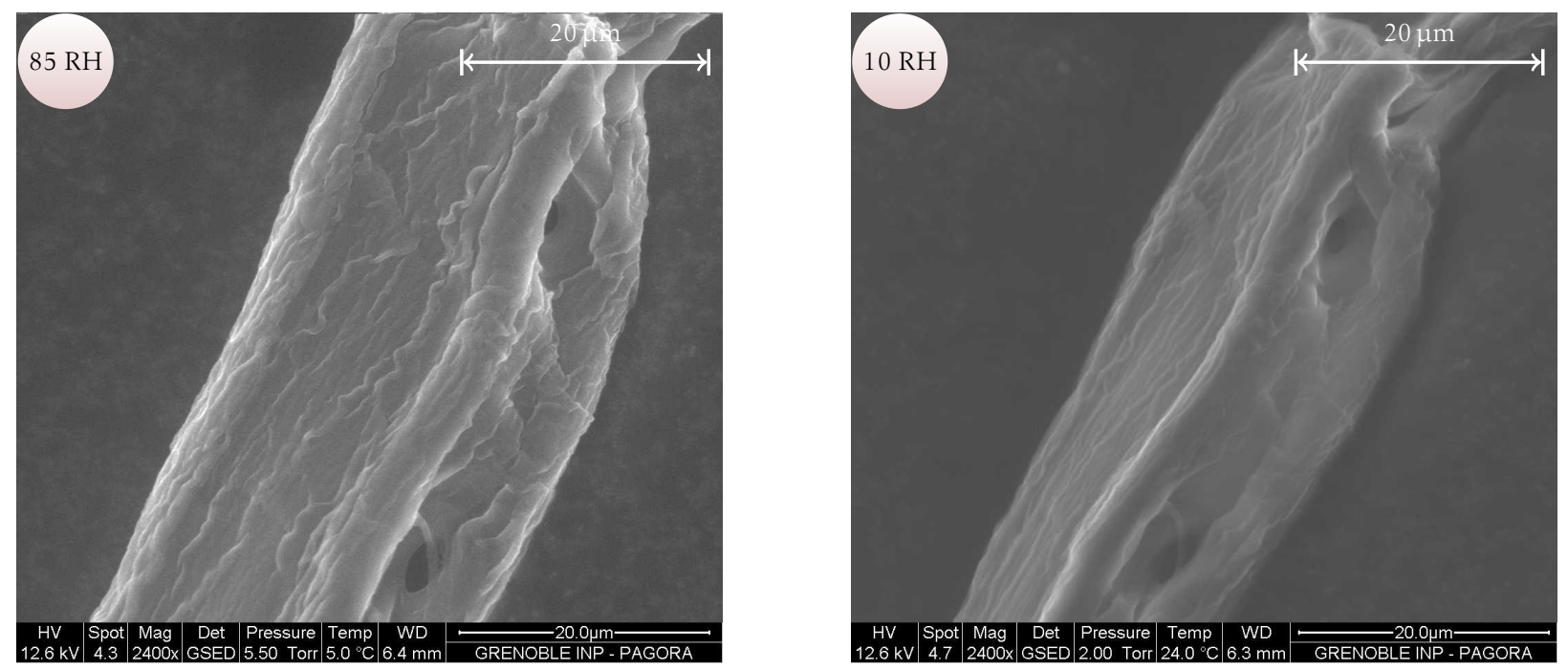

Figure E.34: ESEM images of never dried fibre at $85 \mathrm{RH}$ (left) and at $10 \mathrm{RH}$ (right) 
Aucune conclusion quantitative ne peut malheureusement être faite à cause des variations mesurées qui sont liées à la dispersion des propriétés des fibres et également à l'effet de vrillage lors du séchage.

\section{Etude de la porosité}

L'hornification, déterminée par les mesures de FSP, décrit la fermeture des pores mais il n'est pas possible d'avoir des informations sur leurs tailles. Etant donné que le gonflement des fibres est déterminé à l'état humide, il est nécessaire de faire de même pour la mesure de la porosité. La technique de chromatographie inverse d'exclusion stérique (ISEC) a été utilisée. Les macromolécules de masse moléculaire connue passent au travers de la colonne fabriquée à partir des pâtes ou des fibres. Les plus grosses sortent en premières alors que les plus petites mettent plus de temps pour parcourir les petits pores de la colonne (cf. figure E.35).

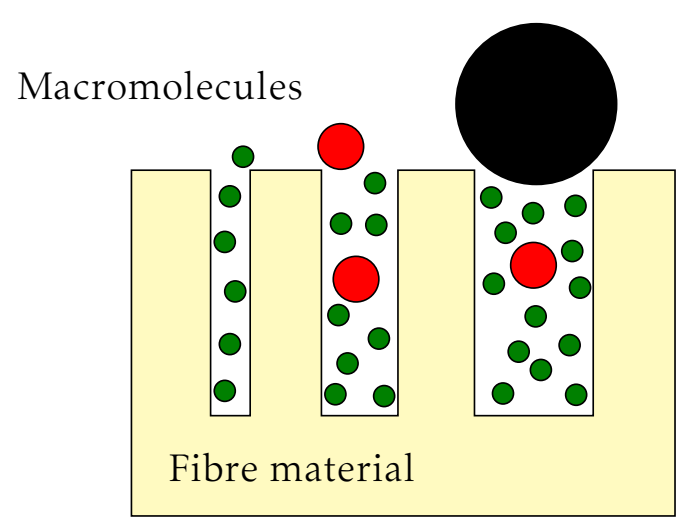

Figure E.35: Accessible pore volumes adapted from [134], where smaller green probe molecules can access pores of all sizes, medium size red probe molecules can partially access certain pores and no pores are accessible for large black probe molecule

En se basant sur les temps de rétention, il est possible de calculer le volume spécifique des pores selon l'équation (E).

$$
\text { Cumulated specific apparent pore volume }(\mathrm{V})=\frac{\left(t_{g}-t_{d}\right)}{m} \cdot v
$$

avec $t_{g}, t_{d}, v$ et $m$ sont respectivement le temps d'élution du glucose, de la solution de dextran, la vitesse et la masse du lit fibreux.

Un système semi-automatique de la fabrication de la colonne a été développé (cf. figure E.36) en se basant sur les travaux de Berthold [42]. 


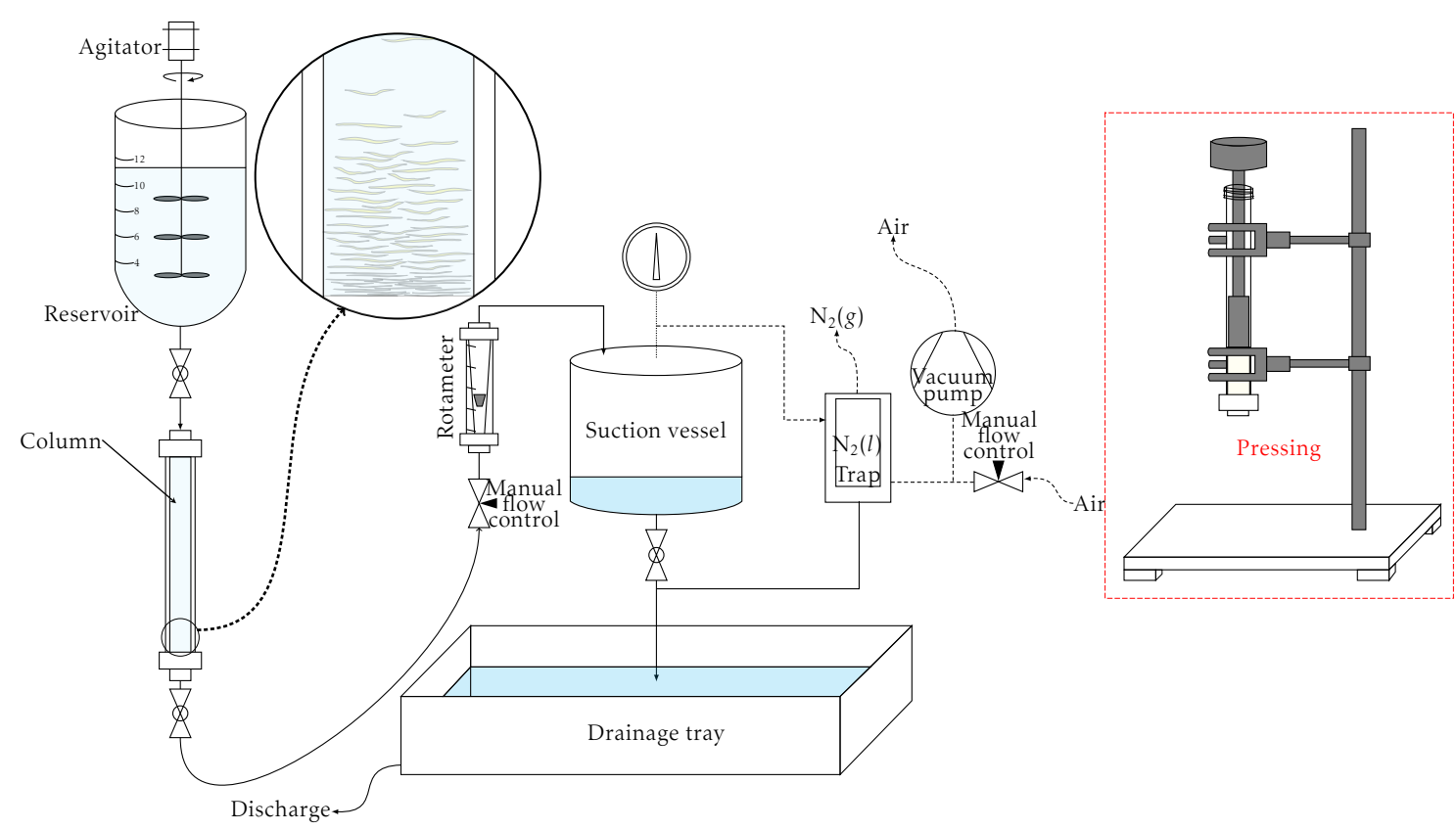

Figure E.36: Fabrication of the column for GPC

Un faible débit sur le filtre présent au bout de la colonne permet aux fibres de se déposer 'tranquillement'. Lorsque la quantité de matière requise s'est déposée, la colonne est ensuite pressée puis intégrée au système chromatographique (cf. figure E.37). Des particules de Dextran calibrées sont utilisées pour sonder les pores de la paroi fibreuse.

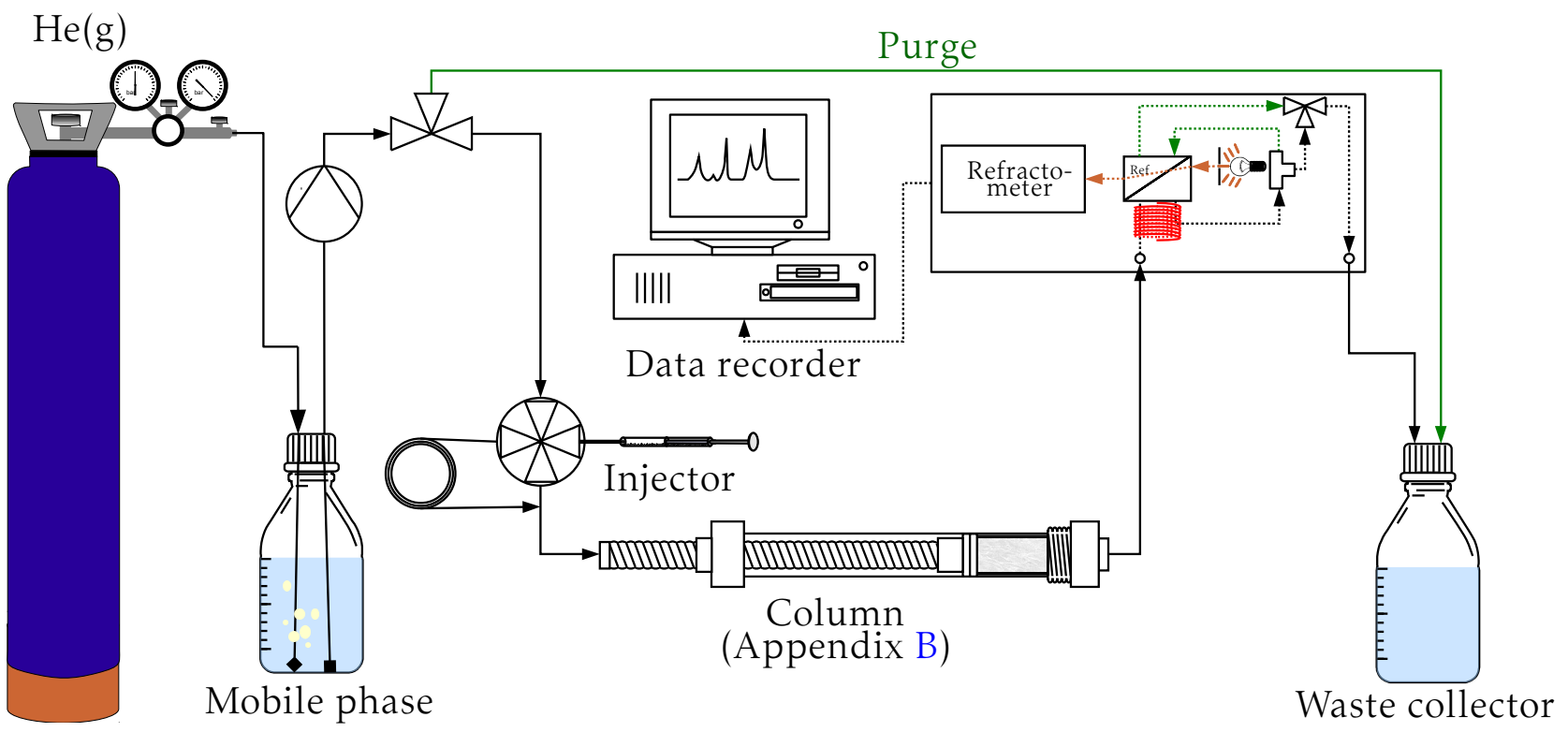

Figure E.37: Experimental set-up for chromatographic measurements 
Après avoir caractérisé le lit fibreux à l'aide des différents sucres, ce dernier est séché à $105^{\circ} \mathrm{C}$ pour déterminer sa masse.

La figures E.38 représente le volume apparent cumulé des pores pour les pâtes recyclées. Celui-ci diminue significativement lors du premier cycle.

La fermeture des pores durant le premier cycle est prédominante pour les macro-pores de taille comprise entre 5.3-13.7 nm (cf. figures E.38 et E.39).

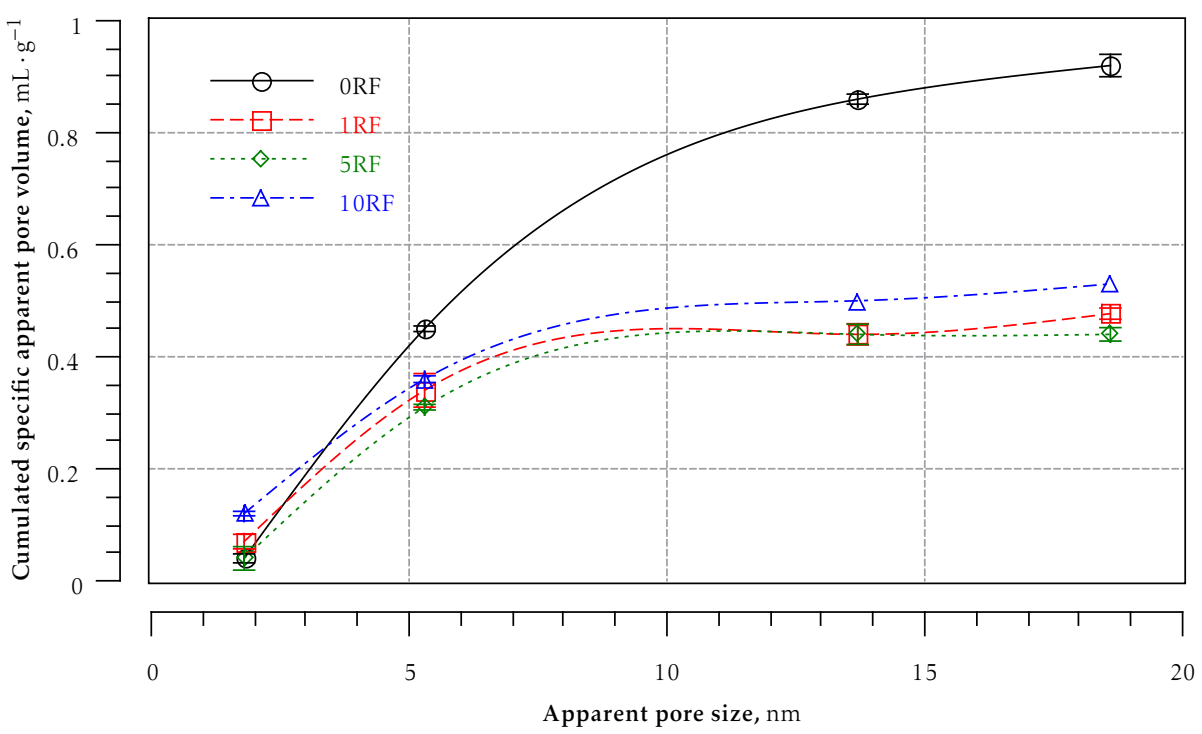

Figure E.38: Cumulated specific apparent pore volume of recycled fibres

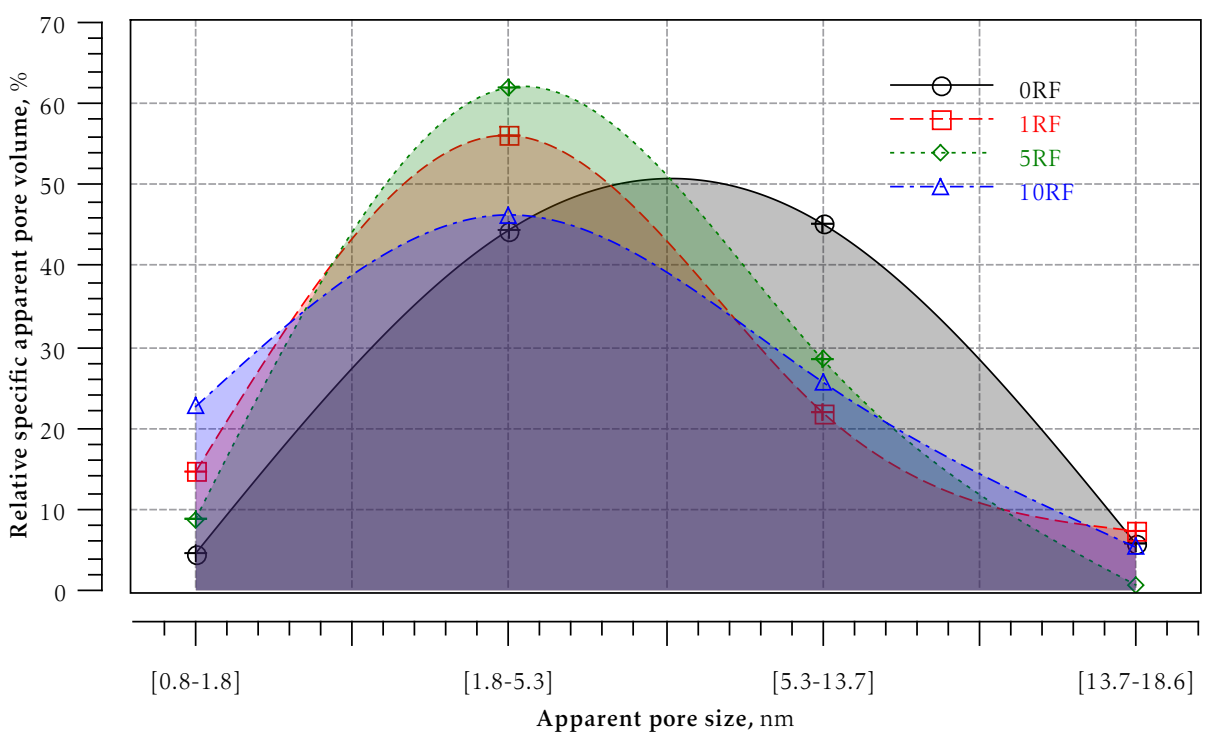

Figure E.39: Cumulated specific apparent pore volume of recycled fibres 


\section{Analyse de la surface}

La figure E.40 montre des images MEB de la surface des fibres en fonction du recyclage. Durant les trois premiers cycles, la surface se compacte avant de se disloquer puis de se délaminer.
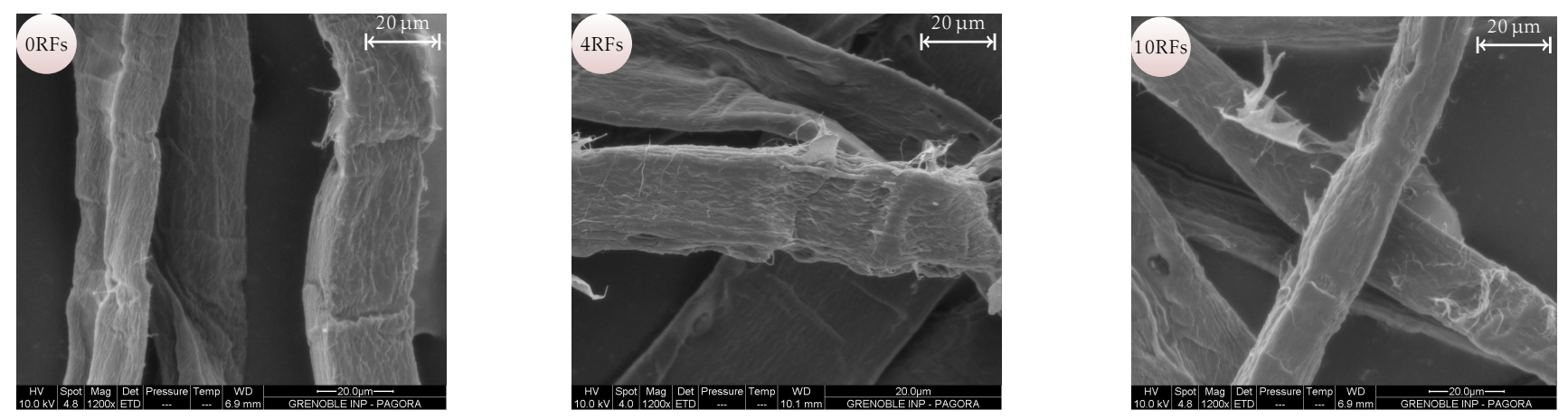

Figure E.40: SEM micrographs showing the surface characteristics of fibres during recycling

La surface des fibres a également été caractérisée par microscopie à force atomique à l'aide du mode QNM (cartographie des propriétés nano-mécaniques) (cf. figure E.41).

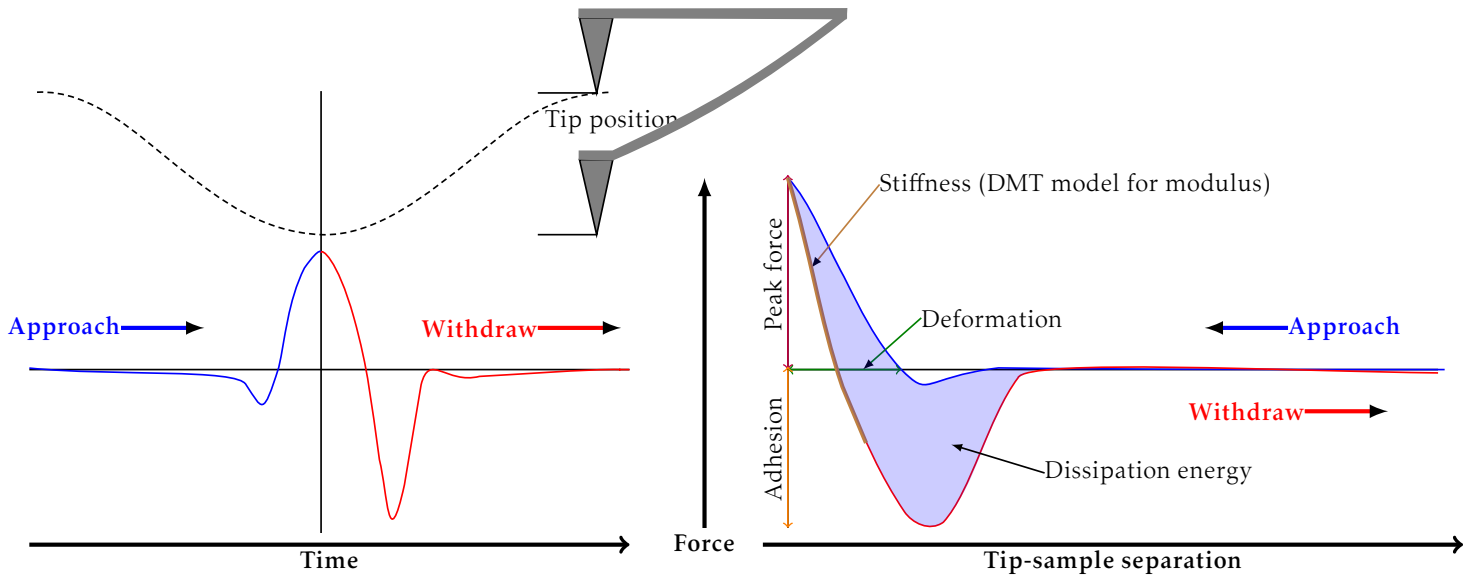

Figure E.41: PeakForce $\mathbb{B}$ Quantitative Nanomechanical Property Mapping (QNM)

La figure E.42 montre les effets du recyclage sur l'adhésion entre la pointe et la surface des fibres. L'adhésion de surface, décrite en termes de dissipation d'énergie, augmente avec le recyclage et avec l'accroissement du set point (qui correspond à la force appliquée). 


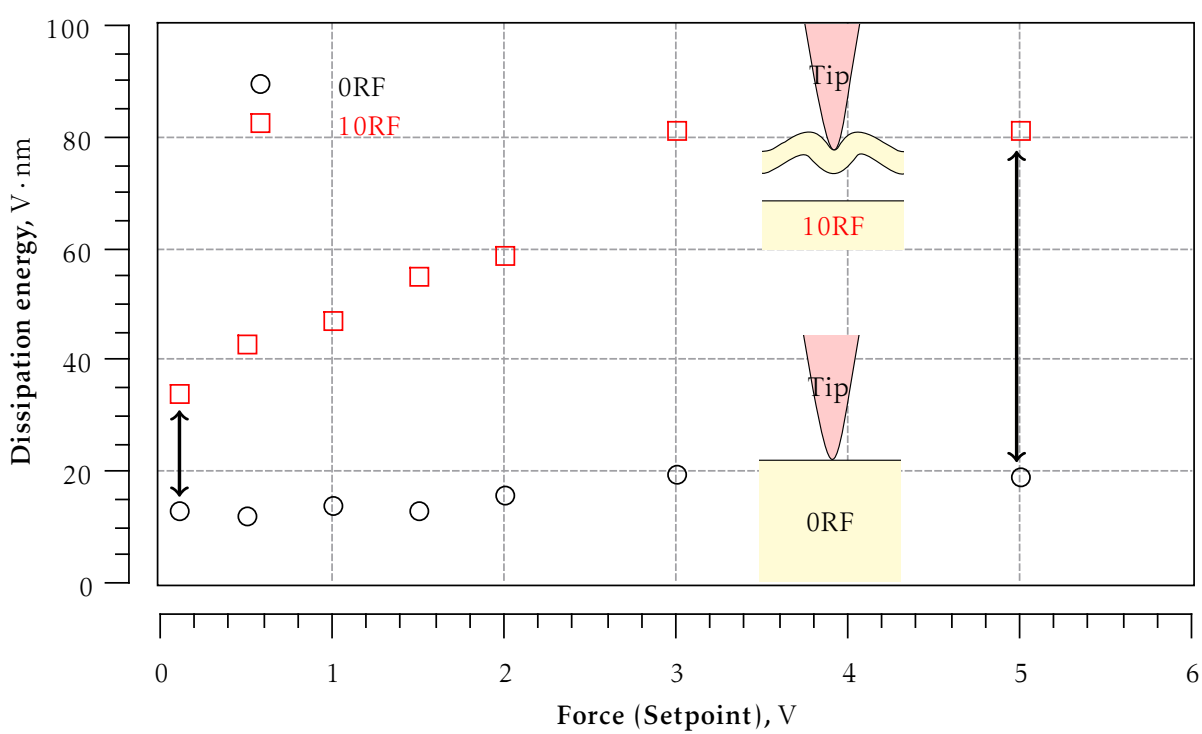

Figure E.42: Effect of recycling on fibre surface adhesion

L'augmentation de l'adhésion de surface est probablement due à la délamination partielle de la paroi fibreuse. Dans le cas des fibres recyclées, l'aire de contact entre la pointe AFM et la fibre est relativement élevée. Des transformations de la paroi des fibres recyclées dues au raffinage par exemple peuvent être envisagées. Le raffinage de ces dernières élimine par pelage la couche externe, et provoque de la fibrillation interne et externe.

La longueur de rupture à l'état humide (WBL) décrit la longueur de feuille nécessaire qui se rompt sous son propre poids. Cette propriété est importante dans le cas des faibles séchages (20 à 30\% d'humidité). Elle est généralement contrôlée par la tension superficielle, la friction de surface et l'entrelacement des fibres. Les bandes humides sont réalisées sur les appareils de préparation de formette (Rapid-Köthen) et placées immédiatement dans l'appareil schématisé sur la figure E.43. 


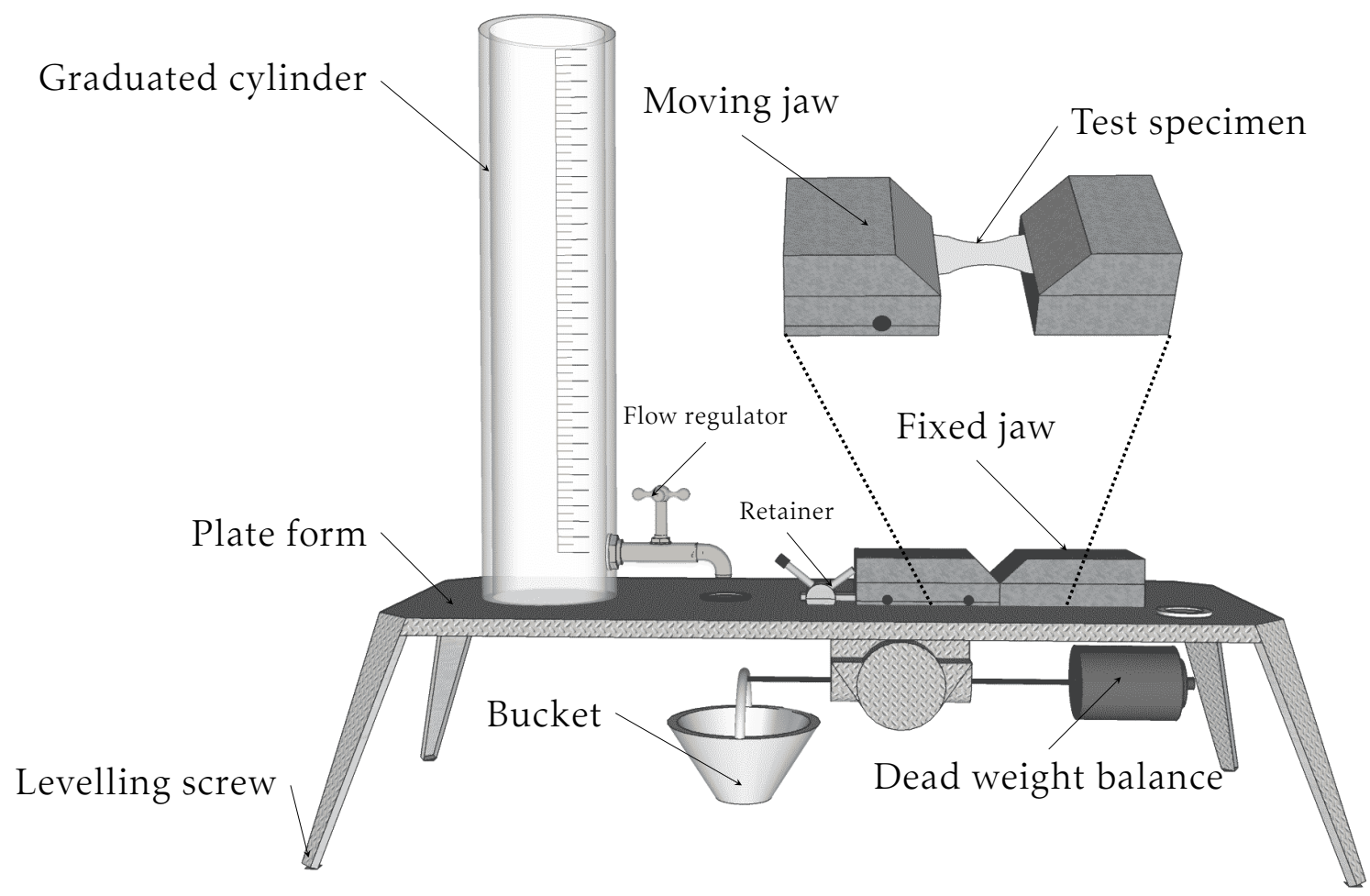

Figure E.43: Wet breaking length measurement device

L'évolution de la longueur de rupture humide en fonction du recyclage est illustrée sur la figure E.44. Une légère augmentation est visible qui peut être générée par l'augmentation de la friction de surface. Ceci est en accord avec les analyses AFM et MEB.

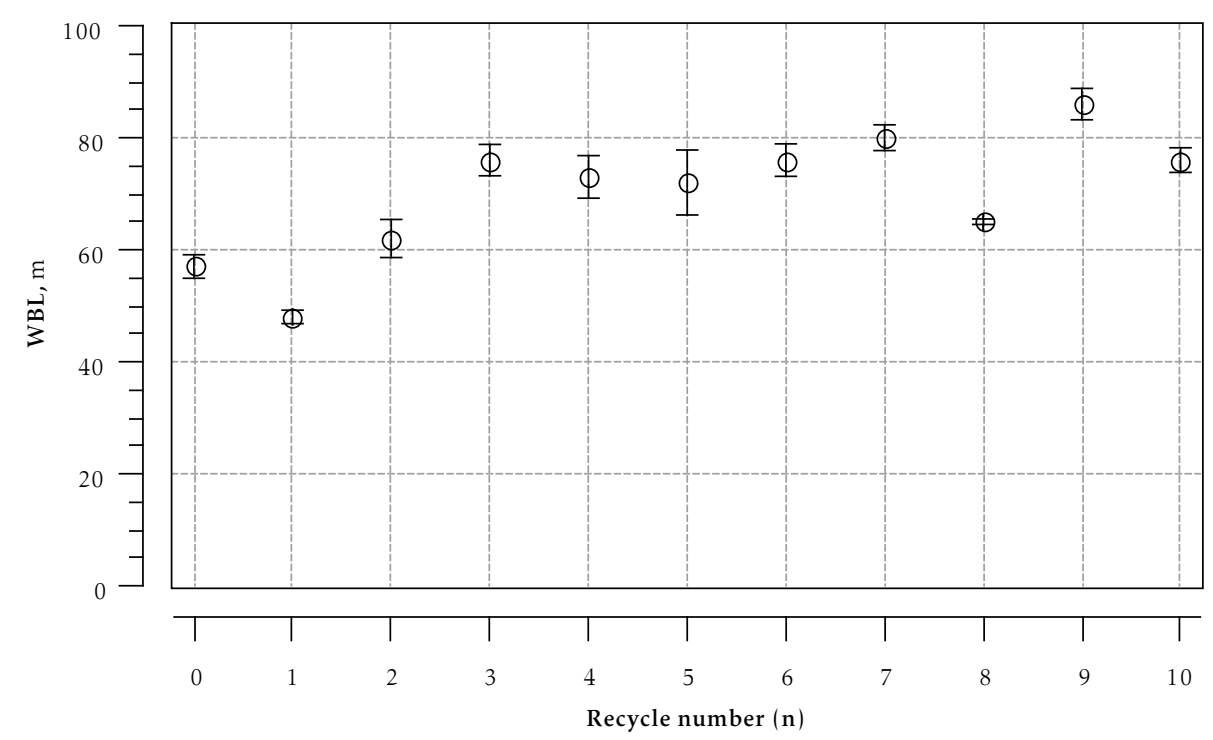

Figure E.44: Evolution of wet breaking length of fibres during recycling 
Les points faibles de la paroi fibreuse peuvent être considérés comme des dislocations dans celle-ci. Ces défauts locaux permettent de pouvoir les hydrolyser par voie chimique ou enzymatique [31, 32, 96]. Ander et Daniel [29] quantifient ces points faibles en définissant un indice de clivage chimique des fibres (cf. equation (E.8), [30]).

$$
\text { Cleavages per fibre }=\frac{\left\langle l_{l}\right\rangle}{\left\langle l_{l c}\right\rangle}-1
$$

avec $\left\langle l_{l}\right\rangle$ et $\left\langle l_{l c}\right\rangle$ sont respectivement les longueurs pondérées en longueur des fibres non clivées et clivées.

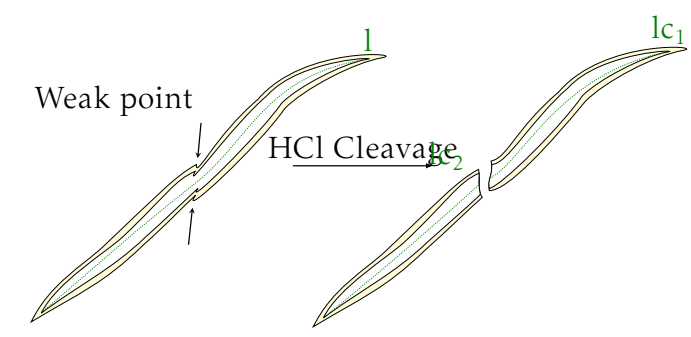

Figure E.45: Cleavage of fibre by $\mathrm{HCl}, l$ and $l c$ are the length of uncleaved and cleaved fibres

La courbe d'évolution de l'indice de clivage présente trois régions distinctes (cf. figure E.46). Dans la première région, l'indice de clivage augmente alors qu'il reste constant dans la région II pour augmenter à nouveau dans la région III.

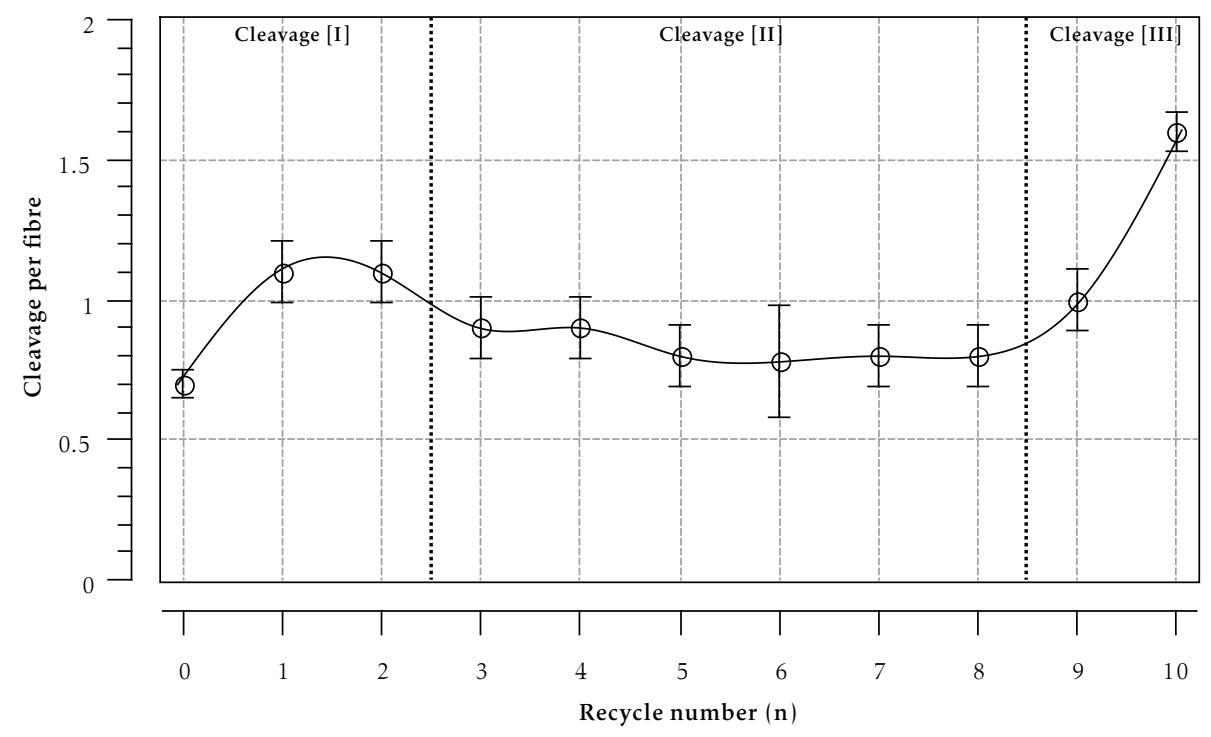

Figure E.46: Cleavages per fibre of fibres during recycling

La figure E.47 montre une possible modification de la paroi fibreuse lors du 
recyclage et du clivage. La couche externe joue un rôle prépondérant durant le traitement acide. L'augmentation significative de l'indice de clivage dans la région III peut être attribuée à l'accroissement du nombre de dislocations et/ou à l'accessibilité de l'acide dans les zones de dislocations.

Dislocation $\mathrm{P} / \mathrm{S} 1 \quad \mathrm{~S} 2 \quad \mathrm{~S} 3$
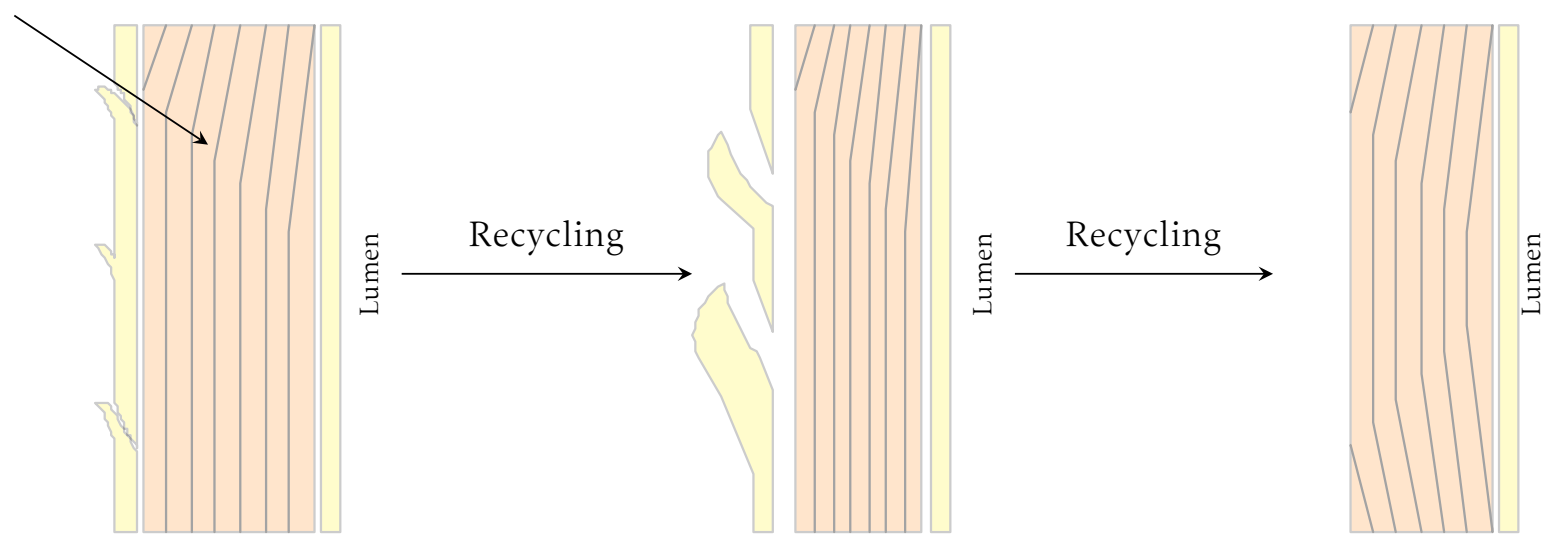

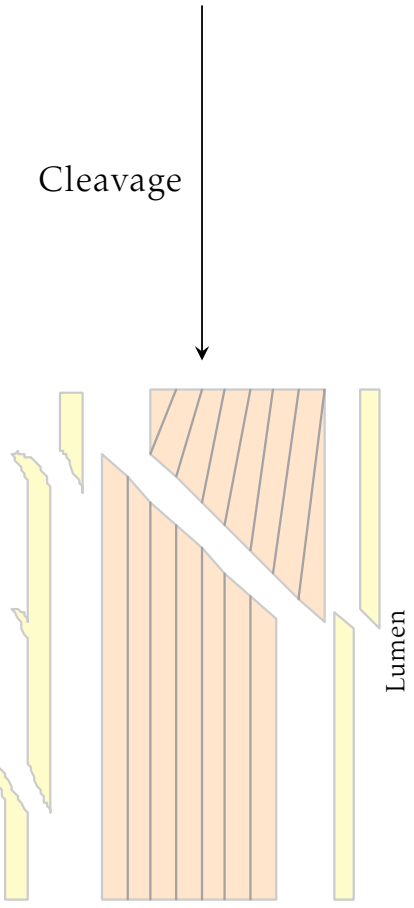

$[\mathrm{I}]$

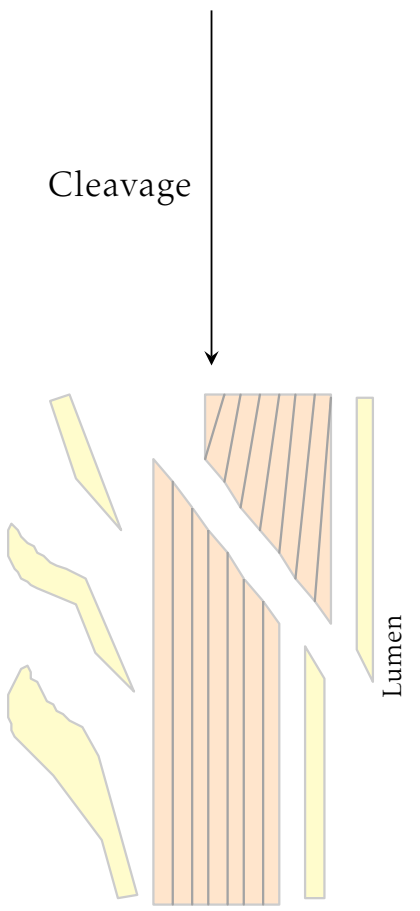

[II]

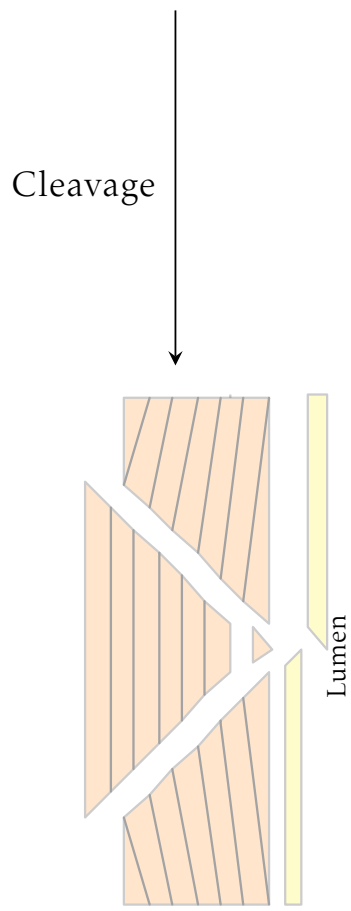

[III]

Figure E.47: Schematic diagram of probable transformations of fibre cell wall during recycling by $\mathrm{HCl}$ cleavage

L'indice de clivage n'est pas un indicateur absolu du nombre de points faibles car il dépend des conditions opératoires. Cependant son utilisation est un bon moyen de comparaison de différentes pâtes.

La présence de nombreux points faibles diminue la résistance des fibres [174]. 
Une synthèse des méthodes de mesure du module élastique de la cellulose native a été présentée par Cintrón et al. [60]. Page et al. ont montré que les fibres après raffinage qui ont un fort module d'Young, possèdent également une forte résistance à la traction [172].

\section{Résistance à la traction des fibres individuelles}

Pour réaliser les tests de traction (cf. figure E.48), les extrémités de la fibre sont collées sur le support avec de la colle Epoxy. Ensuite, le support est mis en place entre les mors de l'analyseur mécanique dynamique (DMA). Le support est alors coupé et une tension est exercée jusqu'à ce que la charge soit trop importante et engendre la rupture de la fibre. La fibre est alors découpée à la lame de rasoir près du point de colle afin que sa section puisse être déterminée par observation au MEB en faisant l'hypothèse qu'elle ne varie pas sur la longueur de la fibre.

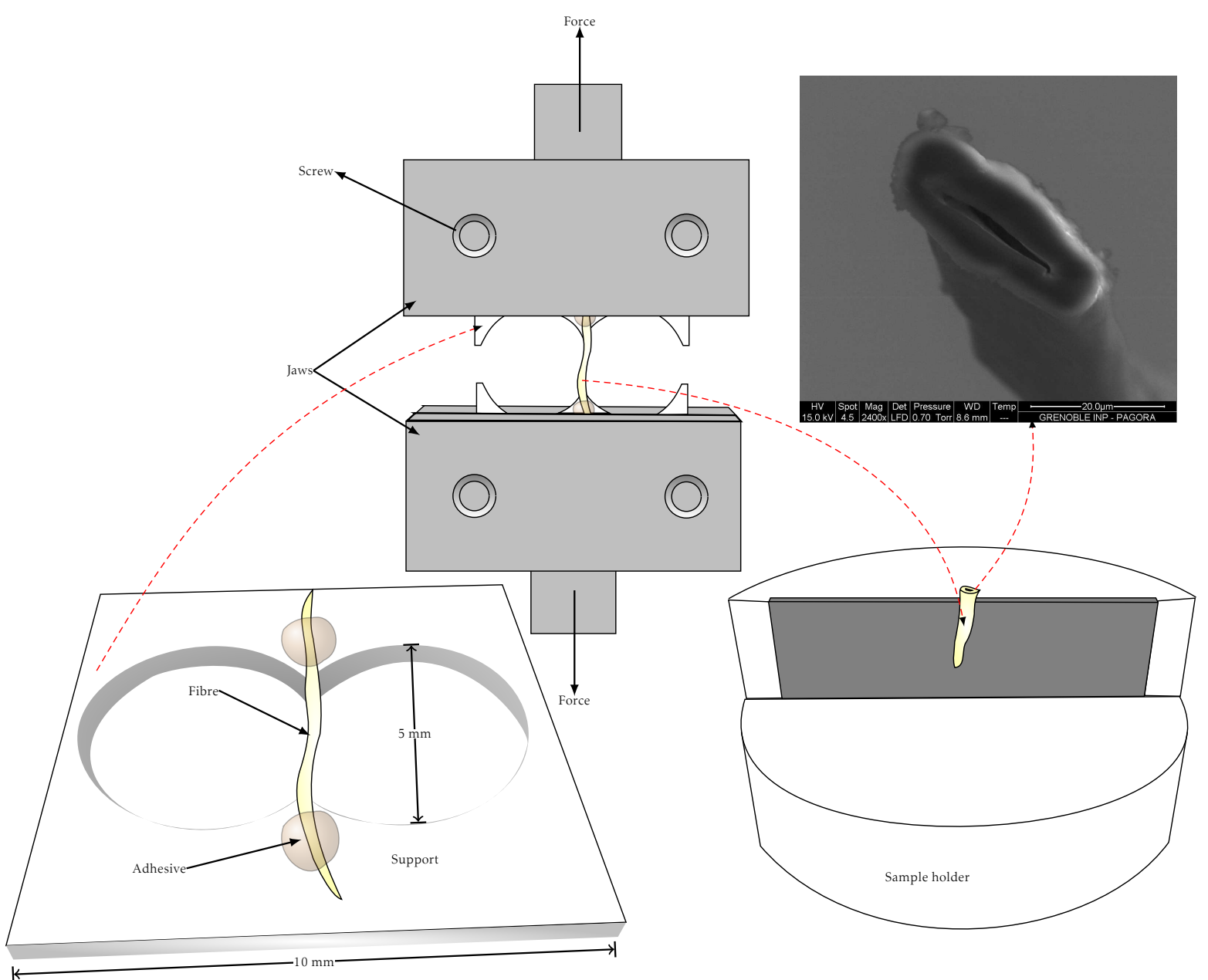

Figure E.48: Single fibre tensile test 
Les courbes de résistance à la traction en fonction de l'élongation sont présentées sur la figure E.49 pour des fibres jamais séchées et ayant subies dix cycles de séchage/hydratation.
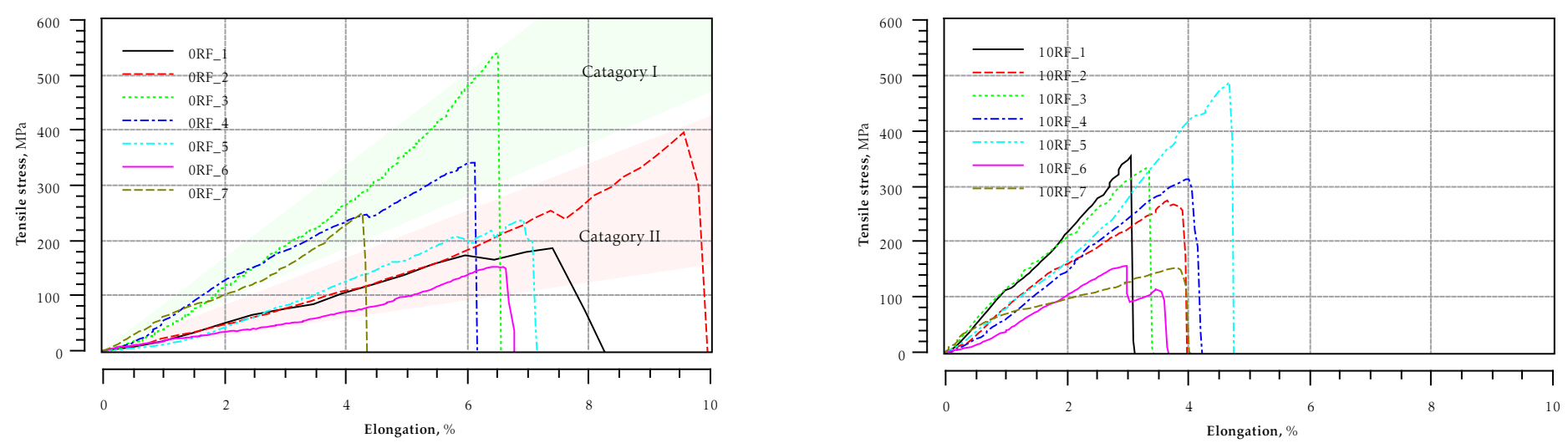

Figure E.49: Stress-strain curve of air dried never dried fibres (left) and ten times recycled fibres (right)

Le module élastique est calculé à partir de la pente à l'origine de la courbe de traction. L'aire sous la courbe correspond à l'énergie absorbée. La non-linéarité des courbes obtenues provient de la présence des dislocations dans la paroi fibreuse. Les résultats expérimentaux sont présentés dans le tableau E.5.

Table E.5: Effect of recycling on single fibre tensile properties

\begin{tabular}{l|cc} 
& 0RF & $10 \mathrm{RF}$ \\
\hline Elongation, \% & $6.8 \pm 0.8$ & $4.1 \pm 0.6$ \\
Stress at break, GPa & $0.32 \pm 0.06$ & $0.27 \pm 0.04$ \\
TEA, $\times 10^{-11} \mathrm{~J} \cdot \mathrm{m}^{-2}$ & $2.1 \pm 0.3$ & $1.2 \pm 0.2$ \\
Elastic modulus, GPa & $5.27 \pm 1.56$ & $8.54 \pm 1.31$
\end{tabular}

Le module élastique des fibres après dix cycles a été augmenté de 62\%, alors que la déformation, la contrainte à la rupture et l'énergie à la rupture ont chuté de $40 \%, 16 \%$ et $43 \%$, respectivement. L'augmentation du module est vraisemblablement liée à la densification de la paroi des fibres (agrégation des micro-fibrilles) et à une forme plus rectiligne des fibres. La diminution des autres propriétés sont quant à elles liées aux points faibles présents dans la paroi des fibres. On peut dire que la fibre devient plus résistante et fragile 
avec le recyclage. Ces résultats sont en accord avec ceux présentés précédemment dans lesquels une augmentation du module élastique et une diminution de l'allongement à la rupture ont été rapportés [122, 78].

\section{Propriétés des formettes réalisées à partir des pâtes recyclées}

\section{Propriétés structurales des formettes}

Le volume massique des formettes est l'inverse de la masse volumique

$$
\text { Volume massique }\left(\mathrm{cm}^{3} \cdot \mathrm{g}^{-1}\right)=\frac{\hbar}{\mathrm{G}}
$$

avec $¥(\mu \mathrm{m})$ représente l'épaisseur de la formette et $\mathrm{G}$ son grammage.

La masse volumique de la cellulose est d'environ $1540 \mathrm{~kg} \cdot \mathrm{m}^{-3}$ alors que la masse volumique d'un papier varie de 300 à $1000 \mathrm{~kg} \cdot \mathrm{m}^{-3}$ [83].

L'évolution du volume massique lors du recyclage est représentée dans la figure E.50. Le volume massique augmente jusqu'au deuxième recyclage avant de se stabiliser.

Les données portent sur un jeu de six mesures de l'épaisseur et une mesure de grammage pour trois formettes.

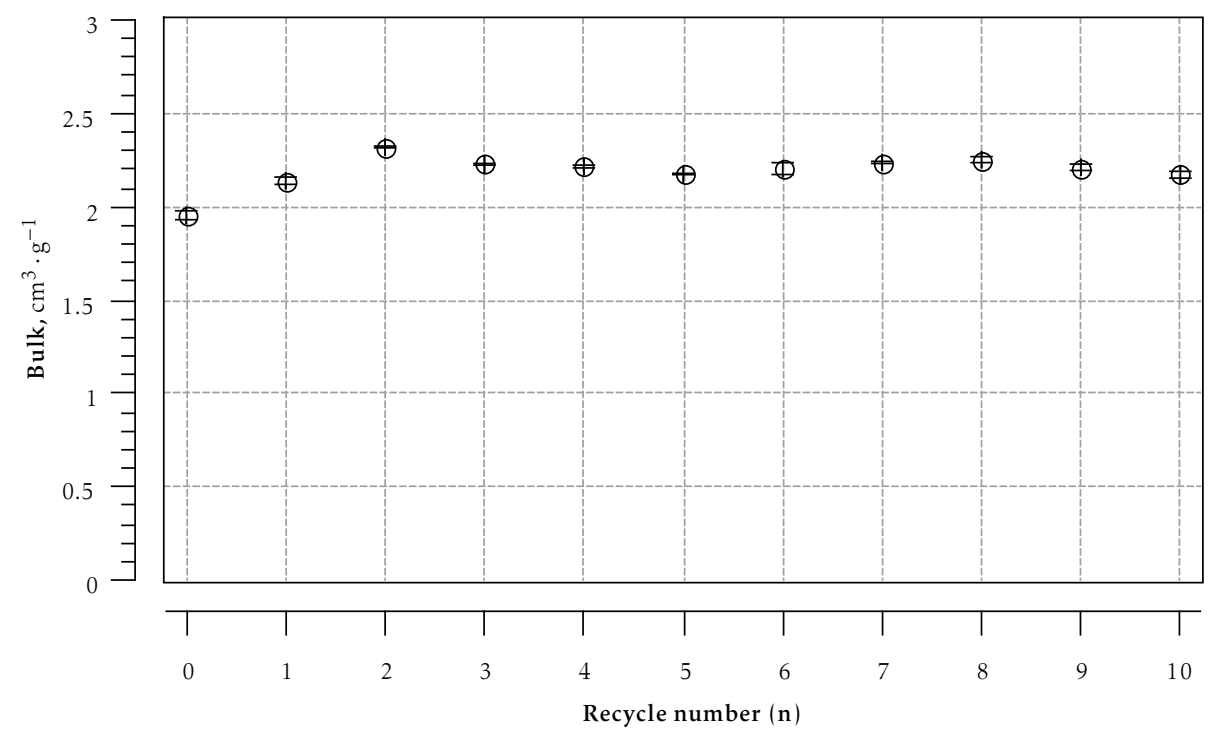

Figure E.50: Bulk of handsheets made from recycled pulps 


\section{Structure 3D des fibres des formettes réalisées à partir des pâtes recyclées}

Les structures tridimensionnelles (3D) permettent d'accéder à de nombreuses informations. La structure 3D des papiers a été précédemment simulée en fonction des propriétés des matières premières afin de prédire les propriétés physiques d'usage [164].

Néanmoins, la structure réelle des papiers est beaucoup plus complexe. Par conséquent, un modèle simpliste ne permet pas de rendre compte de la complexité du réseau fibreux. En raison des avancées technologiques, de nouvelles techniques expérimentales ont été développées afin de caractériser la structure du papier de façon plus fine. La tomographie se réfère à la visualisation 3D d'objets. L'imagerie 3D d'échantillons de tailles supérieures à quelques centimètres est appelée 'computer tomography' (CT). La micro-tomographie est la notation utilisée pour l'acquisition d'images 3D d'échantillons ayant une taille caractéristique de quelques centimètres. Le mode d'imagerie utilisé pour l'imagerie aux rayons X est basé sur le contraste d'absorption. Elle est par exemple employée dans le domaine médical afin de différencier les os des tissus. En comparaison aux microscopes électroniques (SEM), la micro-tomographie aux rayons $\mathrm{X}(\mu \mathrm{CT})$ ne nécessite pas un matériau de contraste ou une résine Epoxy pour l'analyse [196].

La découpe des échantillons pour les essais SEM, peut endommager les fibres et la résine peut modifier la structure 3D. La $\mu \mathrm{CT}$ est une technique non destructive [196, 33].

Dans la méthode $\mu \mathrm{CT}$, les rayons X sont projetés sur l'échantillon. En fonction de la masse volumique et de la nature du matériau, une proportion des rayons est absorbée, alors que le reste est transmis à travers l'échantillon. Le porte échantillon tourne et une série de projections est obtenue. Ces sinogrammes sont filtrés et une méthode de projection inverse est utilisée afin d'obtenir une coupe 2D de l'image. Un volume est obtenu à partir d'une série de projections des images acquises.

Les échantillons de papier sont fixés sur un tube capillaire à l'aide de colle. Le capillaire est ensuite fixé sur le porte échantillon (cf. figure E.51). Le porte échantillon est alors placé dans la chambre de mesure afin de l'équilibrer en termes de température et d'humidité. 


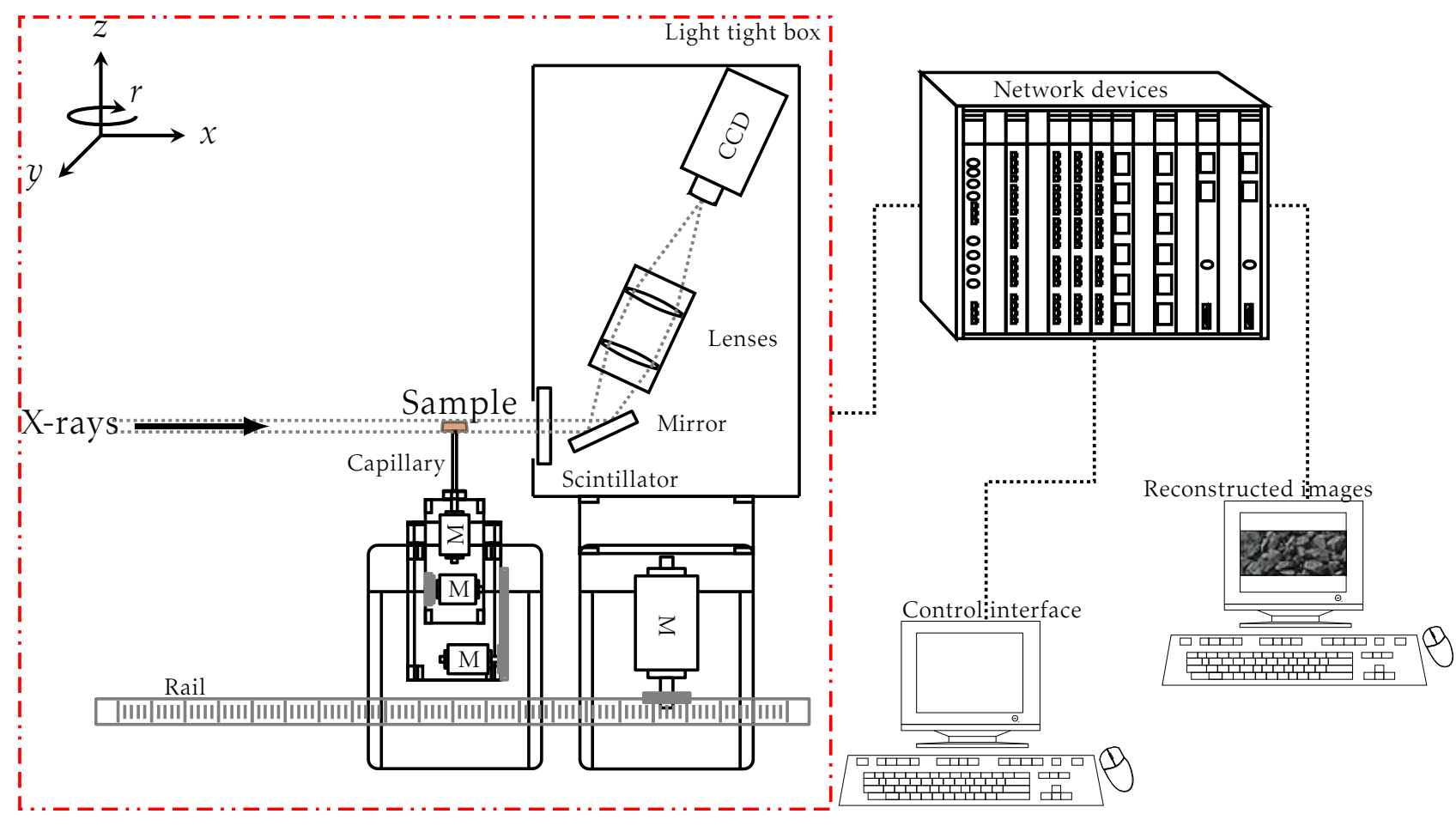

Figure E.51: Experimental set-up of ID-19 at ESRF

Un exemple de résultats obtenus par microtomographie est présenté dans la figureE.52.
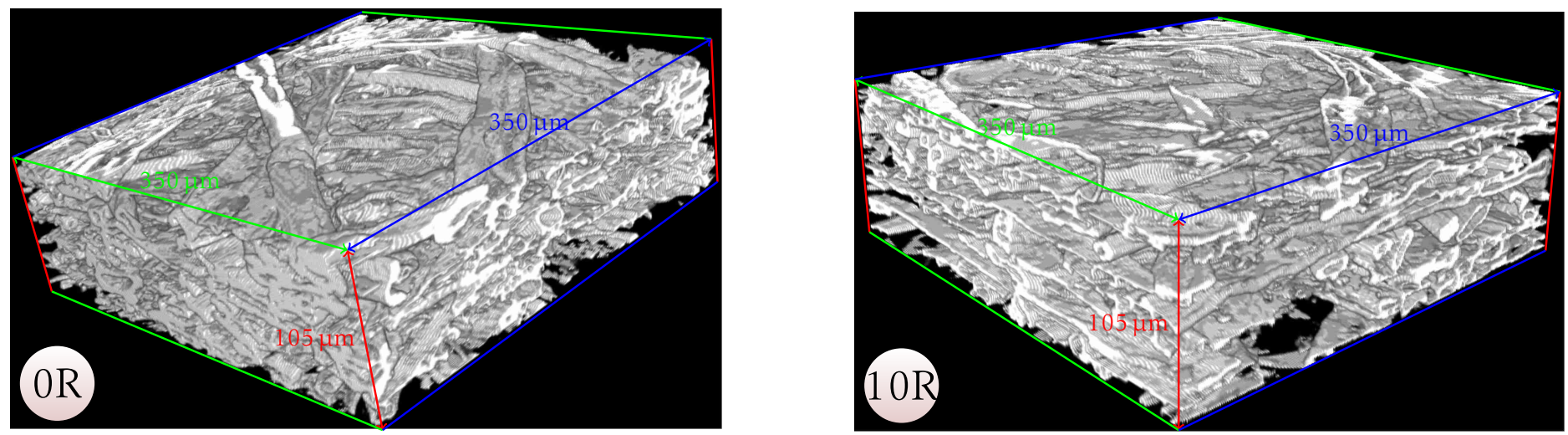

Figure E.52: Microtomographs of handsheets produced from never dried (left) and ten times recycled (right) pulps

A partir de la collection des images, il est possible d'obtenir des profils de porosité par seuillage comme illustré dans la figure E.53. De plus, l'aire de l'intersection des fibres, l'indice de l'écrasement de la fibre, des rapports d'aspects peuvent être analysés à partir de ces données. 


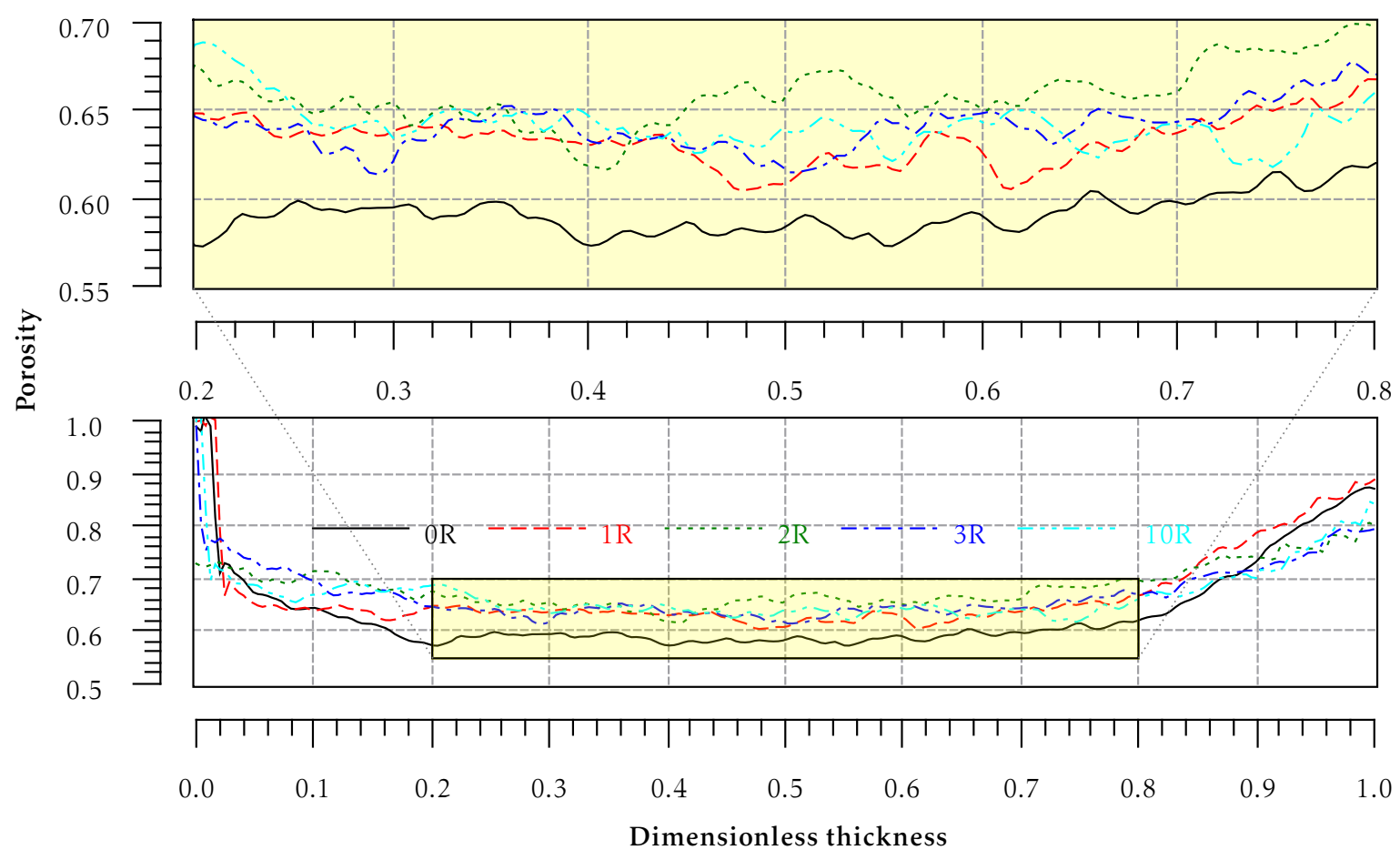

Figure E.53: Porosity profiles of handsheets made from recycled pulps

\section{Indice de traction des formettes réalisées à partir des pâtes re- cyclées}

Les propriétés de traction des échantillons testés ont été mesurées en utilisant un appareil de traction (Lorentzen \& Wettre). Une bande de papier, maintenue entre deux mâchoires est sollicitée avec un taux de déformation spécifié. La force par unité de largeur requise pour rompre l'échantillon est appelée force de traction, alors que l'indice de traction représente la force de traction par unité de grammage. La largeur des échantillons était de $15 \mathrm{~mm}$ et la vitesse de la mâchoire supérieure de $10 \mathrm{~mm} \cdot \mathrm{min}^{-1}$ [5].

L'effet du recyclage sur l'indice de traction est représenté sur la figure E.54. 


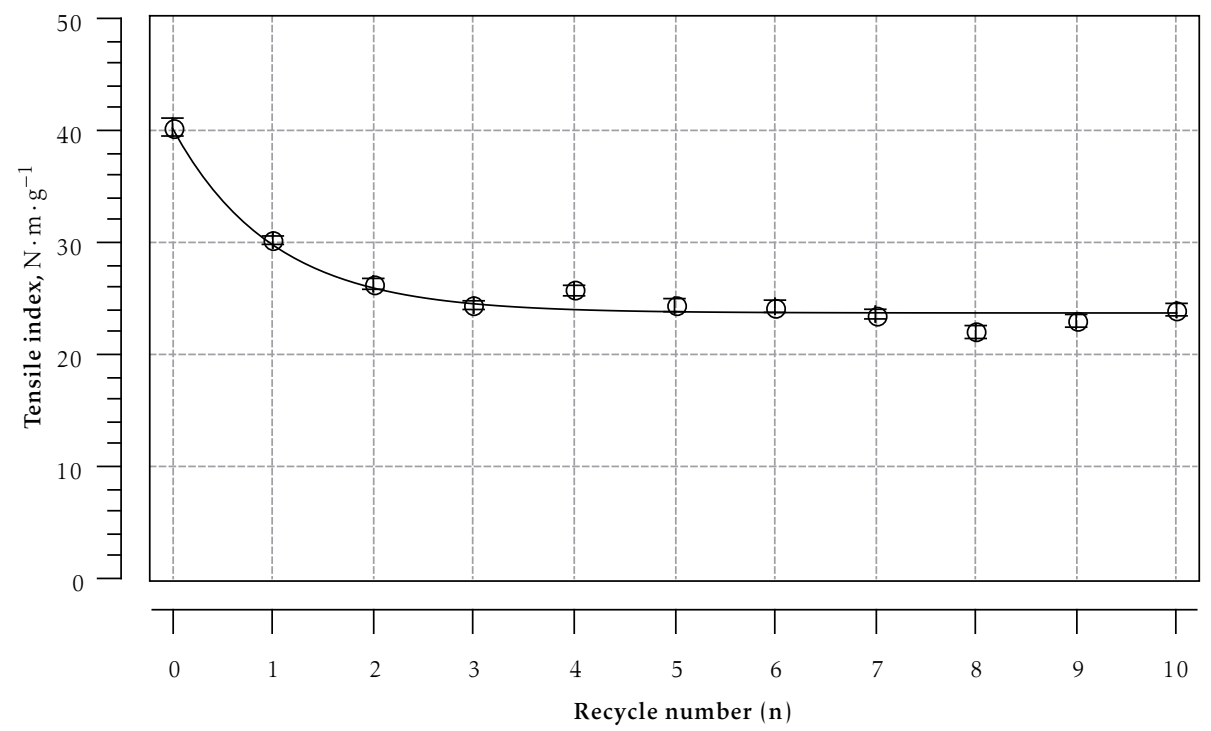

Figure E.54: Tensile index of handsheets made from recycled pulps

L'indice de traction des échantillons testés décroit exponentiellement avec le recyclage. Comme la résistance des fibres n'augmentait pas, comme indiqué par les tests de résistance à mors jointifs, la diminution de résistance trouve son origine dans la perte de résistance des liaisons. La diminution de résistance à la traction était plus importante lors des premiers cycles, en raison de la perte des éléments fins et la diminution de la flexibilité des fibres. Par conséquent, le nombre et la qualité des liaisons sont réduits initialement. L'augmentation de l'aire liée et de l'indice d'effondrement des fibres avec le recyclage (voir soussection 5.1.1) démontre que la qualité des liaisons est détériorée.

\section{Déformation à la rupture des formettes issues des pâtes recy- clées}

La déformation à la rupture est définie comme le maximum de déformation qu'un papier peut subir avant sa rupture. Il consiste ainsi en un pourcentage de modification de sa longueur initiale, lors de la rupture. L'effet du recyclage sur l'élongation est présenté sur la figure E.55. 


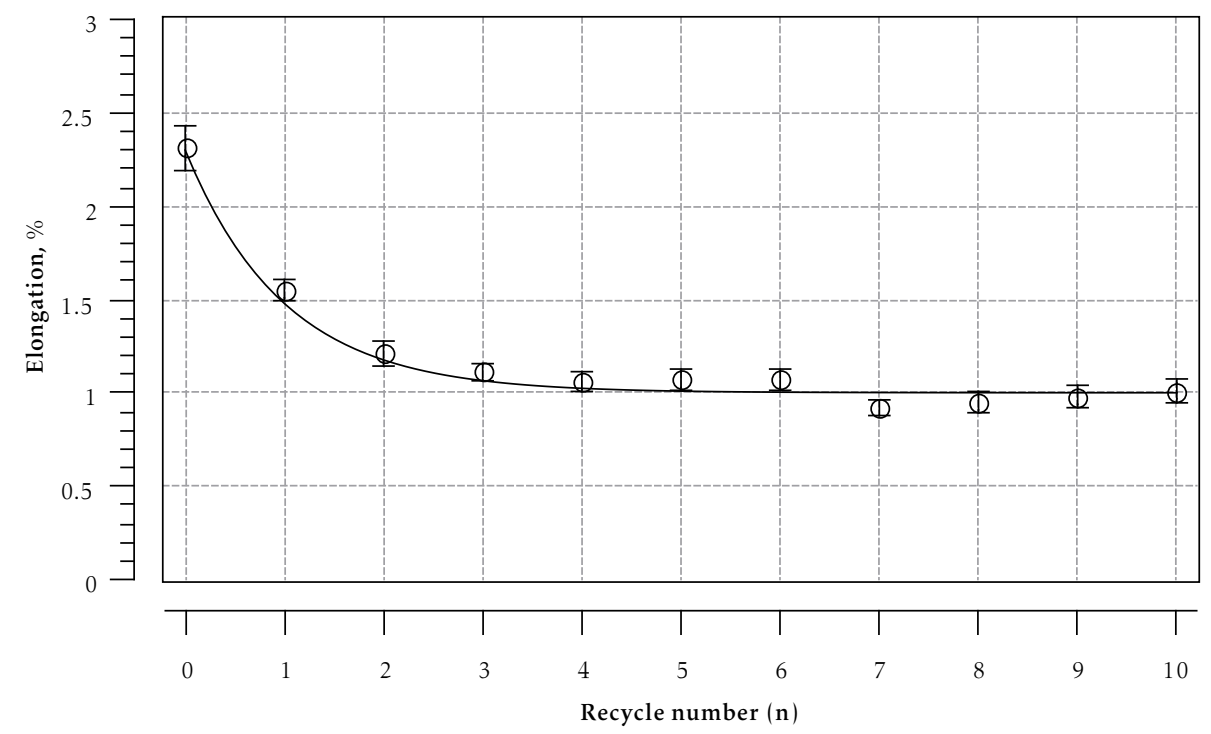

Figure E.55: Elongation of handsheets made from recycled pulps

La déformation à la rupture des échantillons testés diminue exponentiellement avec le recyclage. La diminution était plus importante lors des premiers recyclages ce qui peut être attribué à la perte de flexibilité des fibres et à l'activation du réseau. La réduction de la déformation indique une fragilisation du réseau fibreux. La contrainte transférée aux points faibles du réseau, qui provoque sa rupture, arrive plus tôt.

\section{Module élastique des formettes}

La pente initiale de la courbe contrainte-déformation est appelée module élastique ou module d'Young. La Figure E.56 montre l'effet du recyclage sur le module d'Young. 


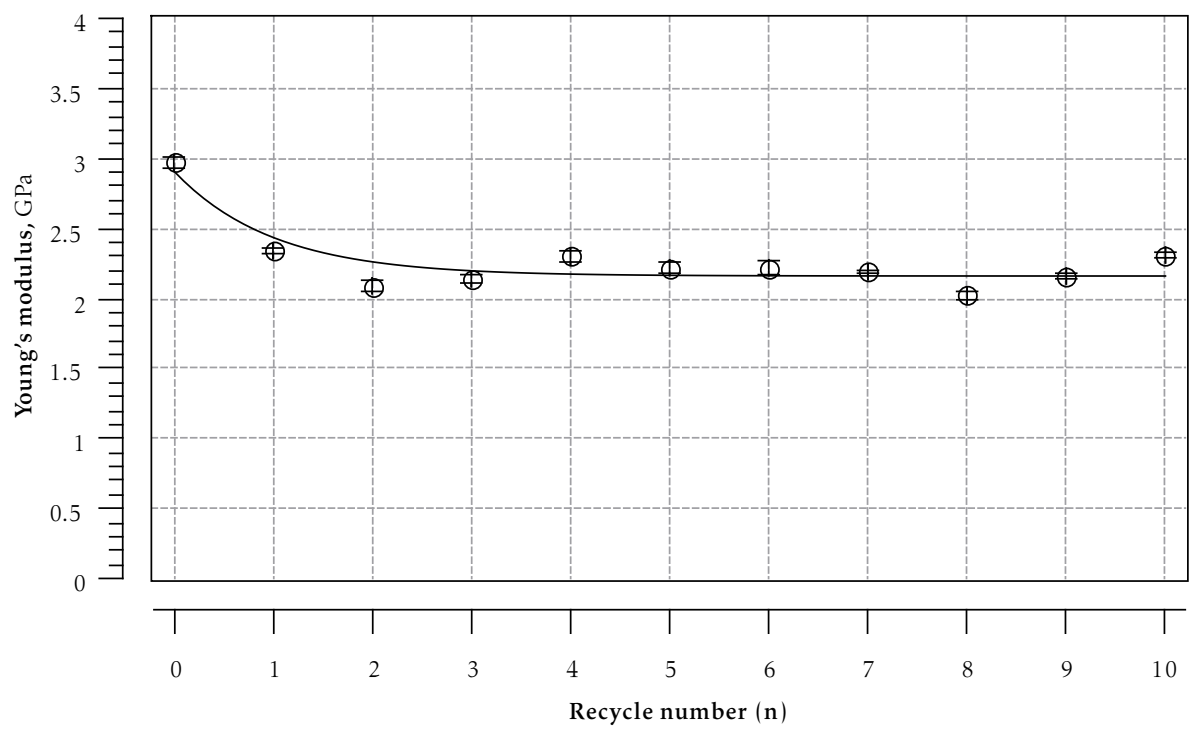

Figure E.56: Young's modulus of handsheets made from recycled pulps

Le module d'Young diminue exponentiellement avec le recyclage. La diminution de ce module était plus importante lors des premiers recyclages.

\section{L'activation du réseau fibreux lors de séchage contraint}

Le coefficient d'activation, $\langle A{ }\rangle$, lors du séchage a été utilisé précédemment pour corréler les propriétés des papiers produits à partir de pâtes raffinées à différents niveaux [182].

Il décrit l'activation du réseau fibreux et peut être défini empiriquement par l'expression suivante

$$
\left\langle\mathrm{A}_{s}\right\rangle=\left(\frac{\langle\mathrm{CWT}\rangle \cdot\langle\mathrm{CI}\rangle}{\left\langle l_{l}\right\rangle}\right)^{\left(\frac{1}{\langle\mathrm{WRV}\rangle}\right)}
$$

où $\langle\mathrm{CWT}\rangle,\langle\mathrm{CI}\rangle,\left\langle l_{l}\right\rangle$ et $\langle\mathrm{WRV}\rangle$ représente l'épaisseur moyenne de la paroi $(\mu \mathrm{m})$, l'indice de courbure moyen (\%), la longueur moyenne pondérée en longueur $(\mathrm{mm})$ et la valeur de rétention de l'eau $\left(\mathrm{mL} \cdot \mathrm{g}^{-1}\right)$, respectivement.

L'effet du recyclage sur l'activation du réseau est représenté sur la figure E.57. 


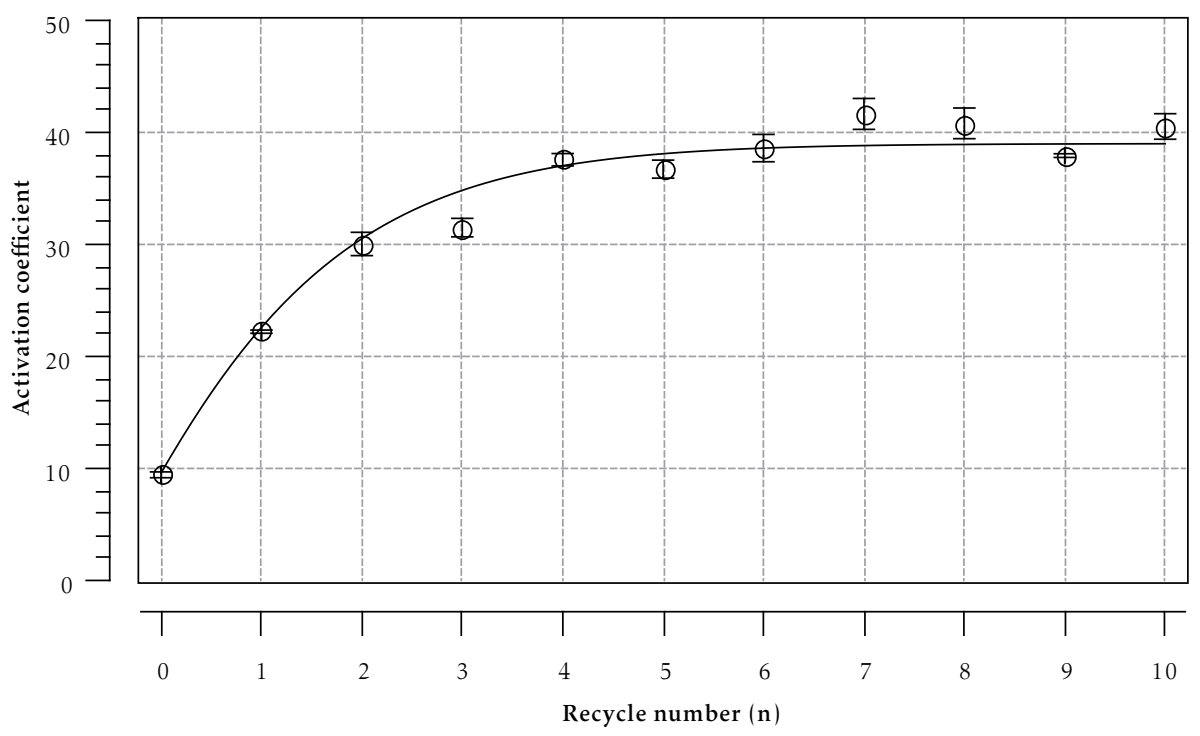

Figure E.57: Fibre network activation of recycled pulps

Le coefficient d'activation augmente avec le recyclage. L'augmentation du coefficient d'activation lors des premiers cycles est significative, atteignant la valeur asymptotique de 40, après quatre recyclages (cf. figure E.57). Comme la longueur de la fibre ne change pas avec le recyclage, alors, d'après la définition du coefficient d'activation du réseau fibreux, la diminution de son potentiel est due à la diminution de l'indice de courbure et du WRV. Une corrélation négative existe entre l'indice de traction et le coefficient d'activation (cf. figure E.58). Les fibres deviennent plates, droites et racornies durant le recyclage. Leur potentiel d'activation diminue avec le recyclage.

Le modèle de l'activation du réseau fibreux peut être utilisé pour différencier des pâtes recyclées durant les premiers cycles. La valeur asymptotique de 40 indique vraisemblablement la perte maximale de l'activation du réseau fibreux pour les conditions de recyclages mises en œuvre dans cette étude. 


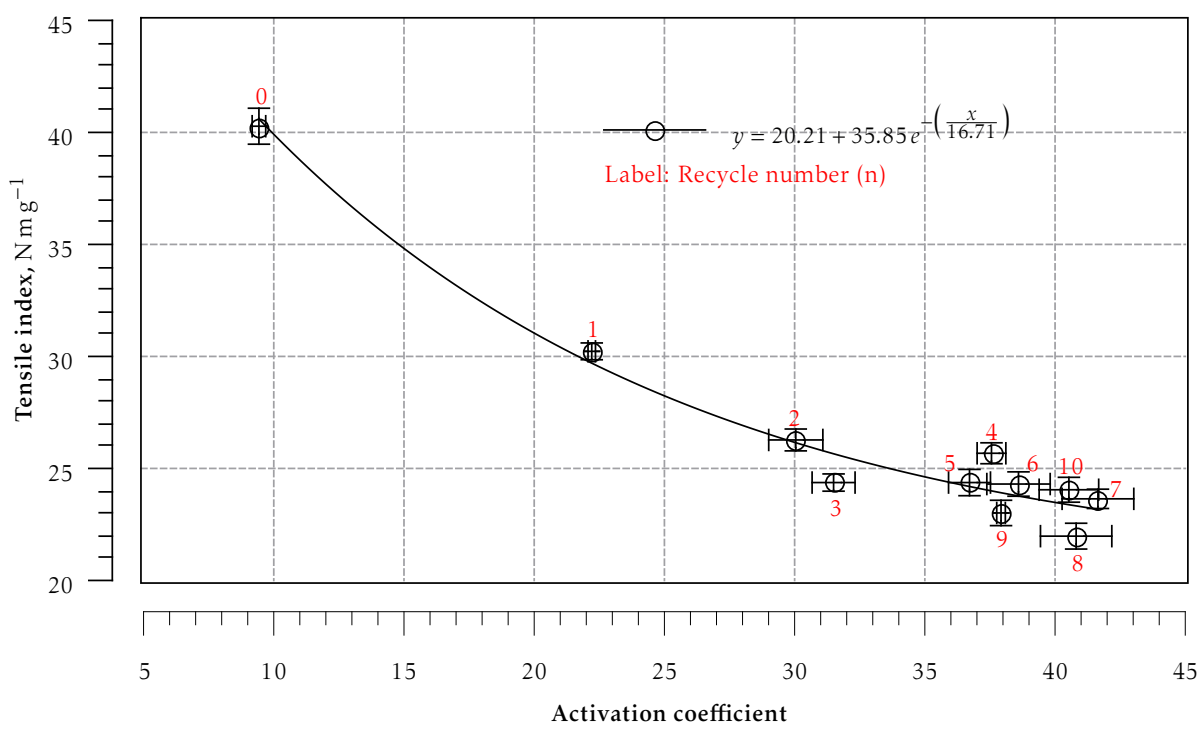

Figure E.58: Tensile index as a function of activation coefficient

\section{Propriétés des formettes réalisées à partir de mélanges de pâtes recyclées}

La pâte recyclée n'est que rarement incorporée dans des sortes de papier de qualité supérieure et son utilisation est une voie vers la réduction des couts. Les pâtes recyclées ont été mélangées avec de la pâte vierge, jamais séchée, afin d'étudier l'évolution des propriétés physiques des formettes ainsi obtenues; Les formettes ont été réalisées à partir de 25\%,50\% et 75\% de pâtes recyclées, puis stockées dans une salle conditionnée à $23^{\circ} \mathrm{C}$ et $50 \mathrm{HR}$ pour une période de 24 heures avant d'effectuer les essais physiques [3].

Les évolutions de la résistance à la traction permettent d'illustrer l'ensemble de nos résultats expérimentaux réalisés à partir des mélanges de pâtes recyclées. L'effet de la quantité et de la nature (nombre de cycles) de la pâte recyclée sur la résistance à la traction est présenté sur la figure E.59 . 


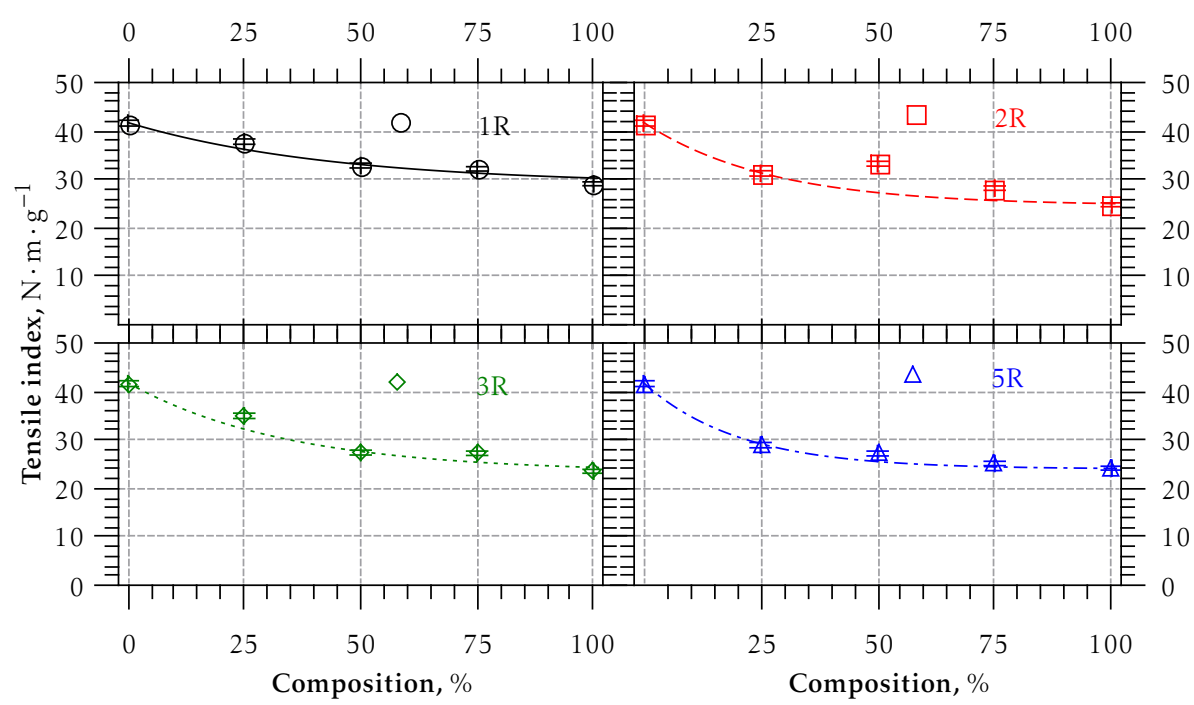

Figure E.59: Tensile index of handsheets made from recycled pulp blends

\section{Conclusions}

Le potentiel maximal des fibres pour la fabrication de papiers est encore actuellement en cours d'étude. De nombreux efforts ont été nécessaires pour améliorer l'utilisation de fibres recyclées. De part, des campagnes d'information pour le grand public, l'adoption de système de sollicitations et l'amélioration de la technologie de production, la collecte, le tri, l'utilisation de fibres recyclées a augmenté depuis quelques ces dernières années. Toutefois, le taux d'utilisation de fibres recyclées dans les papiers graphiques est encore négligeable. La limitation de l'utilisation de fibres recyclées pour des papiers de hautes qualités de papiers ne peut être résolue qu'après avoir évalué les changements physiques dans les fibres, les pâtes et papiers associée à de multiples recyclages. Dans cette étude, la pâte, jamais séchée initialement, a été recyclée dix fois. La caractérisation des pâtes et des fibres a été réalisée au cours des recyclages. Il a été constaté que les propriétés chimiques des pâtes n'ont pas changé. Toutefois, le processus de recyclage a modifié la morphologie des fibres. La diminution de la largeur de la fibre a été principalement causée par le retrait du bois de printemps.

La courbure et les coudes des fibres ont également été réduits. Les fibres deviennent plates et des irrégularités-défauts dans la fibre disparaissent. La paroi des fibres a été densifiée et la contribution des fibres de bois final est relativement plus élevée concernant le retrait de la paroi des fibres. 
Le remouillage in situ et le séchage des fibres dans un microscope électronique à balayage environnemental a permis de vérifier les résultats obtenus lors de l'analyse morphologique.

L'analyse de surface des fibres a révélé le clivage des couches des parois P/S1 lors du recyclage.

L'adhérence de surface des fibres augmente avec le recyclage comme indiqué par l'augmentation de la dissipation de l'énergie mesurée par microscopie à force atomique. L'augmentation de la longueur de rupture humide a également indiqué une augmentation de la surface de frottement et des forces capillaires avec le recyclage.

\section{Perspectives}

Les comportements des fibres de printemps et d'été lors de cycles sont différents et nécessiteraient une étude plus approfondie afin de distinguer leurs contributions sur les modifications des propriétés des fibres. Bien que les mesures de fibres uniques soient délicates, d'avantage de fibres devraient être testées et l'évolution des propriétés mécaniques des fibres ainsi que l'angle des microfibrilles établie en fonction du nombre de cycles de recyclage.

La surface des fibres est modifiée, comme démontré par des observations par microscopie électronique ainsi que par un microscope à force atomique. Néanmoins, la quantification de l'évolution de l'adhésion de surface et la rigidité avec le recyclage a besoin d'être établie en utilisant par exemple le microscope à force atomique. Les résultats obtenus par tomographie à rayons X nécessitent un approfondissement car la modification des changements structuraux en fonction de la nature des fibres, de printemps ou d'été, n'a pas été établie.

Nous avons constaté ici que l'effet du premier séchage sur la structure était important. Par conséquent, l'opération unitaire de séchage dans les usines non intégrées doit être revue. Il est connu que le racornissement des fibres d'une pâte contenant plus d'hémicelluloses est plus faible, et par conséquent leur recyclabilité est meilleure. Avec l'avènement de la bioraffinerie, en particulier l'extraction des hémicelluloses des pâtes, conduira à la réduction de la recyclabilité.

L'étude a portée ici sur l'influence des cycles de séchage et de pressage. Il conviendrait d'étudier l'influence du raffinage des pâtes sur le racornissement des fibres dans un travail futur. 


\section{References}

[1] ISO 5264-1:1979 - Pulps - Laboratory beating - Part 1: Valley beater method. Standard test method, International Organization for Standardization, Geneva, Switzerland, 1979. 37, 71

[2] ISO 3696:1987 - Water for analytical laboratory use - Specification and test methods. Standard test method, International Organization for Standardization, Geneva, Switzerland, 1987. 51

[3] ISO 187:1990 - Paper, board and pulps - Standard atmosphere for conditioning and testing and procedure for monitoring the atmosphere and conditioning of samples. Standard test method, International Organization for Standardization, Geneva, Switzerland, 1990. 113, 137, 211

[4] ISO 1974:1990 - Paper - Determination of tearing resistance (Elmendorf method). Standard test method, International Organization for Standardization, Geneva, Switzerland, 1990. 131

[5] ISO 1924-2:1994 - Paper and board - Determination of tensile properties - Part 2: Constant rate of elongation method. Standard test method, International Organization for Standardization, Geneva, Switzerland, 1994. 126, 206

[6] ISO 536:1995 - Paper and board - Determination of grammage. Standard test method, International Organization for Standardization, Geneva, Switzerland, 1995. 113

[7] SCAN-CM 15:88, Viscosity in cupri-ethylenediamine solution. SCANtest standards, Scandinavian Pulp, Paper and Board testing committee, Stockholm, 1998. 72 
[8] ISO 5267-1:1999 - Pulps - Determination of drainability - Part 1: Schopper-Riegler method. Standard test method, International Organization for Standardization, Geneva, Switzerland, 1999. 43

[9] T 279 pm-99, Effective fiber length index by zero/short - span tensile testing. Standard test method, TAPPI Press Atlanta, Ga, 1999. 125

[10] ISO 2758:2001 - Paper - Determination of bursting strength. Standard test method, International Organization for Standardization, Geneva, Switzerland, 2001. 130

[11] T $223 \mathrm{~cm}-01$, Pentosans in wood and pulp. Standard test method, TAPPI Press Atlanta, Ga, 2001. 72

[12] ISO 5264-2:2002 - Pulps - Laboratory beating - Part 2: PFI mill method. Standard test method, International Organization for Standardization, Geneva, Switzerland, 2002. 35

[13] ISO 302:2004 - Pulps - Determination of Kappa number. Standard test method, International Organization for Standardization, Geneva, Switzerland, 2004. 73

[14] ISO 5263-1:2004 - Pulps - Laboratory wet disintegration. Standard test method, International Organization for Standardization, Geneva, Switzerland, 2004. 35, 71, 113

[15] ISO 5269-2:2004 - Pulps - Preparation of laboratory sheets for physical testing - Part 2: Rapid-Köthen method. Standard test method, International Organization for Standardization, Geneva, Switzerland, 2004. 71, 113

[16] ISO 534:2005 - Paper and board - Determination of thickness, density and specific volume. Standard test method, International Organization for Standardization, Geneva, Switzerland, 2005. 114

[17] T $233 \mathrm{~cm}-06$, Fiber length of pulp by classification. Standard test method, TAPPI Press Atlanta, Ga, 2006. 36

[18] ISO 2471:2008 - Paper and board - Determination of opacity (paper backing) - Diffuse reflectance method. Standard test method, International Organization for Standardization, Geneva, Switzerland, 2008. 134 
[19] ISO 2470-1:2009 - Paper, board and pulps - Measurement of diffuse blue reflectance factor - Part 1: Indoor daylight conditions (ISO brightness). Standard test method, International Organization for Standardization, Geneva, Switzerland, 2009. 134

[20] T $221 \mathrm{~cm}-09$, Drainage time of pulp. Standard test method, TAPPI Press Atlanta, Ga, 2009. 17

[21] Cepi sustainability report 2011 - european paper industry - part of a sustainable future. http://www.cepi-sustainability.eu/uploads/ Sustainability_Report_WEB.pdf, 2012. [Online; accessed 02-September2012]. 172, 173

[22] M. D. Abramoff, P. J. Magelhaes, and S. J. Ram. Image processing with ImageJ. Biophotonics International, 11(7):36-42, 2004. 87, 102, 109

[23] L. G. Aggebrandt and O. Samuelson. Penetration of water-soluble polymers into cellulose fibers. Journal of Applied Polymer Science, 8(6):28012812, November 1964. 13, 20

[24] Y. Agyei. Deforestation in sub-saharan Africa. African Technology Forum, 8(1), May 2009. 3, 171

[25] F. W. Ahrens and H. Xu. Effect of pulp drying history on pressing and drying. In 1999 TAPPI Engineering/Process and Product Quality Conference, volume 2, pages 1009-1024. Tappi Press Atlanta, Ga, August 1999. 33

[26] R. Alén. Structure and chemical composition of wood. In Forest Products Chemistry, volume 3 of Papermaking Science and Technology, pages 11-57. Fapet Oy, Jyvöskylö, Finland, 1998. 9

[27] S. D. Alexander, R. Marton, and S. D. McGovern. Effect of beating and wet pressing on fiber and sheet properties: I. Individual fiber properties. Tappi, 51(6):277-283, 1968. 22, 67

[28] B. Alince and T. G. M. van de Ven. Porosity of swollen pulp fibres evaluated by polymer adsorption. In C. F. Baker, editor, The fundamentals of papermaking materials: Transactions of the 11th Fundamental Research Symposium, volume 2, pages 771-788. Pira International, Cambridge, UK, September 1997. 13 
[29] P. Ander and G. Daniel. Morphology of spruce fibre dislocations as studied by balloon swelling and acid cleavage - light and electron microscope observations. In U. Schmitt, P. Ander, J. Barnett, A. M. Emons, P. Saranpää, and S. Tschegg, editors, Wood Fibre Cell Walls: Methods to Study Their Formation, Structure and Properties, COST Action E20. OPOCE, EC, Brussels, 2003. 22, 61, 199

[30] P. Ander, G. Daniel, C. Garcia-Lindgren, and A. Marklund. Characterization of industrial and laboratory pulp fibres using $\mathrm{HCl}$, cellulase and FiberMaster analysis. Nordic Pulp E Paper Research Journal, 20(1):115120, 2005. 22, 199

[31] P. Ander, G. Daniel, B. Pettersson, and U. Westermark. Possible applications of cellobiose oxidizing and other flavine adenine dinucleotide enzymes in the pulp and paper industry. In T. W. Jeffries and L. Viikari, editors, Enzymes for Pulp and Paper Processing, volume 655 of ACS symposium series, chapter 23, pages 297-307. November 1996. 21, 22, 199

[32] P. Ander and K. Nyholm. Deformations in wood and spruce pulp fibres: Their importance for wood and pulp properties. In S. E. StanzlTschegg and A. Reiterer, editors, Proceedings of the International Symposium on Wood Machining - Properties of Wood and Wood Composites Related to Wood Machining, Vienna, Austria, September 2000. ChristianDoppler-Laboratory for Fundamentals of Wood Machining. 21, 199

[33] C. Antoine, P. Nygard, Ø. W. Gregersen, R. Holmstad, T. Weitkamp, and C. Rau. 3D images of paper obtained by phase-contrast X-ray micro tomography: Image quality and binarisation. Nuclear Instruments E Methods in Physics Research Section a-Accelerators Spectrometers Detectors and Associated Equipment, 490(1-2):392-402, September 2002. 115, 204

[34] R. Aravamuthar and J. S. Greaves. Effect of multiple recycles on wheat straw fibers. In Pulping Conference, Proceedings of the Technical Association of the Pulp and Paper Industry, volume 1, pages 485-510, Atlanta, Ga, 1998. Tappi Press Atlanta, Ga. 33

[35] M. Babiak and J. Kúdela. A contribution to the definition of the fiber saturation point. Wood Science and Technology, 29(3):217-226, 1995.18

[36] E. L. Back. Thermal auto-crosslinking in cellulose material. Pulp E Paper Canada, 68(4):165-171, 1967. 33 
[37] J. R. Barnett and V. A. Bonham. Cellulose microfibril angle in the cell wall of wood fibres. Biological Reviews of the Cambridge Philosophical Society, 79(2):461-472, May 2004. 8, 175

[38] A. D. Bawden and R. P. Kibblewhite. Effects of multiple drying treatments on kraft fibre walls. Journal of Pulp and Paper Science, 23(7):J340J346, 1997. 33

[39] A. Bergander. Local variability in chemical and physical properties of spruce wood fibers. Doctoral thesis, KTH, Pulp and Paper Technology, 2001. 8

[40] A. Bergander and L. Salmén. Cell wall properties and their effects on the mechanical properties of fibers. Journal of Materials Science, 37(1):151156, Januray 2002. 8

[41] J. Berthold, J. Desbrieres, M. Rinaudo, and L. Salmén. Types of adsorbed water in relation to the ionic groups and their counterions for some cellulose derivatives. Polymer, 35(26):5729-5736, 1994. 167

[42] J. Berthold and L. Salmén. Inverse size exclusion chromatography (ISEC) for determining the relative pore size distribution of wood pulps. Holzforschung, 51(4):361-368, January 1997. 13, 20, 21, 51, 193

[43] J. L. Bienfait. Relation of the manner of failure to the structure of wood under compression parallel to the grain. J. Agric. Res, 33(2):183-194, 1926. 21

[44] F. A. Blouin, L. F. Martin, and S. P. Rowland. Gel-permeation properties of cellulose: Part III: measurement of pore structure of unmodified and of mercerized cottons in fibrous form. Textile Research Journal, 40(9):809$813,1970.21$

[45] F. C. Bond. The third theory of comminution. In Transactions of the American Institute of Mining, Metallurgical and Petroleum Engineers, volume 193, pages 484-494. 1952. 25

[46] R. Booker and J. Sell. The nanostructure of the cell wall of softwoods and its functions in a living tree. European Journal of Wood and Wood Products, 56(1):1-8, January 1998. 6 
[47] J. Bouchard and M. Douek. The effect of recycling on the chemical properties of pulps. Journal of Pulp and Paper Science, 20(5):J131-J136, May 1994. 73

[48] A. A. Brancato. Effect of Progressive Recycling on Cellulose Fiber Surface Properties. Doctoral thesis, School of Chemical and Biomolecular Engineering, December 2008. 94

[49] J. Brändström, S. L. Bardage, G. Daniel, and T. Nilsson. The structural organisation of the S1 cell wall layer of norway spruce tracheids. IAWA Journal, 24(1):27-40, 2003. 6

[50] K. Bredereck, A. Bluher, and A. Hoffmannfrey. Determination of the pore structure of cellulose fibres by exclusion chromatography. Papier, 44(12):648-656, December 1990. 21

[51] C. A. Bronkhorst and K. A. Bennett. Deformation and failure behavior of paper. In R. E. Mark, J. Borch, and C. Habeger, editors, Handbook of Physical Testing of Paper, volume 1, pages 314-419. CRC Press, 2 edition, September 2001. 122

[52] W. B. Campbell. The mechanism of bonding. Tappi, 42(12):999-1001, 1959. 27

[53] B. Cao, U. Tschirner, and S. Ramaswamy. Impact of pulp chemical composition on recycling. Tappi Journal, 81(12):119-127, December 1998. 33

[54] G. Carlsson and T. Lindstorm. Hornification of cellulosic fibres during wet pressing. Svensk Papperstidning-Nordisk Cellulosa, 87(15):R119R125, October 1984. 33

[55] L. A. Carlsson and T. Lindstrom. A shear-lag approach to the tensile strength of paper. Composites Science and Technology, 65(2):183-189, 2005. 122

[56] P. C. Carman. Fluid flow through granular beds. Transactions of the Institution of Chemical Engineers, 15:150-166, 1937. 18

[57] A. Chakraborty, M. M. Sain, M. T. Kortschot, and S. B. Ghosh. Modeling energy consumption for the generation of microfibres from bleached kraft pulp fibres in a PFI mill. BioResources, 2(2):210-22, May 2007. 26 
[58] Y. M. Chen, J. Q. Wan, and Y. W. Ma. Effect of noncellulosic constituents on physical properties and pore structure of recycled fibre. APPITA Journal, 62(4):290-296, July 2009. 33

[59] Y. M. Chen, Y. Wang, J. Q. Wan, and Y. W. Ma. Crystal and pore structure of wheat straw cellulose fiber during recycling. Cellulose, 17(2):329-338, April 2010. 33

[60] M. S. Cintrón, G. P. Johnson, and A. French. Young's modulus calculations for cellulose I $\beta$ by MM3 and quantum mechanics. Cellulose, 18:505516, 2011. 23, 201

[61] Confederation of European Paper Industries. Key statistics 2010 - European pulp and paper industry. http://www.cepi.org/docshare/ Common $/$ GetFile.asp? PortalSource $=961 \&$ DocID $=33814 \& \mathrm{mfd}=$ off $\&$ pdoc $=1$, June 2011. [Online; accessed 3-April-2012]. 33, 151

[62] S. R. Corson. Influence of fibre and fines fractions on thermomechanical pulp properties. Doctoral thesis, The Norwegian Institute of Technology, 1979. 27,61

[63] European Recovered Paper Council. European declaration on paper recycling, 2006-2010. http://www.recoveredpaper-id.eu/uploads/ MonitReport09.pdf. [Online; accessed 16-March-2012]. 32

[64] European Recovered Paper Council. Five years of raising the bar in paper recycling. http://www.paperforrecycling.eu/uploads/Modules/ Newsmanager/erpc-monitoring-report-2010-final-2.pdf. [Online; accessed 16-March-2012]. 32

[65] Paper Industry Association Council. Paper \& paperboard recovery. http: //www.paperrecycles.org/stat_pages/recovery_rate.html, 2011. [Online; accessed 16-March-2012]. 32

[66] W. F. Cowan. Zero/short span tensile testing can determine basic paper properties. Pulp Paper, 60:84-86, 1986. 123, 138

[67] W. F. Cowan and E. J. K. Cowdrey. Evolution of paper strength components by short-span tensile analysis. Tappi, 57(2):90-93, 1974. 124

[68] R. L. Crawford. Lignin biodegradation and transformation. Wiley Interscience publication, New York, April 1981. 9 
[69] D. Curtil. Le schopper s'automatise, dynamic freeness Tester-DFT3000. http://cerig.efpg.inpg.fr/dossier/EFPG-innovations/page14.htm, 2007. [Online; accessed 16-March-2012]. 46

[70] D. Da Silva Perez, D. Reich, D. Moineau, G. Eymin-Petot-Tourtollet, A. Guillemain, and Petit-Conil. On-line measurement of pulp and fibre quality : sampling and analytical devices. In COST E41 workshop, Proceedings, Bled, Slovenia, September 2007. 17

[71] G. D. Danilatos. Optimum beam transfer in the environmental scanning electron microscope. Journal of Microscopy, 234(1):26-37, 2009. 81

[72] H. Darcy. Les fontaines publiques de la ville de Dijon : exposition et application des principes à suivre et des formules à employer dans les questions de distribution d'eau. V. Dalmont (Paris), 1856. 17, 158

[73] G. de Silveira, P. Forsberg, T. E. Conners, and S. Banerjee. Scanning electron microscopy: A tool for the analysis of wood pulp fibers and paper. In T. E. Conners and S. Banerjee, editors, Surface Analysis of Paper, chapter 2, pages 47-71. CRC Press, 1995. 81

[74] J. M. B. F. Diniz, M. H. Gil, and J. A. A. M. Castro. Hornification - its origin and interpretation in wood pulps. Wood Science and Technology, 37(6):489-494, April 2004. 33

[75] J. C. Domec and B. L. Gartner. How do water transport and water storage differ in coniferous earlywood and latewood? Journal of Experimental Botany, 53(379):2369-2379, December 2002. 5

[76] I. Duchesne and J. C. Daniel. The ultrastructure of wood fibre surfaces as shown by a variety of microscopical methods - A review. Nordic Pulp E Paper Research Journal, 14(2):129-139, 1999. 13

[77] I. Duchesne and J. C. Daniel. Changes in surface ultrastructure of norway spruce fibres during kraft pulping - visualisation by field emission-sem. Nordic Pulp E Paper Research Journal, 15(1):54-61, 2000. 7, 8, 13, 175

[78] D. F. Dumbleton. Longitudinal compression of individual pulp fibers. Tappi, 55(6):461-467, 1972. 110, 203 
[79] Environmental Paper Network Paper Calculator. Environmental impact estimates. http://calculator.environmentalpaper.org/home, April 2012. [Online; accessed 3-April-2012]. 151, 152

[80] FAO. ForesSTAT of food and agriculture organization of the united nations. http://faostat.fao.org/site/626/default.aspx\#ancor, 2011. [Online; accessed 16-March-2012]. 30, 151, 171, 172

[81] B. D. Favis and D. A. I. Goring. A model for the leaching of lignin macromolecules from pulp fibres. Journal of Pulp and Paper Science, 10:J139J143, 1984. 13, 176

[82] B. D. Favis, W. Q. Yean, and D. A. I. Goring. Molecular weight of lignin fractions leached from unbleached kraft pulp fibers. Journal of Wood Chemistry and Technology, 4(3):313-320, September 1984. 13

[83] C. Fellers. Paper physics. In M. Ek, G. Gellerstedt, and G. Henriksson, editors, Pulp and Paper Chemistry and Technology: Paper Products Physics and Technology, volume 4, pages 25-67. Walter de Gruyter \& Co, August 2009. 114, 203

[84] D. Fengel. Ultrastructural behaviour of cell wall polysaccharides. Tappi, 53(3):497-503, 1970. 7, 8, 175

[85] D. Fengel and D. Grosser. Chemische zusammensetzung von nadel- und laubhölzern. European Journal of Wood and Wood Products, 33(1):32-34, 1975. 9

[86] D. Fengel and M. Stoll. Über die Veränderungen des Zellquerschnitts, der Dicke der Zellwand und der Wandschichten von Fichtenholz-Tracheiden innerhalb eines Jahrringes. Holzforschung, 27(1):1-7, February 1973. 6, 7

[87] D. Fengel and G. Wegener. Wood: Chemistry, Ultrastructure, Reactions. Walter De Gruyter Inc, June 1989. 8

[88] Foex Indexes. Market comments - general economy and pix european and chinese indices. http://www.foex.fi/index.php? mact $=$ News, cntnt 01 , detail,0\&cntnt01 articleid=195\&cntnt01 origid $=$ 15\&cntnt01 returnid=81, April 2012. [Online; accessed 3-April-2012]. 151,173 
[89] O. L. Forgacs. Structural weaknesses in softwood pulp tracheids. Tappi, 44(2):112-119, February 1961. 21

[90] A. Frey-Wyssling. Röntgenometrische Vermessung der Sunmikroskopische Räume in Gerüstsubtanzen Mikrofibrillen. Protoplasma, 27(1):372411, 1937. 7, 175

[91] H. W. Giertz. Ännu ett sätt att se på malningsprocessen. Norsk Skogsindustri, 18(7):239-248, 1964. 29

[92] H. W. Giertz. The influence of beating on individual fibers and the causal effects on paper properties. In International Symposium on Fundamental Concepts of Refining, pages 87-92, Appleton, Wisconsin, USA, September 1980. Institute of Paper Chemistry. 25

[93] A. A. Gorbunov, L. Y. Solovyova, and V .A. Pasechnik. Fundamentals of the theory and practice of polymer gel-permeation chromatography as a method of chromatographic porosimetry. Journal of Chromatography $A$, 448:307-332, January 1988. 21

[94] D. A. I. Goring. A speculative picture of the delignification process. In Cellulose Chemistry and Technology, volume 48 of ACS Symposium Series, chapter 19, pages 273-277. American Chemical Society, August 2011. 12

[95] J. Grignon and A. M. Scallan. Effect of $\mathrm{pH}$ and neutral salts upon the swelling of cellulose gels. Journal of Applied Polymer Science, 25(12):28292843, 1980. 51

[96] N. Gurnagul, D. H. Page, and M.G. Paice. The effect of cellulose degradation on the strength of wood pulp fibres. Nordic Pulp E Paper Research Journal, 7(3):152-154, 1992. 21, 199

[97] N. Gurnagul and R. S. Seth. Wet-web strength of hardwood kraft pulps. Pulp E Paper Canada, 98(9):44-48, September 1997. 27

[98] H. Dolmetsch u. H. Dolmetsch. Über die Beziehung zwischen Kristalliten, Elementarfibrillen u. zugänglichen Bereichen in Cellulosefasern Insbesondere in Holzfaserzellwänden. Das Papier, 22(1):1-11, 1968. 20

[99] L. Hagel and P.L. Dubin. Pore size distributions. In Aqueous SizeExclusion Chromatography, volume 40 of Journal of chromatography library, chapter 5, pages 119-155. Elsevier, 1988. 56 
[100] P. Hakkila. Structure and properties of wood and woody biomass. In Forest resources and sustainable management, page 129. Published in cooperation with the Finnish Paper Engineers' Association and TAPPI, 1998. 7,9

[101] N. Hartler. Aspects on curled and microcompressed fibers. Nordic Pulp E Paper Research Journal, 10(1):1-7, 1995. 21

[102] T. Hattula and Niemi H. Sulphate pulp fibre flexibility and its effect on sheet strength. Paperi Ja Puu-Paper and Timber, 70(4):356-361, 1988. 25

[103] A. Heikkurinen. Single fiber properties. In Pulp and Paper Testing, volume 17 of Papermaking Science and Technology, chapter 2. Fapet Oy, Jyvöskylö, Finland, 1998. 24

[104] R. R. A. Higham. In A handbook of papermaking: The technology of pulp, paper and board manufacture, page 400. Business Books, 2 edition, 1968. 6

[105] H. S. Hill, J. Edwards, and L. R. Beath. Curlated pulp - A new approach to pulp processing. Tappi, 33(1):36-44, January 1950. 17

[106] R. G. Holdich. Crushing and classification. In Fundamentals of particle technology, pages 113-122. Midland Information Technology and Publishing, 2002. 26

[107] R. A. Horn. Morphology of wood pulp fiber from softwoods and influence on paper strength. United States Department of Agriculture, Forest Service, (242):1-11, 1974. 14

[108] R. C. Howard and W. Bichard. The basic effects of recycling on pulp properties. Journal of Pulp and Paper Science, 18(4):151-159, July 1992. 33

[109] M. A. Hubbe, R. A. Venditti, and O. J. Rojas. What happens to cellulosic fibers during papermaking and recycling? A review. BioResources, 2(4):739-788, November 2007. 33

[110] E. L. Hult. DCP/MAS ${ }^{13}$ C-NMR spectroscopy applied to structure and interaction studies on wood and pulp fibers, KTH, Pulp and Paper Technology, 2001. 7, 175 
[111] E. L. Hult, P. T. Larsson, and T. Iversen. Cellulose fibril aggregation - an inherent property of kraft pulps. Polymer, 42(8):3309-3314, April 2001. 33

[112] Marja-Sisko Ilvessalo-Pföffli. In Fiber Atlas: Identification of Papermaking Fibers, page 15. Springer, 1 edition, May 1995. 6

[113] K. Ishikiriyama, M. Todoki, T. Kobayashi, and H. Tanzawa. Pore size distribution measurements of poly(methyl methacrylate) hydrogel membranes for artificial kidneys using differential scanning calorimetry. Journal of Colloid and Interface Science, 173(2):419-428, August 1995. 167

[114] S. Janardhnan and M. Sain. Bio-treatment of natural fibers in isolation of cellulose nanofibres: Impact of pre-refining of fibers on bio-treatment efficiency and nanofiber yield. Journal of Polymers and the Environment, 19:615-621, 2011. 36

[115] P. Jandera and G. Henze. Liquid chromatography, 1. Fundamentals, history, instrumentation, materials. In Ullmann's Encyclopedia of Industrial Chemistry, volume 21, pages 85-138. Wiley-VCH, 6 edition, 2002. 20

[116] H. F. Jang, R. Amiri, R. S. Seth, and A Karnis. Fiber characterization using confocal microscopy - collapse behavior of mechanical pulp fibers. Tappi Journal, 79(4):203-210, April 1996. 120

[117] G. Jayme. Mikro-Quellungsmessungen an Zellstoffen. Der PapierFabrikant/Wochenblatt für Papierfabrikation, 6:187-194, 1944. 33

[118] G. Jayme and M. Harders-Steinhauser. Über die Erkennung von Dichte Unterschieden in Cellulosefasern durch Doppelfärbung. Das Papier, 9(21/22):507-510, 1955. 22

[119] G. Jayme and G. Hunger. Electron microscope 2- and 3-dimensional classification of fibre bonding. Transactions of the Symposium Held at Oxford, September 1961. British Paper and Board Makers' Association, Technical Section. 28

[120] G. Jayme and G. Hunger. Rearrangement of microfibrils in dried cellulose and the implication of this structure alteration on pulp properties. Fundamentals of Papermaking Fibres, Transactions of the Symposium, pages 263-271, London, 1958. BP\&BMA. 33 
[121] G. Jayme and L. Rothamel. Development of a standard centrifugal method for determining the swelling values of pulps. Das Papier, 2(11):7$18,1948.18$

[122] C. A. Jentzen. The effect of stress applied during drying on some of the properties of individual pulp fibers. Doctoral thesis, Institute of Paper Science and Technology, January 1964. 29, 110, 203

[123] T. Johansson, H. Paulapuro, and T. C. Maloney. Removal of water from the cell wall during drying. In PITA Water Removal Conference 1997, volume 39, pages 39-47, 1998. 13

[124] T. Kang. Role of External Fibrillation in Pulp and Paper Properties. Doctoral thesis, Helsinki University of Technology, Laboratory of Paper and Printing Technology, Espoo, September 2007. 18

[125] C. T. Keith and W. A. Jr. Côté. Microscopic characterization of slip lines and compression failures in wood cell walls. Forest Products Journal, 18(3):67-74, 1968. 22, 177

[126] R. J. Kerekes. CANFOR Pulp refining seminar Part I: Basics. http://www.temap.com/assets_main/documents/CanforSeminarI_ USMay10x.pdf. [Online; accessed 16-March-2012]. 25

[127] R. J. Kerekes. Characterizing refining action in PFI mills. Tappi Journal, 4(3):9-13, March 2005. 35

[128] A. J. Kerr and D. A. I. Goring. The role of hemicellulose in the delignification of wood. Canadian Journal of Chemistry, 53(6):952-959, March 1975. 12

[129] R. P. Kibblewhite. Fractures and dislocations in the walls of kraft and bisulphite pulp fibres. Cellulose chemistry and technology, 10(4):497-503, July/August 1976. 21

[130] R. P. Kibblewhite and D. Brooks. Factors which influence the wet-web strength of commercial pulps. APPITA Journal, 28(4):227-231, 1975. 16

[131] F. Kick. Das Gesetz der proportionalen Widerstände und seine Anwendungen. Felix, A., 1885. 25 
[132] H. Kjellgren. Influence of paper properties and polymer coatings on barrier properties of greaseproof paper. PhD thesis, Karlstad University, Faculty of Technology and Science, 2007. 25

[133] J. H. Klungness and D. F. Caufield. Mechanisms affecting fiber bonding during drying and aging of pulps. Tappi Journal, 65(12):94-97, December 1982. 33

[134] A. Kongdee, T. Bechtold, E. Burtscher, and M. Scheinecker. Inverse size exclusion chromatography - a technique of pore characterisation in textile materials. Lenzinger Berichte, 82:96-101, 2003. 193

[135] E. J. Kontturi. Surface chemistry of cellulose: From natural fibres to model surfaces. Doctoral thesis, Eindhoven University of Technology, Espoo, February 2005. 93

[136] J. E. Lain. Fibre saturation point by inverse gel filtration. Paperi ja Puu, 59(11):735-736, 1977. 21

[137] P. R. Larson. A biological approach to wood quality. Tappi, 45(6):443448, 1962. 5

[138] P. R. Larson. Wood formation and the concept of wood quality, volume 1. Bull. 74, School of Forestry, Yale University, 1969. 5

[139] P. Larsson. Dimensional Stability of Paper : Influence of Fibre-Fibre Joints and Fibre Wall Oxidation. Doctoral thesis, KTH, Fibre Technology, 2008. 98

[140] P. T. Larsson, K. Wickholm, and T. Iversen. A CP/MAS ${ }^{13}$ C-NMR investigation of molecular ordering in celluloses. Carbohydrate Research, 302(1-2):19-25, July 1997. 7

[141] J. Lenz, J. Schurz, and E. Wrentschur. Vergleichende Untersuchungen zur Faserbildung aus Lösungen von Cellulose in organischen Lösungsmitteln und Cellulolsederivaten in Natronlauge. Das Papier, 42(12):663-679, 1988. 20

[142] T. Q. Li, U. Henriksson, and L. Ödberg. Determination of pore sizes in wood cellulose fibers by $2 \mathrm{H}$ and $1 \mathrm{H}$ NMR. Nordic Pulp E Paper Research Journal, 8(3):326-330, 1993. 13, 20 
[143] J. K. Lin, M. R. Ladisch, J. A. Patterson, and C. H. Noller. Determining pore size distribution in wet cellulose by measuring solute exclusion using a differential refractometer. Biotechnology and Bioengineering, 29(8):976-981, June 1987. 53

[144] T. Lindstörm, L. Wågberg, and T. Larsson. On the nature of joint strength in paper - A review of dry and wet strength resins in paper manufacturing. In I'Anson S. J., editor, Advances in paper science and technology: transactions of the 13th Fundamental Research Symposium, volume 1, pages 457-562. Pulp and Paper Fundamental Research Society, Bury, UK, 2005. 28

[145] A. A. Lossada, J. C. Formento, and Pino A. L. Refining selected actions on chemical pulps in a PFI beater. Part I : Effects of radial compression stresses. Paperi ja Puu, 83(4):326-331, 2001. 18

[146] J. Lumiainen. Refining of chemical pulp. In J. Gullichsen and H. Paulapuro, editors, Papermaking: Stock preparation and wet end, volume 8 of Papermaking Science and Technology, pages 87-122. Fapet Oy, Jyväskylä, Finland, January 2000. 25

[147] S. O. Lundqvist. Application of SilviScan for optimal utilization of forests in Sweden. In Presentation at the Marcus Wallenberg Prize Seminar, National museum. Stockholm, October 2001. 14

[148] G. Lunn. Chromatography, liquid. In Kirk-Othmer Encyclopedia of Chemical Technology, volume 6, pages 440-468. John Wiley \& Sons, Inc., 5 edition, 2004. 20

[149] L. M. Lyne and W. Gallay. Measuement of wet strength. Tappi, 37(12):694-698, March 1954. 27

[150] T. C. Maloney. On the pore structure and dewatering properties of the pulp fiber cell wall. Doctoral thesis, Helsinki University of Technology, Espoo, May 2000. 13, 20, 33

[151] T. C. Maloney, T. Q. Li, U. Weise, and H. Paulapuro. Intra- and inter-fibre pore closure in wet pressing. APPITA Journal, 50(4):301-306, July 1997. 33 
[152] T. C. Maloney and H. Paulapuro. Thermoporosimetry of pulp fibers. In The Science of Papermaking Transactions of the 12th Fundamental Research Symposium, pages 897-926, September 2001. 169

[153] T. C. Maloney, A. Todorovic, and H. Paulapuro. The effect of fiber swelling on press dewatering. Nordic Pulp E Paper Research Journal, 13(4):285-291, 1998. 33, 167, 168

[154] L. F. Martin, F. A. Blouin, and S. P. Rowland. Characterization of the internal pore structures of cotton and chemically modified cottons by gel permeation. Separation Science, 6(2):287-296, 1971. 21

[155] L. F. Martin and S. P. Rowland. Gel permeation properties of cellulose. I. Preliminary comparison of unmodified and crosslinked, decrystallized cotton cellulose. Journal of Polymer Science Part A-1: Polymer Chemistry, 5(10):2563-2578, 1967. 21

[156] R. Marton and S. D. McGover. Relation of crystallite dimensions of fibrillar orientation to fiber properties. pages 153-158, New York, 1970. Tappi. 8

[157] M. Matsuo, C. Sawatari, Y. Iwai, and F. Ozaki. Effect of orientation distribution and crystallinity on the measurement by X-ray diffraction of the crystal lattice moduli of cellulose i and ii. Macromolecules, 23(13):3266$3275,1990.23$

[158] J. W. Mc Bain. Die Hauptprinzipien der Kolloidchemie. KolloidZeitschrift, 40(1):1-9, September 1926. 20

[159] B. A. Meylan and B. G. Butterfield. Three-Dimensional Structure of Wood: A Scanning Electron Microscope Study. Syracuse Wood Science Series. Syracuse University Press, 1972. 6, 174, 175

[160] J. L. Minor. Hornification - its origin and meaning. Progrress in Paper Recycling, 3(2):93-95, February 1994. 33, 122

[161] U. B. Mohlin and C. Alfredsson. Fibre deformation and its implications in pulp characterization. Nordic Pulp and Paper Research Journal, 5:172179, December 1990. 41

[162] U. B. Mohlin, J. Dahlbom, and J. Hornatowska. Fiber deformation and sheet strength. Tappi Journal, 79(6):105-111, June 1996. 11 
[163] CBC News. N.B. fossils show origins of wood. http://www.cbc.ca/news/ canada/new-brunswick/story/2011/08/12/nb-origins-of-wood-found. html, August 2011. [Online; accessed 16-March-2012]. 4

[164] N. Nilsen, M. Zabihian, and K. Niskanen. KCL-PAKKA: A tool for simulating paper properties. Tappi Journal, 18(5):163-166, May 1998. 115, 204

[165] T. Nishino, K. Takano, and K. Nakamae. Elastic modulus of the crystalline regions of cellulose polymorphs. Journal of Polymer Science Part B: Polymer Physics, 33(11):1647-1651, 1995. 23

[166] K. Nyholm, P. Ander, S. Bardage, and G. Daniel. Dislocations in pulp fibres - their origin, characteristics and importance - A review. Nordic Pulp E Paper Research Journal, 16(4):376-384, 2001. 22

[167] Food \& Agriculture Organization. State of the World's Forests 2011. Food \& Agriculture Organization of the United Nations (FAO), Rome, Italy, February 2011. 3, 4, 31

[168] C. Ottestam, P. Engstrand, M. Htun, B. Sjögren, and K. Ölander. Modified wrv method for measuring the swelling properties of mechanical pulps. In 1991 International Mechanical Pulping Conference Proceedings, pages 87-89. Tappi Press Atlanta, Ga, June 1991. 18

[169] L. Paavilainen. The possibility of fractionating softwood sulfate pulp according to cell wall thickness. APPITA Journal, 45(5):319-326, 1992.7

[170] D. H. Page. A theory for the tensile strength of paper. Tappi, 52(4):674681, 1969. 121

[171] D. H. Page. The beating of chemical pulps - The action and the effects. In 9th Fundamental Research Symposium on Fundamentals of paper-making, volume 1 of Fundamentals of paper-making, pages 1-37. Mechanical Engineering Publications Limited, Bury Saint Edmunds and London, Cambridge, England, 1989. 25, 56

[172] D. H. Page. The mechanism of strength development of dried pulps by beating. Svensk Papperstidning, 88(3):R30-R35, February 1989. 23, 110, 201 
[173] D. H. Page. A quantitative theory of the strength of wet webs. Journal of Pulp and Paper Science, 19(4):J175-J176, July 1993. 27

[174] D. H. Page, F. El-Hosseiny, K. Winkler, and R. Bain. The mechanical properties of single wood-pulp fibres. Part1: A new approach. Pulp $\mathcal{E}$ Paper Magazine of Canada, 73(8):72-76, 1972. 22, 200

[175] D. H. Page, F. Elhosseiny, K. Winkler, and A. P. S. Lancaster. Elastic modulus of single wood pulp fibers. Tappi, 60(4):114-117, April 1977. 23, 24

[176] D. H. Page, R. S. Seth, B. D. Jordan, and M. C. Barbe. Curl, crimps, kinks and microcompressions in pulp fibres their origin, measurement and significance. In Paper making raw materials: their interactions with the production process and their effect on paper properties. Transactions of the 8th Fundamental Research Symposium, volume 1, pages 183-227. Mechanical Engineering Publications Limited, Oxford, London, 1985. 16, 24

[177] A. J. Panshin and C. DeZeeuw. Textbook of Wood Technology: Structure, Identification, Properties, and Uses of the Commercial Woods of the United States and Canada. McGraw-Hill Inc., USA, 4th revised edition, May 1980. 6,9

[178] R. Passas, G. Eymin, C. Voillot, G. Tarrajat, G. Caucal, and B. Khelifi. MorFi: analyseur morphologique des fibres. Récents Progrès en Génie des Procédés, 15(78):259-264, 2001. 37

[179] R. Patt, O. Kordsachia, and R. Süttinger. Pulp. In Ullmann's Encyclopedia of Industrial Chemistry, volume 30, pages 477-540. Wiley-VCH, 6 edition, 2002. 10

[180] J. Porath and P. Flodin. Gel filtration: a method for desalting and group separation. Nature, 183(4676):1657-1659, June 1959. 20

[181] B. R. Porter and M. L. Rollins. Changes in porosity of treated lint cotton fibers. I. Purification and swelling treatments. Journal of Applied Polymer Science, 16(1):217-236, January 1972. 20

[182] I. Pulkkinen and V. Alopaeus. The effect of fiber dimensions on fiber network activation and tensile strength. Holzforschung, 66(1):111-117, August 2012. 30, 129, 209 
[183] E. Retulainen, K. Niskanen, and N. Nilsen. Fibers and bonds. In Paper Physics, volume 16 of Papermaking Science and Technology, pages -. Fapet Oy, Jyväskylä, Finland, January 1998. 26, 29

[184] R. P. Rittinger. Lehrbuch der Aufbereitungskunde. Verlag von Ernst \& Korn, Berlin, Germany, 1867. 25

[185] A. A. Robertson. Some observations on the effects of drying papermaking fibres. Pulp and Paper Magazine, 3(65):T161-T168, 1964. 33

[186] W. Robinson. The microscopical features of mechanical strains in timber and the bearing of these on the structure of the cell-wall in plants, volume 210. Printed and published for the Royal society by Harrison and sons, 1920. 21,22

[187] J. C. Roux, G. Joris, and G. Caucal. Raffinage séparé ou en mélange, quels outils à mettre en œuvre pour optimiser la composition fibreuse, réduire la consommation énergétique pour la régularité de la qualité. Revue A.T.I.P., 56(3):9-16, 2002. 46

[188] J. C. Roux, G. Joris, and G. Caucal. Optimising interactivity of chemicals and processes in the wet end. In 4th major 2 day PIRA International Conference and exhibition plus industry breifing: Starch technology in papermaking, Radisson SAS Hotel, Nice, France, May 2004. PIRA International. 13, 46

[189] A. W. Rudie, J. Morra, J. M. St. Laurent, and K. L. Hickey. The influence of wood and fiber properties on mechanical pulping. Tappi Journal, 77(6):86-90, June 1994. 7

[190] G. Ruffini. Improvement of bonding of wood pulps by the presence of acidic groups. Svensk Papperstidning, 69(3):72-76, 1966. 33

[191] U. Sahlberg, Lennart Salmén, and A. Oscarsson. The fibrillar orientation in the S2-layer of wood fibres as determined by X-ray diffraction analysis. Wood Science and Technology, 31(2):77-86, April 1997. 8

[192] I. Sakurada, Y. Nukushina, and T. Ito. Experimental determination of the elastic modulus of crystalline regions in oriented polymers. Journal of Polymer Science, 57(165):651-660, 1962. 23 
[193] L. Salmén. Structure and properties of fibres. In Pulp and Paper Chemistry and Technology: Paper Chemistry and Technology, volume 3, pages 15-22. Walter de Gruyter \& Co, August 2009. 9

[194] L. Salmén. Structure and properties of fibres. In M. Ek, G. Gellerstedt, and G. Henriksson, editors, Pulp and Paper Chemistry and Technology: Paper Chemistry and Technology, volume 3, pages 29-30. Walter de Gruyter \& Co, August 2009. 24

[195] L. Salmén and Berthold. Effects of mechanical and chemical treatments on the pore-size distribution in wood pulps examined by inverse sizeexclusion chromatography. Journal of Pulp and Paper Science, 23(6):J245J253, 1997. 33

[196] E. J. Samuelsen, P. J. Houen, Ø. W. Gregersen, T. Helle, and C. Raven. Three-dimensional imaging of paper by use of synchrotron X-ray microtomography. Journal of Pulp and Paper Science, 27(2):50-53, February 2001. 115, 204

[197] A. M. Scallan. The structure of the cell wall of wood - A consequence of anisotropic inter-microfibrillar bonding. Wood Science, 6(3):266-271, January 1974. 11, 12

[198] A. M. Scallan and J. E. Stone. The effect of component removal upon the porous structure of the cell wall of wood. II. Swelling in water and the fiber saturation point. Tappi, 50(10):496-501, October 1967. 13, 18

[199] A. M. Scallan and A. C. Tigerström. Swelling and elasticity of the cell walls of pulp fibres. Journal of Pulp and Paper Science, 18(5):188-193, September 1992. 25, 33, 110

[200] R. S. Seth. The effect of fiber length and coarseness on the tensile strength of wet webs: a statistical geometry explanation. Tappi Journal, 99(3):99102, March 1995. 11, 27

[201] R. S. Seth. The importance of fibre straightness for pulp strength. Pulp E Paper Canada, 107(1):34-42, 2006. 29

[202] R. S. Seth and D. H. Page. The mechanism of the strength and extensibility of wet webs. Paperi Ja Puu-Paper and Timber, 65(12):797, 1983. 27 
[203] H. Sihtola, B. Kyrklund, L. Laamanen, and I. Palenius. Comparison and conversion of viscosity and DP-Values determined by different methods. Paperi ja Puu, 4(a)(45):225-232, 1963. 72

[204] J. Silvy, G. Romantier, and R. Chiodi. Méthodes pratiques de côntrole du raffinage. Revue ATIP, 22(1):31-53, 1968. 49

[205] J. Silvy, G. Sarret, and F. Jestin. Evaluation des fractions d'eau de rétention extrafibres et intra-fibres dans la mesure du W.R.V. Application au Contrôle du Raffinage des pâtes á papier. In Atti del Congresso Europeo di Tecnica Cartaria, pages 169-185, Venice, Italy, September 1964. EUCEPA Gruppo di Studi Europeo TAPPI. 18

[206] Eero Sjöström. Wood Chemistry: Fundamentals and Applications. Academic Press, 2 edition, 1993. 7

[207] American Chemical Society. In Chemical technology, volume 14 of 1-6, page 171. American Chemical Society, January 1984. 7

[208] R. M. Soszynski and R. J. Kerekes. Elastic interlocking of nylon fibers suspended in liquid. part 1. Nature of cohesion among fibers. Nordic Pulp E Paper Research Journal, 3(4):180-184, 1988. 11

[209] B. Steenberg. Beating process studied by fiber swelling. Svensk Papperstidning, 50(11B):155-163, 1947. 22

[210] J. E. Stone and A. M. Scallan. Influence of drying on the pore structure of the cell wall. In Consolidation of the paper web: transactions of the symposium held at Cambridge, pages 145-174. British Paper and Board Makers' Association, 1966. 33

[211] J. E. Stone and A. M. Scallan. A structural model for the cell wall of water swollen pulp fibres based on their accessibility to macromolecules. Cellulose Chemistry and Technology, (2):342-358, 1968. 11, 19, 33, 178

[212] J. E. Stone and A. M. Scallan. The effect of component removal upon the porous structure of the cell wall of wood. Part III. A comparison between the sulphite and kraft processes. Pulp E Paper Canada, (69):69-74, 1968. 11 
[213] J. E. Stone, A. M. Scallan, and B. Abrahamson. Influence of beating on cell wall swelling and internal fibrillation. Svensk Papperstidning, 19(10):687-694, 1968. 13, 58

[214] J. E. Stone, E. Treibere, and B. Abrahamson. Accessibility of regenerated cellulose to solute molecules of the molecular weight of 180 to $2 \times 10^{6}$. Tappi, 52(1):108-110, 1969. 20, 33

[215] R. Stratton and A. Colson. Fiber wall damage during bond failure. Nordic Pulp and Paper Research Journal, 8:245-257, June 1993. 89

[216] A. Šturcová, G. R. Davies, and S. J. Eichhorn. Elastic modulus and stresstransfer properties of tunicate cellulose whiskers. Biomacromolecules, 6(2):1055-1061, 2005. 23

[217] H. D. Tiemann. Effect of moisture upon the strength and stiffness of wood. volume 63-71 of Bulletin (United States. Forest Service), pages 114116. U.S. Department of Agriculture, Forest Service, 1906. 18

[218] M. S. Tswett. Physikalisch-chemische Studien über das chlorophyll. Die Adsorptionen. Berichte der Deutschen Botanischen Gesellschaft, (24):316323, 1906. 20

[219] J. Tyrvainen. Newsgrade TMP from three different norway spruce (Picea abies) wood assortments in mill-scale: Their different qualities could be used to make specific paper products. Pulp E Paper Canada, 98(10), October 1997.7

[220] D. M. Updegraff. Semimicro determination of cellulose in biological materials. Analytical Biochemistry, 32(3):420-424, December 1969. 9

[221] A. Vainio and H. Paulapuro. Interfiber bonding and fiber segment activation in paper. BioResources, 2(3):442-458, May 2007. 27

[222] J. A. Van den Akker, A. L. Lathrop, M. H. Voelker, and L. R. Dearth. Importance of fibre strength in paper. Tappi, 41(8):416-425, January 1958. 122

[223] J. A. Van den Akker, W. Å. Wink, and R. H. Van Eperen. Instrumentation studies. LXXXIX. Tearing strength of paper. III. Tearing resistance by the in-plane mode of tear. Tappi, 50(9):466-470, 1967. 131 
[224] C. P. Wade. Preparation of whole-fiber cotton gel-filtration chromatography columns. Journal of Chromatography A, 268(0):187-195, 1983. 21

[225] X. Wang. Improving the Papermaking Properties of Kraft Pulp by Controlling Hornification and Internal Fibrillation. Doctoral thesis, Helsinki University of Technology, Laboratory of Paper and Printing Technology, Espoo, Finland, September 2006. 169

[226] X. Wang and K. R. Braaten. Growth rings and spruce pulpwood sorting. Nordic Pulp E Paper Research Journal, 12(3):196-202, September 1997. 7

[227] A. B. Wardrop and H. E. Dadswell. The occurrence, structure and properties of certain cell wall deformations. Council For Scientific and Industrial Research, (221):14-40, 1947. 22, 177

[228] R. H. Waring and S. W. Running. Section I - Introduction to analysis of seasonal cycles of water, carbon, and minerals through forest stands. pages 17 -144. Academic Press, San Diego, 3 edition, 2007. 5

[229] G. Weingarten. Sulle superficie di discontinuità nella teoria della elasticità dei corpi solidi. Rendiconti Lincei, 10(5):57-60, 1901. 21

[230] U Weise. Hornification - mechanisms and terminology. Paperi Ja PuuPaper and Timber, 80(2):110-115, 1998. 98

[231] U. Weise, L. E. Hiltunen, and H. Paulapuro. Verhornung von Zellstoff und Maßnahmen zu ihrer Reversion. Das Papier, 52(10A):V14-V19, 1998. 33

[232] U. Weise and H. Paulapuro. Changes of fibre dimensions during drying. In 1995 International Paper Physics Conference, pages 121-124, Niagaraon-the-Lake, Canada, September 1995. Technical Section, Canadian Pulp \& Paper Association. 13

[233] U. Weise and H. Paulapuro. The effect of drying and re-wetting cycles on fibre swelling. In Fourth European Workshop on Lignocellulosics and Pulp, pages 389-394, Stresa, Italy, September 1996. 33

[234] M. Wennerblom, A. D. Frövi, A. M. Olsson, and L. Salmén. Short communication softening properties of earlywood and latewood of spruce. Nordic Pulp E Paper Research Journal, 11(4):279-280, December 1996. 7 
[235] U. Westermark, O. Lindbrandt, and I. Eriksson. Lignin distribution in spruce (picea abies) determined by mercurization with SEM-EDXA technique. Wood Science and Technology, 22(3):243-250, 1988. 9

[236] K. Wickholm. Structural Elements in Native Celluloses. Doctoral thesis, KTH, Pulp and Paper Technology, 2001. 7, 8

[237] K. Wickholm, P. T. Larsson, and T. Iversen. Assignment of non-crystalline forms in cellulose I by CP/MAS ${ }^{13} \mathrm{C}-\mathrm{NMR}$ spectroscopy. Carbohydrate Research, 312(3):123-129, November 1998. 7

[238] A. P. Wilkins. The nomenclature of cell wall deformations. Wood Science and Technology, 20:97-109, 1986. 22

[239] J. M. Willis, W. Q. Yean, and D. A. I. Goring. Molecular weights of lignosulphonate and carbohydrate leached from sulphite chemimechanical pulp. Journal of Wood Chemistry and Technology, 7(2):259-268, 1987. 13

[240] J. W. Wilson and R. W. Wellwood. In Intra-increment chemical properties of certain western Canadian coniferous species, W.A. Côte Jr (ed.). Syracuse University Press, New York, 1965. 7, 8

[241] P. Zugenmaier. Introduction. In T. E. Timell and R. Wimmer, editors, Crystalline Cellulose and Derivatives: Characterization and Structures, Springer Series in Wood Science, page 4. Springer Berlin Heidelberg, 2008. 72 


\section{Index}

3D fibre structure, 115

Cost analysis, 151

3D pore structure, 115

Cryo-FE-SEM, 13

Angiosperms, 4

CSF, 17

Annual ring, 5

Apparent pore volume, 20, 58

Atomic force microscope, 92

Axial shrinkage, 28

Basis weight, 113

Bound water, 167

Brightness, 134, 149

Broken ends, 17, 68

Broken fibre content, 42

Bulk, 137

Burst index, 130, 145

Calliper, 114

Campbell-effect, 27

Cantilever, 93

Capillary effect, 50

Cell wall thickeness, 33

Cell wall ultra-structure, 6

Cellulose, 9

Centrifugation, 50

Chromatographic system, 52

Cleavages per fibre, 22

Column, 51

Computer Tomography, 115

Deforestation rate, 3

Degree of polymerisation based on viscosity, 72

Delignification, 10, 13

Dewatering time, 47

Dextran, 55, 56

DFT 3000, 46

Dissipation energy, 94

DSC, 167

DT, 17

Dynamic mechanical analyser, 108

Earlywood, 5

Elongation, 127

environmental impacts, 151

ESRF, 116

Fibre curl, 16, 64

Fibre kink, 16

Fibre length, 15, 39

Fibre segment activation, 28

Fibre width, 16, 40, 63, 105

Fibrillation, 17, 45, 48, 68

Fines, 17, 36, 48-50

Forests, 2 
Fractionator, 36, 37

FSP, 18, 55

Fuel-wood, 3

GFC, 20, 54, 55

Giertz effect, 29

GPC, 20

Gymnosperms, 4

Handsheet former, 113

Hardwoods, 4

$\mathrm{HCl}, 22$

Heartwood, 5

Hemicellulose, 9

Hornification, 33, 100

ISEC, 20

Jentzen effect, 29

Kappa number, 72

Lactones, 33

Lateral shrinkage, 28

Latewood, 5

Latwood, 68

Lignin, 9, 13, 72

Liquid chromatography, 20

Lumen, 6

MHS equation, 72

Microfibril angle, 89

Microfibrils, 7

MicroFibrils Angle, 8

Microtomography, 115

Morphological analysis, 37, 73

Network activation coefficient, 129, 130

Non-wood, 10

Opacity, 135, 149
P/S1 peeling, 92

$\mathrm{P} / \mathrm{S} 1$ stitching, 91

Page theory, 121

PeakForce QNM, 93

Pentosans, 33, 72

PFI, 35, 48, 55, 62

Pore closure, 33

Pore size distribution, 20, 58

Porosimetry, 58

RCF, 18, 50

Recycling rate, 32

Refining, 25, 61

Refining effect, 25, 60

Refractive index, 19, 53

RI detector, 53

RIA, 53, 54

Round-wood, 3

Sapwood, 5

Scattering coefficient, 136, 150

SEC, 20

Shear-lag model, 122

Shrinkability, 102

Single fibre tensile strength, 108

Softwoods, 4

Solute exclusion, 13, 55

Sparging, 51

Specific filtration resistance, 48,49

SR, 17

Strength loss, 33

TEA, 128

Tear index, 131, 147

Tensile index, 126, 130, 139

Thermoporosimetry, 167

Tracheids, 6

Valley beater, 36, 49, 71 
Viscometer, 72

Viscosity, 72

Viscosity radius, 56

Waste papers, 30

WBL , 59-61, 69

Weak points, 22, 69

Wet zero-span breaking length, 124, 138

Wood, 4

Wood structure, 4

WRV, 18, 49, 50, 69

X-rays Microtomography, 115

Young's modulus, 127, 128

Zero-span breaking length, 123, 138 
